$$
\text { HASL--300-Vol.1-28.ed. }
$$

HASL.30O

\title{
THE PROCEDURES MANUAL
}

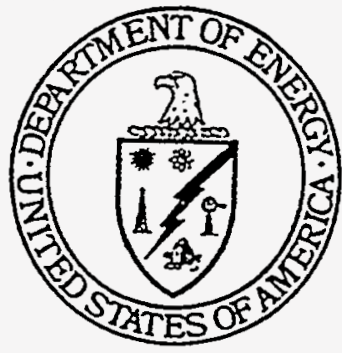
OF THE ENVIRONMENTAL MEASUREMENTS LABORATORY

\author{
Volume l, 28th Edition
}

February 1997

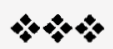

Mitchell D. Erickson, Director

PROCESSED FROM BEST AVALLABLE COPY

$\$$

Nancy A. Chieco, Editor

U. S. Department of Energy 201 Varick St., 5th Floor

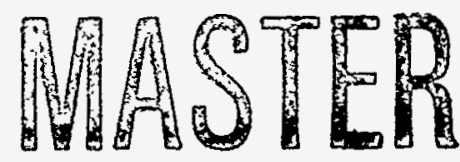
New York, NY 10014-4811 
This report supersedes: HASL-300, 27th edition issued November 1990, revised 1992; 26th edition issued 1983; 25th edition issued 1982; supplement 8 issued 1981; supplement 7 issued 1979; supplement 6 issued 1978; supplement 5 issued 1977; supplement 4 issued 1976; supplement 3 issued 1972 (revised 1975). NYO-4700 issued 1957 (revised 1960, 1962); supplements 1 and 2 issued 1963.

\section{DISCLAIMER}

"This report was prepared as an account of work sponsored by an agency of the United States Government. Neither the United States Government nor any agency thereof, nor any of their employees, makes any warranty, express or implied, or assumes any legal liability or responsibility for the accuracy, completeness, or usefulness of any information, apparatus, product, or process disclosed, or represents that its use would not infringe privately owned rights. Reference herein to any specific commercial product, process, or service by trade name, trademark, manufacturer, or otherwise, does not necessarily constitute or imply its endorsement, recommendation, or favoring by the United States Government or any agency thereof. The views and opinions of authors expressed herein do not necessarily state or reflect those of the United States Government or any agency thereof."

This report has been reproduced directly from the best available copy.

Available to DOE and DOE Contractors from the Office of Scientific and Technical Information, P.O. Box 62, Oak Ridge, TN 37831; prices available from (423) 576-8401.

Available to the public from the U.S. Department of Commerce, Technology Administration, National Technical Information Service, 5285 Port Royal Road, Springfield, Virginia 22161, (703) 487-4650. 


\section{DISCLAIMIER}

Portions of this document may be illegible in electronic image products. Images are produced from the best available original docoment. 


\section{Preface}

This 28th edition supersedes all previous versions. The Manual was originally issued in 1957 as a U. S. Atomic Energy Commission Report (NYO-4700). In 1972, substantive changes were made, and the designation became HASL-300 (Health and Safety Laboratory). When the Laboratory's name was changed in 1977 to the Environmental Measurements Laboratory (EML), the Manual was retitled to the EML Procedures Manual (Supplement 6, issued 1978). In subsequent years, parts of HASL-300 were updated as necessary.

Volume I of this edition covers existing technology and procedures currently in use at EML. Some older procedures have been updated and new procedures have been added. Procedures no longer in use at EML, but still valid and used at other laboratories, appear in Volume II.

The Manual includes contributions from most of the EML scientific staff. We welcome any questions, corrections or information you may have concerning this publication. Points of contact are listed in the text. Additional information and updates are also available on EML's Web Site (www.eml.doe.gov). The special contributions of Sylvia Kendall for desktop publishing support and of Jenny May-Maiello for graphic design are also acknowledged. 


\section{QUALITY ASSURANCE}

U.S. Department of Energy 201 Varick Street, 5th Floor New York, NY 10014-4811 


\section{Quality Assurance}

1.1 Overview

1.2 Scope $1-2$

1.3 Definitions $1-2$

1.5.1 Introduction

1.5.2 Responsibilities of Field Workers and Laboratory Workers ................................ 1-4

1.5.3 Responsibilities of First Line Supervisors ............. 1-4

1.5.4 Responsibilities of Individual Project Leaders ............ 1-5

1.5.5 Responsibilities of Division Directors and Program

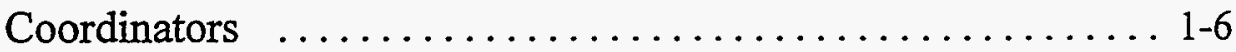

1.5.6 Responsibilities of the QA Officer $\ldots \ldots \ldots \ldots \ldots \ldots \ldots \ldots \ldots \ldots \ldots \ldots \ldots \ldots \ldots$

\subsection{Field Measurements and Sampling $\ldots \ldots \ldots \ldots \ldots \ldots$ 1-8}

1.6.1 Introduction $\ldots \ldots \ldots \ldots \ldots \ldots \ldots \ldots \ldots \ldots \ldots \ldots \ldots \ldots \ldots \ldots \ldots \ldots \ldots, 8$

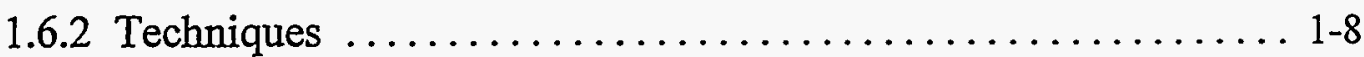

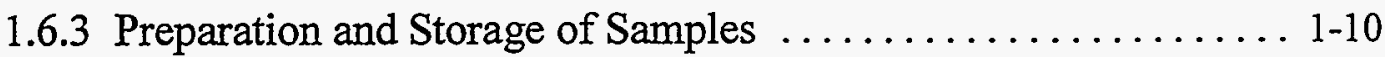



1.7 Chemical and Radiochemical Analyses ........... 1-12

1.7.1 Introduction $\ldots \ldots \ldots \ldots \ldots \ldots \ldots \ldots \ldots \ldots \ldots \ldots \ldots \ldots \ldots \ldots \ldots, 1-12$ 




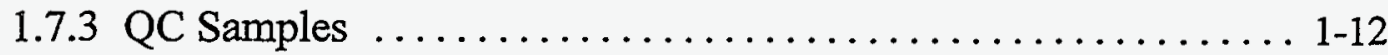

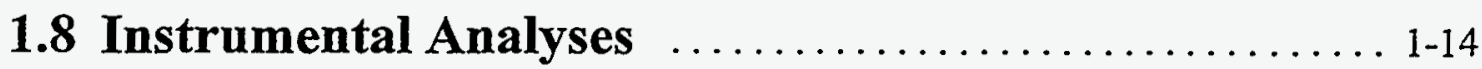

1.8.1 Introduction $\ldots \ldots \ldots \ldots \ldots \ldots \ldots \ldots \ldots \ldots \ldots \ldots \ldots \ldots \ldots \ldots \ldots .1-14$

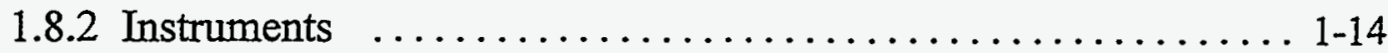

1.8.3 Calibrations .................................. 1-15

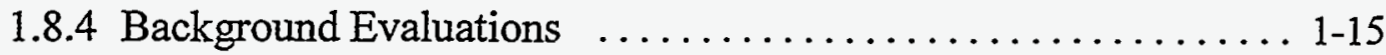

1.8.5 Checks of the Stability of the Instrument $\ldots \ldots \ldots \ldots \ldots \ldots .1-16$

1.9 Data Reduction, Storage, and Reporting $\ldots \ldots \ldots \ldots 1-16$

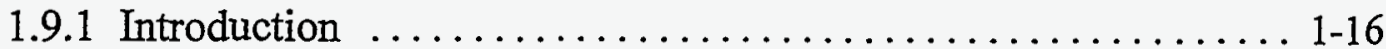

1.9.2 Field and Laboratory Records $\ldots \ldots \ldots \ldots \ldots \ldots \ldots \ldots \ldots . \ldots \ldots$

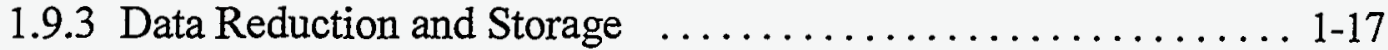

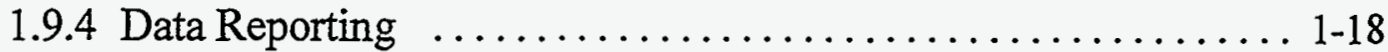

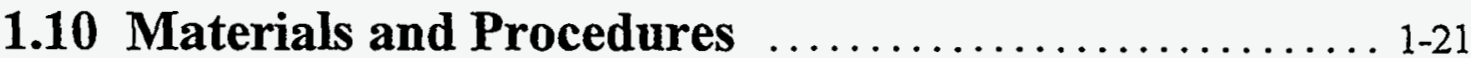

1.10.1 Introduction $\ldots \ldots \ldots \ldots \ldots \ldots \ldots \ldots \ldots \ldots \ldots \ldots \ldots \ldots \ldots, 1-21$

1.10.2 Standards and Reference Materials Library $\ldots \ldots \ldots \ldots \ldots . . .1-21$

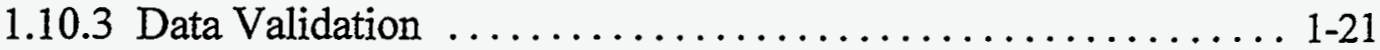

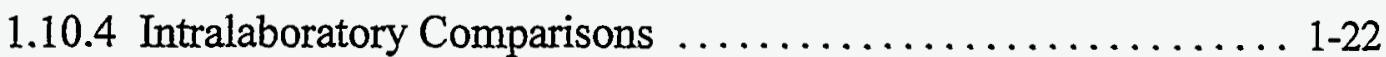

1.10 .5 Interlaboratory Comparisons $\ldots \ldots \ldots \ldots \ldots \ldots \ldots \ldots \ldots \ldots \ldots \ldots \ldots . .22$

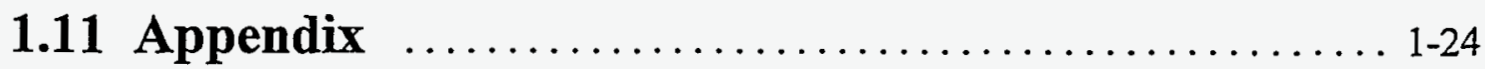




\section{QUALITY ASSURANCE}

\subsection{OVERVIEW}

The Management of the Environmental Measurements Laboratory (EML) is fully committed to the maintenance of an effective quality assurance (QA) program in order that all work carried out by EML will be of high quality. The EML Institutional Quality Assurance Program Plan (EM-6-2) describes the QA procedures used at the Laboratory. Guidelines for the design of the EML Institutional Quality Assurance Program Plan are provided for by: DOE Order 5700.6C, "Quality Assurance;" DOE-ER-STD-6001-92, "DOE Standard: Implementation Guide for Quality Assurance Programs for Basic and Applied Research;" and Chicago Operations Office Order CH 5700.6C, "Quality Assurance." The EML QA program has been designed to conform to those guidelines.

The management system of the QA program at EMI includes the entire staff, from the Laboratory Director, through the Deputy Director and the Division Directors to the project leaders and first line supervisors, and finally to the scientist or engineer in the laboratory or in the field. The QA Officer oversees the functioning of the Institutional QA Program (see Section 1.5).

An important aspect of the EML QA program is the written documentation of QA and quality control (QC) procedures that are used in the performance of research projects. A Project QA Plan is prepared for each project that is contained in a field work proposal, describing or referencing the procedures used during the project and the quality of results from these procedures that is required for the successful performance of the project. Twice each year, the Division Director or Program Coordinator who oversees a project submits to the EML QA Officer a brief evaluation of the current level of quality of performance on that project. The QA Officer selects a few of these projects and makes a detailed examination of the evidence of quality that was used to evaluate them. The documentation of the quality of the work is described in this section, and in subsequent sections of this Manual. Under normal circumstances, all research reports contain a section in which the QA procedures used and the results obtained are discussed. When they believe that it will be beneficial, Division Directors may require the submission of 
special QA reports for specific projects within their divisions. QA reports can be used during assessments of a project by the Division Director or by the QA Officer to assure that $\mathrm{QA}$ requirements are being met. For a $\mathrm{QA}$ program to be effective, corrective action must always be taken when substandard results are detected, and subsequent follow-up audits must be made to verify that any problems have been solved. The Division Directors oversee the planning, performance, and documentation of any needed remedial actions required as the result of an audit.

The most important requirement for the success of the QA program is the commitment in the Laboratory that our goal is to always perform high quality work. This requires both a degree of dedication of the staff and an absolute honesty in data preparation.

\subsection{SCOPE}

The policies, procedures, guidelines, and implementation practices at EML with regard to its QA program are presented in this section. Also, outlined are the responsibilities of EML personnel to ensure the quality of the data at all phases of the project from planning to the reporting of the data.

\subsection{DEFINITIONS}

Three definitions related to QA practices are given below. A more complete list may be found in Section 1.11.

QA involves all those planned and systematic actions necessary to provide adequate confidence that a facility, structure, system, or component will perform satisfactorily and safely in service.

QC, which is included within QA, comprises all those actions necessary to control and verify the features and characteristics of a material, process, product, or service to specified requirements. 
Audit/Appraisal is a planned and documented activity performed in accordance with procedures to determine, by examination and evaluation of objective evidence, the adequacy of and extent to which applicable elements of the QA program have been developed, documented, and effectively implemented in accordance with specified requirements.

\subsection{STAFF QUALIFICATIONS}

The first step in establishing a QA program is the securing of personnel who are competent to perform the technical procedures, and training them so that they are thoroughly familiar with the instruments and procedures that they will use. This is as important as are written QC procedures and record keeping. Technical personnel should understand the nature of the physical and chemical properties that affect the measurement procedures that they perform, so that they may recognize and interpret any deviations from the expected behavior. When technical personnel are to perform a procedure in which they are not experienced, they are first trained, then tested in order to demonstrate that they can perform the procedure with an acceptable level of uncertainty.

\subsection{STAFF RESPONSIBILITIES}

\subsubsection{INTRODUCTION}

All individuals and all levels of management at EML have responsibilities within the QA program. Although the Laboratory Director has overall supervision of and responsibility for the program, and the QA Officer oversees its functioning, the Deputy Director, Division Directors, first line supervisors, project leaders, field workers and laboratory workers are responsible for the quality of the work that is performed under their control. The specific responsibilities that are described below are applicable in most instances. However, each research project has its own individual characteristics, and Division Directors may reassign specific responsibilities within particular projects to attain the most efficient organization. 


\subsubsection{RESPONSIBMITIES OF FIELD WORKERS AND LABORATORY WORKERS}

The field workers and laboratory workers are responsible for the quality of their performance of assigned tasks. They must be thoroughly familiar with the QA aspects of the procedures that they follow. For the most part, these procedures are described in later sections of this Manual. All personnel must carefully adhere to those procedures, must report to their supervisors any problems that caused deviation from these procedures or results from standard procedures or expected results, and must undertake to correct identified problems. They are responsible for the prevention of the loss of quality during handling of the equipment and materials that they use in their work, and for the routine maintenance, calibration and the general care of their equipment. They maintain records of instrument calibrations and of the results of the QC procedures that they perform.

\subsubsection{RESPONSIBUITIES OF FIRST LINE SUPERVISORS}

First line supervisors ensure that the personnel under their supervision are aware of, and fulfill their responsibilities for the QA aspects of the tasks that they perform. They ensure that only authorized personnel perform the research operations, that proper care is taken of equipment and materials to avoid loss of quality, and that approved sampling and measurement procedures (most of which are described in Sections 2, 3, and 4) are used. First line supervisors are responsible for the specification and supervision of appropriate QA procedures to be performed by the personnel under their supervision, and they periodically review the procedures that are in use to ensure their adequacy for determining the quality of the results that will be obtained from current and future operations. They see that logbooks and other records, such as calibrations and the results of QC procedures, are maintained properly, that appropriate information is recorded, and that any changes that are made in documents that give instructions or describe procedures are reviewed and approved. They test the validity of measurement data that they report, and ensure that corrective actions are taken when problems arise, and that follow-up verification is obtained of the success of those actions.

First line supervisors ensure that measuring and test equipment and materials are traceable to national standards or to equivalent calibration standards, and that they are cared for properly by those who are using them. They implement documented QA 
procedures to ensure that the quality of items and services procured to accomplish tasks under their supervision meets appropriate QA criteria, and they ensure that documents needed to verify the quality of these items and services are retained in a recoverable form. They design and implement documented QA procedures to cover the calibration and continuing operation of such equipment and the confirmation of the suitability of such supplies. They ensure that defective equipment is not used until it has been repaired, and that records are kept of the repair of equipment.

\subsubsection{RESPONSIBUITIES OF INDIVIDUAL PROJECT LEADERS}

Project leaders must participate in the preparation of the Project QA Plan, in which are specified the level of quality required for each phase of their research projects, and the QC methods to be used to ensure that the required levels of quality are attained. They must continually evaluate the specified levels of uncertainty and the levels that are actually being attained, and they must maintain retrievable records that furnish evidence that shows whether the quality of data produced by their project conforms to the requirements of the project. Project leaders ensure that the field measurements, sample collection and handling, laboratory analysis, data analysis and reporting of results are performed properly to prevent the loss of the quality of the data. They must be actively involved in monitoring the quality of all aspects of the work that is being done on their projects, and they review and approve documents and changes in documents that describe the QA requirements of work done on these projects. They must seek to obtain corrective actions if these are needed, and must initiate and provide follow-up verification of any such corrective actions.

When equipment, supplies or services are purchased for use on their projects, project leaders must design and implement documented QA procedures to cover the calibration and operation of the equipment and the confirmation of the suitability of the supplies or services. Project leaders are responsible for determining that documents needed to verify the quality of such equipment, supplies or services are retained in a recoverable form. They must also continually monitor the quality of equipment, supplies and services that are actually in use on their projects. When contractors are hired to perform some aspects of a research project, the project leader must see that the contract documents include specifications of the quality of the work that must be done by the contractor, and must 
design and implement a documented QA program to cover the contractor's performance under the contract.

Project leaders have the overall responsibility for the quality of the final products from their projects, and especially for the quality of the data and interpretations published under their projects. They ensure that measurement data that they have received are validated and that inaccurate information is corrected before the research results are submitted for publication. They are responsible for the proper care and protection of measurement data that they have received. Project leaders normally prepare the progress reports and the final publications of project accomplishments and they must verify the accuracy of the published data, and must include a QA section within these reports. The project leader is responsible for the timely submission of $\mathrm{QA}$ reports if these are required for the project. The frequency and content of these reports are to be specified by the appropriate Division Director. Normally such reports should list the data from QC measurements, and should include a discussion of the adequacy of the QC results in light of the project requirements. This discussion should include a review of corrective measures taken and of the results obtained for any problems discussed in the previous report, and it should describe any corrective measures that are being taken for current problems. The project leader must see that retrievable records that furnish evidence of the quality of the work done on the project are specified, prepared and maintained, and must be sure that any and all documentation required for possible QA audits is available.

\subsubsection{RESPONSIBILITIES OF DIVISION DIRECTORS AND PROGRAM COORDINATORS}

Division Directors and Program Coordinators provide ongoing supervision of QA for work within their divisions and programs. They develop specific QA objectives, involving documented procedures, for the laboratory worker and field worker, communicate these objectives to the workers, and then ensure that these documented operating procedures and protocols are followed, and that the desired quality of work is maintained. They periodically review the QA procedures that are in use to ensure their adequacy for determining the quality of the results that will be obtained from current and future operations. Division Directors and Program Coordinators must approve any changes in documents that detail procedures that are used within their divisions. They also review the procedures used to select items and services procured for their divisions 
and programs, and they ensure that QA requirements for these items and services are being met and are properly documented.

The Division Directors and Program Coordinators have the ultimate responsibility for the quality of the products delivered by each of the projects within their divisions and programs, and must be actively involved in ensuring that the required quality of work is achieved in all aspects of these projects. They must be active in the review, criticism, and correction of the work of persons who are working on their projects. Twice a year, Division Directors and Program Coordinators supply the EML QA Officer with brief written evaluations of each project for which they are responsible. Division Directors and Program Coordinators must ensure that any QA reports that they require for a project are submitted on time and are complete. They oversee the procedures used in the peer review of reports originating in their division, and they ensure that these procedures are rigorous enough to detect and eliminate faulty data and interpretations. They ensure that all research reports undergo peer review within EML before publication in EML reports or submission to scientific journals. They encourage project leaders to submit reports of their research to peer-reviewed scientific journals whenever this is feasible. Division Directors and Program Coordinators perform QA reviews of projects under their supervision semiannually, and ensure that any needed corrective actions are taken.

\subsubsection{RESPONSIBUITIES OF THE QA OFFICER}

The EML QA Officer keeps informed about and investigates any problems that arise in the quality of the work that is performed at the Laboratory, and keeps the Laboratory Director informed. Twice a year the QA Officer receives brief evaluations from the Division Directors and Program Coordinators of the quality of work that is being performed on the projects that are under their supervision. The QA Officer selects a few of these projects for more detailed investigation, choosing some at random, others based upon their importance to the Laboratory, and others because of their quality history. The QA Officer maintains written records of the semiannual evaluations submitted by the Division Directors and Program Coordinators and of any subsequent investigations, as well as of any other QA appraisals or investigations performed at the Laboratory. The QA Officer also ensures that the EML Institutional Quality Assurance Program Plan is kept current. 


\subsection{FIELD MEASUREMENTS AND SAMPLING}

\subsubsection{INTRODUCTION}

Some measurements of environmental parameters, such as the intensity and composition of environmental radiation, are performed in the field, while others, such as the concentration of specific radionuclides in samples of soil or vegetation, are performed in the laboratory on samples that were collected in the field. In either situation the procedures used in the field work must be performed correctly if the measurements are to produce valid results. There are many potential pitfalls involved in this initial step, and precautions must be taken to avoid them to ensure the validity of the final data. Before sampling is begun, all project personnel should be informed of the procedures to be used, so that it is certain that the material and the amount of material to be sampled or the property to be measured are appropriate at all phases of the project.

\subsubsection{TECHNIQUES}

A field measurement or a collected sample must be representative of the parameter or material that is to be analyzed. However, obtaining a representative measurement or sample of environmental parameters or materials is often not straightforward. Most environmental parameters and materials vary with location and time. For example, cosmic-ray intensities vary with time, and various organs of plants and animals differ in their contents of organic and inorganic constituents. Soil typically contains particles of various sizes, and the chemical composition and surface reactivity of these particles often vary as a function of particle size. Water bodies, such as lakes and the ocean, are commonly stratified, with variations in physical and chemical compositions from one layer to another, and usually have a surface film in which many reactive chemical constituents are strongly concentrated. The atmosphere also is stratified, and air parcels differ from place to place in their concentrations of trace gases and suspended particles. Thus, even at a single site, the composition of air will vary with time, and especially as the wind shifts. The intensity of radiation from radionuclides within the soil varies from location to location. When possible, statistical tests should be applied to determine the number of observations or samples that are needed to achieve the required level of uncertainty. 
Before measurements of environmental radiation are begun, the appropriateness of the measurement location must be ascertained. If radiation from the soil is to be measured, the representativeness of the soil at the measurement location must be checked. It must be verified that there are no objects in the vicinity of the radiation detector that can affect the readings that the detector gives.

If samples are to be collected, before sampling is begun the researcher must define clearly the material that is to be sampled to be certain that the sample will be representative of that material. If vegetation is to be sampled, will the sample include the roots of the plant, and if so, must all soil that is clinging to the roots be meticulously removed? If a sample of the atmospheric aerosol that is representative of air over a large region is to be collected, does wind direction matter, at what height above ground is the sample to be taken, and is there danger that nearby structures or activities will affect the composition of the sample? The sample should be representative of the complete material, but it must not be contaminated by extraneous materials. The sampling process itself can affect the validity of the sample. Thus, a rain gauge may distort the flow of winds in its vicinity and cause less rain to fall into its opening than would fall onto the same area of open soil. A water sampler lowered into a lake may carry water from a near surface layer into a lower layer that is being sampled. Therefore, the details of sampling and of sample handling must be considered carefully before sampling is performed in order that the entire research effort not be jeopardized by the careless omission of some needed safeguard.

Because the intensity of environmental radiation and the composition and physical characteristics of environmental materials often vary with location over short distances, and may vary significantly with time, it is usually advisable to do replicate measurements or sampling. The analysis of the data from such measurements or samples can provide a measure of the random error of the entire operation. When a material that is being sampled is clearly heterogeneous, as are many soils and biological materials, replicate samples should be taken. When the intensity of radiation or the composition of the material is expected to change significantly with time, it is usually advisable to take a series of measurements or samples over the period of interest. This replicate or serial measurement or sampling can yield a more useful picture of the characteristics of interest than would data for a single measurement or sample. The measurement or sampling interval and frequency are often dictated by the research objectives of the project. If portions of samples are to be retained in storage, this should be considered in planning how much material is to be collected, and the treatment it is to receive. 


\subsubsection{PREPARATION AND STORAGE OF SAMPLES}

Many types of measurements of environmental materials, such as vegetation, water, and atmospheric particles, require that a sample of the material be collected and returned to the laboratory for analysis. These samples may require some form of pretreatment before they can be analyzed. For example, vegetation samples may be dried or ashed, and water samples may be filtered. Where possible, groups of samples that are expected to contain high concentrations of an analyte are processed independently from those with low concentrations to minimize the possibility of cross contamination. Pretreatment normally alters the physical state of the sample, and also sometimes alters its chemical state. In planning the pretreatment, the researcher considers the nature of the measurement that is to be made and the state that the sample must be in to undergo that measurement. The possibility should also be considered that the results obtained from the sample might lead to a desire to reanalyze it for the same property, or to analyze it for some other property. Hence, it will often be desirable to collect more sample than will be processed, and to process more sample than will be used in the analysis (if the analytical process is such as to consume the sample).

Another consideration in planning and in carrying out the pretreatment of samples is the reference state of the samples that will be used in reporting the results of the measurements. For example, samples of vegetation, of soil or of sediment are usually weighed before being analyzed, and the results are reported with reference to unit weight and/or the unit area from which the samples were collected. Normally these types of samples are weighed directly after collection (wet weight), dried and weighed again (dry weight) before they are analyzed. Under this protocol, the researcher must ensure that all samples are dried to the same extent to report results per unit dry weight. But this restriction is lifted if results are reported per unit weight or per unit area of sample collection.

Decisions concerning the pretreatment process to be used can be complicated if the process is such that it may alter the property of the sample that is to be measured. For example, if a volatile component, such as iodine, is to be measured in vegetation, the pretreatment of the vegetation sample must not be such as to volatilize that component. 
Project leaders are responsible for their own samples until the project is complete. Upon completion of a project, any portions of samples that are to be saved should be entered into and stored in an archival system.

\subsubsection{CODING AND RECORD KEEPING}

At the time of sample collection or of field measurement, samples and resulting data sets are normally given code numbers that serve to identify them during subsequent analytical, calculation and data reporting stages. Sometimes a coding system separate from that used for field measurements or sample collection is used for the analysis stage, either for the convenience of the analysts or to aid in ensuring the "blindness" of the QC samples or measurements. A number of factors enter into the designing of these codes. There is always an advantage in keeping the codes as simple as possible to minimize the probability that errors will be made in transferring data. However, the code should be distinctive enough to distinguish each set of samples and data from other, unrelated samples and data that pass through the laboratory. Care must be taken to mark samples and field data clearly to minimize the possibility of misreading of labels and notes. Especially during the sampling or field measurement phase, it is often desirable to use codes that contain information on the site and/or time of sampling or measurement, or upon the nature of the sample or measurement.

In designing protocols for recording field measurements or sampling and subsequent analytical data, the researcher must consider whether the recorded data will be sufficiently complete for the possible future uses that may be made of the final analytical results. Normally, much more detailed information is available during the field measurement or sampling and analytical phases than is expected to be of importance for the interpretation of the results, and only the information that appears to be immediately useful is recorded. At times, as the final data have been studied, it has become evident that some of the unrecorded information might have been useful in interpreting those results. To avoid the possible loss of useful information, the researcher should make a habit of keeping detailed field and laboratory notes, either in a bound notebook or another recoverable medium. Researchers should think through as many possibilities as they can based on their experience before designing the systems and codes of data recording. 


\subsection{CHEMICAL AND RADIOCHEMICAL ANALYSES}

\subsubsection{INTRODUCTION}

The EML QA program for chemical and radiochemical analyses has long been welldefined and carefully followed. QC measures are an integral part of each analytical procedure, and quantitative estimates of analytical uncertainties are made and reported routinely.

\subsubsection{APPROVED PROCEDURES}

There are often several chemical or radiochemical techniques that may be used to accomplish a particular analysis. In most instances, past experience at EML or amongst the scientific community generally has shown that one of these techniques is to be preferred to the others, at least for a particular type of sample and with the concentration of the constituent of interest falling within a specific range. In these instances, the particular analytical procedure to be used is specified in Section 4 of this Manual. The details of these procedures are documented in those sections, and they are updated periodically. At times it may be necessary for the analyst to modify a procedure when working with a particular set of samples. Such modifications must be noted so that the exact procedures that were used can be identified if questions arise at a later time. In order to have a written record of all pertinent information relating to the analysis of all samples, analysts should record their daily progress or results in a laboratory notebook or other recoverable medium.

\subsubsection{QC SAMPLES}

Whenever possible, the project leader, as part of the external QC program, should submit QC samples to the analyst along with routine samples in such a way that the analyst does not know which of the samples are the QC samples. These external QC samples, which usually include duplicate and blank samples, should test sample collection and preparation as well as sample analysis whenever this is possible. In addition, analysts are expected to run internal QC samples that will indicate to them 
whether the analytical procedures are in control. Both the external and internal QC samples should be prepared in such a way as to duplicate the chemical matrix of the routine samples, insofar as this is practical. The QC samples that are routinely used consist of five basic types: blank samples, replicate samples, reference materials, control samples and "spiked" samples. Special definitions of these terms related to QA are defined in the Appendix.

Blank samples are analyzed to give a measure of any contamination of the sample that is occurring during the course of the collection, preparation or analysis. The analyst commonly introduces blank samples into the sample stream. Often these are "reagent blanks" that are prepared by starting with deionized water or with an empty sample container and going through all of the normal procedures involved in the analysis; i.e., adding all of the reagents at the proper points. Whenever possible, the matrix of blank samples should be the same as that of the samples being analyzed. The data for the routine samples are usually corrected by subtracting from their measured values the value of the blank. It must be remembered, however, that blank measurements of only the analytical processes cannot be used to evaluate contamination that occurs during the collection and preparation of the sample.

Replicate samples are obtained sometimes by repeating the collection as well as the analysis of samples, but often only replicate aliquots of the same laboratory sample are analyzed. Repeated sampling of a heterogeneous solid environmental material, such as soil, may not yield truly replicate samples. If such materials are taken into solution before being measured, replicate subsamples of the solution are often analyzed. Commonly, individual samples are measured more than once, and for nondestructive techniques, such as gamma-ray spectroscopy on whole samples, replicate measurements may involve exactly the same sample, renumbered and resubmitted for analysis.

The third type of QC sample, the reference material or standard reference material, is used primarily to calibrate the measurement method or apparatus.

The concentration of the control sample, or check standard, has an overall analytical uncertainty that is known well enough for this control sample to be used in place of a standard reference material to insure that the measurement method is in control. Such control samples should have the same matrix and the same range of concentrations as do the routine samples when this is possible. 
Spiked samples, the fifth type of QC sample, are prepared by adding a known amount of the constituent of interest to blank samples or to samples that have already been analyzed, to provide samples with known concentrations. They may be used to estimate chemical yields of analytical processes. Where the presence of other constituents in a sample may be affecting the response of an instrument to the constituent of interest, multiple spiking may be done. In this process, a series of samples are spiked by the addition of increasing amounts of the constituent of interest. The measured values are inspected to determine whether the instrument yielded a linear response to the increasing concentrations, and whether the rate of increase of the response was that expected from the differences in concentration between samples.

\subsection{INSTRUMENTAL ANALYSES}

\subsubsection{INTRODUCTION}

Virtually all measurements performed at EML require the use of an electronic instrument to provide quantitative data. This is true of measurements of radiation, of radioactive materials and of non-nuclear measurements. Thus, it is of crucial importance to the quality of the work done at EML that all analysts be thoroughly familiar with the proper procedures to be followed in calibrating, operating and caring for the instruments that they must use. Usually this requires that the analyst understand at least the general scientific principles that are the basis of the measurement.

\subsubsection{INSTRUMENTS}

To maximize the quality of the research data produced at EML, every effort is made to keep abreast of the state of the art in instrumentation used in environmental research and, where it is financially possible, to obtain the best available instruments. To keep instruments performing efficiently, a schedule of preventive maintenance is followed where appropriate. A record of instrument performance is maintained, and any modifications made in the instruments, whether permanently or for a particular project, are 
documented. Any such modifications in any instrument must conform to the safety standards and practices that are specified in the EML Safety Manual.

\subsubsection{CALIBRATIONS}

Provisions have been made for the periodic, quantitative assessment of the performance of most instruments used by EML. For many instruments, calibration standards are available. These standards are measured to obtain a curve that relates the intensity of the signal from the instrument to the concentration of the substance or the intensity of the property being measured. In other instances, this calibration consists of a one point check using a single standard reference instrument, source, material or sample. For the quality of the measurements to be optimized, the analyst must use the appropriate standards, calibration procedures and frequency of calibration, and must keep a record of the traceability of the standardization.

\subsubsection{BACKGROUND EVALUATIONS}

Commonly, instruments will provide a background signal that may be the result of minor fluctuations in an electrical field. Also, phenomena or materials that produce effects that are similar to the effects produced by the parameter or substance of interest may occur in the environment of the detector and may produce a signal from the detector. It is then necessary to measure this background signal so that the data from routine measurements may be corrected for its presence. Even when the signal from the measured parameter or samples is high relative to the background and the background correction is small, the background signal should be measured regularly to be sure that it has not changed and that the instrument has not become defective or contaminated. The analyst must be sure that the procedures that are in use to measure background are adequate in nature and are performed with the needed frequency. A record must be kept of the measured backgrounds and this record should be analyzed statistically so that data may be properly corrected, and also so that variations resulting from instrument problems or from contamination can be detected and eliminated. 


\subsubsection{CHECKS OF THE STABMITY OF THE INSTRUMENT}

The analyst must always be on guard against any instability of the instruments that are being used to produce analytical data. All electronic components are subject to variations because of changes in environmental factors, such as temperature and humidity, and to degradation over time, and even new equipment may contain weak components. The records of instrument calibrations and of background measurements, including control charts, are the main data base used to judge the stability of an instrument. The occasional remeasurement of a set of samples that showed a range of instrumental response may be useful in providing another check on the performance of an instrument.

\subsection{DATA REDUCTION, STORAGE, AND REPORTING}

\subsubsection{INTRODUCTION}

The quality of the data reported by EML depends not only upon the care with which sampling and analysis are performed, but also upon the care with which calculations of the resulting data are performed, and upon the manner in which the data are presented in reports. A key aspect of a QA program is maintaining records that document every step of the process that leads to the data that ultimately are reported.

\subsubsection{FIELD AND LABORATORY RECORDS}

A measurement is useful if it is representative of the environmental material or parameter that is under study. The field notes taken during measurement or sampling normally provide a basis for judging the representativeness of a sample or a field measurement. Similarly, the laboratory notes made during the analysis of a sample serve as a basis for judging the quality of the analysis, indicating whether any problems arose during the analytical procedures that might have adversely affected their outcome. For this reason, every effort should be made during field measurement or sampling and during sample preparation and analysis to record all aspects of the procedure that might reasonably be expected to affect the outcome of the analysis. As a general rule, every possibly relevant variable that is amenable to quantification should be recorded, even if 
only by a check mark on a form. These records may be needed not only by the person who is writing them, and not only for the time period during which they are being written, but in many instances they may be needed by other persons and at some future time. It is important, therefore, that the notes be both legible and clear in meaning, so that others who read them will be able to reconstruct the events that are referred to.

It is often convenient to preserve electronically the data records from those instruments which provide digital electronic output signals. When this is done, great care must be taken to ensure that blocks of data are thoroughly annotated to identify the time and location and all other important circumstances concerning the measurements. Often the best way to do this is to organize blocks of data into files with distinctive file names, and to keep a logbook with the supporting information for each file. Alternatively, if electronic editing capabilities are available, comments can be entered into the data file itself.

\subsubsection{DATA REDUCTION AND STORAGE}

When a new project is initiated, the project leader and appropriate Division Directors should discuss the policies to be followed concerning the manner and length of time of storage of data that will be produced during that project. There are types of data, such as those related to possible legal actions involving the U.S. Government, that should be stored indefinitely. When data are stored electronically, backup files must be prepared to eliminate the possibility that the data may inadvertently be lost. When it begins to become evident that a medium on which data are stored, such as a particular type of magnetic disk or tape, is in danger of becoming obsolete, and there is thus a danger that the data will become irretrievable, the data should be copied to a better medium.

Whenever data are to be transferred or stored, an appropriate control procedure should be established to minimize the danger that human error will compromise the quality of the data. Data reduction is normally accomplished electronically using computer programs, both to avoid the drudgery of repeated "hand" calculations and to avoid calculation errors. For small numbers of unusual measurements or samples, however, hand calculations may be more efficient than writing a computer program of limited applicability. When unfamiliar calculations are performed, whether by hand or by using 
computer programs, care must be taken to be sure that the logic built into the calculation is correct. Calculations are often checked by performing them on data that lead to a known result.

\subsubsection{DATA REPORTING}

When data are reported, an estimate of their uncertainty must be given. Contained below are the guidelines and definitions of the terms that are used at EML for reporting the errors and uncertainties of data. The meaning of reported uncertainties must be indicated either by stating exactly what they represent or by describing how they were calculated, because a simple $\mathrm{X} \pm \mathrm{Y}$ statement may be interpreted in any one of a number of ways. The statement of uncertainty should include estimates of all significant sources of error involved, whether these result from the field measurement or sampling phase, the analysis phase or the data reduction phase, if they will affect the final result within the number of significant figures reported.

When data are reported, the reporting format must be commensurate with their expected use. Tables of data allow the full presentation of values and of their estimated uncertainties. Graphical presentation typically allows better visualization of the data. With both tabular and graphical presentations, it is important to assume that nothing will be immediately obvious to the reader, and the column headings or legends must include all information that is necessary in order to understand the data that are presented.

When data are presented, it is important to report only the appropriate number of significant figures. Usually the data should be carried to additional figures during preliminary calculations, and then the final result should be rounded off to the proper number of significant figures. When the tables are printed by a computer, the format that is used may result in too many decimal places being reported for some samples. If this happens, the table should be edited to limit all data to the appropriate number of figures. One approach to determining the number of significant figures to be reported (Sanderson et al., 1980 ) first determines the number of significant figures in the uncertainty, and rounds the reported value to the same decimal place as the uncertainty. In this approach, it is assumed that the rounded-off standard deviation reported should not differ from the calculated value of the standard deviation by more than $20 \%$. For example, if the calculated standard deviation is 0.1635 , it should be reported as 0.16 , which differs from 
the original by only $2 \%$. Rounding off to 0.2 should be avoided because it differs from the original by over $22 \%$. The value of the measurement is then rounded such that its last significant figure will be in the same decimal place as that of the error.

When data are presented in memoranda or internal EML reports, it is usually both possible and desirable to include a complete discussion of the QA data that are pertinent to the measurements. The information to be presented may include, in addition to the uncertainties, various statistical tests and indications of analytical sensitivity, such as the lower limit of detection (LLD), the instrumental detection limit (IDL), the method detection limit (MDL), the limit of detection (LOD) (Currie, 1988), the specificity (identification), and purity (absence of contaminant). The specificity and purity may be estimated from the resolution of a signal or from the goodness of fit to known quantities, such as energy, wavelength or rate of radioactive decay. Reporting the results for QC samples, replicates and blank samples also provides important information about the quality of data. If a discussion of QA cannot be included in a journal or symposium publication, normally a separate, supporting QA report should be prepared.

Terms such as "below detection limits" should not be reported in place of the actual analytical results obtained. Reporting results as "less than" some minimum detectable level also results in some loss of statistical information, and may lead to erroneous interpretations. When low activities or concentrations are measured, the actual results obtained, including any negative values, normally should be reported along with the associated overall uncertainties and the measures of analytical sensitivity that are mentioned above. An interpretation of negative values can be included in the text. Waite et al. (1980) and Gilbert and Kinnison (1981) have discussed techniques that may be followed in averaging data sets that contain "less than" values.

A number of terms have been used by the scientific community to describe data quality, but these terms are often given different meanings by different individuals. The definitions that follow are the preferred usage for the purposes of this document and have been taken from Croarkin (1985), Taylor $(1985,1987)$, and Taylor and Oppermann (1986). 
Error - The difference between the true value and the measured value of a quantity or parameter.

Uncertainty - The range of values within which the true value is estimated to lie. It is a best estimate of possible inaccuracy due to both random and systematic errors.

Random Errors - Errors that vary in a nonreproducible way around the limitingmean. These errors can be treated statistically by use of thelaws of probability.

Systematic Errors - Errors that are reproducible and tend to bias a result in one direction. Their causes can be assigned, at least in principle, and they can have both constant and variable components. Generally, these errors cannot be treated statistically.

A statement of uncertainty assigns credible limits to the reported value, stating to what extent that value may differ from its true value. The uncertainty of a measured value can be defined by a statistically determined confidence interval for the random error and by an estimate of the bounds for systematic error; they should be stated separately. When appropriate, they may also be combined into a single range to describe the overall uncertainty. Since there are a variety of methods suggested in the literature for combining random and systematic errors, the particular method used must be explicitly stated. One accepted practice (Croarkin, 1985) is to combine in quadrature, systematic errors that are known to be independent, to add linearly systematic errors that may not be independent, and to combine systematic and random errors linearly. The confidence level chosen must be stated whenever a confidence interval is reported.

Systematic errors which can be determined by calculation or by experiment should be eliminated by an appropriate correction. Estimating the magnitude of some systematic errors may require scientific judgement on the part of the experimenter. All significant sources of error should be identified and reported. 


\subsection{MATERIALS AND PROCEDURES}

\subsubsection{INTRODUCTION}

Certain materials and procedures must be used during routine analyses for the successful performance of the EML QA program. These include standard instruments, reference materials, and standard reference materials that are used to calibrate instruments or to test the quality of measurements. They also include the $\mathrm{QC}$ procedures that are used during the performance of routine analyses and data validation procedures that are used to check the reasonableness of data. In addition, whenever it is possible, EML takes part in intralaboratory comparisons, using more than one operator, instrument or technique, and in interlaboratory comparisons with other government or private laboratories that are making comparable measurements.

\subsubsection{STANDARDS AND REFERENCE MATERIALS LIBRARY}

EML divisions may maintain a library of standard and reference materials that are needed for calibrating the analytical instruments in that division and for use in the internal QC program in that division. Where possible, any sample matrix that is routinely analyzed should be represented among these reference materials. The division staff maintains records to document the history of acquisition and use of those materials. The Division Director, or a designated subordinate, is responsible for keeping this library complete and current.

\subsubsection{DATA VALIDATION}

For many analytical processes, control charts or other QC records are continually updated to enable the operator to determine whether these processes are under control. The operator enters data for blank samples, background measurements and standard or control samples into the records, and onto the appropriate charts. The records and charts are reviewed and evaluated to determine whether the process is in control and the data are acceptable, or if corrective actions must be taken. Chemical yields, rates of 
radioactive decay, energy calibrations, and other operating characteristics must be evaluated routinely and unusual results must be flagged for immediate investigation.

\subsubsection{INTRALABORATORY COMPARISONS}

Whenever it is possible, attempts are made to verify that no systematic error is introduced into the results of analytical procedures because of differences in techniques among analysts, or because of differences in performance between supposedly equivalent instruments. Analyses of selected samples are repeated using different analysts or different instruments, and the results are compared to insure that variations are within the expected range. In addition, where it is possible, measurements are made or samples are analyzed using more than one technique, to be sure that the technique that is routinely in use gives results that are consistent with results from other approved techniques.

\subsubsection{INTERLABORATORY COMPARISONS}

It is a policy at EML to take part in as many interlaboratory comparisons of measurement and analytical techniques as is practical. The EML staff has taken the initiative in organizing many such intercomparisons, as for example for thermoluminescent detectors (TLDs) and radon detectors, and EML has participated in many intercomparisons sponsored by other government or private laboratories or organizations, as in the chemical analysis of precipitation. In addition, EML has provided reference measurements for Department of Energy contractors in the analysis of radionuclides in environmental samples.

\section{REFERENCES}

Croarkin, C.

"Measurement Assurance Programs Part II: Development and Implementation"

National Bureau of Standards Special Publication 676-II, April (1985) 
Currie, L. A.

"Detection in Ánalytical Chemistry"

ACS Symposium Series 361, p. 17 (1988)

Gilbert, R. O. and R. R. Kinnison

"Statistical Methods for Estimating the Mean and Variance from Radionuclide Data

Sets Containing Negative, Unreported or Less-Than Values"

Health Physics, $\underline{40}, 377-390$ (1981)

Sanderson, C. G., L. K. Cohen, A. Goldin, A. N. Jarvis, L. Kanipe, C. Sill,

M. Trautman, and B. Kahn

"Quality Assurance for Environmental Monitoring Programs"

J. E. Watson (Chairman)

in: Upgrading Environmental Radiation Data, Health Physics Society Committee

Report HPSR-1, pp. 5-1 to 5-7, U.S. Environmental Protection Agency Report EPA 520/1-80-012 (1980)

Taylor, J. K.

"Handbook for SRM Users"

National Bureau of Standards Special Publication 260-100, September (1985)

Taylor, J. K.

Quality Assurance of Chemical Measurements

Lewis Publishers, Inc., Chelsea, MI 48118 (1987)

Taylor, J. K. and H. V. Oppermann

"Handbook for the Quality Assurance of Metrological Measurements"

National Bureau of Standards Handbook 145, November (1986)

Waite, D. A., D. H. Denham, J. E. Johnson, D. E. Michels and N. Turnage

"Statistical Methods for Environmental Radiation Data Interpretation"

J. E. Watson (Chairman)

in: Upgrading Environmental Radiation Data

Health Physics Society Committee Report HPSR-1, pp 7-1 to 7-19, U.S.

Environmental Protection Agency Report EPA 520/1-80-012 (1980) 


\subsection{APPENDIX}

\section{DEFINITIONS OF QA TERMS USED IN THE CHEMICAL LABORATORY}

Some terms concerning standards and calibration are defined by Taylor (1985):

A standard is "a substance or material, the properties of which are believed to be known with sufficient accuracy to permit its use to evaluate the same property of another. In chemical measurements, it often describes a solution or substance, commonly prepared by the analyst, to establish a calibration curve or the analytical response function of an instrument."

A primary standard is "a substance or artifact, the value of which can be accepted (within specific limits) without question when used to establish the value of the same or related property of another material."

A check standard is "in physical calibration, an artifact measured periodically, the results of which typically are plotted on a control chart to evaluate the measurement process."

Standardization, in analytical chemistry, is "the assignment of a compositional value to one standard on the basis of another standard."

A standard reference material is "a reference material distributed and certified by the National Institute of Standards and Technology."

A certified reference material (CRM) is "a reference material one or more of whose property values are certified by a technically valid procedure, accompanied by or traceable to a certificate or other documentation which is issued by a certifying body."

A certified value is "the value that appears in a certificate as the best estimate of the value for a property of a reference material." 
A reference material (RM) is "a material or substance one or more properties of which are sufficiently well established to be used for the calibration of an apparatus, the assessment of a measurement method, or for the assignment of values to materials."

Traceability is "the ability to trace the source of uncertainty of a measurement or a measured value."

Calibration is the "comparison of a measurement standard or instrument with another standard or instrument to report or eliminate by adjustment any variation (deviation) in the accuracy of the item being compared."

A calibrant is "a substance used to calibrate or to establish the analytical response of a measurement system."

Intercalibration is "the process, procedures, and activities used to ensure that the several laboratories engaged in a monitoring program can produce compatible data. When compatible data outputs are achieved and this situation is maintained, the laboratories can be said to be intercalibrated."

A standard method is "a method (or procedure) of test developed by a standardswriting organization, based on consensus opinion or other criteria, and often evaluated for its reliability by a collaborative testing procedure."

A reference method is "a method which has been specified as capable, by virtue of recognized accuracy, of providing primary reference data."

Two useful terms defined by the International Institute of Technology (IOLM, 1976) are:

Repeatability of measurements - "The closeness of the agreement between the results of successive measurements of the same quantity carried out by the same method, by the same observer, with the same measuring instruments, in the same laboratory, at quite short intervals of time."

Reproducibility of measurements - "The closeness of the agreement between the results of measurements of the same quantity, where the individual measurements are made: 
- by different methods, with different measuring instruments,

- by different observers, in different laboratories,

- after intervals of time that are quite long compared with the duration of a single measurement, and

- under different normal conditions of use of the instruments employed."

"The term reproducibility is also used when some of the factors listed above are different in the individual measurements; these factors should be specified in detail in each particular case."

Some additional terms that are of use in discussing QA are defined by Taylor (1985):

A technique is "a physical or chemical principle utilized separately or in combination with other techniques to determine the composition (analysis) of materials."

A method is "an assemblage of measurement techniques and the order in which they are used."

A procedure is "a set of systematic instructions for using a method of measurement or of sampling or of the steps or operations associated with such."

A protocol is "a procedure specified to be used when performing a measurement or related operation, as a condition to obtain results that could be acceptable to the specifier."

An analyte is "the specific component measured in a chemical analysis; also called analate."

A sample is "a portion of a population or lot. It may consist of an individual or groups of individuals. It may refer to objects, materials, or to measurements, conceivable as part of a larger group that could have been considered."

An increment is "an individual portion of material collected by a single operation of a sampling device, from parts of a lot separated in time or space. Increments may be either tested individually or combined (composited) and tested as a unit." 
A composite sample is "a sample composed of two or more increments selected to represent a population of interest."

A blind sample is "a sample submitted for analysis whose composition is known to the submitter but unknown to the analyst. A blind sample thus is one way to test proficiency of a measurement process."

Double blind refers to "a sample, known by the submitter but submitted to an analyst in such a way that neither its composition nor its identification as a check sample are known to the latter."

A blank is "the measured value obtained when a specified component of a sample is not present during the measurement. In such a case, the measured value/signal for the component is believed to be due to artifacts, hence should be deducted from a measured value to give a net value due to the component contained in a sample. The blank measurement must be made so that the correction process is valid."

A split sample is "a replicate portion or subsample of a total sample obtained in such a manner that it is not believed to differ significantly from other portions of the same sample."

A duplicate sample is "a second sample randomly selected from a population of interest (see also split sample) to assist in the evaluation of sample variance."

A replicate is "a counterpart of another, usually referring to an analytical sample or a measurement. It is the general case for which duplicate is the special case consisting of two samples or measurements."

A duplicate measurement is "a second measurement made on the same (or identical) sample of material to assist in the evaluation of measurement variance."

'These definitions of "blind sample" and "double blind" are applicable to certain uses in quality assurance, but these terms are also commonly used with other, somewhat different meanings.

${ }^{2}$ This definition of "blank" is applicable to certain uses in $Q A$, but this term is also commonly used with other, somewhat different meanings. 
A control sample is "a material of known composition that is analyzed concurrently with test samples to evaluate a measurement process (see also Check Standard)."

The limiting mean is "the value approached by the average as the number of measurements, made by a stable measurement process, increases indefinitely."

An outlier is "a value which appears to deviate markedly from that for other members of the sample in which it occurs."

The coefficient of variation is "the standard deviation divided by the value of the parameter measured."

The relative standard deviation is "the coefficient of variation, expressed as a percentage."

Validation is "the process by which a sample, measurement method, or a piece of data is deemed to be useful for a specific purpose."

A control chart is "a graphical plot of test results with respect to time or sequence of measurement together with limits within which they are expected to lie when the system is in a state of statistical control."

The control limits are "the limits shown on a control chart beyond which it is highly improbable that a point could lie while the system remains in a state of statistical control."

The warning limits are "the limits shown on a control chart within which most of the test results are expected to lie (within a 95\% probability) while the system is in a state of statistical control."

The terms error, uncertainty, random errors and systematic errors, all of which concern data quality, are defined in Section 1.9.4, Data Reporting. 


\section{REFERENCES TO DEFINITIONS}

International Organization of Legal Metrology

"Vocabulary of Legal Metrology: Fundamental Terms"

Paris, France (1976)

Taylor, J. K.

"Handbook for SRM Users"

National Bureau of Standards Special Publication 260-100, September (1985) 


\section{SAMPLING}

U.S. Department of Energy 201 Varick Street, 5th Floor

New York, NY 10014-4811 
2.2.1 Scope $\ldots \ldots \ldots \ldots \ldots \ldots \ldots \ldots \ldots \ldots \ldots \ldots \ldots \ldots \ldots .2 .2-1$

2.2.2 Aerosols .................................. 2.2-1



2.2.2.2 Total Particle Collections ................... 2.2-3

2.2.2.3 Dichotomous Sampler ................... 2.2-7

2.2.2.4 High Volume Cascade Impactors $\ldots \ldots \ldots \ldots \ldots \ldots \ldots .2 .2-8$

2.2.2.5 Moudi ............................ 2.2-13

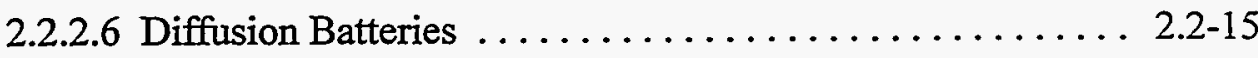

2.2.2.7 Graded Screen Array $\ldots \ldots \ldots \ldots \ldots \ldots \ldots \ldots . . . . .2 .2-23$

2.2.2.8 Mathematical Analysis of Particle Size Data ......... 2.2-26

2.2.3 Radon and Thoron $\ldots \ldots \ldots \ldots \ldots \ldots \ldots \ldots \ldots \ldots \ldots . . .2 .2-34$

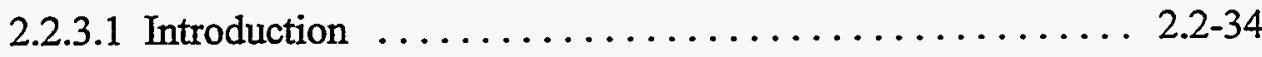

2.2.3.2 Grab Sampling for Radon $\ldots \ldots \ldots \ldots \ldots \ldots \ldots \ldots .2 .2-35$

2.2.3.3 Two-Filter Tube Sampling for Radon and Thoron ...... 2.2-37

2.2.3.4 Continuous Radon Monitoring . . . . . . . . . . . . . . 2. 240

2.2.3.5 Integrating Radon Monitors $\ldots \ldots \ldots \ldots \ldots \ldots \ldots \ldots .2 .2-41$

2.2.3.6 Quality Control ......................... 2.2-44

2.2.3.7 ${ }^{220} \mathrm{Rn}$ and ${ }^{222} \mathrm{Rn}$ in Soil Gas $\ldots \ldots \ldots \ldots \ldots \ldots \ldots \ldots .2 .2-47$

2.2.4 Radon and Thoron Progeny $\ldots \ldots \ldots \ldots \ldots \ldots \ldots \ldots \ldots . . .2 .2-51$

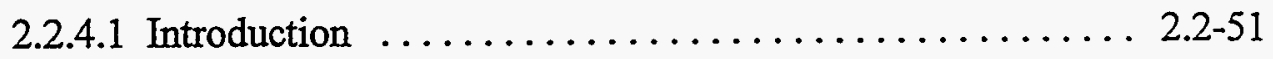

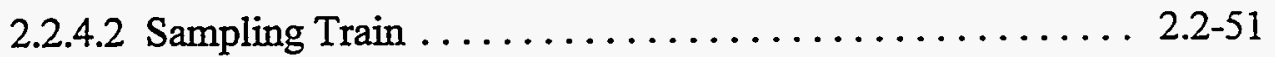

2.2.4.3 Alpha Particle Counting System ............... 2.2-53

2.2.4.4 Modified Kusnetz Method ................... 2.2-54

2.2.4.5 Modified Tsivoglou Method ................. 2.2-56

2.2.4.6 Raabe-Wrenn Least-Squares Method .............. 2.2-59

2.2.5 Atmospheric Tracing . ....................... 2.2-67

2.2.5.1 Introduction $\ldots \ldots \ldots \ldots \ldots \ldots \ldots \ldots \ldots \ldots \ldots \ldots \ldots \ldots \ldots \ldots \ldots .2 .2-67$ 
Page

2.2.5.2 The Programmable Atmospheric Tracer Sampler . . . . . 2. 2-68

2.2.5.3 Adsorbent Tube Bake-Out Procedure . . . . . . . . . 2.2-69

2.2.5.4 Deployment, Sampling, and Retrieval .......... 2.2-71

2.3 Atmospheric Deposition

2.3.1 Scope $\ldots \ldots \ldots \ldots \ldots \ldots \ldots \ldots \ldots \ldots \ldots \ldots \ldots \ldots \ldots .2 .3-1$



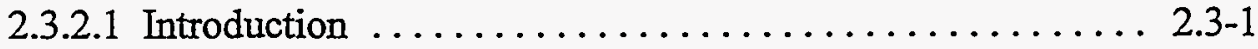

2.3.2.2 Apparatus ............................. 2.3-2

2.3.2.3 Deployment, Sampling, and Retrieval ............ 2.3-2

2.3.3 Ion Exchange Fallout Collector $\ldots \ldots \ldots \ldots \ldots \ldots \ldots \ldots$. 2.3-3

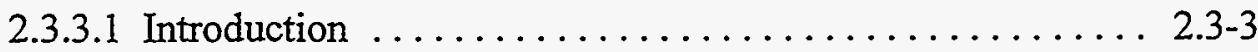



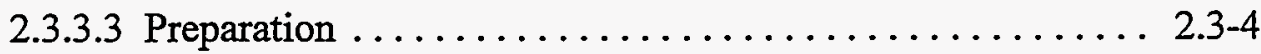

2.3.3.4 Collector Location $\ldots \ldots \ldots \ldots \ldots \ldots \ldots \ldots \ldots \ldots . . .2 .3-4$

2.3.3.5 Site Operator Instructions $\ldots \ldots \ldots \ldots \ldots \ldots \ldots \ldots .2 .3-4$

2.3.4 Wet/Dry Deposition Collector $\ldots \ldots \ldots \ldots \ldots \ldots \ldots \ldots .2 .3-6$

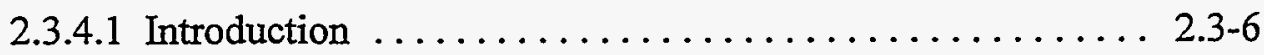

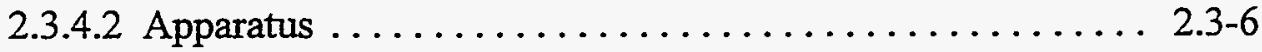

2.3.4.3 Sampling and Deployment................. 2.3-7

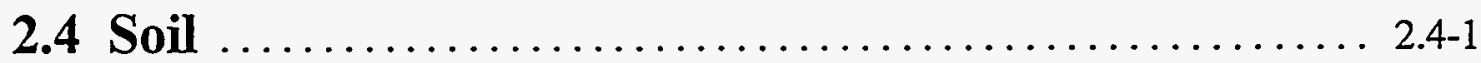

2.4.1 Scope $\ldots \ldots \ldots \ldots \ldots \ldots \ldots \ldots \ldots \ldots \ldots \ldots \ldots \ldots \ldots \ldots .2 .4-1$

2.4.2 Sampling Objectives $\ldots \ldots \ldots \ldots \ldots \ldots \ldots \ldots \ldots \ldots \ldots .2 .4-1$

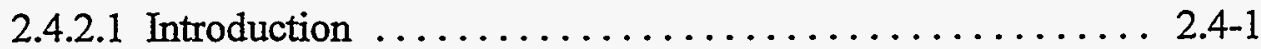

2.4.2.2 Deposition Inventories ...................... 2.4-2

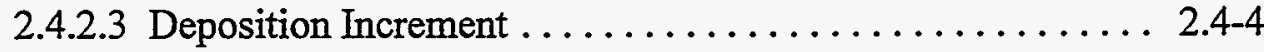

2.4.2.4 Operational or Accidental Releases ............... 2.4-4

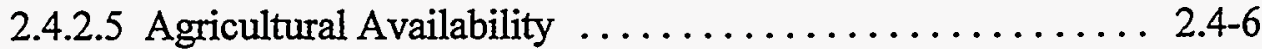

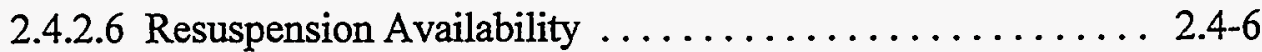

2.4.3 Recommended Procedures $\ldots \ldots \ldots \ldots \ldots \ldots \ldots \ldots \ldots . . \ldots \ldots . .4 .4$

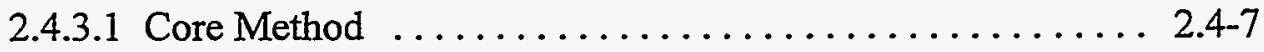




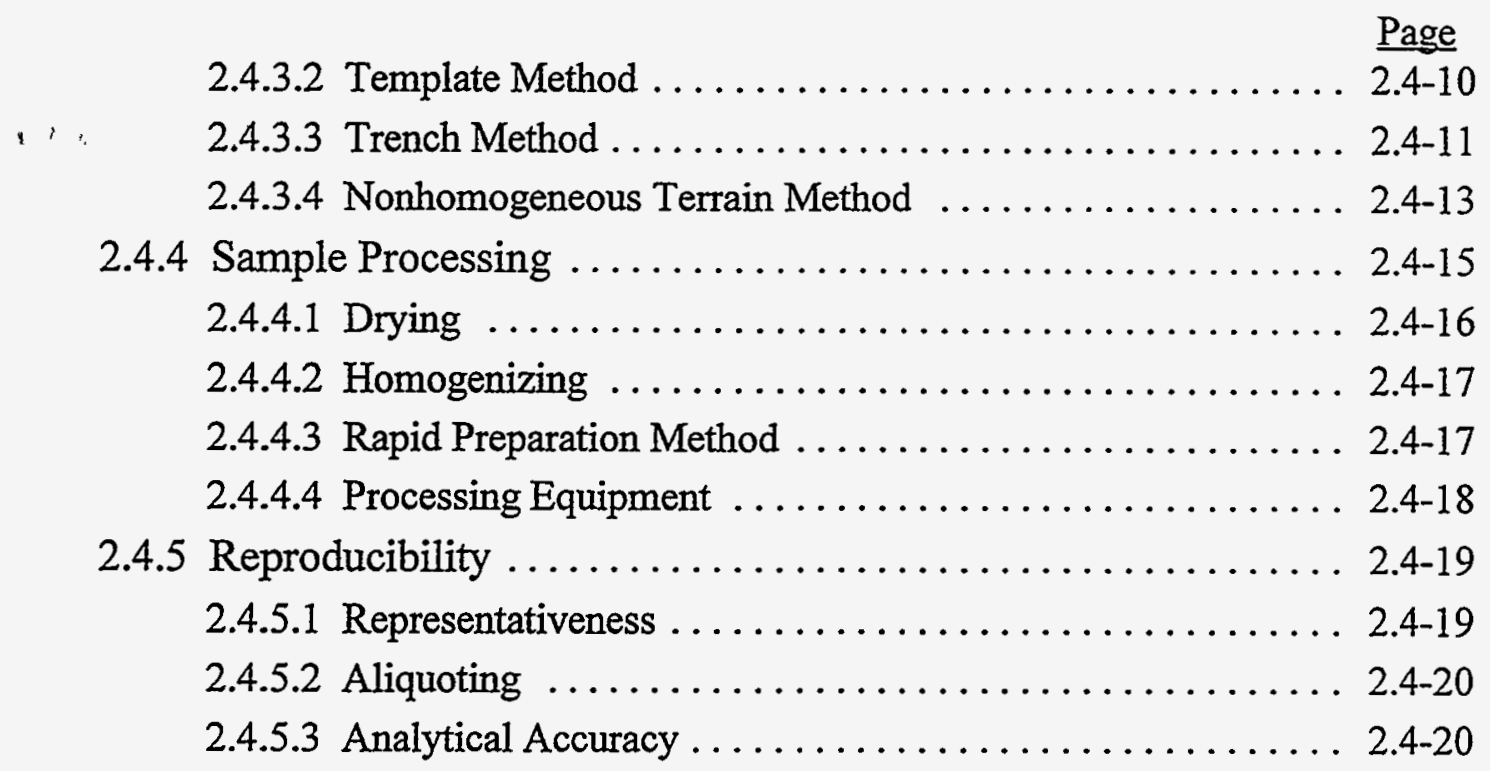

2.5.1 Scope $\ldots \ldots \ldots \ldots \ldots \ldots \ldots \ldots \ldots \ldots \ldots \ldots \ldots \ldots \ldots \ldots \ldots \ldots \ldots \ldots .2 .5-1$

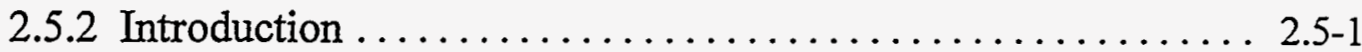

2.5.3 Equipment Design and Application ................ 2.5-2

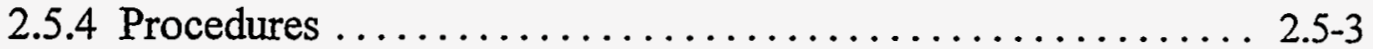

2.5.4.1 General Operation ....................... 2.5-3

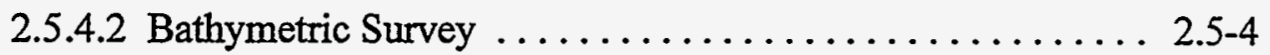

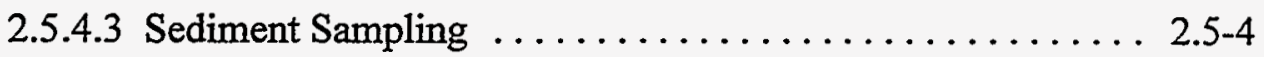

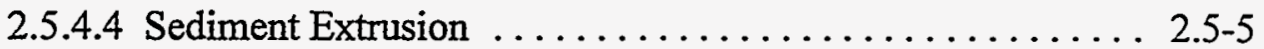

2.6 Uranium and Radium in Water .................... 2.6-1

2.6.1 Scope $\ldots \ldots \ldots \ldots \ldots \ldots \ldots \ldots \ldots \ldots \ldots \ldots \ldots \ldots .2 .6-1$

2.6.2 Apparatus $\ldots \ldots \ldots \ldots \ldots \ldots \ldots \ldots \ldots \ldots \ldots \ldots \ldots \ldots \ldots \ldots \ldots \ldots \ldots \ldots .6 .1$

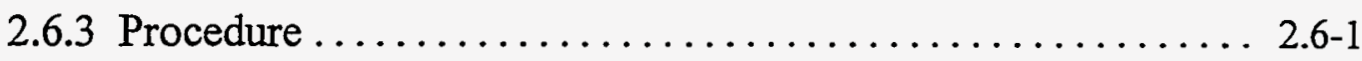

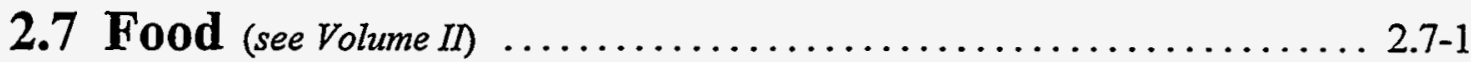




\section{SAMPLING}

\subsection{OVERVIEW}

The purpose of environmental sampling and analysis is to obtain data that describe a particular site at a specific point in time from which an evaluation can be made as a basis for possible action. In this process, the collection of valid samples is the vital first step. Sampling should be done with the same care as the analysis, and both should be done with a rigor that is appropriate for the project at hand. In order for the data to be meaningful, sampling must be carried out with a clear purpose and with an understanding of the problem to be solved and the physical conditions that exist.

At EML, environmental sampling is carried out for purposes such as inventorying a pollutant at a specific point in time, calculating the pollutant transfer coefficients, and reconstructing deposition chronologies. Through these long-term studies, experience has been gained in sampling radioactive fallout, air particulates and gases, and total and rate of deposition. In this section, we describe the procedures developed by EML for environmental sampling. The corresponding analytical procedures are presented later in this Manual.

General guidance on collecting valid samples is given in Section 1.6.2. Unlike chemical or radiometric analyses, it is not possible to set down step-by-step procedures for sampling. For example, a variety of samples may be required for the purpose of establishing relationships between concentrations in different matrices to further the understanding of dynamic processes. Also, the concentration of a particular pollutant in an environmental matrix will change with time and location.

Usually, the crucial decisions in planning a sampling program are how many sites should be sampled and how often they should be sampled. These decisions can only be made based on a knowledge of the degree of variability due to these two factors (see Section 1.6.2). Most sampling programs require exploratory sampling so that the variability with time and location can be assessed in comparison with the required uncertainty. Experience has shown that statistical approaches based on these exploratory samples 
usually lead to the taking of a smaller number of samples than would have otherwise been predicted. Another important consideration is that the number of samples must be consistent with the available analytical facilities.

Many times, the samples received in the laboratory may be representative of the particular conditions to be evaluated, but are not in the proper physical form for analysis. The samples may require reduction in size, drying or some form of homogenizing before subsamples can be taken for analysis. Some general considerations concerning sample preparation are discussed in Section 1.6.3 of this Manual.

The philosophy at EML is usually to collect a sufficient amount of sample so that there is not only enough to measure the constituent of interest, but also enough for reanalysis at a later time (see Section 1.6.3). Storage of samples for later analyses requires judgment in order to avoid loss of constituents to be measured or to avoid undesirable decomposition. EML maintains an extensive library of samples associated with its research programs, in some cases going back over 30 years (Klusek, 1989).

\section{REFERENCE}

Klusek, C. S.

"The EML Soil Inventory and Archive"

U.S. Department of Energy Report EML-519 (1989) 


\subsection{AIR}

Contact Person(s) : Alfred Cavallo and Raymond J. Lagomarsino

\subsubsection{SCOPE}

Described in this section are the equipment and procedures used at EML in sampling for trace amounts of certain gases, liquids or solids dispersed in air. For gases, this involves either "whole air" samplers, or samplers which selectively adsorb the gas of interest. For the liquids and solids (aerosols), the techniques involve separating the particles from air by means of filtration or impaction.

In situ methods in which the sampling and analysis are one combined operation are not described as they are not commonly used at EML. Further information can be found in Air Sampling Instruments for Evaluation of Atmospheric Contaminants, a handbook published by the American Conference of Government Industrial Hygienists (ACGIH, 1989). This handbook, which is updated every 5 to 6 years, includes descriptions of practically every commercially available instrument for sampling trace gases and aerosols. It also has concise theory sections covering basic physics and experimental design pertinent to air sampling.

The locales for sampling in EML programs range from indoor (residential or occupational) to outdoor (surface to upper troposphere).

\subsubsection{AEROSOLS}

INTRODUCTION

Many of EML's research programs require that samples be taken of aerosols, defined as "a system of colloidal particles dispersed in a gas". The suspending gas is normally indoor or outdoor air. Smoke and mist are common examples of aerosols, but frequently 



\section{REFERENCES}

Hinds, W. C.

"Aerosol Technology: Properties, Behavior and Measurement of Air borne Particles"

Wiley-Interscience, New York (1982)

Air Sampling Instruments for Evaluation of Atmospheric Contaminants

Hering, S. (Editor)

American Conference of Governmental Industrial Hygienists (ACGIH), Cincinnati, OH, Seventh Edition (1989)

\section{A. Introduction.}

The procedures discussed in this section are limited to the specific requirements of programs currently conducted at EML and may not be applicable to other studies that require the total collection of air particles.

Since January 1963, EML has conducted the Surface Air Sampling Program (SASP). In 1987, the Remote Atmospheric Measurements Program (RAMP) was initiated as an extension and modification of SASP. The primary objectives of SASP/ RAMP are to identify and study the temporal and spatial distribution of anthropogenic and natural radionuclides in the lower troposphere. These objectives are achieved by filtration of large volumes of air to concentrate the radionuclides in the aerosol prior to sample analyses. The sampling procedures used in SASP/RAMP would not be appropriate if one requires particle size analyses or particle specific activity distributions. The filters used in these programs also will not collect uncharged, unattached radioactive gas molecules.

To sample large volumes of air and obtain total particle collection, it is necessary to use an appropriate filter material and an air mover. In SASP/RAMP, the air mover must be capable of continual operation at high flow rates under harsh environmental 
conditions. To calculate air concentrations of radionuclides, it is necessary to accurately determine the total volume of the air which has been sampled.

\section{B. Filter material for total particle collections.}

The criteria for filter selection are good collection efficiency for submicron particles at the range of face velocities used, high particle and mass loading capacity, low-flow resistance, low cost, high mechanical strength, low-background activity, compressibility, low-ash content, solubility in organic solvents, non-hygroscopicity, temperature stability, and availability in a variety of sizes and in large quantities. In the selection of a filter material, a compromise must be made among the above criteria that best satisfies the sampling requirements. An excellent review of air filter material used to monitor radioactivity was published by Lockhart et al. (1964). Lippmann (1989a) also provides information on the selection of filter materials for sampling aerosols by filtration.

Two filter sizes are presently used in SASP/RAMP, a $20.32 \mathrm{~cm}$ circle and a $20.32 \mathrm{~cm} \times 25.40 \mathrm{~cm}$ rectangle. The polypropylene fiber filter, Dynaweb Grade DW7301L (see Specification 7.17) is used at all SASP and RAMP sites. The filter is composed of a $100 \%$ polypropylene web that is $100 \%$ binderless. Three layers of this web are collated and sandwiched between two sheets of a protective DuPont Reeme ( $100 \%$ polyester) scrim. The top scrim is removed prior to sampling at RAMP sites because after their return to EML for analysis these samples are compressed into pellets and the scrim hinders compression. At all other sites, the filter can be used during sampling with both top and bottom scrim in place.

\section{Air movers.}

A large variety of air movers are commercially available and have been reviewed by Rubow and Furtado (1989).

Many factors must be considered when selecting an air mover. Such factors as portability, power requirements, maximum operational flow rate/temperature/pressure, cost, durability, and maintenance must be considered in the selection of an air mover. It is also important that the air mover itself is not a source of contamination in any study. 
The major factors that were considered in the selection of an air mover for the SASP sites were durability, low maintenance, and a flow rate of $\sim 1 \mathrm{~m}^{3} \mathrm{~min}^{-1}\left[288^{\circ} \mathrm{K}, 101.3 \mathrm{kPa}\right.$ $(760 \mathrm{~mm} \mathrm{Hg})]$ at pressure drops across the filter ranging from $\sim 5 \mathrm{kPa}$ to $\sim 20 \mathrm{kPa}(20-80$ in water).

Two air mover systems are currently used in SASP/RAMP. The SASP sites are equipped with a Roots Blower (see Specification 7.18) connected to a $1 \mathrm{HP}$ electric motor (see Specifications 7.18) by a fan belt. The RAMP sites are equipped with a Fuji ring compressor (see Specification 7.18) in which the air mover is directly connected to a 0.5 HP electric motor. The Roots system must be enclosed in a louvered shelter to protect the pump from direct exposure to precipitation. The Roots system is frequently mounted on a 1-m high metal stand. The Fuji system is enclosed in a custom manufactured aluminum container. The Roots system is heavier than the Fuji system and may be more durable for continual operation in harsh environments. The Roots system exhausts some oil vapors which may be a contaminant in certain studies. The Roots system is designed for $20.32 \mathrm{~cm}$ round filters, while the Fuji system is designed for $20.32 \mathrm{~cm} \times 25.40 \mathrm{~cm}$ rectangular filters.

\section{Flow calibration and sample volume determination.}

To calculate the concentrations of radionuclides in the lower troposphere or concentrations of any air pollutant collected and concentrated by filtration, it is necessary to accurately determine the total volume of air sampled.

Generally, a parameter of the air mover can be related to flow. If the mean flow during a collection period can be determined, the total volume of air sampled can be calculated. Accurate flow measurements and the total integrated sample volume of air can be obtained using a mass flow meter and a totalizer. This direct technique of air flow measurement becomes impractical at remote field locations due to cost and exposure of the flow meter to harsh environments. Other procedures for the measurement of air flow in sampling systems are reviewed by Lippmann (1989b).

We have determined that the best technique to measure flow, at sites equipped with Roots systems, is to determine an empirical relationship between the Roots blower inlet pressure and the flow through the upstream filter. An orifice meter was manufactured at EML to derive this relationship. The orifice meter has been calibrated for flow using 
secondary flow measurement devices that are traceable to a primary standard volume meter. All Roots systems are calibrated using this orifice meter prior to field installation. Periodic calibrations are conducted at the field sites.

During sampling, the pressure at the Roots blower inlet is measured using a Magnehelic gauge. The initial and final pressure values are averaged. The average inlet pressure is then used in the previously described empirical relationship to calculate the average flow. This average flow is multiplied by the collection interval to obtain the total volume sampled. The total volume sampled is adjusted to a pressure of $101.3 \mathrm{kPa}$ $\left(760 \mathrm{~mm} \mathrm{Hg}\right.$ ) and a temperature of $288^{\circ} \mathrm{K}$.

At sites equipped with a Fuji system, the flow is determined using an empirical relationship between the pressure differential across a fixed orifice located in the blower exhaust. The initial and final pressure values are used to calculate a mean flow and a total sample volume as previously described. The relationship between flow and the pressure differential across the fixed orifice plate and in the Fuji flow system was determined using a Hastings Laminar flow element traceable to a primary standard volume meter.

\section{REFERENCES}

Lippmann, $M$.

"Sampling Aerosols by Filtration"

Hering, S. (Technical Editor)

in: Air Sampling Instruments, 7th Edition, American Conference of Governmental Industrial Hygienists, Cincinnati, Ohio, pp. 305-336 (1989a)

Lippmann, $M$.

"Calibration of Air Sampling Instruments"

Hering, S. (Technical Editor)

in: Air Sampling Instruments, 7th Edition, American Conference of Governmental Industrial Hygienists, Cincinnati, Ohio, pp. 73-100 (1989b) 
Lockhart, L., R. Patterson and W. Anderson

"Characteristics of Air Filter Media Used for Monitoring Airborne Radioactivity"

Naval Research Laboratory Report NRL-6054, June (1964)

Rubow, K. and V. Furtado

"Air Moves and Samplers"

Hering, S. (Editor)

in: Air Sampling Instruments, 7th Edition, American Conference of Governmental Industrial Hygienists, Cincinnati, Ohio, pp. 241-274 (1989)

\subsubsection{3}

\section{DICHOTOMOUS SAMPLER}

A Sierra Model 245 automatic dichotomous sampler is used to study the "inhalable" $(<10 \mu \mathrm{m})$ particles in ambient air. The original commercial design was developed and sold by Sierra Instruments Inc., but is now sold by Andersen Instruments, Inc., Sierra Anderson Division, 4801 Fulton Industrial Blvd., Atlanta, GA 30336.

The dichotomous sampler is capable of separating the particles $<10 \mu \mathrm{m}$ (determined by the aerosol $10 \mu \mathrm{m}$ inlet cut-off size) into two fractions. By means of virtual impaction, the sampled particles are separated into two fractions, the fine fraction, $<2.5 \mu \mathrm{m}$, and the coarse fraction between 2.5 and $10 \mu \mathrm{m}$. This technique has the added advantage of eliminating problems associated with particle bounce and re-entrainment that are sometimes experienced in cascade impactor sampling.

The particles are collected on Teflon membrane filters which are ideal for gravimetric analyses of the fine, coarse, and inhalable (sum of fine and coarse) fractions, and for chemical analyses by X-ray fluorescence or other high-resolution chemical techniques.

The experimental procedures used at EML are defined in the instrument manual along with literature references on the development and application of the sampler for studies of PM-10 compliance monitoring, source discrimination, fine-particle monitoring, and visibility monitoring. 


\section{A. Introduction.}

A description of the Sierra Model 235 multistage cascade impactor, which is used to measure the particle size distribution of both indoor and outdoor aerosols, is presented here. The original design was developed and sold by Sierra Instruments, Inc., but now is sold by Andersen Instruments, Inc., Sierra Anderson Division, 4801 Fulton Industrial Blvd., Atlanta, GA 30336. A number of articles are available in the literature that deal with both the theoretical and experimental development of inertial impactors (Marple et al., 1973, 1974; Marple and Willeke, 1979).

At EML, this impactor is used for research studies and is not commonly used for long-term sampling projects. The methods described herein are used for 1-5 day sampling periods, and the units are checked on a daily basis.

\section{B. Operating conditions.}

The sampler is capable of sampling at flow rates ranging from $0.56 \mathrm{~m}^{3} \mathrm{~min}^{-1}$ to $1.68 \mathrm{~m}^{3} \mathrm{~min}^{-1}$. The flow is regulated by a constant flow controller that automatically adjusts to any flow rate change caused by loading conditions or motor variances. In the majority of our outdoor sampling efforts, the unit is operated at a flow rate of $0.85 \mathrm{~m}^{3}$ $\min ^{-1}(30 \mathrm{~cm})$ to prevent overloading and to assure that the flow rate remains constant during the sampling interval.

Configuration. The unit is housed in a standard high-volume sampling shelter without a size selective inlet. Under some conditions a $10 \mu \mathrm{m}$ or $15 \mu \mathrm{m}$ size-selective inlet is used. The impaction substrate is slotted Whatman No. 41 filter paper treated with light mineral oil (Fisher Scientific, 50 Faden Road, Springfield, NJ 07081). The oiled filter minimizes particle rebound and reentrainment, which has been found during previous impactor evaluations to bias impactor size distribution measurements toward smaller sizes (Knuth, 1979a). This method of oil treating the filter is not compatible with mass determination by weighing methods. All of our analyses are done by chemical or radiochemical procedures. 
General maintenance. The impactor plates are washed with alcohol and the slots are cleaned using a cotton swab or soft cloth. Care must be taken so that the integrity of the slot is not altered by using any cleaning material that may damage the slot spacing or edges in any manner. The plates are dried with compressed air and the slots are visibly examined to assure that no foreign material remains in the slot. The impactor plates should be numbered $1-5$ in a corresponding corner of the plates, starting with the largest slotted plate numbered 1 to the smallest plate numbered 5 .

Loading procedures. The loading and unloading of the impactor assembly should be done in a clean environment (clean room or clean bench). The loaded unit can then be transported to the sampling area and installed on site. In handling the filters, reasonable care should be taken to minimize contamination of the sample. Filters should be handled at the extreme comers, and latex gloves or forceps should be used during the following loading procedures.

1. Soak the total number of filter impaction substrates needed for the number of impactors being loaded in mineral oil using a Pyrex or comparable dish. Note: A few extra oiled filters may be required for blank analysis values.

2. After the filters are completely wetted, they are removed from the oil and allowed to drip until all excess oil has run off.

3. Starting with the slotted base plate, place a mineral oil wetted filter on the plate assuring that the paper is centered and all slots are open. Using a glass rod, assure that the paper is in contact with the plate and no air bubbles are present.

4. Carefully place the slotted plate, \#5, on top of the filter. Assure that it is flat, and that it secures the impaction filter \#5 beneath it to the base plate. At this time, the filter paper should be visible through the slots. If not, remove plate \#5 and reposition it 180 degrees. Check again.

5. Continue placing filters and plates \#4 to \#1 as described in Step 4 above.

6. After positioning slotted plate \#1, tighten the two screws that secure the five plates sandwiched to the base plate. At this time, wipe any excess oil from the sides of the 
plate assembly. Note: If there is an extreme amount of oil seeping through the edges of the plates, start over at Step 1 allowing a longer time for the filters to drip dry.

7. The extra wetted filter is carefully folded over on itself once or twice and placed in a polyethylene bag. Attach an appropriate identification label to the bag.

8. The loaded impactor assembly is taken to the sampling site and placed in position over an appropriate back-up filter. For our work, a suitable back-up filter is determined dependent on the analytical procedure to be used. Back-up filters which have been used for different applications are glass fiber, Microsorban, Microdon, and Whatman No. 41.

9. Tighten wing nut assemblies on four corners of the base plate to secure impactor assembly to back-up filter holder.

\section{Sampling procedure.}

1. Start unit and adjust the in-line orifice reading to the desired setting. This setting corresponds to the flow rate determined from a calibration curve of the orifice. Note: A top loading calibrated orifice can be used to verify flow rate in the field.

2. Record all pertinent flow and time information required. Note: If a number of runs will be required for the experiment, a data sheet form should be developed and used for recording data.

3. Whenever possible, the unit should be visibly checked during the sampling period. If an in-line orifice is being used, the manometer reading can be read and recorded at any time during the exposure period without interrupting the sampling procedure.

4. At the end of the sampling period, record all data and if possible recheck the flow rate with the top loading orifice. 


\section{Sample recovery.}

1. Carefully remove the impactor assembly from above the back-up filter. Immediately fold the back-up filter over on itself, soiled side in, twice if required, and place in prelabeled polyethylene bag.

2. Take the impactor assembly to a clean environment and begin the disassembly procedure by carefully removing the top plate, \#1, folding the soiled filter over on itself, soiled side in, and placing it in a prelabeled envelope or polyethylene bag. Note: We have found that polyethylene bags are better than glassine envelopes for storing the oiled filters prior to analysis. A glass rod can be used for creasing the oiled filter when folding.

3. Place the five oiled impaction filter polyethylene bags into a larger polyethylene bag, appropriately labelled, along with the back-up filter and the appropriate blank oiled filter. This is the extra-oiled impaction filter prepared prior to loading the impactor. Note: Although you can usually determine the stage the oiled filter came from by visual determination of the width of the deposit on the filter, care should be taken when removing the filters from the impactor to assure that the filter is correctly identified when placed in the polyethylene bag.

4. The impactor plates can be placed in an alcohol bath to remove any excess oil from the plates prior to cleaning for assembly. Compressed air can be used to clean slots if necessary.

Data analysis. Various methods of describing the particle size distribution of sampled aerosol have been used through the years for impactor data. In order to use any one or more of these methods it is necessary to determine the amount of material collected on each of the impactor stages and back-up filter. At EML, this is usually done by measuring the radioactivity or by chemical analyses.

Two of the most used methods of describing the distribution derived from impactor data are histograms and cumulative plots (Knutson and Lioy, 1989). At EML we use a computer program, UNFOLD.PS, to construct smooth particle size spectra from the 
impactor data. To do this, construct an impactor efficiency file from the curves given in Figure 7 of Knith (1979b) per the instructions given in UNFOLD.PS, then follow the steps in Section 2.2.2.8, C6.

\section{REFERENCES}

Knuth, R. H.

"Comments on Inertial Impactor Calibration and Use"

in: Aerosol Measurements, University of Florida, Gainesville, pp. 108-116 (1979a)

Knuth, R. H.

"Calibration of a Modified Sierra Model 235 Slotted Cascade Impactor"

USDOE Report EML-360 (1979b)

Knutson, E. O. and P. J. Lioy

"Measurements and Presentation of Aerosol Size Distributions"

Lioy, P. J. and M. J. Y. Lioy (Editors)

in: Air Sampling Instruments for Evaluation of Atmospheric Contaminants

American Conference of Governmental Industrial Hygienists, Cincinnati, $\mathrm{OH}$, pp. E1-E12, Seventh Edition (1989)

Marple, V. A. and K. Willeke

"Inertial Impactors"

D. A. Lundgren et al. (Editors)

in: Aerosol Measurements

University of Florida, Gainesville, pp. 90-107 (1979)

Marple, V. A., B. Y. H. Liu, and K. T. Whitby

"Fluid Mechanics of the Laminar Flow Aerosol Impactor"

J. Aerosol Sci., $\underline{5}, 1-16$ (1973)

Marple, V. A., B. Y. H. Liu, and K. T. Whitby

"On the Flow Fields of Inertial Impactors"

J. Fluids Eng., 96, 394-400 (1974) 


\section{A. Introduction.}

Procedures are presented for using the micro-orifice uniform deposit impactor (MOUDI) in determining the particle size distribution of the decay products of radon and/or thoron gas. The MOUDI covers the size range of 50-5000 nm and complements the capabilities of the diffusion batteries (Section 2.2.2.6), which cover a range of 5-500 nm. To sample a broader size range, $0.5-5000 \mathrm{~nm}$, the MOUDI may be used in parallel with the graded screen array, described in Section 2.2.2.7.

The MOUDI consists of two basic assemblies -- the cascade impactor itself, and its housing. The cascade impactor consists of an air inlet, eight impaction stages, and a backup filter, which is located in the base of the impactor. Each stage contains a removable impact plate for the stage above and a nozzle plate for the stage below.

Contained in the housing is a valve for controlling the flow through the impactor, two pressure gauges to monitor the flow, and a mechanism for rotating the impaction stages.

The table below gives the main characteristics of the MOUDI. Further information about the MOUDI can be found in Marple (1991). 
CHARACTERISTICS OF THE MOUDI

(Manufacturer's data)

$\begin{array}{cccc}\text { Stage } & \text { Cut Size, nm } & \begin{array}{c}\text { No. of } \\ \text { Nozzles }\end{array} & \begin{array}{c}\text { Pressure } \\ \text { Reading }\end{array} \\ \text { 3A } & 3200 & 10 & \\ 4 \mathrm{~A} & 1800 & 20 & \\ 5 \mathrm{~A} & 1000 & 40 & \begin{array}{c}1250 \mathrm{~Pa}, \\ \text { upper gauge }\end{array} \\ 6 \mathrm{~A} & 560 & & \\ 7 \mathrm{~A} & 290 & 80 & \\ 8 \mathrm{~A} & 173 & 900 & \\ \mathrm{~B} & 97 & 900 & \\ \mathrm{BB} & 45 & 2000 & \begin{array}{c}45 \mathrm{kPa}, \\ \text { lower gauge }\end{array}\end{array}$

\section{Sampling procedure.}

Preparation. Assemble the MOUDI for sampling by first inserting a filter in the backup filter holder at the base of the MOUDI, then by placing clean impaction plates on each stage starting from bottom to top of the impactor. Coating the plates with silicone spray (to prevent bounce) is necessary only in dry, dusty conditions. Finally, place the cover onto the upper stage.

Checking air flow rate. Start the pump and adjust the pressure reading to $1250 \mathrm{~Pa}$ for the upper gauge and $45 \mathrm{kPa}$ for the lower one. If this cannot be accomplished, stop the pump and disassemble the MOUDI. Reassemble it after checking each stage to see if the gaskets are properly placed and greased. Then repeat the air flow test. If the pressure readings are satisfactory, proceed to sampling.

Caution: After testing the air flow rate, always check the backup filter, which is easily broken by a reverse airflow when the sampling pump is shut off.

Sampling. For radon progeny, the sampling time is usually $5 \mathrm{~min}$, but 10 to 
$20 \mathrm{~min}$ is also used for low radon concentrations. For measurements of thoron progeny, a sampling period of 10 or more hours might be needed. In this case, use the rotating feature by turning on the switch at the base of the MOUDI. Use a stopwatch to time the sampling and keep the watch running to time the interval between sampling and counting.

Alpha counting. After sampling, disassemble the MOUDI starting with the upper stage and working downward to the base. Transfer the impaction plates into the alpha counters, being careful to keep them in order. Alpha count the plates simultaneously and analyze for activity using methods described in Procedure 2.2.4.6.

Generally, 10 alpha counters are needed. To complete sample transfer within $2 \mathrm{~min}$, two experienced operators are needed.

Calculation of particle size. Follow the procedures given in Procedure 2.2.2.8, Sections C. 6 or C.7. Use the calibration curves supplied by the manufacturer.

\section{REFERENCES}

Marple, V. A., Rubow, K. L. and Behm, S. M.

"A Microorifice Uniform Deposit Impactor (MOUDI): Description, Calibration, and Use"

Aerosol Science and Technology, 14, 434-446 (1991)

\section{A. Introduction.}

We describe in this section EML's diffusion batteries (DB), and their use in measuring aerosol particle size distributions. There are five EML designs covering two main applications: 


$\begin{array}{lccc}\text { Type of diffusion battery } & \begin{array}{c}\text { No. of } \\ \text { channels } \\ \text { or stages }\end{array} & \begin{array}{c}\text { Typical } \\ \text { flow rate } \\ \left(\mathrm{L} \mathrm{\text {min } ^ { - 1 } )}\right.\end{array} & \begin{array}{c}\text { Typical } \\ \text { application }\end{array} \\ \text { Multichannel disk-type } & 5 & 3 & \text { Radon progeny } \\ \text { Multistage disk-type } & 12 & 4 & \text { Aitken particles } \\ \text { Multichannel screen-type } & 5 & 25 & \text { Radon progeny } \\ \text { Multistage screen-type } & 11 & 4 & \text { Aitken particles } \\ \text { Multichannel carbon-type } & 5 & 283 & \text { Radon progeny }\end{array}$

The multichannel designs, also called parallel diffusion batteries, are those in which air is drawn simultaneously through side-by-side samplers. Thus, all aerosol samples needed to construct the particle size distribution are collected simultaneously. In the multistage design, also called series, the aerosol samples are collected sequentially. The term Aitken particles refers to outdoor atmospheric particles with diameters $<0.2 \mu \mathrm{m}$. (However, diffusion batteries can be used for somewhat larger particles, either indoors or outdoors.) The term radon progeny particles refers to those few particles, typically 1 in $10^{6}$, which carry short-lived radon progeny atoms. The batteries can also be used for other species, such as sulfate in airborne particles.

The type most commonly used in recent years is the multichannel screen battery, so this will be used for illustration in the discussion to follow. Consult Knutson and Sinclair (1979), Sinclair (1972), Sinclair and Hoopes (1975), and Sinclair et al. (1978) for further information on the other types.

We also use wire screens for a different but related radon progeny aerosol measurement (George, 1972).

\section{B. Description of the multichannel screen diffusion battery.}

Figure 2.1 shows the main components of the multichannel screen diffusion battery, and Figure 2.2 shows one of the components in the cross section. The system consists of five filter-type aerosol sampler units, each preceded by a different number of screens: 


$\begin{array}{lrrrrr}\text { Sampler unit: } & \text { B0 } & \text { B1 } & \text { B2 } & \text { B3 } & \text { B4 } \\ \text { Number of screens: } & 0 & 1 & 5 & 15 & 40\end{array}$

(Unit B0 is also called the reference filter.)

The screen used is a standard industrial twill-weave stainless-steel screen with the following dimensions:

$\begin{array}{ll}\text { Mesh } & 250 \mathrm{~cm}^{-1} \\ \text { Wire diameter } & 20 \mu \mathrm{m} \\ \text { Screen thickness }^{*} & 50 \mu \mathrm{m} \\ \text { Solid fraction } & 0.345 \\ \text { Diameter of flow area } & 10.16 \mathrm{~cm}\end{array}$

${ }^{*}$ As measured with a machinist's micrometer

\section{Sampling procedure.}

In normal use, the five samplers are loaded with clean $0.8 \mu \mathrm{m}$ pore membrane filters (Type AA, Millipore Corp., Bedford, MA) and air is drawn at $25 \mathrm{~L} \mathrm{~min}^{-1}$ through each unit. As the air flows through the screens, particles are selectively removed from the airstream and deposited on the wires. Particles that escape collection by the screens are collected on the filters. For radon progeny the preferred sampling period is $5 \mathrm{~min}$, but 10 or $20 \mathrm{~min}$ can be used if the concentration is low. For other species, the sampling period must be adjusted.

After sampling, the filters are removed and analyzed. When analyzing for radon progeny, any of the three alpha-counting protocols described in Section 2.2.4 can be used. As described there, it is important that the counting begin no more than $2 \mathrm{~min}$ after the end of sampling. This can be accomplished if two experienced operators are on hand at the time when the filters are transferred. 


\section{Calculations.}

Recovery of the particle size distribution from the activity measured on the five filters is possible only with a quantitative knowledge of transport through the screens as a function of the particle size. We currently use the "CKK" equation (Cheng et al., 1980; Cheng and Yeh, 1983):

$$
\Phi=\exp [-\mathrm{n} \cdot \mathrm{m} \cdot \ln (10)]
$$

where

$$
\begin{aligned}
& \Phi=\text { the fractional penetration through a stack of } n \text { screens } \\
& \mathrm{n}=\text { number of screens in the stack } \\
& \mathrm{m}=\mathrm{A}_{0} \mathrm{Pe}^{-2 / 3}+\mathrm{A}_{1} \mathrm{R}^{2}+\mathrm{A}_{2} \mathrm{R}^{2 / 3} \mathrm{Pe}^{1 / 2} \\
& \mathrm{~A}_{0}=1.96, \mathrm{~A}_{1}=3.37, \mathrm{~A}_{2}=1.94 \\
& \mathrm{Pe}=\mathrm{u}_{\mathrm{f}} \mathrm{d}_{\mathrm{w}} / \mathrm{D}, \text { the Peclet number } \\
& \mathrm{R}=\mathrm{d}_{\mathrm{p}} / \mathrm{d}_{\mathrm{w}}, \text { the interception parameter } \\
& \mathrm{D}=\mathrm{kTC} /\left(3 \pi \mu \mathrm{d}_{\mathrm{p}}\right), \text { the diffusion coefficient } \\
& \mathrm{k}=1.38 \mathrm{x} 10^{-23} \mathrm{~J} \mathrm{~K}^{-1}, \text { Boltzmann's constant } \\
& \mathrm{T}=\text { temperature in degrees absolute, normally } 293^{\circ} \mathrm{K} \\
& \mathrm{d}_{\mathrm{w}}=\mathrm{wire}_{\mathrm{p}}=1+\left(\lambda / \mathrm{d}_{\mathrm{p}}\right)[2.514+0.800 \text { exp }(-0.55 \mathrm{~d} \mathrm{p} / \lambda)], \text { the Cunningham slip factor } \\
& \lambda=\text { mean free path of air molecules, normally } 66 \times 10^{-9} \mathrm{~m} \\
& \mathrm{~d}=1 \text { diameter, } \mathrm{m}
\end{aligned}
$$




$$
\begin{aligned}
& \mu=\text { viscosity of air, normally } 1.81 \times 10^{-5} \mathrm{~Pa} \mathrm{sec} \\
& u_{f}=\text { air approach velocity, } \mathrm{m} \mathrm{sec}^{-1} .
\end{aligned}
$$

The values for $A_{0}, A_{1}$, and $A_{2}$ apply to the standard ( 250 mesh $\mathrm{cm}^{-1}$ ) screen - see Cheng and Yeh (1983) for other screens. The equation for the Cunningham factor is from Hinds (1982). In some of our computer programs we use an older equation with slightly different coefficients.

To analyze data from our diffusion batteries, we use one of the following two computer codes, which run on IBM-compatible personal computers:

Unfold.Pas - This is a Pascal program that uses the above-described Twomey algorithm. An alternative algorithm, the expectation-maximization algorithm, described by Maher and Laird (1985), is also available.

NMSimplx.Pas - This program, also in Pascal, makes use of the Nelder-Mead downhill simplex method to fit single or dual lognormal distributions to the data.

The logic involved in the first of these is the same as that in ExMaxDB.Pas, described in Knutson (1989).

\section{REFERENCES}

Cheng, Y. S. and H. C. Yeh

"Performance of a Screen-Type Diffusion Battery"

V. A. Marple and B. Y. H Liu (Editors)

in: Aerosols in the Mining and Industrial Environment

Ann Arbor Science Publishers, Ann Arbor MII (1983)

Cheng, Y. S., J. A. Keating, and G. M. Kanapilly

"Theory and Calibration of a Screen-Type Diffusion Battery"

J. Aerosol Sci., 11, 549-556 (1980) 
George, A. C.

"Measurement of the Uncombined Fraction of Radon Daughters with Wire Screens"

Health Physics, 23, 390-392 (1972)

Hinds, W. C.

Aerosol Technology

John Wiley, NY (1982)

Knutson, E. O.

"Personal Computer Program for Use in Radon/Thoron Progeny Measurements"

USDOE Report EML-517, March (1989)

Knutson, E. O. and D. Sinclair

"Experience in Sampling Urban Aerosols with the Sinclair Diffusion Battery and Nucleus Counter"

Proceedings: Advances in Particle Sampling and Measurement, EPA Report No. EPA600/7-79-065, pp. 98-120, February (1979)

Sinclair, D.

"A Portable Diffusion Battery - Its Application to Measuring Aerosol Size Characteristics"

Am. Ind. Hyg. Assoc. J., 33, 729-735 (1972)

Sinclair, D. and G. S. Hoopes

"A Novel Form of Diffusion Battery"

Am. Ind. Hyg. Assoc. J., 즈, 39-42 (1975)

Sinclair, D., A. C. George and E. O. Knutson

"Application of Diffusion Batteries to Measurement of Submicron Radioactive Aerosols" in: Airborne Radioactivity

American Nuclear Society, La Grange Park, II, Order No. 71001.pp. 103-114 (1978) 


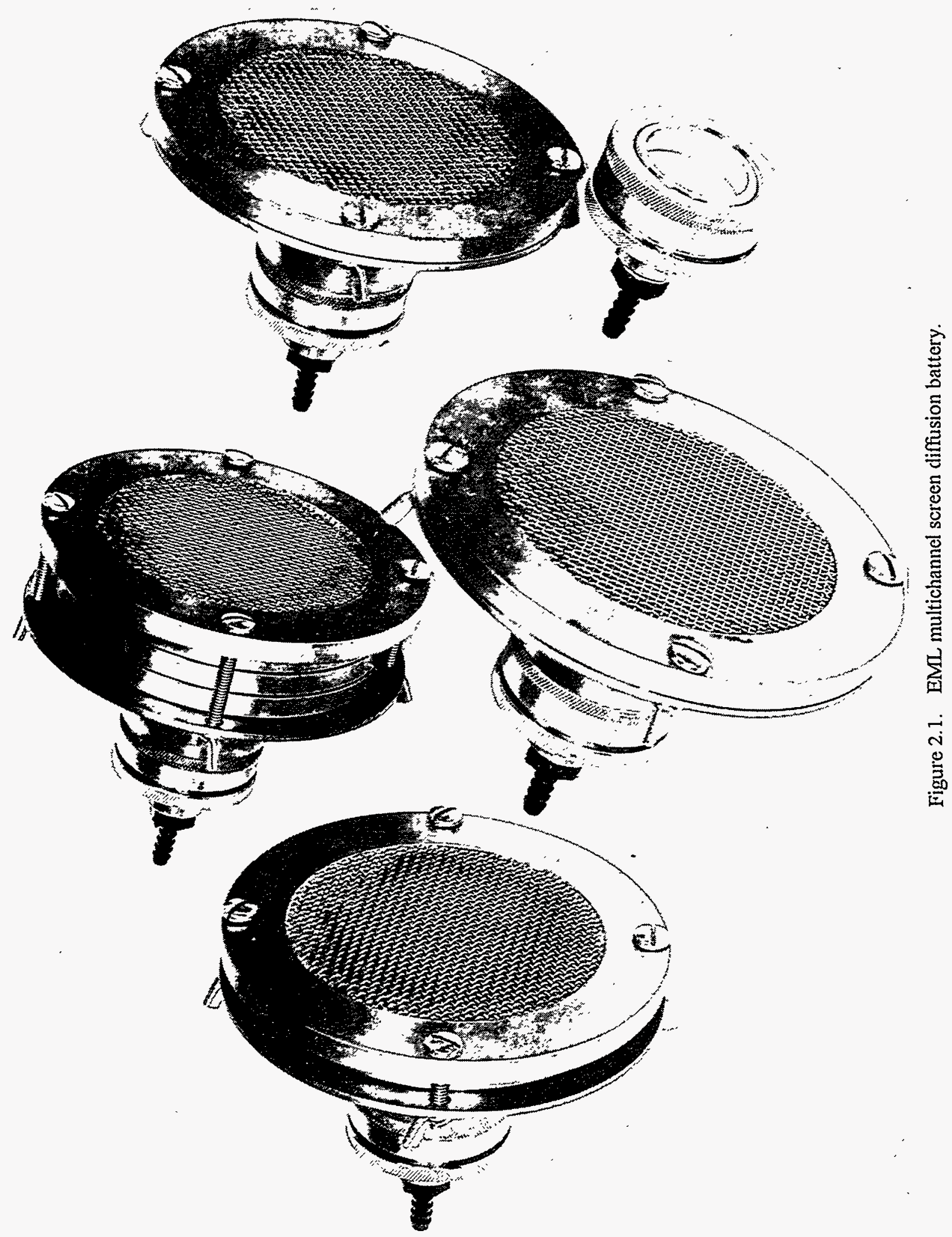









\section{A. Introduction.}

The graded screen array (GSA, also called the graded screen diffusion battery) is used for measuring the particle size of radon progeny in the range below $20 \mathrm{~nm}$. The GSA is not capable of dealing with larger sized aerosol particles, particularly those above $50 \mathrm{~nm}$. Therefore, the GSA should be used simultaneously with one of the diffusion batteries shown in Section 2.2.2.6 or with the MOUDI impactor (see Section 2.2.2.5). For example, the GSA used together with the MOUDI provides size information in the range of $0.5-5000 \mathrm{~nm}$.

It is permissible to use the GSA alone, but only in laboratory studies in which radon progeny or thoron progeny are sampled from a chamber free of larger aerosol particles.

\section{B. Apparatus required.}

1. One set of four circular stainless steel screens, each mounted on a metal ring - the properties of these screens are:

\begin{tabular}{|c|c|c|c|c|}
\hline $\begin{array}{c}\text { Screen } \\
\text { label }\end{array}$ & $\begin{array}{l}\text { Mesh, } \\
\mathrm{cm} \\
\end{array}$ & $\begin{array}{l}\text { Wire diameter, } \\
\mathrm{cm} \\
\end{array}$ & $\begin{array}{c}\text { Screen } \\
\text { thickness, cm }\end{array}$ & $\begin{array}{c}\text { Solid } \\
\text { fraction }\end{array}$ \\
\hline 60 & 23.6 & 0.0187 & 0.0356 & 0.360 \\
\hline 100 & 39.4 & 0.0108 & 0.0249 & 0.308 \\
\hline 200 & 78.7 & 0.0052 & 0.0135 & 0.275 \\
\hline 635 & 250 & 0.0020 & 0.0050 & 0.345 \\
\hline
\end{tabular}


Two ring sizes are available: small and large. The dimensions and recommended uses are shown below.

\begin{tabular}{|c|c|c|c|c|c|c|}
\hline $\begin{array}{c}\text { Size } \\
\text { of } \\
\text { Ring }\end{array}$ & $\begin{array}{l}\text { Outside } \\
\text { diameter, } \\
\mathrm{cm} \\
\end{array}$ & $\begin{array}{l}\text { Inside } \\
\text { diameter, } \\
\mathrm{cm} \\
\end{array}$ & $\begin{array}{l}\text { Ring } \\
\text { thickness, } \\
\mathrm{cm} \\
\end{array}$ & $\begin{array}{l}\text { Flow } \\
\text { area, } \\
\mathrm{cm}^{-2}\end{array}$ & $\begin{array}{l}\text { Typical } \\
\text { flow rate, } \\
\underline{L \text { min }^{-1}}\end{array}$ & $\begin{array}{l}\text { For use at radon } \\
\text { concentration, } \\
\quad \mathrm{Bq} \mathrm{m} \\
\end{array}$ \\
\hline Small & 2.67 & 1.91 & 0.25 & 2.86 & 3 & $>1000$ \\
\hline Large & 5.19 & 3.96 & 0.38 & 12.3 & 10 & $>200$ \\
\hline
\end{tabular}

2. A holder for the size of the screen selected.

3. A supply of Metricel DM-800 filters of the same size as the selected screens, and an open-faced holder.

4. A pair of rotameter-type flow meters, one for the GSA and one for the open-faced filter, and a suction pump.

5. Five drawer-type scintillation alpha counters of the type described in Section 2.2.4, Table 2.4.

6. A personal computer equipped with Keithley-Metrabyte CTM-05 (or equivalent) pulse-counting hardware and the software program ALPHALOG, as described in Section 2.2.4.6.

7. A stopwatch.

\section{Procedure}

1. Set up and check out the alpha-counting equipment, as described in Section 2.2.4.3B.

2. Calibrate both flow meters as described in Section 2.2.4.2A. 
3. Using the alpha counters, check the background activity of the wire screens (the background should be no higher than 0.2 counts $\mathrm{min}^{-1}$ ). This step may be omitted if it is known that the screens have not been used in the past $24 \mathrm{~h}$.

4. Mount the four screens into the holder so that the air will flow through in the sequence 60-100-200-635; orient the mounting rings so that the flush side faces upstream.

5. Set up the filter for sampling, as described in Section 2.2.4.2B; set up the screenholder in the same way.

6. Draw samples simultaneously through the filter and the screens, as described in Section 2.2.4.6.

7. After sampling, transfer the screens and the filter into separate alpha counters, and perform minute-by-minute counting using ALPHALOG, as described in Section 2.2.4.6.

8. Use program RWRENN6.EXE to calculate activity concentrations from the above count data.

9. Use this data to calculate particle size distributions, as described in Section 2.2.2.8. This method has been used to measure the diffusion coefficient of unattached radon progeny in filtered room air (George, 1994).

\section{Quality Control.}

1. For the air sampling and alpha counting steps, follow the quality control procedures outlined in Section 2.2.4.2A and 2.2.4.3B.

2. Whenever possible, take duplicate or triplicate samples from each experimental condition. 


\section{REFERENCES}

George, A. C. and E. O. Knutson

"Particle Size of Unattached Radon Progeny in Filtered Room Air"

Radiation Protection Dosimetry, 56, 119-121 (1994)

\section{A. Introduction.}

This section describes the steps needed to calculate particle size distributions from samples taken with the MOUDI impactor (Section 2.2.2.5), a diffusion battery (Section 2.2.2.6), and a graded screen array (Section 2.2.2.7), used individually or in combination. It can also be used for Sierra impactor data (Section 2.2.2.4).

The mathematical algorithms used in these calculation programs are described by Maher and Laird (1985), Solomon and Ren (1992), Knutson (1991), and Twomey (1975). Early versions of some of these programs are given in Knutson (1989). The full set of updated programs is available on a disk labeled EOKEMLUS.DOE.

\section{B. Equipment and supplies needed.}

1. An input data file (on disk) produced by the program RWRENN6.EXE.

2. Copies (on disk) of the files $A B C^{\wedge} \mathbb{N P} 2 . E X E, N M S I M P L X . E X E$, UNFOLDPS and EMLMOUDI.EFF.

3. An IBM-compatible personal computer, preferably 386 or better.

\section{Procedure.}

1. Prepare the computer for this task as follows: 
a. From the root directory of the computer's C: drive, make a directory called UTH and copy the above. EXE files into the UTIL directory.

b. Include the UTIL directory in the PATH statement.

c. From the root directory, make a directory called (for example) SIZEDATA and copy the above-mentioned input data file and the EMLMOUDI.EFF file into this directory.

d. Change to the SIZEDATA directory.

2. Type $A B C^{\wedge} \mathbb{N P} 2$; when requested, supply:

- the name of the RWRENN6 output file;

- the number of data points in each data set (that is, the number of alpha counters that were used to collect the data);

- whether or not the RWRENN6 program was instructed to analyze for thoron as well as radon progeny;

- $\quad$ answer $Y$ to the question about mapping.

This procedure produces a new file, with name ending in the character ${ }^{\wedge}$, that is ready for use by either NMsimplx or UnfoldPS.

From this point on, the procedure differs according to which sampler or combination of samples was used. 


\begin{tabular}{|c|c|c|}
\hline Sampler. & $\begin{array}{c}\text { Program } \\
\text { to use }\end{array}$ & Option \\
\hline Diffusion battery & NMsimplx & Classical RnP \\
\hline Graded screen array & NMsimplx & Unimodal \\
\hline $\begin{array}{l}\text { Diffusion battery plus } \\
\text { graded screen array }\end{array}$ & Nmsimplx & $\begin{array}{l}\text { Bimodal (fixed, common } \\
\text { geometric standard deviation, } \\
\text { GSD) }\end{array}$ \\
\hline MOUDI impactor & UnfoldPS & \\
\hline $\begin{array}{l}\text { MOUDI plus graded } \\
\text { screen array }\end{array}$ & UnfoldPS & \\
\hline
\end{tabular}

Both of these programs require that certain information be entered from the keyboard. When answering a $\mathrm{Y}$ or $\mathrm{N}$ question it is not necessary to press ENTER. Other responses require pressing the ENTER key to signal completion of the response. For many questions, the proper response will be obvious; these are not discussed in the itemized procedure below.

3. Diffusion Battery Alone. As indicated above, use NMSIMPLX. Supply data as indicated below. Type:

- a mask, such as *.??^, for the input data file,

- the number of the input data file, or 0 to try another mask,

- $Y$ in answer to the question about error terms,

- A to select diffusion battery,

- a digit from 1 to 5 to specify which diffusion battery,

- the flow rate in $\mathrm{L} \mathrm{min}^{-1}$,

- the uncertainty in the flow rate (normally about 3\% of the flow rate itself),

- 5 to select the Classical RnP,

- $Y$ in answer to the question about default starting values,

- 0 in answer to the question about Monte Carlo replications,

- or $\mathrm{N}$, as appropriate, for the default temperature and pressure,

- $\quad \mathrm{Y}$ in answer to each question about acceptable data quality. 
The progress of the calculation can be viewed on the screen as NMsimplx runs. The results will also be stored in an ASCII file with an extension .NM5. The program produces an uncertainty estimate for each of the calculated parameters; this estimate is printed directly below the value of parameter.

4. Graded Screen Array Alone. Use NMsimplx. Type in the data indicated below.

- a mask, such as *.??^, for the input data file,

- the number of the input data file, or 0 to try another mask,

- $\quad Y$ in answer to the question about error terms,

- B to select graded screen array,

- $\quad \mathrm{N}$ in answer to the questions about adding screens and showing rules,

- BCDE X in answer to prompt for GSconfig, ${ }^{1}$

- $\quad Y$ in answer to the question about alpha counting,

- the flow rates in $\mathrm{L} \mathrm{min}^{-1}$,

- the uncertainty in the flow rate(s) (normally about $3 \%$ of the flow rate itself),

- $\quad \mathrm{N}$ to the question about inlet losses,

- the flow area of the screens that were used,

- 1 to select unimodal,

- $\quad \mathrm{N}$ in answer to the question about default starting values,

- $\quad 1$ in answer to the request for a starting diameter,

- $\quad \mathrm{Y}$ or $\mathrm{N}$, as desired, to the question about switching to diffusion coefficient, (if no, answer $\mathrm{Y}$ or $\mathrm{N}$, as appropriate, to the next question about temperature and pressure),

- 0 in answer to the question about Monte Carlo replications,

- $\quad Y$ in answer to each question about acceptable data quality.

The program will run and produce a file as described under Diffusion Battery Alone, except that the file extension will be .NM1.

1 This is the proper response if: the four screens were stacked into one holder during the sampling, such that the air flowed through in the sequence $B-C-D-E$; the filter was in a separate holder; and the data in the input file are in the sequence $B, C, D, E, X$ Otherwise, answer $Y$ to the question about screen rules, and use these rules to decide how to answer the GSconfig prompt. 
5. Diffusion Battery plus Graded Screen Array. Use NMsimplx. Type in the information itemized below.

- a mask, such as *.??^, for the input data file,

- the number of the input data file, or 0 to try another mask,

- $\mathrm{Y}$ in answer to the question about error terms,

- $A B$ to select the combination of diffusion battery and graded screens, ${ }^{1}$

- a digit from 1 to 5 to specify which diffusion battery,

- the diffusion battery flow rate in $\mathrm{L} \mathrm{min}^{-1}$,

- the uncertainty in the above flow rate (normally about $3 \%$ of the flow rate itself),

- $\quad \mathrm{N}$ in answer to the questions about adding screens and showing rules,

- BCDE X in answer to prompt for GSconfig, ${ }^{2}$

- $\quad \mathrm{Y}$ in answer to the question about alpha counting,

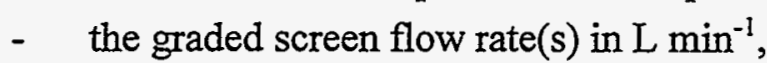

- the uncertainty in the above flow rate(s) (normally about $3 \%$ of the flow rate itself),

- the flow area of the screens that were used,

- 2 to select bimodal with fixed, common GSD,

- $\quad 1.5$ in response to the prompt for a value of GSD,

- $\quad \mathrm{N}$ in answer to the question about default starting values,

- 1100 in answer to the request for a two starting diameters,

- 0 in answer to the question about Monte Carlo replications,

- $\mathrm{Y}$ or $\mathrm{N}$, as appropriate, to the question about temperature and pressure,

- $\quad \mathrm{Y}$ in answer to each question about acceptable data quality.

The output file from the above steps will have the extension .NM2.

1 This is the proper response if the diffusion battery data precedes the graded screen data in the input data file. Otherwise interchange $A$ and $B$.

2 This is the proper response if: the four screens were stacked into one holder during the sampling, such that the air flowed through in the sequence $B-C-D-E$; the filter was in a separate holder; and the data in the input file are in the sequence $B, C, D, E, X$. Otherwise, answer $Y$ to the question about screen rules, and use these rules to decide how to answer the Gsconfig prompt. 
6. MOUDI, alone or with a reference filter. Use UnfoldPS. Before proceeding, review the input data file and make note of which impactor stages are represented in the file. (Often the upper stages are not alpha-counted.) Also review the EMLMOUDI.EFF file and make note of the $50 \%$ cut points of the first and last stage for which there is data in the input file. Type in the following information:

- a mask, such as *.??^, for the input data file,

- the number of the input data file, or 0 to try another mask,

- $\mathrm{Y}$ in answer to the question about error terms,

- C to select impactor,

- $\quad 40$ in answer to the question about smallest size, ${ }^{1}$

- 4000 in answer to the question about largest size, ${ }^{2}$

- 2 to select lognormal basis functions,

- 1.3 in answer to the question about GSD,

- $\quad \mathrm{Y}$ or $\mathrm{N}$, as appropriate, concerning the temperature and pressure,

- EMLMOUDI.EFF in response to the prompt for an info file,

- $\quad 1$ in answer to the question about particle density, ${ }^{3}$

- $\quad \mathrm{Y}$ or $\mathrm{N}$, as appropriate, to the questions about data present in the input file,

- $\mathrm{Y}$ in answer to the questions about data quality,

- $\quad 0$ for the number of Twomey iterations,

- 100 for the number of E-M iterations,

- $\quad 0.00005$ for the convergence criterion,

- $\quad \mathrm{N}$ in answer to the question about skipping negative values,

- $\quad 0$ in response to the prompt about Monte Carlo error study,

- $\quad \mathrm{Y}$ in answer to the question about long-form output,

- ENTER in answer to the request for file name,

- $\quad \mathrm{Y}$ in answer to the question about short-form output,

- ENTER in answer to the request fór file name,

'This value should be no smaller than $80 \%$ of the $50 \%$ cut-point of the last impactor stage for which there is data in the input file.

2 This value should not exceed $120 \%$ of the $50 \%$ cut-point of the first impactor stage for which there is data in the input file.

3 Entering 1 will cause the results to be presented in terms of equivalent unit-density spheres, i.e., the aerodynamic equivalent diameter. Entering the actual density of the particle will cause the results to be presented in terms of the Stokes equivalent diameter. 
- $\quad Y$ in response to the question about 50-line screen.

As UnfoldPS runs, the progress of the current calculation is shown near the bottom of the screen. The upper part of the screen shows the results of the previous calculation. When finished, the long-form output can be found in a file with extension ending in \&, and the short-form output will be in a file with extension ending in.

7. MOUDI plus Graded Screen Array. Use UnfoldPS. Before proceeding, review the input data file and the EMLMOUDI.EFF file as described in the last section. Then type in the following information -

- a mask, such as *.??^, for the input data file,

- the number of the input data file, or 0 to try another mask,

- $Y$ in answer to the question about error terms,

- $\quad$ CB to specify the type of sampler, ${ }^{1}$

- 0.5 in answer to the question about smallest size,

- 4000 in answer to the question about largest size, ${ }^{2}$

- 2 to select lognormal basis functions,

- 1.3 in answer to the question about GSD,

- $\mathrm{Y}$ or $\mathrm{N}$, as appropriate, concerning the temperature and pressure,

- EMLMOUDI.EFF in response to the prompt for an info file,

- $\quad 1.5$ in answer to the question about particle density, ${ }^{3}$

- $\mathrm{Y}$ or $\mathrm{N}$, as appropriate, to the questions about data present in the input file,

- $\quad \mathrm{N}$ in answer to the questions about adding screens and showing rules,

- $\quad$ BC in answer to the prompt for GSconfig, ${ }^{4}$

1 This is the correct response if the impactor data precedes the grades screen data in the input file; otherwise, interchange $B$ and $C$.

2 This value should not exceed $120 \%$ of the 50\% cut-point of the first impactor stage for which there is data in the input file.

3 This value of particle density is approximately correct for indoor aerosols; the results will be $g$ iven in terms of Stokes equivalent diameter, which is appropriate when combining data from impactors and diffusion batteries, or graded screens.

4 This assumes that data are available only from screens $B$ and $C$, and that these two screens were stacked during sampling, such that the air flowed through in the sequence B-C. Otherwise, read the "screen rules" to determine the proper entry for GSconfig. 
- $\quad Y$ in answer to the question about alpha counting,

- the graded screen flow rate in $\mathrm{L} \mathrm{min}^{-1}$,

- the uncertainty in the above flow rate (normally about $3 \%$ of the flow rate itself),

- the flow area of the screens that were used,

- $Y$ in answer to the questions about data quality,

- 0 for the number of Twomey iterations,

- 1000 for the number of E-M iterations,

- 0.00005 for the convergence criterion,

- $\mathrm{N}$ in answer to the question about skipping negative values,

- $\quad 0$ in response to the prompt about Monte Carlo error study,

- $Y$ in answer to the question about long-form output,

- ENTER in answer to the request for file name,

- $Y$ in answer to the question about short-form output,

- ENTER in answer to the request for file name,

- $\quad Y$ in response to the question about 50-line screen.

\section{Quality Control.}

1. Using a text-viewing or text-editing program, examine the output file from NMsimplx or from UnfoldPS. Starting at the end of the file, spot-check the column titled "RawData" to ensure that the input data have been properly matched to the stages of the sampling device. It will be necessary to consult the RWeENN6 output file to determine the proper matching.

2. Examine each block of results in the output file. Any block of data that has more than one negative input datum should be discarded. Examine also the "ChiSqr" value, which is a measure of the internal consistency of the data. If this number is $>25$, the data block should be discarded unless the reason for the high value can be identified and fixed.

3. Similarly if the ChiSqr value is $<1$, the data block should be discarded because the uncertainties in the input data are too large to support calculation of the particle size distribution. 


\section{. REFERENCES}

Knutson, E. O.

"Personal Computer Programs for Use in Radon/Thoron Progeny Measurements"

DOE Report EML-517 (1989)

Knutson, E. O.

"Application of the Expectation-Maximization Algorithm to the Processing of Cascade Impactor Data: The Method of Lognormal Components"

J. Aerosol Sci., 22, 267-270 (1991)

Maher, E. F. and N. M. Laird

"Algorithm for Reconstruction of Particle Size Distributions from Diffusion Battery Data"

J. Aerosol Sci., 16, 557-570 (1985)

Solomon, S. B. and T. Ren

"Counting Efficiencies for Alpha Particles Emitted from Wire Screens"

Aerosol Sci. and Tech., 17, 69-83 (1992)

Twomey, S.

"A Comparison of Constrained Linear Inversion and a Iterative Nonlinear Algorithm Applied to the Indirect Estimation of Particle Size Distributions"

J. Comput. Phys., 18, 188-200 (1975)

\subsubsection{RADON AND THORON}

\subsubsection{1}

\section{INTRODUCTION}

The procedures and instruments commonly used at EML for measuring radon are presented here. The main sampling methods are grab, two filter tube, continuous, and 
integrating. Both active and passive methods are included. These methods are used in laboratory applications as well as in situations requiring mobility and portability.

2.2.3.2

GRAB SAMPLING FOR RADON

Three sizes of scintillation flasks, all based on a design described in George (1976), are used at EML. Typical characteristics are shown in Table 2.1. The flasks are made at EML from methylmethacrylate stock. The bottom plate, made from sheet, is cemented to the body, made from tubing. The top plate, also from sheet, slides into the body and is sealed with an O-ring. The top plate is fitted with two petcock valves. All three flasks are designed to be counted on $12.7-\mathrm{cm}$ phototubes.

As the radon inside the flask decays, most of the radon progeny that are produced plate out on the interior surfaces of the flask. The alpha particles from radon and radon progeny interact with the $\mathrm{ZnS}(\mathrm{Ag})$ atoms to produce scintillations which are detected, amplified, and converted to an electrical signal by the phototube.

\section{A. Flask coating procedure.}

In this procedure, we place a thin phosphor coat on all internal surfaces. The thin coating on the bottom plate adds $15-20 \%$ to the sensitivity. The materials and steps used in this process are listed below.

\section{Coating materials:}

1. Silver activated zinc sulfide phosphor (Wm. B. Johnson Associates, PO Box 472, 216 Edgar Ave., Ronceverte, WV 24970).

2. Bonding solution (Caution-Flammable-Use Hood). Dissolve $30 \mathrm{~mL}$ of silicone fluid (Dow Corning, Midland, MI; Silicone Fluid 200) in $285 \mathrm{~mL}$ of reagent benzene plus $285 \mathrm{~mL}$ of reagent cyclohexane. Stir until the silicone is completely dissolved. This is enough to coat about 100 flasks. 


\section{Coating steps (use a hood):}

1. Clean the flask with a nonabrasive cleaner. [A plastic cleaning foam (REN RP-70 cleaner) has been found to be excellent.]

2. Remove cover, add $50 \mathrm{~mL}$ of bonding solution, replace cover, close both valves, and rotate the flask slowly until all surfaces including the bottom are coated. Open valves and remove cover.

3. Pour out excess bonding mixture into a second clean flask or return to the stock bottle. Let flask air dry for a few minutes.

4. Add 15-20 g of phosphor, replace cover, and close valves.

5. Shake gently until all surfaces are coated. Open valves and remove cover.

6. Pour out the loose powder and blow off excess phosphor from the flask and from the cover with clean air.

7. Replace cover and purge the flask with aged (radon free) air or nitrogen for a few minutes. Close the valves and store the flask for use.

8. When background becomes excessive, wipe out phosphor with clean tissue, clean as above, and rephosphor.

\section{B. Sampling and counting procedures.}

1. Connect a high collection efficiency filter to one valve of the flask to remove particulates and radon progeny. Connect the other valve to an air pump and draw air at 2-10 L min $\mathrm{min}^{-1}$ for 2-5 min for scintillation flasks of $165-2000 \mathrm{~mL}$.

2. Turn off pump and close both valves. An alternate method for filling the flask is to evacuate it down to $133 \mathrm{~Pa}(1 \mathrm{~mm} \mathrm{Hg})$ of pressure prior to filling with the test atmosphere.

3. Count the scintillation flask on a $12 \mathrm{~cm}$ phototube beginning $3 \mathrm{~h}$ after sampling. 
4. To convert the counting rate to radon concentration, use the calibration factor $\left(C_{f}\right)$ obtained from a test in a known radon environment and the appropriate decaycorrection. Each scintillation flask should have its own $\mathrm{C}_{\mathrm{f}}$ which can be influenced by its size and by the phototube assembly. It is very important that the entire system (scintillation flask, and phototube) is calibrated as a unit. Typical $\mathrm{C}_{\mathrm{f}}$ for the three scintillation flasks used at EML are shown in Table 2.1.

5. Determine the radon concentration at the time of sampling by applying the decay correction $\left(\mathrm{D}_{\mathrm{f}}\right)$ :

$$
D_{f}=\exp \left(\frac{-0.693 t}{T_{1 / 2} \text { for radon }}\right)=\exp (-0.0075 t)
$$

where $t$ is the time in hours from the midpoint of sampling to the midpoint of counting, and $T_{1 / 2}$ is the half-life of radon $(91.7 \mathrm{~h})$.

\section{REFERENCE}

George, A. C.

"Scintillation Flasks for the Determination of Low-Level Concentrations of Radon"

Proceedings of Ninth Midyear Health Physics Symposium, Denver, Colorado, February (1976)

\subsubsection{3}

TWO-FILTER TUBE SAMPLING FOR RADON AND THORON

The two-filter tube sampling method described in this section is used for simultaneous measurements of airborne radon and thoron (Knutson et al., 1994). 


\section{A. Description.}

The sampling tube $(9.7 \mathrm{~cm}$ diameter $\times 61 \mathrm{~cm}$ in length) has two filter heads. The front end of the tube is fitted with a $10.5-\mathrm{cm}$ filter holder to accommodate two $10.5-\mathrm{cm}$ high efficiency glass fiber filters mounted in series for the collection of all particulates including radon and thoron progeny. The back end of the tube is fitted with a 4.7-cm filter head assembly to hold a $4.7-\mathrm{cm}$ diameter high efficiency Metricel membrane filter paper. This filter has very low alpha-radioactivity background ( 0.05 counts $\left.\mathrm{min}^{-1}\right)$ and antistatic electrical properties. The background counting rate of the back-end filter is critical in measuring the expected low counting rate from the buildup of radon and thoron progeny originating inside the tube. The sampling flow rate, ranging from $30-50 \mathrm{~L} \mathrm{~min}^{-1}$, is high enough to minimize the loss of ${ }^{216} \mathrm{Po}$ and ${ }^{218} \mathrm{Po}$ atoms to the wall of the tube and to maximize the collection on the back-end filter.

\section{B. Sampling and counting.}

1. Mount two $10.5-\mathrm{cm}$ diameter glass fiber filters in series in the front end of the tube and a single $4.7-\mathrm{cm}$ Metricel membrane filter in the back end of the tube.

2. Connect the intake of an air pump to the back end of the tube and sample for 5-6 $\mathrm{h}$.

3. At the end of sampling, transfer the back-end filter into a 5-cm diameter gross alpha scintillation counter and begin to count as soon as possible in short time intervals (5$10 \mathrm{~min}$ ) for 4-6 h. One convenient way to do this is to make use of a portable personal computer equipped with pulse-counting hardware and the software program ALPHALOG (see Section 2.2.4.6). The sooner the counting begins the better the precision that is obtained for radon measurements; for thoron, the sensitivity increases with counting times of several hours.

\section{Calculations.}

The concentrations of radon and thoron are calculated from the radioactivity measured on the back-end filter using a computer program JWTI, which is based on the recursion formula method of Samuelson (1987). This method is very flexible employing different sampling regimes and counting intervals. The calculated penetration $\left(\mathrm{F}_{f}\right)$ of ${ }^{216} \mathrm{Po}$ from thoron and ${ }^{218}$ Po from radon is governed by the flow rate and the value for the diffusion 
coefficient, $D$, used for these isotopes. The relationship between $D$ and $F_{f}$ is obtained from Table 2.2, using the value of the parameter $\mu$ given by the expression

$$
\mu=\frac{\pi \times \mathrm{D} \times \mathrm{L}}{\mathrm{Q}}
$$

where

$$
\begin{aligned}
& \pi=3.14 \\
& D=\text { diffusion coefficient, } \mathrm{cm}^{2} \sec ^{-1}\left(0.085 \mathrm{~cm}^{2} \mathrm{sec}^{-1}\right) \\
& L=\text { length of tube, } \mathrm{cm}(61 \mathrm{~cm}) \\
& Q=\text { sampling flow rate, } \mathrm{cm}^{3} \mathrm{sec}^{-1}\left(500-800 \mathrm{~cm}^{3} \mathrm{sec}^{-1}\right)
\end{aligned}
$$

The value of $D=0.085 \mathrm{~cm}^{2} \mathrm{sec}^{-1}$ is used for very fresh radon progeny. The concentration of radon or thoron is underestimated by $3 \%, 4.5 \%$, and $6.0 \%$ if the value used for $\mathrm{D}=0.06,0.05$, and $0.043 \mathrm{~cm}^{2} \mathrm{sec}^{-1}$, respectively.

A computer program JWT1 is used to calculate both radon and thoron concentrations in $\mathrm{Bq} \mathrm{m} \mathrm{m}^{-3}$. The lower limit of detection is $5 \mathrm{~Bq} \mathrm{~m}^{-3}$ for both radon and thoron. For concentrations higher than $200 \mathrm{~Bq} \mathrm{~m}^{-3}$, a smaller two-filter tube can be used (Thomas, 1970).

\section{REFERENCES}

Knutson, E. O., A. C. George, P. Shebell and C. V. Gogolak

"EMI Thoron Gas Measurements"

Radiation Protection Dosimetry, 6, 263-266 (1994)

Samuelson, C.

"Applications of a Recursion Formula to Air Sampling of Radon Daughters" Nuclear Instruments and Methods in Physics Research, A262, 457 (1987) 
Thomas, J. W. and P. C. LeClare

"A Study of the Two-Filter Method for Radon-222"

Health Phys., 18, 113 (1970)

\subsubsection{4}

\section{CONTINUOUS RADON MONITORING}

Continuous reading radon monitors are ideal for measuring varying concentrations of radon over a long period of time. The scintillation flask used for radon grab sampling can be adapted to measure radon continuously by sampling at flow rates of $1-2 \mathrm{~L} \mathrm{~min}^{-1}$ as described in Thomas and Countess (1979). Any of the three flasks used at EML can serve this purpose. The flask is mounted on top of the phototube in light-proof housing. Sample air is drawn continuously through the flask by means of a pump, with a high efficiency filter mounted inline to remove dust particles and radon progeny. The number of alpha counts accumulated at the end of each counting interval, usually 30-60 min, is stored in a computer or printed out on paper tape.

The entire system is calibrated in a continuous operation mode to obtain the appropriate $C_{f}$ at a fixed flow rate and at varying radon concentrations. In an atmosphere in which the concentration of radon changes drastically, the calibration of the whole system becomes tedious. However, with the aid of modern desk computers the average radon concentration during the sampling interval can be calculated accurately. This is accomplished by means of an equation that takes into consideration the number of counts obtained in preceding sampling intervals (Thomas and Countess, 1979).

The $\mathrm{C}_{\mathrm{f}}$ from four continuous 2-L scintillation flask radon monitors used in the EML radon calibration facility at flow rates of $1 \mathrm{~L} \mathrm{~min}^{-1}$ range from $0.0019-0.0020$ counts $\mathrm{min}^{-1}$ per $\mathrm{Bq} \mathrm{m} \mathrm{m}^{-3}$ (4.2-4.5 counts $\mathrm{min}^{-1}$ per $\left.\mathrm{pCi} \mathrm{L}^{-1}\right)$. The range in factors results from differences in phototubes and scintillation flasks. 


\section{REFERENCE}

Thomas, J. W. and R. J. Countess

"Continuous Radon Monitor"

Health Phys., 36, 734 (1979)

\subsubsection{5}

\section{INTEGRATING RADON MONITORS}

The activated carbon monitor is used to obtain the average radon concentration over a 1-3 day period of passive sampling. The original design (George, 1984) was based on the M11 charcoal canister. Although there are currently many variants of the activated carbon monitor, the device described below is the one most used at EML.

\section{A. Description.}

This monitor consists of the following materials: (1) a metal can with a lid (1.2-cm diameter by $2.9-\mathrm{cm}$ deep); (2) $75 \mathrm{~g}$ of 8-16 mesh activated carbon (Calgon, Pittsburgh, PA); (3) metal screen ( $80 \mathrm{mesh}$ ) to keep the carbon in place; (4) removable internally expanding retaining ring to secure the screen; and (5) a strip $(35 \mathrm{~cm})$ of vinyl tape for sealing the lid to the can when closed. After loading and after each use, the carbon monitor is heated at $100^{\circ} \mathrm{C}$ for a minimum of $10 \mathrm{~h}$ to drive off any previously adsorbed radon and water.

The counting system consists of: (1) an $8.0-\mathrm{x}-8.0 \mathrm{~cm} \mathrm{NaI}(\mathrm{Tl})$ detector inside a 9-cm lead shield coupled to a compact multichannel pulse-height analyzer and a printer. The counting system is operated with the discriminator set to include gamma energies between 0.23 and $0.72 \mathrm{MeV}$, spanning the peaks of ${ }^{214} \mathrm{~Pb}(0.242,0.294$, and $0.352 \mathrm{MeV})$ and ${ }^{214} \mathrm{Bi}(0.609)$ $\mathrm{MeV}$. The normal counting period is $10 \mathrm{~min}$.

To determine the counting efficiency of the $\mathrm{NaI}$ detection system, a monitoring device is sealed after spiking with a known quantity of radon or ${ }^{226} \mathrm{Ra}$. This becomes the standard source with the proper counting geometry. A different standard source will be required 
for any change in the configuration of the monitor. Typically, the efficiency of the EML canister and counter combination is 0.117 counts per radon atom decay.

\section{B. Calibration.}

The monitors must be calibrated because the response depends on the length of exposure, relative humidity during the exposure, and on the type of carbon used. (Temperature effects encountered indoors are, however, insignificant because of the limited temperature range encountered.) Calibration is performed for several relative humidities from $20-90 \%$ and for 1-3 days of exposure. This calibration is required whenever new samplers are built using a new batch of activated carbon.

Each combination of exposure time and humidity yields a $C_{f}$ calculated from the following equation:

$$
C_{f}=\frac{1000 N}{60 E R n D_{f}}
$$

where

$$
\begin{aligned}
1000= & \mathrm{L} \mathrm{m}^{-3}, \\
\mathrm{~N}= & \text { net counting rate (counts } \left.\mathrm{min}^{-1}\right), \\
60= & \sec \mathrm{min}^{-1}, \\
\mathrm{E}= & \mathrm{NaI} \text { detector efficiency, typically } 0.117 \text { counts } \sec ^{-1} \mathrm{~Bq}^{-1} \mathrm{Rn}(0.26 \\
& \text { counts min } \left.\mathrm{pC}^{-1}\right), \text { and } \\
\mathrm{Rn}= & \text { radon concentration in Bq } \mathrm{m}^{-3}, \\
\mathrm{D}_{\mathrm{f}}= & \text { decay factor from the midpoint of exposure to the midpoint of } \\
& \text { counting (see Equation } 1, \text { Section } 2.2 .3 .2) .
\end{aligned}
$$

Typical results are shown in Table 2.3. The amount of water adsorbed, shown in the left column, was determined from the difference in weights before and after exposure. 


\section{Use of device.}

Before exposure the monitoring device is heated to regenerate it. Exposure times longer than 3 days should be avoided because water competes with radon adsorption and the effectiveness of the device as an integrating monitor will diminish.

To calculate the concentration of radon from the measured count rate and measured water uptake, Equation 2 is applied after solving for radon:with the symbols as

$$
=\frac{1000 \mathrm{~N}}{60 \mathrm{EC}_{\mathrm{f}} \mathrm{D}_{\mathrm{f}}}
$$

defined before. Use Table 2.3 to obtain the $C_{f}$. The $1 \sigma$ counting error is calculated from the following expression:

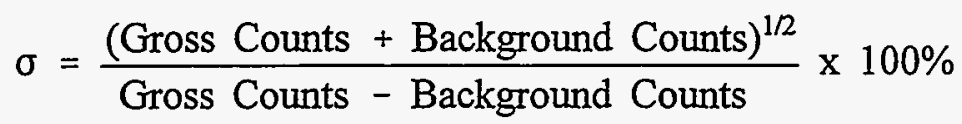

When exposed for 3 days and counted 3 days later, the device described here has a lower limit of detection of $7.4 \mathrm{~Bq} \mathrm{~m}^{-3}$. Other versions of the device that contain better adsorptive carbon can measure radon concentration levels at $3.7 \mathrm{~Bq} \mathrm{~m}^{-3}$ or less.

\section{REFERENCE}

George, A. C.

"Passive Integrated Measurement of Indoor Radon Using Activated Carbon"

Health Phys., $\underline{46}, 867$ (1984) 
To verify the measurement accuracy of all monitoring devices for radon and thoron, periodic intercomparison measurements are made with pulse ionization chambers (Fisenne and Keller, 1985). The pulse ionization chambers are the primary calibration instruments traceable directly to the NIST through use of their standard ${ }^{226} \mathrm{Ra}$ reference solutions. The accuracy of the thoron concentrations is obtained through intercomparisons (Knutson et al., 1994).

\section{REFERENCES}

Fisenne, I. M. and H. W. Keller

"The EML Pulse Ionization Chamber System for Radon-222 Measurements" USDOE Report EML-437, March (1985)

Knutson, E. O., A. C. George, P. Shebell and C. V. Gogolak

"EML Thoron Gas Measurements"

Radiation Protection Dosimetry, 6, 263-266 (1994)

TABLE 2.1

TYPICAL CHARACTERISTICS OF THE THREE SIZES OF EML SCINTILLATION FLASKS

\begin{tabular}{llll}
\hline Flask volume $(\mathrm{L})$ & 0.165 & 0.415 & 2.00 \\
$\mathrm{C}_{\mathrm{f}}$ : & & & \\
$\begin{array}{l}\text { counts } \mathrm{min}^{-1} \mathrm{~Bq} \mathrm{~m} \\
\text { counts } \mathrm{min}^{-1} \mathrm{pCi} \mathrm{L}^{-1}\end{array}$ & 0.022 & 0.054 & 0.135 \\
& 0.8 & 2.0 & 5.0 \\
LLD $\left(\mathrm{Bq} \mathrm{m}^{-3}\right)$ & 7.4 & 3.7 & 3.0 \\
\hline
\end{tabular}


TABLE 2.2

VALUES OF $F_{\mathrm{f}}$ AS A FUNCTION OF $\mu=\pi \times \mathrm{D} \times \mathrm{L} / \mathrm{Q}$

\begin{tabular}{llll}
\hline$\mu$ & & & \\
& & & \\
& & & $F_{f}$ \\
0.005 & 0.877 & 0.25 & 0.420 \\
0.008 & 0.849 & 0.30 & 0.384 \\
0.010 & 0.834 & 0.35 & 0.349 \\
0.020 & 0.778 & 0.40 & 0.324 \\
0.030 & 0.737 & 0.45 & 0.302 \\
0.040 & 0.705 & 0.50 & 0.282 \\
0.050 & 0.678 & 0.60 & 0.248 \\
0.060 & 0.654 & 0.70 & 0.220 \\
0.070 & 0.633 & 0.80 & 0.220 \\
0.080 & 0.614 & 0.90 & 0.178 \\
0.090 & 0.596 & 1.00 & 0.162 \\
0.100 & 0.580 & 1.50 & 0.110 \\
0.120 & 0.551 & 2.00 & 0.083 \\
0.140 & 0.525 & 2.50 & 0.067 \\
0.160 & 0.502 & 3.00 & 0.056 \\
0.180 & 0.481 & 4.00 & 0.042 \\
0.200 & 0.462 & 5.00 & 0.033 \\
& & & \\
\hline
\end{tabular}


TABLE 2.3

\section{$C_{f}:$ EFFECTIVE VOLUME SAMPLED AS A FUNCTION OF EXPOSURE TIME AND WATER ADSORBED}

\begin{tabular}{|c|c|c|c|}
\hline \multirow{2}{*}{$\begin{array}{l}\text { Water adsorbed } \\
\text { (g) }\end{array}$} & \multicolumn{3}{|c|}{$\begin{array}{l}\text { Effective sample volume }(L) \\
\text { for various exposure times }\end{array}$} \\
\hline & 1 day & 2 days & 3 days \\
\hline 0.500 & 144 & 216 & 259 \\
\hline 0.700 & 143 & 213 & 255 \\
\hline 1.000 & 142 & 210 & 251 \\
\hline 1.500 & 140 & 204 & 246 \\
\hline 1.700 & 138 & 202 & 242 \\
\hline 2.000 & 137 & 199 & 238 \\
\hline 2.500 & 135 & 196 & 233 \\
\hline 3.000 & 132 & 190 & 229 \\
\hline 3.500 & 131 & 187 & 225 \\
\hline 4.000 & 130 & 184 & 220 \\
\hline 4.500 & 127 & 181 & 216 \\
\hline 5.000 & 125 & 179 & 207 \\
\hline 6.000 & 121 & 167 & 199 \\
\hline 7.000 & 118 & 161 & 190 \\
\hline 8.000 & 114 & 153 & 181 \\
\hline 9.000 & 109 & 144 & 173 \\
\hline 10.000 & 105 & 135 & 164 \\
\hline 11.000 & 101 & 127 & 156 \\
\hline 12.000 & 096 & 121 & 151 \\
\hline
\end{tabular}


${ }^{220} \mathrm{Rn}$ AND ${ }^{222} \mathrm{Rn}$ IN SOIL GAS

\section{APPLICATION}

This section describes the procedures used to determine soil gas ${ }^{220} \mathrm{Rn}$ and ${ }^{222} \mathrm{Rn}$ concentrations using commercially-available scintillation cells and photomultiplier tube apparatus (referred to as "222 $\mathrm{Rn}$ detector" in the following procedure).

\section{A. Apparatus.}

1. Scintillation cells (Scintrex, Ltd., 222-T Snidercroft Rd., Concord, ON, Canada, nee EDA, Model \#RDX-013, or equivalent).

2. Photomultiplier tube and counting apparatus (Scintrex, Ltd., 222-T Snidercroft Rd., Concord, ON, Canada, Model \#RDA-200, or equivalent).

3. Stainless-steel sampling tubes, $0.95 \mathrm{~cm} \mathrm{ID}$, various lengths.

4. Portable battery-operated pump (Du Pont E. I. De Nemours and Co., Route 87 S., PO Drawer Z, Fayetteville, NC 28302, Model \#2500B, or equivalent) capable of $\sim 2.0 \mathrm{~L} \mathrm{~min}^{-1}$ at $\Delta \mathrm{p}$ of $10 \mathrm{kPa}$.

5. Flow meter, $0-5 \mathrm{~L} \mathrm{~min}{ }^{-1}$ (Cole-Parmer, or equivalent).

6. Pressure gauge, $0-25 \mathrm{kPa}$ (Dwyer Instruments, Inc., PO Box 373-T, Michigan City, $\mathbb{N}$ 46360 , or equivalent).

7. Short section of Tygon tubing (or equivalent) filled with dessicant held in place by glass wool, fitted at one end with an in-line filter holder containing Millipore (or equivalent) $0.8 \mu \mathrm{m}$ filter to remove progeny from initially entering scintillation cell.

8. Computer program RNTNCAL.FOR (Hutter, unpublished) or equivalent, for calculating ${ }^{220} \mathrm{Rn}$ and ${ }^{222} \mathrm{Rn}$ concentrations from scintillation cell counts. 


\section{B. Procedure.}

1. Emplace sampling tubes into the soil using reamer to pound, ensuring no soil is retained in tube, or alternatively, drill hole using auger and insert tube, tamping soil to minimize cavities along tube walls.

2. Obtain a 5-min background count for each scintillation cell to be used before the sample is drawn at the measurement site.

3. Obtain the soil gas sample using a portable battery-operated pump, according to the apparatus arrangement shown in Figure 2.3, with a flow rate of between 1 and $3 \mathrm{~L}$ $\mathrm{min}^{-1}$ for $1 \mathrm{~min}$. Measure and record the flow rate and the volume of the sample tubing. The sample is drawn directly into scintillation cells that have been previously inserted into the ${ }^{222} \mathrm{Rn}$ detector.

4. Turn the pump off, remove the tubes to the scintillation cell and screw on the cap to the ${ }^{222} \mathrm{Rn}$ detector.

5. Obtain a 1-min count (see Notes 1 and 2).

6. Remove the scintillation cell from the ${ }^{222} \mathrm{Rn}$ detector, and obtain another sample using a different scintillation cell, or see Note 3 to reuse a cell.

7. Obtain a 5 - or 10 -min count at least $5 \mathrm{~min}$ after the soil gas sample was drawn into the scintillation cell, and no longer than 3 or 4 days. Typically, these counts are obtained either on-site at the end of collecting all samples, or within an hour to two at a convenient (i.e., warm and dry) site.

8. Calculate the concentration of ${ }^{220} \mathrm{Rn}$ and ${ }^{222} \mathrm{Rn}$, correcting for decay of ${ }^{220} \mathrm{Rn}$ during sampling (Hutter, in press). This requires knowledge of the flowrate and sample tube volume. A computer program, RNTHCAIC.FOR, is available to do this calculation. 


\section{Notes:}

1. The time between the end of pumping and the start of counting must be as short as possible ( $<90 \mathrm{~s} ; 11 / 2$ half-lives of ${ }^{220} \mathrm{Rn}$ ). Typically, this delay time is about $10 \mathrm{sec}$. Record the time between the end of pumping and the start of counting to within $\pm 1 \mathrm{~s}$.

2. The counts $\mathrm{min}^{-1}$ from the sample must turn out to be at least 3 times the background. Obtain a new sample if this requirement is not met, either using a different scintillation cell, or by waiting until the background decreases.

3. If the 5 -min count is obtained $5 \mathrm{~min}$ after sampling is ended, the cell can then be purged with outdoor air and a new background count obtained, to be reused for the next sample, provided that the new background check shows the sample counts $\mathrm{min}^{-1}$ to be at least 3 times the background.

\section{QA/QC.}

Duplicate samples, defined as measurements performed on the same sampling hole on the same day $\sim 10$ to $15 \mathrm{~min}$ apart using a different scintillation cell, are performed at a rate of 1 in 10 for the purpose of assessing measurement precision. Second countings of samples are performed at approximately the same rate in order to ensure cell integrity with regard to leakage and to assess statistical counting uncertainties.

Calibrations of scintillation cells $/{ }^{222} \mathrm{Rn}$ detector apparatus are performed on at least a semiannual basis using the EML Radon Gas and Radon/Thoron Progeny Facilities for Testing and Research (see Section 6.2) or other radon chambers. At this time, it is useful to check for leaks in the scintillation cells by testing for consistency in the count rate, and by taking into consideration decay over several days. Approximately 1 in 5 cells obtained from a commercial vendor have been found to leak. Nearly all leaks can be sealed using epoxy around the window and all valve connections. 


\section{REFERENCES}

Hutter, A. R.

"A Method for Determining Soil Gas ${ }^{220} \mathrm{Rn}$ (Thoron) Concentrations" Health Physics, 68 835-839 (1995)

Hutter, A. R.

${ }^{1220} \mathrm{Rn} /{ }^{222} \mathrm{Rn}$ Ratios as Indicators of Soil Gas Transport"

(unpublished data)

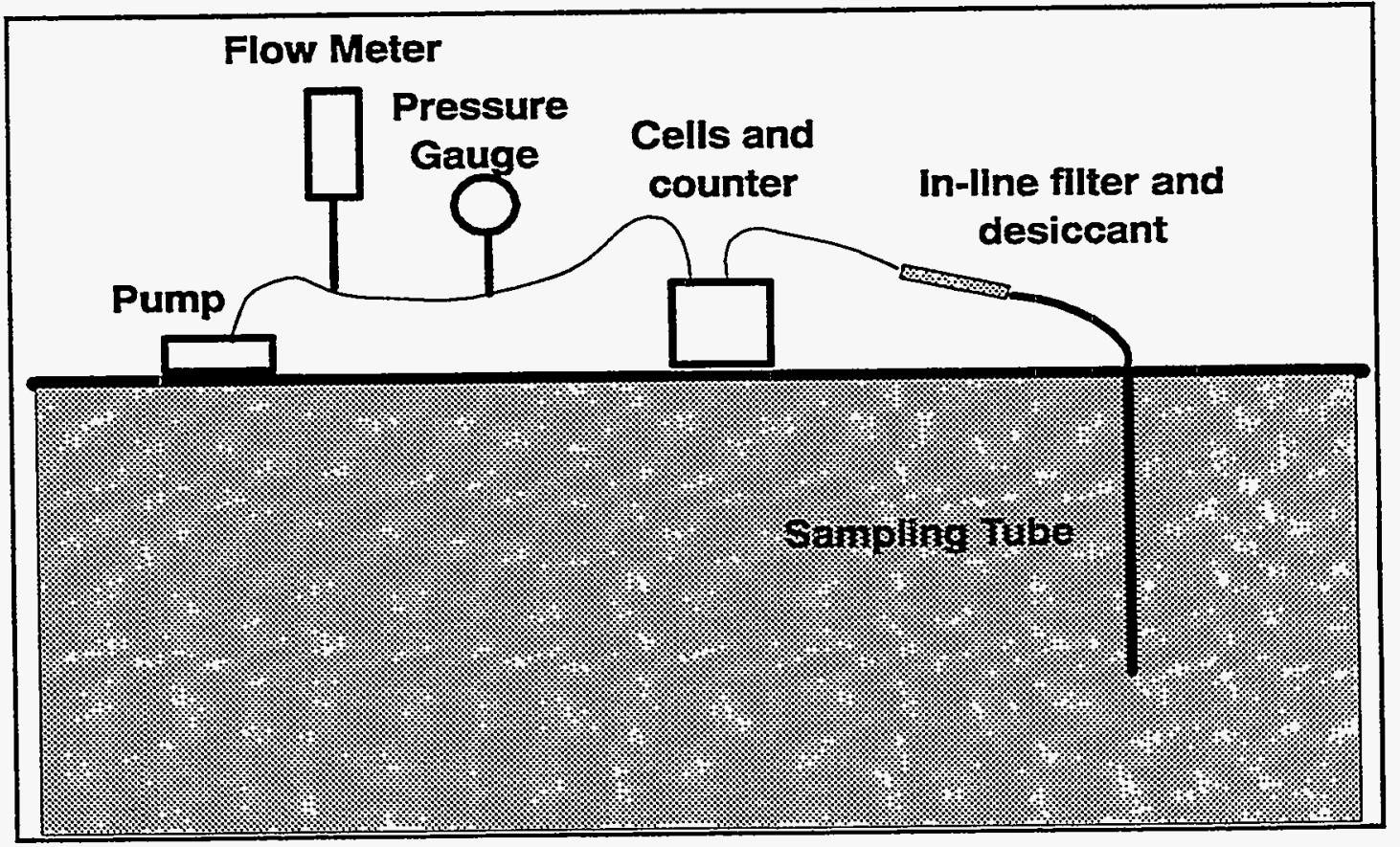

Figure 2.3. Schematic of soil gas sampling arrangement. 


\title{
2.2.4 RADON AND THORON PROGENY
}

\author{
2.2.4.1
}

INTRODUCTION

Presented here are the three methods commonly used at EML for determining the concentration of radon and thoron progeny in air. All three methods - modified Kusnetz, modified Tsivoglou, and Raabe-Wrenn least squares - are based on collection in a known volume of air through a filter in a known time period, and on counting the radioactivity on the filter after sampling. The methods described are suitable for use in the laboratory, in homes, and in the workplace. The same sampling and counting equipment are used, but with different counting and calculational procedures.

Units: when specifying the concentration of individual progeny nuclides, the Systeme Internationale (SI) unit is $\mathrm{Bq} \mathrm{m}^{-3}$; when specifying the potential alpha energy concentration (PAEC) of radon or thoron progeny, the SI unit used is $\mathrm{J} \mathrm{m}^{-3}$.

These procedures have also been adopted by the World Health Organization (George, 1993).

SAMPLING TRAIN

All three methods require a sampling train such as that shown in Figure 2.4.

\section{A. Air pump and flowmeter.}

A portable air pump capable of moving $10-20 \mathrm{~L} \mathrm{~min}^{-1}$ through a high efficiency filter is required. The pump should be rugged, light weight, quiet, and operate from the voltage line in the building. A calibrated flowmeter to measure the flow rate through the filter holder assembly should be attached upstream of the intake of the pump. Calibration of the flowmeter is air density dependent and care should be taken if used at different 
altitudes. The pump flowmeter train assembly should be calibrated at the same elevation, and at the same resistance that is caused by the filter and by the other component parts in the sampling train as shown in Figure 2.4. Never measure the air flow rate from the exhaust of the pump because of possible leakage in the pump housing.

\section{B. Air filters and filter holder.}

Use membrane filters with nominal pore size $<0.8 \mu \mathrm{m}$, or glass fiber with a collection efficiency of $>99.9 \%$. Membrane filters exhibit negligible alpha-particle absorption during alpha counting. Glass fiber filters allow deeper penetration of particles, but with the recommended flow rates, face velocity $\left(<100 \mathrm{~cm} \mathrm{sec}^{-1}\right)$, and short sampling period, particle burial in the filter is negligible. Any other type of filter medium should be investigated for good surface deposition characteristics. In general, small pore filters exhibit the best surface collection, but also present the highest resistance to air flow. To compensate for this, use a higher capacity pump or a larger diameter filter and filter holder. The diameter of the filter can either be 2.5 or $4.7 \mathrm{~cm}$ depending on the size of the filter holder and the detector counter used. The connecting tubing between pump and filter holder should be a thick-walled pressure type hose. Filter holders should be of the open faced type to prevent the loss of radon progeny particles due to plate out upstream from the filter. The biggest error in measuring radon progeny by collection on a filter is often from a defective filter holder. To prevent this, make sure the filter holder-filter assembly is airtight and has the proper O-ring seal.

\section{Timing device.}

Sample collection timing is very important in the short sampling periods used for short-lived radionuclides. Errors may be significant if operators rely on their wristwatch and on their memory. 


\section{A. Description.}

Table 2.4 shows the main characteristics of the two alpha-particle counters most used at EML to measure the alpha activity deposited on a filter. Both counters are designed for gross alpha (total alpha) counting, in which the alpha particles from ${ }^{218} \mathrm{Po}(6 \mathrm{MeV})$ and ${ }^{214} \mathrm{Po}(7.7 \mathrm{MeV})$ are counted without discrimination.

Both counting systems consist of a simple scintillation disc ( $\mathrm{ZnS}$ silver activated screen) mounted on or close to the face of a photomultiplier tube, and placed in close proximity to the filter. [In the TH-29-B (see Table 2.4), the scintillator is a separate Mylar disk coated on one side with $\mathrm{ZnS}$; be sure the coated side faces the filter.] The photo-multiplier tube and preamplifier are housed in light-tight housing and are connected to a stable high voltage supply and a scaler.

\section{B. Calibration.}

Table 2.5 shows the characteristics of the certified alpha standard source most used at EML to periodically measure the efficiency of the counting systems. The counting efficiency is the ratio of the counting rate (in counts $\mathrm{sec}^{-1}$ ) of the instrument to the known activity (in $\mathrm{Bq}$ ) of the standard source. The EML counters described above have an efficiency of about $48 \%$, but this figure should always be remeasured after the counters are moved to a field site.

Checking calibration is also a good quality assurance step. Measured efficiencies which are outside the range $47-49 \%$, or which are not reproducible, are an indication of equipment malfunction and these counters should not be used.

Because the counters are energy-independent, efficiencies determined with the ${ }^{241} \mathrm{Am}$ or ${ }^{239} \mathrm{Pu}$ sources (alpha energies $5.53 \mathrm{MeV}$ and $5.15 \mathrm{MeV}$, respectively) are believed to apply to radon/thoron progeny as well. For very precise work, the efficiency as measured above should be multiplied by the factor shown in Table 2.5 to correct for backscatter. 
This correction takes into account the fact that the alpha emission from the front face of a metal plate is slightly higher than from the same activity deposited on a filter.

\subsubsection{4}

MODIFTED KUSNETZ METHOD

The simplest method is the Modified Kusnetz, in which a 5 min air sample is taken on a high efficiency filter to be counted for 5-10 min any time between 35-95 min after the end of sampling. This method differs from the original method (Kusnetz, 1956) in that a scaler is used in place of a ratemeter and the counting time is longer.

\section{A. Procedure.}

Before sampling and counting a sample, the operator should ensure that the entire sampling train is in good working order and that the counting system is calibrated and running properly. The counting efficiency of the system and its background counting rate should be determined as discussed earlier.

Using a 2.5 or $4.7 \mathrm{~cm}$ open-faced filter holder, mount a membrane or glass fiber filter. Attach the filter holder upstream of a calibrated flowmeter with the proper hose and connect to the intake of an air pump.

Sample air from the environment to be tested for $5 \mathrm{~min}$ at a rate of $10-20 \mathrm{~L} \mathrm{~min}^{-1}$. After sampling, remove the filter containing the radon progeny from the filter holder and transfer it to the counting system for analysis. There is no urgency to do this since the operator has $35 \mathrm{~min}$ to commence counting. During that waiting period he/she may wish to take another sample in another part of the building using a new filter and the same filter holder and sampling equipment.

The modified Kusnetz method requires a single count of the filtered sample usually for $10 \mathrm{~min}$ in the counting interval from 35-45 min after the end of sampling, or at any other 10-min intervals between 35-85 min after the end of sampling. 


\section{B. Calculation.}

The potential alpha energy concentrations (PAEC) can be calculated from the equation:

$$
\text { PAEC in } n J m^{-3}=N /\left(E \times T_{s} \times Q \times T_{f}\right)
$$

where

$$
\begin{aligned}
& \mathrm{N}=\text { net counts } \mathrm{min}^{-1} \\
& \mathrm{E}=\text { fractional counter efficiency }\left(\text { counts } \mathrm{sec}^{-1} \mathrm{~Bq}^{-1}\right) \\
& \mathrm{T}_{\mathrm{s}}=\text { sampling time in } \mathrm{min}, \\
& \mathrm{Q}=\text { sampling flow rate in } \mathrm{m}^{3} \mathrm{~min}^{-1}, \text { and } \\
& \mathrm{T}_{\mathrm{f}}=\text { time factor given in Table } 2.6 .
\end{aligned}
$$

The relative standard deviation of the measurement in \% can be calculated from the following equation.

$$
\sigma_{p}=\frac{\sqrt{\frac{\text { gross count }(\mathrm{cpm})}{\text { time counted }(\mathrm{min})}+\frac{\text { background }(\mathrm{cpm})}{\text { time counted }(\mathrm{min})}}}{\text { gross counts }(\mathrm{cpm})-\text { background }(\mathrm{cpm})} \times 100
$$

Example: An air sample was collected for $T_{s}=5 \mathrm{~min}$, at a flow rate of $Q=10 \mathrm{~L} \mathrm{~min}^{-1}$ $\left(0.01 \mathrm{~m}^{3} \mathrm{~min}^{-1}\right)$. The counting was done from $35-45 \mathrm{~min}$ after the end of sampling. The total alpha count in that interval was 4200 counts. The counter background was 0.1 counts $\mathrm{min}^{-1}$ measured for a period of $25 \mathrm{~min}$. The counter efficiency, $\mathrm{E}$, was $0.48 . \mathrm{T}_{\mathrm{f}}$ at $40 \mathrm{~min}$, which is the midpoint of $35-45 \mathrm{~min}$, is 7.21 obtained from Table 2.6. The total count in that interval was 4200 counts. 
From Equation (1)

$$
\text { PAEC }=\frac{(4200 / 10-0.1)}{(0.48 \times 5 \times 0.01 \times 7.21)}=\frac{420}{0.173}=2420 \mathrm{~nJ} \mathrm{~m}
$$

The relative standard deviation of the PAEC measurement is

$$
\sigma_{p}=\frac{\sqrt{\frac{420}{10}+\frac{0.1}{25}}}{420-0.1}=0.015(1.5 \%)
$$

The modified Tsivoglou method is more complicated than the Kusnetz, but it yields additional information: the concentration of individual radon progeny (Thomas, 1972).

\section{A. Procedure.}

The sampling procedure is identical to that used for the modified Kusnetz method. (Actually all three methods can be used with the same filter containing sampled radon progeny.)

After the end of sampling, remove the filter containing radon progeny from the filter holder and transfer it to the counting system for analysis. The standard counting intervals are 2-5, 6-20, and 21-30 min after the end of sampling. These intervals allow adequate time to transfer the filter into the counting system and record the counts from the different counting intervals. The technician or the person who performs the test is required to stand by for $30 \mathrm{~min}$ unless he uses automated counting equipment operated by a computer. 


\section{B. Calculation.}

The equations needed to calculate the air concentrations from a 5 min sample and for the standard counting intervals are:

$$
\begin{aligned}
& C_{1}=\frac{\left[6.247 N_{t}(2-5)-3.028 N_{t}(6-20)+2.857 N_{t}(21-30)\right]}{E Q} \\
& C_{2}=\frac{\left[0.056 N_{t}(2-5)-0.776 N_{t}(6-20)+1.836 N_{t}(21-30)\right]}{E Q} \\
& C_{3}=\frac{\left[-0.832 N_{t}(2-5)+1.224 N_{t}(6-20)-1.389 N_{t}(21-30)\right]}{E Q} \\
& C_{p}=\frac{\left[2.011 N_{t}(25) 1.372 N_{t}(620)+3.954 N_{t}(2130)\right]}{E Q}
\end{aligned}
$$

where

$\mathrm{C}_{1}, \mathrm{C}_{2}, \mathrm{C}_{3}=$ the concentrations of ${ }^{218} \mathrm{Po},{ }^{214} \mathrm{~Pb}$, and ${ }^{214} \mathrm{Bi}$, respectively $\left(\mathrm{Bq} \mathrm{m} \mathrm{m}^{-3}\right)$,

$$
C_{p}=\text { the PAEC }\left(\mathrm{nJ} \mathrm{m}^{-3}\right)
$$

$N_{t}(\ldots)=$ the net counts in the intervals $2-5,6-20$, and $21-30$,

$$
\begin{aligned}
& \mathrm{E}=\text { fractional counter efficiency }\left[\left(\text { counts } \min ^{-1}\right) /\left(\text { disintegrations } \min ^{-1}\right)\right] \\
& \text { and }
\end{aligned}
$$

$$
\mathrm{Q}=\text { sampling air flow rate, } \mathrm{L} \mathrm{min}^{-1} \text {. }
$$

These coefficients were derived using $3.05 \mathrm{~min}$ for the half life of ${ }^{218} \mathrm{Po}$ (Martz et al., 1988). The half lives used for ${ }^{214} \mathrm{~Pb}$ and ${ }^{214} \mathrm{Bi}$ were 26.8 and $19.9 \mathrm{~min}$, respectively (ICRP, 1983).

The standard deviation equations which calculate the precision for each radionuclide are: 


$$
\begin{aligned}
& S_{1}=\frac{1}{E Q}\left[39.0 N_{t}(2-5)+9.17 N_{t}(6-20)+8.16 N_{t}(21-30)\right]^{1 / 2} \\
& S_{2}=\frac{1}{E Q}\left[0.003 N_{t}(2-5)+0.062 N_{t}(6-20)+3.37 N_{t}(21-30)\right]^{1 / 2} \\
& S_{3}=\frac{1}{E Q}\left[0.69 N_{t}(2-5)+1.50 N_{t}(6-20)+1.93 N_{t}(21-30)\right]^{1 / 2} \\
& S_{p}=\frac{1}{E Q}\left[4.04 N_{t}(2-5)+1.88 N_{t}(6-20)+15.6 N_{t}(21-30)\right]^{1 / 2}
\end{aligned}
$$

where

$$
\begin{aligned}
\mathrm{S}_{1}, \mathrm{~S}_{2}, \mathrm{~S}_{3} & =\text { standard deviation of the }{ }^{218} \mathrm{Po},{ }^{214} \mathrm{~Pb} \text {, and }{ }^{214} \mathrm{Bi}\left(\mathrm{Bq} \mathrm{m} \mathrm{m}^{-3}\right), \\
\mathrm{S}_{\mathrm{p}} & =\text { standard deviation of the } \operatorname{PAEC}\left(\mathrm{nJ} \mathrm{m}^{-3}\right)
\end{aligned}
$$

$\mathrm{N}_{\mathrm{t}}(\ldots), \mathrm{E}$ and $\mathrm{Q}$ were defined earlier.

The coefficients in the above four equations are the squares of the corresponding coefficients in Equations 3-6. Terms involving the uncertainty of the background count rate have been omitted because they are seldom significant.

A computer program based on the optimizing technique of Nazaroff (1984) (WWN5, for a PC; see Table 2.7) has been written to do the calculations in Equations 3-10. Results can be acquired within $15 \mathrm{sec}$ after the end of counting. The program can also do these calculations from any three-count radon progeny measurement.

The two outlined counting procedures can be applied to the same filter sample simply by using two counting regimes in sequence: three counting intervals $[(2-5),(6-20)$, and (21-30)] for the modified Tsivoglou method; a single counting interval from 35-45 min after the end of sampling (modified Kusnetz method). This provides a useful check against errors in transcribing data and in calculations. 
As used here, the term "Raabe-Wrenn least squares method" refers to a certain configuration of alpha counters, a computer, hardware, and software for measuring radon decay product concentrations (Raabe and Wrenn, 1969). This sytem can handle up to 10 samples simultaneously, which is needed when measuring the particle size distribution of radon progeny particles (Sections 2.2.2.5 - 2.2.2.7). The method is also convenient for single samples.

\section{A. Components.}

1. Up to 10 , as needed, drawer-type gross alpha counters, Model TH-29-B or equivalent.

2. A portable personal computer with two free 8-bit expansion slots.

3. Two CIO-CTR05 computer interface boards (Computer Boards 44 Wood Ave., Mansfield, MA 02048, 508-261-1123; FAX 508-261-1094) or equivalent, installed in adjacent slots of the above computer (item 2). One board must be set to address 0300 (hex) and the other to address 0304 (hex).

4. Two 37-pin D-connectors and hoods (Wire Pro, Inc., types 17-10370 and 17-1371; available from Newark Electronics, 212-963-0289). Install five female K-LOC connectors (King Electronics, Tuckahoe, NY, 914-793-5000) through the side panel of each hood, and wire them to the pins corresponding to the five CIO-CTR05 counter inputs. Note: the D-connectors must be made up in complimentary pairs so both can be connected to the CIO-CTR05 boards at the same time.

5. Up to 10 , as needed, RG-174 coaxial cables with K-LOC connectors (King Electronics, Tuckahoe, NY, 914-793-5000).

6. Copies of the computer programs ALPHALOG (or ALPHALG2) and RWRENN6; see Table 2.7. 


\section{B. Procedure.}

1. Set up apparatus; it is best to arrange the alpha counters in a line, five on either side of the computer. Install cables and power-packs.

2. Start the computer and make a directory with a name appropriate for the project you are working on. Copy ALPHALOG and RWRENN6 into this directory."

3. Start ALPHALOG; ensure that the computer's time and date are set correctly; when prompted, mlun the test using the internal $1000 \mathrm{~Hz}$ pulser (a 10-second counting period is appropriate) to verify that the boards have been properly installed.

4. Skip over the "Enter parameters" step (it is usually more convenient to enter the parameters during counting).

5. Set the counting period (normally 60 seconds) and the number of intervals (normally 1000) and press $Y$ in answer to the "Ready to Start" prompt. The program will go into a preview mode, in which counts are collected and displayed - but not saved every $10 \mathrm{sec}$.

6. Collect the filter or particle size samples as described in Sections 2.2.2.5, 2.2.2.6, 2.2.2.7, or 2.2.4.2.

7. Promptly transfer the filters or other sampling substrates into the alpha counters; be sure that the substrates are placed "dirty side up" and $\mathrm{ZnS}$ scintillator disks are placed "shiny side up."

8. Verify from the computer preview screen that all channels are collecting counts; then with an eye on the stopwatch, start official counting by hitting ENTER. Make note of the time at which counting was started.

9. Once the counting is well underway, choose option [F7] to record the parameters associated with the sample. Enter information when prompted.

' Or copy these two programs into a directory entitled EOKPROGS and place EOKPROGS into the PATH statement. 
10. After sufficient data has been collected, press [Shift-F10] to stop; $40 \mathrm{~min}$ of data is sufficient for calculating radon progeny, but $240-300 \mathrm{~min}$ is needed for calculating thoron progeny.

11. If the parameters were entered during the data collection period (step 9), press $Y$ to proceed directly to the program RWRENN6, which calculates the decay product concentrations. If not, press $\mathbf{N}$, then correct the parameters using an edit program such as MSDOS EDIT. Then run RWRENN6.

\section{REFERENCES}

George, A. C.

"Measurement of Airborne Radon-222 Daughters by Filter Collection and Alpha Radioactivity Detection"

B. Seifert, H. J. van de Wiel, B. Dodet, I. K. O'Neill (editors)

in: Environmental Carcinogens - Methods of Analysis and Exposure Measurement Indoor Air, WHO, IARC Scientific Publications No. 109, Lyon, Vol. 12, pp. 173-179, March (1993)

\section{ICRP}

Radionuclide Transformations: Energy and Intensity of Emissions.

Annals of the International Commission Radiological Protection, ICRP Publication 38, Vol. 11-13 (1983)

\section{Kusnetz, H. L.}

"Radon Daughters in Mine Atmospheres. A Field Method for Determining Concentrations"

Am. Ind. Hyg. Assoc. Q., 17, 85-88 (1956)

Martz, D. E., R. T. Harris, and G. H. Langner

"The Half-Life of ${ }^{218} \mathrm{Po}$ "

Report UNC-J-002-88, UNC Geotech, Grand Junction, CO (1988) 
Nazaroff, W. W.

"Optimizing the Total-Alpha Three-Count Technique for Measuring Concentrations of Radon Progeny in Residences"

Health Phys., 46, 395-405 (1984)

Raabe, O. G. and M. Wrenn

"Analysis of the Activity of Radon Daughter Samples by Weighted Least Squares" Health Phys., $\underline{17}$, 593-605 (1969)

Thomas, J. W.

"Measurement of Radon Daughters in Air"

Health Phys., 23, 783-789 (1972)

TABLE 2.4

DRAWER-TYPE ALPHA PARTICLE COUNTERS USED AT EML

\begin{tabular}{lcc}
\hline Designation & TH-29-B & Greybox \\
Built by & EML & EML \\
Date of design & 1981 & 1975 \\
Photomultiplier & & \\
$\quad$ tube diameter $(\mathrm{cm})$ & 5.0 & 12.7 \\
Largest filter & 5 & 10 \\
$\quad$ accommodated $(\mathrm{cm})$ & None & None \\
Window material & & 3 \\
Filter-to-window & 2 & 48 \\
$\quad$ spacing (mm) & 48 & 50 \\
Typical efficiency $(\%)$ & & \\
Estimated dead-time & 50 & \\
$\quad$ each pulse $(\mu \mathrm{s})$ & &
\end{tabular}


TABLE 2.5

ALPHA-EMITTING STANDARD USED AT EML

Identifying marks $\ldots \ldots \ldots \ldots \ldots \ldots \ldots \ldots$

When obtained ..................... Fall 1981

Where obtained $\ldots \ldots \ldots \ldots \ldots$ Oak Ridge National Laboratory

Active material . . . . . . . . . . . . ${ }^{241} \mathrm{Am}$

Size of active deposit $(\mathrm{mm}) \ldots \ldots \ldots \ldots \ldots$

Certifying agency $\ldots \ldots \ldots \ldots \ldots \ldots$ NIST

Certified activity (Bq)

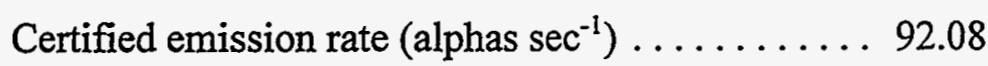

Mounting material .............. Stainless steel

Diameter of mount $(\mathrm{mm}) \ldots \ldots \ldots \ldots \ldots . \ldots 25.4$

Estimated ratio of emission to activity . . . . . 0.504

Condition as of February 1988 .......... Good

Efficiency multiplier ............... 0.992 
TABLE 2.6

TME FACTORS FOR USE IN THE MODIFIED

KUSNETZ METHOD

\begin{tabular}{|c|c|}
\hline $\begin{array}{l}\text { Time after sampling } \\
\text { (min) }\end{array}$ & $\begin{array}{l}\text { Time } \\
\text { factor }\end{array}$ \\
\hline 40 & 7.21 \\
\hline 42 & 7.02 \\
\hline 44 & 6.83 \\
\hline 46 & 6.63 \\
\hline 48 & 6.44 \\
\hline 50 & 6.25 \\
\hline 52 & 6.06 \\
\hline 54 & 5.87 \\
\hline 56 & 5.67 \\
\hline 58 & 5.48 \\
\hline 60 & 5.29 \\
\hline 62 & 5.10 \\
\hline 64 & 4.90 \\
\hline 66 & 4.71 \\
\hline 68 & 4.52 \\
\hline 70 & 4.32 \\
\hline 72 & 4.18 \\
\hline 74 & 4.04 \\
\hline 76 & 3.94 \\
\hline 78 & 3.75 \\
\hline 80 & 3.61 \\
\hline 82 & 3.51 \\
\hline 84 & 3.32 \\
\hline 86 & 3.17 \\
\hline 88 & 3.03 \\
\hline 90 & 2.88 \\
\hline
\end{tabular}

*Midpoint of counting interval. 
TABLE 2.7

COMPUTER PROGRAMS USED AT EML IN CONNECTION WITH RADON PROGENY MEASUREMENTS

Name Language Machine Method Comments

\begin{tabular}{|c|c|c|c|}
\hline ALPHALOG.PAS & Pascal & $\mathrm{PC}$ & $\begin{array}{l}\text { Simultaneous collection of } \\
\text { data from up to } 10 \text { alpha } \\
\text { counters }\end{array}$ \\
\hline WWN5.PAS & Pascal & $\begin{array}{l}\text { Modified } \\
\text { Tsivoglou }\end{array}$ & $\begin{array}{l}\text { Nazaroff equations (1984) for } \\
\text { any combination of sampling } \\
\text { and counting times, including } \\
\text { counting while sampling }\end{array}$ \\
\hline
\end{tabular}

RWRENN6.BAS GW BASIC PC Least squares Raabe and Wrenn (1969) 


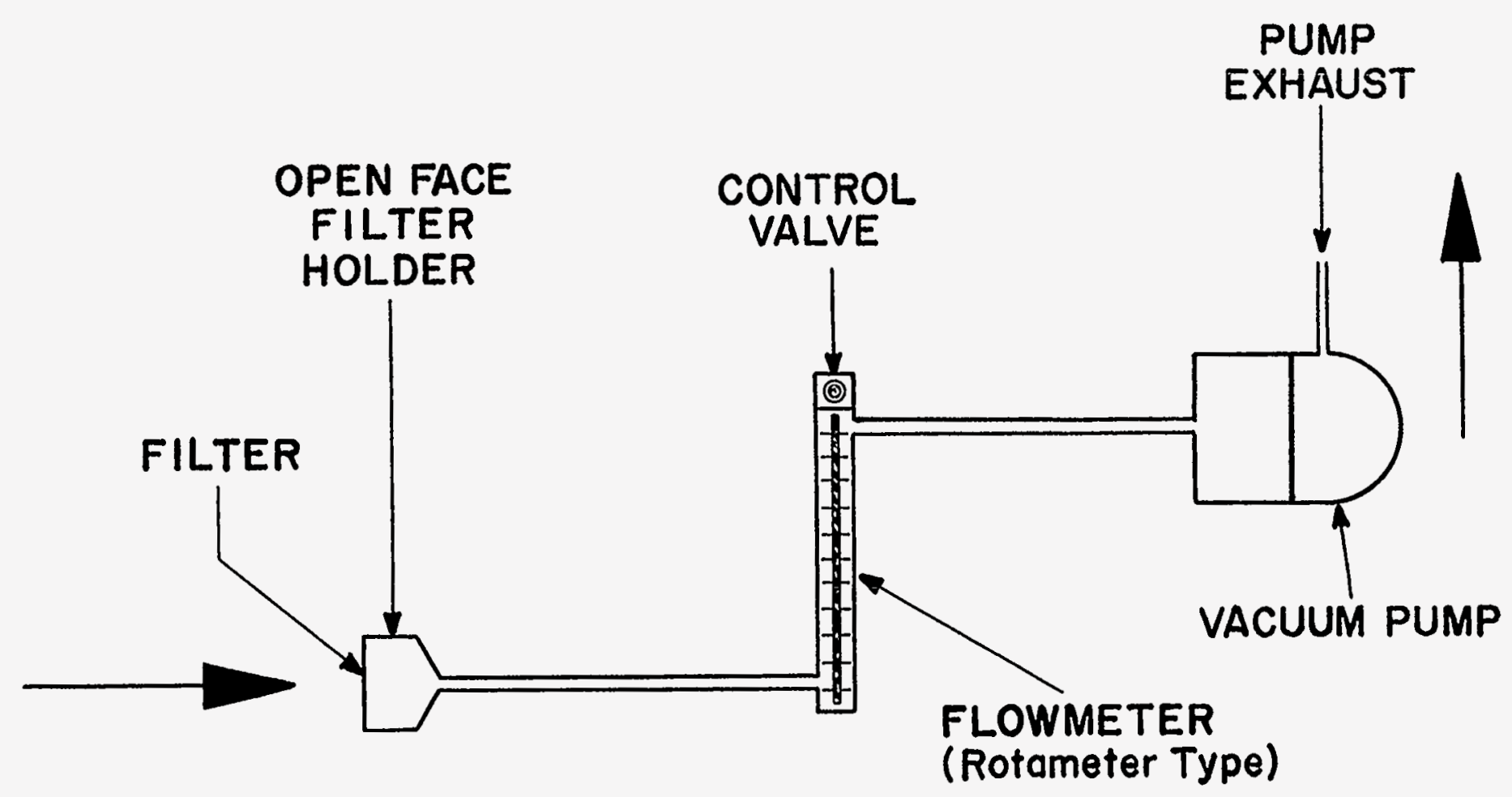

Figure 2.4. Sampling train for radon progeny measurements. 


\subsubsection{ATMOSPHERIC TRACING}

\subsubsection{1}

\section{INTRODUCTION}

This section describes the apparatus and procedures used by EML to sample tracer gases used in studies of long range atmospheric transport. The analysis of the collected samples is described separately in Section 4.2. Atmospheric tracing is done primarily to test meteorological models that predict the atmospheric transport and dispersion of pollutants over distances as far as $3000 \mathrm{~km}$ from the source(s). Among other things, these models are used in assessing the potential for "acid precipitation" in different regions and under different meteorological conditions.

The tracer gases used in EML atmospheric tracing experiments are:

\begin{tabular}{|c|c|c|}
\hline Tracer Gas & Acronym & $\begin{array}{l}\text { Global Background } \\
\left(\mathrm{fL} \mathrm{L} \mathrm{L}^{-1}\right) \\
\end{array}$ \\
\hline perfluoromethylcyclohexane & $\mathrm{PMCH}$ & 3.3 \\
\hline total perfluorodimethylcyclohexane & $\mathrm{PDCH}$ & 28.8 \\
\hline $\begin{array}{l}\text { perfluoromethylcyclopentane } \\
\text { ortho(cis)perfluoro- }\end{array}$ & PMCP & 3.2 \\
\hline dimethylcyclohexane & OC-PDCH & 0.15 \\
\hline perfluorotrimethylcyclohexane & PTCH & $<0.5$ \\
\hline
\end{tabular}


The programmable atmospheric tracer sampler (PATS) (Model RD113, Gilian Instrument Company, Dawes Highway, Wayne, NJ 07470) is used as the primary sampler for all perfluorocarbon tracers. The unit, shown in Figure 2.5, is housed in a waterproof $36 \mathrm{~cm} \times 25 \mathrm{~cm} \times 20 \mathrm{~cm}$ container and weighs $\sim 7 \mathrm{~kg}$. It consists of two sections: the lid (air flow module, AFM) and the base (power control module, PCM).

The lid holds 23 sampling tubes, each containing $150 \mathrm{mg}$ of Ambersorb adsorbent (Rohm and Haas). The Ambersorb absorbs the tracer from the sample air flowing through the tube. The air flow may be directed through a preselected tube by means of a multiple port switching valve (Scanivalve, 10222 San Diego Mission Road, San Diego, CA 92120) which is controlled by the PCM.

The base of the PATS contains a constant flow pump which draws air through the selected sampling tube. The constant flow pump is based on the EML tethered air pump system (Latner, 1986), and may be set to draw 10, 20,30, 40 or $50 \mathrm{~cm}^{3} \mathrm{~min}^{-1}$. The base also contains programmable timer controls that allow for single or multiple start and stop times over a 7-day period. These controls are also used for automatic analysis when the unit is coupled to a gas chromatograph.

Two liquid crystal displays in the base indicate the time of day, the day of the week, and the tube number, and a digital printer records this information at each tube change. As a precaution against printer failure, this information is also stored in an integrated memory circuit module (Polito, 1987) mounted on each lid. The memory module is used during the gas chromatographic analysis phase (see Section 4.2), and this information is stored in a computer for inclusion into a data base.

Power is supplied by an internal rechargeable battery which can operate the unit up to 30 days. Longer periods of operation are possible if $110 \mathrm{~V}$ A.C. power is available. All lids are interchangeable and usable with any base, so that after 23 samples have been collected, a new lid may be substituted to continue sampling. 


\section{A. Introduction.}

Programmable atmospheric tracer sampler (PATS) adsorbent tubes are decontaminated (baked out) by resistance heating of each tube at $>400^{\circ} \mathrm{C}$. This method is applicable to all PATS lids. Up to 10 lids may be baked out at one time (Figure 2.6).

\section{B. Special apparatus.}

1. Constant current power supply, Power Mate Corporation Model BPA 2086-V or equivalent with special interface.

2. Micromaster programmable controller, Model No WP6201-AA-AA or equivalent.

3. Matheson Model 3800 gas pressure regulator or equivalent (two regulators required).

4. PATS bases and lids (up to 10 ).

5. Primeline (Soltec) two channel strip chart recorder or equivalent.

C. Special gases.

1. Nitrogen, ultra high purity (UHP) $(99.999 \%)$ or equivalent.

2. Nitrogen, Matheson Purity (99.995\%) or equivalent.

\section{Procedure.}

1. Attach each lid to each base whose batteries have been fully charged. Disconnect all battery chargers.

2. Insert a jumper in the receptacle labelled Analysis Connector of each base to disable the sampling pump. 
3. Attach the UHP nitrogen line to the To Equalization port of each lid. Set the equalization nitrogen gas pressure to $207 \mathrm{kPa}$ (30 psig).

4. Plug each lid AFM electrical connector into each base receptacle labelled AFM Connector.

5. Set the clock time and day of the week of each PATS base and power up each unit. The clock time is set at the thumbwheel switch marked Set/Time Alarm and the day of the week by placing the appropriate Day Select switch to On.

6. Manually cycle through each of the 23 tubes using the base manual Valve Step switch. Cycle each lid at least twice to assure seating of the Scanivalve. Leave the tube number of each unit at " 00 " and turn off the power to each unit.

7. Attach the Matheson Purity Nitrogen gas line to the To PCM connector of each lid.

8. Set the UHP nitrogen pressure to $138 \mathrm{kPa}(20 \mathrm{psig})$ and turn the nitrogen gas on. Adjust the flow to $\sim 30 \mathrm{~cm}^{3} \mathrm{~min}^{-1}$.

9. For each base, set the thumbwheel switch marked Duration to 0016 and the Sample Quantity switch to "24". This sets the switching time between tubes and the number of tubes to be sequentially decontaminated. There are only 23 tubes in each lid, however, the base must switch to the 24th tube in order to complete the bake-out cycle.

10. Plug all the electrical jacks into the Desorption Power lid connector.

11. Turn the constant current power supply on and set the current to $16.0 \mathrm{~A}$.

12. Turn on the dual channel strip chart recorder to record the desorption voltage and current.

13. Quickly turn each unit on, push the PATS base Operate switch to Off, bring the Alarm toggle switch from Manual to Multi and then back to Manual. Bring the Operate toggle switch to Run. This procedure should be completed within $1 \mathrm{~min}$ for all 10 bases so that all units will start at the same time. 
14. At the next minute, tube number 1 of each unit will switch into position, the current will be turned on and the tube will be heated to $>400^{\circ} \mathrm{C}$ for a $15-\mathrm{min}$ period. The contaminants will be desorbed into the nitrogen carrier gas stream. At the end of the 15-min heating period the current will turn off. At $16 \mathrm{~min}$, the next tube will advance into position and the heating cycle will be repeated until all 23 tubes have been decontaminated.

15. Disconnect all wires and gas lines. Insert a $1 / 2$ hole septum over all lid inlet and outlet ports and disconnect the lid plug from the base.

\subsubsection{4}

DEPLOYMENT, SAMPLING, AND RETRIEVAL

Information on the deployment, sampling and retrieval may be found in the report by Draxler and Heffter (1986), which gives a detailed description of one tracing experiment.

\section{REFERENCES}

Dietz, R. N.

"Overview of Perfluorocarbon Tracer (PFT) Technology Applied to Quality Assurance of ANATEX Tracer Data"

Anatex Model Evaluation Workshop, Orlando, Florida, Oct. 20 and 21 (1987)

Draxler, R. and J. L. Heffter

"Across North America Tracer Experiment (ANATEX), Comprehensive Plan"

National Oceanic and Atmospheric Administration, Air Resources Laboratory, Silver

Spring, MD (November, 1986)

Latner, N.

"TAPS: Tethered Air Pump System"

USDOE Report EML-456 (1986) 
Lovelock, J. E. and G. J. Ferber

"Exotic Tracers For Atmospheric Studies"

Atmospheric Environment, 16, 1467-1471 (1982)

Polito, $\mathrm{M}$.

"A Data Acquisition System for Atmospheric Tracer Studies" USDOE Report EML-500 (1987) 


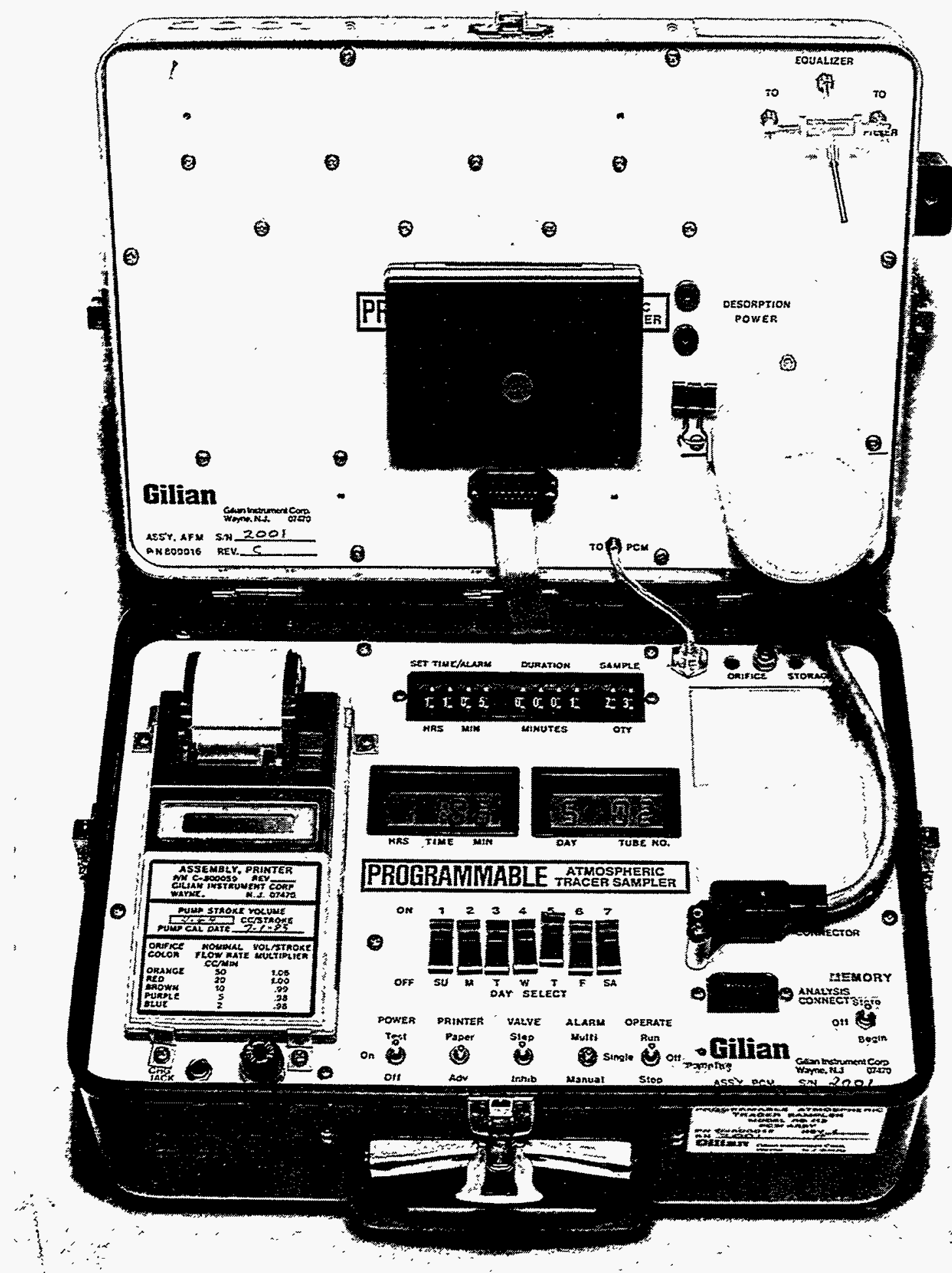

Figure 2.5. The programmable atmospheric tracer sampler. 


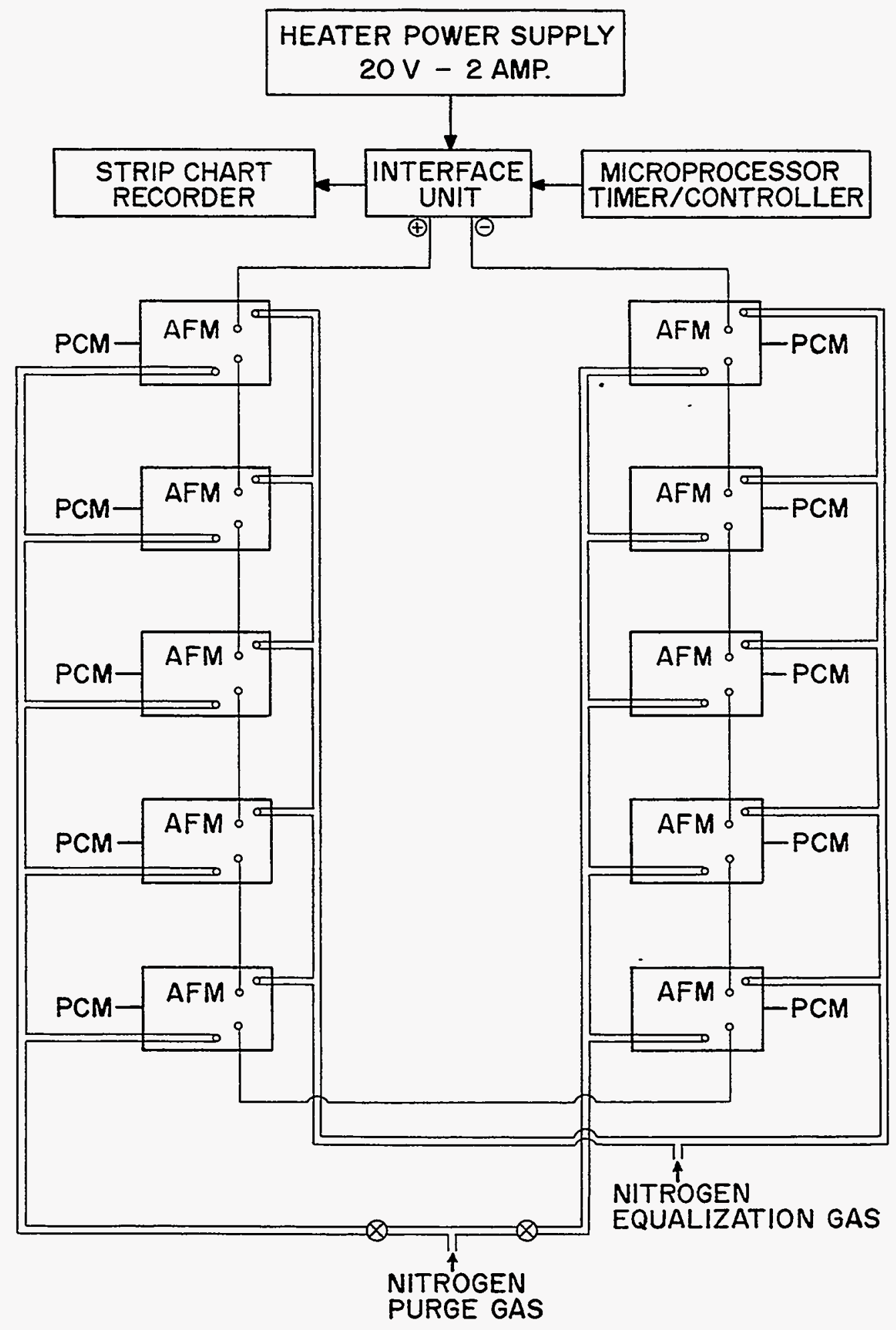

Figure 2.6. Configuration of the PATS lid absorbent tube bake-out system. 


\title{
2.3 ATMOSPHERIC DEPOSITION
}

\author{
Contact Person(s) : Mathew A. Monetti
}

\subsubsection{SCOPE}

EML collects radioactive debris that is dispersed into the atmosphere from nuclear accidents and from the testing of nuclear weapons using two deposition collectors. These collectors enable us to measure radioactive deposition at locations far removed from the source. The collectors and collection methods described here have been used successfully for many years. Presently there are about 75 sites worldwide at which monthly collections are made. These samples are routinely radiochemically analyzed for ${ }^{90} \mathrm{Sr}$. Following a known or suspected release of radioactivity, sampling and analysis protocol can be altered in order to determine the arrival time and composition of the debris.

We provide all the necessary supplies and instructions, in the appropriate language, and maintain written communication with site operators.

\subsubsection{POT COLLECTOR}

\subsubsection{1}

\section{INTRODUCTION}

The determination of ${ }^{90} \mathrm{Sr}$ and other radioisotopes contained in fallout can be carried out on the total material collected in a high-walled pot. The collection of fallout is simple, but the transfer of all the material in the pot must be done with great care in order to collect all of the radioactive material and to avoid a buildup of contamination in the pot, which may influence a subsequent sample. 
EML's collectors are stainless-steel pots, $0.076 \mathrm{~m}^{2}$ in area, but many other highwalled vessels are equally suitable. The requirements include smooth surfaces that do not absorb the radionuclides and rounded corners for easy cleaning and durability. The pot collector is shown in Figure 2.7.

\subsubsection{3}

\section{DEPLOYMENT, SAMPLING, AND RETRIEVAL}

For our program, the pot collector is placed in an open area away from buildings or overhanging trees and shrubbery. An acceptable location is atop a one or two story building with a flat roof not shaded by other buildings or trees. The pot is placed outside on the first of the month and left there for one month. (During months of heavy rainfall, it may be necessary to remove and evaporate some of the water prior to the end of the month.)

At the end of the month, the water in the pot is evaporated to $\sim 200 \mathrm{~mL}$ and transferred to a 1-L polyethylene bottle. Distilled water is added to the pot and, using a rubber spatula, the sides and bottom of the pot are scrubbed. The slurry is transferred to the bottle and the washing is repeated with $1: 1 \mathrm{HNO}_{3}$, again scrubbing and transferring the slurry. Then the pot is washed a final time with distilled water and scrubbed. It is extremely important to transfer all the solid material in the pot to the bottle. If necessary more than one polyethylene bottle may be used for each month's collection. The bottle(s) should be carefully labeled with the site location, sample dates, and the monthly precipitation amount from the nearest recording station. 


\subsubsection{ION EXCHANGE FALLOUT COLLECTOR}

2.3.3.1

INTRODUCTION

Like the pot collector, the ion exchange collector is designed for measuring fallout at sites removed from the source. At sites where personnel and facilities are at a minimum, the ion exchange collector is used. The collectors are exposed for monthly intervals and the collected fallout may then be shipped to a laboratory for analysis. The collector can be operated without a laboratory facility and consists of a funnel, an ion-exchange column, and a leveling device (all constructed of polyethylene) mounted in a wooden housing. The ion-exchange column is packed with paper pulp, ion-exchange resin and a glass wool plug, and is saturated with water. A utility light fixture with a $100-\mathrm{W}$ bulb and a 30-m heavy duty extension cord is provided to heat the device in cold climates. The ion exchange fallout collector is shown in Figure 2.7. A descriptive diagram of the collector is shown in Figure 2.8.

1. One plywood housing

2. One 30-cm polyethylene funnel

3. Two loaded resin columns, with caps

4. Two tapered caps

5. One leveling tube

6. One threaded T-connection

7. One piece of plastic tubing

8. One wash bottle

9. One set spare caps for mailing

10. One $100-W$ heating unit, where necessary 
The polyethylene ion-exchange column contains glass wool, paper pulp, and cation exchange resin. The glass wool is the standard laboratory material. The paper pulp is prepared from Whatman No. 41 filter paper or S\&S No. 289 paper pulp by mixing in a blender with distilled water.

Dowex 50W-X12 cation exchange resin (Bio-Rad Laboratories, Inc., 1000-T Alfred Nobel Dr., Hercules, CA 94547) 50-100 mesh in hydrogen form is used. The resin is washed with deionized water.

Glass wool is added to the bottom of each column and cation exchange resin and paper pulp are added in that order. Approximately $17 \mathrm{~cm}$ of glass wool, $50 \mathrm{~mL}$ of wet settled resin and $4 \mathrm{~cm}$ of paper pulp are packed into a column filled with distilled water. Care is taken to prevent the formation of air pockets.

\subsubsection{4}

\section{COLLECTOR LOCATION}

The collector should be located with the same criteria used for selecting a rain gauge site. These criteria are generally that the collector should be able to receive precipitation in an open area free of buildings, trees, and other obstructions that might shelter the collector. In many locations a flat roof is available - this is a suitable location, when the collector cannot be placed in an open area at ground level.

\subsubsection{5}

\section{SITE OPERATOR INSTRUCTIONS}

1. Remove the ion-exchange column from its mailing tube and retain the tube for return of sample. 
2. With the ion-exchange column inverted (small end up), remove the small cap and replace with the open tapered cap.

3. Force the short end of the J-shaped tube over the tapered cap and tape the other end to the column so that the small flexible hose is approximately level with the top of the tube.

4. Turn the column upright (large end up) and remove the large cap. Screw the column to the polyethylene funnel and place in the wooden housing. (Retain both caps for return of sample.)

5. Lead the long flexible tube, which serves as an overflow, through the hole in the side of the housing.

6. If subfreezing weather is anticipated during the month, hang the heating element from the eyelet in the housing and switch on during periods when snow or ice may accumulate in the collector.

7. The collector is left exposed continuously for 1 month, beginning on the first day of the month.

8. It is important that the resin column be kept saturated with water. If a dry period extends for several days, examine the column and add water if necessary. A plastic wash bottle is provided for this purpose. In most areas, tap water is satisfactory. However, in a few localities where rain water is collected directly for household use, distilled water should be used.

9. At the end of the observation period, use the wash bottle to rinse down any dust that has accumulated in the funnel and into the column, and wipe the inside of the funnel thoroughly with a wet tissue, which is then added to the column.

10. Remove the ion-exchange column from the assembly and replace the caps. (The new column can be installed at this time.) 
11. Note: The column label should contain the station name, the month of exposure, and the total amount of precipitation recorded by the station during the month.

12. Pack the column in the original mailing tube and return to the analytical laboratory.

\subsubsection{WET/DRY DEPOSITION COLLECTOR}

INTRODUCTION

We examine the wet phase and dry phase of deposition to further understand atmospheric pollution deposition processes, and the physical and chemical characteristics of these pollutants. Our measurements of fallout from weapons tests were largely directed towards stratospheric fallout, which is primarily deposited by way of precipitation scavenging. Dry deposition processes are also important and, therefore, it is desirable to measure wet and dry deposition separately.

\subsubsection{2}

APPARATUS

EML's wet/dry deposition collector is a two-bucket system with a movable peaked cover designed to expose the wet and cover the dry bucket at the onset of precipitation, and vice versa (Volchok and Graveson, 1976). There is a conductivity sensor on each side of the roof. The sensor base plate electrode is separated from the parallel bar electrode above by a thin (about $0.8 \mathrm{~mm}$ ) air space. Any electrical flow between the electrodes activates the motor in the base of the unit and the counter-weighted lid moves to cover the dry bucket. When the circuit is broken, the cover returns. Heaters are mounted below the sensors and are activated when the temperature drops below $4^{\circ} \mathrm{C}$ to melt snow or ice accumulations on the sensor, or when the instrument is in the wet 
collection mode to evaporate moisture from the sensor. Thus, when properly adjusted, the wet collector will be exposed only during periods of continuous precipitation.

\subsubsection{3}

SAMPLING AND DEPLOYMENT

In the past, we have used stainless-steel pots to collect radioactive debris from weapons tests, and heavy polyethylene buckets to collect trace metals. Since many of EML's sites are remote from the Laboratory, we have adopted a commercial container which has a positive closure to prevent leakage during shipment. The opening in the plastic bucket is about $30 \mathrm{~cm}$ in diameter. The sampling containers for these wet/dry collectors are polyethylene buckets particularly well-suited for shipping because of the design of the lip and cover [Freund Can Co., 167 West 84th St., Chicago, $\Pi 60620$ (As-1137/5541 plastic pail with cover)]. On the underside rim of the cover there is a groove with an O-ring seal. When the cover is securely hammered onto the bucket with a rubber mallet, no leakage will occur. The cover is destroyed when it is removed from the bucket at the Laboratory. The sample buckets are shipped in fiber mailing boxes.

In 1978, EML started to use Aerochem Metrics Model 201 wet/dry collectors (Bushnell, FL) at some of our sampling sites. This collector is similar in concept and design to EML's collectors. Both the EMIL and the Aerochem Metrics collectors are shown in Figure 2.9. The major mechanical difference is the Aerochem Metrics clutch system and switching system. EML's collector uses a shear pin to prevent damage to the motor when the lid is frozen in place. The Aerochem unit has a clutch on the motor drive of the covering lid. This clutch eliminates the problems caused by frequent shear pin breakage common to the EML units at windy or cold sites. The switches on the motor box of the Aerochem Metric unit are mercury-wetted switches, whereas microswitches are used in our units. 


\section{REFERENCE}

Volchok, H. L. and R. T. Graveson

"Wet/Dry Fallout Collection"

in: Proceedings of the Second Federal Conference on the Great Lakes, Great Lakes

Commission, pp. 259-264 (1976) 


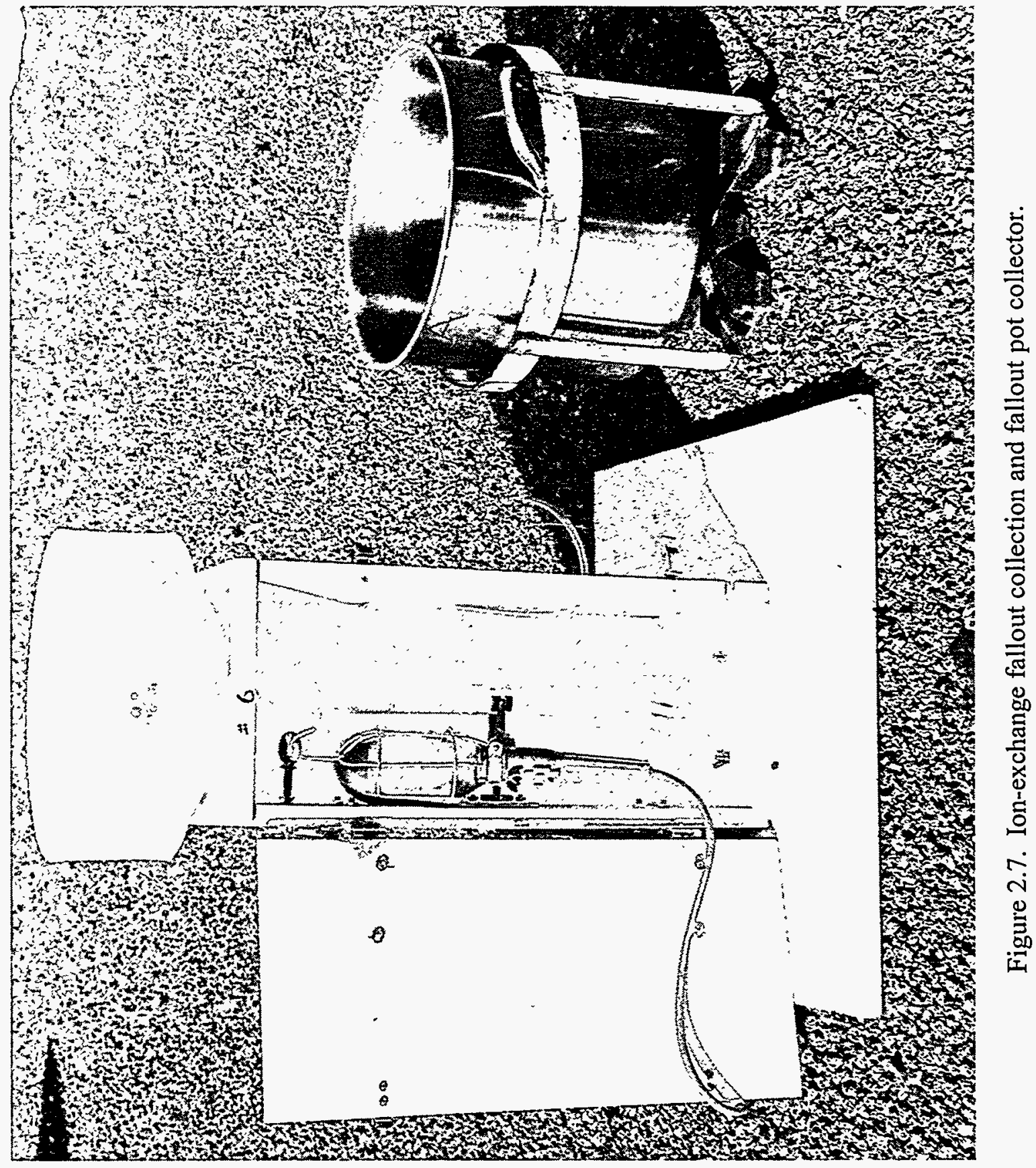




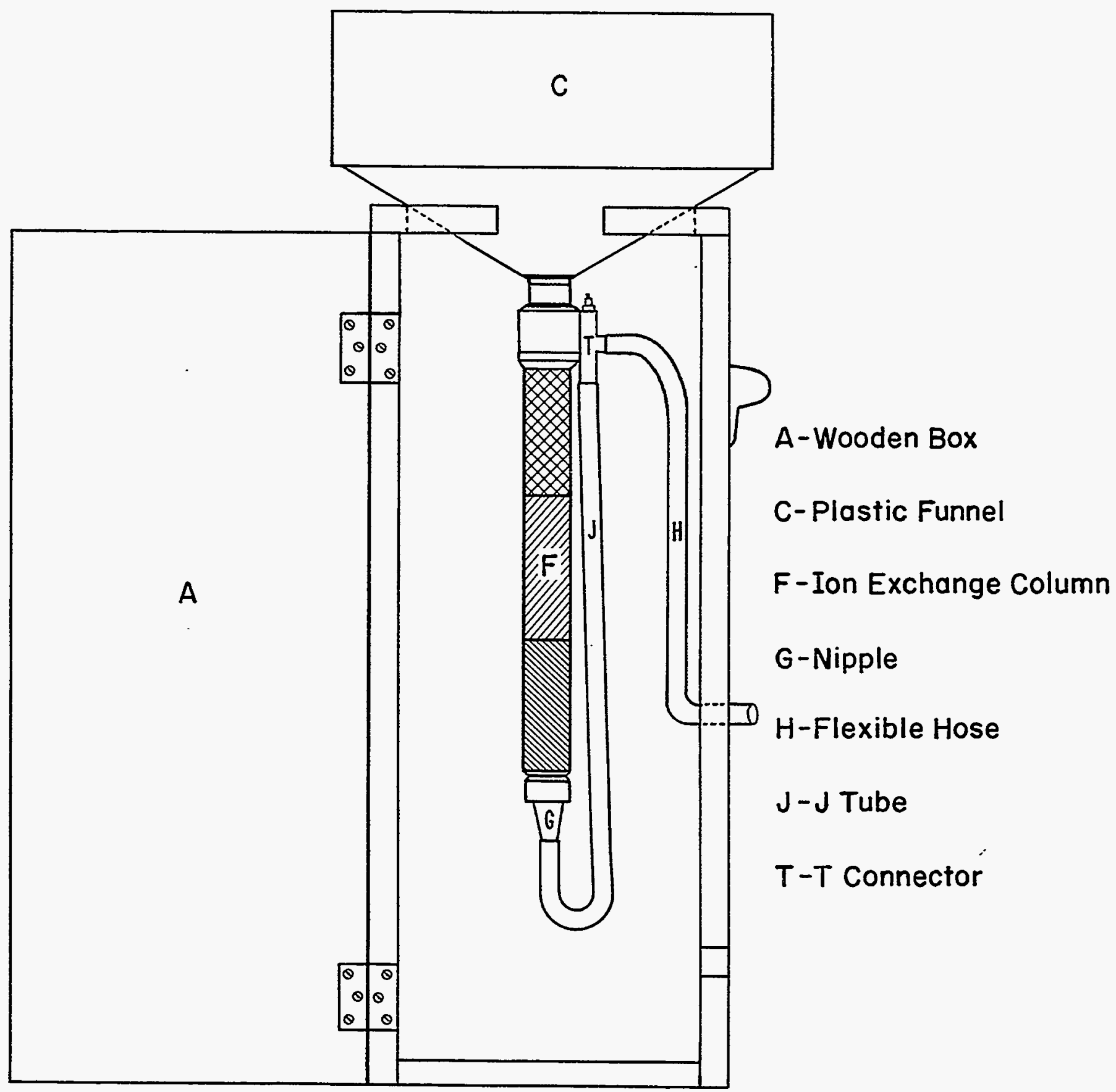

Figure 2.8. Ion-exchange fallout collector. 


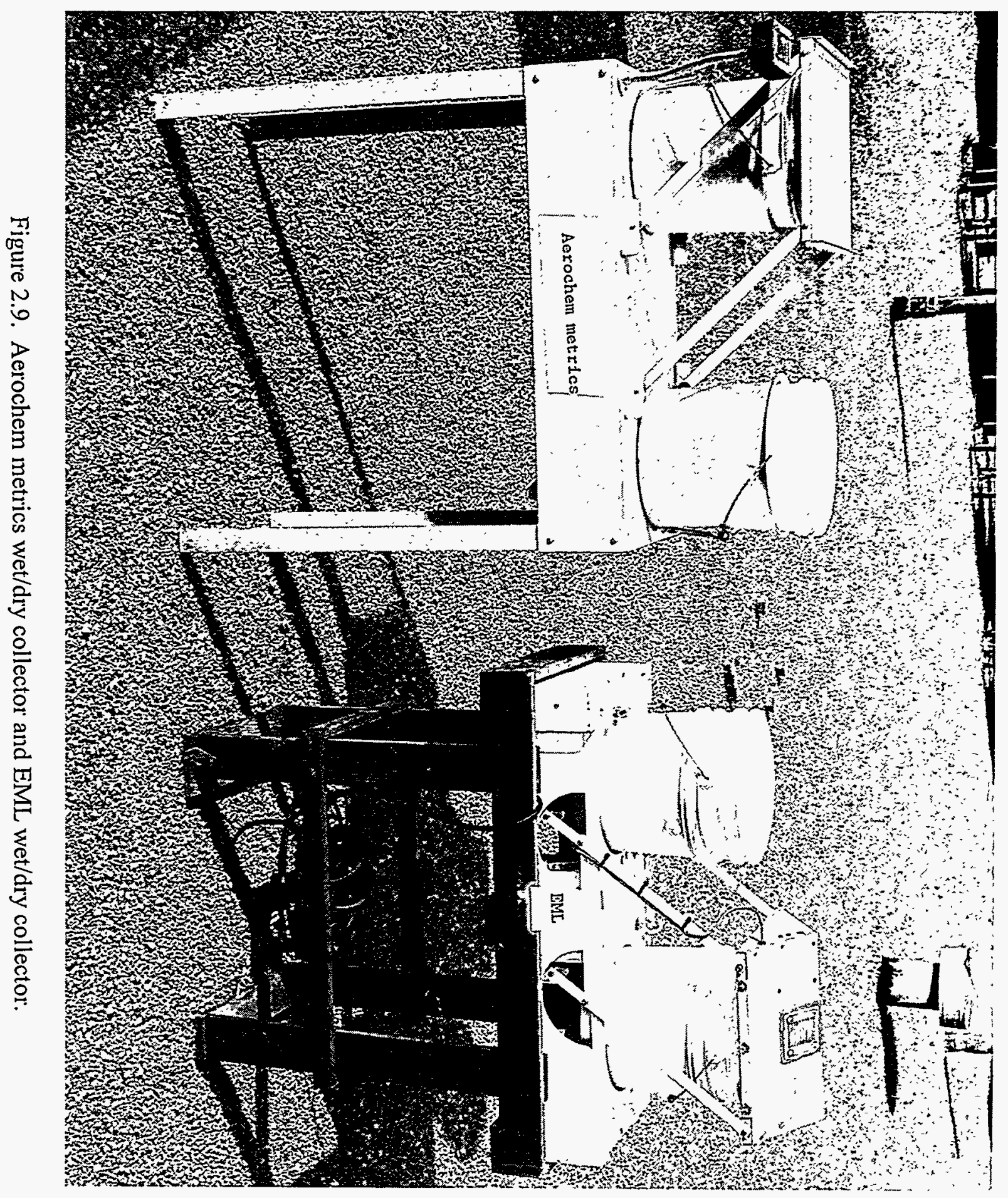




\subsection{SOIL}

Contact Person(s) : Catherine S. Klusek

\subsubsection{SCOPE}

Presented in this section are possible uses of soil sampling and some recommended procedures for this sampling and for the preparation of the samples. An attempt is made to point out some of the problems that exist in site selection, and the sampling methods that are available.

\subsubsection{SAMPLING OBJECTIVES}

\subsubsection{1}

INTRODUCTION

The sampling of soil is a useful approach to determine the accumulated amounts of airborne long-lived radioactive and stable contaminants that deposit on the ground. Soil sampling is, however, of questionable value in attempting to estimate small increments of deposition over a period of a few years or less. It is not recommended as a routine method of environmental monitoring except in preoperational surveys.

Historically, soil sampling procedures for radionuclides were modifications of techniques used in agriculture and engineering. Often, not enough emphasis is placed on the importance of a proper sampling method to accurately represent the total pollutant being sampled. The objectives of the project should be well-defined and the degree of precision required should be established before sampling. The purpose of the project (deposit, resuspension, root uptake) should dictate the type of sampling (total inventory, surface sampling, depth profile) used. Site characteristics, such as soil type, topography, source, and current distribution of the contaminant must be taken into account when designing the study. Other factors to be considered in the design stage are the uniformity 
of the contamination, the required accuracy necessary to provide reasonable results, and the minimization of cross-contamination.

\subsubsection{2}

\section{DEPOSITION INVENTORIES}

The most direct use of soil measurements is for estimating the inventory of the material deposited over a given area. EML has used soil analyses in estimating the deposition of ${ }^{90} \mathrm{Sr}$ (Hardy et al., 1968) and plutonium (Hardy et al., 1973; Krey et al., 1976a) on a worldwide basis, and in estimating the deposition of ${ }^{90} \mathrm{Sr}$ (Hardy et al., 1972), ${ }^{137} \mathrm{Cs}$ (Beck and Krey, 1980) and plutonium (Hardy, 1976) northeast of the Nevada Test Site. Such inventories require the selection of a sufficient number of representative sites, with the density of the sites depending on the accuracy sought. A statistical sampling scheme is generally not employed in sampling global fallout because the aerial distribution and particle size are nearly uniform over large areas (i.e., latitude bands). Low-level baseline sampling would require the careful selection of sites that serve to integrate fallout over time.

The most useful measures of the concentration of deposited material in soil relate to the amount per unit area. Sampling is therefore carried out in such a way that the weight of the material collected can be directly related to the area sampled and the depth of the sampling. The analytical results from a weighed aliquot of the soil sample can then be readily related to area concentration.

An additional requirement is that the sample is taken to a sufficient depth so that all of the deposited material is sampled. Without previous knowledge of the depth of penetration, an excessive sampling depth must be selected. This results in dilution of the radionuclide or other contaminant of interest. If time and cost considerations allow, it is recommended that a depth profile be taken (see Section 2.4.3.3). An optimum depth for sampling, which should contain $90-95 \%$ of the total material of interest, can then be selected. In our worldwide ${ }^{90} \mathrm{Sr}$ network we have increased our depth of sampling over the years from a few centimeters to $30 \mathrm{~cm}$. Even so, at some sites typified by calcareous soils, the ${ }^{90} \mathrm{Sr}$ has moved deeper than $30 \mathrm{~cm}$. Sampling parameters should be reevaluated and decisions made on a site basis. 
Criteria for site selection. When the accumulated deposition over a given time period is to be estimated by soil sampling, it is necessary that the area selected for sampling has been undisturbed for at least the time interval that is of interest. As the time interval increases, it becomes increasingly more difficult to obtain an accurate history of the site. In studies of cumulative fallout deposition, areas undisturbed since the early 1950 s are sought. Institutional property will have certain advantages over private property in these cases.

The second criterion, that of representativeness of the sample site, depends on the surroundings and the meteorological or climatological factors of the area. This generally requires that the site selected should be at the center of a large, flat open area.

Accumulative areas at the foot of slopes, in low spots or in flooded areas are not suitable. The site should not be near enough to buildings or trees to be sheltered during blowing rains. The sampling location should be $100 \mathrm{~m}$ or more from dusty roads. A large area of collection is desirable to make the sample more representative. A surface area of 500$1000 \mathrm{~cm}^{2}$ is adequate when a composite of 10 cores is taken over a reasonable distance $(\sim 30 \mathrm{~cm}$ apart).

The third criterion, that the deposited material remain in place, generally requires that the area be vegetated and have moderate to good permeability. There should be little or no runoff during heavy rains and no overwash at any time. The soil should have a base exchange capacity adequate to keep the contaminant from being readily leached into the ground water. A good grass turf aids in absorption of water and reduces the likelihood of runoff. Such sites are frequently found on smooth ridge crests, level virgin land, and in well-kept lawns and grounds around institutional buildings.

At EML, we recommend short-cropped grass sods as the most suitable sampling areas. Higher stands of vegetation may bias the collections by acting as a filter to remove airborne material in excess of what would normally be deposited. In wooded areas, the nonuniform distribution of tree canopies will lead to unevenness in deposition of fallout materials. Soils having high earthworm activity should be avoided because of uneven mixing of the soil to considerable depths. Rodent activity also makes an area unsuitable for sampling (Alexander et al., 1960). 


\section{DEPOSITION INCREMENT}

Incremental estimation of a contaminant through soil sampling and analysis should be undertaken only after careful planning. Because of the large uncertainties in sampling and the inherent variability in soil, estimating short-time increments of deposition history or deposition changes of small degrees is not recommended. When monitoring global fallout, short-term changes in radiation concentrations are generally small compared to the variability in the local radionuclide distribution. Direct collections of deposition or of airborne material are much more specific and yield more information with respect to the time when contamination occurred.

\subsubsection{4}

\section{OPERATIONAL OR ACCDENTAL RELEASES}

Following an acute release of a contaminant or an accident at a specific facility, surface soil sampling soon after the event can be used to define the contamination contours or distribution pattern. This would require sampling only the top $5 \mathrm{~cm}$ of soil, including the vegetation. Our experience indicates that attempts to sample a shallower depth results in less reproducible samples. In many areas, a site meeting the criteria for an optimal sample has a root mat extending several centimeters into the ground, and it is rarely possible to remove a core $<5 \mathrm{~cm}$ in depth intact.

In the case of accidental or operational releases, consideration must be given to liquid effluent as well as airborne particulate deposition. The amount deposited may vary with direction and distance from the release point. Airborne particle dispersal is affected by particle size and meteorological patterns. Gaussian plume models which take into account meteorology, stack height, topography, and deposition velocity of the particles are used to map local dispersion patterns. The horizontal dispersion of liquid effluent releases is influenced by the composition and quantity of the liquid, the topography, the soil type, and the properties of the contaminant of interest. 
Sampling techniques used in evaluating acute releases are more site dependent and methods used for fallout deposition may not be appropriate. Differences in the methods are dictated by the nature of the distribution of the contaminant in the soil, the range of particle sizes, and the generally higher levels of releases. Soil sampling in locally contaminated areas, such as Rocky Flats, can be inventoried by EML methods where the contaminant was initially made airborne in micron size particles from the source (Hardy and Krey, 1971).

Criteria for site selection. Wind roses and atmospheric dispersion calculations provide useful guidance in selecting appropriate soil sampling locations. Figure 2.10 shows a suggested distribution of sampling sites covering the area surrounding a plant, with emphasis on the downwind direction. This approach to soil sampling was used by EMI at Rocky Flats (Krey, 1976; Krey et al., 1976b). Samples are commonly collected from a regular grid or a radial pattern, at least close to the facility. When using such a systematic sampling design, care should be taken to ensure that the repetitive spacing of the sampling points does not introduce an error in the results. Consideration should also be given to the likelihood that a grid point can not be used for sampling. Some alternative random location or selection procedure should be established during the planning stages. Soil sampling within a $16 \mathrm{~km}$ radius should give an adequate preliminary picture of levels around the plant. It is also suggested that one or more samples be taken close to the center of the most heavily populated area in the vicinity of the plant. When sampling at facilities that release activity via tall stacks, sample locations at considerable distance from the potential release point may be indicated.

The same site selection techniques can be used for a preoperational survey around a plant. When choosing sampling locations at this early stage, it is desirable to select areas that can be resampled at a later time, should it become necessary. Samples should not be taken from the identical location since the sampling depletes the soil and alters the concentration of the pollutant under investigation. Rather, a sampling plot of several square meters should be established and samples removed from different areas of the plot. 
When evaluating soil for uptake availability of a contaminant of interest, it is not necessary to measure the total deposit but only the amount in the root zone that would be available to the plant or crop of interest. In most cases this would be the depth of the plowed layer. In addition to root zone concentration, the extent to which the nuclide or contaminant is chemically available for uptake must be considered. The availability of the particular contaminant to the plant is rarely $100 \%$. The processes influencing the mobility and availability of radionuclides in soil are complex and have been discussed by several researchers (Schulz, 1965; Russell et al., 1971; Eisenbud, 1987). Routine procedures for soil sampling will not be appropriate for this type of study because of the distribution of the nuclide. Field studies of environmental levels of radionuclides in agricultural soils have confirmed the nonuniformity of the concentration distribution due to mixing of the soil during cultivation and depletion by plant uptake and harvesting (Hardy and Bennett, 1977).

\section{RESUSPENSION AVAMABLITY}

There is no standard method to sample for availability of a contaminant in the soil for resuspension. The direct measurement of the airborne contaminant is the only sound approach to the problem of evaluating exposure to resuspended material. The practical problems of the mechanics of sampling make it difficult to take a very shallow soil sample with reliability. In trying to sample $1 \mathrm{~cm}$ or less it is very difficult to take into account the variation in the ground contour and to reproduce a particular sample. EML has tested other techniques, such as pressing gummed film to the surface, but these also have many mechanical difficulties (Krey et al., 1977). Another approach is to measure a depth profile at the site and plot the contamination as a function of depth. This has been found in practice to be an exponential, and extrapolation of the curve to zero depth might give an index of suspendability. This approach is limited by the error introduced if the shape of the curve as it approaches the surface deviates markedly from an exponential 
function. Resuspension concepts and prediction of the degree to which resuspension may occur have been reviewed (Healy, 1980; Sehmel, 1980).

\subsubsection{RECOMIMENDED PROCEDURES}

A few soil sampling methods will be discussed in this section. Variations on these techniques or alternate methods (ASTM, 1983a; Bernhardt, 1976; EPA, 1979, 1983; Gilbert, 1987) may be necessary to accommodate site specific characteristics or the objectives of the project. The standard EML procedure for collecting soil is described in the section on core method below, i.e., $10-8.9 \mathrm{~cm}$ diameter cores spaced $0.5 \mathrm{~m}$ apart on a flat grassy lawn are collected to a depth of $5 \mathrm{~cm}$, representing an area of $620 \mathrm{~cm}^{2}$.

In all the procedures discussed, it is important to consider the potential for crosscontamination. If depth profiles are taken, it may be necessary to decontaminate or discard tools as different levels of contamination are sampled.

\subsubsection{1}

CORE METHOD

The procedure described here is designed to obtain samples that will measure the total amount of an initially airborne contaminant that has fallen out in a given area. The criteria for selecting an optimal site have been discussed in Section 2.4.2.2.

All analytical values must be related to the surface area sampled. The surface area and depth define the volume; the weight of the volume of dry soil defines the field bulk density. These data are necessary to convert to radioactivity concentration per unit surface area (i.e., $\mathrm{Bq} \mathrm{m}^{-2}$ ).

A recommended procedure is described as follows. 
1. Following the selection of an undisturbed site which meets the criteria previously discussed, lay out a straight line transect about $4.5 \mathrm{~m}$ long. If the site is to be resampled at a later time, record distances to fixed landmarks to identify the relative location of the transect or adopt a systematic scheme or grid.

2. If the vegetation cover is not to be included with the soil sample, or is to be kept as a separate sample, the vegetation is removed to the surface level.

3. Using the $5 \mathrm{~cm}$ depth top soil cutter, press it into the ground without twisting or disturbing the grass cover or surface soil. This may best be accomplished by stepping on the rim of the cutter with both shoe heels. If more force is required, a rubber mallet may be used. Gently twist the handle of the cutter to cleanly remove the top soil plug. Place the core in a plastic sampling bag.

4. Repeat the process until the desired number of cores have been sampled. We recommend 10 cores for providing a representative sample (Alexander et al., 1961). Samples of 20 and 10 core composites were collected in about 10 areas and no significant differences were found in the estimated ${ }^{90} \mathrm{Sr}$ deposit. Compositing soil samples provides a larger sample volume and possibly a more representative sample of the area. Compositing is discussed further in Bernhardt (1976) and Gilbert (1987). Take 10 top soil cores in a straight line about $30 \mathrm{~cm}$ apart, placing the cores in a plastic bag. (The total area sampled is $620 \mathrm{~cm}^{2}$.)

5. Sometimes it may not be possible to remove a $5 \mathrm{~cm}$ depth plug cleanly because of a thick root mat. If the top soil and bottom soil are to be combined, a $10 \mathrm{~cm}$ or $15 \mathrm{~cm}$ deep cutter may be used to remove the top soil by pounding it part way into the ground with the rubber mallet, until it is possible to remove the core intact.

6. Next, take the subsoil samples down to the desired depth with the auger. A slight downward pressure with slow turning will guide the auger. When the cylinder is about 3/4 full, remove the auger slowly and either tap out or scrape out the soil with a large flat blade knife. Continue to use the auger until the desired depth has been sampled. If rocks or roots impede the auger, it may be possible to carefully remove them. They should be included with the sample. If, however, this destroys the core, the sample should not be used. It is a useful practice to place the soil from the core as it is removed into a plastic pail until the entire depth is removed. Then, if the core is 
not suitable, it may be poured back into the hole. Only after the entire sample is successfully removed is the soil added to the sampling bag. Repeat the procedure for the remaining cores.

7. After collection, label the plastic bag containing the sample, fold, and seal with a heavy duty stapler. If a portable scale is available, the wet weight can be taken in the field. Then place the sample in a canvas bag and tie firmly. The label should include the date, location, and depth.

8. The holes should be filled with top soil to prevent an accident. A new grass cover will develop in a few weeks. If immediate restoration is necessary, plugs may be cut from a piece of sod.

Using the above procedure, a site can be sampled in two increments, $0-5 \mathrm{~cm}$ and $5-30 \mathrm{~cm}$. This is useful in areas where most of the contamination is in the surface cut of the soil. In other sampling situations using cores of 10 and $15 \mathrm{~cm}$ depth will provide incremental samples: $0-5,5-10,10-15$, and $15-30 \mathrm{~cm}$. When attempting this type of incremental sampling, attention must be given to two sources of error: contamination by fall-in of soil from the upper layers of more highly contaminated soil as the subsequent cores are taken from the hole and error in depth due to compaction.

\section{A. Limitations.}

Implicit when using the above sampling method is the need to take the sample deep enough so that all the radionuclide deposited is collected. The extent of vertical penetration will depend primarily upon the soil type with other factors, such as amount of annual precipitation and chemical form of the nuclide, playing a confounding role. [See Section 3.3 Field Gamma-Ray Spectrometry for a discussion of the use of in situ spectrometry to aid in establishing the depth of penetration.]

There is no simple satisfactory way of sampling powdery, dry, loose, single grain soils by this core method. It is best to take samples when the soil has enough moisture to be coherent even if this requires wetting the area to be sampled by sprinkling. An alternate method for sampling loose soils is to leave the corer in place and scoop out the contents. 
Only one composite depth can be taken however, since once the corer is removed the integrity of the core is lost.

\section{B. Sampling equipment for the core method.}

Tools for sampling may be of any material and type that will take a core of equal area through its entire length. The following equipment are used at EML:

1) Barrel auger - Standard Type No. R-HEO, $8.2 \mathrm{~cm} \mathrm{ID}$, with T-handle, Arts Machine Shop, American Falls, ID.

2) Top soil cutters - 5, 10, $15 \mathrm{~cm}$ depth, $8.9 \mathrm{~cm} \mathrm{ID}$. Made from $0.155 \mathrm{~cm}$ thick coldrolled steel. One end sharpened on a lathe, the other end fitted with a welded handle.

3) Other equipment -

Long, flat blade knife for removing cores from auger

Measuring tape $(\sim 8 \mathrm{~m})$

File for sharpening top soil cutters

Rubber mallet

Plastic bucket (5 L).

Although the core method is preferred, there are areas where the rocks will make it impossible to use this technique. An alternate method we employ in these areas is to cut out a $900 \mathrm{~cm}^{2}$ sample using a $31.6 \mathrm{~cm}$ square-template for guidance. The soil and rocks are removed with chisels and scoops down to the desired depth. The rocks are included and weighed with the sample. The large rocks can be discarded after removing loose dirt. The remaining smaller rocks are crushed as part of the sample.

This method is comparable to the ring method used by the Nevada Applied Ecology Group (NAEG) for sampling sandy and rocky soils. Here a $12.7 \mathrm{~cm} \mathrm{D}$ ring with a lip to assure constant depth of penetration is used. The soil adjacent to the exterior of the ring 
is removed to the depth of the ring. The confined volume of soil is then transferred to a plastic bag using an appropriate tool. Depth profile may be drawn using this method by repeating the steps for each subsequent depth to be sampled. Removal of soil exterior to the ring is necessary to minimize the possibility of cross-contamination of the deeper, less radioactive fractions (Fowler et al., 1974). A minimum of five separate samples $\left(633 \mathrm{~cm}^{2}\right)$ are recommended along a transect and composited for analysis.

\section{A. Sampling equipment for the template method.}

Tools for sampling may be of any material that will maintain a rigid shape and straight edge. The sampling equipment used at EML consists of:

1) A square template $20 \mathrm{~cm}$ or $30 \mathrm{~cm}$ on the inner edge made of $0.157-\mathrm{cm}$ thick coldrolled steel, with holes at the corners,

2) Pins or long nails of corresponding diameter to the corner holes of the template to anchor the template to the surface,

3) Chisels, knives, and small shovels.

The trench method is used to determine the depth of penetration of a radionuclide or contaminant or to establish a detailed depth profile. The most suitable area for taking soil profiles is one where there are no rocks and stones, and very few pebbles. The procedure works well in sandy loam, loam or loamy sand types of soil (Hardy, 1974; Fowler et al., 1974). Use of this method in unsuitable areas will result in cross-contamination of lower profile cuts with higher specific activity subsoil from upper layers. Cross contamination effects of worms and burrowing insects, cracks from swelling, and other biological and physical disturbances must be considered.

A recommended procedure is described as follows. 
1. As far as grass cover and terrain are concerned, the site selection criteria previously described apply. As in the core sampling method, the depth profile samples are taken so that the weight and depth of the material collected can be directly related to the area.

2. If the vegetation represents a seasonal growth, it should be clipped to $2.5-5 \mathrm{~cm}$ over a measured area.

3. Lay a tarpaulin (about $0.6 \mathrm{~m}^{2}$ ) on the ground near the clipped area. Dig a trench of an appropriate size for ease of access (about $60 \mathrm{~cm}$ wide by $90 \mathrm{~cm}$ long by $60 \mathrm{~cm}$ deep, about $15-25 \mathrm{~cm}$ deeper than the desired sampling depth) immediately adjacent to the clipped area, placing the dirt on the tarpaulin. Usually the sod can be cut out in blocks making it easy to replace after sampling.

4. The face of the trench (adjacent to the clipped area) is smoothed from side to side with a flat blade shovel or mortar trowel, making it perpendicular to the surface.

5. A metal flat-bottomed three-sided pan with sharpened edges on the open side $(15 \mathrm{~cm}$ $\times 15 \mathrm{~cm} \times 5 \mathrm{~cm}$ deep; $230 \mathrm{~cm}^{2}$ ) is pressed into the face from ground surface to $5 \mathrm{~cm}$. Remove the first cut and seal in a small plastic bag.

6. Cut away the top soil on either side of the cut to make a shelf about $35-\mathrm{cm}$ long by $15-\mathrm{cm}$ wide and $5 \mathrm{~cm}$ deep from the surface. Lightly brush away any particles that may have fallen on the shelf.

7. Again, push the open-end cutting pan into the side and cut out the next incremental sample. Continue this procedure until the desired depth is reached. The actual depth of each cut can be determined by placing a two by four on the surface and measuring to each subsurface.

8. When all the samples have been taken, fill the trench with dirt on the tarpaulinand replace the sod taken from the trench. 


\section{A. Limitations.}

A depth profile is only useful for finding the relative vertical distribution of the radionuclide. Since only $100-300 \mathrm{~cm}^{2}$ of surface area at one spot is sampled when taking depth increments, the integrated deposit is not necessarily representative of the area.

The trench method is more time consuming and more difficult than taking core samples. Therefore, researchers rarely sample and composite more than two samples per trench and rarely take duplicate profiles. However, if care is taken, there will be very little cross contamination and the data collected in terms of the depth profile will be more accurate.

Some consideration should be given to the problems introduced by sampling across soil horizons. Soil horizons differ in chemical and physical properties.

\section{B. Sampling equipment for the trench method.}

The sampling equipment used by EML consists of:

1) Three-sided square pan with cutting edges on the open side (15 $\mathrm{cm} \times 15 \mathrm{~cm} \times 5 \mathrm{~cm}$ deep made of $0.157 \mathrm{~cm}$ thick cold-rolled steel, welded at the corners);

2) Mortar trowel;

3) Long flat-blade knife;

4) $1.2 \mathrm{~m}$ piece of two by four.

Previous site selection techniques discussed above assure a representative sample by the homogeneous and undisturbed nature of the location. In geographic areas 
characterized by sparse vegetation, wind erosion, and nonuniform drainage, the fallout is expected to be nonuniformly distributed. A procedure for recovering a representative soil sample from complex terrain for the purpose of determining the integrated radionuclide inventory deposited in the area is described in Krey and Klusek (1995). The following protocol is used in such a nonhomogeneous terrain area.

1. In the area of interest, select a site that is visually representative of the area. An optimal area would be without excessive rills, washout areas, and gullies that would indicate areas of intense erosion. If the area is very diverse in landscape, consideration should be given to subdividing the area and taking samples in each area.

2. Determine the number and types of covers (strata). Some common strata are: open pavement, trees, shrubs, grass, rocks. Some judgment is required with small trees and large bushes. Vegetation should be assigned as a cover type if there is a significant difference in the character or amount of accumulation of soil at their base. It is preferable to preassign the vegetation to a category before attempting to estimate the linear percentage to avoid changing the category's characteristic in midcount.

3. Select, at random, a starting point and mark off a $100 \mathrm{~m}$ straight line transect in a randomly selected direction.

4. Using a measure that is a reasonable small unit, i.e., every $0.1 \mathrm{~m}$, count off the number of meters intersected by each stratum. Estimate the proportion of each cover along the total transect.

5. Determine the number and method of sampling to be used in each identified stratum. Samples should be taken in every stratum that represents more than $5 \%$ of the total transect. For each cover type to be sampled:

a) Sample (systematically) by the core method at a fixed interval (e.g., $6 \mathrm{~m}$ ) along the transect using the closest sampling location within $\pm 0.5 \mathrm{~m}$ of the mark.

b) Sample (randomly) each cover type by the template method. It is only feasible to take two to four samples by this method. 
6. The samples taken in a stratum are combined. At this point the soil samples (one per stratum) can be analyzed:

a) independently to obtain information pertinent to each stratum and the total inventory estimated by summing the proportional contribution from each stratum;

b) the soil samples can be composited based on the areal proportion and only this composite sample for the site is analyzed.

\subsubsection{SAMPLE PROCESSING}

The procedures to be followed to process a soil sample to obtain a representative subsample for analysis depend to some extent on the nuclide of interest, the size of the sample, and the amount of processing already undertaken in the field. Any one of, or a combination of, the following procedures may be employed. The soil may be crushed to reduce the size of stones, sieved to remove sample content above a desired size, blended to obtain a more homogenous distribution of particle sizes, or milled to reduce the particle size of the soil. If the sample was sieved or split in the field or a small sample was taken, the preparation process may be eliminated. For some purposes, it is possible to remove large nonporous stones and not grind them to size, but they must be weighed separately and an appropriate allowance made. As a general rule, at EML samples are air-dried, crushed to break up large rocks, blended to allow a representative aliquot to be removed, and only this subsample is pulverized. The pulverizing reduces the soil to a standard particle size. Other preparation protocols are addressed in ASTM (1983b).

Global fallout is relatively homogeneous in particle size and distribution in the sample. When sampling accidental or operational releases, the procedures described here may not be adequate. Care must be taken that the subsample taken for analysis accurately represents the total sample. This will depend on the size and the degree of heterogeneity. Multiple subsampling and multiple analysis may be the only technique available to adequately define the content of radionuclides in heterogeneous samples. 
Care must be taken in all stages of processing to avoid contamination from previously prepared higher concentration samples. In addition to careful cleaning of the equipment between samples it is sometimes advisable to run blank samples (e.g., sand) between the samples being processed. Another technique to minimize cross-contamination is to order the processing of samples starting with the lowest level samples first. This is difficult to know in an exact sense, but generally deeper depth samples will have a lower content than surface samples.

2.4.4.1

DRYING

A recommended procedure is described as follows.

1. The "excludable" fraction of the sample should be defined, dependent on the contaminant of interest. Vegetation, root mat, large organic pieces, stones, etc., in some cases can be discarded. If this is the case, the sample can be screened using a 6 $\mathrm{mm}$ sieve or a 10 mesh screen. The appropriate steps of drying process (e.g., Step 3 and/or 5) can then be skipped.

2. If facilities are available, the samples can be dried at $100^{\circ} \mathrm{C}$ overnight. Oven drying has the advantage that most materials will become brittle enough to mill properly. Otherwise, spread out the sample on a plastic sheet or in trays and allow to air dry. This will take 3 days to 2 weeks. Turning the soil will facilitate the drying process especially when the drying area does not allow the soil to be spread in a thin layer. However, turning is not advised when the rising dust might cause contamination of other samples.

3. Break up soil aggregates and pull apart the top soil plugs consisting of vegetation and root mat. Using scissors or clippers, cut up the vegetation so that it is distributed homogeneously throughout the sample.

4. When completely dry, weigh the entire sample.

5. Remove large rocks, weigh separately, and discard. 
When using equipment in this stage of preparation close attention must be paid to cross-contamination. Machinery should be dismantled and decontaminated between samples.

1. If the sample contains small rocks or pebbles:

a) crush the entire soil sample, reducing the sample to $6.35 \mathrm{~mm}$, or

b) sieve the entire sample through a 12.7-mm screen.

2. Then blend the entire sample for 15-30 min.

3. Spread out the sample, mark off quarters, and take scoop-fulls from each quarter in a consecutive manner until about $3 \mathrm{~kg}$ has been collected.

4. Pass this subsample of soil through a grinder, ball mill, sieve or pulverizer. The pulverizer used at EML reduces the soil to $<1.3 \mathrm{~mm}$ (15 mesh equivalent). Transfer to a wide-mouth polyethylene bottle.

5. When ready for analysis, roll the bottle to mix the sample thoroughly.

$$
\text { 2.4.4.3 }
$$

\section{RAPID PREPARATION METHOD}

An alternate soil sample preparation procedure is employed to allow rapid processing and aliquoting when gamma-ray spectrometric analysis for short-lived radionuclides is desired. This procedure was developed to provide timely data from surface soil samples collected following the Chernobyl reactor accident. After weighing the sample in the wet state (as received), the entire sample is sieved through a steel mesh screen with square openings, $1.27 \mathrm{~cm}$ on edge. Rocks and pebbles of greater size are brushed and discarded. 
Vegetation and root mat are cut to a size (about $0.6 \mathrm{~cm}$ ) that permits them to pass through the sieve. The mixture of wet soil and vegetation is homogenized by hand for several minutes before aliquots of about $100 \mathrm{~g}$ are removed. If desired, the remainder of the sample can then be dried and processed as previously described.

In experiments involving 75 sets of duplicate wet samples, $87 \%$ of the samples agreed with each other to within two standard deviations of the propagated Poisson counting uncertainties. This indicates a reasonable degree of homogenization of the wet sample. In addition, the ratio of the deposition values for wet samples to dry pulverized samples was close to unity for most radionuclides. The homogenization of the wet samples is not as efficient in mixing the infrequent particles enriched in refractory nuclides, resulting in a consistent bias toward higher wet values (Krey et al., 1986).

The following equipment are used at EML to process soil:

1) Scale - capacity of $50 \mathrm{~kg}$;

2) Sieves - various screen sizes;

3) Splitter - sample reducer, Humbaldt Mfg. Co., Norridge, $\Pi$. 60656;

4) Crusher - jaw crusher, Morse Bros. Machinery Co., Denver, CO 80214;

5) Blender - twin shell pin intensifier blender, 16 qt capacity, Patterson-Kelly Co., Inc., East Stroudsburg, PA 18301;

6) Pulverizer - pulverizing (hammer) mill with \#50 Screen, Weber Brothers \& White Metal Works, Inc., Hamilton, MI 49419;

7) Drying oven. 


\subsubsection{REPRODUCIBHITY}

The accuracy of the estimated deposit at any site is related to the representativeness of the soil sampling and aliquoting, and to the quality of the analysis. The sampling design should prevent biases and allow errors to be readily determined. A QA program should be maintained at all stages of the project. A detailed discussion of QA for soil sampling is presented in Barth et al. (1989) and van Ee et al. (1990).

\subsubsection{1}

\section{REPRESENTATIVENESS}

If the criteria for selecting a soil sampling site are satisfied, the EML sampling method has been shown to provide reasonable estimates of local and regional fallout. Integrated fallout of ${ }^{137} \mathrm{Cs},{ }^{90} \mathrm{Sr}$, and ${ }^{239,240} \mathrm{Pu}$ have been shown to be quite uniform within metropolitan areas characterized by the same annual precipitation. The deposition variability of these radionuclides was $<15 \%$ (Hardy, 1975). In extensive studies of ${ }^{90} \mathrm{Sr}$ deposition, 50 paired sites, $2-40 \mathrm{~km}$ apart, showed an average difference expressed as a percent of the mean of $<10 \%$ (Hardy and Krey, 1971).

Where it can be assumed that there is little gradient in the cumulative fallout within a city, duplicate soil collection and measurement has inferred a precision of sampling and analysis of $\pm 8 \%$ for ${ }^{137} \mathrm{Cs}$ (Beck and Krey, 1980), $\pm 9 \%$ for ${ }^{239,240} \mathrm{Pu}, \pm 3 \%$ for the ${ }^{240} \mathrm{Pu} /{ }^{239} \mathrm{Pu}$ atom ratio, and $\pm 4 \%$ for the ${ }^{241} \mathrm{Pu} /{ }^{239} \mathrm{Pu}$ atom ratio (Krey and Beck, 1981) for the determination of these values at any given site.

The statistical analyses of common sampling designs, such as random or systematic sampling, are discussed in a familiar text such as Cochran (1977).

In cases where the contaminant may not be uniform in size or concentration, the resulting "hot spots" will be found in some but not all the samples. Uneven physical distribution of particles or particles with a large range of sizes and concentrations complicates the collection of a representative sample and subsampling. Gilbert (1987) discusses different approaches for sampling nonuniformly distributed contamination. 
Analyses of Utah soils have shown that the prepared soils are sufficiently homogeneous, after following the procedures described above (air-dry, crush, blend, and pulverize), that duplicate aliquots agree within two standard deviations of the counting rate for ${ }^{137} \mathrm{Cs}$ (Beck and Krey, 1980) and the radioassay value for ${ }^{238} \mathrm{Pu},{ }^{239,240} \mathrm{Pu}$, and ${ }^{241} \mathrm{Am}$ (Krey and Beck, 1981). A mean deviation of 7\% was found for 426 pairs of duplicate soil aliquots, representing the subsampling and analytical errors for ${ }^{90} \mathrm{Sr}$ over an 8-y period (Hardy and Krey, 1971).

\subsubsection{3}

ANALYTICAL ACCURACY

\section{A. Soil standards.}

Two standard reference materials are available through the National Institute of Standards and Technology (NIST, formerly the National Bureau of Standards), SRM 4353 Rocky Flats Soil Number 1 and SRM 4355 Peruvian Soil. SRM 4355 has nonmeasurable radioactivity concentrations for many fallout radionuclides and is intended for use as a blank. Upper limits of the radioactive concentrations are given and can be used to monitor laboratory contamination and background counting rates.

Secondary soil standards, that is, large quantity soil samples that have been dried, blended and pulverized and aliquots of which have been analyzed by inter- and intralaboratory comparisons, are used at EML as the best possible substitute for a primary standard soil sample for artificial radioactivity. There is no satisfactory way to add a radionuclide to a soil sample so that it represents the chemical and physical form as it exists in the field. 


\section{B. Blanks.}

Soils collected in 1943, and therefore containing no artificial radionuclides, have served as a quality control blank sample throughout EML's soils programs to monitor contamination by laboratory handling, reagents, and other possible sources.

\section{REFERENCES}

Alexander, L. T., E. P. Hardy, and H. C. Hollister

"Radioisotopes in Soils: Particularly with Reference to Strontium-90"

R. S. Caldecott and L. A. Snyder (Editors)

in: Proceedings of a Symposium on Radioisotopes in the Biosphere, University of

Minnesota, Minneapolis, MN, pp. 3-22 (1960)

Alexander, L. T., R. H. Jordan, R. F. Dever, E. P. Hardy, G. H. Hamada, L. Machta, and R. J. List

"Strontium-90 on the Earth's Surface "

USAEC Report TID-6567, Office of Technical Information, Washington, D.C., February (1961)

ASTM

"Sampling Surface Soils for Radionuclides"

Annual Book of American Society of Testing Materials, C-998-83, Vol. 12.01, p. 792 (1983a)

ASTM

"Soil Sampling Preparation for the Determination of Radionuclides"

Annual Book of American Society of Testing Materials, C-999-83, Vol. 12.01, p. 796 (1983b)

Barth, D. S., B. J. Mason, T. H. Starks, and K. W. Brown

"Soil Sampling Quality Assurance User's Guide"

USEPA Report EPA/600/8-89-046, p. 268, March (1989) 
Beck, H. L. and P. W. Krey

"Cesium-137 Inventories in Undisturbed Utah Soils - Interim Report on Radionuclides in Soils of Populated Areas"

USDOE Report EML-375, June (1980)

Bernhardt, D. E.

"Evaluation of Sample Collection and Analysis Techniques for Environmental Plutonium"

USEPA Report ORP/LV-76-5, Las Vegas, NV (1976)

Cochran, W. G.

Sampling Techniques

John Wiley \& Sons, Inc. (1977)

Eisenbud, $\mathrm{M}$.

Environmental Radioactivity

Academic Press, Inc., New York, Third Edition, pp. 84-104 (1987)

EPA

"Sample Collection Manual: Guidelines for Collecting Field Samples"

Environmental Protection Agency, Office of Pesticides Programs, Washington, DC, March (1979)

EPA

"Preparation of Soil Sampling Protocol: Techniques and Strategies"

Environmental Protection Agency, Office of Research and Development, Report 600/

4-83-020, Washington, DC, August (1983)

Fowler, E. B., R. O. Gilbert, and E. H. Essington

"Sampling of Soils for Radioactivity: Philosophy, Experience and Results"

in: Atmosphere-Surface Exchange of Particulate and Gaseous Pollutants

ERDA Symposium Series 38, CONF-740921, pp. 709-727 (1974)

Gilbert, R. O.

Statistical Methods for Environmental Pollution Monitoring

Van Nostrand Reinhold Company, New York (1987) 


\section{Hardy, E. P.}

"Depth Distribution of Global Fallout ${ }^{90} \mathrm{Sr},{ }^{137} \mathrm{Cs}$, and ${ }^{239,240} \mathrm{Pu}$ in Sandy Loam Soil"

USAEC Report HASL-286, pp. 2-10, October (1974)

\section{Hardy, E. P.}

"Regional Uniformity of Cumulative Radionuclide Fallout"

USAEC Report HASL-288, pp. 2-9, January (1975)

Hardy, E. P.

"Plutonium in Soil Northeast of the Nevada Test Site"

USERDA Report HASL-306, pp. 51-85 (1976)

Hardy, E. P. and B. Bennett

"Radionuclide Uptake by Cultivated Crops"

USERDA Report HASL-321 pp. 19-38 (1977)

Hardy, E. P. and P. W. Krey

"Determining the Accumulated Deposit of Radionuclides by Soil Sampling and Analysis" Los Alamos Scientific Laboratory Report LA-4756, pp. 37-42, August (1971)

Hardy, E. P., P. W. Krey, and H. L. Volchok

"Global Inventory and Distribution of Fallout Plutonium"

Nature, 241, 444-445 (1973)

Hardy, E. P., H. L. Volchok, and P. W. Krey

"Strontium-90 Fallout in Utah"

USAEC Report HASL-257, pp. 71-94, July (1972)

Hardy, E. P., M. Meyers, J. S. Allen, and L. T. Alexander

"Strontium-90 on the Earth's Surface"

Nature, 219, 584-587 (1968) 
Healy, J. W.

"Review of Resuspension Models"

W. C. Hanson (Editor)

in: Transuranics Elements in the Environment

USDOE Technical Information Center Report DOE/TIC-22800, pp. 209-235 (1980)

Krey, P. W.

"Remote Plutonium Contamination and Total Inventories from Rocky Flats"

Health Phys., 30, 209-214 (1976)

Krey, P. W. and H. L. Beck

"The Distribution Throughout Utah of ${ }^{137} \mathrm{Cs}$ and ${ }^{239+240} \mathrm{Pu}$ From Nevada Test Site

Detonations"

USDOE Report EML-400, November (1981)

Krey, P. W. and C. S. Klusek

"Soil Sampling in Complex Terrain"

J. Radioanalytical Nuc. Chem., 197, 79-98 (1995)

Krey, P. W., E. P. Hardy, and L. E. Toonkel

"The Distribution of Plutonium and Americium with Depth in Soil at Rocky Flats" USERDA Report HASL-318, April (1977)

Krey, P. W., E. P. Hardy, C. Pachucki, F. Rourke, J. Coluzza, and W. K. Benson "Mass Isotopic Composition of Global Fall-Out Plutonium in Soil"

in: Transuranium Nuclides in the Environment, IAEA-SM-199/39, pp. 671- 678 (1976a)

Krey, P. W., E. P. Hardy, H. L. Volchok, L. E. Toonkel, R. H. Knuth, R. Coppes, and T. Tamura

"Plutonium and Americium Contamination in Rocky Flats Soils, 1973"

USAEC Report HASL-304, March (1976b) 
Krey, P. W., C. S. Klusek, C. G. Sanderson, K. M. Miller, and I. K. Helfer

"Radiochemical Characterization of Chernobyl Fallout in Europe"

H. L. Volchok and N. Chieco (Editors)

in: A Compendium of the Environmental Measurements Laboratory's Research Projects

Related to the Chernobyl Nuclear Accident,

USDOE Report EML-460, pp. 155-213, October (1986)

Russell, R. S., B. O. Bartlett, and R. S. Bruce

"Appraisal of the Long-Term Nuclide Contamination Hazard"

D. W. Bensen and A. H. Sparrow (Editors)

in: Survival of Food Crops and Livestock in the Event of a Nuclear War

AEC Symp. Ser. 24 (CONF-700909), Natl. Tech. Info. Service, U.S. Dept. of Commerce, Springfield, VA, pp. 548-565 (1971)

Schulz, R. K.

"Soil Chemistry of Radionuclides"

Health Phys., 11, 1317-1324 (1965)

Sehmel, G. A.

"Particle Resuspension: A Review"

Environment International, 4, 107-127 (1980)

van Ee, J. J., L. J. Blume, and T. H. Starks

"A Rationale for the Assessment of Errors in the Sampling of Soils"

USEPA Report EPA/600/4-90/013, p. 57, May (1990) 

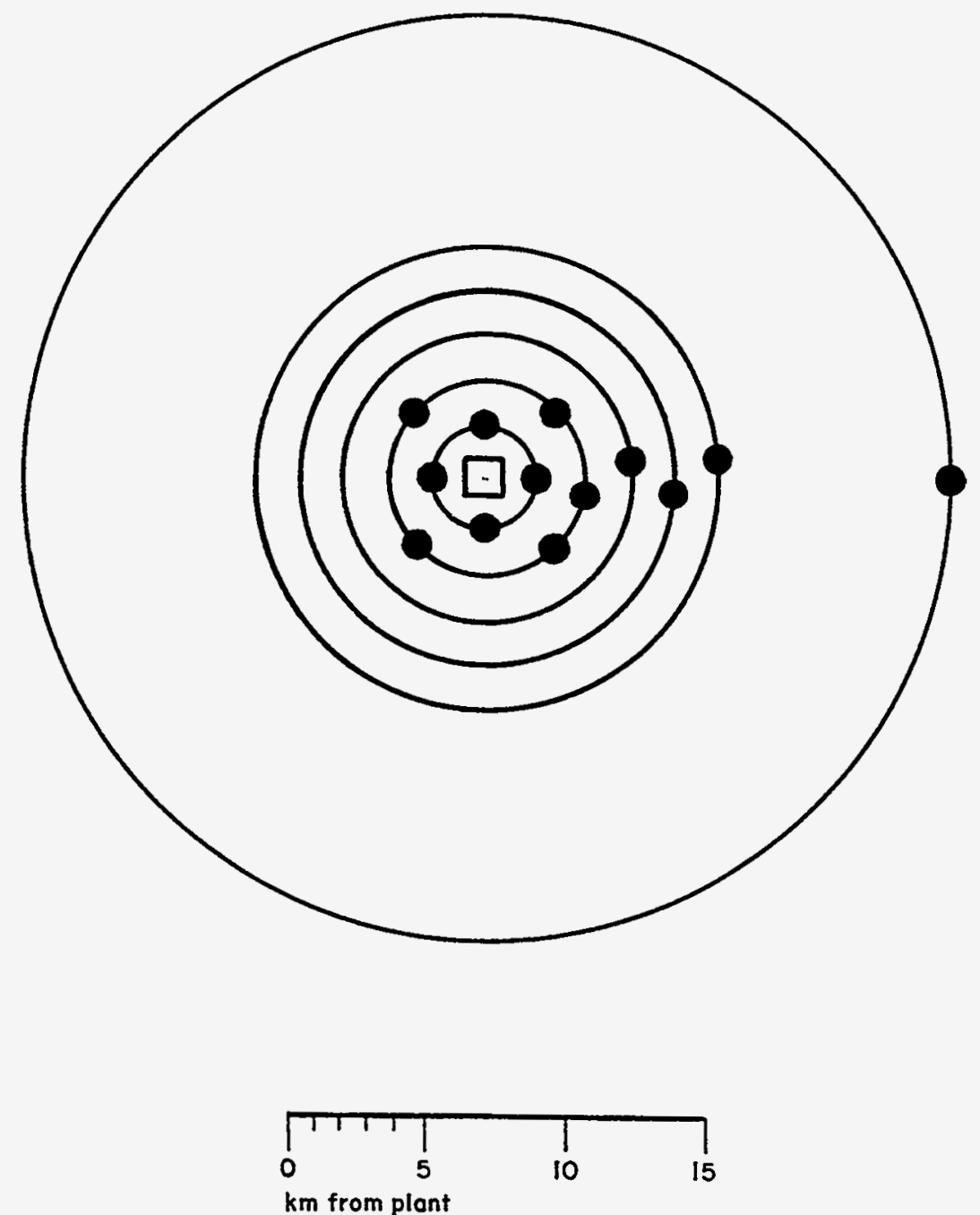

Figure 2.10. A suggested distribution of sampling sites covering the area surrounding a plant, with emphasis on the downwind direction. 


\title{
2.5 SEDIMENT
}

\author{
Contact Person : Mathew A. Monetti
}

\subsubsection{SCOPE}

Discussed in this section are the methodologies used at EML for obtaining sediment cores from inland lakes and reservoirs for reconstructing the deposition histories of energy-related pollutants. Topics included are the relationship of lake bathymetry to core quality, a general description of the operation of the corer from our specially designed catamaran, and the procedures for extruding the cores. The catamaran itself is discussed in Section 6.7.

\subsubsection{INTRODUCTION}

Lake and reservoir sediments offer unique substrates for investigating the occurrence of many energy-related pollutants since they are the main "sink" for materials entering watersheds and may be dated by radioactive methods so as to provide a depositional history (MARC, 1985). Furthermore, accurate coring, e.g., minimal disturbance of sediments, is of great importance to paleolimnological investigations, especially those aimed at reconstructing the deposition history and/or inventories of pollutants deposited through atmospheric processes. Errors caused by a poorly performing coring system or by taking sediment cores from locations in lakes that are not representative of atmospheric deposition (e.g., areas affected by sediment focusing or excessive erosion) can often lead to an erroneous interpretation of actual events (Heit and Miller, 1987).

To properly assess geochronologies of sediments in a meaningful way, methodologies must be available for taking large volume, undisturbed sediment cores, so that multiple parameter analyses can be performed at critical core depths. As stated in Burke (1968), for the commonly used 4-7 cm diameter sediment corers, increased sample size can be obtained only by homogenization of longer segments of core, with consequent blurring of stratigraphic details. For isotopic dating procedures, the demand for really large samples (>200 g wet weight) presents an even more severe problem. Only by increasing the area of the column sampled can one improve the sensitivity in the study of sediment horizons. 
Unfortunately, most larger area coring devices are of such size and weight that they can only be used on oceanographic-sized vessels, making them unfit for studies of most lakes and reservoirs.

As further stated in Burke (1968), the study of vertical profiles of the concentrations of fallout radionuclides (which have been available for sedimentation only since the early $1950 \mathrm{~s}$ in most places) also demands efficient sampling of the topmost layers of sediments and their recovery in situ. Some evidence from studies of more conventionally collected cores indicates that these upper layers are commonly lost, either by washout at the top of the core tube or by being swept away from the point of impact by the shock wave of rapidly falling corers (Sachs and Raymond, 1965). Also, as stated in McIntyre (1971), a peculiarity of many gravity corers is that the length of the core retrieved may be considerably less than the penetration depth of the core barrel into the sediment. This is likely to cause a vertical redistribution of the sediment and hence an erroneous chronology. Also core tubes having diameters $<10 \mathrm{~cm}$ are subject to loss of surface sediment (McIntyre, 1971).

\subsubsection{EQUIPMENT DESIGN AND APPLICATION}

We have developed a large diameter $(21 \mathrm{~cm})$, slow penetration sediment corer, and a $27 \mathrm{ft}(7 \mathrm{~m}$ ) transportable research vessel ("Sedimental Journey") to avoid the pitfalls described above. This equipment, whose design and construction are described in detail in Section 6.7, has allowed us to take large volume, undisturbed cores from many locations in the U.S. and measure increments of sediment as small as $1 \mathrm{~cm}$ for a suite of toxic trace substances and environmental tracers indicative of both natural and anthropogenic origins. In fact, sediment cores taken with the tripod-sphincter corer have been shown to be comparable in quality to those taken with the much larger "Soutar type" box corers which, because of their size, can only be used successfully on oceanographic vessels (Burke et al., 1983).

Examples of highly resolved sediment cores that can be obtained with our sediment sampling equipment are shown in Figures 2.11-2.13. Plotted in these figures is the ${ }^{137} \mathrm{Cs}$ activity per unit area $\left(\mathrm{Bq} \mathrm{m}^{-2}\right)$ versus depth $(\mathrm{cm})$ for sediment cores taken from Cayuga Lake, Ithaca, NY (Figure 2.11), and Deer Creek Reservoir, Provo, UT (Figure 2.12). Clearly, the ${ }^{137} \mathrm{Cs}$ distributions closely follow the historic deposition 
pattern of ${ }^{137} \mathrm{Cs}$ from atmospheric weapons testing shown in Figure 2.13. The two easily distinguished ${ }^{137} \mathrm{Cs}$ peaks follow the major cycles of fallout from atmospheric weapons testing in 1959 and 1963 . Furthermore, there is a rapid drop of ${ }^{137} \mathrm{Cs}$ activity in sediments younger than 1963 following the Nuclear Test Ban Treaty between the U.S. and U.S.S.R. Lastly, the activity after the onset of atmospheric weapons testing follows closely that of historic fallout patterns with little debris diffused or mixed below the depth corresponding to 1951 .

Further confirmation of our ability to take quality cores with this system is shown by the use of the SNAP-9A (Systems for Nuclear Auxiliary Power) Pu isotope signature. In 1964, the U.S. satellite, SNAP-9A, which used ${ }^{238} \mathrm{Pu}$ as a heat source for generating power, disintegrated after entering the atmosphere of the Southern Hemisphere. As a result, there was a sharp rise in the stratospheric concentration of ${ }^{238} \mathrm{Pu}$ relative to ${ }^{239+240} \mathrm{Pu}$ in 1964 in the Southern Hemisphere. The pulse of ${ }^{238} \mathrm{Pu}$ from SNAP-9A did not reach the surface of the Northern Hemisphere until 1966, at which time a distinct rise in the ratio of ${ }^{238} \mathrm{Pu}$ to ${ }^{239+240} \mathrm{Pu}$ activity also occurred. It is the increase in this ratio which is used as a geochronological marker for establishing the year 1966 in a sediment core. This is clearly shown in Figure 2.14 where the ${ }^{238} \mathrm{Pu} /{ }^{239+240} \mathrm{Pu}$ activity ratio increases between 13 and $11.5 \mathrm{~cm}$ deep in the sediment core from Cayuga Lake, NY.

The remainder of this section is a description of the procedures that are used by EML to take sediment cores from inland lakes and reservoirs using the Sedimental Journey and tripod corer.

\title{
2.5.4 PROCEDURES
}

\author{
2.5.4.1
}

GENERAL OPERATION

Procedures for the preparation and operation of the Sedimental Journey are described in detail in Section 6.7. The basic coring strategy is to launch the boat and determine the best sampling sites through a bathymetric survey of the lake or reservoir, obtain the 
cores, extrude the cores immediately after their retrieval, and, finally, remove the boat from the water with the trailer.

Bathymetric surveys are always conducted prior to coring since such data are usually not available. It has been our experience that even when bathymetry is available, it is usually inaccurate or out of date and hence misleading. This can be a severe problem because the lack of accurate bathymetric data will almost certainly result in a failure to locate suitable coring locations within a lake or reservoir.

In general, it has been our aim to take replicate cores from those portions of lake basins which are the least likely to be affected by processes known to disturb sediments, such as, excess terrestrial runoff (erosion), sediment slumping, sediment resuspension, and sediment focusing (Davis and Ford, 1982; Edgington and Robbins, 1977; Heit and Miller, 1987). In this regard, the bathymetric survey is used to select sites located in relatively large, hypolimnetic basins, as far away as possible from shoreline areas prone to erosion. Inlet and outlet areas, as well as locations at the confluence of rivers or close to dams are also avoided.

For our bathymetric survey work we use a Lowrance graph recorder sonar unit. This device is capable of accurately graphing a lake bottom to a depth of $300 \mathrm{~m}(900 \mathrm{ft})$. Our location is determined using a micrologic explorer Loran system.

\section{SEDIMENT SAMPLING}

All of the sediment cores are taken with a sphincter corer with a tripod modification capable of taking a $21 \mathrm{~cm}$ diameter, $90 \mathrm{~cm}$ long core. The design and mechanics of the corer are described in detail in Section 6.7. 
The device uses the barrel and nose-cone of the original 21-cm sphincter corer (Burke, 1968), but is mounted in a tripod (see Section 6.7.4). The operation of the sphincter is not changed by this design, the tripod frame only provides stability to keep the corer in a vertical position while it penetrates. This design also allows the corer to be lowered to the bottom very slowly, with minimal sediment disturbance. The core barrel is driven into the sediment only by the force of weights mounted on the weight stand (see Section 6.7.4). Varying the amount of this weight gives control over penetration into various sediment types. It should be noted that the tripod corer is "manageable" on small boats and is easily disassembled for transporting or shipping.

The corer is lowered at $100 \mathrm{~m} \mathrm{~min}^{-1}\left(75 \mathrm{~m} \mathrm{~min}^{-1}\right.$ in fairly active weather) until it is 5 $\mathrm{m}$ from the bottom (observable with the Sonar unit). It is then slowed to $10 \mathrm{~cm} \mathrm{~min}^{-1}$, or as slow as the winch will operate, for placement on the bottom. The feet of the tripod contact the sediment first. The barrel continues to descend, penetrating the sediment by the force of the weights contained in the weight stand. The core barrel stops its downward travel when the weight stand comes in contact with the guide ring (see Section 6.7.4.2), or when the resistance of the sediment is sufficient to stop the downward penetration. When the weight stand comes to a stop, the weight is taken off the springloaded release pin and the pin retracts (see Section 6.7.4). This should be completed within $60 \mathrm{sec}$ after the corer reaches the bottom. The pull on the wire to retrieve the corer begins immediately and closes the diaphragms of the core catcher and the top valve. Stress is taken off the closure wires by a stop which engages the bottom of the release mechanism where force is exerted during retrieval.

The corer is pulled out of the sediment at the slowest winch speed, or about $10 \mathrm{~cm} \mathrm{~min}^{-1}$. In the past, pullout forces have not exceeded $4450 \mathrm{~N}$ (1000 lb force) and are generally somewhat lower. After pullout the corer can be retrieved at a rate of $50 \mathrm{~m} \mathrm{~min}^{-1}$.

\subsubsection{4}

SEDIMENT EXTRUSION

More than one method has been employed to extrude sediments from the core barrels since EML started coring in the early 1970s. In all of these methods, the sediments are always extruded immediately after retrieval. Water retained above the core is siphoned or 
pipetted off and may be reserved for analyses. In most cases, contiguous $1 \mathrm{~cm}$ increments are taken from the top of the core to $40 \mathrm{~cm}$, and $2 \mathrm{~cm}$ sections are taken thereafter. The outer $\sim 1 \mathrm{~cm}$ of each layer of sediment is always discarded, resulting in $\sim 19 \mathrm{~cm}$ diameter sections. We have found that if this outer ring of sediment is not eliminated, contamination may result from surface sediments "dragging down" to subsurface depths via friction with the core barrel wall or through other physical processes. Lastly, glassware and covers used for sample storage are prewashed with 1:1 $\mathrm{HNO}_{3}$, rinsed with double-deionized water, followed by acetone and methyl-alcohol washes. All of the glassware is dried and sealed under laminar flow clean stations until the time of use. Precleaned glass jars are used, which can be obtained from several suppliers.

Prior to extrusion, the length of the core and its distance from the top of the barrel are measured and noted. After the excess water is removed by siphon or pipette, the sediment is then carefully removed in $1 \mathrm{~cm}$ increments with a large Teflon-coated spoon bent into an " $\mathrm{L}$ " shape. The accuracy of this procedure is ensured by placing the sediment in precalibrated 16-oz (500 $\mathrm{mL})$ wide-mouth glass jars, and noting the actual extrusion depth relative to the initial location of the top of the core as measured immediately after core retrieval. In addition, the core barrels are marked at $5 \mathrm{~cm}$ increments for further calibration; thus, errors in extrusion are identified.

Once the surface sediment is removed, the remaining sediment is usually firm and is not easily disturbed. The rest of the core can be sectioned in the following manner. A stainless steel piston is fitted inside the top of the core barrel and contacts the sediment. While this piston is held in place, the core is laid down on its side, the bottom core catcher is removed, and another piston is placed into the bottom of the core. The core is then listed upright again and placed on an extruding stand. Next, the top piston is removed and the bottom one is pushed up through the core barrel until the sediment becomes flush with the top of the barrel. The remainder of the sediment is extruded at 1 to $2 \mathrm{~cm}$ intervals by pulling the barrel down to designated marks on the attached scale. The outer ring is removed from the protruding sediment; the rest of the interval is sliced and packed into sampling jars. Sediment is sectioned in this way to a desired depth or to the bottom of the core. A description of the construction and design of the extruder used to section the core is given in Section 6.7.4. 
The reproducibility of this technique is demonstrated in Figure 2.15, which compares the ${ }^{137} \mathrm{Cs}$ distribution in two sediment cores (A and B), taken from separate but nearby locations (within $0.5 \mathrm{~km}$ ) in Cayuga Lake, Ithaca, NY. Although the sedimentation rate was slightly different for the two locations (Heit et al. 1986), as would be expected from bathymetric variations within a lake basin, even for nearby locations, the ${ }^{137} \mathrm{Cs}$ distribution patterns were remarkably similar with only small differences occurring among the major peaks which represent fallout from atmospheric weapons testing in 1959 and 1963. This agreement $\left( \pm 2 \mathrm{~cm}\right.$ ) between the patterns of ${ }^{137} \mathrm{Cs}$ distributed in these independent cores attests to the reproducibility of our current method of sediment extrusion.

\section{REFERENCES}

Burke, J. C.

"A Sediment Coring Device of 21-cm Diameter with Sphincter Core Retainer" Limnology and Oceanography, $\underline{13}$, 714-718 (1968)

Burke, J. C., R. E. Hamblin, and S. A. Casso

"Tripod Modification Of Sphincter Corer: Construction, Operation, Core Extrusion and Sampling Efficiency"

Woods Hole Oceanographic Institution Technical Report WHOI-83-36, Woods Hole, MA 02543, pp. 1-13 (1983)

Davis, M. B. and M. S. Ford

"Sediment Focusing In Mirror lake, New Hampshire"

Limnology and Oceanography, 27, 137-150 (1982)

Edgington, D. N. and J. A. Robbins

"Patterns Of Deposition of Natural and Fallout Radionuclides to Limnological Processes" J. O. Nriagu (Editor) in: Environmental Biogeochemistry - Metals Transfer and Ecological Mass Balances Ann Arbor Science, Ann Arbor, MI, pp. 705-729, Vol. 2 (1977) 
Heit, M. and K. M. Miller

"137 Cs Sediment Depth Profiles And Inventories In Adirondack Lake Sediments"

Water, Air and Soil Pollution, $\underline{3}$, 243-265 (1987)

Heit, M., Y. L. Tan, K. M. Miller, J. Quanci, C. Marinetti, and S. Silvestri

"The Sediment Chronology and Polycyclic Aromatic Hydrocarbon Concentrations and Fluxes in Cayuga Lake, NY"

U.S. Department of Energy Report EML-451 (1986)

MARC: Monitoring and Assessment Research Centre

"Historical Monitoring"

MARC Report 31, University of London, London, England (1985)

McIntyre, A. D.

"Efficiency of Benthos Sampling Gear"

N. A. Holme and A. D. Mclntyre (Editors)

in: Methods for the Study of Marine Benthos

International Biological Program Handbook No. 16, Blackwell Scientific Publications, pp. 140-146 (1971)

Sachs, P. L. and S. O. Raymond

"A New Unattached Sediment Sampler"

J. Marine Res., 23, 44-53 (1965) 

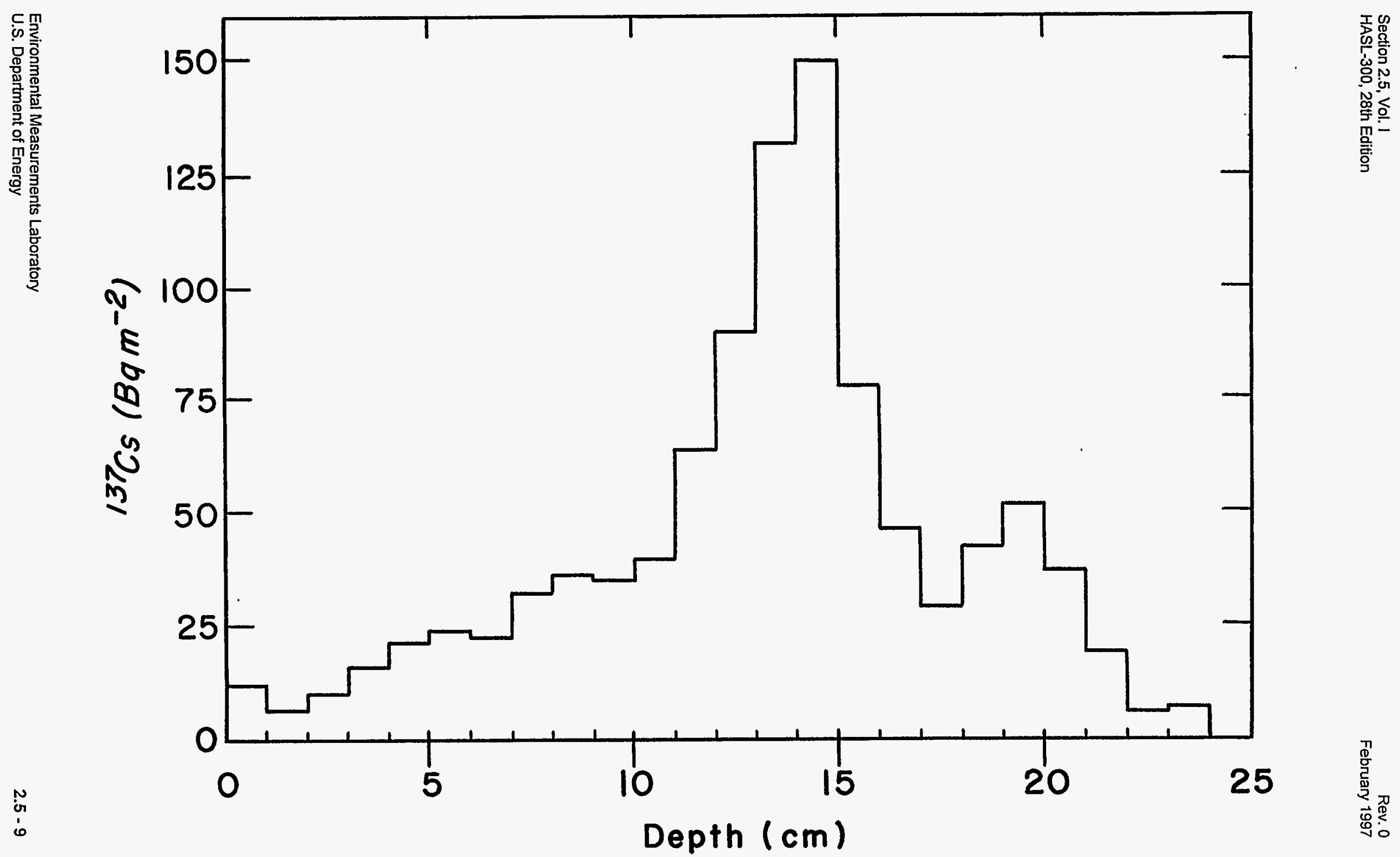

Figure 2.11. ${ }^{137} \mathrm{Cs}$ activity per unit area versus sediment depth for Cayuga Lake, New York. 


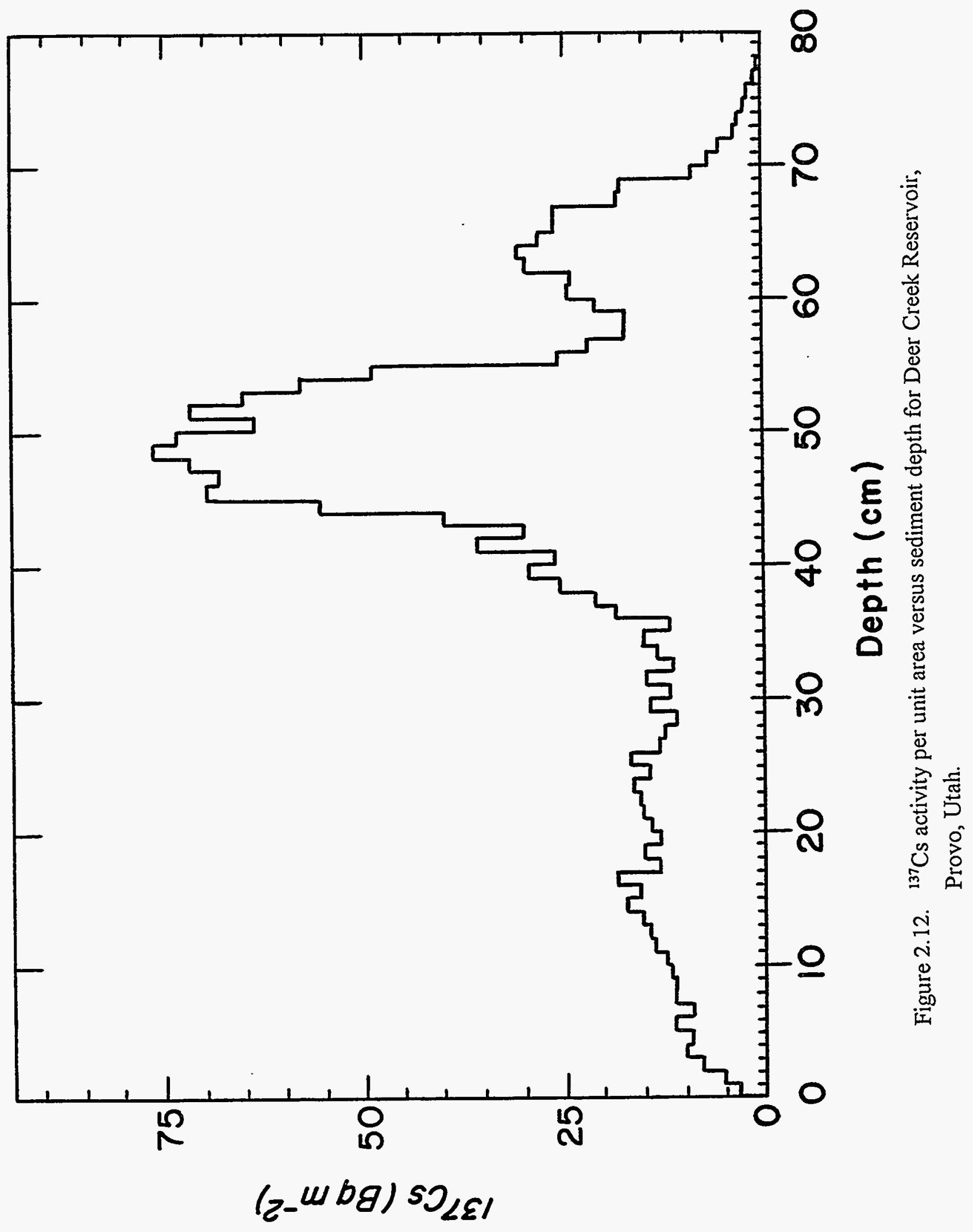




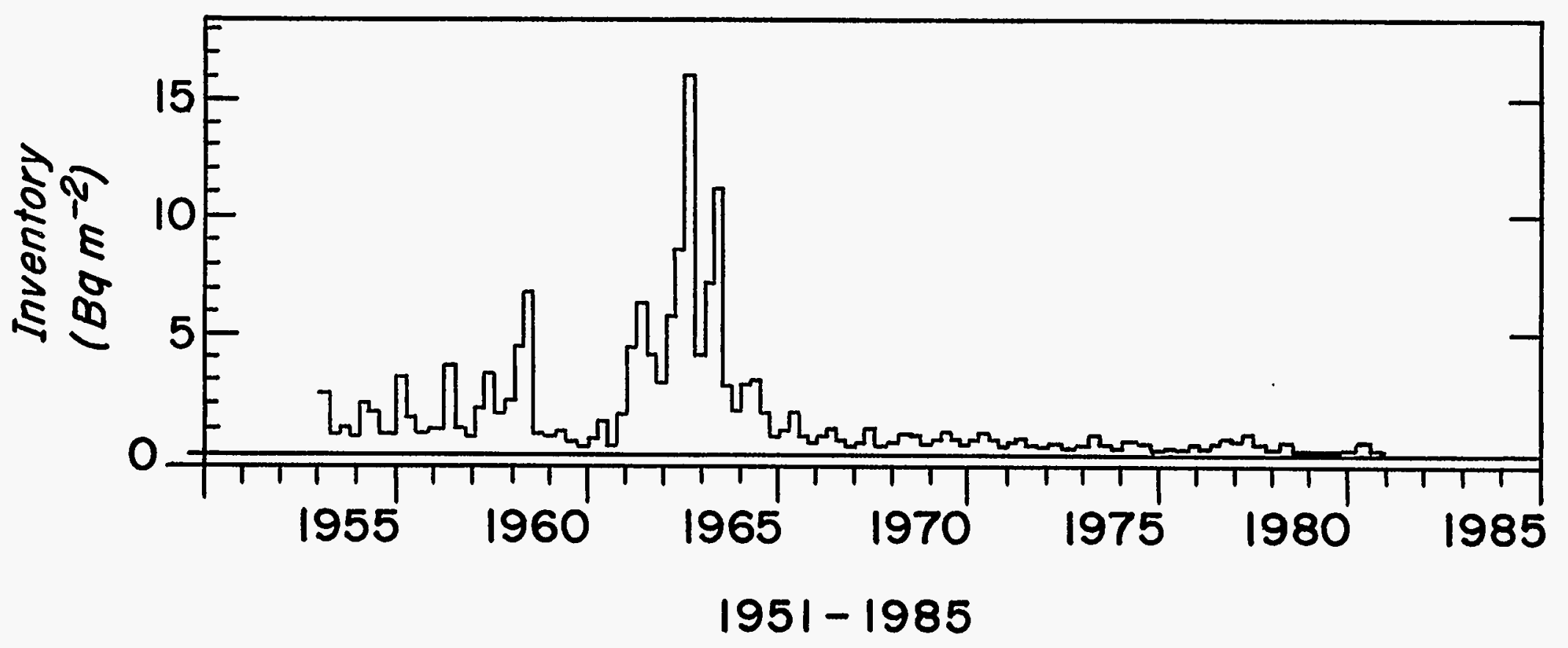

Figure 2.13. Historic deposition patterns of ${ }^{137} \mathrm{Cs}$ in the $40^{\circ}-50^{\circ}$ latitude band from atmospheric weapons testing. 


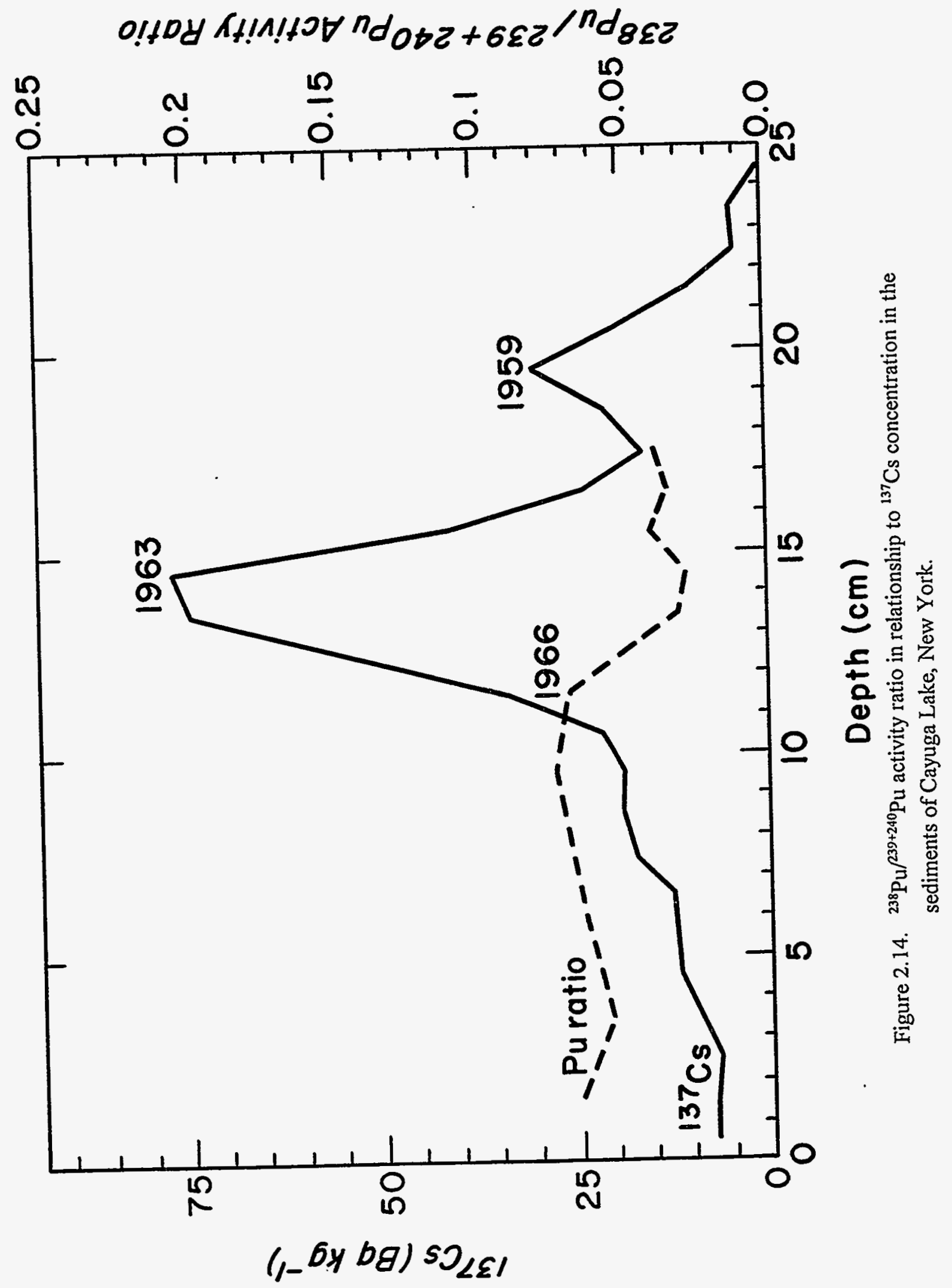




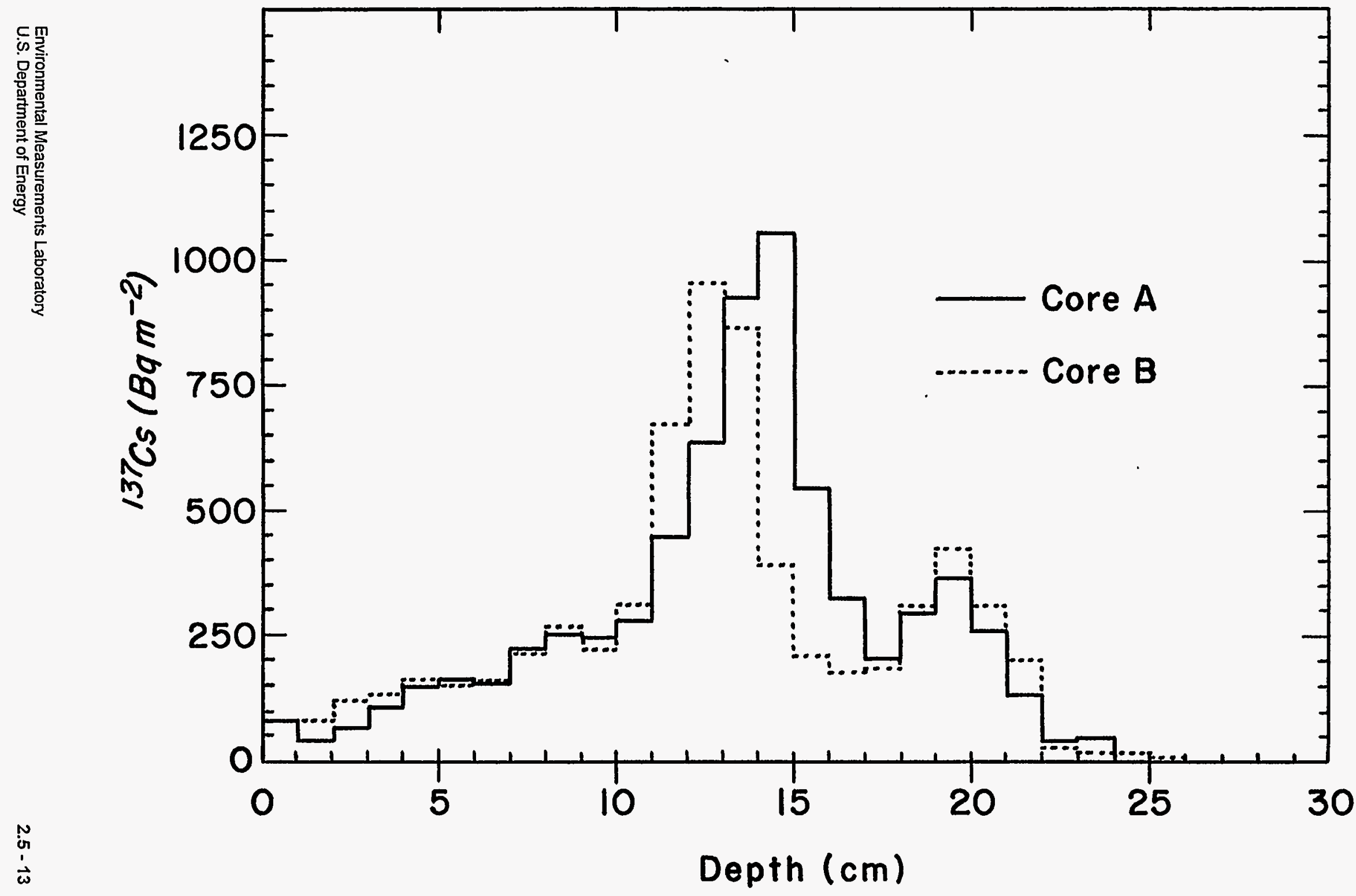

Figure 2.15. ${ }^{137} \mathrm{Cs}$ distribution in two sediment cores taken from nearby locations in Cayuga Lake, New York. 


\title{
2.6 URANIUM AND RADIUM IN WATER
}

\author{
Contact Person(s) : Isabel M. Fisenne
}

\subsubsection{SCOPE}

A simple method for the field collection of uranium and radium in $10 \mathrm{~L}$ of fresh water (tap, well, river) is described. Similar collection methods for radium are in use elsewhere, notably at the New York University Institute of Environmental Medicine.

This method has been used to collect uranium and radium from fresh water samples at environmental levels. The use of the ion exchange collector concentrates the uranium and radium in the water samples and permits shipment of the samples within national and international regulations. Water samples collected with this system have been sent to EML from locations within the continental U.S., the middle East, and Africa.

\subsubsection{APPARATUS}

The ion exchange collector is a modified version of the fallout collector described in Section 2.3.3. This unit consists of a funnel and ion exchange column constructed of polyethylene. The funnel is welded to a threaded cap which is attached to the top of the ion exchange column. The bottom of the column is threaded for a tapered fitting, which in turn has a small cap at the end. The funnel and the tapered fitting are replaced with standard bottle caps for return shipment to EML.

\subsubsection{PROCEDURE}

The column is packed with a plug of glass wool, a $1 \mathrm{~cm}$ plug of Whatman No. 41 paper strip (Note 1), $150 \mathrm{~mL}$ of mixed anionic-cationic resin (Note 2), and a top plug of filter paper strip. The threaded tapered fitting (outlet end of the column) is taped to the column to assure leak-proof operation during sampling. 
The column is attached to a funnel marked for a $10 \mathrm{~L}$ volume, it is then placed in a stand and $10 \mathrm{~L}$ of the water to be sampled is poured into the funnel. The bottom cap is removed from the tapered fitting, allowing gravity flow of the water. The collection time is about $3 \mathrm{~h}$.

Upon return to EML, the resin and paper pulp are pushed out of the column body into a 250-mL platinum crucible. The glass wool is discarded. The resin may be analyzed sequentially for isotopic uranium and radium (see Procedure Se-01, Section 4).

Notes:

1. Filter paper pulp must not be used, as the flow rate through the column is too slow. Narrow strips cut from filter discs are recommended.

2. The resin is an equal mixture of Bio-Rad AG1-X4 in the $\mathrm{H}^{+}$form (20-50 mesh) and Bio-Rad AG 50-X8 (20-50 mesh). 


\subsection{FOOD}

(see Volume II)

Environmental Measurements Laboratory 


\section{RADIATION MEASUREMENTS}

U.S. Department of Energy 201 Varick Street, 5th Floor New York, NY 10014-4811 
Page

\section{Radiation Measurements}

3.2.1 Scope $\ldots \ldots \ldots \ldots \ldots \ldots \ldots \ldots \ldots \ldots \ldots \ldots \ldots \ldots \ldots .2 .2-1$

3.2.2 PIC System Design .......................... 3.2-1

3.2.2.1 Ion Chamber ............................ 3.2-1

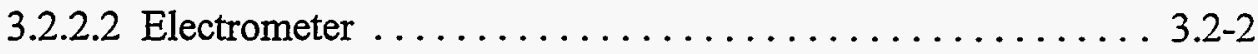

3.2.2.3 Readout Systems $\ldots \ldots \ldots \ldots \ldots \ldots \ldots \ldots \ldots \ldots . . .2 .2-3$

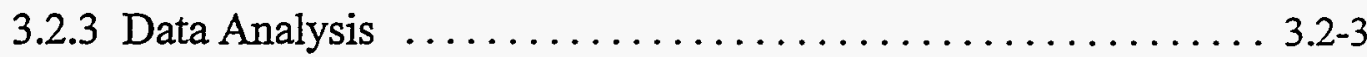

3.2.4 Calibration $\ldots \ldots \ldots \ldots \ldots \ldots \ldots \ldots \ldots \ldots \ldots \ldots \ldots \ldots \ldots \ldots \ldots \ldots .2-4$

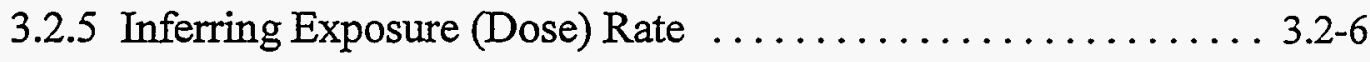

\subsection{Field Gamma-Ray Spectrometry ................ 3.3-1}

3.3.1 Scope $\ldots \ldots \ldots \ldots \ldots \ldots \ldots \ldots \ldots \ldots \ldots \ldots \ldots \ldots \ldots .3 .3-1$

3.3.2 Instrumentation $\ldots \ldots \ldots \ldots \ldots \ldots \ldots \ldots \ldots \ldots \ldots \ldots \ldots \ldots \ldots \ldots \ldots \ldots \ldots \ldots \ldots .3 .2$

3.3.3 Site Selection and Instrument Setup ................ 3.3-3

3.3.4 Calibration ................................... 3.3

3.3.5 Spectrum Analysis $\ldots \ldots \ldots \ldots \ldots \ldots \ldots \ldots \ldots \ldots \ldots . . .3 .3 .8$

3.3.6 Inventory Measurements $\ldots \ldots \ldots \ldots \ldots \ldots \ldots \ldots \ldots \ldots \ldots \ldots \ldots \ldots \ldots .3 .10$

3.3.6.1 Application ............................. 3.3-10

3.3.6.2 Homogeneous Terrain $\ldots \ldots \ldots \ldots \ldots \ldots \ldots \ldots \ldots . .3 .3-11$

3.3.6.3 Nonhomogeneous Terrain $\ldots \ldots \ldots \ldots \ldots \ldots \ldots \ldots . . .3 .3-12$

3.3.7 Spectral Stripping for Germanium Detectors $\ldots \ldots \ldots \ldots \ldots$ 3.3-14

3.3.7.1 Application ............................ 3.3-14

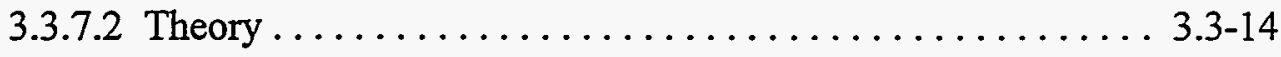

3.3.7.3 Determination of Stripping Parameters ( $f$ and $r$ ) $\ldots \ldots \ldots$ 3.3-15

3.3.7.4 Stripping Operation $\ldots \ldots \ldots \ldots \ldots \ldots \ldots \ldots \ldots \ldots . . .3 .3-16$ 


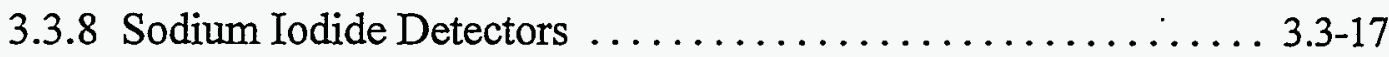



3.3.8.2 Equipment $\ldots \ldots \ldots \ldots \ldots \ldots \ldots \ldots \ldots \ldots \ldots \ldots \ldots \ldots \ldots \ldots \ldots .3-17$

3.3.8.3 Peak Analysis ........................... 3.3-18

3.3.8.4 Energy Band Analysis $\ldots \ldots \ldots \ldots \ldots \ldots \ldots \ldots \ldots . .3 .3-18$

3.3.8.5 Total Spectrum Energy Analysis $\ldots \ldots \ldots \ldots \ldots \ldots \ldots .3 .3-20$

\subsection{Remote Gamma-Ray Spectrometry Stations .......... 3.4-1}

3.4.1 Scope $\ldots \ldots \ldots \ldots \ldots \ldots \ldots \ldots \ldots \ldots \ldots \ldots \ldots \ldots \ldots . .4 .4-1$

3.4.2 Components ................................. 3.4

3.4.3 Instrumentation Assembly . ...................... 3.4

3.4.4 EML Quality Control .......................... 3.4

3.5 Thermoluminescence Dosimetry ................. 3.5-1

3.5.1 Scope $\ldots \ldots \ldots \ldots \ldots \ldots \ldots \ldots \ldots \ldots \ldots \ldots \ldots \ldots \ldots . . .6 .5 .1$

3.5.2 Special Apparatus $\ldots \ldots \ldots \ldots \ldots \ldots \ldots \ldots \ldots \ldots \ldots \ldots . . .6 .5-1$

3.5.3 Predeployment Preparation of Chips $\ldots \ldots \ldots \ldots \ldots \ldots \ldots .3 .5-4$

3.5.4 Environmental Deployment .................... 3.5-5

3.5.5 Readout and Calibration $\ldots \ldots \ldots \ldots \ldots \ldots \ldots \ldots \ldots \ldots . . .6 .6 .6$

3.5.6 Analysis of Results .......................... 3.5

\subsection{Bonner-Sphere Neutron Spectrometry ............ 3.6-1}

3.6.1 Scope $\ldots \ldots \ldots \ldots \ldots \ldots \ldots \ldots \ldots \ldots \ldots \ldots \ldots \ldots . .6 .6 .6 .1$

3.6.2 General Description $\ldots \ldots \ldots \ldots \ldots \ldots \ldots \ldots \ldots \ldots \ldots . .6 .6 .1$

3.6.3 Personnel and Training $\ldots \ldots \ldots \ldots \ldots \ldots \ldots \ldots \ldots \ldots .6 .6 .2$

3.6.4 Methodology .............................. 3.6-2

3.6.4.1 Description of the System .................. 3.6-2

3.6.4.2 The Bonner Spheres ....................... 3.6-3

3.6.4.3 The Response Functions . . . . . . . . . . . . . . 


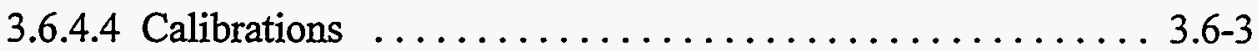

3.6.4.5 Electronics ......................

3.6.5 Neutron Spectrum Unfolding . . . . . . . . . . . . . . 3.6-4

3.6.6 Acceptable Solution ....................... . . . . 


\section{RADIATION MEASUREMENTS}

\subsection{OVERVIEW}

Since about 1950, the Environmental Measurements Laboratory has carried out major research programs directed at the study of ionizing radiation and natural and man-made radionuclides in the working and in the public environment. Among these studies have been: (1) the long-term investigation of the atmospheric distribution and ground deposition of radionuclides from global fallout generated by nuclear weapons tests; (2) the evaluation of the radiation exposure of human populations from these nuclides as well as those deposited locally by the Nevada weapons tests, those released from nuclear facilities (including the reactor accidents at Three Mile Island and Chernobyl), and those normally present from natural sources; and (3) the determination of radiation worker exposures at particle accelerators, nuclear reactors, and other nuclear facilities. An important component of these studies has been the development and improvement of techniques for low-level radiation measurement and data interpretation. In the following sections, five highly generic measurement systems are described that have been developed and/or refined at EML, and that have found wide application in environmental radiation studies. In each case, an essential part of the system is the methodology associated with detector calibration, measurement procedure(s), and data analysis and interpretation. The indicated references provide additional details and examples of applications for the interested reader. 


\title{
3.2 IONIZATION CHAMBERS
}

\author{
Contact Person(s) : Kevin M. Miller
}

\subsubsection{SCOPE}

This section describes the design, analysis procedures, calibration, and use of pressurized ionization chambers (PIC). These instrument systems are used at EML in the assessment of the penetrating component (gamma plus cosmic-ray secondaries) of the environmental radiation field. Accurate and highly precise measurements of the total exposure rate (or dose rate in air) are made of such sources as:

1. cosmic-ray secondary radiation (high energy muons, photons, and electrons) in the lower atmosphere;

2. natural background radiation from primordial radionuclides (and progeny) in the soil and air;

3. anthropogenic isotopes associated with

a. direct radiation from nuclear facilities,

b. gaseous effluents from nuclear operations,

c. fallout deposition,

d. residual radioactivity at sites undergoing clean-up.

\subsubsection{PIC SYSTEM DESIGN}

ION CHAMBER

The chamber selected as our standard consists of a $25-\mathrm{cm}$ diameter stainless-steel sphere with a wall thickness of $2.37 \mathrm{~g} \mathrm{~cm}^{-2}$ filled to a pressure of $2.5 \mathrm{MPa}$ (25 atmospheres) with ultrapure argon gas. The collecting electrode is a $5-\mathrm{cm}$ diameter hollow 
' 
Direct observation of the electrometer output voltage is made with a standard voltmeter. To obtain precise measurements, signal integration is performed by one of two methods listed below.

\section{A. Survey mode.}

For real-time results, a voltage-to-frequency converter with scaler and calibrated digital readout is used to provide readings with selectable integration times of 4,40 , or $400 \mathrm{sec}$. This readout system is generally used with the $18-\mathrm{cm}$ diameter chambers in the form of a two-unit package called a SPICER (small pressurized ionization chamber for environmental radiation; Latner et al., 1983). The system is battery powered and can be either held in hand or tripod mounted. Figure 3.1 shows the system in this latter mode of operation.

\section{B. Monitor mode.}

The second read-out method is designed for continuous remote monitoring. It consists of an analog to digital converter, timing and control circuitry, and a magnetic tape cassette recorder (Memodyne, Model 201) housed in a weather proof aluminum box (Cassidy et al., 1974). The ion chamber and electrometer are packaged in a similar box which sits atop the recorder box during operation in the field as shown in Figure 3.2. The standard system records the digitized electrometer output voltage every $10 \mathrm{sec}$, and is capable of storing up to 17 days of data on a single cassette. Some units have been modified to allow the option of recording every 10,20 or $40 \mathrm{sec}$. An alkaline cell battery pack provides power for 8 weeks of operation.

\subsubsection{DATA ANALYSIS}

The magnetic tape cassette is read out with a Memodyne $3122 \mathrm{ABV}$ reader interfaced to a Hewlett-Packard 9826 computer using a specially developed analysis program (Gogolak, 1982). The analysis procedure is as follows: 
1. A $6 \mathrm{~h}$ record of the $10-\mathrm{sec}$ data is read into the computer and displayed on a screen.

2. The data is averaged over 5-min intervals, corrected for the zero reading of the electrometer and converted to exposure rate via a chamber specific calibration factor.

3. A printout of the 5-min averages as well as the average over each hour is made. The standard deviation (SD) of the 5-min data for each hour is also computed and printed.

4. Subsequent 6-h records are analyzed and at the end of the tape a summary plot of the hourly data is made. Bad data sections are then edited and a final printout and plot of the corrected hourly average and SD data is obtained. The hourly average maximum and minimum and daily average are also printed for each day along with the average over the entire period.

5. Permanent storage of the corrected 5-min data is made on a diskette.

6. If desired, the data can be analyzed for any dose due to the passage of a plume (Gogolak and Miller, 1974). The analysis routine examines the SD of the 5-min data in each hour and if it is sufficiently high $\left(>1.7 \mathrm{nGy} \mathrm{h}^{-1}\right)$ it is assumed that a fluctuating plume component was present. A search is then made on each side of the plume hour for the nearest three background hours indicated by a standard deviation that is sufficiently low $\left(<1 \mathrm{nGy} \mathrm{h} \mathrm{h}^{-1}\right)$. The average background is computed from these $6 \mathrm{~h}$ and subtracted from the total dose in the plume hour to yield the net plume dose.

\subsubsection{CALIBRATION}

Calibration of the PIC is performed using a National Institute of Standards and Technology (NIST) certified sealed $37 \mathrm{MBq}{ }^{226} \mathrm{Ra}$ source and a shadow shield technique (De Campo et al., 1972). The shadow shield method is necessary because it removes the contribution of the room scatter component which we have found to be on the order of $30 \%$ of that from the primary beam. At the same time, it also corrects for any other constant factors included in the PIC output such as room background, electrometer zero offset, and $\alpha$ and stress currents. The calibration procedure is as follows: 
1. The source is placed in a low mass holder at a height of about $1 \mathrm{~m}$ above the floor and at a distance of 4, 5, or $6 \mathrm{~m}$ to the PIC (geometric center), which is at the same height on a low mass stand.

2. A $30-\mathrm{cm}$ thick lead shield with a cross section measuring $10-\mathrm{x}-10 \mathrm{~cm}$ is interposed on a low mass stand so as to intercept all primary rays from the source to all parts of the PIC through the full thickness of the shield. Alignment is checked with a string with a dummy source in place. Slight overshielding (a larger shadow) on the order of $1-2 \mathrm{~cm}$ is used as this results in a negligible error as opposed to undershielding which could produce a significant error. The use of a shield with a cross section that is roughly half that of the PIC is recommended because the shadow size cast will require the shield to be placed near the midpoint between the source and the PIC thus minimizing the production and interception of lead fluorescent $\mathrm{X}$-rays and also allowing a proper alignment which is not too critical to shield placement.

3. The source is placed in its holder and the output signal of the PIC is averaged over a 10- to $30-\mathrm{min}$ time period.

4. The lead shield is removed (leaving its stand in place so as to change the scattering conditions as little as possible), and the PIC output signal is averaged again over a 10 to 30 -min period.

5. The difference between the average signals (reading with shield not in place minus reading with shield in place) is divided by the exposure rate delivered by the source at that distance to yield the Ra primary beam calibration factor. This calibration factor must be adjusted by a small amount for the energy spectrum that will be encountered for a particular radiation field. For our standard $25 \mathrm{~cm}-2.5 \mathrm{MPa}$ chamber, this correction yields a $3 \%$ higher sensitivity for a normal background radiation field, while for the $18 \mathrm{~cm}-3.7 \mathrm{MPa}$ chamber it is $1 \%$ higher.

6. Background readings are taken in the calibration room and in a whole body counter in both the negative and positive high voltage modes of operation to verify that the system does not have stress currents present and that there is no high internal background. 


\subsubsection{INFERRING EXPOSURE (DOSE) RATE}

The total PIC current can be expressed as

where

$$
R=k_{c} I_{c}+k_{t} I_{t}+R^{\prime}
$$

$\mathrm{k}_{\mathrm{c}}=$ the calibration factor for cosmic radiation

$I_{c}=$ the cosmic radiation exposure rate

$\mathrm{k}_{\mathrm{t}}=$ the calibration factor for terrestrial radiation

$I_{t}=$ the terrestrial radiation exposure rate

$R^{\prime}=$ the $\alpha$ particle current from contamination in the steel shell $(\sim 2 \mathrm{fA}$ for the $25-\mathrm{cm}$ chamber and $1 \mathrm{fA}$ for the $18-\mathrm{cm}$ chamber)

For our standard chamber, the values of $\mathrm{k}_{\mathrm{c}}$ and $\mathrm{k}_{\mathrm{t}}$ are only $1 \%$ different so that for most applications the total exposure rate $\left(I_{c}+I_{t}\right)$ is inferred by simply subtracting the $\alpha$ current from the total current and dividing by the factor corresponding to the dominant component of the radiation field. (In a strict sense, the quantity "exposure rate" is only applied to $\gamma$ rays of certain energies. However, for environmental radiation fields it is convenient to extend the meaning to include the exposure rate equivalent of ionization in free air due to cosmic rays.) For expressing the exposure rate in SI units, the appropriate quantity would be $\mathrm{C} \mathrm{kg}^{-1} \mathrm{~s}^{-1}$ or $\mathrm{A} \mathrm{kg}^{-1}$. Since this is a rather unfamiliar unit, we prefer to convert the exposure rate to dose rate in air when reporting data in SI units.

A more accurate estimate of $I_{t}$ is derived by substituting a value for $I_{c}$ in the above equation. To do this, the altitude of a measurement site is determined using a topographical map or the barometric pressure is measured. The corresponding cosmicray exposure rate (dose rate in air) is inferred using the data presented in Figure 3.3. It should be noted that $I_{c}$ will vary with the 11 year solar cycle, being a few percent higher (lower) at solar minimum (maximum) (O'Brien, 1972).

Although not common with the small freely suspended MOSFET electrometer, stress currents can result from mechanical pressure on the ionization chamber insulator. The presence of these unidirectional currents is checked by reversing the high voltage polarity on the ion chamber shell. After correcting for the electrometer zero offset, the readings 
should agree if no stress current is present. If the readings do differ, the true reading is just the arithmetic average of the two.

\section{REFERENCES}

Cassidy, M. E., S. Watnick, V. C. Negro, D. C. Freeswick and R. T. Graveson

"A Computer-Compatible Field Monitor System"

IEEE Transactions Nuclear Science, NS-21, 461 (1974)

De Campo, J. A., H. L. Beck and P. D. Raft

"High Pressure Argon Ionization Chamber Systems for Measurements of Environmental Exposure Rates"

USAEC Report HASL-260, December (1972)

Gogolak, C. V.

"Collection and Analysis of Environmental Radiation Data Using a Desktop Computer" USDOE Report EML-398, April (1982)

Gogolak, C. V. and K. M. Miller

"Method for Determining Radiation Exposure Rates due to a Boiling Water Reactor

Plume from Continuous Monitoring Ionization Chambers"

Health Physics, 27, 132-134 (1974)

Latner, N., K. Miller, S. Watnick and R. T. Graveson

"SPICER: A Sensitive Radiation Survey Instrument"

Health Physics, 44, 379-386 (1983)

Negro, V. C., S. Watnick and P. D. Raft

"A Temperature-Compensated Electrometer for Environmental Measurements"

IEEE Transactions Nuclear Science, NS-21, 805 (1974)

O'Brien, $\mathrm{K}$.

"The Cosmic Ray Field at Ground Level"

In: The Natural Radiation Environment II

USERDA CONF-720805-P1, Vol. 1, pp. 15-54 (1972) 


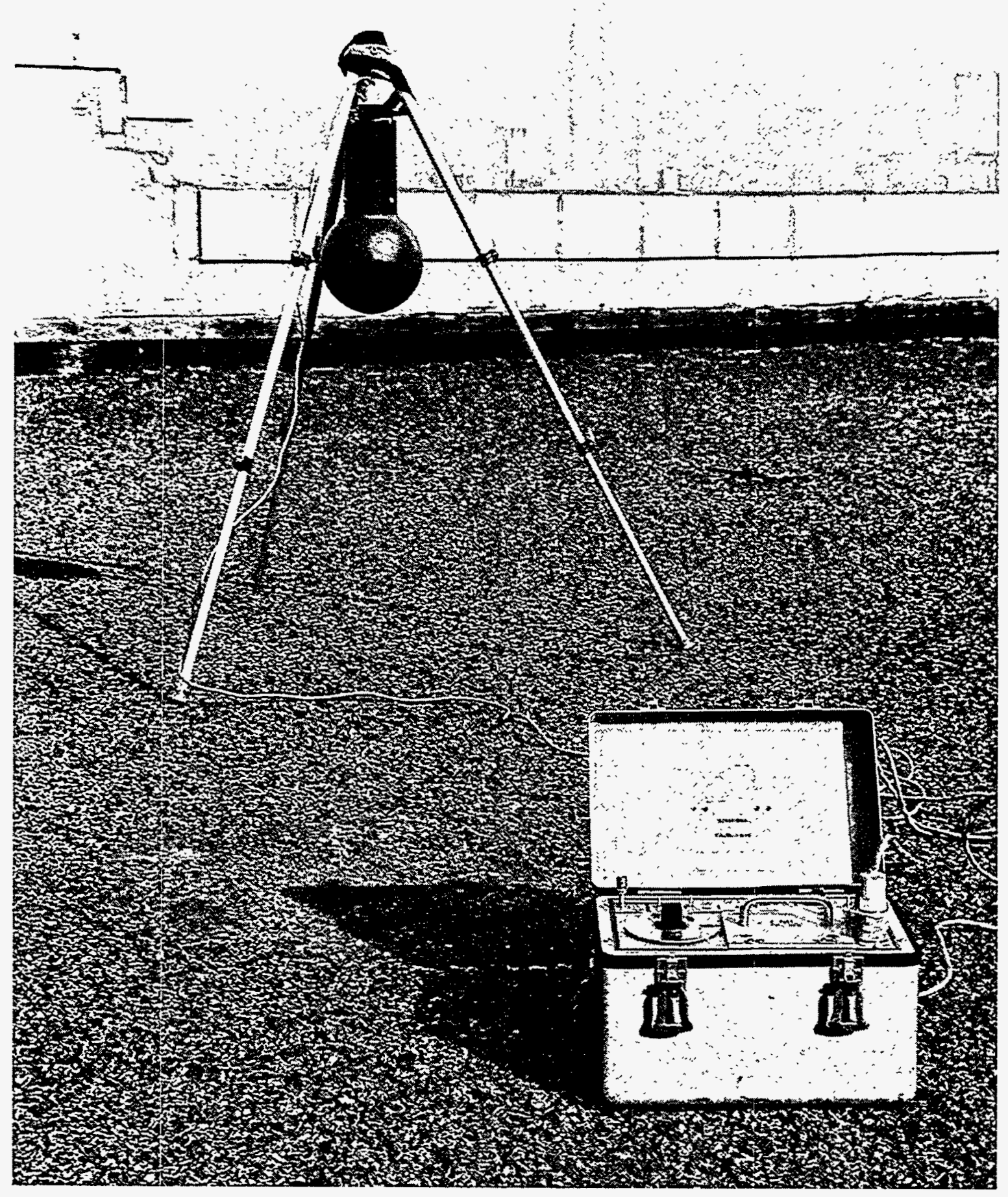

Figure 3.1. SPICER system set up for field measurements showing a tripod mounted ion chamber with an electrometer unit connected via cable to the digital readout 


\title{
3.3 FIELD GAMMA-RAY SPECTROMETRY
}

\author{
Contact Person(s) : Kevin M. Miller
}

\subsubsection{SCOPE}

This section describes the instrumentation, setup, calibration and analysis for EML's field (in situ) $\gamma$-ray spectrometry using high resolution $\mathrm{Ge}$ detectors. The specific application to inventory measurements is described, as well as a spectral stripping routine for total exposure rate measurements. Methods for low resolution $\mathrm{NaI}$ detectors are also given.

Field spectrometry is used at EML for the rapid identification of radionuclides in the environment. When the source geometry is taken into account, the concentrations or inventories (activity per unit area) of these radionuclides in the soil can be inferred along with the contribution to the above ground exposure rate. Applications have included:

1. the measurement of natural background and weapons test fallout emitters (Lowder et al., 1964a, b; Beck, 1966; Beck et al., 1964);

2. indoor radiation studies (Miller and Beck, 1984);

3. analysis of power reactor plumes (Gogolak, 1984);

4. the determination of aged fallout levels in terrain with mixed ground cover (Miller and Helfer, 1985).

5. site characterization for environmental restoration (Miller et al., 1994). 

and used for sample analysis. Once cooled down and detached from the feeder dewar, the hand-held cryostat (1.2 L) can be used for as long as $24 \mathrm{~h}$ before refilling is required. For studies involving low energy, a recently acquired detector measures $75 \%$ in relative efficiency and is of $\mathrm{N}$-type Ge with a beryllium window. It has a 3-L dewar and can be hand-held although it is best suited for tripod mounting.

Generally, measurements are made in the field using a portable battery-powered computer-based spectroscopy system. High voltage and preamplifier power are supplied to the detector by the system. Some detectors feature low power preamplifiers which provide for extended operational time when using battery power in the field. A spectroscopy grade amplifier is also contained within the system. The complete spectrometer system can be carried and operated by one person.

\subsubsection{SITE SELECTION AND INSTRUMENT SETUP}

The detector is placed $\sim 1 \mathrm{~m}$ above the ground with the analyzer and operator positioned a few meters away. The site chosen should be a flat, relatively even and an open area. Terrain that has obstructions such as boulders, large felled or standing trees, and any man-made structures should be avoided as these will block the $\gamma$ flux from the underlying soil. Extreme ground roughness will result in anomalies since the soil surface area close to the detector is increased, while the surface contribution from large distances is reduced. For measurements of fallout radionuclides, the area must be undisturbed in that water and wind erosion as well as human activity, such as cultivating, would tend to upset the distribution of any deposited activity. Figure 3.4 shows an example of equipment placement at a typical field site.

When selecting a site for measurement, the source geometry must be taken into account. An unshielded detector, placed at $1 \mathrm{~m}$ above the ground, samples the photon flux from a volume of soil out to a radius on the order of $10 \mathrm{~m}$ and down to a depth of about $30 \mathrm{~cm}$, depending upon the photon energy. Figure 3.5 shows a pictorial representation of the relative ground area contributions to the primary (uncollided) flux at a height of $1 \mathrm{~m}$ for a medium energy (662 keV) source with a typical exponential depth profile in the soil. This effective "field of view" varies, being somewhat larger (smaller) for higher (lower) energy sources. Also, activity that is closer to the soil surface will produce a wider field of view. In effect, a field spectrum samples an area of several 
hundred square meters, thus averaging out the local inhomogeneities in the distribution of the radionuclides. The source being measured is essentially a giant soil sample and counting statistics for a given spectral absorption peak are obtained in a fraction of the time required for counting a small collected sample.

A good practice to follow is to make a series of short measurements in an area to ensure that there is approximate uniformity before collecting a longer spectrum and obtaining the desired counting statistics. When making measurements of natural background, or when the exposure rate is dominated by the man-made emitters under study, this check can be performed with an ionization chamber or a suitably sensitive survey ratemeter in that uniform exposure rate readings would imply a spatially invariant source distribution relative to the detector. If the particular radionuclides under study contribute just a small fraction to the total exposure rate, then the corresponding peak area count rates should be checked with the spectrometer to ensure uniformity.

\subsubsection{CALIBRATION}

A complete description of field $\gamma$-ray spectrometry can be found in Beck et al. (1972) and Miller and Shebell (1995). To summarize, the exposure rate in air above the ground is related to the absorption peak counting rate registered by the detector by

$$
\frac{N_{f}}{I}=\frac{N_{0}}{\phi} \frac{N_{f}}{N_{0}} \frac{\phi}{I}
$$

where

$N_{o} / \phi$ is the counting rate from a particular spectrum absorption peak due to a unit primary photon flux density of energy $E$ incident on the detector along the detector axis (normal to the detector face).

$N_{f} / N_{o}$ is the correction required to account for detector angular response, and

$\phi / \mathrm{I} \quad$ is the primary photon flux density with an energy $\mathrm{E}$ at the detector resulting from the decay of a particular radionuclide per unit exposure rate at the 
detector from all primary and scattered photons originating from this nuclide and any others present from its radioactive decay series.

The first two terms depend on the particular detector; $\phi / I$ values depend only on the source composition and geometry and can be used for any spectrometer calibration.

In a like manner, the concentration or inventory of a particular nuclide is related to absorption peak counting rate by

$$
\frac{N_{\mathrm{f}}}{\mathrm{A}}=\frac{\mathrm{N}_{0}}{\phi} \frac{N_{\mathrm{f}}}{N_{0}} \frac{\phi}{\mathrm{A}}
$$

where $\phi / A$ is the total photon flux density at the detector location per unit concentration or inventory of the nuclide.

The three factors to compute $\mathrm{N}_{\mathrm{f}} / \mathrm{I}$ or $\mathrm{N}_{\mathrm{f}} / \mathrm{A}$ are discussed separately.

\section{A. $\mathrm{N}_{0} / \phi$.}

The response to unit flux at normal incidence is evaluated for a detector using various $\gamma$-ray point sources. A complete energy response curve from $40 \mathrm{keV}$ to $3 \mathrm{MeV}$ can be inferred with a set made up with the reasonably long lived isotopes ${ }^{152} \mathrm{Eu},{ }^{241} \mathrm{Am},{ }^{137} \mathrm{Cs}$, ${ }^{60} \mathrm{Co}$, and ${ }^{228} \mathrm{Th}$. The measurement procedure is as follows:

1. Position the source at a distance of at least $1 \mathrm{~m}$ and at normal incidence to the detector face.

2. Calculate the uncollided flux density at the detector effective crystal center, which is obtained by dividing the $\gamma$ emission rate by $4 \pi r^{2}$. The value of $r$ is the distance from the source to the crystal effective center. This can be taken to be the geometric center for high energy ( $>1 \mathrm{MeV}$ ) rays and the crystal face for low energy ( $<0.1 \mathrm{MeV})$ $\gamma$ rays. For the energy range between these two values, an estimate of average penetration distance can be made based on the absorption coefficient of the crystal. The $\gamma$ flux density is also corrected for air and source holder attenuation.

3. Collect a spectrum and determine the full absorption peak count rate. 
4. Collect a spectrum without the source present and subtract out from the previously measured count rate any contribution to the peak from background emitters.

5. Divide the corrected count rate by the flux density to determine $\mathrm{N}_{0} / \phi$.

6. Perform this measurement at different energies with either simultaneous or separate runs.

7. Plot the values of $N_{0} / \phi$ versus energy on a log-log scale and fit the data to a smooth curve. Figure 3.6 shows $N_{o} / \phi$ as function of energy above $200 \mathrm{keV}$ for the detectors listed in Table 3.1. In the energy range shown, the response can be fit to a straight line on a $\log -\log$ plot to within $\pm 3 \%$.

\section{B. $\mathbf{N}_{\mathbf{f}} / \mathbf{N}_{0}$.}

The uncollided $\gamma$-ray flux for a soil half space source geometry is not limited to angles normal to the detector face. Therefore, the complete flux density response calibration must account for the fact that a cylindrical Ge crystal when oriented with the axis of symmetry perpendicular to the ground plane has a variable altitudinal (zenithangle) response. The correction factor, $\mathrm{N}_{\mathrm{f}} \mathrm{N}_{0}$, is determined from point source calibrations as functions of energy and angle in the vertical plane and can be calculated from

$$
\frac{N_{f}}{N_{0}}=\int_{0}^{\frac{x}{2}} \frac{\phi(\theta)}{\phi} \frac{N(\theta)}{N_{0}} d \theta
$$

where

$\phi(\theta) / \phi$ is the fraction of the total primary flux at zenith angle $\theta$ for a given source energy and geometry, and

$N(\theta) / N$ is the response of the detector at angle $\theta$ for the same energy $\gamma$ ray relative to the response at normal incidence.

The procedure for determining the values of $N_{f} / N_{0}$ is as follows: 
1. Measure the full absorption peak count rate (minus any background contribution to the peak) using a point source at a fixed distance of at least $1 \mathrm{~m}$ to the crystal at $15^{\circ}$ intervals between incident angles of $0^{\circ}$ (normal to detector face) and $90^{\circ}$.

2. Plot the relative response $N(\theta) / N$ versus angle and fit the data to a smooth curve.

3. Evaluate Equation 3 numerically for at least three different source distributions in the soil (surface plane, $3 \mathrm{~cm}$ relaxation depth, and uniform). The angular flux distribution data can be found in Beck et al. (1972).

4. Repeat Steps 1-3 for several other energies and plot the resultant values of $N_{f} / N_{0}$ versus energy. The data points can be fit to a smooth curve for each source depth distribution.

As noted before, a longer crystal would tend to yield a higher count rate in the field, meaning that the value of $N_{f} N_{0}$ would be $>1$. For the source distributions generally encountered, more than $80 \%$ of the uncollided flux is incident between $\theta=30^{\circ}-90^{\circ}$ ( $\theta$ measured from the detector axis normal to the ground interface). Uniformity of the zenith angular response in this range to within a few percent assures that the value of $\mathrm{N}_{\mathrm{f}} \mathrm{N} \mathrm{N}_{\mathrm{o}}$ will not vary significantly with changes in the distribution of flux. In general, a more uniform response is achieved with a crystal where the diameter is close to the length dimension. However, the variation in $\mathrm{N}_{\mathrm{f}} \mathrm{N}_{0}$ for a detector where the crystal length/diameter is as high as 1.3 or as low as 0.7 would not be expected to be more than about $20 \%$ for energies $>200 \mathrm{keV}$. Figure 3.7 shows angular correction factor data at three different energies for several detectors in a downward facing geometry and a uniform source depth profile.

\section{C. $\phi / \mathrm{I}$ and $\phi / \mathrm{A}$.}

The $\phi / \mathrm{I}$ (and $\phi / \mathrm{A}$ ) factors are derived from $\gamma$-ray transport calculations. Tabulations of these data along with other pertinent information on the make-up of the environmental $\gamma$ radiation field can be found in Beck et al. (1972), Beck and de Planque (1968), and Beck (1972). A complete set of exposure rate values (I/A) for close to 200 common fission and activation isotopes at various exponential depth distributions in the soil can be found in Beck (1980). 


\section{Notes:}

1. The inference of exposure rates from nuclides located in the ground does not require a precise knowledge of the distribution with depth or of the exact soil density or composition. This property results because the observed peak count rate in a field spectrum is essentially a measure of the uncollided flux, and although this quantity and the exposure rate produced by it and the associated scattered flux varies significantly with the source depth distribution and soil characteristics, the ratio of these two quantities, $\phi / I$, does not. Thus, even a crude estimate of source distribution should not lead to a sizeable error in the exposure rate.

2. In lieu of a complete experimental calibration of a Ge detector for field spectrometry, generic factors may be substituted at energies $>200 \mathrm{keV}$ (Helfer and Miller, 1988). The only parameters needed to apply this semiempirical calibration method are the manufacturer's quoted efficiency at $1332 \mathrm{keV}(5-45 \%)$, the crystal length/diameter ratio (0.5-1.3), and the detector orientation in the field (upward or downward facing). The accuracy of the derived factors is estimated to be $\pm 10 \%$ for energies $>500 \mathrm{keV}$ and $\pm 15 \%$ for energies between 200 and $500 \mathrm{keV}$.

\subsubsection{SPECTRUM ANALYSIS}

In many situations, the built-in peak area estimate features of state-of-the-art analyzers are used in providing quick results in the field. Prominent peaks are identified in a bench-mark spectrum and the appropriate regions of interest are set up. On certain analyzers, function keys are programmed using the net peak area, counting time and calibration factor $\left(N_{f} / I\right.$ and $\left.N_{f} / A\right)$ to provide instantaneous readout of exposure rate and concentration or inventory.

For more complete data reduction, a small computer is interfaced to the analyzer to run a spectrum analysis program. If desired, a totally portable system may be configured using a battery-powered laptop computer. Our standard analysis program (Gogolak and Miller, 1977; Gogolak, 1982) performs the following:

1. Based on a two point energy calibration as set by the operator, certain peaks which are characteristic of typical environmental spectra are identified, namely: 
a. the $186,295,352,609,1120$ and $1765 \mathrm{keV}$ peaks in the ${ }^{238} \mathrm{U}$ series;

b. the $583,911,966$ and $2615 \mathrm{keV}$ peaks in the ${ }^{232} \mathrm{Th}$ series;

c. the $1460 \mathrm{keV}$ peak of ${ }^{40} \mathrm{~K}$;

d. the $662 \mathrm{keV}$ peak of ${ }^{137} \mathrm{Cs}$.

These peaks are defined by set energy bands where the left and right channel markers are representative of the Compton continuum.

2. The counts between the energy boundaries for each of the above peaks are summed. The background counts in three channels on each side of a peak are averaged and the result is used as an estimate of the baseline under the peak. This is multiplied by the number of channels in the peak and subtracted out from the total counts in the peak band to yield the net peak counts.

3. Detector specific calibration factors are applied to convert from peak count rate to exposure rate and concentration or inventory.

4. A printout is made listing count rates, converted quantities and associated statistical counting errors.

5. Permanent storage of the spectrum is made on either magnetic tape or diskette.

6. As an option, an automated search is performed to identify any peaks present in the spectrum. Data such as nuclide, half-life, $\gamma$-ray intensity and associated energy are printed out using a library of nearly 400 principal $\gamma$-ray energies that are seen in the environment (Section 5, this Manual). Any peak can be quickly analyzed by using an optional automated continuum strip.

7. The program enters an interactive phase where the operator examines any additional peaks, checks the results of the automated routine, or investigates any unusual or unexpected features of a spectrum. 


\subsubsection{INVENTORY MEASUREMENTS}

\subsubsection{1}

APPLICATION

A field $\gamma$-ray spectrum can also provide an estimate of the amount of activity per unit area of soil surface for nuclides which have been deposited on the ground. To do this, a knowledge of the source distribution in the soil is required in order to relate the measured total absorption peak count rate to the incident unscattered photon flux and then to the activity in the soil in a manner analogous to that used for natural emitters.

The activity profile with depth for deposited nuclides in undisturbed soils can be represented by an exponential function,

$$
S=S_{0} e^{-(\alpha / p) p z}
$$

where

$\mathrm{S}$ is the activity per $\mathrm{cm}^{3}$ at depth $\mathrm{z} \mathrm{cm}$,

$S_{0}$ is the activity per $\mathrm{cm}^{3}$ at the soil surface,

$\alpha$ is the reciprocal of the relaxation length in $\mathrm{cm}^{-1}$, and

$\rho$ is the in situ soil density $\left(\mathrm{g} \mathrm{cm}^{-2}\right)$.

The cumulative activity, or inventory I, integrated to depth $z^{\prime}$ is, then,

$$
I=\int_{0}^{z^{\prime}} \mathrm{dz}=\frac{S_{0}}{\alpha}\left[1-\mathrm{e}^{-(\alpha / \rho) p z^{\prime}}\right]=I_{0}\left[1-e^{(\alpha / \rho) p z^{\prime}}\right]
$$

where $I_{0}$ is the total inventory integrated to infinite depth. 
In the case of a freshly deposited nuclide, the depth parameter is infinite corresponding to a plane source distribution and a relaxation length of zero. In practice, the effects of ground roughness bury the source somewhat. Even on what appears to be flat terrain, we apply an $\alpha / \rho$ value of $6.25 \mathrm{~cm}^{2} \mathrm{~g}^{-1}$, which at a typical soil density of $1.6 \mathrm{~g} \mathrm{~cm}^{-3}$ corresponds to a relaxation depth of $0.1 \mathrm{~cm}$. Values of $\alpha / \rho$ for aged global fallout ${ }^{137} \mathrm{Cs}$ have been found to range from a high of 1.0 for an evergreen forest floor to a low of 0.03 for a flood irrigated lawn. At a soil density of $1.6 \mathrm{~g} \mathrm{~cm}^{-3}$, these correspond to relaxation lengths of 0.6 and $21 \mathrm{~cm}$, respectively. More typical values of $\alpha / \rho$ tend to range from 0.05-0.1 for open field sites and 0.2-0.5 for wooded or desert areas.

Values of the unscattered flux and its angular distribution at $1 \mathrm{~m}$ above the ground have been tabulated for exponentially distributed sources in the soil for various energies and $\alpha / \rho$ values (Beck et al., 1972). Using Equation 2, where the term A now represents the inventory (activity per unit area), the detector response can be calculated for a particular nuclide as a function of $\alpha / \rho$. If the nuclide has two reasonably strong $\gamma$ lines well separated in energy, the value of $\alpha / \rho$ can be inferred from the ratio of the measured fluxes.

In the case of a monoenergetic source such as ${ }^{137} \mathrm{Cs}$, the value of $\alpha / \rho$ can be determined experimentally as follows:

1. A $62 \mathrm{~cm}^{2}$ or similar large area corer and auger is used to extract soil samples from different depth intervals (see Section 2.4 ) depending upon the expected activity distribution. For example, if the profile is expected to be shallow, the depth intervals can be $0-2.5,2.5-5$, and $5-30 \mathrm{~cm}$, while if it is expected to be deep, intervals of $0-5$ $\mathrm{cm}, 5-10 \mathrm{~cm}$, and $10-30 \mathrm{~cm}$ can be used. More than one core can be taken, in which case the samples from the same depth are composited. The depth of the soil core should be sufficient to include essentially all of the deposited activity so that $I_{0}$ can be determined.

2. An aliquot of a sample from each depth increment is counted on a high resolution $\mathrm{Ge}$ detector to determine the concentration of the radionuclide of interest. 
3. The activity per unit area for each depth is computed from the product of the concentration and the sample mass for that depth increment divided by the area of the sample.

4. A fit to Equation 5 is then applied, the variables being $I_{0}-I$, the integrated activity per unit area and $\rho z^{\prime}$, the gross in situ mass per unit area down to depth $z^{\prime}$. Graphically, this can be performed by plotting the $\log \left(I_{0}-I\right)$ versus $\rho z$ and fitting a straight line through the points, weighting the points near the surface more heavily since this is where most of the activity is contained. The slope of this line is just $\alpha / \rho$.

Note:

Although the inventory is determined using the soil sample data itself, it is quite useful to corroborate this estimate with the field spectrum. The soil cores may represent an area of a few hundred square centimeters, while the field data represent the average over several hundred square meters.

\subsubsection{3}

\section{NONHOMOGENEOUS TERRAIN}

For areas where the fallout is suspected of being unevenly distributed across the ground, representative inventory measurements can still be made by relying on the fact that a field spectrum averages out the local inhomogeneities in the source geometry. This more generalized method is applied to cases such as the measurement of aged fallout in the desert southwest of the U.S. where blowsand tends to shift from bare soil and collect under vegetative cover. A complete description can be found in Miller and Helfer (1985). The basic steps include:

1. Soil samples in depth increments are collected from the different types of ground cover present (grass, brush, bare soil, etc.).

2. The samples are counted and a depth profile for each type of ground cover is obtained using the procedure outlined above for homogeneous terrain. 
3. If the depth profiles are significantly different, the approximate percentage of ground cover of each type is determined within a $15 \times 15 \mathrm{~m}$ square centered at the detector. This can be performed using a combination of tape measurements and eye estimates to approximate the dimensions of any patches of grass or shrubs and trees out to their drip line, or to where there is an obvious change in soil characteristics, and then calculating the total area of each particular type of cover.

4. The conversion factor to apply to the peak count rate in order to obtain an inventory estimate is given by

$$
<g\rangle=\left[\sum_{i} \frac{x_{i}}{g_{i}}\right]^{-1}
$$

where

$\langle\mathrm{g}\rangle$ is the average full absorption peak count rate to inventory conversion factor for an infinite half space source distribution with randomly spaced segments of different types of ground cover, each of which has its own characteristic nuclide depth distribution and inventory (inventory per unit count rate),

$x_{i}$ is the fraction of the total inventory associated with the $i$-th type of ground cover,

$\mathrm{g}_{\mathrm{i}}$ is the full absorption peak count rate to inventory conversion factor for an infinite half-space source distribution with a measured depth profile characteristic of the $\mathrm{i}$-th type of ground cover (inventory per unit count rate).

Note:

The value of $<g>$ exhibits a dependence on the relative inventory mix for the different ground covers which can only be determined through soil sampling. Nonetheless, the value of $\langle g\rangle$ must fall within the range of the individual values of $g_{i}$. Generally, this range is not large. Although the inventory may typically vary by a factor of two for the different ground covers, the variation in the conversion factor for the different depth profiles associated with these ground covers will average $<30 \%$. This is particularly true for sites where the fallout is near the soil surface because the conversion factor does not vary strongly with the depth profile for values of $\alpha / \rho>0.2$. 


\subsubsection{SPECTRAL STRIPPING FOR GERMANIUM DETECTORS}

\subsubsection{1}

APPLICATION

A stripping operation can be applied to a Ge detector spectrum which has been collected in free air in order to obtain the $\gamma$ flux density as a function of energy Miller, 1984). This flux spectrum can be readily converted to an independent estimate of the free air exposure rate or be used to make energy response corrections to other radiation measuring devices. This technique has particular applications to indoor radiation measurements, where the flux distribution is not easily predicted because of the complex and generally unknown $\gamma$ source geometry.

\section{THEORY}

A count registered by the detector can be caused by the full or partial absorption of an incident photon or by the passage of a cosmic ray produced charged particle. In order to obtain a measure of the incident photon flux spectrum, the partial absorption and cosmicray events must be subtracted out and then the full absorption efficiency curve of the detector can be applied.

The stripping operation can be expressed as:

$$
N_{1}^{\prime}=N_{1}-\sum_{j=i+1}^{L} f_{i j}\left(r_{j}-1\right) N_{j}^{\prime}-N_{c}
$$

where

$N_{i}^{\prime}, N_{j}^{\prime}$ are the counts in an energy band due to the total absorption of incident $\gamma$ flux,

$\mathrm{N}_{\mathrm{i}}$ is the observed counts in an energy band due to all sources,

$\mathrm{L}$ is the energy band containing the highest energy $\gamma$ line (generally

$$
2.615 \mathrm{keV} \text { ), }
$$


$f_{i j}$ is the fraction of the continuum counts at energy band $i$ due to the partial absorption of incident $\gamma$ flux at energy band $j$,

$r_{j}$ is the ratio of total counts to full absorption peak counts for incident flux at energy band $j$, and

$\mathrm{N}_{\mathrm{c}}$ is the counts in band $\mathrm{i}$ due to cosmic-ray events, assumed to be constant in any energy band and given by

$$
N_{C}=\left(E_{2}-E_{1}+1\right)^{-1} \sum_{i=E_{1}}^{E_{2}} N_{i}
$$

where

$E_{1}, E_{2}$ are the lower and upper energy bands of a pure cosmic-ray energy (generally 3-4 $\mathrm{MeV}$ ).

DETERMINATION OF STRIPPING PARAMETERS ( $f$ and $r$ )

Factor $f$ - For the factor $f$, a multiple step function fit is used wherein the region below the Compton edge is divided into 10 equal size energy bands and the region above the Compton edge is divided into four equal bands. The fraction of the total counts in the continuum in each band can be experimentally determined for a particular detector by examining the shape of the continuum below a full absorption peak from a monoenergetic $\gamma$ source. Although the shape of the continuum is a function of the energy and incident angle of the $\gamma$ ray, it is not overly sensitive to these variables, particularly at the higher energies which contribute the most to the exposure rate.

Factor $\mathrm{r}$ - In the same manner that the full absorption peak efficiency is determined for a detector (see Procedure 3.3.4 for $\mathrm{N}_{\mathrm{o}} / \phi$ ), the total efficiency can be determined and its ratio computed. However, effectively monoenergetic sources must be used and room background must be subtracted from the entire spectrum. Like the full absorption peak efficiency, the total efficiency varies with incident angle for a cylindrical detector. However, the ratio of the two, $r$, varies more smoothly and is not a strong function of the incident angle. A single angular correction factor can therefore be applied which is representative of the higher $\gamma$ energies which weigh heavily in the exposure rate 
computation. This factor can be obtained by assuming an isotropic radiation field and averaging over the entire $4 \pi$ solid angle. Although, in practice, the radiation field may not be isotropic, a reasonable approximation to this ideal can be created by accumulating a spectrum with the detector oriented in different directions, thus averaging out the angular dependence. To accomplish this, we routinely count for equal lengths of time with the detector pointing in six different directions, that is, each way along three orthogonal axes.

\section{STRIPPING OPERATION}

The stripping operation is performed on a portable $\mathrm{PC}$ using Basic and takes $<1 \mathrm{~min}$ to complete. It proceeds as follows:

1. The spectrum is calibrated using two energy-channel points supplied by the operator.

2. The spectrum is compressed from 4000 channels to 400 channels for faster computation.

3. The spectrum is stripped of the cosmic-ray events.

4. The spectrum is stripped of partial absorption events for equal size energy bands of 10 $\mathrm{keV}$ each and starting at the band containing the highest energy $\gamma$ line (generally $2.615 \mathrm{keV}$ ), where $N^{\prime}=N-N_{c}$. Succeeding lower energy bands can then be computed based on the $N_{j}^{\prime}$ values from any higher band.

5. The spectrum of counts which remains after the stripping operation is completed is converted to the incident flux density energy distribution by applying the full absorption peak efficiency of the detector. As in the case of the factor $r$, an angular correction based on the assumption of isotropic incidence must be applied. 
6. The $\gamma$ exposure rate is computed by integrating across the spectrum the product of the energy, flux density, and mass energy absorption coefficient for air at each energy band.

\section{Note:}

Intercomparisons between the spectral stripping method and our own instruments and standard procedures, as well as with those of the Japanese Atomic Energy Research Institute, have shown good agreement (Nagaoka, 1987). Based on these measurements, we conservatively estimate an upper limit in the total systematic error of $\pm 5 \%$ when this method is applied to spectra at background levels. The statistical error was determined to be $< \pm 2 \%$ (at the $1 \sigma$ level) for a 1-min spectrum at typical background levels.

\subsubsection{SODIUM IODIDE DETECTORS}

\subsubsection{1}

APPLICATION

Although no longer used routinely by EML for field $\gamma$-ray spectrometry, NaI detectors are nonetheless capable of yielding satisfactory results particularly for natural background measurements. Like the case for Ge detector measurements, full absorption analysis can be applied to a NaI field spectrum. In addition, there is the technique of "energy band" analysis as well as "total spectrum energy" analysis. A complete description of these methods can be found in Beck et al. (1972).

The NaI detectors (usually 4-x-4 in cylindrical crystals attached to matched photomultiplier tubes) are coupled through an emitter-follower preamplifier. Either a battery powered portable or $120 \mathrm{~V}$ AC vehicle-based analyzer with at least 400 channels 
is used to collect the spectra. NaI detectors are usually covered (in addition to the manufacturer's standard thin aluminum or stainless steel window) by a 6-mm bakelite shield to reduce the $\beta$-ray contribution to the Compton continuum as well as to moderate thermal stresses. The NaI detector is transported in a rugged, foam-cushioned box to minimize mechanical and thermal shock.

PEAK ANALYSIS

For $\mathrm{NaI}$ spectrometry, the $\mathrm{N}_{\mathrm{o}} / \phi$ should be obtained with source energies as close as possible to those environmental sources to be evaluated, usually the energies associated with the ${ }^{238} \mathrm{U}$ and ${ }^{232} \mathrm{Th}$ series, ${ }^{40} \mathrm{~K}$, and ${ }^{137} \mathrm{Cs}$. The resulting $\mathrm{N}_{\mathrm{o}} / \phi$ versus $\mathrm{E}$ data do not represent the usual $\mathrm{NaI}$ response curve, because the analysis of absorption peaks does not lead to accurate estimates of the actual peak areas, unless one engages in a complex computer program for spectral stripping. NaI peak areas are determined in essentially the same manner as those from Ge detectors, though only a few absorption peaks are measurable due to the relatively poor resolution. Cross-calibration of a $\mathrm{NaI}$ detector with the more accurate Ge detector can be very helpful.

\subsubsection{4}

\section{ENERGY BAND ANALYSIS}

When the naturally-occurring nuclides associated with the ${ }^{238} \mathrm{U}$ and ${ }^{232} \mathrm{Th}$ series and ${ }^{40} \mathrm{~K}$ predominate at a field site, the simplified "energy band" method of analysis can be applied. In this method, the spectrum energy is calculated as the product of the counts per channel times the energy represented by each channel in bands of channels. The energy bands are centered on the $1.46 \mathrm{MeV}^{40} \mathrm{~K}$, the $1.76 \mathrm{MeV}^{214} \mathrm{Bi}$, and the $2.62 \mathrm{MeV}^{208} \mathrm{Tl}$ peaks. The response in each bin can then be represented by the following equations. 


$$
\begin{gathered}
E_{1}=u_{1} U+k_{1} K+t_{1} T+I_{1} \\
E_{2}=u_{2} U+k_{2} K+t_{2} T+I_{2} \\
E_{3}=t_{3} T+I_{3}
\end{gathered}
$$

where $E_{1}, E_{2}$ and $E_{3}$ are the measured "energy" values for some arbitrary counting period; $\mathrm{U}, \mathrm{K}$ and $\mathrm{T}$ represent the exposure rates to be measured; the constants represent the distribution of spectrum energy per unit exposure rate among the three energy bands; and $I_{1}, I_{2}$ and $I_{3}$ are the cosmic-ray contributions. The three equations relating the $U, K$ and Th exposure rates to the energy bands are determined by solving Equation 9 and evaluating the coefficients from regression analyses of a large number of field spectra for which exposure rates had been determined from absorption peak analyses.

The following approximate equations apply for a nominal $10.16 \times 10.16 \mathrm{~cm} \mathrm{NaI}$ detector, shielded with 6-mm Bakelite, though calibration of individual detectors is preferred.

$$
\begin{gathered}
U=0.4 E_{1}^{\prime}-0.2 E_{3}^{\prime} \\
K=0.08 E_{1}^{\prime}-0.06 E_{2}^{\prime}-0.02 E_{3}^{\prime} \\
T=0.3 E_{3}^{\prime}
\end{gathered}
$$

and

$$
I=E_{\mathrm{T}}{ }^{\prime} / 37
$$

where $I$ is the total exposure rate from natural radioactivity in soil, and

$$
\begin{aligned}
& 1.32 \mathrm{MeV}<\mathrm{E}_{1}{ }^{\prime}<1.60 \mathrm{MeV}, \\
& 1.62 \mathrm{MeV}<\mathrm{E}_{2}{ }^{\prime}<1.90 \mathrm{MeV}, \\
& 2.48 \mathrm{MeV}<\mathrm{E}_{3^{\prime}}<2.75 \mathrm{MeV}, \\
& 0.15 \mathrm{MeV}<\mathrm{E}_{\mathrm{T}^{\prime}}<3.40 \mathrm{MeV},
\end{aligned}
$$


the $\mathrm{E}$ values being in $\mathrm{GeV}$ per 20 -min counting period. The primes indicate that the energy contributions from cosmic rays must be subtracted before Equations 10 are used.

Energy band analysis is performed quickly in the field by interfacing the multichannel analyzer to a portable computer. Using interactive software (Gogolak and Miller, 1977), the basic steps include:

1. The spectrum is read into the computer and the operator provides a two point energy calibration (usually the positions of the $1460 \mathrm{keV}^{40} \mathrm{~K}$ and $2615 \mathrm{keV}^{208} \mathrm{Tl}$ peaks) and the altitude to the nearest thousand feet (for the cosmic-ray corrections).

2. The above equations are applied.

3. A printout is made of the total exposure rate and the contributions for ${ }^{40} \mathrm{~K}$ and the ${ }^{238} \mathrm{U}$ and ${ }^{232}$ Th series.

TOTAL SPECTRUM ENERGY ANALYSIS

The exposure rate in air is proportional to $\int^{\infty} \phi(E)\left(\mu_{\mathrm{e}} / \rho\right) E d E$, where $\phi(E)$ is the flux of $\gamma$ rays of energy $E$ and $\mu_{e} / \rho$ is the mass absorption coefficient in air. Between a few hundred $\mathrm{keV}$ and several $\mathrm{MeV}, \mu_{\mathrm{e}} / \rho$ is fairly constant. Also for low energies, the probability of an incident photon being totally absorbed by a large $\mathrm{NaI}$ detector is fairly high (on the order of $50-100 \%$ from $100 \mathrm{keV}$ to $1 \mathrm{MeV}$ ). About $75 \%$ of the exposure rate from the soil is due to emitters between $100 \mathrm{keV}$ and $1500 \mathrm{keV}$. This and the fact that the spectrum of $\gamma$ rays from natural emitters is fairly invariant to the exact proportions of $U$, Th and $\mathrm{K}$ in the soil, indicates that the total "spectrum energy" is a reasonable measure of free air exposure from natural radioactivity in the soil. A large NaI or similar detector measures the flux to a fairly high degree of accuracy and, even though sensitivity decreases somewhat at higher energies due to the escape of secondary scattered photons, this decrease tends to be compensated by correspondingly smaller values of $\mu_{\mathrm{e}} / \rho$ for energies above $1 \mathrm{MeV}$ relative to values below $1 \mathrm{MeV}$. 
Unlike many NaI hand-held survey instruments, which depend on the assumption that the counting rate above some bias level is proportional to the exposure rate, the total energy technique requires only that the counts in a channel be proportional to $\phi(E)\left(\mu_{\mathrm{e}} / \rho\right)$ for that energy, and is, therefore, less sensitive to spectral changes. For example, a NaI survey meter might indicate that the exposure rate from a unit flux of $60 \mathrm{keV}$ photons as being almost equal to the exposure rate from a unit flux of $1460 \mathrm{keV}$ photons. This would occur since the $1460 \mathrm{keV}$ pulse would be recorded due to the high probability of a Compton collision in the detector even though many of the secondaries would escape the crystal. In the total energy technique, the higher energy counts are weighted by the energy deposited and reflect their relative contribution to the exposure rate more correctly. The slightly larger total absorption at $60 \mathrm{keV}$ reflects the larger value of $\left(\mu_{\mathrm{e}} / \rho\right)$ relative to higher energy $\gamma$ rays.

The spectrum "energy" calibration factors for $10.16 \times 10.16 \mathrm{~cm}$ detectors are determined in two ways:

1. The detectors are exposed to a known point source of ${ }^{226} \mathrm{Ra}$ in the laboratory and the measured exposure rate is compared to an ionization chamber reading. The measurement should be corrected to account for the fact that the rays from the point source are incident along the detector axis.

2. A comparison of measurements of "spectrum energy" from actual field spectra is made with simultaneous ionization chamber measurements for different environmental radiation fields.

The two methods give essentially the same calibration factors.

\section{REFERENCES}

Beck, H. L.

"Environmental Gamma Radiation from Deposited Fission Products, 1960 - 1964"

Health Physics, 12, 313-322 (1966) 
Beck, H. L.

"The Physics of Environmental Gamma Radiation Fields"

J. A. S. Adams, W. M. Lowder, and T. F. Gesell (Editors)

In: The Natural Radiation Environment II, CONF-720805-P1, pp. 101-134 (1972)

Beck, H. L.

"Exposure Rate Conversion Factors for Radionuclides Deposited on the Ground" USDOE Report EML-378, July (1980)

Beck, H. L. and G. de Planque

"The Radiation Field in Air Due to Distributed Gamma-Ray Sources in the Ground" USAEC Report HASL-195, May (1968)

Beck, H. L., W. J. Condo and W. M. Lowder

"Spectrometric Techniques for Measuring Environmental Gamma Radiation"

USAEC Report HASL-150, October (1964)

Beck, H. L., J. A. De Campo and C. V. Gogolak

"In Situ $\mathrm{Ge}(\mathrm{Li})$ and NaI(Tl) Gamma-Ray Spectrometry for the Measurement of Environmental Radiation"

USAEC Report HASL-258, July (1972)

Gogolak, C. V.

"Collection and Analysis of Environmental Radiation Data Using a Portable Desktop Computer"

USDOE Report EML-398, April (1982)

Gogolak, C. V.

"Rapid Determinations of Noble Gas Radionuclide Concentrations in Power Reactor Plumes"

Health Physics, 46, 783-792 (1984)

Gogolak, C. V. and K. M. Miller

"New Developments in Field Gamma-Ray Spectrometry"

USDOE Report EML-332, December (1977) 
Helfer, I. K. and K. M. Miller

"Calibration Factors for Germanium Detectors Used for Field Spectrometry"

Health Physics, 55, 15-29 (1988)

Lowder W. M., H. L. Beck, and W. J. Condo

"Spectrometric Determination of Dose Rates from Natural and Fall-Out

Gamma-Radiation in the United States, 1962-63"

Nature, 202, 745 (1964a)

Lowder W. M., W. J. Condo and H. L. Beck

"Field Spectrometric Investigations of Environmental Radiation in the U.S.A."

In: The Natural Radiation Environment, University of Chicago Press, Chicago, II, pp. 597-616 (1964b)

Miller, K. M.

"A Spectral Stripping Method for a Ge Spectrometer Used for Indoor Gamma Exposure Rate Measurements"

USDOE Report EML-419, July (1984)

Miller, K. M. and H. L. Beck

"Indoor Gamma and Cosmic Ray Exposure Measurements Using a Ge Spectrometer and Pressurized Ionisation Chamber"

Radiation Protection Dosimetry, 7, 185-189 (1984)

Miller, K. M. and I. K. Helfer

"In Situ Measurements of ${ }^{137} \mathrm{Cs}$ Inventory in Natural Terrain"

In: Environmental Radiation '85, Proceedings of the Eighteenth Midyear Topical

Symposium of the Health Physics Society, pp. 243-251 (1985)

Miller, K. M. and P. Shebell

"In Situ Gamma-Ray Spectrometry - A Tutorial for Environmental Radiation Scientists" USDOE Report EML-557, October (1995)

Miller, K. M., P. Shebell and G. A. Klemic

"In Situ Gamma-Ray Spectrometry for the Measurement of Uranium in Surface Soils" Health Physics, $\underline{67}$, 140-150 (1994) 


\section{Nagaoka, T.}

"Intercomparison Between EML Method and JAERI Method for the Measurement of Environmental Gamma Ray Exposure Rates"

Radiation Protection Dosimetry, 18, 81-88 (1987) 
Table 3.1

Ge DETECTOR SPECIFICATIONS

\begin{tabular}{|c|c|c|c|c|c|c|c|c|c|}
\hline Manufacturer & $\begin{array}{l}\text { Serial } \\
\text { No. }\end{array}$ & Code & Type & $\begin{array}{l}\text { Cryostat } \\
\text { orientation }\end{array}$ & $\begin{array}{c}\text { Efficiency } \\
(\%)\end{array}$ & $\begin{array}{c}\text { Resolution } \\
\text { at } 1332 \\
(\mathrm{keV})\end{array}$ & $\begin{array}{c}\text { Dimensions } \\
\text { DxL } \\
(\mathrm{mm})\end{array}$ & $\mathrm{L} / \mathrm{D}^{+}$ & $\begin{array}{c}\text { PeakJ } \\
\text { Compton }\end{array}$ \\
\hline \multirow[t]{5}{*}{$\begin{array}{l}\text { Princeton } \\
\text { Gamma-tech }\end{array}$} & 484 & P1 & $\mathrm{Ge}(\mathrm{Li})$ & $\begin{array}{l}4 \mathrm{~L} \text {, } \\
\text { downward }\end{array}$ & 2.9 & 1.70 & $36 \times 20$ & 0.56 & 23.0 \\
\hline & 514 & $\mathrm{P} 2$ & $\mathrm{Ge}(\mathrm{Li})$ & $\begin{array}{l}4 \mathrm{~L} \text {, } \\
\text { downward }\end{array}$ & 12.2 & 2.43 & $43 \times 44$ & 1.02 & 30.0 \\
\hline & 1039 & P3 & $\mathrm{Ge}(\mathrm{Li})$ & $\begin{array}{l}17 \mathrm{~L} \text {, } \\
\text { upward }\end{array}$ & 27.9 & 2.36 & $59 \times 47$ & 0.80 & 35.9 \\
\hline & 1545 & P4 & $\mathrm{Ge}(\mathrm{Li})$ & $\begin{array}{l}17 \mathrm{~L} \text {, } \\
\text { upward }\end{array}$ & 22.3 & 2.10 & $56 \times 54$ & 0.96 & 49.5 \\
\hline & 1030 & P5 & P-type Ge & $\begin{array}{l}2 \mathrm{~L} \text {, all } \\
\text { attitude }\end{array}$ & 21.7 & 1.77 & $59 \times 35$ & 0.59 & 52.0 \\
\hline \multirow[t]{4}{*}{$\begin{array}{l}{ }^{* *} \mathrm{EG} \& \mathrm{G} \\
\text { Ortec }\end{array}$} & $23-\mathrm{N}-37 \mathrm{VB}$ & OI & N-type Ge & $\begin{array}{l}30 \mathrm{~L} \text {, } \\
\text { upward }\end{array}$ & 35.3 & 1.96 & $55 \times 65$ & 1.18 & 59.4 \\
\hline & $25-\mathrm{N}-1514$ & $\mathrm{O} 2$ & N-type Ge & $\begin{array}{l}30 \mathrm{~L} \text {, } \\
\text { upward }\end{array}$ & 35.4 & 1.73 & $55 \times 73$ & 1.31 & 67.9 \\
\hline & 26-P-70P & $\mathrm{O} 3$ & P-type Ge & $\begin{array}{l}1.8 \mathrm{~L} \text {, all } \\
\text { attitude }\end{array}$ & 45.0 & 1.80 & $60 \times 79$ & 1.31 & 73.0 \\
\hline & 33-TN30860A & - & N-type Ge & $3 \mathrm{~L}$, all-attitude & 75.0 & 1.95 & $71 \times 79$ & 1.11 & 74.8 \\
\hline
\end{tabular}

"Princeton Gamma-Tech, Inc., 1200 State Road, Princeton, NJ 08540

*EG \& G Ortec, 100 Midland Road, Oak Ridge, TN 37830

${ }^{+} \mathrm{Ge}$ crystal length/diameter ratio 


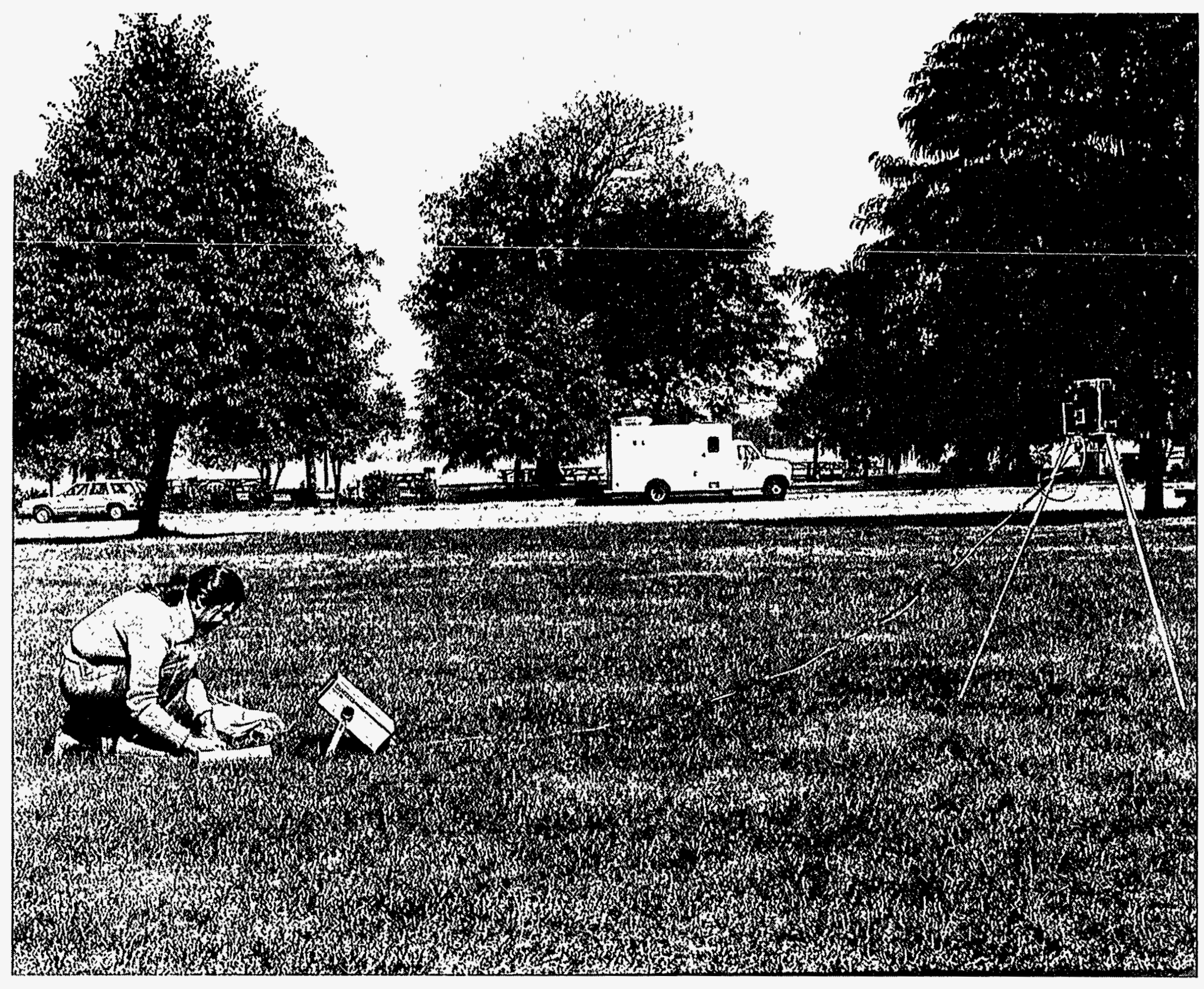

Figure 3.4. Typical field site for conducting in situ $\gamma$-ray spectrometry showing placement of tripod mounted portable Ge detector and battery-powered multichannel analyzer. 
Figure 3.5. Schematic representation of the relative ground area contributions to the primary flux at $1 \mathrm{~m}$ above the ground for an exponentially distributed source with an energy of $662 \mathrm{keV}$ and where $\alpha / \rho=0.21 \mathrm{~cm}^{2} \mathrm{~g}^{-1}$. 


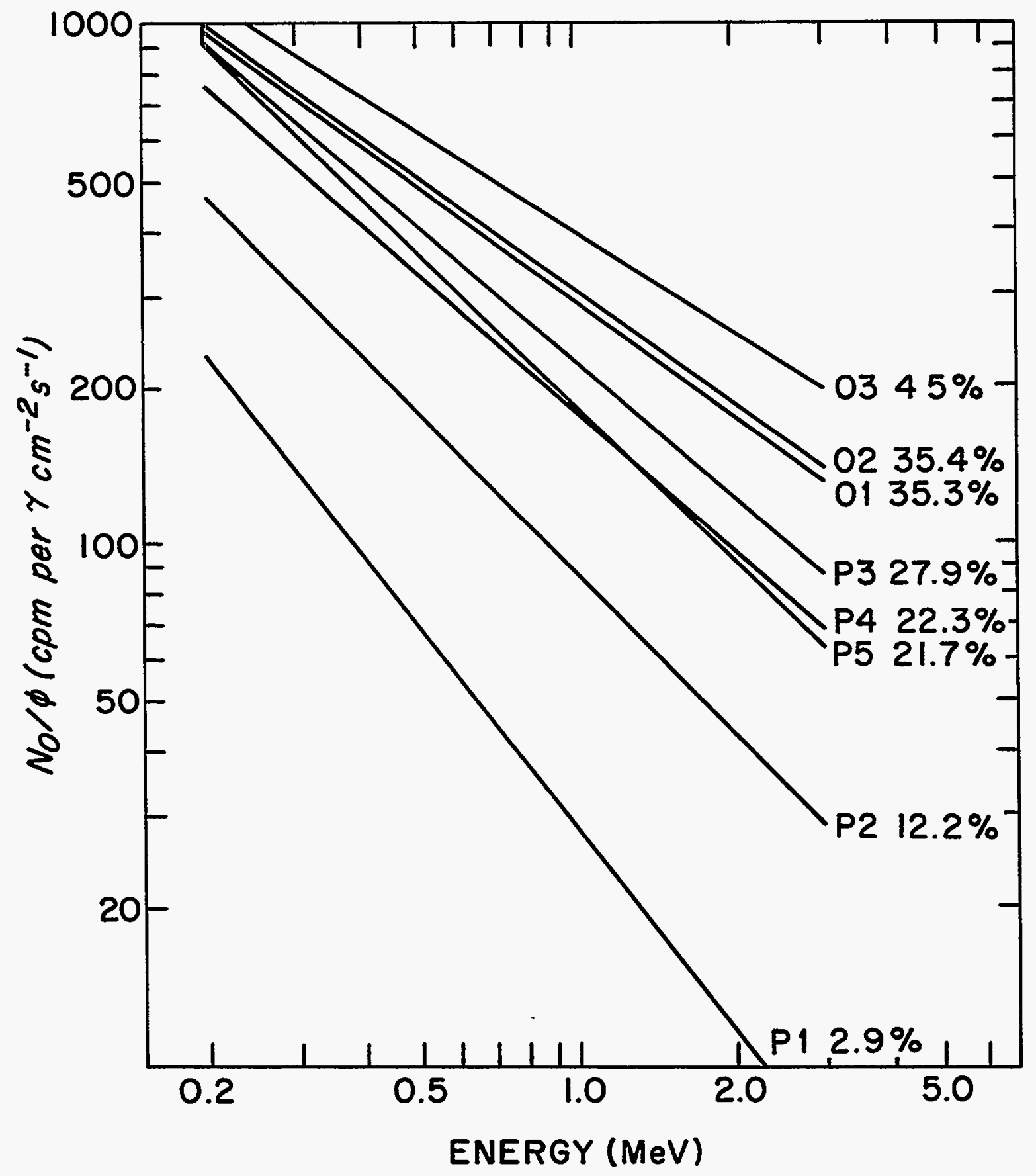

Figure 3.6. Count rate per unit incident flux at normal incidence $\left(\mathrm{N}_{\mathrm{o}} / \phi\right)$ as a function of energy for seven of the detectors listed in Table 3.1. 


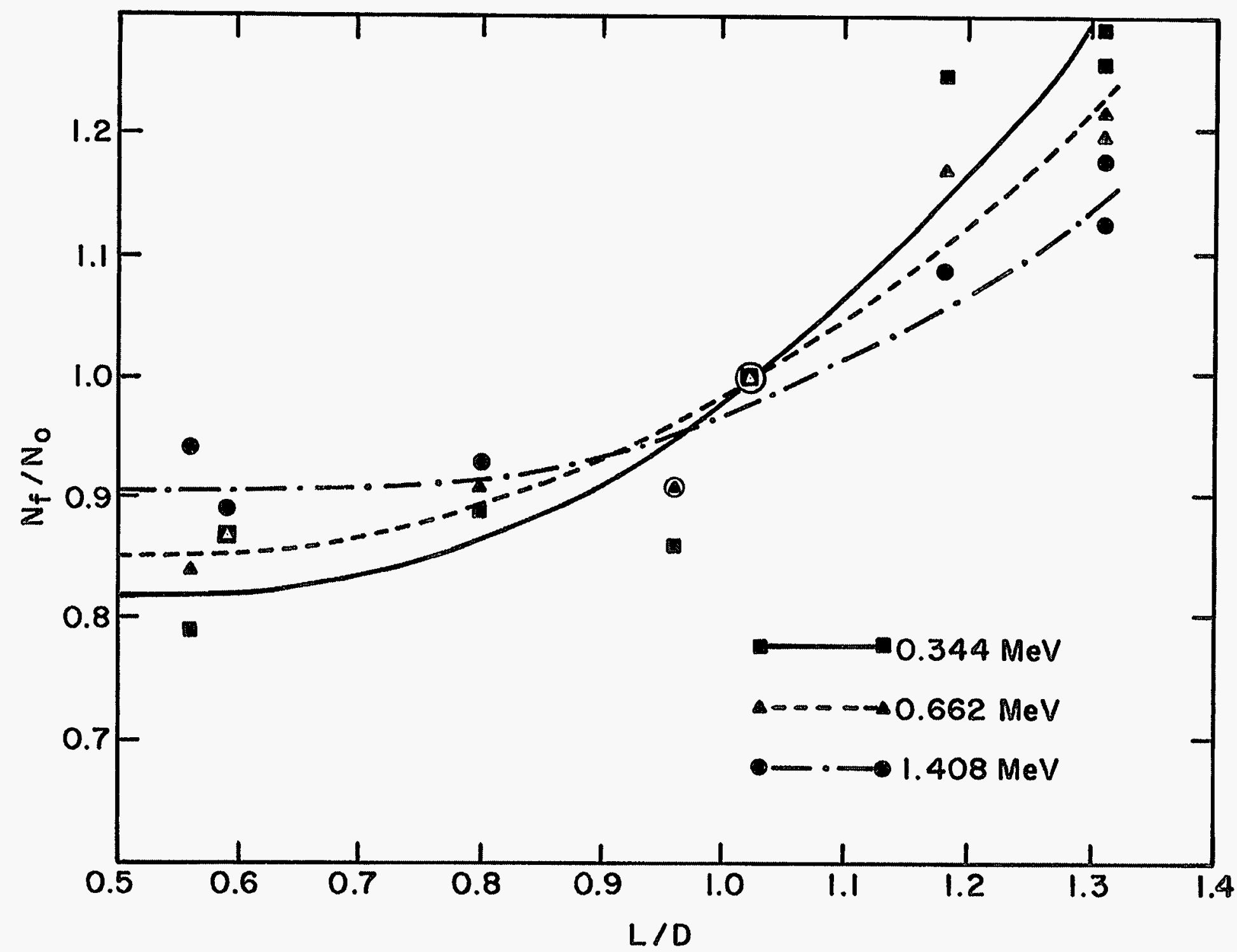

Figure 3.7. Angular correction factor $\left(\mathrm{N}_{\mathrm{f}} / \mathrm{N}_{0}\right)$ as a function of Ge crystal length/diameter (L/D) ratio at three different energies for a downward facing detector for a uniform with depth source profile in the soil. 


\title{
3.4 REMOTE GAMMA-RAY SPECTROMETRY STATIONS
}

\author{
Contact Person(s) : Colin G. Sanderson
}

\subsubsection{SCOPE}

EML's global studies are comprised of a Global Fallout Program, a Surface Air Sampling Program, and a Remote Atmospheric Measurements Program (Sanderson et al., 1994). These programs currently represent EML's effort to sample, measure, and study radionuclides in the lower troposphere and their deposition on the earth's surface (Larsen et al., 1994).

Using remote atmospheric measurement systems (RAMS) in RAMP, EML is able to measure gamma-ray emitting radionuclides having short half-lives, such as ${ }^{7} \mathrm{Be}$, that have been collected by drawing air through highly efficient filters, see Procedure 2.2.2.2. The gamma-ray spectrum is transmitted to ARGOS communications systems flown aboard polar-orbiting National Oceanic and Atmospheric Administration (NOAA) satellites, retransmitted to ground stations, and recovered via a telephone link by EML's computer. The recovered sodium iodide $(\mathrm{NaI})$ gamma-ray spectrum is automatically resolved using a linear least squares program with a library of 18 possible gamma-ray emitting radionuclides. The last background spectrum received is also included in the library. When the analysis is complete, the results are written to a report file and a computer mail message is issued to report the completion of the analysis.

Once a RAMS station has been set up, its weekly operation requires $<1 \mathrm{~h}$ of an operator's time. Each week one sample, one calibration, and one background is counted. Each month the weekly air filter samples are sent to the University of Miami and the computer floppy disk is sent to EML. 



\section{F. Laptop computer.}

A computer with backlite LCD display, either dual floppy, one fixed and one floppy disk, at least one RS232 and one parallel port, $512 \mathrm{~K}$ memory, and simple $12-\mathrm{V} \mathrm{DC}$ operation are required.

\section{G. Satellite transmitter and antenna.}

A 2 W, 401.650 MHz transmitter, $30.5 \mathrm{~m}$ of low-loss cable and an omnidirectional quadrafilar helix antenna are used to transmit the gamma-ray data to the ARGOS satellites. Polar Research SITRA-1 or SITRA-2 or equivalent is used.

Figure 3.8 shows a block diagram of EML's second generation NaI RAMS.

\subsubsection{INSTRUMENTATION ASSEMBLY}

\section{A. Lead shield and $\mathrm{NaI}$ detector.}

Place the removable bottom of the environmental chamber on a table or floor that will support about $200 \mathrm{~kg}$. The shield parts are numbered for assembly. Place the lead base (1) on the indicated circle. Place lead ring (2) on top of (1) and lead ring (3) on top of (2). Align these rings so that the sample drawer fits properly. Continue to install lead rings (4), (5), and (6). Next, install the small copper insert, the NaI gamma-ray detector with preamplifier attached, and then the large copper housing. Add lead rings (7), (8), (9), (10), and (11). Finally, place the lead plug (P) on top of the shield and place the environmental chamber over the completed shield.

\section{B. Equipment rack.}

Place the RAMP system power supply on the top shelf of the equipment rack, passing the cables through the hole in the back of the shelf. Place the MCA on the center shelf and connect the cables from the NaI gamma-ray detector preamplifier to the "0 TO 1250 V POSITIVE H.V.," "PULSE INPUT," and "PREAMP POWER." Place the serial/parallel two-way converter to the left and to the back of the bottom shelf. 
Hang the transmitter on the two screws on the back of the equipment rack. Connect the printer cable to the computer printer port. The other end of this cable is connected to the PARALLEL side of the serial/parallel two-way converter.

Connect a 25-pin RS232C cable to the SERIAL side of the serial/parallel two-way converter and to the 25-pin connector on the transmitter. Use tape to secure this cable to the back of the equipment rack.

Connect the 9-pin end of an RS232C cable to the computer, connect the other end of this cable to the 25-pin I/O Port RS232C on the MCA. Connect the antenna cable to the transmitter and the ARGOS antenna.

\section{*** WARNING ***}

The power switch on the back of the RAMP system power supply must be in the down (OFF) position and the ARGOS antenna connected to the transmitter before proceeding. The transmitter will be damaged if power is applied without a load on the antenna connector. Connect one end of the $\mathrm{AC}$ power cord to the inside of the environmental chamber, connect the other end to the multioutput $\mathrm{AC}$ extension box.

Connect the RED power plug from the RAMP system power supply to the computer.

Connect the BLACK power plug with the hole in the center from the RAMP system power supply to the MCA.

\section{$* * *$ WARNING ***}

Very carefully connect the BLACK miniature phone power plug from the RAMP system power supply to the serial/parallel two-way converter. It is possible to cause a short circuit and blow an internal fuse if this plug is not inserted properly.

Connect the AMP lock power connectors to the transmitter.

Connect the AC power cord of the RAMP system power supply to the multioutput $\mathrm{AC}$ extension box. 
Power the RAMP system power supply with the switch on the back of the unit.

Power the MCA and perform setup as follows:

Press SETUP on the MCA.

Press the UP ARROW.

Press YES eight times or until BAUD RATE=9600 appears again.

Press the UP ARROW.

Press YES six times or until PARITY $=8+$ NONE appears again.

Insert the SYSTEM DRIVE A disk into drive A on the right side of the computer. Insert a formatted data disk into drive $B$ on the left side of the computer.

Power the computer. The switch is toward the back on the right side. The computer will self-test, load, and execute "IRAMP," the RAMS control program.

The system is now ready for operation. Set the clock as described in Appendix $A$ and perform the steps described in Appendix B. In the event of problems, go to Appendix C for system troubleshooting. Appendix $D$ gives the schedule of routine operation.

\subsubsection{EML QUALITY CONTROL}

All data records being received from Service ARGOS are monitored by EML personnel daily to ensure that all sites are transmitting valid data. This includes almost continuous monitoring of EML VAX mail messages by EML personnel during the normal work day. All completed spectra received from Service ARGOS are also examined daily. Calibration spectra are computed and plotted as received. Background spectra are plotted and visually compared with previous background spectra. The results of the analyses of the sample spectra are examined for quality of fit and for the likelihood of fission products. The cause of excessively high quality of fit or fission product likelihood is determined, corrected, and a reanalysis is then performed.

All hard copy and computer files, both original data and results of analyses, are maintained at EML. 


\section{REFERENCES}

Larsen, R. J., H.-N. Lee, M. Monetti and C. G. Sanderson

"EML's Global Sampling Programs"

Proc. of the Conference on Atmospheric Chemistry, American Meteorological Society, Boston, MA (1994)

Sanderson, C. G., N. Latner and R. J. Larsen

"Environmental Gamma-Ray Spectrometry at Remote Sites with Satellite Data

Transmission"

Nuclear Instruments and Methods, A239, 271-277 (1994)

Latner, N., C.G. Sanderson, V.C. Negro, S. Wurms and N. Chiu

"AUTORAMP - An Automatic Unit for Unattended Aerosol Collection, Gamma-Ray Analysis and Data Transmission from Remote Locations" 


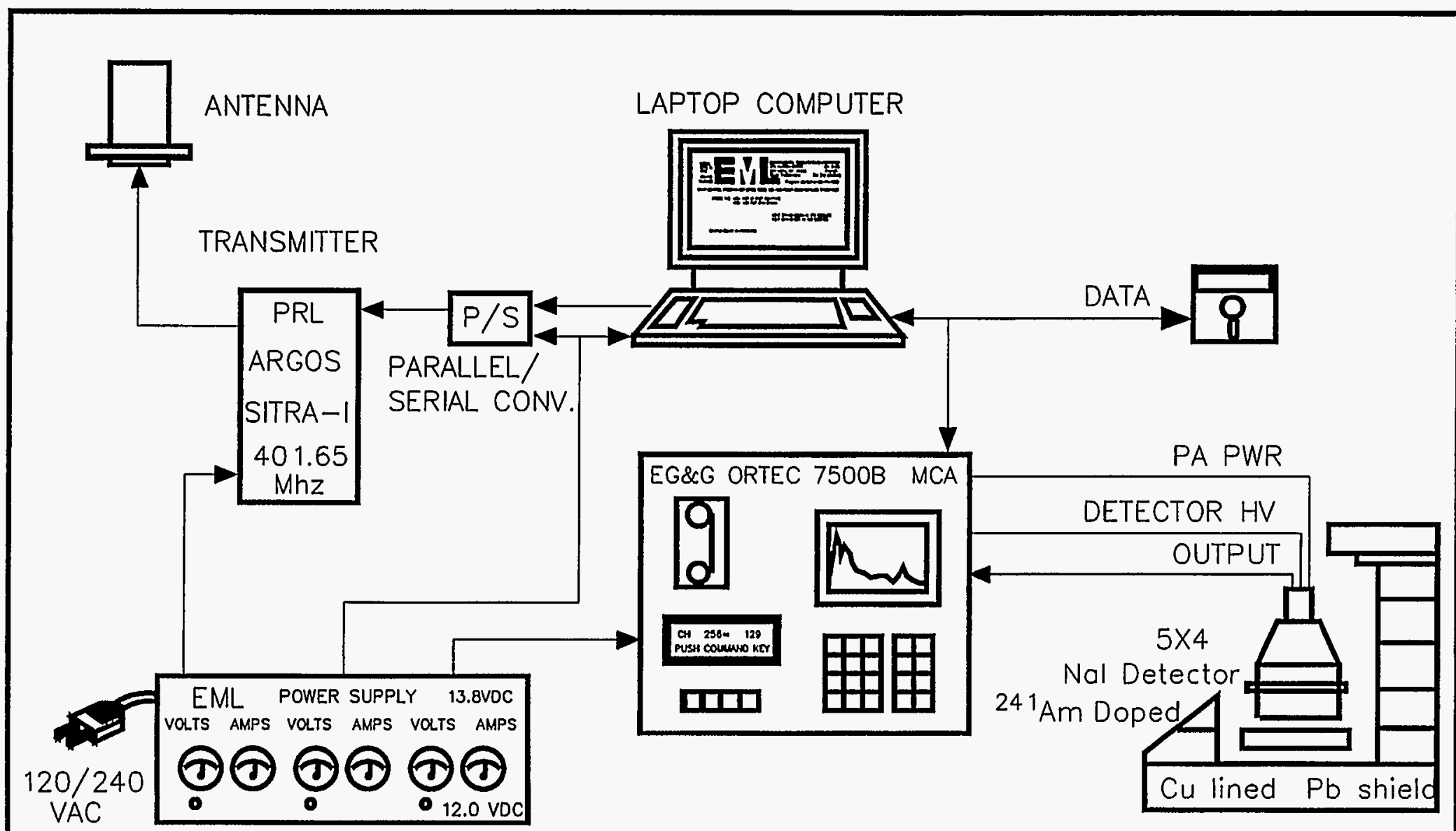

$\stackrel{\omega}{\dot{s}}$

Figure 3.8. Block diagram of EML's second generation NaI RAMS. 


\section{OPERATION PROTOCOL}

\section{CONTROL PROGRAM MENUS}

When the RAMP control program starts, there are four possible operations.

$$
\begin{aligned}
& I \text { - for initial setup, } \\
& C \text { - for system clock reset, } \\
& G \text { - for } M C A \text { gain check, or } \\
& A \text { - for data acquisition. }
\end{aligned}
$$

\section{INTTIAL SETUP}

Press the 'T' key on the computer. Enter the local date and time in the format as shown on the screen. Press ENTER. If the entry is correct, press the ' $\mathrm{Y}$ ' key on the computer. If the entry is incorrect, press the ' $\mathrm{N}$ ' key on the computer and reenter the correct local date and time.

If the 'Initial Setup' is entered in error, press the ' $Q$ ' key to quit this routine.

The default values displayed can be accepted by pressing ENTER. Press the ' $Y$ ' key to accept this value, or ' $N$ ' to enter a new value.

\section{CLOCK RESET}

It is very important that the date and time displayed in the upper-left corner of the screen is correct to within $1 \mathrm{~h}$. If the date and time should change by more than $1 \mathrm{~h}$, it must be reset. Press the ' $C$ ' key on the computer and enter the current local date and time 
in the format as shown on the screen. Press ENTER. If the entry is correct, press the ' $Y$ ' key on the computer. If the entry is incorrect, press the ' $\mathrm{N}$ ' key on the computer and reenter the correct local date and time.

\section{DATA ACQUISITION - CALIBRATION}

Press the ' $\mathrm{A}$ ' key on the computer for data acquisition. When the ' $\mathrm{A}$ ' key is pressed, there will be five possible operations.

$$
\begin{aligned}
& S \text { - for sample count, } \\
& B \text { - for background count, } \\
& C \text { - for calibration count, } \\
& G \text { - for MCA gain check, or } \\
& P \text { - to return to previous menu. }
\end{aligned}
$$

The first set of data obtained by the system must be a calibration count. Place a Coleman lantern mantle into the drawer of the RAMS counter and start data collection by pressing the ' $\mathrm{C}$ ' key on the computer. The computer will automatically set up the analyzer and begin data collection. The calibration data should be collected for at least $4 \mathrm{~h}$. Stop the calibration count by pressing the ' $S$ ' key, then the ' $Y$ ' key, and then the ' $T$ ' key. The calibration data will then be recorded on the floppy disk and transmitted to the ARGOS satellite. Remove the Coleman lantern mantle from the drawer of the RAMS counter.

\section{DATA ACQUISITION - BACKGROUND}

Press the ' $\mathrm{A}$ ' key on the computer for data acquisition. When the ' $\mathrm{A}$ ' key is pressed there will be five possible operations.

$S$ - for sample count,

$B$ - for background count,

$C$ - for calibration count,

$G$ - for MCA gain check, or

$P$ - to return to previous menu. 
Background data must be collected after the calibration and before any samples. Remove the Coleman lantern mantle from the drawer of the RAMS counter and replace with a BACKGROUND filter. Start data collection by pressing the 'B' key on the computer. The computer will automatically set up the analyzer and begin data collection. The background data should be collected at least overnight. Stop the background count by pressing the ' $\mathrm{S}$ ' key, then the ' $\mathrm{Y}$ ' key and then the ' $\mathrm{T}$ ' key on the computer. The background data will then be recorded on the floppy disk and transmitted to the ARGOS satellite.

\section{DATA ACQUISITION - SAMPLE}

Press the 'A' key on the computer for data acquisition. When the 'A' key is pressed, there will be five possible operations.

$$
\begin{aligned}
& S \text { - for sample count, } \\
& B \text { - for background count, } \\
& C \text { - for calibration count, } \\
& G \text { - for MCA gain check, or } \\
& P \text { - to return to previous menu. }
\end{aligned}
$$

After one calibration and one background data set have been collected, exposed air filter samples can be counted.

Prepare for sample data collection by pressing the ' $S$ ' key. Enter the following information when requested on the computer screen.

Date \& time that sample collection started.

Date \& time that sample collection ended.

Reading of running time meter when sample collection started.

Reading of running time meter when sample collection ended.

Reading of pressure meter when sample collection started.

Reading of pressure meter when sample collection ended.

Temperature when sample collection started.

Temperature when sample collection ended. 
Please refer to Appendix D for a schedule of when SAMPLES, CALIBRATIONS, and BACKGROUNDS should be counted.

When instructed, place the folded sample air filter into the drawer of the RAMS counter and start data collection by pressing any key on the computer. The computer will automatically set up the analyzer and begin data collection. 


\title{
APPENDIX B
}

\section{FIELD QUALITY CONTROL}

\author{
POWER SUPPLY METERS
}

Each voltmeter should read about $14 \mathrm{~V}$.

The MCA ammeter should read about $0.7 \mathrm{~A}$ with the display OFF and about $1.0 \mathrm{~A}$ with the display ON.

The laptop ammeter should read about $0.3 \mathrm{~A}$ with the display OFF and about $0.5 \mathrm{~A}$ when the display is $O N$.

The transmitter/converter ammeter should read about $0.3 \mathrm{~A}$. Every $3 \mathrm{~min}$ when the transmitter is active this ammeter will read about $0.8 \mathrm{~A}$ for a second. This meter deflection is a good indication that the transmitter is operating.

\section{LIGHT EMITTING DIODE (LED) INDICATORS}

A red LED on the parallel/serial converter should be on, as it indicates the unit is powered.

A green LED on the MCA indicates that the battery is charging properly. If this LED should become red, a fault condition exists and EML must be notified by TELEX or FAX, but no operator action is required.

The three LEDs on the power supply should be on, as they indicate that the battery chargers are operating. 


\section{APPENDIX C}

\section{SYSTEM TROUBLESHOOTING}

Once the system has been set up and is running, it should continue to do so indefinitely. There are a number of error traps built into the computer software program. If an error occurs and is trapped by the program, a message will be displayed indicating what action the operator should take.

Power for the transmitter/converter is protected with a $5 \mathrm{~A}$ internal fuse. If the LED on the serial/parallel two-way converter is not illuminated, and the transmitter/ converter meters indicate no voltage, this fuse may need to be replaced. In order to replace this fuse, the cover for the RAMP system power supply must be removed. The fuse holder is at the back of the left side of the unit. Spare fuses have been supplied with the backup equipment.

If none of the power supply LEDs are lit, the ammeters read 0 and the voltmeters read between 12 and $13 \mathrm{~V}$, then the $\mathrm{AC}$ fuse at the back of the unit must be replaced to restore the $\mathrm{AC}$ (main) power. The batteries inside the power supply will continue to provide power for the RAMS for about $8 \mathrm{~h}$.

If the computer does not respond, press 'CTRL, 'ALT,' and 'DEL' at the same time. This will reset the computer and restart the program. Press the ' $\mathrm{A}$ ' key, then either the ' $\mathrm{C}$,' 'B' or 'S' key, as appropriate.

If the computer has entered the DOS operating mode as indicated by the DOS prompt $A>$, type 1RAMP and press ENTER to restart the program. Press the ' $A$ ' key, then either the ' $\mathrm{C}$, ' 'B' or 'S' key, as appropriate.

If the MCA has been turned off or has lost power for a long time, it will be necessary to reset the high voltage and to recycle the baud rate and parity so that communications with the computer can be established. Proceed as follows:

Press SETUP on the MCA. 


\section{Press the UP ARROW.}

Press 'YES' eight times or until 'BAUD RATE=9600' appears again.

Press the UP ARROW.

Press 'YES' six times or until 'PARITY $=8+N O N E$ ' appears again.

Press END.

Use 'CTRL,' 'ALT,' and 'DEL.' (This will reset the computer and restart the program.)

When the RAMP control program starts, there will be four possible operations.

$$
\begin{aligned}
& I \text { - for initial setup, } \\
& C \text { - for system clock reset, } \\
& G \text { - for MCA gain check, or } \\
& A \text { - for data acquisition. }
\end{aligned}
$$

Press the 'I' key on the computer. Accept the default values displayed by pressing ENTER. Press the ' $Y$ ' key to accept these values. The high voltage will automatically be set to the correct value by the initial setup routine.

Computer software problems can develop if: 1) the MCA amplifier gain is beyond the range that the computer can correct; 2) the MCA gain setting when the unit was packaged for shipment was preset to an invalid value; and 3) the internal temperature of the environmental chamber was not set at $88^{\circ} \mathrm{F}$. The MCA gain can be checked and adjusted as follows.

Press the ' $G$ ' key on the computer keyboard. "THE CURRENT GAIN VALUE IS" message will be displayed. After a few seconds, the current gain value will also be displayed. If the gain is very different from the preset value, enter the preset value and press the ENTER key on the computer. Press ENTER on the computer to leave the current gain value unchanged.

The MCA gain can also be reset automatically by the software. The procedure is as follows:

From the initial menu, press ' $\mathrm{T}$ ' for initialization.

Enter the correct local date and time.

When prompted for a GAIN value, enter the word RESET. 
Follow the instructions on the computer screen.

If the data that is being transmitted is not received by EML in New York City, EML will inform site operators by TELEX or FAX and ask operators to TELEX or FAX the status of the RAMS. After EML has determined the correct resolution of the problem, instructions will be communicated to site operators. 


\section{APPENDIX D}

\section{SCHEDULE FOR ROUTINE OPERATION}

MONDAY - Transmit the SAMPLE count started on Friday by sequentially pressing the $S, Y$, and $T$ keys on the computer.

Remove the exposed sample filter from the RAMP counter, write the date of transmission on the filter label under Notes, and mail the filter to the University of Miami.

Place the Coleman lantern mantle into the RAMP counter and start data collection by sequentially pressing the $\mathrm{A}$ and $\mathrm{C}$ keys on the computer.

WEDNESDAY - Transmit the CALIBRATION count started on Monday by sequentially pressing the $\mathrm{S}, \mathrm{Y}$, and $\mathrm{T}$ keys on the computer.

Remove the Coleman lantern mantle from the RAMP counter; store mantle.

Place the unexposed background filter into the RAMP counter and start data collection by sequentially pressing the $\mathrm{A}$ and $\mathrm{B}$ keys on the computer.

Change RAMP air filter and record pump data as outlined in Appendix A; store filter.

FRIDAY - Transmit the BACKGROUND count started on Wednesday by sequentially pressing the 'S', 'Y,' and ' $\mathrm{T}$ ' keys on the computer.

Remove the unexposed background filter from the RAMP counter; store filter. 
Fold and place the exposed filter sample collected on Wednesday into the RAMP counter and start data collection by pressing the ' $\mathrm{A}$ ' and ' $\mathrm{S}$ ' keys on the computer. Enter the data requested by the computer.

MONTHLY - At the end of each month, replace the data disk in Drive B of the computer and mail the old disk to EML. 


\title{
3.5 THERMOLUMINESCENCE DOSIMETRY
}

\author{
Contact Person(s): Gladys Klemic
}

\subsubsection{SCOPE}

Research at EML is directed toward advancements in environmental applications of thermoluminescence dosimetry (TLD). This includes investigations of problems associated with low-level measurements of the gamma- and cosmic-ray natural background (de Planque and Gulbin, 1985; Julius and de Planque, 1984; Gulbin and de Planque, 1983, 1984), as well as techniques for the separation of neutron and gamma components from artificial sources (Klemic et al., 1996). EML organizes the International Intercomparisons of Environmental Dosimeters, a voluntary program of testing and research on integrating dosimeters for the measurement of environmental radiation (Klemic et al., 1995; Maiello et al., 1990a,b, 1995; de Planque and Gesell, 1986) that attracts participants from around the world.

Consistent procedures for the preparation, packaging, recordkeeping and readout, along with many QC checks, are necessary to obtain reliable results. The procedures described here have been shown to result in high quality measurements that are suitable for low-level environmental radiation monitoring and for TLD research purposes (see also American National Standards Institute Report, ANSI N545-1975, and the future release of ANSI N13.37, which will replace it).

\subsubsection{SPECIAL APPARATUS}

\section{TLD phosphors:}

Lithium fluoride dosimeters - ${ }^{7} \mathrm{LiF}: \mathrm{Mg}, \mathrm{Ti}$ chips are used for most applications. They are $3.2 \times 3.2 \times 0.89 \mathrm{~mm}, 24 \mathrm{mg}$ chips manufactured by Harshaw/Bicron Co., 6801 Cochran Rd., Solon, OH 44139-3395 (TLD-700). 
Aluminum oxide dosimeters $-\mathrm{Al}_{2} \mathrm{O}_{3}: \mathrm{C}$ chips are presently used only for research purposes. They are $5 \mathrm{~mm}$ in diameter and $1 \mathrm{~mm}$ thick and are manufactured by Victoreen, Inc., 6000 Cochran Rd., Solon, OH 44139-3395 (Model 2600-80).

Calcium fluoride chips and bulb dosimeters - EML's historical experience has included bulb-type dosimeters and other types of chips such as $\mathrm{CaF}_{2}: \mathrm{Mg}$ (Gulbin and de Planque, 1983; Gulbin and de Planque, 1984); however, these phosphors are not presently in use and will not be covered here.

\section{Annealing equipment:}

High temperature furnace - A furnace with constant temperature capability up to $1000^{\circ} \mathrm{C}$ (manufactured by Blue M Electric Co., Blue Island, $\mathbb{I L}$ 60406) is used for high temperature annealing.

Low temperature furnace - This furnace is maintained at $100^{\circ} \mathrm{C}$ for low temperature annealing (manufactured by Fisher Isotemp, Pittsburgh, PA 15219).

Planchet - An EML-designed, platinum-plated copper planchet is used for chip annealings. The tray is indented so that chips may be identified by placement (see Figure 3.9).

Brass heat sink - A brass heat sink, $5 \times 5 \times 21 \mathrm{~cm}$, is mounted on an aluminum base that is placed on steel unistrut bars to allow uniform cooling of chips (see Figure 3.9).

\section{Controlled lighting:}

Gold fluorescent lights with no ultraviolet emissions.

Dark room shades.

Incandescent bulbs, including a $25-\mathrm{W}$ red bulb for minimal lighting.

\section{4. ${ }^{137} \mathrm{Cs}$ source:}

A $2 \mathrm{Ci}$ collimated NIST traceable ${ }^{137} \mathrm{Cs}$ source is used for calibration. Usual exposure distance is $2 \mathrm{~m}$, where the beam is uniform within a radius of at least $3.0 \mathrm{~cm}$.

\footnotetext{
*A new controlled atmoshpere oven (manufactured by GS Lindberg/Blue M., Watertown, WD has recently been installed for research but it is not yet in regular use.
} 


\section{TLD readers:}

The TLD reader room is air-conditioned to maintain a constant temperature $\left(\sim 20^{\circ} \mathrm{C}\right)$ year-round. Three TLD readers are presently in use:

EML reader - This reader was built by EML and has been in use since 1971. It uses linear pan heating and measures the TL signal with a high-gain, low-noise photomultiplier tube cooled to $15^{\circ} \mathrm{C}$ below room temperature. An internal light source is used to verify the electronic stability of the system. Power is applied to a heating element silver-soldered to a thin silver heater pan. The chip is positioned manually and centered in the pan by a removable platinum-plated, stainless-steel disk with a central hole for the TLD. This disk suppresses the infrared signal from the heater pan and provides a reproducible geometry for chip placement. Heating parameters are adjustable up to a maximum temperature of about $550^{\circ} \mathrm{C}$. Purified nitrogen gas flows through the heating chamber at a rate of about $2.4 \mathrm{~L} \mathrm{~min}^{-1}$ to suppress any nonradiation induced TL signal. A strip chart records the glow curve and temperature profile with a selected glow peak region of interest indicated by pen offsets. The integral "counts" in this region is indicated on a numerical display.

Victoreen Model $2800 M$ - A commercially available reader with many of the same features as the EML reader using updated technology, it will eventually replace the aging EML reader. Readout data can be sent to a printer or personal computer.

Automatic hot gas reader (TNO) - Built by Radiologische Dienst TNO at the Netherlands Organization for Applied Research, this reader is different from the other two, both in heating methods and chip handling. It uses three jets of preheated nitrogen gas (about $190^{\circ} \mathrm{C}$ constant temperature, not a linear profile) for reproducibility and speed. Chips are handled automatically at a rate of 150 per hour up to 1000 chip readings. Readout parameters and output are controlled and stored by a personal computer. This reader is especially suitable for large-scale studies and routine monitoring.

\section{Lead shield:}

A $10-\mathrm{cm}$ thick lead shield is used to store TLDs after preparation or before readout. 


\subsubsection{PREDEPLOYMENT PREPARATION OF CHIPS}

\section{A. Batch preselection.}

Before chips are to be used for measurements, a set (typically 200 to 300 chips) from a single manufacturing batch is tested for uniformity as follows:

1. Clean and anneal chips as described in Section B.

2. Give chips a single exposure to ${ }^{137} \mathrm{Cs}$ (typically on the order of $70 \mu \mathrm{Gy}$ in air, or $8 \mathrm{mR})$.

3. Read out the chips (see Table 1 for readout parameters).

4. Assign chips with readings that agree to within $30 \%$ of each other to the same group. Outliers should be flagged and removed from the group. (Note: A tighter criterion of 5-10\% may be used for special applications.)

5. Assign each chip a unique identification number to be permanently retained.

\section{B. Cleaning/annealing.}

1. Manually clean individual chips before each use with methanol and a cotton swab, handling chips with forceps or a vacuum pen under appropriate lighting conditions (see Table 3.2) (Freeswick and Shambon, 1970).

2. Anneal chips as follows:

LiF: $1 \mathrm{~h}$ at $400^{\circ} \mathrm{C}, 1$-min cool-down on heat sink, followed by $2 \mathrm{~h}$ at $100^{\circ} \mathrm{C}$. $\mathrm{Al}_{2} \mathrm{O}_{3}: 10 \mathrm{~min}$ at $400^{\circ} \mathrm{C}$.

3. Cool chips to room temperature on heat sink.

4. Store chips in the lead shield if they are not going to be used immediately. Keep careful records of the date and time that the chips are placed in the lead shield, as well as when they are removed for deployment. 


\section{Dosimeter packaging.}

Packaging should be light-tight, moisture proof, and thick enough to provide electronic equilibrium and to shield against environmental beta radiation. The EML dosimeter consists of a commercially available black lucite box in which additional black lucite pieces have been bonded in place to provide depressions for holding the individual chips (see Figure 3.10). It has outside dimensions of $2.9 \times 2.9 \times 0.9 \mathrm{~cm}$. Each chip is surrounded by lucite with a minimum thickness of $2.8 \mathrm{~mm}$ (about $320 \mathrm{mg} \mathrm{cm}^{-2}$ ). A dosimeter usually consists of five chips, though the container can hold up to 10 chips. To ensure water-tightness, the lucite box is placed inside a thin plastic bag which is heat sealed or secured with fiber tape.

\subsubsection{ENVIRONMENTAL DEPLOYMENT}

Environmental TLDs are usually deployed for 1-3 months. A preselected uniform batch (see Section 3.5.3.A) with enough TLDs to cover at least three monitoring cycles is maintained exclusively for use in environmental monitoring (i.e., while one set is in the field, there are enough dosimeters for two replacement sets). A set of environmental dosimeters for a monitoring cycle includes six control dosimeters prepared with the field dosimeters, as described in Section 3.5.3.B. All dosimeters are stored in the lead shield after preparation until they can be deployed, noting date and time. Two of the controls will be used to determine the exposure rate in this shield (STORAGE CONTROLS) and are thus stored there for the duration of the field cycle. The other four are used for calibration of the system (CALIBRATION CONTROLS) and will be discussed in Section 3.5.5.D.

If the field site is far from the laboratory, it is necessary to use additional controls to account for exposure received in transit (TRANSIT CONTROLS). These controls must be kept with the field dosimeters at all times except during field deployment, when they must be kept somewhere where the exposure rate is known or can be measured independently.

The dosimeter is hung $1 \mathrm{~m}$ above the ground, away from large structures that may provide shielding or additional exposure (see Figure 3.11). Usually the dosimeter is left hanging freely to rotate in the wind for isotropic angular exposure. At the end of the field 
cycle, the dosimeters are returned to the lead shield until they are read out, and a replacement set is deployed in the field.

\subsubsection{READOUT AND CALIBRATION}

\section{A. Reader checkout.}

1. Prepare the reader for use by setting the appropriate reader parameters as shown in Table 3.2.

2. Check the reader's dark current, the heating chamber current (background of empty chamber), and the response of the reader to the internal light source.

3. Read out a test chip several times to check for anomalous results.

\section{B. Preread annealing.}

1. Remove the FELD dosimeters from the lead shield, noting the date and time.

2. Perform a preread anneal for LiF chips at $100^{\circ} \mathrm{C}$ for $10 \mathrm{~min}$. (No preread anneal is used for $\mathrm{Al}_{2} \mathrm{O}_{3}$ chips.)

\section{Initial readout.}

1. Read out one chip from each FIELD dosimeter. After it returns to room temperature, the chip is read a second time to measure the background of the system.

2. Temporarily discontinue readout at this point to prepare the calibration dosimeters.

\section{Calibration.}

Four dosimeters are used to calibrate the system during readout. Two of these are from the set originally prepared at the start of the field cycle, and two are taken from the set of replacement dosimeters just prepared. Thus, while each prepared set includes four 
calibration dosimeters, two will be used right away to calibrate the returning field set and the other two are stored in the lead shield for the duration of the field cycle to be used at the following readout. The calibration is performed as follows:

1. Examine the range of reader net counts from the initial readout of each field dosimeters to determine the calibration exposures to use. Choose three calibration exposures to bracket the readings given by the field dosimeters and to provide a check on the linearity of the system. (The approximate counts per unit exposure is known from previous readouts.)

2. Remove the CALIBRATION CONTROLS from the lead shield, noting the date and time of retrieval.

3. Give one CALBBRATION CONTROL an exposure that is expected to yield net counts in the range of the lowest result found for the FIELD dosimeters. Another CALIBRATION CONTROL is given an exposure corresponding to the highest reading, and the other two receive an exposure in the middle of this range.

\section{E. Resuming readout.}

1. Remove the STORAGE CONTROL dosimeters from the lead shield, noting the date and time.

2. Anneal all of the control dosimeters in the case of LiF TLDs (the four CALIBRATION CONTROLS as well as the two STORAGE CONTROLS) for $10 \mathrm{~min}$ at $100^{\circ} \mathrm{C}$.

3. Intersperse the STORAGE CONTROL and CALIBRATION CONTROL dosimeters among the FIELD dosimeters. Read out one chip from each dosimeter before going to the next chip in any dosimeter. (This provides a QC check against any variations in the system during readout.)

\subsubsection{ANALYSIS OF RESULTS}

An interactive program that runs on a personal computer (written in Fortran) handles the data analysis. A sample input file is shown in Appendix A. It includes for each 
dosimeter: date and times of preparation, deployment, return, and readout; gross counts; background counts; and time of exposure to the cesium source. The program determines the field exposure by correcting for exposure received in storage as measured by the storage controls and then converting counts to exposure by using the calibration controls. The user is given the option of rejecting outliers, and the statistical error is propagated throughout each step in the calculation. The program returns the net field exposure and exposure rate and the field deployment time for each field dosimeter. It also gives information about the exposure rate in storage, the calibration factor, and any rejected readings. A sample output file is shown in Appendix A. Details of the calculations are given below.

\section{A. Computing net counts and standard error.}

The mean net counts for each dosimeter is:

$$
\mathrm{X}=\sum_{\mathrm{i}=1}^{\mathrm{n}}\left(\mathrm{x}_{\mathrm{i}}\right) \div \mathrm{n}
$$

where

$$
\begin{aligned}
& \mathrm{x}_{\mathrm{i}}=\text { gross counts }- \text { background counts of } i \text { th chip. } \\
& \mathrm{n}=\text { number of chips per dosimeter ( } \mathrm{n}=5 \text { usually). }
\end{aligned}
$$

The standard error of the mean for each dosimeter is:

$$
\operatorname{err}_{x}=\frac{\sigma}{\sqrt{n}}=\sqrt{\frac{\sum_{i-1}^{n}\left(x_{i}-X\right)^{2}}{n(n-1)}}
$$

\section{B. Outlier check.}

The program checks for outliers among the individual chip readings in a dosimeter. Two different criteria are used:

1. "Extreme Values" - Individual chip net counts that are $>150 \%$ or $<50 \%$ of the dosimeter average $\mathbf{X}$ are flagged. This test would find missing chips or noise spikes. 
2. " $r$ Test" - This is an outlier test for gaussian distributions (Proschan, 1969). The net counts for the five chips are ranked in order of smallest to the largest, such as $x_{1}, x_{2}$, $x_{3}, x_{4}, x_{5}$. If the ratio $\mathrm{r}=\left(x_{2}-x_{1}\right) \div\left(x_{5}-x_{1}\right)$ is larger than 0.780 , chip $x_{1}$ is flagged. Similarly if the ratio $\mathrm{r}=\left(x_{5}-x_{4}\right) \div\left(x_{5}-x_{1}\right)$ is larger than 0.780 , chip $x_{5}$ is flagged. This is a more sensitive test that can identify outliers that would be missed by the extreme value test. While it is redundant for values beyond $150 \%$ of the mean, it could miss the very low extreme values so both tests are needed. (Proschan describes more sensitive tests to apply when more than 7 chips are used.)

In either criterion, the user is then given the option of omitting flagged chips from the analysis. No chips are automatically rejected: the final decision is made by the user. (In practice data are rarely rejected, and then usually for obvious reasons.) If a chip is rejected, the mean net counts and standard error are recalculated for that dosimeter, and a note appears in the output file.

\section{Storage exposure rate correction factor.}

$$
\mathrm{S}=(1 / 2)\left(\mathrm{X}_{\mathrm{SC} 1}+\mathrm{X}_{\mathrm{SC} 2}\right) \div \mathrm{t}_{\mathrm{SC}}
$$

where

$\mathrm{S}=$ storage correction factor,

$\mathrm{X}_{\mathrm{SCl}}=$ mean net counts for STORAGE CONTROL \#1,

$\mathrm{X}_{\mathrm{SC} 2}=$ mean net counts for STORAGE CONTROL \#2, and

$\mathrm{t}_{\mathrm{SC}}=$ time storage controls were in lead shield (calculated by subroutine from dates in input file)

The standard error is propagated through this calculation as:

$$
\mathrm{err}_{\mathrm{S}}=\frac{1}{2 \mathrm{t}_{\mathrm{SC}}} \sqrt{\left(\mathrm{err}_{\mathrm{SC} 1}^{2}+\mathrm{err}_{\mathrm{SC} 2}^{2}\right)}
$$

With err $\mathrm{SCl}_{\mathrm{S}}$ and $\mathrm{err}_{\mathrm{SC2}}$ being the standard error of the storage controls as defined in Section $\mathrm{A}$ above. (The error associated with the storage time is negligible, estimated at $<0.01 \%$.) 


\section{Calibration factor.}

$$
\mathrm{C}=\left(\frac{1}{4}\right) \sum_{\mathrm{i}=1}^{4}\left(\mathrm{X}_{\mathrm{CCi}}-\mathrm{St}_{\mathrm{CCi}}\right) \div \text { exposure }_{\mathrm{CCi}}
$$

where

$$
\begin{aligned}
& \mathrm{C}=\text { calibration factor, } \\
& \mathrm{X}_{\mathrm{CCi}}=\text { mean net counts of CALIBRATION CONTROL \#i } \\
& \mathrm{t}_{\mathrm{CCi}}=\text { time ith CALIBRATION CONTROL was kept in lead shield (calculated by } \\
& \text { subroutine from dates in input file), } \\
& \text { exposure }_{C C i}=\text { cesium exposure given to ith CALIBRATION CONTROL (calculated from } \\
& \text { decay corrected known source strength and time of exposure). }
\end{aligned}
$$

The standard error propagated through this calculation is then:

$$
\operatorname{err}_{C}=\frac{1}{4} \sqrt{\sum_{i=1}^{4}\left(\frac{\operatorname{err}_{C C i}^{2}+\operatorname{err}_{S}^{2} t_{C C i}^{2}}{\text { exposure }_{C C i}^{2}}\right)}
$$

(Uncertainties in cesium calibration exposure are treated as systematic rather than statistical errors and are treated separately.)

The program also performs a linear regression on the corrected counts vs. exposure for the four calibration dosimeters. The goodness of fit is a check on the linearity of the system and the slope may be compared to $C$.

\section{E. Field exposure.}

The field site exposure $(f)$ is a function of the quantities calculated above. The algorithm used by the program to calculate field site exposure may be summarized as:

$$
F=f\left(X_{F}, t_{F}, S, C\right)=\frac{\left(X_{F}-S t_{F}\right)}{C}
$$

where

$$
\mathrm{X}_{\mathrm{F}}=\text { mean FIELD dosimeter counts }
$$




$$
\begin{gathered}
\mathrm{t}_{\mathrm{F}}=\text { time FIELD dosimeters were kept in a lead shield (calculated by } \\
\text { subroutine from dates in input file), }
\end{gathered}
$$

\section{F. Error analysis.}

Total uncertainty $=$ statistical error at $95 \%$ confidence + estimated systematic error

While the standard error was propagated through all the above calculations using the analytical propagation of error formulas, for the last step it is easier to calculate the statistical error numerically as shown below (Bevington and Robinson, 1992).

Statistical err $=2.776 \sqrt{\left(a^{2}+b^{2}+c^{2}\right)}$

where

$$
2.776=95 \% \text { confidence interval for } 5 \text { chips }
$$

(4 degrees of freedom)

$$
\begin{aligned}
& a=\frac{\left(\left[X_{F}+e r_{F}\right]-S t_{F}\right)}{C}-\frac{\left(X_{F}-S t_{F}\right)}{C} \\
& b=\frac{\left(X_{F}-\left(S+e r r_{F}\right) t_{F}\right)}{C}-\frac{\left(X_{F}-S t_{F}\right)}{C} \\
& c=\frac{\left(X_{F}-S t_{F}\right)}{\left(C+e r r_{C}\right)}-\frac{\left(X_{F}-S t_{F}\right)}{C}
\end{aligned}
$$

Systematic error is estimated on a case-by-case basis and added linearly to the statistical error for reporting the final results. The estimated systematic uncertainty associated with EML's cesium source is $2.5 \%$. 


\section{G. Situations involving transit controls.}

In cases where TRANSIT CONTROLS are required, the field exposure (Section E) calculation would instead be:

where

$$
\mathrm{F}=\left(\mathrm{X}_{\mathrm{F}}-\mathrm{T}\right) / \mathrm{C}
$$

\section{$\mathrm{T}=$ mean TRANSIT dosimeter counts corrected for exposure received in storage.}

If it happens that the TRANSIT CONTROLS are stored in the same lead shield as the other storage controls during the field cycle (time $=t_{F}$ ), then

$$
\mathrm{T}=\left(\mathrm{X}_{\mathrm{T}}-\mathrm{St} \mathrm{t}_{\mathrm{F}}\right)
$$

where

$$
\mathrm{X}_{\mathrm{T}}=\text { mean TRANSIT dosimeter counts. }
$$

More likely the TRANSIT CONTROLS will be stored in a different lead shield near the field site, in which case the storage exposure must be measured by some other means.

\section{REFERENCES}

"American National Standard Performance, Testing, and Procedural Specifications for Thermoluminescence Dosimetry (Environmental Applications)" American National Standards Institute Report, ANSI N545-1975 (1975)

Bevington, P. R. and D. K. Robinson

"Data Reduction and Error Analysis for the Physical Sciences"

Second Edition, McGraw-Hill, Inc., New York, p. 49 (1992) 
de Planque, G. and T. F. Gesell

"Environmental Measurements with Thermoluminescence Dosemeters - Trends and Issues"

Radiation Protection Dosimetry, 17, 193-200 (1986)

de Planque, G. and J. F. Gulbin

"The Effects of Moisture on LiF TLDs and Its Consequences for Environmental Measurements"

Proceedings 18th Midyear Health Physics Symposium, pp. 307-315, January (1985)

Freeswick, D. C. and A. Shambon

"Light Sensitivity of LiF Thermoluminescent Dosimeters"

Health Physics, 19, 65 (1970)

Gulbin, J. F. and G. de Planque

"An Investigation of Field Fading in LiF (TLD-700) in an Environmental Monitoring Program"

Radiation Protection Dosimetry, $\underline{5}, 155-199$ (1983)

Gulbin, J. F. and G. de Planque

"Ten Years of Residential TLD Monitoring"

Radiation Protection Dosimetry, $\underline{6}, 299-303$ (1984)

Julius, H. W. and G. de Planque

"Influence of Annealing and Readout Procedures on Fading and Sensitivity Changes in

LiF for Temperatures and Humidities Typical for Environmental and Personnel

Dosimetry"

Radiation Protection Dosimetry, 6, 253-256 (1984)

Klemic, G.A., N. Azziz and S.A. Marino

"The Neutron Response of $\mathrm{Al}_{2} \mathrm{O}_{3}: \mathrm{C},{ }^{7} \mathrm{LiF}: \mathrm{Mg}, \mathrm{Cu}, \mathrm{P}$, and ${ }^{7} \mathrm{LiF}: \mathrm{Mg}, \mathrm{Ti}$ TLDs"

Radiation Protection Dosimetry, 65, 221-226 (1996)

Klemic, G.A., J. Shobe, T. Gesell and P. Shebell

"Results of the Tenth International Intercomparison of Environmental Dosemeters"

Radiation Protection Dosimetry, 58, 133-142 (1995) 
Maiello, M., J. F. Gulbin, G. de Planque and T. F. Gesell

"8th International Intercomparison of Environmental Dosimeters"

Radiation Protection Dosimetry, 32, 91-98 (1990a)

Maiello, M., J. F. Gulbin, G. de Planque, and H. Thompson Heaton

"Ninth International 'Mini' Intercomparison of Environmental Dosemeters: A Further Analysis of the Data"

Radiation Protection Dosimetry, 58, 167-175 (1995)

Proschan, F.

in: Precision Measurement and Calibration

$\mathrm{Ku}, \mathrm{H}$. H. (ed.)

Selected NBS Papers on Statistical Concepts and Procedures

NBS Special Publication 300, Vol. 1, pp. 349-354 (1969) 
TABLE 3.2

SUMMARY OF TLD PARAMETERS USED

\begin{tabular}{|c|c|c|c|}
\hline \multicolumn{4}{|c|}{ ANNEALING } \\
\hline & Prede & yment & Prereadout \\
\hline LiF & $\begin{array}{c}400^{\circ} \mathrm{C} \\
100^{\circ} \mathrm{C} \\
\end{array}$ & $\begin{array}{l}1 \mathrm{~h} \\
2 \mathrm{~h}\end{array}$ & $100^{\circ} \mathrm{C} \quad 10 \mathrm{~min}$ \\
\hline $\mathrm{Al}_{2} \mathrm{O}_{3}$ & $400^{\circ} \mathrm{C}$ & $10 \mathrm{~min}$ & none \\
\hline
\end{tabular}

\begin{tabular}{|c|l||}
\hline \multicolumn{2}{|c|}{ LIGHTING CONDITIONS } \\
\hline $\mathrm{LiF}$ & no UV: gold fluorescent or incandescent \\
\hline $\mathrm{Al}_{2} \mathrm{O}_{3}$ & darkroom \\
\hline
\end{tabular}

\begin{tabular}{||c|c|c|c|c||}
\hline \multicolumn{5}{|c|}{ READ OUT Linear Heating: Victoreen Reader } \\
\hline & Starting Temp & Heating Rate & Heating Time & $\begin{array}{c}\text { Integration } \\
\text { Period* }\end{array}$ \\
\hline $\mathrm{LiF}$ & $100^{\circ} \mathrm{C}$ & $10^{\circ} \mathrm{C} \mathrm{sec}$ & $30 \mathrm{sec}$ & $\sim 10-20 \mathrm{sec}$ \\
\hline $\mathrm{Al}_{2} \mathrm{O}_{3}$ & $100^{\circ} \mathrm{C}$ & $10^{\circ} \mathrm{C} \mathrm{sec}$ & $20 \mathrm{sec}$ & $\sim 5-20 \mathrm{sec}$ \\
\hline
\end{tabular}


Table 3.2 (Cont'd)

\begin{tabular}{||c|c|c|c||}
\hline \multicolumn{4}{|c|}{ READ OUT Linear Heating: EML } \\
\hline & Heating Rate & $\begin{array}{c}\text { Maximum } \\
\text { Temperature }\end{array}$ & $\begin{array}{c}\text { Integration Period* } \\
\text { (adjusted visually) }\end{array}$ \\
\hline $\mathrm{LiF}$ & $10^{\circ} \mathrm{C} \mathrm{sec}^{-1}$ & $\sim 330^{\circ} \mathrm{C}$ & peaks $3,4 \& 5$ \\
\hline $\mathrm{Al}_{2} \mathrm{O}_{3}$ & $10^{\circ} \mathrm{C} \mathrm{sec}^{-1}$ & $\sim 300^{\circ} \mathrm{C}$ & whole peak \\
\hline
\end{tabular}

\begin{tabular}{||c|c|c||}
\hline \multicolumn{2}{||c|}{ READ OUT Constant Temperature Hot Gas: TNO } \\
\hline & Heating Time & Integration Period* \\
\hline $\mathrm{LiF}$ & $11 \mathrm{sec}$ & $1.3-10.0 \mathrm{sec}$ \\
\hline $\mathrm{Al}_{2} \mathrm{O}_{3}$ & $13 \mathrm{sec}$ & $1.7-12.0 \mathrm{sec}$ \\
\hline
\end{tabular}

*Integration period adjusted as needed to include entire peak. 


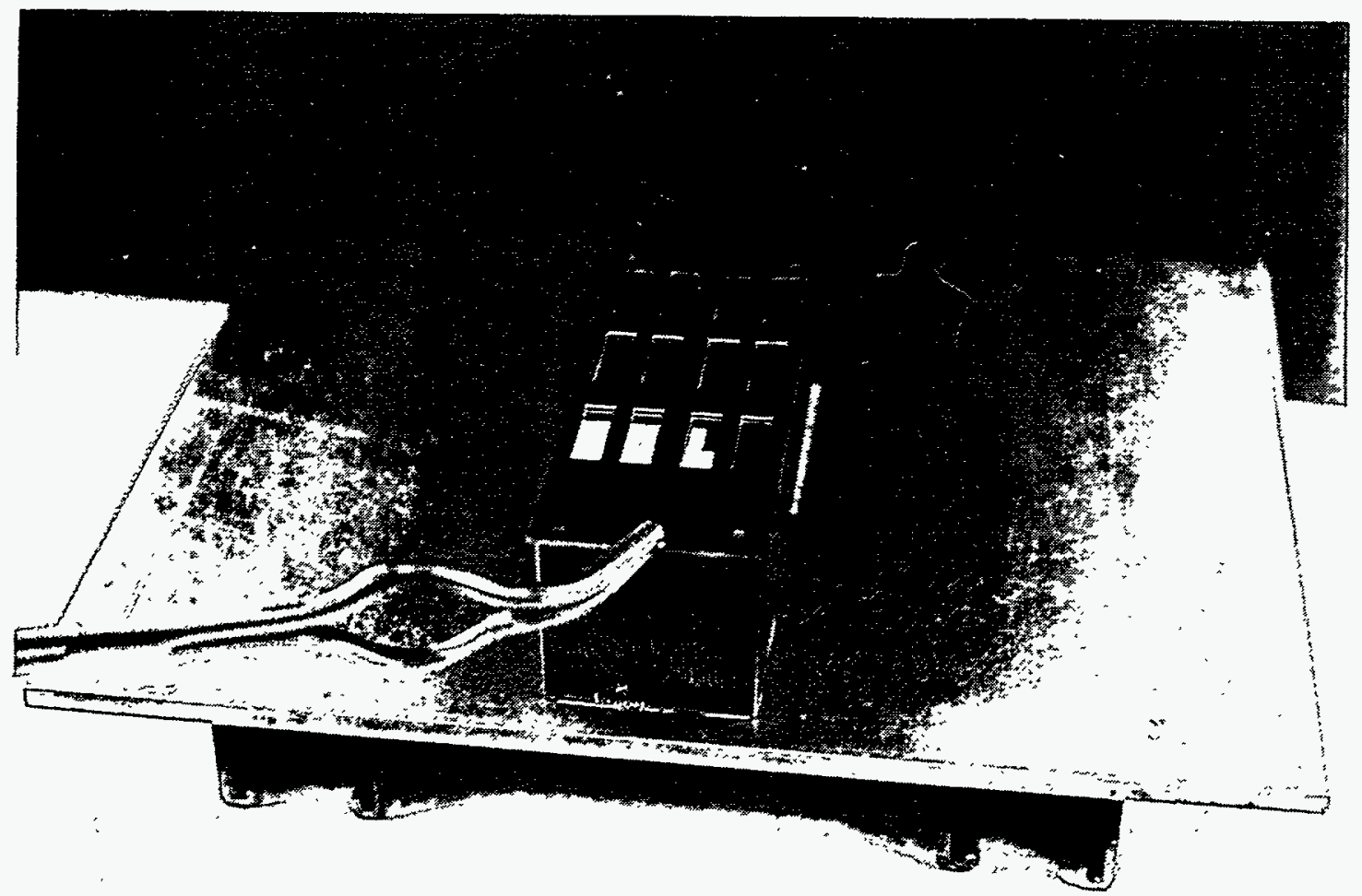

Figure 3.9. EML-designed planchet used for chip annealings, shown on brass heat sink. Chip identity is retained by position in planchet. 

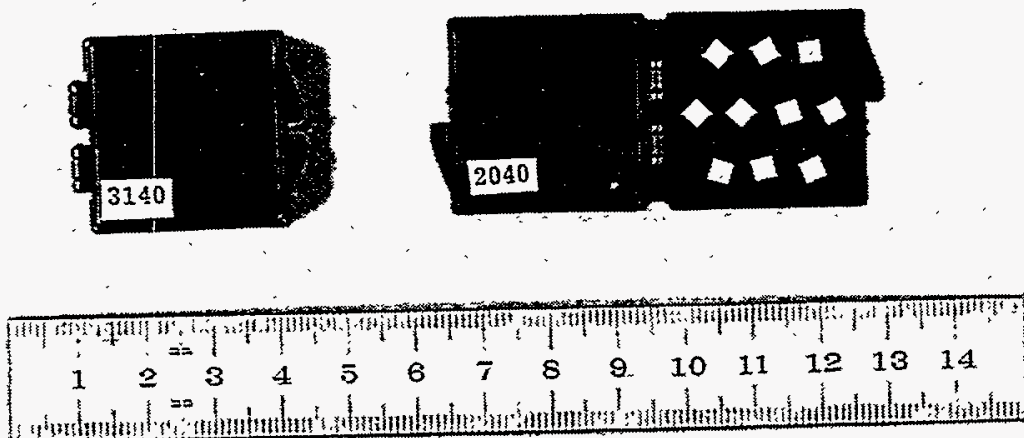

Figure 3.10 EML's light-tight lucite dosimeter package $\left(320 \mathrm{mg} \mathrm{cm}^{-2}\right)$, shown here in closed and open positions with $10 \mathrm{LiF}$ chips. 




Figure 3.11 EML dosimeter deployed at field site. Lucite box is sealed in plastic bag to protect from moisture, and hung $\sim 1 \mathrm{~m}$ above ground. 


\section{APPENDIX A}

SAMPLE INPUT FILE Environmental TLD Measurements

\begin{tabular}{|c|c|c|c|c|c|c|c|c|}
\hline SITE & ID & $\begin{array}{l}\text { DNTO LEAD } \\
\text { SHIELD }\end{array}$ & DEPLOYED & $\begin{array}{l}\text { RET. LEAD } \\
\text { SHIELD }\end{array}$ & READ OUT & $\begin{array}{r}\text { Cour } \\
\text { GROSS } \\
\end{array}$ & BKD & $\begin{array}{l}\text { Cs Expos } \\
\text { min }\end{array}$ \\
\hline Shrm & 3140 & $\begin{array}{c}02 / 21 / 93 \\
19: 23\end{array}$ & $\begin{array}{c}02 / 26 / 93 \\
15: 55\end{array}$ & $\begin{array}{c}04 / 27 / 93 \\
07: 37\end{array}$ & $\begin{array}{c}10 / 20 / 93 \\
09: 45\end{array}$ & $\begin{array}{l}430 \\
425 \\
417 \\
414 \\
447\end{array}$ & $\begin{array}{l}36 \\
32 \\
34 \\
30 \\
38\end{array}$ & 0.00 \\
\hline Roof & 3100 & $\begin{array}{c}02 / 21 / 93 \\
19: 23\end{array}$ & $\begin{array}{c}03 / 01 / 93 \\
15: 20\end{array}$ & $\begin{array}{c}05 / 25 / 93 \\
12: 45\end{array}$ & $\begin{array}{c}10 / 20 / 93 \\
09: 45\end{array}$ & $\begin{array}{l}534 \\
507 \\
556 \\
536 \\
550\end{array}$ & $\begin{array}{l}35 \\
33 \\
28 \\
36 \\
36\end{array}$ & 0.00 \\
\hline Chstr & 3110 & $\begin{array}{c}02 / 21 / 93 \\
19: 23\end{array}$ & $\begin{array}{c}02 / 23 / 93 \\
14: 45\end{array}$ & $\begin{array}{c}05 / 19 / 93 \\
12: 00\end{array}$ & $\begin{array}{c}10 / 20 / 93 \\
09: 45\end{array}$ & $\begin{array}{l}808 \\
812 \\
806 \\
803 \\
783\end{array}$ & $\begin{array}{l}29 \\
36 \\
29 \\
30 \\
34\end{array}$ & 0.00 \\
\hline Chstr & 3120 & $\begin{array}{c}02 / 21 / 93 \\
19: 23\end{array}$ & $\begin{array}{c}02 / 23 / 93 \\
14: 45\end{array}$ & $\begin{array}{c}05 / 19 / 93 \\
12: 00\end{array}$ & $\begin{array}{c}10 / 20 / 93 \\
09: 45\end{array}$ & $\begin{array}{l}811 \\
802 \\
764 \\
739 \\
764\end{array}$ & $\begin{array}{l}30 \\
30 \\
34 \\
38 \\
33\end{array}$ & 0.00 \\
\hline Sphr & 3130 & $\begin{array}{c}02 / 21 / 93 \\
19: 23\end{array}$ & $\begin{array}{c}03 / 01 / 93 \\
15: 20\end{array}$ & $\begin{array}{c}05 / 25 / 93 \\
12: 45\end{array}$ & $\begin{array}{c}10 / 20 / 93 \\
09: 45\end{array}$ & $\begin{array}{l}319 \\
349 \\
333 \\
338 \\
347\end{array}$ & $\begin{array}{l}35 \\
31 \\
30 \\
30 \\
31\end{array}$ & 0.00 \\
\hline $\begin{array}{l}\text { Stor } \\
\text { Ctl }\end{array}$ & 3200 & $\begin{array}{c}02 / 21 / 93 \\
19: 23\end{array}$ & $\begin{array}{c}00 / 00 / 00 \\
00: 00\end{array}$ & $\begin{array}{c}00 / 00 / 00 \\
00: 00\end{array}$ & $\begin{array}{c}10 / 20 / 93 \\
09: 45\end{array}$ & $\begin{array}{l}265 \\
257 \\
264 \\
267 \\
253\end{array}$ & $\begin{array}{l}29 \\
32 \\
27 \\
32 \\
29\end{array}$ & 0.00 \\
\hline $\begin{array}{l}\text { Stor } \\
\text { Ctl }\end{array}$ & 3190 & $\begin{array}{c}02 / 21 / 93 \\
19: 23\end{array}$ & $\begin{array}{c}00 / 00 / 00 \\
00: 00\end{array}$ & $\begin{array}{c}00 / 00 / 00 \\
00: 00\end{array}$ & $\begin{array}{c}10 / 20 / 93 \\
09: 45\end{array}$ & $\begin{array}{l}241 \\
269 \\
329 \\
244 \\
239\end{array}$ & $\begin{array}{l}32 \\
36 \\
32 \\
32 \\
32\end{array}$ & 0.00 \\
\hline Calib 1 & 3150 & $\begin{array}{c}02 / 21 / 93 \\
19: 23\end{array}$ & $\begin{array}{c}00 / 00 / 00 \\
00: 00\end{array}$ & $\begin{array}{c}00 / 00 / 00 \\
00: 00\end{array}$ & $\begin{array}{c}10 / 20 / 93 \\
09: 45\end{array}$ & $\begin{array}{l}478 \\
511 \\
471 \\
489 \\
486\end{array}$ & $\begin{array}{l}31 \\
32 \\
35 \\
39 \\
36\end{array}$ & 3.50 \\
\hline Calib 2 & 3160 & $\begin{array}{c}02 / 21 / 93 \\
19: 23\end{array}$ & $\begin{array}{c}00 / 00 / 00 \\
00: 00\end{array}$ & $\begin{array}{c}00 / 00 / 00 \\
00: 00\end{array}$ & $\begin{array}{c}10 / 20 / 93 \\
09: 45\end{array}$ & $\begin{array}{l}656 \\
643 \\
675 \\
618 \\
643\end{array}$ & $\begin{array}{l}31 \\
30 \\
34 \\
41 \\
34\end{array}$ & 6.0 \\
\hline Calib 3 & 3000 & $\begin{array}{c}04 / 23 / 93 \\
13: 00\end{array}$ & $\begin{array}{c}00 / 00 / 00 \\
00: 00\end{array}$ & $\begin{array}{c}00 / 00 / 00 \\
00: 00\end{array}$ & $\begin{array}{c}10 / 20 / 93 \\
09: 45\end{array}$ & $\begin{array}{l}614 \\
598 \\
610 \\
666 \\
597\end{array}$ & $\begin{array}{l}30 \\
34 \\
32 \\
38 \\
30\end{array}$ & 6.0 \\
\hline Calib 4 & 3050 & $\begin{array}{c}04 / 23 / 93 \\
13: 00\end{array}$ & $\begin{array}{c}00 / 00 / 00 \\
00: 00\end{array}$ & $\begin{array}{c}00 / 00 / 00 \\
00: 00\end{array}$ & $\begin{array}{c}10 / 20 / 93 \\
09: 45\end{array}$ & $\begin{array}{l}771 \\
798 \\
791 \\
777 \\
796\end{array}$ & $\begin{array}{l}28 \\
31 \\
32 \\
32 \\
29\end{array}$ & 9.0 \\
\hline
\end{tabular}




\section{APPENDIX A (Cont'd)}

\section{SAMPLE OUTPUT FILE}

Environmental TLD Measurements (with statistical uncertainty at $1 \sigma$ )

\begin{tabular}{crrrrrr}
\hline SITE & \multicolumn{2}{c}{ DEPLOYMENT } & NETmR & EXPOSURERATE $\mu R / h$ & FIELD h \\
Shrm & $2-26-93$ & $4-27-93$ & 9.12 & $6.37 \pm .25(3.9 \%)$ & 1431.70 \\
Roof & $3-01-93$ & $5-25-93$ & 14.53 & $7.13 \pm .22(3.1 \%)$ & 2037.42 \\
Chstr & $2-23-93$ & $5-19-93$ & 24.68 & $12.11 \pm .29(2.4 \%)$ & 2037.25 \\
Sphr & $3-1-93$ & $5-25-93$ & 6.73 & $3.30 \pm .17(5.2 \%)$ & 2037.42
\end{tabular}

Stor. Corr. Factor: $.0448 \pm .00150 \mathrm{cts} / \mathrm{h}(3.4 \%)$

Ave. Calibration Factor: $25.028 \pm .428 \mathrm{cts} / \mathrm{mR}$ (1.7\%)

Lead Shield Exp. Rate: $1.789 \pm .067 \mathrm{micro}-\mathrm{R} / \mathrm{h}(3.8 \%)$

$\begin{array}{lrrrrrrrr}\text { SITE } & \text { SN } & \text { Net Cts } & \text { Store h } & \text { Cor. Cts } & \text { err } & \text { \% err } & \text { sd } & \text { \%sd } \\ \text { Shrm } & 3140 & 422.60 & 4342.67 & 228.19 & \pm 8.0 & (1.1 \%) & 10.5 & 4.58 \\ \text { Roof } & 3100 & 531.00 & 3736.95 & 363.71 & \pm 9.5 & (1.5 \%) & 17.3 & 4.75 \\ \text { Chstr } & 3110 & 784.90 & 3737.12 & 617.60 & \pm 10.0 & (1.1 \%) & 26.3 & 4.26 \\ \text { Sphr } & 3130 & 335.80 & 3736.95 & 168.51 & \pm 8.3 & (1.8 \%) & 13.6 & 8.08 \\ \text { Stor Ctl } & 3200 & 255.40 & 5774.34 & 255.40 & \pm 3.4 & (1.3 \%) & 7.5 & 2.94 \\ \text { Stor Ctl } & 3190 & 261.60 & 5774.34 & 261.60 & \pm 17.0 & (6.5 \%) & 38.0 & 14.53 \\ \text { Calib 1 } & 3150 & 482.40 & 5774.34 & 223.90 & \pm 11.2 & (1.5 \%) & 15.9 & 7.12 \\ \text { Calib 2 } & 3160 & 645.00 & 5774.34 & 386.50 & \pm 12.5 & (1.4 \%) & 20.0 & 5.17 \\ \text { Calib 3 } & 3000 & 614.20 & 4316.72 & 420.95 & \pm 13.2 & (1.9 \%) & 25.8 & 6.13 \\ \text { Calib 4 } & 3050 & 782.20 & 4316.72 & 588.95 & \pm 8.8 & (0.8 \%) & 13.3 & 2.26\end{array}$


APPENDIX A (Cont'd)

SAMPLE OUTPUT FILE continued

..........CALIBRATION INFORMATION..........

$\begin{array}{cccccc}\text { Name } & \text { SN } & \text { Expos mR } & \text { cts/mR } & \text { err } & \text { \%err } \\ \text { Calib 1 } & 3150 & 9.229 & 24.260 & \pm 1.216 & (5.0 \%) \\ \text { Calib 2 } & 3160 & 15.821 & 24.429 & \pm 0.787 & (3.2 \%) \\ \text { Calib 3 } & 3000 & 15.821 & 26.607 & \pm 0.836 & (3.1 \%) \\ \text { Calib 4 } & 3050 & 23.732 & 24.817 & \pm 0.371 & (1.5 \%)\end{array}$

Fit to line:

$$
\begin{aligned}
\text { slope } & =25.084(\mathrm{cts} / \mathrm{mR}) \\
\mathrm{y} \text { intcpt } & =-0.05930 \\
\text { morm } & =28.031 \\
\text { ierr } & =0
\end{aligned}
$$

The following packets had outlier data rejected:

(none) 


\title{
3.6 BONNER-SPHERE NEUTRON SPECTROMETRY
}

\author{
Contact Person(s) : Paul Goldhagen
}

\subsubsection{SCOPE}

Described here are EML's moderated multisphere neutron spectrometers, also known as Bonner sphere neutron spectrometers, and the general principles of their use in neutron spectrometry. These spectrometers are used to measure neutron fields:

1. That result from cosmic-ray interactions in the atmosphere (Hajnal et al., 1971; Nakamura et al., 1987);

2. In the containment of pressurized nuclear reactors (Hajnal et al., 1979; Nakamura et al., 1984; Sanna et al., 1980);

3. Around unmoderated (fast) reactors (Griffith et al., 1984; Hoots and Wadsworth, 1984).

4. In plutonium facilities (Harvey and Hajnal, 1993).

5. Fusion test reactors (Kugel et al., 1994a,b).

\subsubsection{GENERAL DESCRIPTION}

The primary Bonner sphere spectrometer system consists of 12 moderated polyethylene spheres equipped with boron-trifluoride proportional counters. The spectrometers are usable either in parallel or in the independent mode. In the parallel mode, all the spheres are exposed simultaneously to the neutron field, while in the independent mode they are exposed sequentially. Sufficient detail is given here for the basic methodology; the references cited should be consulted for additional information (Aldrich et al., 1981; Awschalom and Sanna, 1985; Cross and Ing, 1987). 
At EML, neutron spectrometry is carried out on an occasional basis, usually to answer specific research requirements or questions. Consequently, there are not rigidly defined procedures. Considerable expertise and judgement are required to perform Bonner sphere spectrometry, and this section should be considered as a guide rather than a specific procedure.

\subsubsection{PERSONNEL AND TRAINING}

The procedures described in this section should be conducted and/or supervised by an experienced physicist or nuclear engineer and that individual should be thoroughly familiar with the content of the references cited in this section.

\subsubsection{METHODOLOGY}

3.6.4.1

DESCRIPTION OF THE SYSTEM

Bonner sphere spectrometers have been used extensively in radiation protection practices to determine neutron spectral distributions around particle accelerators, nuclear power stations and other nuclear facilities, as well as in cosmic-ray neutron research for over two decades. The Bonner sphere spectrometer system is very useful since it is simple, portable, has an isotropic response, covers a wide energy range, and the data can be unfolded and interpreted fairly easily (Bramblett et al., 1960).

One drawback of this method is the low energy resolution. This is partially due to the fact that the statistical fluctuations in the number of collisions in the neutron slowing down processes are large, and the capture reactions are completely indistinguishable from one another. This results in loss of information about the primary neutron energy and, consequently, low resolution. 
The 12 Bonner spheres are: one bare and one $0.075-\mathrm{cm} \mathrm{Cd}$ covered $5.08-\mathrm{cm}$ diameter boron-trifluoride spherical proportional counter, and 10 spherical polyethylene moderators of 7.62-cm, $7.85-\mathrm{cm}, 10.01-\mathrm{cm}, 10.24-\mathrm{cm}, 12.75-\mathrm{cm}, 14.94-\mathrm{cm}, 15.24-\mathrm{cm}, 19.96-$ $\mathrm{cm}, 25.04-\mathrm{cm}$ and $30.07-\mathrm{cm}$ diameters, respectively, with spherical proportional counters placed at the centers.

3.6.4.3

THE RESPONSE FUNCTIONS

The Bonner sphere spectrometer response functions must be calculated. The calculations should be performed using different numerical methods (such as those incorporated in the ANISN and MORSE codes), different evaluated neutron cross section sets, and different energy bining. The response functions of the boron-trifluoride counter equipped Bonner spheres are calculated using DTF-IV and by ANISN transport codes in the adjoint mode. The calculated detector response functions are considered sufficiently accurate if the results obtained using different cross section sets and transport codes differ by only a few percent for the entire energy region (Burgart and Emett, 1972; Maerker et al., 1971).

\subsubsection{4}

\section{CALIBRATIONS}

The Bonner sphere spectrometer calibrations, whenever possible, should be performed with the National Institute of Standards and Technology (NIST) monoenergetic neutron beams, and the normalization of the overall response functions should be performed using ${ }^{252} \mathrm{Cf}$ spontaneous fission neutron sources. At EML, the ${ }^{252} \mathrm{Cf}$ calibrations are performed in an open air calibration facility, where the air and ground scattering contribution in the worst case amounts to only $3 \%$ at $1 \mathrm{~m}$ source-to-detector separation (Hajnal et al., 1970; Hunt, 1984a,b). 
Since the boron-trifluoride counters are not sensitive to $B$ or $\gamma$ radiation, a simple electronic setup is sufficient: a proportional counter is connected to a preamplifier and the signal is fed to a linear amplifier, followed by a discriminator and a scaler to record the number of capture reactions. The scaler readouts can be recorded on tape, or in an appropriate logbook.

\subsubsection{NEUTRON SPECTRUM UNFOLDING}

The result of a set of measurements with Bonner spheres is a set of 12 count rates for the 12 detector configurations. A mathematical method known as unfolding is used to obtain a neutron spectrum from these data. This is accomplished using a computer program entitled TWOGO developed at EML (Hajnal, 1981).

The relative count rates obtained for a set of 12 counts made with the Bonner spheres are a function of the energy distribution of the neutron field. The unfolding code provides a reasonable estimate of neutron fluence rate as a function of energy, i.e., a neutron spectrum (Ing and Makra, 1978). The user of TWOGO provides an estimate of the shape, called trial vector, of the neutron spectrum and the computations performed by TWOGO iteratively adjust the spectrum to fit or be consistent with the data. The computations rely on the use of the data available in the literature regarding the response of the detector in its various configurations, as a function of neutron energy. Estimates of the relative errors of individual counts are used as weighting factors during the iterative fitting process.

Several indices are computed with the TWOGO program that serve to measure the goodness of fit of the unfolding process. These indices are based on determining the degree of agreement between the data obtained by observation. That is, count rates due to neutrons for each of the detector configurations, and synthetic counts or estimates of these same parameters obtained by folding together the response matrix and the spectrum obtained by the unfolding process. 


\subsubsection{ACCEPTABLE SOLUTION}

A solution can be called exact, approximate, or appropriate (Gold, 1964). Exact solutions may have zero errors, and might look reasonable. However, they may have unphysical characteristics, such as oscillations, and the "tail-wags-the-dog syndrome" might appear. Usually, the unfolded data should not be expected to have too good a fit, at least not better than the error of the input data. In general, the trial vectors should contain the features of the neutron spectra one can expect from the physics of the problem. Similarly, the smoothing functions, if any, have to be properly chosen. Appropriate solutions can be obtained from good measurements, and considerable experience is needed to judge just when a reasonable spectral solution is reached.

\section{REFERENCES}

Aldrich, J. M., D. L. Haggard, G. W. R. Endres, J. J. Fix, F. M. Cummings, M. R. Thorson and R. L. Kathren

"Evaluating Existing Radiation Fields"

Pacific Northwest Laboratory Report PNL-3536, Richland, WA (1981)

Awschalom, M. and R. S. Sanna

"Application of Bonner Sphere Detectors in Neutron Field Dosimetry"

Radiation Protection Dosimetry, 10, 89 (1985)

Bramblett, R. L., R. I. Ewing and T. W. Bonner

"A New Type of Neutron Spectrometer"

Nuclear Instruments and Methods, 2, 1 (1960)

Burgart, C. E. and M. B. Emmett

"Monte Carlo Calculations of the Response Functions of Bonner Ball Neutron

Detectors"

Oak Ridge National Laboratory Report ORNL-TM-3739, Oak Ridge, TN (1972) 
Cross, W. G. and I. Ing

"Neutron Spectrometry"

In: The Dosimetry of Ionizing Radiation

Kase, K. R., B. E. Bjarngard and F. A. Attix (Editors)

Academic Press, Vol. II, pp. 91-169, Orlando, FL (1987)

Gold, R.

"An Iterative Unfolding Method for Response Matrices"

USAEC Report ANL-6984, Argonne National Laboratory, Argonne, II (1964)

Griffith, R. V., C. J. Huntzinger, and J. H. Thorngate

"Neutron Spectra as a Function of Angle at Two Meters from the Little Boy

Assembly"

Lawrence Livermore National Laboratory Report UCRL-90178, Livermore, CA (1984)

Hajnal, F.

"An Iterative Nonlinear Unfolding Code: TWOGO"

USDOE Report EML-391 (1981)

Hajnal, F., J. E. McLaughlin and R. Oeschler

"Technique for Determining Moderated-Neutron Instrument Characteristics"

USAEC Report HASL-222 (1970)

Hajnal, F., J. E. McLaughlin, M. S. Weinstein and K. O'Brien

"Sea-Level Cosmic-Ray Neutron Measurements"

USAEC Report HASL-241 (1971)

Hajnal, F., R. S. Sanna, R. M. Ryan and E. H. Donnelly

"Stray Neutron Fields in the Containment of PWRs"

International Atomic Energy Agency Report IAEA-SM-242/24, STU/PUB/527,

Vienna (1979)

Harvey, W. F. and F. Hajnal

"Multisphere Neutron Spectroscopy Measurements at the Los Alamos National

Laboratory Plutonium Facility"

Radiation Protection Dosimetry, 50, 13-30 (1993) 
Hoots, S. and D. Wadsworth

"Neutron and Gamma Dose and Spectra Measurements on the Little Boy Replica" Lawrence Livermore National Laboratory Report UCRL-90095, Livermore, CA (1984)

Hunt, J. B.

"The Calibration of Neutron Sensitive Spherical Devices in Non-Isotropic Neutron Fields"

Radiation Protection Dosimetry, 9, 105 (1984a)

Hunt, J. B.

"The Calibration of Neutron Sensitive Spherical Devices"

Radiation Protection Dosimetry, $\underline{8}, 239$ (1984b)

Ing, H. and S. Makra

"Compendium of Neutron Spectra in Criticality Accident Dosimetry"

International Atomic Energy Agency Report, IAEA Technical Report Series No. 180, Vienna (1978)

Kugel H. W., G. Ascione, S. Elwood, J. Gilbert, L. P. Ku, J. Levine, K. Rule, N. Azziz, P. Goldhagen, F. Hajnal and P. Schebell

"Measurements of TFTR D-T Radiation Shielding Efficiency"

in: Fusion Engineering and Design, Proceedings of the Third International Symposium of Fusion Nuclear Technology, Los Angeles, CA., September (1994a)

Kugel, H. W., J. Gilbert, D. Hwang, M. Lewis, J. Levine, L. P. Ku, K. Rule, F. Hajnal, N. Azziz, P. Goldhagen, G. Klemic and P. Shebell

"TFTR Radiation Cont ir and Shielding Efficiency Measurements During D-D Operations"

in: Fusion Technology, Proceedings of the Eleventh Topical Meeting on the Technology of Fusion Energy, American Nuclear Society, September (1994b)

Maerker, R. E., L. R. Williams, F. R. Mynatt and N. M. Greene

"Response Functions for Bonner Ball Neutron Detectors"

Oak Ridge National Laboratory Report ORNL-TM-3451, Oak Ridge, TN (1971) 
Nakamura, T., T. Kosako and S. Iwai

"Environmental Neutron Measurements Around Nuclear Facilities with Moderated-

Type Neutron Detector"

Health Physics, 47, 729 (1984)

Nakamura, T., Y. Uwamino, T. Ohkubo and H. Hara

"Altitude Variation of Cosmic Ray Neutrons"

Health Physics, 3, 509 (1987)

Sanna, R. S., F. Hajnal, J. E. McLaughlin, J. F. Gulbin and R. M. Ryan

"Neutron Measurements Inside PWR Containments"

USDOE Report EML-379 (1980) 


\section{ANALYTICAL CHEMISTRY}

U.S. Department of Energy 201 Varick Street, 5th Floor New York, NY 10014-4811 
Page

\section{Analytical Chemistry $\ldots \ldots \ldots \ldots \ldots . . . .1-1$}

\subsection{Overview .......................... 4.1}

\subsection{Atmospheric Tracer Technology $\ldots \ldots \ldots \ldots \ldots$. $4.2-1$}

4.2.1 Scope $\ldots \ldots \ldots \ldots \ldots \ldots \ldots \ldots \ldots \ldots \ldots \ldots \ldots \ldots .4 .2-1$

Procedure:

ATT-01 Gas Chromatographic Determination of

Perfluorocarbon Tracer

4.3.1 Scope

4.3.2 Elemental Analyses

Procedures:

Anions-01-E Anions - Ion Chromatography (see Volume II)

Ca-01-E Calcium - Atomic Absorption Spectrometry (see Volume II)

Ca-02-E Calcium - Permanganate Titration of the Oxalate (see Volume II)

F-01-E Fluoride in Soil and Sediment - Specific Ion Electrode

Measurements (see Volume II)

Hg-01-E Mercury - Atomic Absorption Spectrometry (see Volume II)

$\mathrm{NH}_{3}^{-}-01-\mathrm{E}$ Ammonium in Water and Precipitation Samples Autocolorimetry (see Volume II)

Sr-01-E Strontium - Atomic Absorption Spectrometry (see Volume II)

U-01-E Uranium in Urine - Fluorimetry (see Volume II)

4.3.3 Multielemental Analyses $\ldots \ldots \ldots \ldots \ldots \ldots \ldots \ldots \ldots . . \ldots \ldots .3 . \ldots \ldots$

Procedures:

M-01 Cadmium and Lead in Human Excreta and

Composite Diet Samples - Atomic Absorption

Spectrometry (see Volume II) 
M-02 Precipitation and Lake Water Samples - Physical and Chemical Measurements (see Volume II)

M-03 Trace Metals - Atomic Absorption and/or Emission Spectrometry (see Volume II)

4.4.1 Scope

Procedure:

OR-01 PAH and PCDD/Fs in Sediment - GC//MS

4.5.1 Scope

4.5.2 Radiometrology

Procedures:

A-01-R Alpha Radioassay

B-01-R Beta Radioassay

Ga-01-R Gamma Radioassay

4.5.3 Quality Control and Detection Limits

4.5.4 Radiochemical

Procedures:

Am-01-RC Americium in Soil

Am-02-RC Americium-241 in Soil - Gamma Spectrometry

Am-03-RC Americium in Water, Air Filters, and Tissue (see Volume II)

Am-04-RC Americium in QAP Water and Air Filters - Eichrom's TRU Resin

Am-05-RC Americium in Water and Air Filters (see Volume II)

Am-06-RC Americium and/or Plutonium in Vegetation

Fe-01-RC Iron in Aqueous Samples - Dual-DPM Mode Liquid Scintillation Analysis

${ }^{3} \mathrm{H}-01-\mathrm{RC}$ Tritium in Water - Acid Electrolysis (see Volume II)

${ }^{3} \mathrm{H}-02-\mathrm{RC}$ Tritium in Water - Alkaline Electrolysis (see Volume II) 
${ }^{3} \mathrm{H}-03-\mathrm{RC}$ Organically Labeled Tritium - Combustion Procedure (see Volume II)

${ }^{3} \mathrm{H}-04-\mathrm{RC}$ Tritium in Water - Liquid Scintillation Counting

Pb-01-RC Lead-210 in Bone, Food, Urine, Feces, Blood, Air, and Water

$\mathrm{Pb}-02-\mathrm{RC}$ Determination of ${ }^{210} \mathrm{~Pb}$ in Bone Ash by Measurement of ${ }^{210} \mathrm{Po}$

Po-01-RC Polonium in Water and Urine

Po-02-RC Polonium in Water, Vegetation, Soil, and Air Filters

$\mathrm{Pu}-01-\mathrm{RC}$ Plutonium in Air Filters

$\mathrm{Pu}$-02-RC Plutonium in Soil Samples (see Volume II)

$\mathrm{Pu}-03-\mathrm{RC}$ Plutonium in Soil Residue - Total Dissolution Method

$\mathrm{Pu}-04-\mathrm{RC}$ Plutonium in Tissue (see Volume II)

$\mathrm{Pu}-05-\mathrm{RC}$ Plutonium in Tissue - Solvent Extraction (see Volume II)

$\mathrm{Pu}$-06-RC Plutonium in Urine (see Volume In)

$\mathrm{Pu}-07-\mathrm{RC}$ Plutonium in Large Urine Samples (see Volume II)

$\mathrm{Pu}$-08-RC Plutonium in Vegetation and Tissue - Nitric/Hydrochloric Acid Method (see Volume II)

$\mathrm{Pu}-09-\mathrm{RC}$ Plutonium in Vegetation and Tissue - Nitric/Sulfuric Acid Method (see Volume II)

$\mathrm{Pu}-10-\mathrm{RC}$ Plutonium in Water

$\mathrm{Pu}-11-\mathrm{RC}$ Plutonium Purification - Ion Exchange Technique

$\mathrm{Pu}-12-\mathrm{RC}$ Plutonium and/or Americium in Soil or Sediments

Note: For Plutonium in Vegetation see Am-06-RC.

Ra-01-RC Radium-226 in Bone Ash

Ra-02-RC Radium-226 - Emanation Procedure

Ra-03-RC Radium-226 in Soil, Vegetation Ash, and Ion Exchange Resin

Ra-04-RC Radium-226 in Tap Water, Urine, and Feces

Ra-05-RC Radium-224 in Urine (see Volume II)

Ra-06-RC Radium-226 in Urine and Water

Rn-01-RC Radon-222 in Air and Breath Samples

Sr-01-RC Strontium-89 (see Volume II) 
Sr-02-RC Strontium-90 (see Volume II)

Sr-03-RC Strontium-90 in Environmental Matrices

Sr-04-RC Strontium-90 in Water Containing Other Radioisotopes by Cerenkov Counting

Tc-01-RC Technetium-99 in Water and Vegetation (see Volume II)

Th-01-RC Thorium in Urine (see Volume II)

U-01-RC Enriched Uranium in Urine (see Volume II)

U-02-RC Isotopic Uranium in Biological and Environmental Materials

U-03-RC Isotopic Uranium in Bone Ash

U-04-RC Uranium in Biological and Environmental Materials (see Volume II)

4.5.5 Generic ............................ 4.5-5

Procedures:

G-01 Electrodeposition of the Actinides: Mitchell Method

G-02 Electrodeposition of the Actinides: Talvitie Method

G-03 Microprecipitation Source Preparation for Alpha Spectrometry

4.5.6 Sequential Analyses . . . . . . . . . . . .

Procedures:

Se-01 Isotopic Uranium and Radium-226

Se-02 Isotopic Uranium, Isotopic Thorium and Radium-226

Se-03 Americium, Plutonium and Uranium in Water 


\section{ANALYTICAL CHEMISTRY}

\subsection{OVERVIEW}

During the past 40 years, EML has developed analytical procedures for the determination of specific gases, inorganic and organic constituents, and radionuclides. The procedures in this section are subdivided into three general categories: gases, , organics, and radionuclides. All of the procedures have been written in a detailed manner to provide the user with sufficient information to obtain a quantitative result which is accurate, precise, free from chemical interference and contamination with specified detection capabilities. These procedures are presently used at EML to obtain data for ongoing and future research programs.

The procedures described in the gases subsection have been used primarily for the Laboratory's atmospheric tracer program. These procedures are highly specific for this application, with detection limits on the order of $10^{-15}$.

The procedures described in the organic subsection have been applied primarily to the analysis of certain organic compounds in sediment or soil. Primary emphasis has been the determination of polyaromatic hydrocarbons (PAHs) at low concentrations for pollutant history studies conducted at EML. Separation, purification, and concentration procedures are fully described, including expected analyte recovery for these matrices and sample sizes.

The last subsection is devoted to radionuclide measurements and radiochemical procedures. The radionuclide measurement techniques are described for alpha, beta, and gamma detection types of equipment. Information about background corrections, efficiency determination, and quality control methods and limits of detection are also included. The radiochemical procedures described are designed for various environmental matrices. The sample preparation portion introduces the procedure so that representative, homogeneous, and equilibrated samples are obtained. Next, separation and purification techniques are described to obtain a radiochemically pure sample. 
Measurement techniques, including limits of detection, quality control or special procedural precautions are also included.

Further information about these procedures can be obtained from the individual listed as the principal contact person. 


\subsection{ATMOSPHERIC TRACER TECHNOLOGY (ATT)}

\subsubsection{SCOPE}

For over a decade EML has participated in a number of atmospheric tracer experiments designed to provide dynamic modelers with large data bases to verify, modify, or develop computer simulations of atmospheric transport and diffusion of energy-related pollutants over long distances. The tracer technology involves the release of perfluorocarbon tracers at controlled and known rates from a source(s) into the atmosphere under well-documented meteorological conditions, and measuring the tracers at very low concentrations at various distances from the release point(s).

We will present here the analytical procedure for the determination of the perfluorocarbon tracers (PFTs), perfluoromethylcyclopentane (PMCP), perfluoromethylcyclohexane (PMCH), ortho(cis)dimethylcyclohexane (OC-PDCH), perfluorodimethylcyclohexane isomer (PDCH), and perfluorotrimethylcyclohexane (PTCH). The PFTs are quantitatively determined by gas chromatography/electron capture detection. 


\title{
GAS CHROMATOGRAPHIC DETERMINATION OF PERFLUOROCARBON TRACERS
}

Contact Person: Raymond J. Lagomarsino

\begin{abstract}
APPLICATION
This procedure is applicable to tracers adsorbed on Ambersorb adsorbent contained in stainless steel tubes in the programmable atmospheric tracer sampler (PATS) lids (described in Section 2.2.4.2).

The procedure (Dietz, 1986) involves the heating of the PATS tubes (described in Section 2.2.4.2) to $400^{\circ} \mathrm{C}$ by resistance heating and desorbing the PFTs and ambient air impurities (halocarbons) into the carrier gas. The compounds pass through a temperature programmed precut column (heated by resistance heating) where the high boiling point impurities are retained. The low boiling point impurities and the PFTs are partially separated by the precut column. Further decontamination of the lighter impurities is accomplished by catalytic reduction using a first stage palladium catalyst maintained at $200^{\circ} \mathrm{C}$ and by venting the impurities to the atmosphere by proper timing of the carrier gas switching valves. A trap containing Florisil (FL) adsorbent is opened and the partially purified PFTs are adsorbed. Subsequent rapid resistance heating of the trap to $200^{\circ} \mathrm{C}$ injects the PFTs into the carrier gas of the main chromatographic column system. The desorbed tracers are further purified by passage through two palladium catalyst beds maintained at $200^{\circ} \mathrm{C}$, and a Nafion permeation dryer. The purpose of the dryer is to remove moisture and halogenated acids produced by the catalytic reduction. The PFTs are then chromatographically separated by the main column and detected by the electron capture sensor. Details of the pneumatics configuration are shown in Figure 1.
\end{abstract}




\section{SPECIAL GASES}

1. $5 \%$ ultra high purity (UHP) $(99.999 \%)$ hydrogen in UHP $(99.999 \%) \mathrm{N}_{2}$ carrier gas.

2. Compressed air to operate the valve actuators.

3. UHP (99.999\%) $\mathrm{N}_{2}$.

4. Primary calibration standards consisting of a mixture of the four tracers: perfluoromethylcyclopentane, perfluoromethylcyclohexane, ortho-(cis) perfluorodimethylcyclohexane (with isomers), and perfluorotrimethylcyclohexane.
a. $1.0 \mu \mathrm{L} \mathrm{L}^{-1}$ of each tracer in $\operatorname{UHP}(99.999 \%) \mathrm{N}_{2}$.
b. $10 \mathrm{~nL} \mathrm{~L}^{-1}$ of each tracer in UHP $(99.999 \%) \mathrm{N}_{2}$.
c. $0.1 \mathrm{~nL} \mathrm{~L}^{-1}$ of each tracer in UHP $(99.999 \%) \mathrm{N}_{2}$.
d. $1.0 \mathrm{pL} \mathrm{L}^{-1}$ of each tracer in UHP $(99.999 \%) \mathrm{N}_{2}$.

\section{SPECIAL APPARATUS}

1. Gas chromatograph, modified Varian Vista 6000 or equivalent with Ni-63 electron capture detector and $1 \mathrm{mV}$ recorder or data acquisition system.

a. Main column

b. Precut column

c. Florisil trap

d. Catalyst

e. Carrier gas purifiers
$15 \mathrm{~cm} \times 3.2 \mathrm{~mm}$ : stainless steel packed with $0.1 \%$ SP-1000 on Carbopack C, 80/100 mesh.

$53 \mathrm{~cm} \times 3.2 \mathrm{~mm}$ thin walled stainless steel tubing packed with Unibeads 2 S, 80/100 mesh.

$11.5 \mathrm{~cm} \times 1.6 \mathrm{~mm}$ thin walled stainless steel tubing packed with FL, 60/100 mesh.

$1 \%$ palladium on polyethylenimine $/ \mathrm{SiO}_{2}$ (Royer Pd Catalyst), 20-40 mesh beads.

activated charcoal, $13 \mathrm{X}$ molecular sieve and $\mathrm{O}_{2}$ traps. 
2. PATS, power control module (base).

3. Matheson Model 3800 gas regulators or equivalent.

4. Power Mate Corporation Model BPA 2086-V constant current power supply or equivalent.

5. Primeline (Soltec) two channel strip chart recorder or equivalent to monitor the PATS adsorbent tube desorption current and FL trap desorption temperature.

A. Gas chromatograph operating conditions.

Carrier gas flow

Catalyst temperature

Column temperature

Detector temperature

Compressed air

Nitrogen main column system $-22 \mathrm{~cm}^{3} \mathrm{~min}^{-1}$

precut column system $-15 \mathrm{~cm}^{3} \mathrm{~min}^{-1}$

$200^{\circ} \mathrm{C}$ (Injectors $\mathrm{A}$ and $\mathrm{B}$ )

main column $-160^{\circ} \mathrm{C}$

precut column - temperature programmed $-80^{\circ} \mathrm{C}$

to $160^{\circ} \mathrm{C}$ at $160^{\circ} \mathrm{min}^{-1}$

$200^{\circ} \mathrm{C}$

set at $276 \mathrm{kPa}$ (40 psig) to actuate switching valves

set at $207 \mathrm{kPa}$ (30 psig) for PATS lid Scanivalve equalization pressure 
B. Gas chromatograph relay assignments.

Relay No.

1

2

3

4

5

6

7

8
Assignment

V1, sample valve (SV)

V2, precut valve (PCV) for changing the direction of the precut column (PCC) carrier gas flow.

V3, flow direction valve (FDV) for loading FL trap and purging catalyst $B$.

V4, FL trap valve (FTV).

Desorption power - High heat.

Desorption power - Low heat.

PATS tube/FL trap power routing relay.

Auxiliary power to precut column (PCC) for High temperature. Relay Off for Low temperature. 


\section{Timed relay automation programming.}

\begin{tabular}{|c|c|c|c|c|c|c|}
\hline $\begin{array}{l}\text { Method } \\
\text { No. }\end{array}$ & $\begin{array}{l}\text { Method } \\
\text { time } \\
\text { (min) }\end{array}$ & $\begin{array}{c}\text { Analysis } \\
\text { integrated } \\
\text { time (min) } \\
\end{array}$ & $\begin{array}{l}\text { Relay } \\
\underline{\mathbf{O n}}\end{array}$ & $\begin{array}{l}\text { Relay } \\
\underline{\text { Off }}\end{array}$ & $\begin{array}{c}\text { Aux. temp. }\left({ }^{\circ} \mathrm{C}\right) \\
\text { precut } \\
\text { column) }\end{array}$ & $\underline{\text { Remarks }}$ \\
\hline \multirow[t]{8}{*}{1} & .00 & .00 & & & 85 & \\
\hline & .01 & .01 & 3,7 & 8 & & $\begin{array}{l}\text { V3 (FDV) On, FL Trap } \\
\text { relay On. Auxiliary power } \\
\text { to PCC Off. }\end{array}$ \\
\hline & .02 & .02 & 4 & & & $\begin{array}{l}\text { V4 (FTV) On (FL Trap } \\
\text { Open) }\end{array}$ \\
\hline & .05 & .05 & 5 & & & High heat On FL Trap \\
\hline & .08 & .08 & 6 & 5 & & $\begin{array}{l}\text { Low heat On FL Trap, High } \\
\text { heat Off }\end{array}$ \\
\hline & .35 & .35 & 4,6 & & & $\begin{array}{l}\text { V4 (FTV), (FL Trap Off), } \\
\text { heat Off }\end{array}$ \\
\hline & .40 & .40 & & 7 & & FL Trap relay Off \\
\hline & 3.00 & 3.00 & & & & Total run time \\
\hline \multirow[t]{4}{*}{3} & .00 & 3.00 & & & 80 & \\
\hline & .01 & 3.01 & 2 & & & $\begin{array}{l}\text { V2 (PCV) On, PCC low } \\
\text { Forward }\end{array}$ \\
\hline & .10 & 3.10 & 1,5 & & & V1 (SV) On, High heat On \\
\hline & 2.00 & 5.00 & & & & Total nun time \\
\hline \multirow[t]{7}{*}{4} & .00 & 5.00 & 8 & & 160 & Auxiliary Power to PCC On \\
\hline & .60 & 5.60 & & 3 & & V3 (FDV) Off \\
\hline & .65 & 5.65 & 4 & & & V4 (FTV) (Trap Open) \\
\hline & 1.50 & 6.50 & & 1,5 & & $\begin{array}{l}\text { V1 (SV) OFF, High heat } \\
\text { Off }\end{array}$ \\
\hline & 4.50 & 9.50 & & 4 & & V4 (FTV) (Trap Closed) \\
\hline & 4.60 & 9.60 & & 2 & & $\begin{array}{l}\text { V2 (PCV) Off (precut } \\
\text { Column Back-Flush) }\end{array}$ \\
\hline & 6.20 & 11.20 & & & & Total run time \\
\hline \multirow[t]{2}{*}{5} & .00 & 11.20 & & & 145 & $\begin{array}{l}\text { PCC in Back-Flush, } \\
\text { Catalyst B purge }\end{array}$ \\
\hline & .60 & 11.80 & & & & Total run time \\
\hline
\end{tabular}




\section{Setting the gas chromatograph.}

1. Set the $5 \%$ UHP hydrogen in UHP $\mathrm{N}_{2}$ carrier gas pressure regulator to $690 \mathrm{kPa}$ (100 psig).

2. Set the main column pressure to $276 \mathrm{kPa}(40 \mathrm{psig})$ and adjust the main column flow to $22 \mathrm{~cm}^{3} \mathrm{~min}^{-1}$ using a calibrated flowmeter or soap bubble spyrometer. Set the precut column pressure to $104 \mathrm{kPa}$ (15 psig). This pressure should give carrier gas flows of $15 \mathrm{~cm}^{3} \mathrm{~min}^{-1}$ at vents 1,2 , and 4 .

3. Adjust the Nafion permeation dryer purge gas flow rate at vent 3 to $\sim 30 \mathrm{~cm}^{3} \mathrm{~min}^{-1}$.

4. Input all GC operating conditions and the timed relay program for each method as specified in Section 4.2.3.3. Method 2 is not used because it is a permanent gas chromatograph memory default method.

5. Set the constant current power supply to $14-14.25 \mathrm{~A}$.

\section{E. Determination.}

1. Attach the PATS lid containing the samples to the PATS base. Plug in the lid AFM connector into the base receptacle labelled AFM Connector.

2. Set the base sample Duration rotary switches to $0013(13.0 \mathrm{~min})$ and the Sample Quantity (number of tubes) to 24. This sets the switching time between tubes and the number of tubes to be sequentially analyzed. The number of tubes desired is 23 ; however, the analysis sequence is such that the PFTs from a sample are not injected into the main column until Method 1 of the next analysis sequence. Turn the Power Switch of the base to On.

3. Plug the constant current power supply jack into the lid receptacle labelled Power Desorption.

4. Attach the UHP $\mathrm{N}_{2}$ gas line to the lid connector labelled To Equalization and set the tank regulator pressure at $207 \mathrm{kPa}$ (30 psig). Its purpose is to equalize the pressure to 
prevent the lid Scanivalve plates from separating. Pressurize the Scanivalve and manually step through each of the 23 tubes by rapidly pushing the Valve step switch to assure that the Scanivalve plates are properly seated. Step to tube number " 00 " and leave at that tube number.

5. Pull off the lid filter at the connector labelled To SV and attach the polyurethane tubing purge gas outlet from V1 (SV) to this connector. Attach the V1 (SV) polyurethane tubing carrier purge gas inlet line to the lid connector labelled To PCM. This connects the GC carrier gas lines to the lid.

6. Turn on the constant current power supply and the two-channel strip chart recorder.

7. Turn on the compressed air, set at $276 \mathrm{kPa}$ (40 psig), to operate the valve actuators.

8. To start the analysis cycle, push the PATS base Operate switch to Off, bring the Alarm toggle switch from Manual to Multi then back to Manual and finally move the Operate toggle switch to Run. Tube No. 1 will advance into position, the gas chromatograph will start and run through its automated sequence consisting of four methods.

Method 1: The FDV (V3) and FTV (V4) valves are energized and the FL trap containing the PFTs from the previous sample is heated. The desorbed PFTs are injected into the main column system (Figure 2). The precut column is in the backflush mode.

Method 3: SV (V1) and PCV (V2) are energized to place the precut column carrier gas flow into the forward position. Heat is applied to the PATS sample tube to desorb the sample (Figure 3).

Method 4: The desorbed PFTs pass through the precut column, the column is heated to $160^{\circ} \mathrm{C}$ at a rate of $160^{\circ} \mathrm{C} \mathrm{min}$. V3 is placed in standby and V4 is energized to allow effluent from the precut column to flow through the FL trap where the PFTs are adsorbed (Figure 4). V1, V2, and V4 rotate to standby, forcing the precut column carrier gas path into the back-flush mode. 
Method 5: The precut column temperature is lowered to $145^{\circ} \mathrm{C}$ with continued backflush to elute the residual higher boiling components out of vent 1 to the atmosphere (Figure 5).

9. At the end of $13 \mathrm{~min}$, the next sample tube will step into position. This sequence will continued until all 23 sample tubes have been desorbed and analyzed.

10. Record the chromatogram. A typical chromatogram of an ambient air sample containing tracers is shown in Figure 6.

11. Integrate the tracer peaks corresponding to their appropriate retention times. Apply the appropriate response factor corrections obtained from the analysis of working standards prior to the analysis of each batch of samples. Obtain the tracer volumes from the appropriate calibration curves and convert to concentrations using the sample collection volume.

12. The retention times are based upon the start of the 13-min run. The expected retention times are as follow:

Perfluoromethylcyclopentane (PMCP) - $1.47 \mathrm{~min}$

Perfluoromethylcyclohexane (PMCH)

$-2.25 \mathrm{~min}$

Ortho(cis)perfluorodimethylcyclohexane (OC-PDCH)

$-3.60 \mathrm{~min}$

Meta-para perfluorodimethylcyclohexane (MP-PDCH)*

$-4.17 \mathrm{~min}$

Meta(cis)perfluorodimethylcyclohexane (MC-PDCH)

$-4.43 \mathrm{~min}$

Para(trans)perfluorodimethylcyclohexane (PT-PDCH)

- $4.84 \mathrm{~min}$

Perfluorotrimethylcyclohexane (1-PTCH)

$-9.20 \mathrm{~min}$

Perfluorotrimethylcyclohexane (2-PTCH)

- $9.73 \mathrm{~min}$

*Combination of the meta(trans) and para(cis) perfluorodimethylcyclohexane. 


\section{LOWER LIMIT OF DETECTION}

Lower limit of detection (LLD) is defined here as that amount which gives detector response equal to three times the noise level (ACS, 1980). The LLDs are:

Perfluorocarbon

PMCP

$\mathrm{PMCH}$

$\mathrm{OC}-\mathrm{PDCH}$

MP-PDCH

MC-PDCH

PT-PDCH

PTCH

\section{$\underline{\operatorname{LLD}(\mathrm{fL})}$}

2.7

4.0

2.2

2.1

2.0

4.0

3.9

\section{DATA ACQUISITION AND REDUCTION}

\section{A. Computer hardware.}

1. IBM personal computer AT with a minimum of 512 kilobytes system memory, 20 megabyte hard-disk drive, $51 / 4$ inch floppy disk drive, enhanced graphics adapter card, graphics memory expansion card (64K) or equivalent.

2. IBM enhanced color display monitor offering 640 by 350 screen resolution and keyboard or an equivalent monitor and keyboard.

3. Nelson Series 760 intelligent $A / D$ interface box or equivalent.

4. Citizen Model MSP-20 printer or equivalent.

5. Standard IEEE-488 (GPIB) cable.

6. Dysan 5 1/4 inch high-capacity, 96 TPI floppy diskettes or industry equivalent. 


\section{B. Computer software.}

1. Nelson Analytical Corp. Model 2600 chromatography software package. Other software vendors provide equivalent packages.

2. Nelson Analytical Corp. Model 2660 peak summary tables software (optional).

3. Nelson Analytical Corp. Model 2670 batch reprocessing software (optional).

\section{General procedure.}

The electron capture detector (ECD) signal from the Vista 6000 gas chromatograph is monitored in real time by the Nelson intelligent interface offering 20 bit precision. The interface digitizes the detector signal and stores the values in its memory. When the data are available in the interface and the operating program is the 2600 software ACQUIRE on the IBM AT, the data is transmitted to the computer according to the METGEN method. The software detects peaks, determines their baselines, integrates these peaks, and then calculates the component concentrations. Three data files are created for each sample. They are distinguished by their file extensions: .PTS, .HDR, and .ATB. In the .PTS file, raw chromatographic $\mathrm{mV}$ data are saved in binary form, while in the .HDR file, parameters for sample identification pertaining to that raw data are stored in ASCII form. The peak heights and/or peak areas obtained from integration, as determined by the method, are stored in the .ATB file. Baselines different than those specified in the method may be drawn using the software's timed events capability. The values from the reintegration may be overwritten in the ATB file. A schematic of the data acquisition and data reduction system is shown in Figure 7.

\section{CALIBRATION}

\section{A. Standards preparation.}

Presented here is a procedure for the preparation of PFT standards for both instrument calibration and daily working standards. The daily standards are analyzed prior to the analysis of a batch of samples to determine response factor corrections resulting from 
changes in electron-capture sensitivity. This method is applicable to PATS lids (described in Section 2.2.4.2).

\section{B. Special apparatus.}

1. Matheson Model 3800 gas pressure regulator or industry equivalent.

2. Nupro Model SS-4BMG gas flow regulating valve or industry equivalent. Caution: Low concentrations of tracer material could be adsorbed with other type valves.

3. A complete PATS unit consisting of a base (PCM) and a baked-out lid (AFM).

4. Teledyne-Hastings-Raydist Model HBM-1A bubble spyrometer or industry equivalent, NIST traceable.

5. A $1 \mathrm{~A}$ cylinder containing $1 \mathrm{fL} \mathrm{L}^{-1}$ each of $\mathrm{PMCP}, \mathrm{PMCH}, \mathrm{OC}-\mathrm{PDCH}$ and $\mathrm{PTCH}$ in UHP $\mathrm{N}_{2}$.

\section{Sample preparation.}

1. Plug the battery charger into the port labelled Chg.Jack and a jumper in the receptacle labelled Analysis. The purpose of the jumper is to disable the sampling pump. Set the thumbwheel switches to the following settings:

a. Set Time/Alarm: set to the appropriate time of day.

b. Duration: set to the time in minutes desired for each tube to remain in line position.

c. Sample Quantity: set to 24 . The sample quantity ( 23 in this instance) must always be set for one more tube than is physically present on a lid.

d. Day Select: place to the On position for the appropriate day of the week.

2. Plug the lid connector into the base receptacle marked AFM Connector and turn the Power Switch of the base to the On position. 
3. Set the Matheson purity $\mathrm{N}_{2}$ tank pressure to $207 \mathrm{kPa}$ (30 psig) and attach the $3.2 \mathrm{~mm}$ polyurethane tubing line from the cylinder to the equalization inlet port labelled To Equalization on the lid. Open the $\mathrm{N}_{2}$ supply to pressurize the Scanivalve and manually cycle through each of the 23 tubes. This is done by a rapid push of the Valve step switch. Make sure that the LCD display indicates that tube "00" is in line after this is completed.

4. Attach the $3.2 \mathrm{~mm}$ polyurethane tubing from V2 of the standard gas flow control system (Figure 8) to the lid port labelled To SV.

5. Set the pressure of the standard cylinder containing the PFTs to $207 \mathrm{kPa}$ (30 psig). Slowly open the regulator valve (V1) to permit tracer gases to flow into the PATS lid through the Nupro Flow Control Valve (V2).

6. Connect the $3.2 \mathrm{~mm}$ polyurethane tubing from the outlet of the lid labelled To PCM to a calibrated flowmeter and adjust V2 to obtain $50 \mathrm{~cm}^{3} \mathrm{~min}^{-1}$.

7. Remove the calibrated flowmeter and replace with the bubble spyrometer.

8. Using a stopwatch, time a soap bubble to travel from 0 to the $100 \mathrm{~cm}^{3}$ marks. Record the time and convert minutes and seconds to a pure minute time frame.

9. The quotient of 100 and the time is the flow rate of a mixed tracer gas.

10. If the flow rate is $<49.0$ or above $51.0 \mathrm{~cm}^{3} \mathrm{~min}^{-1}$, fine adjust the needle valve to obtain $\sim 50 \mathrm{~cm}^{3} \mathrm{~min}^{-1}$.

11. Repeat Step 8 at least four times. The precision of the average of the four measurements should be within $2 \%$.

12. Disconnect the tubing from the outlet of the lid to the bubble spyrometer.

13. To load daily working standards, set the Duration to $0002(2 \mathrm{~min})$ to load $100 \mathrm{fL}$ of tracers onto each tube. 
14. A signal must be given to inform the PATS logic to start the time sequence that indexes the first tube into position. To start the sequence push the Operate toggle switch to Off, move the Alarm toggle switch from Manual to Multi to Manual and finally push the Operate switch to Run.

15. The first tube will switch into line and $100 \mathrm{~cm}^{3}$ of PFT gas will flow through the tube over a $2-\mathrm{min}$ period. At the end of the $2-\mathrm{min}$ period, the next tube will switch into position. This cycle will continue for 23 tubes. The actual volume of each PFT adsorbed on each tube is calculated by multiplying the average of the four measurements of flow rate obtained from Step 11 by time and by the value of the standard of each tracer affixed to the standard (fL).

$$
\text { Volume of PFT added }=\sum_{i=1}^{\mathfrak{n}} f_{i} / n \times f L \text { standard } \times \text { time }
$$

where $f_{i}$ is the value of the ith flow measurement in $\mathrm{cm}^{3} \mathrm{~min}^{-1}$.

16. After tube "00" appears on the tube LCD, push the Operate switch to Off and shut all cylinder valves.

17. Disconnect all tubing and place a $1 / 2$ hole septum over the lid inlet and outlet ports.

18. Disconnect the lid plug from the base and remove the entire lid from the base.

19. For preparing standards up to $5 \times 10^{6} \mathrm{fl}$, the same loading procedure is followed by using the appropriate standard for the desired range of tracer quantities and setting the flow rate and time. The PATS base time setting may be increased during a run so that by extending the time, increased tracer quantities may be loaded from one adsorbent tube to the next. For constructing a calibration curve, the standards are prepared in triplicate. Data (peak height or area) obtained from the analysis of the standards in the $10^{2}$ to $10^{6} \mathrm{fL}$ range are fitted by a 6 th-order polynomial. A linear fit is applied below $100 \mathrm{fL}$. 


\section{REFERENCES}

Dietz, R. L.

"Preliminary Operating Manual, Brookhaven National Laboratory Addendum to a Modified Varian Model 6000 GC"

Private Communication (1986)

Subcommittee on Environmental Analytical Chemistry, American Chemical Society (ACS), Committee on Environmental Improvement (1980)

"Guidelines for Data Acquisition and Data Quality Evaluation in Environmental

Chemistry"

Anal. Chem., 52, 2242-2248 (1980) 


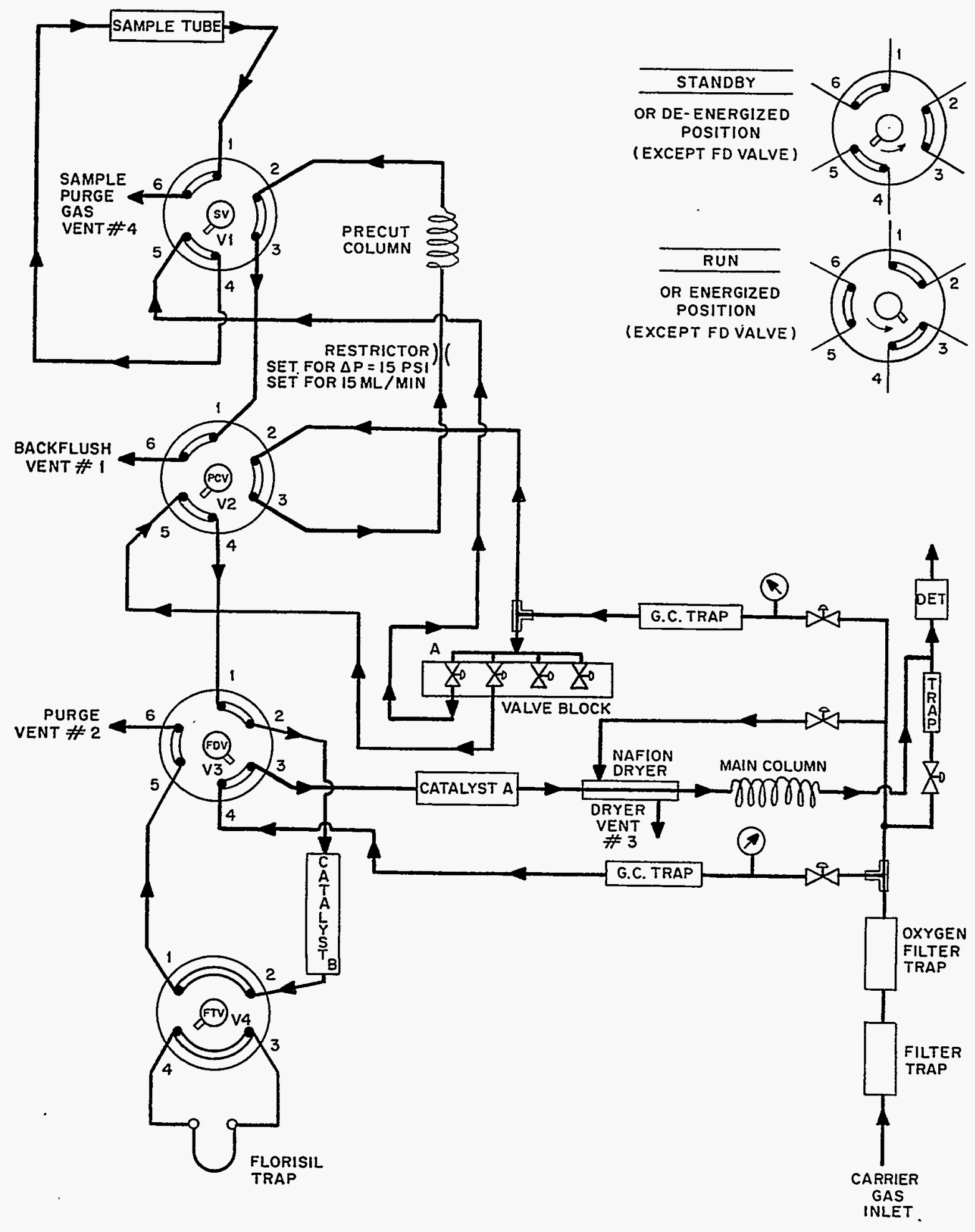

Figure 1. Pneumatics configuration of the perfluorocarbon tracer gas chromatograph. 


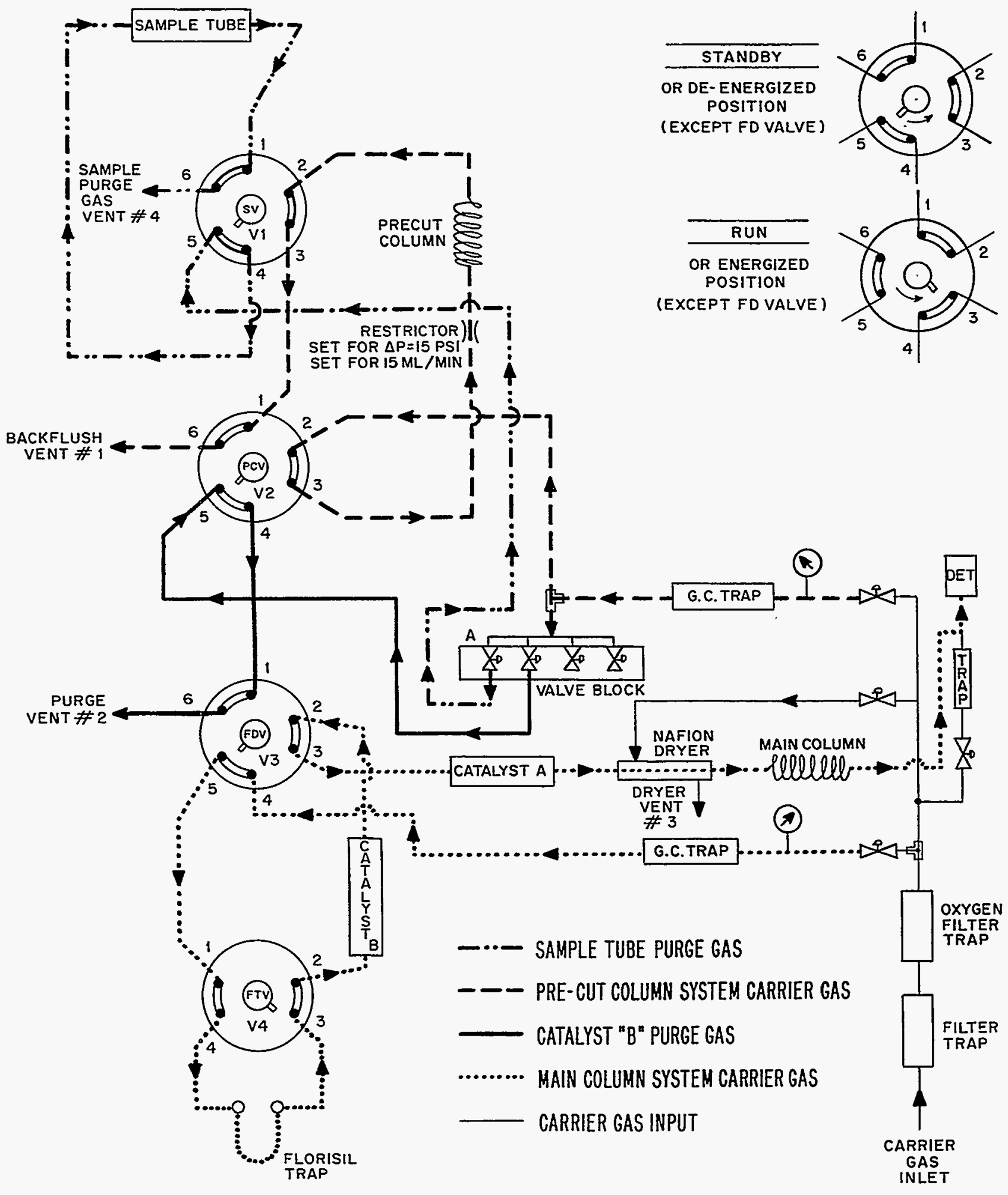

Figure 2. Method 1: carrier gas flow paths - Florisil trap desorption. 


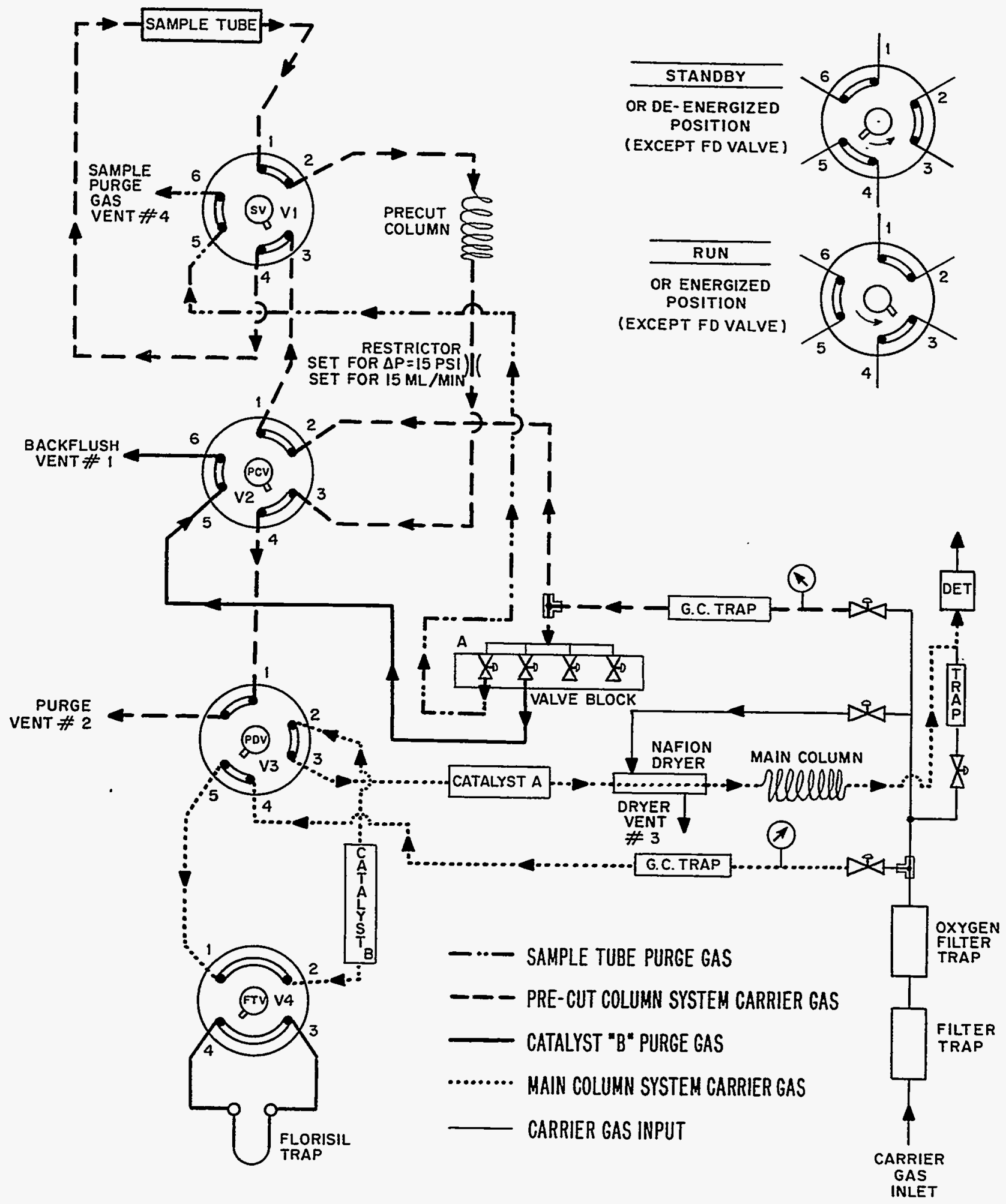

Figure 3. Method 3: carrier gas flow paths - PATS sample desorption. 


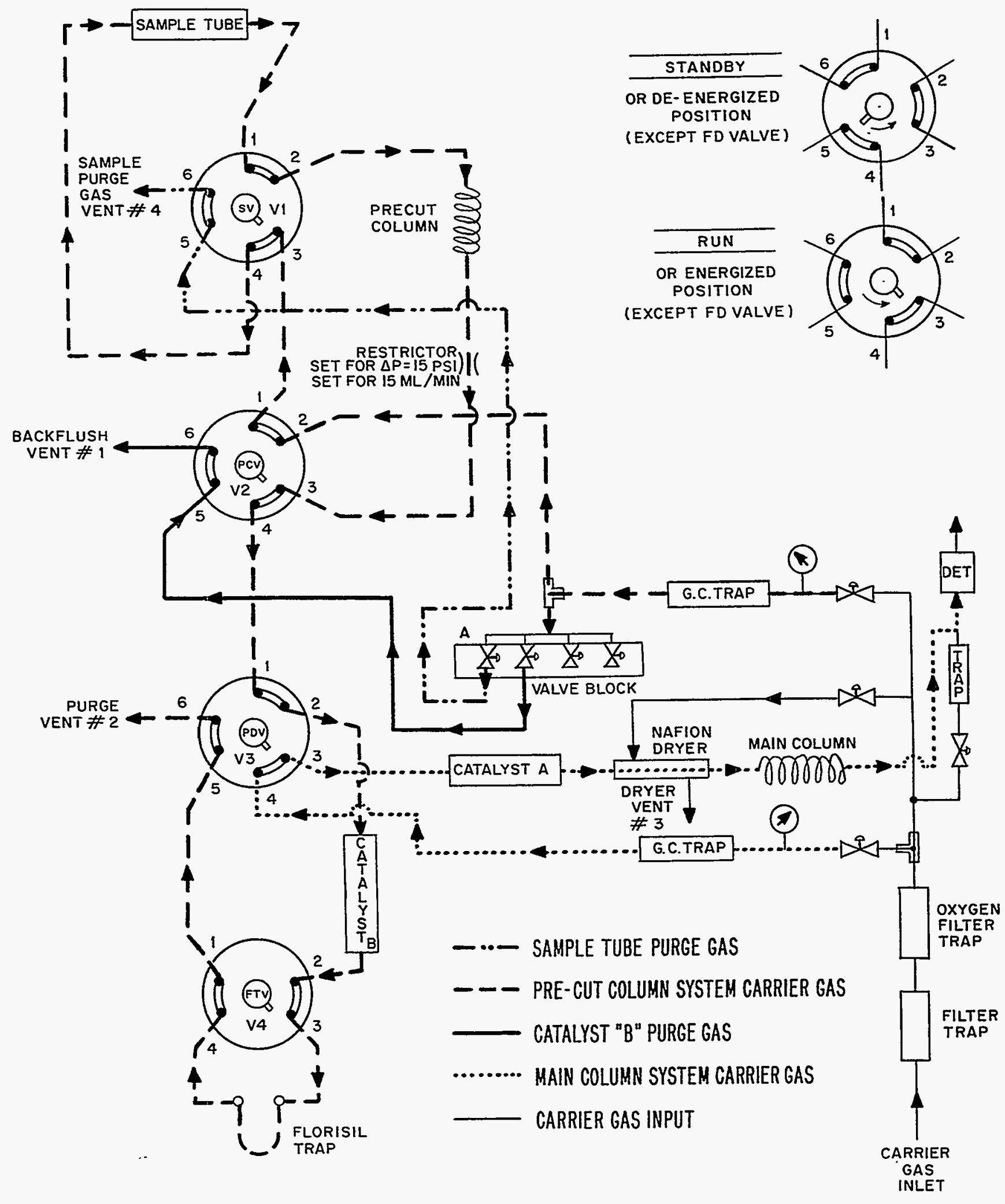

Figure 4. Method 4: carrier gas flow paths - absorption of PFTs on Florisil trap. 


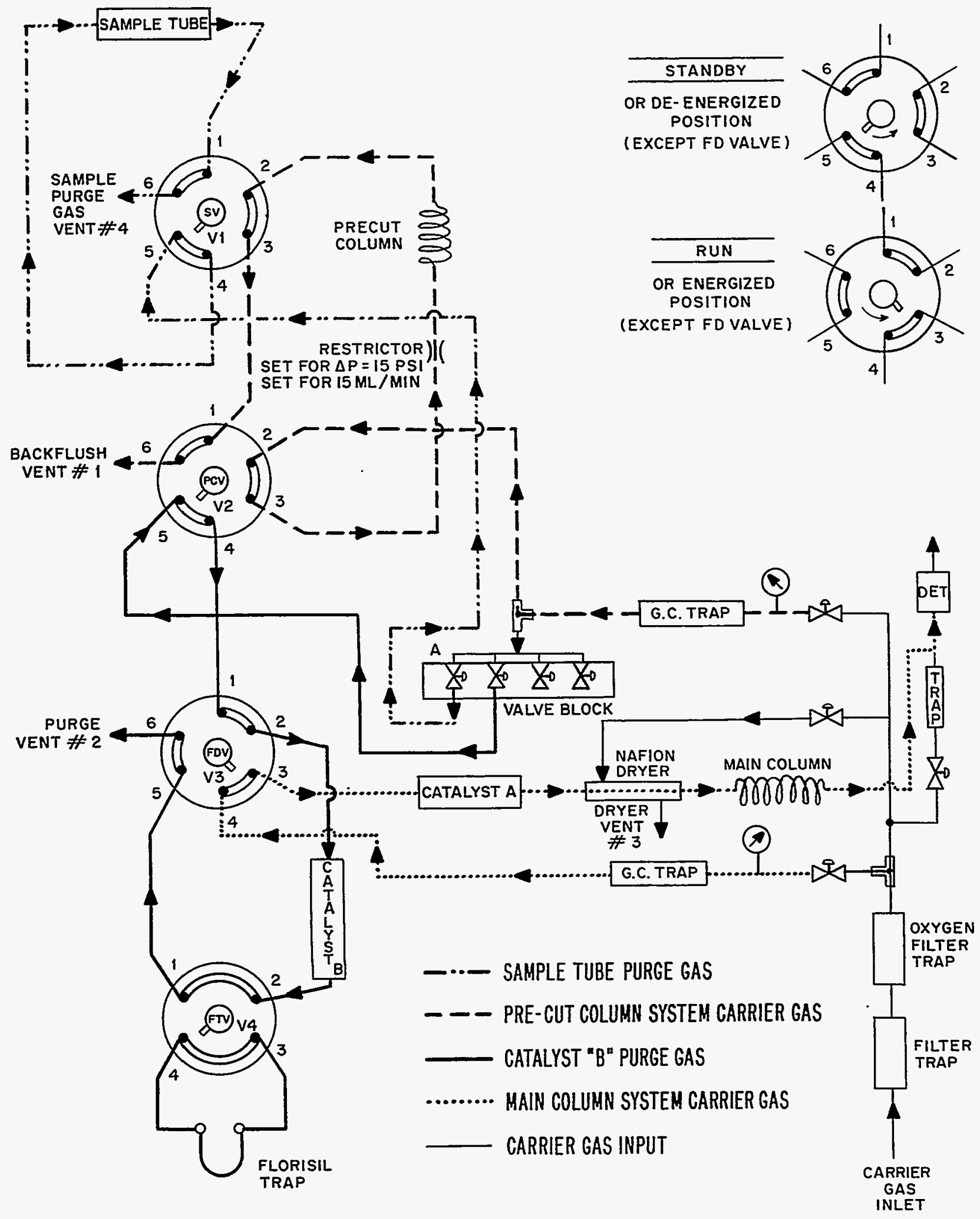

Figure 5. Method 5: carrier gas flow paths - precut column back-flush. 


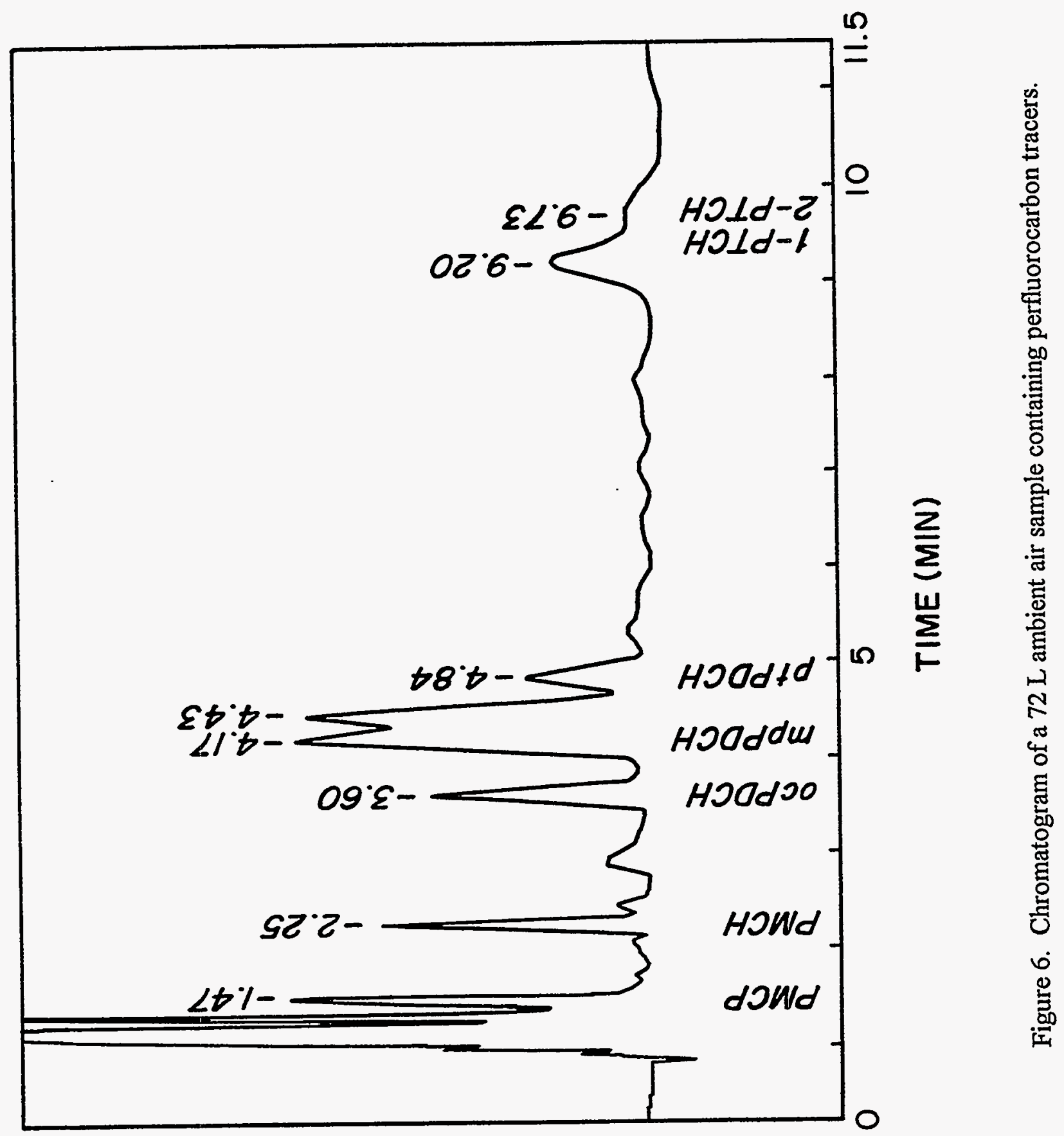

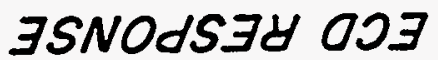




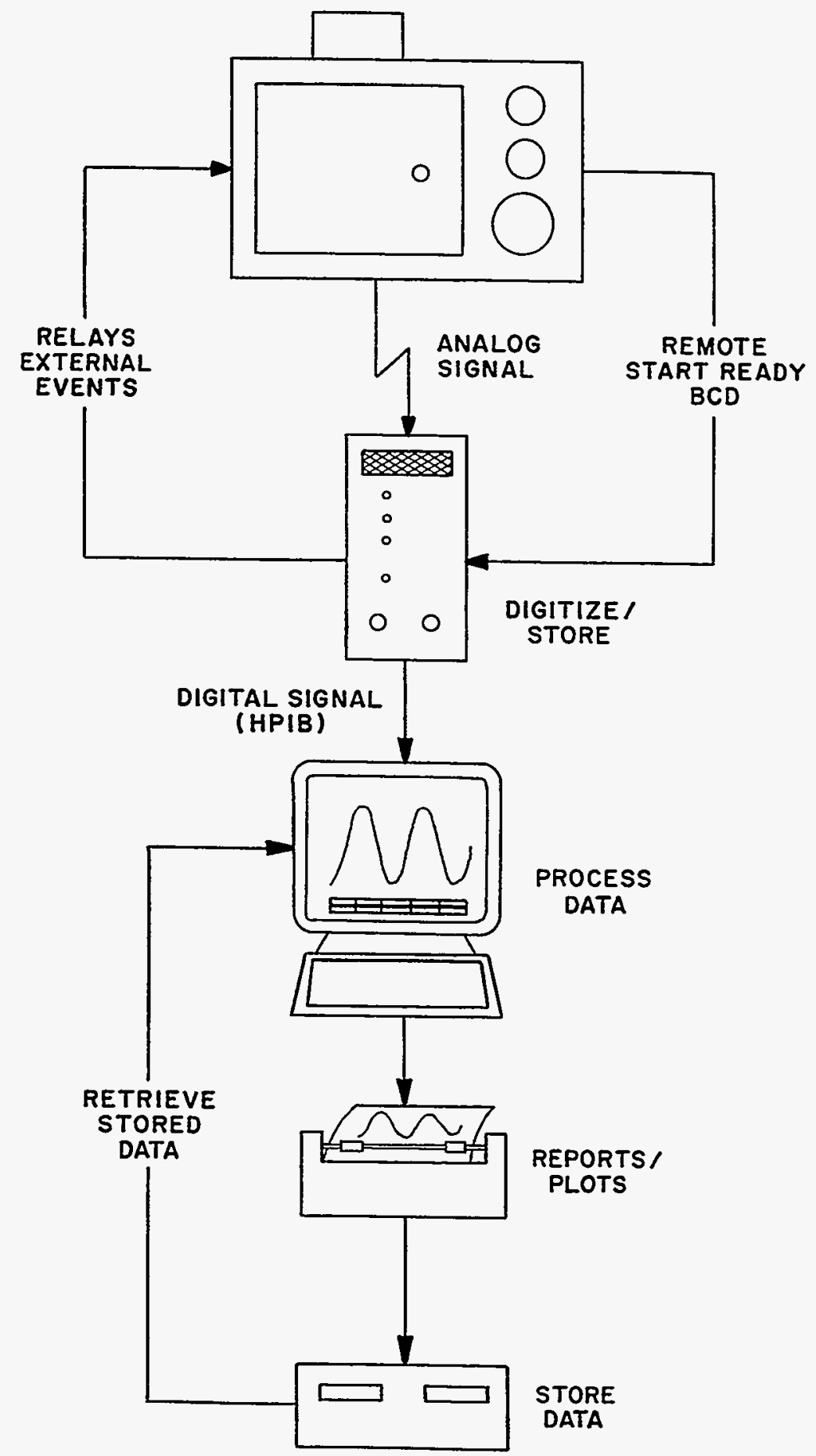

Figure 7. Schematic diagram of the data acquisition and data reduction system. 


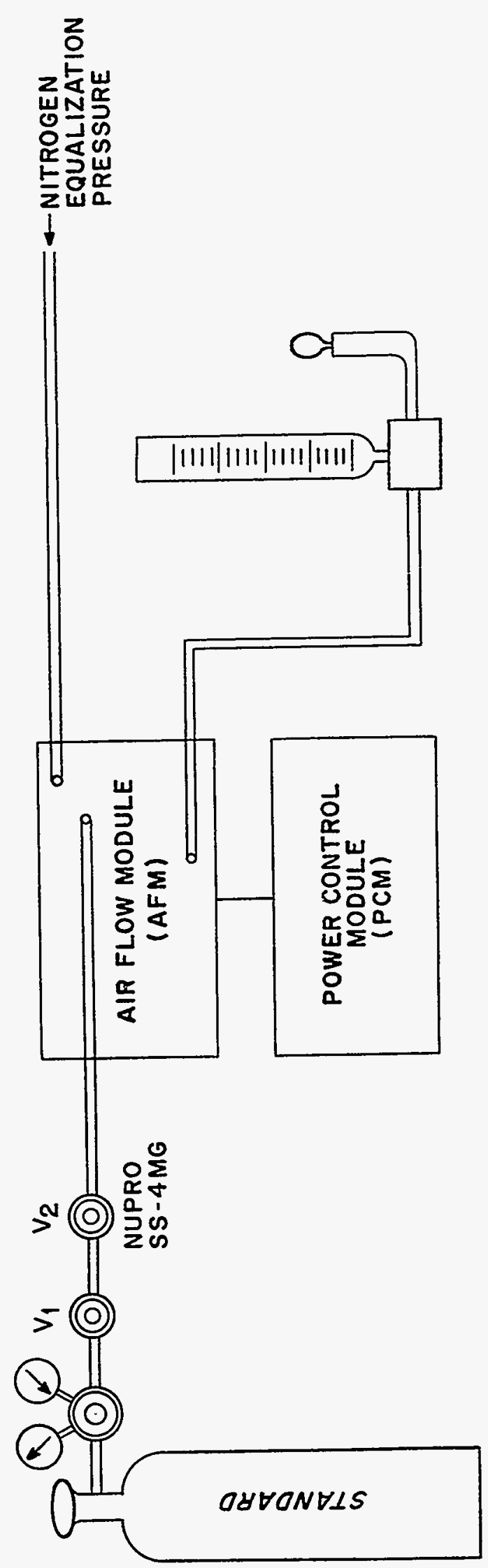




\subsection{INORGANICS}

\subsubsection{SCOPE}

The inorganic procedures previously presented in this section are not currently in use and are now in Volume II. These procedures were adapted for inorganic constituent analyses in air filter extract, water, rain water, and certain soils and sediments. Atomic absorption spectrometry is used for determination of metals, ion chromatography for specific anions, and a specific ion electrode is used for fluoride determination. These procedures have been thoroughly tested; accuracy, precision, and lower limits of detection have been established. Effects from interferences and contamination are detailed in each appropriate procedure. 


\subsubsection{Elemental Analyses}


CALCIUM - ATOMIC ABSORPTION SPECTROMETRY (see Volume II) 
Ca-02-E

CALCIUM - PERMANGANATE TITRATION OF THE OXALATE

(see Volume II) 


\section{F-01-E}

FLUORIE IN SOIL AND SEDIMENT - SPECIFIC ION ELECTRODE MEASUREMENTS

(see Volume II) 
Hg-01-E, Vol. II

Rev. 0

HASL-300, 28th Edition

February 1997

$H g-01-E$

MERCURY - ATOMIC ABSORPTION SPECTROMETRY

(see Volume II) 
$\mathrm{NH}_{3}-01-\mathrm{E}$

AMMONIUM IN WATER AND PRECIPITATION SAMPLES AUTOCOLORIMETRY

(see Volume II) 
STRONTIUM - ATOMIC ABSORPTION SPECTROMETRY

(see Volume II) 
U-01-E, Vol. II

HASL-300, 28th Edition
Rev. 0

February 1997

$U-01-E$

URANIUM IN URINE - FLUORIMETRY

(see Volume II) 


\subsubsection{Multielemental Analyses}


$M-01$

CADMIUM AND LEAD IN HUMAN EXCRETA AND COMPOSITE DIET SAMPLES ATOMIC ABSORPTION SPECTROMETRY

(see Volume In) 
PRECIPITATION AND LAKE WATER SAMPLES PHYSICAL AND CHEMICAL MEASUREMENTS

(see Volume II) 
M-03, Vol. II

HASL-300, 28th Edition

$M-03$

TRACE METALS - ATOMIC ABSORPTION AND/OR EMISSION SPECTROMETRY

(see Volume II) 


\subsection{ORGANICS}

\subsubsection{SCOPE}

The organic pollutants studied at EML are combustion related. Of particular interest are pollutants which are toxic, resistant to natural degradation, and that accumulate in the environment. Polycyclic aromatic hydrocarbons (PAHs) and polychlorinated dibenzo-pdioxins and dibenzofurans (PCDD/Fs) fit into this category and have been analyzed at EML in sediment samples (Tan et al., 1993).

The anthropogenic PAHs in the environment are formed primarily by pyrolysis of carbonaceous materials and the major source is the combustion of fossil fuels. Various combustion processes, especially those involving chlorinated aromatic compounds, generate PCDD/Fs. These compounds are of particular interest to DOE because, besides being combustion originated, they are quite persistent chemicals and can spread throughout the environment. Some PAH and PCDD/F isomers are highly carcinogenic. The study of these compounds in sediments is important as they can reveal the magnitude of contamination, as well as their origins and historical inputs. 
Contact Person(s) : Yulin L. Tan

\begin{abstract}
APPLICATION
This procedure can be applied to the analysis of sediment for three-ring to six-ring polycyclic aromatic hydrocarbons (PAHs) and tetra- to octa- polychlorinated dibenzo-pdioxins and dibenzofurans (PCDD/Fs).

Many organic compounds, including PAHs and PCDD/Fs, that exist in complex compositions in sediments can be extracted into organic solvents such as methylene chloride. From the extract, the PAHs can be isolated from other organics by silica gel adsorption column chromatography and Sephadex LH-20 gel permeation column chromatography, and the PCDD/Fs can be isolated by silica gel adsorption column chromatography, acid/base wash, and alumina adsorption column chromatography. Individual PAHs and PCDD/Fs in these isolated fractions can be further separated and identified with a gas chromatograph/mass spectrometer (GC/MS) by GC retention times and mass spectra. Internal standards of labeled stable isotopes serve the purpose of quantification and quality control.
\end{abstract}

\title{
SPECIAL APPARATUS
}

1. Hewlett-Packard (3000-T Hanover St., Palo Alto, CA 94304) 5988 GC/MS with oncolumn injector.

2. HP 5890 GC/Kratos CONCEPT 1S (Barton Dock Road, Unmston, Manchester, M31 2LD UK) high resolution MS with on-column injector. 
3. Labconco (8111-T Prospect, Kansas City, MO 64132) chemical carcinogen glove box.

4. Cahn Instruments, Inc. (16207-T S. Carmenita Rd., Cerritos, CA 90701), automatic electrobalance.

5. Whatman Soxhlet extraction thimbles, $33 \times 80 \mathrm{~mm}$, cleaned by Soxhlet extraction with $\mathrm{CH}_{2} \mathrm{Cl}_{2}$.

6. Buchi rotary evaporator (Brinkmann, Westbury, NY 11590).

7. Fused silica capillary columns, bonded with DB-5, $30 \mathrm{~m} \times 0.25 \mathrm{~mm}$ i.d., $0.25 \mu \mathrm{m}$ film (J \& W Scientific Inc., Folsom, CA 95630).

8. On-column syringes.

9. Amberized microflex tubes, $5 \mathrm{~mL}$ and $1 \mathrm{~mL}$.

10. Milli-Q reagent grade water system (Millipore Corp., 80-T Ashby Rd., Bedford, MA 01730).

11. Volumetric flasks, $25 \mathrm{~mL}, 150 \mathrm{~mL}, 300 \mathrm{~mL}$.

12. Narrow mouth amber glass bottles, $60 \mathrm{~mL}$, with Teflon-lined screw caps.

13. Glass columns ( $40 \mathrm{~cm} \times 19 \mathrm{~mm}$ i.d.), for gel permeation chromatography, with Teflon stopcocks and $250-\mathrm{mL}$ reservoirs having $24 / 40$ female joints for 24/40 stoppers.

14. Filter paper, Whatman No. $41,2.5 \mathrm{~cm}$, cleaned by Soxhlet extraction with $\mathrm{CH}_{2} \mathrm{Cl}_{2}$.

15. Soxhlet extractors, medium size.

16. Heavy-duty explosion proof centrifuge, Model EXD (Forma Scientific, Inc., Box 649, Millcreek Rd., Marietta, OH 45750). 
17. Baker Bond SPE glass Teflon columns and adapters (J. T. Baker, 222-T Red School Lane, Phillipsburg, NJ 08865).

18. 60-mL separatory funnels with Teflon stopcocks and stoppers.

19. Separatory funnel shaker.

20. Glass columns ( $14 \mathrm{~cm} \times 5.5 \mathrm{~mm}$ i.d.), for alumina column chromatography, with Teflon stopcocks and $50-\mathrm{mL}$ reservoirs.

\section{SPECIAL REAGENTS}

1. Distilled in glass - methylene chloride, hexane, and toluene (Burdick and Jackson, Muskegon, MI 49442).

2. PAH solutions - in the Labconco glove box, put about $100 \mu \mathrm{g}$ each of the following standard PAHs (from various suppliers: Aldrich, P.O. Box 335, Milwaukee, WI 53201; Analabs, North Haven, CT 06473; Ultra Scientific, North Kingston, RI 02852; and Community Bureau of Reference (BCR), Brussels, Belgium) into separate 5-mL amberized microflex tubes:

phenanthrene, anthracene, 1-methylphenanthrene, fluoranthene, pyrene, 1-methylpyrene, benzo(ghi)fluoranthene, benz(a)anthracene, chrysene, triphenylene, benzo(b)fluoranthene, benzo(j)fluoranthene, benzo(k)fluoranthene, benzo(e)pyrene, benzo(a)pyrene, perylene, indeno(1,2,3-cd)pyrene, dibenz(a,c)-anthracene, dibenz(a,h)anthracene, benzo(ghi)perylene.

Dissolve each of the PAHs into $5 \mathrm{~mL}$ of $\mathrm{CH}_{2} \mathrm{Cl}_{2}$.

3. SRM-1647 (priority pollutant PAHs, NIST).

4. PAH standard solution - using the Cahn 25 automatic electrobalance in the Labconco glove box, quantitatively weigh an amount of about $5 \mathrm{mg}$ each of the following PAHs into a 50-mL volumetric flask: 
phenanthrene, anthracene, 1-methylphenanthrene, fluoranthene, pyrene, 1-methylpyrene, benzo(ghi)fluoranthene, benzo(b)fluoranthene, benzo(a)pyrene, benzo(e)pyrene, perylene, indeno(1,2,3-cd)pyrene, dibenz(a,h)anthracene, benzo(ghi)perylene.

Dissolve the weighed PAHs with $50 \mathrm{~mL}$ of toluene so that the concentration of this primary standard solution contains about $100 \mu \mathrm{g} \mathrm{mL}^{-1}$ for each PAH. Transfer the solution to a $60-\mathrm{mL}$ narrow mouth amber glass bottle with a Teflon-lined screw cap and store in a refrigerator. Mix quantitative amounts of the primary standard and SRM-1647. The mixture is analyzed as PAH calibration solutions by measuring the molecular ions of the PAHs with GC/MS according to Steps 1-8 of Determination, Section A. Calibrate the concentrations of each PAH in the standard solution based on the PAH concentrations in SRM-1647. Use the method of standard additions for PAHs that are present in both solutions. For PAHs present only in the standard solution, response factors of neighboring PAHs in the gas chromatogram are used.

5. Labeled PAH solution - Using the Cahn 25 automatic electrobalance in the Laconco glove box, quantitatively weigh an amount about $2 \mathrm{mg}$ each of the following deuterated PAHs into a 50-mL volumetric flask:

anthracene, fluoranthene, pyrene, chrysene, benzo(e)pyrene, perylene, benzo(ghi)perylene

Dissolve the weighed deuterated PAHs in $50 \mathrm{~mL}$ of toluene.

6. PAH calibration solutions - Mix and dilute the labeled PAH solution and the PAH standard solution with toluene to make five calibration solutions. All five calibration solutions contain $5 \mu \mathrm{g} \mathrm{mL}^{-1}$ of each deuterated PAHs with various concentrations of native PAHs at $1 \mu \mathrm{g} \mathrm{mL}^{-1}, 2 \mu \mathrm{g} \mathrm{mL}^{-1}, 5 \mu \mathrm{g} \mathrm{mL}^{-1}, 10 \mu \mathrm{g} \mathrm{mL}^{-1}$, and $25 \mu \mathrm{g} \mathrm{mL}^{-1}$.

7. Native PCDD/F stock solutions containing: 2378-TCDF, 12378-PnCDF, $123478-$ HxCDF, 1234678-HpCDF, OCDF, 2378-TCDD, 12378-PnCDD, 123678-HxCDD, $1234678-\mathrm{HpCDD}$, and $\mathrm{OCDD}, 1 \mathrm{mg} \mathrm{mL}^{-1}$ each in toulene.

8. $\mathrm{PCDD} / \mathrm{F}$ spiking solution - $\mathrm{mix}$ and dilute labeled $\mathrm{PCDD} / \mathrm{F}$ solutions: 
${ }^{13} \mathrm{C}_{6}$ 2378-TCDF, ${ }^{13} \mathrm{C}_{6} 23478-\mathrm{PnCDF}^{13} \mathrm{C}_{6} 123478-\mathrm{HxCDF},{ }^{13} \mathrm{C}_{12}$ 1234678-HpCDF, ${ }^{13} \mathrm{C}_{12}$ 2378-TCDD, ${ }^{13} \mathrm{C}_{12}$ 12378-PnCDD, ${ }^{13} \mathrm{C}_{12}$ 123678-HxCDD, ${ }^{13} \mathrm{C}_{12} 1234678-$ $\mathrm{HpCDD}$, and ${ }^{13} \mathrm{C}_{12}$ OCDD from Cambridge Isotope Laboratory (Woburn, $\mathrm{MA}$ 01810) with toluene so that the spiking solution contains $100 \mathrm{ng} \mathrm{mL}^{-1}$ each of the labeled PCDD/Fs.

9. $\mathrm{PCDD} / \mathrm{F}$ calibration solutions - $\mathrm{mix}$ and dilute the PCDD/F spiking solution and the native $\mathrm{PCDD} / \mathrm{F}$ stock solutions with toluene to make six calibration solutions. All six calibration solutions contain $10 \mathrm{ng} \mathrm{mL}^{-1}$ of each labeled PCDD/Fs in the PCDD/F spiking solution with various concentrations of each of the native $\mathrm{PCDD} / \mathrm{Fs}$ listed in Step 7 at $1 \mathrm{ng} \mathrm{mL}^{-1}, 2 \mathrm{ng} \mathrm{mL}^{-1}, 5 \mathrm{ng} \mathrm{mL}^{-1}, 10 \mathrm{ng} \mathrm{mL}^{-1}, 25 \mathrm{ng} \mathrm{mL}^{-1}$, and $50 \mathrm{ng} \mathrm{mL}^{-1}$.

10. Sulfuric acid, ACS reagent (Fisher Scientific, 52 Faden Rd., Springfield, NJ 07081).

11. $\mathrm{KOH}$ solution, $20 \%$ by weight in Milli-Q water.

12. Copper powder - electrolytic purified (Fisher Scientific, 52 Faden Rd., Springfield, NJ

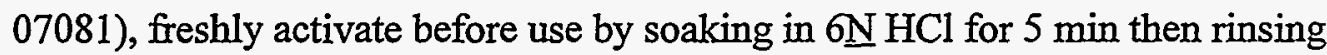
thoroughly with Milli-Q water, followed by methanol.

13. Sephadex LH-20, Sigma Chemicals Co. (P.O. Box 14508, St. Louis, MO 63178) swell overnight in 1:1 methanol-methylene chloride before column packing.

14. Silica gel, 100-200 mesh, EM Science (480-T Democrat Rd., Gibbstown, NJ 08027), Grade 923 , cleaned by Soxhlet extraction with methylene chloride, activated at $120^{\circ} \mathrm{C}$ overnight, then stored in a desiccator.

15. ICN alumina $B$, activity 1 (ICN Pharmaceuticals, Inc., 3300-T Hyland Ave., Costa Mesa, CA 92626) cleaned by Soxhlet extraction with methylene chloride, activated at $150^{\circ} \mathrm{C}$ overnight, then stored in a desiccator.

16. Glass beads, $0.5 \mathrm{~mm}$, cleaned by Soxhlet extraction with $\mathrm{CH}_{2} \mathrm{Cl}_{2}$.

17. Zero gases, $\mathrm{He}, \mathrm{H}_{2}$, air, and $\mathrm{N}_{2}$ (Matheson Gas Products, 30-T Seaview Dr., P.O. Box 1587, Secaucus, NJ 07096). 
18. Perfluorotributylamine, Hewlett-Packard calibration compound.

\section{SAMPLE PREPARATION}

\section{A. Sediment handling.}

1. Centrifuge the watery sediment and decant off the excess water.

2. Dry the sediment in a freeze dryer.

3. Pulverize the dried sediment in a mortar and pestle and then sieve with a No. 40 $(0.425 \mathrm{~mm})$ sieve to remove stones and extraneous material. The fines are collected for analyses.

\section{B. Extraction.}

1. Place the fine sediment in a cleaned Whatman extraction thimble, $33 \times 80 \mathrm{~mm}$.

2. Spike appropriate amounts of labeled PAH and labeled PCDD/F solutions close to those of the estimated analytes.

3. Soxhlet extract the sample with $\mathrm{CH}_{2} \mathrm{Cl}_{2}$ for $30 \mathrm{~h}$.

4. Concentrate the extract to dryness with a rotary evaporator at room temperature.

\section{Silica gel adsorption chromatography and sulfur removal.}

1. Pack $250 \mathrm{mg}$ of clean activated silica gel in an empty SPE glass Teflon column between two Teflon fritted disks. Tap gently for a tight packing and make sure the top of the column is evenly packed and closely held by the Teflon-fritted disk.

2. Pack $1000 \mathrm{mg}$ of cleaned and activated silica gel in another empty SPE column as above.

3. Reactivate the two columns overnight at $120^{\circ} \mathrm{C}$. 
4. Prior to using, allow the columns to cool in a desiccator.

5. Place a piece of Whatman No. 41 filter paper, ca. $1 \mathrm{~cm}$ I.D., onto the top of the Teflon disk of the $250 \mathrm{mg}$ silica gel column, add about $0.5 \mathrm{~g}$ of activated copper powder, place a second piece of filter paper on top of the powder.

6. Transfer the dried residue (Step B4 of Sample Preparation) onto the copper powder topped $250 \mathrm{mg}$ silica gel column with $2 \times 0.5 \mathrm{~mL}$ of $\mathrm{CH}_{2} \mathrm{Cl}_{2}$. Dry the wetted column thoroughly with dry $\mathrm{N}_{2}$ after each transfer.

7. Wet the $1000 \mathrm{mg}$ column with hexane.

8. Attach sampled $250 \mathrm{mg}$ column to the top of the $1000 \mathrm{mg}$ column with a Teflon column adapter.

9. Wash the two-column assembly with $3 \mathrm{~mL}$ of hexane, and discard the wash.

10. Elute the columns with $10 \mathrm{~mL}$ of hexane followed by $7 \mathrm{~mL}$ of $60 \% \mathrm{CH}_{2} \mathrm{Cl}_{2}$ in hexane into two separate $25-\mathrm{mL}$ round bottom flasks for PCDD/F and $\mathrm{PAH}$ fractions, respectively.

11. Evaporate the PAH fraction to dryness at $35^{\circ} \mathrm{C}$ under reduced pressure with a rotary evaporator.

\section{Acid/base wash.}

1. Transfer the PCDD/F fraction in $10 \mathrm{~mL}$ of hexane from Step 10 of Section $\mathrm{C}$, into a $60-\mathrm{mL}$ separatory funnel, add $10 \mathrm{~mL}$ of concentrated $\mathrm{H}_{2} \mathrm{SO}_{4}$.

2. Shake the funnel and contents with a separatory funnel shaker for $2 \mathrm{~min}$.

3. Centrifuge the funnel for $30 \mathrm{~s}$.

4. Drain off the lower aqueous layer.

5. Add second $10 \mathrm{~mL}$ of $\mathrm{H}_{2} \mathrm{SO}_{4}$ into the funnel and repeat Steps 2-4. 
6. Add $10 \mathrm{~mL}$ of Milli-Q water, shake briefly by hand, and repeat Steps 3-4.

7. Add $10 \mathrm{~mL}$ of $20 \% \mathrm{KOH}$ solution into the funnel, and repeat Steps $2-4$.

8. Add second $10 \mathrm{~mL}$ of $20 \% \mathrm{KOH}$ solution and repeat Steps $2-4$ once and Step 6 twice.

9. Transfer the hexane layer into a $25-\mathrm{mL}$ round bottom flask and concentrate to dryness with a rotary evaporator at $35^{\circ} \mathrm{C}$.

\section{E. Alumina column chromatography.}

1. Place a small amount of glass wool at the bottom of the glass column for alumina column chromatography.

2. Pack $2.5 \mathrm{~mL}$ of activated alumina into the column and reactivate overnight at $150^{\circ} \mathrm{C}$.

3. Prior to use, cool the column in a desiccator. Once cool, add a 3-mm thick layer of $\operatorname{dry} \mathrm{Na}_{2} \mathrm{SO}_{4}$.

4. Transfer the residue from Step 9 of Section $\mathrm{D}$ with $2 \times 0.5 \mathrm{~mL}$ of hexane onto the top of the $\mathrm{Na}_{2} \mathrm{SO}_{4}$ layer. Drain the column until the meniscus reaches the sodium sulfate layer after each transfer.

5. Wash the column with $45 \mathrm{~mL}$ of hexane and discard the wash.

6. Elute the column with $4 \mathrm{~mL}$ of $60 \% \mathrm{CH}_{2} \mathrm{Cl}_{2}$ in hexane.

7. Collect the eluent and concentrate to dryness on a rotary evaporator at room temperature.

\section{F. Gel permeation chromatography.}

1. Place a piece of Whatman No. 41 filter paper, $17 \mathrm{~mm}$ in diameter, on the fritted glass disc of the gel permeation column. Wet the filter paper with a few drops of methanol so that the paper adheres to the fritted glass disc. 
2. Pour in a layer of about $1 \mathrm{~mL}$ of glass beads to hold the filter paper onto the fritted glass disc.

3. Fill half the column with the eluting solvent, 1:1 methanol-methylene chloride.

4. Pour in the swelled Sephadex LH-20 to form a 37-cm tall column. After the solvent is drained to the top of the gel, place a piece of Whatman No. 41 filter paper, $17 \mathrm{~mm}$ in diameter, on top of the column and add a 1-cm thick layer of glass beads.

5. Transfer, dropwise, the PAH fraction (Step 11 of Section C) with three to five portions of $0.5 \mathrm{~mL}$ each of the eluting solvent onto the column. Spread the drops evenly over the top of the column and drain off each transferring solution before adding the succeeding portions.

6. Elute with 1:1 methanol-methylene chloride and discard the first $75 \mathrm{~mL}$ of the eluent. Collect the following $30 \mathrm{~mL}$ of eluent and dry it with a rotary evaporator at room temperature.

7. Regenerate the column by washing with an additional $50 \mathrm{~mL}$ of eluting solvent. Keep the column in the solvent and seal the reservoir with a 24/40 stopper. 


\section{DETERMINATION}

\section{A. PAHs.}

1. Set the HP5988 GC/MS parameters as follows:

\section{$\underline{\text { Parameters }}$}

GC Column

Oven temperature program

Carrier gas

Injection mode

Ionization mode

Emission

Electron energy

Ion source temperature
Description

J \& W Scientific, Inc., fused silica capillary column (see Special Apparatus)

from initial $60^{\circ}$ to $150^{\circ} \mathrm{C}$, at $32^{\circ} \mathrm{C} \mathrm{min}$, then from $150^{\circ}$ to $280^{\circ} \mathrm{C}$ at $4^{\circ} \mathrm{C} \mathrm{min}^{-1}$, and hold at $280^{\circ} \mathrm{C}$ for $30 \mathrm{~min}$

$\mathrm{He}$, head pressure 20 psi.

On-column

Electron impact

$300 \mu \mathrm{A}$

$70 \mathrm{eV}$

$150^{\circ} \mathrm{C}$

2. Tune the MS with a calibration compound, perfluorotributylamine.

3. Set the MS at scan mode and mass scan range at 50-500.

4. Inject $0.5 \mu \mathrm{L}$ of each PAH solutions (Step 2 of Special Reagents).

5. Immediately start the GC oven temperature program, and acquire the data during the GC run.

6. Determine the $\mathrm{GC}$ retention times of each $\mathrm{PAH}$ from the total ion chromatograms. 
7. Switch the MS to the SIM mode, set the masses of the molecular ions of labeled and native PAHs in calibration solutions (Step 6 of Special Reagents) to be acquired in their retention windows.

8. Inject $1 \mu \mathrm{L}$ of each PAH calibration solution and acquire the ion abundances of the selected ions at the SIM mode. Repeat five times.

9. Establish the calibration curve for each native PAH by plotting the molecular ion abundance ratios of native to labeled PAHs vs. their concentration ratios in the calibration solutions. For native PAHs without labeled counterparts, data from neighboring labeled PAHs in the total ion chromatograms are used. The slopes of these curves are the response factors, $F$.

10. Repeat Step 3.

11. Redissolve the residue of the PAH fraction (Step 6 of Section F) into a small amount of methylene chloride and inject $1 \mu \mathrm{L}$ of the solution into the GC/MS.

12. Repeat Step 5.

13. Identify the PAHs in the PAH fraction by their retention times and mass spectra, considering there are superimposed spectra of spiked labeled PAHs on some of the native PAHs.

\section{Repeat Step 7.}

15. Inject $1 \mu \mathrm{L}$ solution of $\mathrm{PAH}$ fraction and repeat Step 5 .

16. Calculate the molecular ion abundance ratios of native to labeled PAHs, $r$.

17. Determine the concentrations of native PAHs, $\mathrm{C}$, from the weight of the sample, $\mathrm{W}$, amount of spiked labeled PAHs, $w$, the ion abundance ratios and response factors, $F$, as:

$$
\mathrm{C}=\frac{\mathrm{W} \cdot \mathrm{T} \cdot \mathrm{F}}{\mathrm{W}}
$$


B. PCDD/Fs.

1. Set the Kratos 1S CONCEPT parameters as follows:

$\underline{\text { Parameters }}$

GC column

Oven temperature program

Carrier gas

Injection mode

Ionization mode

Electron energy

Source temperature

Trap current

Resolution

\section{Description}

J \& W Scientific, Inc.; fused silica capillary column (see Special Apparatus)

Hold at initial $80^{\circ} \mathrm{C}$ for $1 \mathrm{~min}$, program from $80-200^{\circ} \mathrm{C}$ at $30^{\circ} \mathrm{C} \mathrm{min}^{-1}$, then go to $280^{\circ} \mathrm{C}$ at $5^{\circ} \mathrm{C} \mathrm{min}^{-1}$ and to $300^{\circ} \mathrm{C}$ at $15^{\circ} \mathrm{C}$ $\min ^{-1}$

$\mathrm{He}$, head pressure 20 psi

On column

Electron impact

$30-40 \mathrm{eV}$

$250^{\circ} \mathrm{C}$

$500 \mu \mathrm{A}$

$10,000(10 \%$ valley definition)

2. Tune the MS with a calibration compound, perfluorotributylamine.

3. Under the GC oven temperature program, acquire the mass spectra of the native PCDD/Fs in stock solution (Step 7 of Special Reagents) at scan mode, mass range 50-500. Establish the retention windows of PCDD/Fs with the acquired spectra and published results (Ryan et al., 1991).

4. Set the MS at the SIM mode and acquiring ions with masses $M$ (molecular mass with all ${ }^{35} \mathrm{Cl}$ ) and $\mathrm{M}+2$ (molecular mass with one ${ }^{37} \mathrm{Cl}$ and the rest ${ }^{35} \mathrm{Cl}$ ) for both native and labeled tetra- to penta- PCDD/Fs isomers and $\mathrm{M}+2$ and $\mathrm{M}+4$ (molecular mass with two ${ }^{37} \mathrm{Cl}$ and the rest ${ }^{35} \mathrm{Cl}$ ) for both native and labeled hexa- to octa- PCDD/Fs isomers in their respective retention windows. 
5. Inject $0.5 \mu \mathrm{L}$ of each PCDD/F calibration solutions (Step 9 of Special Reagents), start the GC oven temperature program, and acquire the ion abundances of the selected ions at the SIM mode.

6. Establish the calibration curves for each native PCDD/F by plotting the ion abundance ratios of native to labeled $\mathrm{PCDD} / \mathrm{F}$ vs. their concentration ratios in the calibration solutions. The slopes of these curves are response factors, $F_{1}$.

7. Redissolve the residue of PCDD/F fraction (Step 7 of Alumina Column Chromatography) into a small amount of toluene and inject $0.5 \mu \mathrm{L}$ of the solution into the GC/MS.

8. Repeat Steps 4.

9. Inject $0.5 \mu \mathrm{L}$ of solution made in Step 7 and start GC/MS run as Step 5.

10. Identify the $\mathrm{PCDD} / \mathrm{Fs}$ in the $\mathrm{PCDD} / \mathrm{F}$ fraction by their retention times (Ryan et al., 1991) and ion abundances.

11. Calculate the ion abundance ratios of native to labeled PCDD/Fs, $r_{1}$.

12. Determine the concentrations of native PCDD/Fs, $C_{1}$, from the weight of the sample, $\mathrm{W}$, amount of spiked labeled PCDD/Fs, $\mathrm{w}_{1}$, the ion abundance ratios of native to labeled $\mathrm{PCDD} / \mathrm{Fs}, \mathrm{r}_{1}$, and $\mathrm{F}_{1}$, as:

$$
C_{1}=\frac{w_{1} \cdot r_{1} \cdot F_{1}}{W}
$$

\section{LOWER LIMIT OF DETECTION}

The recovery of PAHs and PCDD/Fs from the whole sample preparation is $\sim 50 \%$.

The detection limit varies from 1 to $3 \mathrm{pg}$ for three-ring to six-ring PAHs on the HP5988 GC/MS, and 0.2 to $0.5 \mathrm{pg}$ for tetra- to octa-PCDD/Fs on the HP5890 GC/Kratos CONCEPT 1S MS. 


\section{REFERENCES}

Ryan, J. J., H. B. S. Copnacher, L. G. Panopio, B. P.-Y. Lau, and J. A. Hardy

"Gas Chromatographic Separations of All 136 Tetra- to Octa-Polychlorinated-p-dioxins and Polychlorinated Dibenzofurans on Nine Different Stationary Phases"

J. of Chromatography, $\underline{541}, 131-183$ (1991)

Tan, Y. L., A. Kong, and Y. Chiu

"Sample Preparation for Analyzing Polycyclic Aromatic Hydrocarbons and

Polychlorinated Dibenzo-p-dioxins and Dibenzofurans in Sediment by Gas

Chromatography/Mass Spectrometry"

Estuaries, 16, 427-432 (1993) 


\subsection{RADIOCHEMICAL}

\subsubsection{SCOPE}

Described in this section are the radiochemical procedures currently in use at EML. These procedures have been constantly updated for the past 40 years to reflect current separation technology and measurement techniques. The radiochemical procedures are usually written for a distinct environmental matrix since chemical interferences and contamination levels vary according to sample type. 


\subsubsection{Radiometrology}




\section{CALIBRATION OF ALPHA-EMITTING RADIONUCLIDE SOLUTIONS AND SOURCES}

\section{A. Application.}

The methods for primary and secondary calibrations of $\alpha$-emitting solutions and sources are described for detection systems in use at EML.

\section{B. Description of the system.}

Primary calibrations of $\alpha$-emitting radionuclide solutions and sources are performed at EML with a $2 \pi$ windowless (internal) gas flow proportional detection system. A commercially obtained stainless steel counter with a three-position turntable and gas flow indicator (a bubbler) is capable of accepting samples up to $5.08 \mathrm{~cm}(2 \mathrm{in})$ diameter. A heavy walled cylindrically shaped active volume contains a loop anode of stainless steel wire. An EML designed and built preamplifier is connected directly to the top of the detector. The preamplifier acts as an impedance matching device for pulses from the detection volume to an electronic scaler. High voltage from a 0-5000 V regulated power supply is applied to the anode through a cable connection on the preamplifier. The signal from the anode passes through the preamplifier and a cable into an electronic scaler. The duration of the measurement interval is controlled with an electronic timer. Methane ( $99.99 \%$ chemically pure) is used as the counting gas.

Any source to be measured must be of a conducting material. In practice, all sources measured at EML in this system are on metal backings, usually platinum, stainless steel or aluminum. 


\section{Traceability to the National Institute of Standards and Technology (NIST).}

Each day the detection system is used, its operating characteristics are checked with a NIST standard reference material (SRM) $\mathrm{U}_{3} \mathrm{O}_{8}$ source. After thoroughly flushing the system with the counting gas, a voltage plateau is determined with the NIST source. Generally, the plateau is $500-600 \mathrm{~V}$ in length at applied voltages of $2300-2900(+)$. The slope of the voltage plateau is checked and should be $<1 \%$ per $100 \mathrm{~V}$ with methane as the counting gas. The operating voltage selected is $100 \mathrm{~V}$ below the upper "knee" of the plateau. The NIST source is measured for $2000 \mathrm{sec}$, sufficient for a $1 \mathrm{~s}$ Poisson standard deviation (SD) of the measurement of $<0.5 \%$. The background count rate is determined at the same operating potential for $2000 \mathrm{sec}$. The background count should be $4-5$ counts or about 0.003 counts $\sec ^{-1}$ (cps). The net count rate obtained by subtracting the background count rate from the total count rate of the standard source measurement is compared with the certified NIST value. (Total count rate refers to the [sample + background] count rate.) The NIST certified value is given in terms of $\alpha$ particles $\sec ^{-1}$ in a $2 \pi$ configuration. This means that the $\alpha$ backscatter factor is part of the certified value and no correction needs to be made for this source characteristic. The EML value obtained must agree with the NIST certified value (within the error of the measurement) before other sources are measured.

As a further check of this procedure, when NIST $\alpha$-emitting SRM sources are purchased, they are treated as unknowns and measured as described above. The value obtained at EML is then compared with the certified value.

Failure to obtain a flat or a long voltage plateau or failure to agree with the certified source value are immediate indicators of problems in the detection system which must be remedied before proceeding with additional measurements.

\section{EML source preparation.}

A system for the primary calibration of $\alpha$-emitting solutions and sources is maintained at EML because all the nuclides required for in-house programs may not be available as NIST SRMs at any given time and it is cost effective. Both NIST SRM solutions and sources are initially expensive and the cost of recalibration by NIST is often equal to or more than the original cost. Since all EML $\alpha$-emitting solutions and sources are recalibrated at least once a year, the cost of NIST recertification would be prohibitive. 
Electrodeposited sources of $\alpha$-emitting radionuclides are prepared on $17 \mathrm{~mm}$ diameter virgin platinum discs. These sources, once calibrated, are used as standards to determine the detection efficiencies of $\alpha$-scintillation counters and solid-state $\alpha$-spectrometry systems. In general, the activity of these sources is $15-20 \mathrm{~Bq}(450 \mathrm{pCi}$ or $1000 \mathrm{dpm})$. The procedure for obtaining a "working standard" source is to measure the NIST SRM $\mathrm{U}_{3} \mathrm{O}_{8}$ source and background as described above, obtain a voltage plateau for the source to be calibrated, and measure the source at the proper plateau voltage for $2000 \mathrm{sec}$. The net count rate of the source is converted to an activity unit using the detection efficiency of $52 \%$. A Poisson error term is calculated and attached to the activity value.

The calibration of an $\alpha$-emitting solution differs from that for electrodeposited sources only in the method of source preparation. It is the practice at EML that sources from the solution to be calibrated be prepared in triplicate. The source mounts are $24 \mathrm{~mm}$ diameter virgin platinum discs. The solution to be calibrated must be essentially carrierfree. The activity of the solution should be on the order of $160-1600 \mathrm{~Bq} \mathrm{~g}^{-1}$. To minimize $\alpha$ absorption in the source, a weighed amount of a Teflon suspension is transferred to the disc from a polyethylene transfer pipette (see Specification 7.11). About $30 \mu \mathrm{g}$ of Teflon beads are delivered in the 0.1-g aliquot. A weighed aliquot of the solution to be calibrated is dispensed fro the transfer pipette directly into the Teflon suspension bead. As a check on possible self-absorption in the dried source, aliquot weights are varied from 0.1-0.2 $\mathrm{g}$ in the triplication procedure. The sources are slowly dried near a heat lamp.

After the $2 \pi$ proportional detection system has been checked as described above, the first source is heated in an alcohol burner flame just until the Teflon vaporizes. This drives off any residual waters of hydration which could cause self-absorption in the source. The flamed source is immediately placed in the methane atmosphere. A voltage plateau is obtained and the source is measured as described above. This procedure is repeated for the remaining sources. The activity and Poisson counting error are calculated for each source and corrected for the aliquot weight to obtain the activity $\mathrm{g}^{-1}$ of solution. A Gaussian mean and SD are calculated from the triplicate source measurements. The Gaussian SD is usually $<1 \%$.

Dilutions of the calibrated solution may then be prepared by weight for use in various EML programs. 


\section{ALPHA SCINTILLATION MEASUREMENTS}

\section{A. Application.}

The procedure was developed for $\alpha$ measurements of air dust samples and chemical precipitates. Perhaps its largest application has been in the determination of ${ }^{222} \mathrm{Rn}$ and ${ }^{220} \mathrm{Rn}$ concentrations from air filter samples. Routine applications include the determination of the $\alpha$ nuclide concentrations of low-level solutions and measurement of total $\alpha$ activity of radiochemically separated samples and investigations of materials for commercial uses.

The advantages of the use of $\mathrm{ZnS}(\mathrm{Ag})$ on Mylar phosphor discs were enumerated by Hallden and Harley (1960) and are paraphrased here.

Samples are measured with uniform high efficiency, since the sample is in direct contact with the phosphor and photomultiplier tube (PMT).

The phosphor sees only the sample and the face of the PMT, yielding a lower background than other $\alpha$-scintillation systems and proportional counters.

Samples are completely enclosed, readily handled and stored without loss of sample integrity or cross contamination.

Counter maintenance is negligible, since the system cannot be contaminated under ordinary conditions.

Samples need not be conducting as with internal gas proportional counters.

Filter samples are held flat without curling or buckling producing a uniform counting configuration.

\section{B. Description of the detection systems.}

Essentially all $\alpha$ scintillation detection systems use $\mathrm{ZnS}(\mathrm{Ag})$ as the phosphor. There are basically two types of $\alpha$ scintillation counters in use at EML for total $\alpha$ measurements, those accommodating samples using $\mathrm{ZnS}(\mathrm{Ag})$ on Mylar (Hallden and Harley, 1960; see 
Specification 7.11), and those with the phosphor applied as a powder to the face of a PMT tube (Curran and Baker, 1948).

The EML $\alpha$-scintillation counters were designed and built by EML's Instrumentation Division (now the Technical Program Services Division). The EML $\alpha$-scintillation counters accommodate the $2.54 \mathrm{~cm}$ ( 1 in) diameter nylon ring and disc sample mounting system (see Specification 7.2). The sample to be measured is placed on the nylon disc. A $2.4 \mathrm{~cm}$ (15/16 in) diameter die-cut phosphor disc is placed against the sample, a strip of Mylar film (see Specification 7.3) is overlaid and the assembly is locked together with a nylon ring.

A spring-loaded $2.54 \mathrm{~cm}$ (1 in) diameter PMT is housed in a light tight, anti-magnetic shield, facing downward. The sample ring and disc assembly is placed in a space in a pullout drawer. The drawer is closed and a knob turned to the "up" position, placing the sample assembly in direct contact with the PMT. An emitter follower network is built on the base of the PMT. The high voltage supply for the PMT is an integral part of the EML Instrumentation Division's double width nuclear instruments modules (NIM) scaler-timer assembly.

It should be noted that the $\alpha$-scintillation phosphor discs were and are intended for single use, that is, they and most of the samples are disposable as a unit.

\section{Calibration of the instruments.}

The detection efficiencies of the EML $\alpha$-scintillation counters are determined by measuring electrodeposited "working standard" sources prepared at EML. The sources are traceable to the NIST through the process described in Section C, Traceability to NIST.

Measurements with single emitter sources with energies ranging from 3-6 MeV show that the detector response is independent of the energy of the $\alpha$ particle. Sources of ${ }^{226} \mathrm{Ra}$ and ${ }^{228} \mathrm{Th}$ in equilibrium with their short-lived progeny were measured in the $2 \pi$ proportional counter and then mounted and measured in the $\alpha$-scintillation detection systems. After applying the detection efficiency obtained from the "working standards", the values obtained by $\alpha$-scintillation counting agreed with those of the $2 \pi$ proportional 
counter within the error of the measurements. This implies that the detection efficiencies of the $\alpha$-scintillation counters are independent up to $9 \mathrm{MeV}$.

Background count rates are determined for the materials commonly used in EML programs. These include platinum discs and cellulose, polystyrene and glass fiber filters. To determine the "true" background count rates of the systems, so called "nylon background" measurements are performed in which the phosphor is placed in direct contact with the nylon disc. This is especially useful when assessing the performance of each new batch of phosphors. Typical background count rates for these materials are shown below.

Material

Nylon

Polystyrene filter

Cellulose filter

Glass fiber filter

Platinum disc (17 $\mathrm{mm}$ dia.)
Background Count Rate (cpm)

$$
\begin{aligned}
& 0.001 \pm 0.001 \\
& 0.001 \pm 0.001 \\
& 0.002 \pm 0.002 \\
& 0.100 \pm 0.010 \\
& 0.012 \pm 0.002
\end{aligned}
$$

The background count rates are determined for measurement intervals of $1000 \mathrm{~min}$ or more.

The average detection efficiency determined from measurements of a "working standard" source electrodeposited on platinum is $51 \%$. The source is measured twice weekly for a 100-min counting interval.

Quality control charts are maintained for the background count rates and the detection efficiencies of the $\alpha$-scintillation counters. The means and SDs of each type of measurement are calculated on a monthly basis. 


\section{SOLID-STATE ALPHA SPECTROMETRY}

\section{A. Application.}

The solid-state $\alpha$-spectrometry systems are used primarily to determine the concentrations of radionuclides in chemically separated samples. Virtually all radiochemical analyses for $\alpha$-emitting radionuclides are performed with an isotopic tracer.

For the transuranic nuclide determinations, the following tracers are used at EML: ${ }^{236} \mathrm{Pu}$, ${ }^{242} \mathrm{Pu}$, and ${ }^{243} \mathrm{Am}$. Tracers used in the determination of naturally-occurring radionuclides are ${ }^{208} \mathrm{Po},{ }^{209} \mathrm{Po},{ }^{229} \mathrm{Th}$ and ${ }^{232} \mathrm{U}$.

The systems are also used to determine the radiopurity of radionuclide solutions.

\section{B. Description of the detection systems.}

The solid-state $\alpha$-spectrometry systems used at EML are divided into two groups of four detectors, one group is devoted to transuranic measurements and the other to natural radionuclide measurements. Both groups have several elements in common. All components of the systems are commercially available except as noted.

Four vacuum chambers are served by a single beltless vacuum pump. The interior diameters of the vacuum chambers allow samples to be placed as far as $5 \mathrm{~cm}$ from the detector. The sample holder, which may be moved vertically, has been modified at EML to an open drawer-slide arrangement. The drawer is locked in place and the slide, with a $2.54 \mathrm{~cm}$ diameter by $2 \mathrm{~mm}$ deep indentation to accommodate the sample mount, is removable. The sample is placed on a mount, the mount is placed in the indentation, and the slide is then replaced in the open drawer arrangement. This sample holder arrangement eliminates problems of source to detector geometry. The two kinds of solidstate detectors in use at EML will be discussed below. Voltage to the detectors is supplied by individual regulated power supplies. Each detector has a charge sensitive preamplifier and a linear amplifier. Signals from the detectors are directed through a router into a 4096 multichannel analyzer. One analyzer serves four detectors with data collection divided into four 1024 channel segments. A hard copy of the data is obtained with a high speed printer. 
All $\alpha$-spectrometry data are reduced "by hand" which ensures immediate and individual review of the spectra. By this practice, difficulties with the spectrometry systems or the samples are quickly noted and remedied.

The solid-state $\alpha$ detectors are operated at about $10 \mathrm{keV}$ per channel, covering an energy range of about 3-13 MeV. By this practice, all $\alpha$ emitters present in a sample can be identified from their energies. (Virtually all $\alpha$ emitters, both natural and artificial are within this energy range.)

Because EML is primarily involved in the measurement of low-level environmental samples, the most common measurement period by $\alpha$ spectrometry is $5000 \mathrm{~min}$. This time unit will be used in describing some of the characteristics of the detection systems.

The solid-state detectors in use at EML for research programs are conventional 500 $\mathrm{mm}^{2}$ active area silicon surface barrier detectors. The resolution of the detectors, as measured routinely with EML prepared "working standard" sources, is $40-45 \mathrm{keV}$ (FWHM). The systems are calibrated with "mixed $\alpha$ standards" prepared by microprecipitation with $\mathrm{NdF}_{3}$ (Hindman, 1983; Sill, 1981) on polystyrene filters. The background count rates determined with "blank" micro-precipitates in the energy regions of interest are generally 1 to 2 counts $5000 \mathrm{~min}^{-1}$. The emitter mixture of the standards is ${ }^{238,239,242} \mathrm{Pu},{ }^{244} \mathrm{Cm}$. The standard and sample measurements are performed at a distance of $1 \mathrm{~mm}$ from the lip of the detector housing. At this distance the average detection efficiency is $40 \%$.

The detection efficiencies and resolution obtained with microprecipitated standards were checked against those obtained with electrodeposited standards and no differences were found.

The multichannel analyzers used for $\alpha$-spectrometry measurements have been thoroughly tested by EML's Instrumentation Division to ensure stability over long measurement periods (5000-10,000 $\mathrm{min})$. The stability of the systems is aided by placing them in a temperature controlled room. It is important to note that the room temperature needs to be stable. It is fluctuations in temperature that cause the detector response to shift or drift. 
A commercially available hardware/computer controlled system is used for routine measurements. There are eight vacuum modules, each housing a $500 \mathrm{~mm}^{2}$ ion implant detector. To accommodate the software requirements of the system, the detectors are calibrated with $\mathrm{NdF}_{3}$ microprecipitated "working standards" containing only two emitters, ${ }^{242} \mathrm{Pu}$ and ${ }^{244} \mathrm{Cm}$. The resolution of the detectors is about $50 \mathrm{keV}$ FWHM and the detection efficiency about $40 \%$. Background count rates are similar to our research systems.

\section{B. Calibration of the alpha-spectrometry systems.}

The detection efficiencies of the $\alpha$-spectrometry systems are determined by measuring microprecipitated "working mixed standards" prepared at EML. The activity of the standards is determined by $\alpha$-scintillation counting on systems calibrated with sources traceable to NIST through the process described in Section C, Traceability to NIST.

Measurements with single emitter sources with energies ranging from 3-6 MeV have shown that the energy and detection efficiency responses of silicon surface barrier detectors are constant. Sources of ${ }^{228} \mathrm{Th}$ in equilibrium with its short-lived progeny were measured and the activities of the individual progeny were the same within the error of the measurements. This provided empirical information that the energy response of the detectors is constant up to $9 \mathrm{MeV}$ and implies that the detection efficiencies are also constant.

It is the practice at EML to measure the background count rate of the detectors each weekend and during the week if the work load permits. Typical background count rates for the detectors are given above. The background count rates of the systems invariably increase with time due to recoil atoms depositing on the face of the solid-state detectors. It has been the experience at EML that the background count rates become unacceptable after two years of use. The only effective method of reducing the background count rates is to place the detectors in a high vacuum provided by a liquid nitrogen cooled diffusion pumping system over a weekend.

Each "working mixed standard" source has a total activity of about $15 \mathrm{~Bq}$. The standards source is measured for $50 \mathrm{~min}$ before and after each sample measurement. The standard source measurements provide three important pieces of information: the detection efficiency, the detector resolution, and the energy calibration. Deviations from 
normal operating conditions in any or all of these characteristics are immediately investigated. These quality control records are very important in establishing the norm for each detector system.

\section{REFERENCES}

Curran, S. C. and W. R. Baker "Photoelectric Alpha-Particle Detector"

Rev. Sci. Instrum., 19, 116 (1948)

Hallden, N. A. and J. H. Harley

"An Improved Alpha-Counting Technique"

Anal. Chem., 32, 1861-1862 (1960)

Hindman, F. D.

"Neodymium Fluoride Mounting for Spectrometric Determination of Uranium, Plutonium and Americium"

Anal. Chem., 55, 2460-2461 (1983)

Sill, C. W. and R. L. Williams

"Preparation of Actinides for Alpha Spectrometry without Electrodeposition" Anal. Chem., 53, 412-415 (1981) 


\section{CALIBRATION OF BETA-EMITTING RADIONUCLIDE SOLUTIONS}

\section{A. Application.}

The methods for the primary calibration of emitting radionuclide solutions and routine measurements are described for detection systems in use at EML.

\section{B. Description of the system.}

Primary calibrations of $\beta$-emitting radionuclide solutions are performed at EML with gas flow $4 \pi \beta$-proportional and $4 \pi \beta$-proportional $-4 \pi \gamma$ coincidence detection systems. The commercially obtained aluminum proportional counters have a stainless steel wire anode in each half-cylinder. The total interior volume is $50 \mathrm{~cm}^{3}$. The source mount is an aluminum washer, $3.8 \mathrm{~cm}$ in diameter with a $1.8-\mathrm{cm}$ diameter center hole and four $0.3-\mathrm{cm}$ holes in the rim to allow gas to flow between the halves with the source mount in place. The counting gas is $99.99 \%$ chemically pure methane, and the flow through the chamber is monitored with a bubbler. An EML designed and built emitter follower preamplifier is connected directly to the anodes. Signals from each half of the chamber are summed prior to further amplification. The amplifier is arranged to deliver triggered pulses to an electronic scaler. High voltage is supplied by a $0-5000 \mathrm{~V}$ regulated power supply. The chambers exhibit rather long $(600 \mathrm{~V})$ and flat $(<0.5 \%$ per $100 \mathrm{~V})$ counting plateau. The $4 \pi \beta$ detection system is unshielded and has a background rate of 0.7 counts $\sec ^{-1}$ at $3500 \mathrm{~V}$, the usual operating voltage. 
In the $4 \pi \beta-4 \pi \gamma$-coincidence detection system, the chamber is mounted between two $7.6 \times 12.7 \mathrm{~cm} \mathrm{NaI}(\mathrm{TI})$ crystals. The entire detection assembly is lead shielded. The upper crystal is pneumatically raised and lowered to permit access to the chamber. The signals from the two crystals are summed, amplified, and processed through a single channel analyzer, and simultaneously recorded into a scaler and a multichannel analyzer. High voltage to the crystals is supplied by a single $0-1500 \mathrm{~V}$ regulated power supply. As described above, the signals from the chamber are summed and amplified, but then split into the variable coincidence gate and a scaler. The output signals from the variable coincidence gate unit are fed into a third scaler. The three scalers are controlled by a master timer.

A detailed discussion of the $4 \pi \beta-\gamma$-coincidence method can be found in the National Council on Radiation Protection and Measurements Report No. 58 (NCRP, 1985).

\section{Traceability to the National Institute of Standards and Technology (NIST).}

Traceability to NIST is difficult since suitable standard reference material (SRM) sources are not available for many radionuclides. Our approach over the years has simply been to recalibrate NIST SRM solutions or solutions obtained from commercial vendors who are traceable to NIST using EML source preparation techniques and detection systems. Except for discrepancies caused by differences in the use of decay scheme parameters, the EML results are in agreement with the certified values within the error of the measurements.

\section{EML source preparation.}

Two kinds of supporting films for the source mounts are used in the proportional counters, which are described below.

The first type of film support is prepared from clear vinyl paint ${ }^{*}$ and is used with pure beta emitters with a maximum energy $>0.25 \mathrm{MeV}$. It has been established experimentally, using NIST and commercial vendor certified standard solutions of ${ }^{45} \mathrm{Ca}$,

- Flat Vinyl Ink 3900-99-Clear, obtained from: Colonial Printing Ink Co., 180-T East Union Ave., East Rutherford, NJ 07073 
that the detection efficiency of this emitter is $100 \%$ for the source mounts described below.

About $0.1 \mathrm{~mL}$ of paint is dropped onto a 1-cm deep layer of clean cold water in a $50 \mathrm{~cm} \times 75 \mathrm{~cm}$ tray, painted matte black on the interior surfaces. The paint spreads across the surface of the water and the film thickness is estimated visually, by color. A clear or light gray film is $\sim 5 \mu \mathrm{g} \mathrm{cm}^{-2}$, white is $10 \mu \mathrm{g} \mathrm{cm}^{-2}$, and yellow is $20 \mu \mathrm{g} \mathrm{cm}^{-2}$, which has been determined experimentally. At EML, the $10 \mu \mathrm{g} \mathrm{cm}^{-2}$ films are used as they provide the necessary strength, while still remaining extremely thin. The film is lifted from the surface of the water with a wire loop and placed over the aluminum washer. This thin film planchette is dried and then given a thin gold coating of about $10 \mu \mathrm{g} \mathrm{cm}^{-2}$ on one side by vacuum evaporation in a small sputterer unit. Self- absorption in the source mount is minimized using a technique developed at EML (Hallden and Fisenne, 1963). A 0.1-g aliquot of a Teflon bead suspension in water ${ }^{*}\left(100 \mu \mathrm{g}\right.$ of Teflon $\left.\mathrm{g}^{-1}\right)$ is placed onto the nonconducting side of the film. The radioactive solution to be standardized is delivered by weight from a polyethylene transfer pipette, into the Teflon bead suspension on the film. In general, a 0.1-g weight of the radionuclide solution is used in the preparation of a source. The source is air dried and then gold coated to produce an entirely conducting mount.

The second kind of source mount is a $2-\mu \mathrm{m}$ thick film of polycarbonate ${ }^{* *}$ that is glued to the aluminum washer. These mounts are used for measurements by $4 \pi \beta-4 \pi \gamma-$ coincidence counting, which will correct for the absorption within the film. The remainder of the source preparation procedure is the same as described for $4 \pi \beta$ measurements. (Note: The thin vinyl films will only tolerate an $\mathrm{HCl}$ solution of $<1 \underline{\mathrm{N}}$. The stronger polycarbonate will withstand $\mathrm{HCl}$ concentrations of up to $\mathrm{N}$. In addition, the polycarbonate source mounts can be dried gently under a heat lamp.) The graph below shows the $4 \pi \beta$ counter detection efficiency versus $\beta$ - or $\beta+$ endpoint energies for sources mounted on polycarbonate films.

The primary radionuclide solution should be essentially carrier-free. The primary radionuclide solution to be calibrated is diluted to a concentration on the order of

* Vanflex Teflon Emulsifier \#30; obtained from: Interchem Corp., 120 Route 1TN, Suite 115-T, Paramus, NJ 07652

** KIMFOIL; obtained from: Kimberly-Clark Corp., Schweitzer Division, Lee, MA 01238 
$1700 \mathrm{~Bq} \mathrm{~g}^{-1}$. The calibration is performed with triplicate source measurements. The voltage plateau for each source is determined in order to select the proper counting voltage. The activity and Poisson counting error are calculated for each source and corrected for the aliquot weight to obtain the activity $\mathrm{g}^{-1}$. Other corrections appropriate to the particular radionuclide decay scheme are made from the $4 \pi \beta-\gamma$-coincidence measurements. A Gaussian mean and SD are calculated for the triplicate sources.

Appropriate dilutions of the calibrated solution are prepared for distribution within EML for various programs.

\section{LOW BACKGROUND GAS-FLOW PROPORTIONAL COUNTING SYSTEM}

\section{A. Application.}

The only routine beta analysis conducted at EML is the determination of ${ }^{90} \mathrm{Sr}$ by measurement of ${ }^{90} \mathrm{Y}$. These measurements are performed in the LB4100 ${ }^{\mathrm{TM}}$ (Oxford Instruments Inc., Nuclear Measurements Group, 601 Oak Ridge Turnpike, Oak Ridge, TN 37831-2560) low background counting system. This system is also utilized for gross alpha/gross beta determination in water and air filter samples.

\section{B. Description of the detection system.}

The LB4100 is a four drawer, 16 detector, low background counting system that is intended for gross counting of $\alpha, \beta$ and low energy $x$-ray emitters. The detectors are of the gas-flow proportional type. The detector slide accepts samples prepared in solid form on disks either $2.54 \mathrm{~cm}$ or $5 \mathrm{~cm}$ in diameter. Two drawers (of four detectors each) are equipped with $5 \mathrm{~cm}$ detectors, which are used for gross alpha/gross beta determinations. The other two drawers are equipped with $2.54 \mathrm{~cm}$ detectors that are used for counting the $90 Y$ samples. The detectors are mounted on a $2.54 \mathrm{~cm}$ Whatman No. 42 filter paper and are covered with Mylar fastened with a ring and disc assembly.

Each sample detector is the signal source for a counting channel. Each detector activates a counting channel independently of the others. Each channel has its own time control. The channels are individually deadtime corrected. The interface between the counter and the user is a computer and its keyboard. 


\section{Calibration of the detection system for ${ }^{90} \mathrm{Y}$ determinations.}

The detectors are checked for energy plateaus and source $\left({ }^{90} \mathrm{Sr}\right)$ efficiencies prior to use or when the gas tank is changed. The background is also measured prior to use with yttrium oxalate blanks. If the instrument is idle for an extended period of time, crosstalk and yttrium oxalate efficiencies are checked. The counters are standardized with ${ }^{90} \mathrm{Y}$ oxalate with detection efficiencies of $\sim 40 \%$.

\section{LIQUID SCINTILLATION MEASUREMENTS}

\section{A. Application}

The conventional liquid scintillation (LS) counting systems used at EML are designed to detect and measure low-energy (e.g., ${ }^{3} \mathrm{H},{ }^{14} \mathrm{C}$ ) to high-energy beta particles (i.e., ${ }^{90} \mathrm{Y}$,

${ }^{106} \mathrm{Rh}$ ), and alpha particles. Samples containing mixtures of radionuclides that emit either alpha, beta, or conversion electrons are detected and quantified using variations of the LS counting technique. Depending on the LS cocktail, the alpha detection efficiency is generally $>95 \%$, whereas the beta detection efficiency is dependent on energy, spectral shape and cocktail. Typically, beta particles with maximum energies $>0.250 \mathrm{MeV}$ are detected with $>90 \%$ counting efficiency.

One variation of the LS counting technique used at EML is Cerenkov counting (Scarpitta and Fisenne, 1996), which is an adjunct to LS counting that does not require a LS cocktail. Cerenkov counting in aqueous samples is applicable to beta particles with endpoint energies in excess of $0.263 \mathrm{MeV}$. The Cerenkov counting efficiency (CCE) is typically $30 \%$ per $\mathrm{MeV}$ for beta particles with endpoint energies above the Cerenkov threshold; alpha particles are not detected.

Another variation of the $\mathrm{LS}$ technique utilized at EML is photon-electron rejecting alpha liquid scintillation spectrometry (PERALS), which is designed specifically for lowlevel liquid scintillation alpha detection where beta/gamma interferences are eliminated and a high degree of sensitivity is desired. 
The three counting methods described above require a clear liquid sample that can be counted in a short period of time ( 1 to $2 \mathrm{~h}$ ). Presented in the following sections are descriptions of these methods.

\section{B. Description of the detection system}

EML's Packard Tri-Carb-2250CA LS instrument is a multitasker unit for quantitative detection of alpha, beta, positron, and conversion electron radiation. The system may be preprogrammed to select any of the 15 unique programs stored on the computer disk. The instrument can be operated in one of four counting modes: 1) conventional LS, 2) dualDPM, 3) full spectrum analysis, or 4) efficiency tracing. The system provides computer controlled data reduction of counting results after each sample or after every batch of samples identified by the user.

The scintillation detector well is located underneath the sample changer. Samples are automatically lowered into the detector well. The detector assembly consists of two facing bi-alkali high performance photomutiplier tubes, each coupled to an optical chamber. The shielding assembly consists of a light-tight detection chamber, magnetic shielding and $5 \mathrm{~cm}$ of lead to reduce the effect of external radiation.

Spectrum analysis forms the basis of the Packard LS system. Typically, a beta particle will take a few nanoseconds to dissipate all of its energy in the scintillation solution. This results in an analog pulse rising to its maximum amplitude and falling to zero. The amplitude of the analog pulse is converted to a digital value. The conversion is achieved in a high speed analog to digital converter (ADC), and the digital value, which represents the beta particle energy, is the memory slot of a $0-2000 \mathrm{keV}$ spectrum analyzer. The conversion by the ADC is linear so that during the measurement of a sample, the spectrum analyzer will accumulate counts representing the complete energy spectrum of the radionuclide. The spectrum analyzer is calibrated in units of $\mathrm{keV}$, and the user can select one of three regions of interest over a range of $0-2000 \mathrm{keV}$.

Quenching, in general terms, refers to interferences with any of the steps of energy transferred from the solvent (where most of the energy from the ionizing radiation is initially deposited) to the scintillant and subsequent light transmission to the PMT. When a sample is quenched, the spectrum usually is shifted toward the lower energy regions, resulting in an increase in counts (i.e., apparent increase in counting efficiency) in any 
low energy preset regions of interests. In most cases, a correction for this effect may be performed.

A quench-efficiency curve must be established for a given nuclide and scintillator cocktail if the sample differs in chemical composition from the reference standard used to calibrate the instrument. A series of quenched samples, containing a known constant activity for the nuclide of interest, are prepared with a measured volume of scintillation cocktail. Successively increasing amounts of a chemical quenching agent are added to the samples. The samples are then measured for spectrum analysis. A description of the quench corrections and dual-dpm analysis can be found in Scarpitta and Fisenne, (1996).

\section{Calibration of the system.}

The LS stability and operational acceptance criteria are checked first before running any standards or samples. This is accomplished by counting the background, and a ${ }^{3} \mathrm{H}$ and ${ }^{14} \mathrm{C}$ standard provided by the instrument manufacturer using an appropriate spreadsheet program. The count rate is then compared with the quality control charts developed at EMI for this instrument. Descriptions of the cocktail selection, determination of window settings, and detection efficiencies can be found in Scarpitta and Fisenne (1996).

\section{Cerenkov Counting}

Cerenkov radiation occurs when charged particles pass through a dielectric medium (liquid) where there is an exchange of energy from the charged particle to the molecules of the medium (Haberer, 1966). The exchange energy produces local electronic polarizations in the medium if the charged particle is moving at velocities comparable to the velocity of light in the medium. When these polarized molecules return to their normal state, the excess energy is released as electromagnetic radiation known as Cerenkov radiation.

A threshold energy exists for the production of Cerenkov radiation and it is a function of the refractive index, $n$, of the medium. For water $(n=1.33)$ the lower energy limit of electrons for the production of Cerenkov radiation is $0.263 \mathrm{MeV}$ (Berger and King, 1985 ). With beta emitters, the maximum energy, $E_{\max }$, has to substantially exceed this value because of the broad beta energy spectrum. Photoelectrons and Compton electrons 
from gamma emitting nuclides, having energies in excess of the threshold energy, can also be measured by Cerenkov counting. Some nuclides which can be measured by Cerenkov counting are ${ }^{32} \mathrm{P},{ }^{35} \mathrm{~S},{ }^{36} \mathrm{Cl},{ }^{40} \mathrm{~K},{ }^{59} \mathrm{Fe},{ }^{89} \mathrm{Sr},{ }^{90} \mathrm{Sr} / \mathrm{Y},{ }^{106} \mathrm{Rh}(\mathrm{Ru}),{ }^{137} \mathrm{Cs},{ }^{210} \mathrm{~Pb} / \mathrm{Bi}$, ${ }^{234 \mathrm{~m}} \mathrm{~Pa}$ (progeny of ${ }^{238} \mathrm{U}$ via ${ }^{234} \mathrm{Th}$ ), and ${ }^{99} \mathrm{Tc}$.

Cerenkov counting is an adjunct to LS analysis that does not require a scintillating cocktail. Various wavelength shifters (Haberer, 1966; Karamanos et al., 1975) have been used to enhance the CCE by $10-20 \%$ using commercially available LS spectrometers. Wave shifters are organic substances that shift the Cerenkov photon energies from a region of low detector sensitivity to a region of high detector sensitivity.

Standardized solutions, containing the equivalent of about $17 \mathrm{~Bq}(1000 \mathrm{dpm})$, are dispensed gravimetrically, in triplicate, into either $20 \mathrm{~mL}$ plastic or low borosilicate glass vials. Each vial was counted for 15-30 min with window settings of 0-50 keV for Cerenkov counting so that the 1 sigma counting error is $<2 \%$.

For Cerenkov counting, water is typically used as the solvent. The average instrument background, using $20-\mathrm{mL}$ plastic vials containing $3-18 \mathrm{~mL}$ of ultra-pure water is $0.225 \pm$ 0.018 counts sec$^{-1}$ (cps) for a $0-50 \mathrm{keV}$ region of interest. The average background count rate for glass vials $(0.346 \pm 0.010 \mathrm{cps})$ is about $30 \%$ higher than that obtained using plastic vials. Based on replicate background measurements, the lower limit of detection for a $1 \mathrm{~h}$ count at the $95 \%$ confidence level, using water as a solvent, is $0.024 \mathrm{cps}(1.44$ $\mathrm{cpm})$ and $0.028 \mathrm{cps}(1.70 \mathrm{cpm})$ for plastic and glass vials, respectively. For specific alpha/beta applications, see Scarpitta and Fisenne (in press).

\section{E. PERALS Spectrometry}

EML's PERALS spectrometer (Ordela, Inc., 1009 Alvin Weinberg Drive, Oak Ridge, TN 37830) which is a single sample stand-alone Nim Bin type unit. The unit is interfaced to a personal computer which is capable of functioning as a multichannel analyzer. The PERALS technique employs a LS detector with a pulse-shape discriminator for beta-gamma rejection (>98\%) and direct extraction of the alpha activity of interest into $2 \mathrm{~mL}$ of a water insoluble organic phase containing the scintillator. The discriminator takes advantage of the longer decay time of light produced by alpha particle interactions to select the alpha pulses for spectrometry and reject those from beta-particle or gamma-ray events. 
The choice of commercially available extractants (ETRAC Corporation) and chemical composition of the aqueous phase allows for selectivity and flexibility in sample preparation (McDowell and McDowell, 1994). The degree of beta/gamma rejection allows an instrument background (with a blank scintillator in the detector) of $0.001 \mathrm{cpm}$ or less over the entire alpha energy range (3-5 MeV). The advantages of the PERALS method are that it: 1) is extremely sensitive, 2) has a high alpha counting efficiency $(100 \%), 3)$ requires a count time of $1-4 \mathrm{~h}$ per sample or less depending on the sample activity, and 4 ) generates a very small amount of organic waste ( $<2 \mathrm{~mL}$ per sample).

\section{REFERENCES}

Berger, S. and M. King

"A Simple Method for Quantifying ${ }^{32} \mathrm{P}$ in Submicroliter Samples"

Biotechniques, 9, 38-46, Jan.-Feb. (1985)

Haberer, $\mathrm{K}$.

"Measurement of Beta Activities in Aqueous Samples Utilizing Cerenkov Radiation"

Packard Technical Bulletin No. 16, Packard Instrument Co., Downers Grove, $I$, January (1966)

Hallden, N. A. and I. M. Fisenne

"Minimizing Self-absorption in 4-Counting"

Int. J. Appl. Rad. and Isot., 14, 529-532 (1963)

Karamanos, R., J. Bettany and D. Rennie

"Lead-210 Assay in Soil and Plant Material Using Cerenkov Radiation"

Canadian. J. Soil Sci., 55, 407-13 (1975)

McDowell, W. J. and B. L. McDowell.

Liquid Scintillation Alpha Spectrometry.

CRC Press, 2000 Corporate Blvd., N.W. , Boca Raton, FL (1994)

National Council on Radiation Protection and Measurements

A Handbook of Radioactivity Measurements Procedures

NCRP Report No. 58, Bethesda, MD (1985) 
Scarpitta, S. C. and I. M. Fisenne

"Cerenkov Counting as a Complement to Liquid Scintillation Counting"

Appl. Radiat. Isot., 47, 795-800 (1996)

Scarpitta, S. C. and I. M. Fisenne.

"Calibration of a Liquid Scintillation Counter for Alpha, Beta and Cerenkov Counting" USDOE Report EML-583, July (1996) 


\begin{abstract}
APPLICATION
This procedure is used for the nondestructive measurement of $\gamma$-ray emitting radionuclides from a variety of environmental matrices by high resolution germanium (Ge) detector $\gamma$-ray spectrometry and $\mathrm{NaI}(\mathrm{Tl})$ detector $\gamma$-ray spectrometry. It is applicable to nuclides emitting $\gamma$-ray with energies $>20 \mathrm{keV}$ for Ge detectors and $50 \mathrm{keV}$ for $\mathrm{NaI}(\mathrm{Tl})$ detectors. For typical counting systems and sample types, activity levels of about $40 \mathrm{~Bq}$ are easily measured and sensitivities as low as $.002 \mathrm{~Bq}$ can be achieved for many nuclides. Count rates in excess of 2000 counts sec$^{-1}$ (cps) should be avoided because of electronic limitations. High count rate samples can be accommodated by dilution or by increasing the sample to detector distance.
\end{abstract}

The procedure is used for either qualitative, quantitative or relative determinations. In tracer work, the results may be expressed by comparison with an initial concentration of a given nuclide which is taken as $100 \%$. For radioassay, the results may be express-ed in terms of known standards for the radionuclides known to be present. In addition to the quantitative measurement of $\gamma$-ray radioactivity, $\gamma$-ray spectrometry can be used for the identification of specific emitters in a mixture of radionuclides. General information on radioactivity and the measurement of radiation has been published. Information on the specific application of $\gamma$-ray spectrometry is also available in the literature. 


\section{DESCRIPTION OF THE SYSTEM}

Gamma-ray spectra are measured at EML with modular equipment consisting of a detector, an amplifier, a pulse-height analyzer, memory, and a permanent data storage device. Lithium-drifted germanium, $\mathrm{Ge}(\mathrm{Li})$, or high purity $\mathrm{Ge}$ detectors (p-type or $\mathrm{n}$-type) are used for the analysis of complex $\gamma$-ray spectra because of their excellent energy resolutions. These Ge detectors, however, are characterized by high cost and require cooling with liquid nitrogen. Thallium activated sodium-iodide crystals, $\mathrm{NaI}(\mathrm{Tl})$, can be operated at ambient temperatures and are often used at EML as $\gamma$-ray detectors in spectrometer systems. However, their energy resolutions limit their use to the analysis of single nuclides or simple mixtures of a few nuclides.

Upon completion of the $\gamma$-ray assay, the spectral data are interpreted and reduced to nuclide activities in $\mathrm{Bq}$ (disintegrations per second) or related units suited to the particular application. At this time, the spectral data may be inspected on the CRT to identify the $\gamma$-ray emitters present. This is accomplished by reading the channel number from the $\mathrm{x}$-axis and converting to $\gamma$-ray energy by multiplying by the appropriate $\mathrm{keV} /$ channel (system gain). If the system is calibrated for $1 \mathrm{keV}$ per channel with channel zero representing $0 \mathrm{keV}$, the energy will be equal to the channel number. The channel number or $\gamma$-ray energy in $\mathrm{keV}$ is usually displayed on the CRT. Identification of nuclides is aided by catalogs of $\gamma$-ray spectra and other nuclear data tabulations. Because of the reduced spectral resolution obtained with $\mathrm{NaI}(\mathrm{Tl})$. detectors, this technique can only be applied to samples of single nuclides or very simple combinations of nuclides.

Data reduction of spectra taken with Ge spectrometry systems is usually accomplished by integration of the photopeaks above a definable background (or baseline), and by subsequent activity calculations using a library that includes data such as nuclide name, half-life, $\gamma$-ray energies and associated abundance (intensity or branching ratios). Computer programs for data reduction of $\mathrm{NaI}(\mathrm{Tl})$ detector data have been used extensively at EML. Data reduction of spectra involving mixtures of nuclides is usually accomplished by least-square fitting routines to a library of standard spectra of the individual nuclides acquired under individual conditions.

Variation of the physical geometry of the sample and its relationship with the detector will produce both qualitative and quantitative variations in the $\gamma$-ray spectrum. To 
adequately account for these geometry effects, calibrations are designed to duplicate all sample counting conditions including source-to-detector distance, sample shape and size.

Electronic problems, such as erroneous deadtime correction, loss of resolution, and random summing, may be avoided by keeping the gross count rate below $2000 \mathrm{cps}$ and also by keeping the deadtime of the analyzer below 5\%. Total counting time is governed by the radioactivity of the sample, the detector-to-source distance, and the acceptable Poisson counting uncertainty.

In complex mixtures of $\gamma$-ray emitters, the degree of interference of one nuclide in the determination of another is governed by several factors. If the $\gamma$-ray emission rates from different radionuclides are similar, interference will occur when the photopeaks are not completely resolved and overlap. If the nuclides are present in the mixture at markedly different levels of activity, nuclides of higher energies that are predominant can cause serious interferences with the interpretation of minor, less energetic, $\gamma$-ray photopeaks. The complexity of the analysis method is due to the resolution of these interferences and, thus, one of the main reasons for computerized systems.

Cascade summing may occur when nuclides that decay by a $\gamma$-ray cascade are analyzed. Cobalt-60 is an example; 1172 and $1333 \mathrm{keV} \gamma$-ray from the same decay may enter the detector to produce a sum peak at $2505 \mathrm{keV}$ or a count in the continuum between the individual peaks and the sum peak; thus, causing the loss of counts from one or both of the other two peaks. Cascade summing may be reduced by increasing the source-to-detector distance. Summing is more significant if a well-type detector is used.

Random summing is a function of counting rate and occurs in all measurements. The random summing rate is proportional to the total count squared and the resolving time of the detection system. For most systems, random summing losses can be held to $<1 \%$ by limiting the total counting rate to $1000 \mathrm{cps}$.

The density of the sample is another factor that can affect quantitative results. Errors from this source can be avoided by preparing the standards for calibration in solutions or other matrices with a density comparable to the sample being analyzed. Another approach is to apply attenuation corrections to all calibration standards and samples based on sample weight, known volume, $\gamma$-ray path length and average atomic number of the sample matrix. 


\section{APPARATUS}

A $\gamma$-ray spectrometer consists of the following components:

\section{A. Detector assembly.}

1. Germanium detector - The detector should have a volume of at least $50 \mathrm{~cm}^{-3}$, with a full width at one half the peak maximum (FWHM) $<2.2 \mathrm{keV}$ at $1332 \mathrm{keV}$, certified by the manufacturer. A charge-sensitive preamplifier using low noise field effect transistors should be an integral part of the detector assembly. A convenient support should be provided for samples of the desired form. Vertical systems allow the standard/sample to be placed directly on the detector end cap.

2. $\mathrm{NaI}(\mathrm{Tl})$ detector - The sodium iodide crystal, activated with about $0.1 \%$ thallium iodide, should contain $<5 \mu \mathrm{g} \mathrm{g}^{-1}$ of $\mathrm{K}$ and should be free of other radioactive materials. The crystal should be attached and optically coupled to a multiplier phototube. (The multiplier phototube requires a preamplifier or a cathode follower compatible preamplifier with the amplifier.) The resolution (FWHM) of the assembly for the photopeak of ${ }^{137} \mathrm{Cs}$ should be $<7 \%$ for a $75-\mathrm{mm}$ by $75-\mathrm{mm}$ detector.

3. Shield - The detector assembly should be surrounded by an external radiation shield made of massive metal, equivalent to $102 \mathrm{~mm}$ of $\mathrm{Pb}$ in $\gamma$-ray attenuation capability. It is desirable that the inner walls of the shield be at least $127 \mathrm{~mm}$ in distance from the detector surfaces to reduce backscatter. If the shield is made of $\mathrm{Pb}$ or $\mathrm{a} \mathrm{Pb}$ liner, the shield must have a graded inner shield of $1.6 \mathrm{~mm}$ of $\mathrm{Cd}$ or tin lined with $0.4 \mathrm{~mm}$ of copper, to attenuate the $88 \mathrm{keV} \mathrm{Pb} \gamma$-rays. The shield must also have a door or port for inserting and removing samples.

4. High-voltage power/bias supply - The bias supply required for Ge detectors usually provides a voltage up to $5000 \mathrm{~V}$ and 1 to $100 \mu \mathrm{A}$. NaI(Tl) detectors require a highvoltage power supply of a range of usually from 500 to $3000 \mathrm{~V}$ and up to $10 \mathrm{~mA}$ to operate the multiplier phototube. The power supply should be regulated to $0.1 \%$ with a ripple of not more than $0.01 \%$. Line noise caused by other equipment should be removed with filters and additional regulators. 
5. Amplifier - A spectroscopy amplifier compatible with the preamplifier and with the pulse-height analyzer should be used.

\section{B. Data acquisition and storage equipment.}

1. Data acquisition - A multichannel pulse-height analyzer (MCA) or stand-alone analog-to-digital converter $(\mathrm{ADC})$ under software control of a separate computer, performs many functions that are required for $\gamma$-ray spectrometry. An MCA or computer collects the data, provides a visual display, and outputs final results or raw data for later analysis. The four major components of an MCA are the ADC, the memory, control, and input/output. The $\mathrm{ADC}$ digitizes the analog pulses from the detector amplifier. The magnitude of these pulses is proportional to the energy of the photon deposited in the detector. The digital result is used by the MCA to select a memory location (channel number) which is used to store the number of events which have occurred with that energy. Simple data analysis and control of the MCA is accomplished with microprocessors. These processors control the input/output, channel summing over set regions of interest, and system energy calibration, etc.

2. Data storage - Because of the use of microprocessors, modern MCAs provide a wide range of input and output $(\mathrm{I} / \mathrm{O})$ capabilities. Typically, these capabilities include the ability to transfer any section of data to one or more of the following: terminal, line printer, cassette tape, floppy or hard disk, X-Y plotter, and to computer interfaces via a serial or parallel port.

\section{SAMPLE/STANDARD CONTAINERS}

Sample mounts and containers must have a convenient reproducible geometry. Considerations include commercial availability, ease of use and disposal, and the containment of radioactivity for protection of the personnel and working environment from contamination. The evaporation of liquid samples to dryness is not necessary and liquid samples up to $1 \mathrm{~L}$ may be used. Massive samples may cause significant selfabsorption of low energy $\gamma$-rays and may degrade the higher energy $\gamma$-rays. A $\beta$ absorber consisting of about $6 \mathrm{~mm}$ of $\mathrm{Al}, \mathrm{Be}$, or plastic may be used for samples that have a significant $\beta$ activity and high $B$ energies. 


\section{CALIBRATION AND STANDARDIZATION}

This section describes the analysis of mixtures of radionuclides with $\mathrm{Ge}$ detectors or single or simple mixtures of radionuclides with $\mathrm{NaI}(\mathrm{Tl})$ detectors. If complex mixtures of radionuclides are to be analyzed with $\mathrm{NaI}(\mathrm{Tl})$ detectors, refer to page 2 of this procedure.

\section{A. Preparation of apparatus.}

Follow the manufacturer's instructions, limitations, and cautions for the setup and the preliminary testing of all of the spectrometry equipment to be used in the analysis. This equipment would include, as applicable: detector, power supplies, preamplifiers, amplifiers, multichannel analyzers, and computing systems.

Place an appropriate volume of a standard or a mixed standard of radionuclides in a sealed container and place the container at a desirable and reproducible source-to-detector distance. For environmental analysis, most standards/samples are counted at the detector end cap. The standard should provide about $100 \mathrm{cps}$ in the peaks of interest and should be made up of standard sources traceable to a nationally certified laboratory. In all radionuclide measurements, the volumes, shape, physical and chemical characteristics of the samples, standards and their containers must be as identical as practicable for the most accurate results.

\section{B. Energy calibration.}

The energy calibration (channel number of the MCA versus the $\gamma$-ray energy) of the detector system is accomplished at a fixed gain using standards containing known radionuclides. The standards should be in sealed containers and should emit at least four different $\gamma$-ray energies covering the range of interest, usually $50 \mathrm{keV}$ to $2000 \mathrm{keV}$ in order to test for system linearity. Some commercially available nuclides suitable for energy calibration are: ${ }^{210} \mathrm{~Pb}, 46.5 \mathrm{keV} ;{ }^{241} \mathrm{Am}, 59.5 \mathrm{keV} ;{ }^{109} \mathrm{Cd}, 88 \mathrm{keV} ;{ }^{141} \mathrm{Ce}, 145 \mathrm{keV}$; ${ }^{51} \mathrm{Cr}, 320 \mathrm{keV} ;{ }^{137} \mathrm{Cs}, 662 \mathrm{keV} ;{ }^{54} \mathrm{Mg}, 835 \mathrm{keV} ;{ }^{22} \mathrm{Na}, 511$ and $1275 \mathrm{keV} ;{ }^{88} \mathrm{Y}, 898$ and $1836 \mathrm{keV} ;{ }^{60} \mathrm{Co}, 1173$ and $1332 \mathrm{keV}$; equilibrated ${ }^{226} \mathrm{Ra}, 186,352,609,1120$, and 1765 $\mathrm{keV}$. A mixed $\gamma$-ray standard for energy and efficiency calibration is also commercially available. This standard can be obtained in solid form in a user supplied container. The radionuclide purity of the standards should be verified periodically to ensure against 
accidental contamination or the presence of long-lived impurities by comparing the observed spectra with the spectra published in the literature.

A MCA should be calibrated to cover the range of interest. If the range is 50 to 2000 $\mathrm{keV}$, the gain of the system should be adjusted until the ${ }^{137} \mathrm{Cs}$ photopeak, $662 \mathrm{keV}$, is about one-third full scale. Leaving the gain constant, locate at least three other photopeaks of different energies, covering the same range. Determine and record the MCA channel number corresponding to the maximum count rate for each of the four energies. Germanium detectors will have a linear relationship if the equipment is operating properly. Similarly, MCAs and NaI(TI) detectors being produced today are capable of producing an almost linear energy response. Samples should not be analyzed if this relationship is not obtained. Follow the appropriate manufacturer input instructions for the determination of the slope and intercept. During each day in which the spectrometry system is being used to analyze samples, the above sequence of operation shall be repeated using at least two different energies. If the slope and intercept are essentially unchanged, the energy calibration data remain valid. If an appreciable change in the slope or intercept is evident, the entire calibration procedure must be rerun.

\section{Photon detection efficiency calibration.}

Accumulate an energy spectrum using sealed, calibrated radioactivity standards in a desired and reproducible counting geometry. At least 10,000 net counts (total counts minus the compton continuum and ambient background) should be accumulated in each full-energy $\gamma$-ray peak of interest.

Correct the radioactivity standard source $\gamma$-ray emission rate for the decay from the time of standardization to the time at which the count rate is measured.

Calculate the full-energy peak efficiency Ef as follows:

where

$$
\mathrm{Ef}=\mathrm{Np} / \mathrm{Ng}
$$

$\mathrm{Ef}=$ full-energy peak efficiency (counts per $\gamma$-ray emitted),

$\mathrm{Np}=$ net $\gamma$-ray count in the full-energy peak of interest (cps), and 


$$
\mathrm{Ng}=\gamma \text {-ray emission rate }\left(\text { rays } \sec ^{-1}\right)
$$

If the standard source is calibrated as to activity, the $\gamma$-ray emission rate is given by:

$$
\mathrm{Ng}=\mathrm{A}^{*} \mathrm{Pg}
$$

where

$\mathrm{A}=$ number of nuclear decays per second, and

$\mathrm{Pg}=$ probability per nuclear decay for the $\gamma$-ray.

For Ge detectors, plot the values for the full-energy peak efficiency versus $\gamma$-ray energy. The plot will allow the determination of efficiencies at energies for which standards are not available, and will show that the algorithms used in computerized systems are providing valid efficiency calibrations.

Once the efficiencies have been determined, it is unnecessary to recalculate them unless there is a change in resolution, geometry, or system configuration.

\section{SAMPLE MEASUREMENTS}

After the spectrometer system has been set up, the energy and efficiency calibrations are performed, then the unknown sample can be measured.

Following the general concepts of quantitative analytical chemistry, transfer the sample to the specimen container and position it in the same manner as was done during system calibration.

Measure the sample for a period of time long enough to acquire a $\gamma$-ray spectrum which will meet the minimum acceptable counting uncertainty. 


\section{PEAK AREA CALCULATIONS}

Spectral data obtained with a Ge detector are only corrected for background when these peaks may alter the final results. In many experiments, the background may not affect the results but is still monitored to ensure the integrity of the system.

The underlying aim of this procedure is to subtract the continuum or baseline from the spectral data where it underlies a photopeak of interest. For operator-directed calculations, the choice of the baseline level may be straightforward. The simplest way, using a plot of the spectral data, is to draw a straight line, using judgement and experience, that best describes the baseline. Then the baseline data can be read directly from the plot and subtracted.

Photopeaks lying on a sloping baseline or one with curvature will be analyzed, independent of the method, with increased uncertainty. Use of data from these peaks should be limited to those cases where there is no other alternative. Photopeaks that overlap with each other will also increase the uncertainty of the final result.

In order to determine nuclide concentrations, the photopeak areas corrected for background and interferences are divided by the count time and efficiency for the energy of the $\gamma$-ray being calculated to give photons $\sec ^{-1}$ for the peak of interest. If, as is the case for some nuclides, the branching ratio is not accurately known and a direct calibration was made with the same nuclide, the branching ratio and efficiency will be one number that converts $\mathrm{cps}$ to $\mathrm{Bq} \mathrm{sec} \mathrm{s}^{-1}$ for the nuclide and photopeak of interest. If not, the photons $\mathrm{sec}^{-1}$ are converted to disintegrations $\mathrm{sec}^{-1}$ by dividing the photons $\mathrm{sec}^{-1}$ by the photons per disintegration, for the nuclide and photopeak of interest. The results are then corrected for attenuation or decay, or both.

Canberra Industries MicroSampo Version 2.0 (a commercial software package) is used at EML to perform these calculations.

While the uncertainty due to counting and calibration may represent a significant proportion of the total uncertainty in the measurement, systematic uncertainties should be determined and included in the above calculation. Systematic uncertainties include, but 
are not limited to, reproducibility of sample position, peak analyses, decay calculations, background subtraction, pulse pile-up, cascade summing corrections, and self-absorption corrections.

\section{ANALYSIS OF COMPLEX MIXTURES OF NUCLIDES WITH NAI(TI) DETECTORS}

Because of the inherent energy resolution of $\mathrm{NaI}(\mathrm{TI})$ detectors, $\boldsymbol{\gamma}$-ray peaks in complex mixtures of nuclides may not be separated sufficiently for quantification as outlined above. It may not even be possible to visually locate individual peaks if their energies are similar or their intensity is too low in relation to other $\gamma$-rays present in the spectrum. Complex mixtures of as many as 10 to 20 radionuclides can be quantified mathematically with computer programs using linear least squares techniques.

When using these techniques, care should be given to the following parameters.

\section{A. System gain and zero energy channel.}

The exact gain and zero energy channel of the spectrometer must be monitored and recorded. If the computer analysis program performs gain and/or baseline (zero energy channel) corrections on sample data, then the library of standards data must be obtained under uniform and precise calibration conditions.

\section{B. Library standards.}

The least squares analysis technique is a linear combination of all of the data contained in the standards library. Therefore, the standards library must contain a spectra of every component in the sample; in addition, these spectra must be obtained from the purest radionuclides available.

\section{Counting of library standards.}

All $\gamma$-ray spectra will contain a background component. The activity of the library standards must be high enough so that this background component will be insignificant, even though all computer programs make some kind of a background correction. 
The duration of the counting period for the standard library spectra should be long enough to obtain statistically valid data, but it should be short enough so that analyzer gain and baseline drifts are insignificant.

The activity of the library standards should be chosen so that the counting rates of the predominant photopeaks are all about the same.

A very important data evaluation technique to be used with a least squares program is a superimposed plot of the original sample data and the computed spectral data. A plot of residuals (the difference between the original and computed spectra) is also very important. The residuals plot is very sensitive to errors that are caused by omitting radionuclides present in the sample from the library standards.

\section{SAMPLE MEASUREMENTS}

After the spectrometer system has been set up, the energy calibrations performed, and individual pulse-height spectra for nuclides expected to be present in samples are obtained, then the unknown specimens can be measured and quantified.

Following the general concepts of quantitative analytical chemistry, transfer the sample to the specimen container and position it in the same manner as was done during system calibration. Measure the sample for a period of time long enough to acquire a $\gamma$ ray spectrum which will meet the minimum acceptable counting uncertainty.

\section{COMPUTER CALCULATIONS}

WLSQ is an EML least squares Fortran computer program that can be run on a VAX or IBM-compatible PC to resolve complex spectra.

Systematic uncertainties include, but are not limited to: reproducibility of sample position, peak analyses, decay calculations, background substraction, pulse pile up, cascade summing operations, and self-absorption corrections. 
The uncertainty obtained from the least squares analysis can be substituted for the uncertainty in counting and should be included to obtain an overall uncertainty of the analysis. The uncertainties obtained from the least squares analysis are the square roots of the diagonal elements of the inverse matrix used to solve the linear set of simultaneous equations representing the sample spectra.

\section{QUALITY CONTROL}

The following quality control procedures are required so that the $\gamma$-ray spectrometers maintain their energy calibrations. In addition, the systems are to be monitored so that degradation in performance will be noticed as soon as possible.

\section{A. Daily calibration checks.}

The energy calibration of each Ge $\gamma$-ray detector is determined daily with a mixed nuclide source consisting of ${ }^{241} \mathrm{Am}$ and ${ }^{60} \mathrm{Co}$.

In order to maintain an energy calibration of $0.5 \mathrm{keV} /$ channel, count the Am-Co source to obtain well-defined peaks. The $59.5 \mathrm{keV} \gamma$-ray line from ${ }^{241} \mathrm{Am}$ should fall in channel 119. The $1332.5 \mathrm{keV} \gamma$-ray line from ${ }^{60} \mathrm{Co}$ should fall in channel 2665.

If the ${ }^{241} \mathrm{Am}$ and ${ }^{60} \mathrm{Co}$ peaks do not fall in the correct channels, first adjust the DC offset of the amplifier so that the $59.5 \mathrm{keV} \gamma$-ray line falls in channel 119. Then adjust the fine gain of the amplifier so that the $1332.5 \mathrm{keV} \gamma$-ray line falls in channel 2665.

Recount the Co-Am calibration standard to verify the peak positions and readjust the amplifier if necessary.

The energy calibration of each $\mathrm{NaI}(\mathrm{Tl}) \gamma$-ray detector is determined daily with a nuclide source consisting of ${ }^{207} \mathrm{Bi}$.

In order to maintain an energy calibration of $10 \mathrm{keV} / \mathrm{channel}$, count the ${ }^{207} \mathrm{Bi}$ source to obtain well-defined peaks. The $570 \mathrm{keV} \gamma$-ray line should fall in channel 57. The 1064 $\mathrm{keV} \gamma$-ray line should fall in channel 106. 
If the ${ }^{207} \mathrm{Bi}$ peaks do not fall in the correct channels, first adjust the DC offset of the amplifier so that the $570 \mathrm{keV} \gamma$-ray line falls in channel 57. Then adjust the fine gain of the amplifier so that the $1064 \mathrm{keV} \gamma$-ray line falls in channel 106.

Recount the ${ }^{207} \mathrm{Bi}$ calibration standard to verify the peak positions and readjust the amplifier if necessary.

\section{B. Weekly efficiency calibration and resolution checks.}

Each week the same ${ }^{137} \mathrm{Cs}$ calibration standard is counted, recorded, analyzed, and the date is entered into a permanent data base for each $\gamma$-ray spectrometry system.

Count the ${ }^{137} \mathrm{Cs}$ calibration standard in the same manner as unknown samples. Record the data for permanent storage and perform the usual data reduction analysis.

Enter the results of the analysis (Bq) and the resolution of the ${ }^{137} \mathrm{Cs}$ peak (full width at half maximum in $\mathrm{keV}$ ) in the $\gamma$-quality control data base.

Report any deviation from the expected values before samples are analyzed. If remedial action is necessary, the cause and solution of the problem must be recorded in the laboratory logbook. A complete recalibration must be performed if any remedial actions have been taken. 


\subsubsection{Quality Control and Detection Limits}




\subsubsection{QUALITY CONTROL AND DETECTION LIMITS}

\section{QUALITY CONTROL}

Quality control (QC) must be included in all aspects of a radiochemical measurement, from the collection of samples to the reporting of data. The exact $\mathrm{QC}$ practices that are to be used as part of the radiochemical procedures will vary somewhat, depending upon the chemical and radiological characteristics of the analyte. Some of the general requirements of a $\mathrm{QC}$ system for procedures are described here. Where the peculiarities of an analyte necessitate that modifications be made in these requirements, these modifications are discussed in the subsection after the analytical procedure for that analyte. The QC procedures described here are termed "internal" in that they are initiated by the analyst. Similarly, "external" QC procedures should be initiated by project leaders introducing QC samples "blind" into the sample stream. Data from both the internal and external QC procedures must be maintained and tabulated by the party initiating the procedures to document the continuing adequacy of the analytical work.

The QC practices used during radiochemical analyses are intended to assure the analyst that the determinations are under control. They involve continuous testing of those processes that influence the extent to which the results of the analyses remain within the required limits of precision and accuracy. The QC samples that are analyzed consist of five types: instrument calibration standards, blank samples, control samples, "spiked" samples, and replicate samples.

Instrument calibration standards are certified reference materials used primarily to calibrate the measurement apparatus. Standards for radioactivity measurements are discussed in Section 4.5.2. A key requirement of such materials is that they be traceable to the National Institute of Standards and Technology or to other recognized organizations.

Blank samples are commonly "reagent blanks" that are prepared by beginning with deionized water or an empty sample container. All appropriate reagents are added to the sample in the proper sequence and the normal steps involved in the analysis are followed. Ideally, the blank samples would be the same matrix as the routine sample but without the 
analyte of interest. Normally, the activity of each routine sample is corrected by subtracting the reagent blank activity from it to obtain net activity. All the uncertainties of the measurements obtained throughout the analytical procedure should be propagated when calculating the uncertainty of the final result. However, very often only the Poisson errors of the counts of the reagent blank and of the sample are propagated when they are the most significant contributors to the total uncertainty.

Control samples contain known concentrations of the analyte. If possible, they should be the same matrix as the routine samples, and they should have concentrations in the same range as the routine samples. They usually are samples that are included by the analyst in the sample batches to be analyzed, and their values should be known with an uncertainty better than that which will be required of measurements of the routine samples.

"Spiked" samples are prepared by adding a known amount of the radionuclide of interest to blank samples or to samples that have already been analyzed in order to provide a matrix with a known activity.

Replicate samples usually consist of two or more aliquots of homogeneous solid, liquid or gas samples. Individual samples that are measured by nondestructive techniques, such as $\gamma$-ray spectrometry, may be measured more than once to obtain replication of the data.

Analytical instruments, such as analytical balances, must be calibrated regularly. The standards that are used to calibrate any instrument should be traceable to the National Institute of Standards and Technology (NIST), when possible. A record of instrument performance must be maintained by the instrument operator. A record must be kept of any modifications that are made in an instrument, and such modifications must be approved by the analyst's supervisor beforehand and must conform to the safety standards and practices that are specified in the EML Safety Manual.

For most radiochemical procedures, QC samples are added to make up between $10-20 \%$ of the sample stream. It is good analytical practice to process high-level and low-level samples in independent batches whenever possible to minimize the possibility of cross contamination. When samples of very low activity are to be analyzed, blank sample analyses and instrument background measurements should be increased. 
The best estimates of a reagent blank or blank sample activity, instrument background count rate and detection efficiency are obtained from the mean value of replicate determinations. Whenever possible, the mean and standard error of the replicate determinations should be used in calculating a final value.

\section{ESTIMATING THE LOWER LIMIT OF DETECTION}

Counting data are like other analytical data in that a series of measurements of background, for example, should show a Gaussian distribution like that in Figure 1. The standard deviation (SD) for the distribution will give an estimate of how well additional background measurements will approach the original mean. In most environmental analyses, however, we cannot afford the luxury of multiple analyses or even duplicates. Fortunately, counting data follow the Poisson distribution and it is possible to estimate the SD from a single measurement. This SD of counting can be used in the same way as the Gaussian SD to establish a confidence interval about the mean value. For example, if the confidence interval chosen is $\pm 1 \mathrm{SD}$, additional measurements should fall into this interval $68 \%$ of the time. An interval of $\pm 2 \mathrm{SD}$ would contain the new value $95 \%$ of the time.

Thus, if we establish a background with its associated SD for a counting system, this should give us a way of estimating our lower limit of detection (LLD). For example, a sample count $>1$ SD above background should indicate the presence of activity $84 \%$ of the time. In the other $16 \%$, there would be a false positive. If the limit of $2 \mathrm{SD}$ is used, the values would be $97.5 \%$ real and $2.5 \%$, false with $3 \mathrm{SD}, 99.85 \%$ and $0.15 \%$. This seems to be very simple, but it does not consider the fact that the sample counts also would have a distribution.

The interaction of the two distributions becomes more important as environmental analyses tend toward lower levels of radioactivity. As the activity decreases, the counter background or method blank has to be subtracted from the gross count, and estimating the precision of these measurements and also the limit of detection for a procedure becomes slightly more difficult. Where the total count on a sample approaches the background count, you have a situation as in Figure 2 where the two distributions overlap. [This form of showing the detection limit for counting was given by Healy (1956).] 


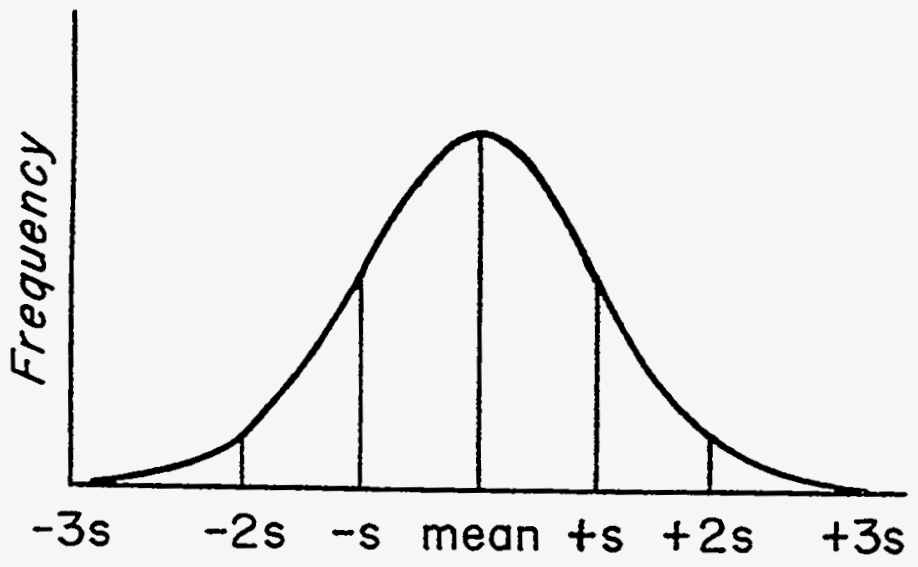

Figure 1. Distribution curve where the relative areas show the fraction of results that should be included within $\pm s(0.68), \pm 2 s(0.95)$ and $\pm 3 s(0.997)$.



Figure 2. Intersecting distributions for background and sample count rates. 
Pasternack and Harley (1971) developed a procedure for calculating what they defined as the LLD, the smallest amount of sample activity that will yield a net count for which there is a confidence at a predetermined level that activity is present. This concept was only practical for $\gamma$ counting in the original form since they required that the number of counts be sufficient for the Poisson distribution to approach the Gaussian distribution so that Gaussian statistics could be used.

However, the approximation is valid down to a few total counts. Thus, the calculations can be applied to any detection system.

The LID can be approximated as

$$
\mathrm{LLD} \cong\left(\mathrm{k}_{\alpha}+\mathrm{k}_{\beta}\right) \mathrm{s}_{0}
$$

where

$\mathrm{k}_{\alpha}$ is the value for the upper percentile of the standardized normal variate corresponding to the preselected risk for concluding falsely that activity is present $(\alpha)$,

$\mathrm{k}_{B}$ is the corresponding value for the predetermined degree of confidence for detecting the presence of activity $(1-\beta)$, and

$s_{0}$ is the estimated standard deviation for the net sample activity.

A still shorter approximation may be made if the values of $\alpha$ and $B$ are set at the same level and if the gross activity and background are very close. In this case:

$$
s_{0}=\sqrt{s_{\text {gross }}^{2}+s_{\text {bkg }}^{2}}=s_{b} \sqrt{2}
$$

The equation then becomes,

$$
L L D \cong 2 \mathrm{ks}_{\mathrm{bkg}} \sqrt{2}
$$


The values of $\mathrm{k}$ for common $\alpha$ 's are:

\begin{tabular}{|c|c|c|c|}
\hline$\alpha$ & $1-\beta$ & $\mathrm{k}$ & $\underline{2 \mathrm{k}} \sqrt{2}$ \\
\hline 0.01 & 0.99 & 2.327 & 6.59 \\
\hline 0.02 & 0.98 & 2.054 & 5.81 \\
\hline 0.05 & 0.95 & 1.645 & 4.66 \\
\hline 0.10 & 0.90 & 1.282 & 3.63 \\
\hline 0.20 & 0.80 & 0.842 & 2.38 \\
\hline 0.50 & 0.50 & 0.000 & 0.00 \\
\hline
\end{tabular}

Routinely, an $\alpha$ value of 0.05 is used at EML for calculating the LLD. The LLD will be provided at the end of each procedure, if appropriate.

The simplest possible case is one where the sample activity is zero. It is sometimes not realized that if a series of counts is taken on such a system, that half of the net values should be less than zero. Negative counts are not possible, of course, but when there is a blank or a background, the entire scale is shifted up and the situation becomes one where half of the sample counts on a zero activity sample would be less than background. This negative net count occurs frequently in low-level measurements, causing considerable concern, but such results are to be expected.

Thus, if we have a counter with a background of 0.003 counts $\mathrm{sec}^{-1}$ (cps) and we count the sample and background for $24000 \mathrm{sec}$, then

$$
s_{b}=\sqrt{72}=8.5
$$

If we set $\alpha$ at 0.05 , accepting a $5 \%$ chance of a false positive result for a sample having no activity,

$$
\operatorname{LLD}=(4.66)(8.5)=40 \text { counts }
$$

or $\sim 0.002$ cps. 
Since the simplified formula used sets $\mathrm{k}_{\alpha}=\mathrm{k}_{\mathrm{B}}$, we are also accepting the chance that we will detect activity when it is present, $95 \%$ of the time, but will miss it $5 \%$ of the time.

If a $50 \%$ chance of finding activity is accepted, the LLD is zero. This is to be expected from the previous qualitative description.

The original paper of Pasternack and Harley (1971) applied the LID concept to multicomponent $\gamma$ spectrometry with $\mathrm{NaI}$ detectors. They noted particularly the decrease in sensitivity as the number of components in the mixture increased, and observed a decrease when the number of radionuclides in the library was increased, even when the actual sample contained only a single component.

Multicomponent $\alpha$ spectrometry can be performed in accordance with the procedures of Fisenne et al. (1973). Solid state $\alpha$-spectrometry calculations are simpler, since overlapping spectra are less common. The basic principle is that an interfering radionuclide effectively increases the background for the nuclide sought.

Examples of LLD calculations for three typical measurement situations are given for illustrative purposes in the appendix.

\section{ZERO LEVELS}

\section{A. Introduction.}

When the measurement of background yields a positive value, its variance and SD may be calculated based on Poisson counting statistics. In any subsequent measurement of a sample, the net activity and its SD may be evaluated to determine whether significant radioactivity is present.

A special case arises when neither counter background nor sample activity is detectable over a reasonably long counter interval. It is of practical interest to be able to quantitate the upper limit of radioactivity that could be present in this sample and yet yield this result, since increasingly more environmental nuclide measurements may fall into this category. 
The measurement and identification of low levels $(<0.0001 \mathrm{~Bq}) /$ sample $)$ of $\alpha$-emitting nuclides by spectrometry represents a specific example. This measurement is performed with a silicon surface barrier or ion implant detector. Any background counts measured in these detectors usually fall within the energy intervals corresponding to naturally occurring nuclides, primarily radium and its progeny. The background count rate for these detectors usually ranges from $0-5$ counts per $6 \times 10^{4} \mathrm{sec}$ in any of the energy intervals containing peaks of the $U$ series, $\mathrm{Pu}$ isotopes and most other $\alpha$ emitters of interest.

Thorium-232 is a special case, since the energy interval at which it appears (3.9 MeV) normally exhibits very low background. For example, a measurement for ${ }^{232}$ Th yielded zero counts in $3 \times 10^{5} \mathrm{sec}$ and zero background counts over a similar count interval. It is of value to calculate rigorously an upper limit of ${ }^{232} \mathrm{Th}$ activity which could be present in such a sample. Two examples are given here. One, where the counting interval is fixed and the other where the count time can be calculated based upon a desired fixed value for the upper limit of activity in the sample (Harley and Fisenne, 1976).

\section{B. Predetermined count interval.}

We may apply the binomial distribution to a group of $\mathrm{N}_{\mathrm{o}}$ atoms to calculate the probability that there will be no $\alpha$ decays in counting interval, $t$.

The binomial distribution for the probability of $\mathrm{x}$ number of disintegrations out of a total of $\mathrm{N}_{0}$ atoms is:

$$
\operatorname{Pr}(\mathrm{x})=\frac{\mathrm{N}_{0} !}{\left(\mathrm{N}_{0}-\mathrm{x}\right) ! \mathrm{x} !} \mathrm{p}^{\mathrm{x}} \mathrm{q}^{\left(\mathrm{N}_{0}-\mathrm{x}\right)}
$$

where

$$
\begin{aligned}
\operatorname{Pr}(\mathrm{x}) & =\text { probability of } \mathrm{x} \text { disintegrations during time, } \mathrm{t} \\
\mathrm{N}_{\mathrm{o}} & =\text { initial number of radioactive atoms, } \\
\mathrm{p} & =\text { probability that an atom will decay in time, } \mathrm{t}, \text { and } \\
\mathrm{q} & =\text { probability that an atom will not decay in time, } t .
\end{aligned}
$$


Since the probability, $\mathrm{q}$, that an atom will not decay is:

then,

$$
q=e^{-\lambda t}
$$

and

$$
p=\left(1-e^{-\lambda t}\right)
$$

$$
\operatorname{Pr}(\mathrm{x})=\frac{\mathrm{N}_{0} !}{\left(\mathrm{N}_{0}-\mathrm{x}\right) ! \mathrm{x} !}\left(1-\mathrm{e}^{-\lambda t}\right)^{\mathrm{x}}\left(\mathrm{e}^{-\lambda t}\right)^{\mathrm{N}_{0}-\mathrm{x}}
$$

The probability of no disintegrations in time $t$ is then:

$$
\operatorname{Pr}(0)=\frac{N_{0} !}{N_{0} 0 !}\left(1-e^{-\lambda t}\right)^{0}\left(e^{-\lambda t}\right)^{N_{0}}=e^{-\lambda N_{0} t}
$$

Because the detector efficiency is $<100 \%$, there is also the possibility that one or more disintegrations occur but that they are not detected. If the detector efficiency is $G$ and one disintegration occurs, the probability of no count being detected is $(1-G)$. If two disintegrations occur, the probability of zero counts is $(1-G)^{2}$. In general, whenever $x$ disintegrations occur, the probability that zero counts will be seen is $(1-G)^{x}$. Then the probability that one or more of $N_{o}$ atoms present disintegrates during time, $t$, but that none is detected is:

$$
\operatorname{Pr}(1) \cdot(1-G)+\operatorname{Pr}(2) \cdot(1-G)^{2}+\operatorname{Pr}(3) \cdot(1-G)^{3}+\ldots
$$

From (1), the probability of one disintegration occurring is:

$$
\operatorname{Pr}(1)=\frac{N_{0} !}{\left(N_{0}-1\right) ! 1 !}\left(1-e^{-\lambda t}\right)^{1}\left(e^{-\lambda t}\right)^{N_{0}-1}
$$

The probability of two disintegrations occurring is:

$$
\operatorname{Pr}(2)=\frac{N_{0} !}{\left(N_{0}-2\right) ! 2 !}\left(1-e^{-\lambda t}\right)^{2}\left(e^{-\lambda t}\right)^{N_{0}-2}
$$


and so on.

If the assumption can be made that $\mathrm{t}$ is small and that $\mathrm{N}_{\mathrm{o}}$ is much greater than one, then $\operatorname{Pr}(1), \operatorname{Pr}(2)$, etc., simplify to:

$$
\begin{aligned}
& \operatorname{Pr}(1) \cong N_{0}(\lambda t)^{-1}\left(e^{-\lambda N_{0} t}\right) \\
& \operatorname{Pr}(2)=\frac{N_{0} N_{0}}{2 !}(\lambda t)^{2}\left(e^{-\lambda N_{0} t}\right) \\
& \operatorname{Pr}(3)=\frac{N_{0} N_{0} N_{0}}{3 !}(\lambda t)^{3}\left(e^{-\lambda N_{0} t}\right)
\end{aligned}
$$

The probability for observing zero counts is the sum of the probabilities for no disintegration to occur plus those for the occurrence of one or more disintegrations which are not detected.

$$
\begin{aligned}
P= & \operatorname{Pr}(0)+\operatorname{Pr}(1) \cdot(1-G)+\operatorname{Pr}(2) \cdot(1-G)^{2}+\operatorname{Pr}(3) \cdot(1-G)^{3}+\ldots \\
& \cong \mathrm{e}^{-\lambda N_{0} t}+\lambda N_{0} t e^{-\lambda N_{0} t}(1-G)+\frac{\left[\lambda N_{0} t(1-G)\right]^{2}}{2 !} e^{-\lambda N_{0} t}+\ldots \\
& \cong e^{-\lambda N_{0} t}\left[1+\frac{\lambda N_{0} t(1-G)}{1 !}+\frac{\left[\lambda N_{0} t(1-G)\right]^{2}}{2 !}+\frac{\left[\lambda N_{0} t(1-G)\right]^{3}}{3 !}+\ldots\right] \\
& \cong e^{-\lambda N_{0} t} \cdot e^{+\lambda N_{0} t(1-G)} \\
& \cong e^{-\lambda N_{0} t G}
\end{aligned}
$$

$\lambda N_{0} t G$ is the number of counts to be expected during the interval $t$.

We would like the occurrence of zero counts, when activity is actually present, to have a small probability. If this probability is arbitrarily set to 0.05 so that zero counts will be detected only $5 \%$ of the time, when $N_{0}$ atoms are actually present and the counting interval is $t$ minutes, then $(6 \mathrm{~b})$ becomes: 


$$
\begin{gathered}
e^{-\lambda N_{0} t G}=0.05 \\
\lambda N_{0} t G=-\ln (0.05) \\
\left(\lambda N_{0}\right)=A_{0}=-\frac{1}{t G} \ln (0.05) \\
A_{0}=\frac{3}{t G}
\end{gathered}
$$

where $\lambda \mathrm{N}_{0}=\mathrm{A}_{\mathrm{o}}$ is the upper limit of sample activity in $\mathrm{Bq}$ at the $95 \%$ confidence level, when $t$ is in seconds and $G$ is expressed as fractional efficiency.

\section{Predetermined upper limit of activity.}

It may be of interest to establish the counting time required to determine that a chosen upper limit of activity is present. Solving (7) for the counting time yields:

$$
\mathrm{t}=\frac{3}{\mathrm{~A}_{0} \mathrm{G}}
$$

As an example, the measured data for the ${ }^{232} \mathrm{Th}$ sample will be used. The efficiency of the surface barrier detector is 0.30 and zero counts were recorded in $3 \times 10^{5} \mathrm{sec}$. From (7):

$$
\mathrm{A}_{0}=\frac{3}{\left(3 \times 10^{5}\right)(0.3)}=3 \times 10^{-5} \mathrm{~Bq}
$$

The upper limit of activity in this sample is thus $3 \times 10^{-5} \mathrm{~Bq}$ at the $95 \%$ confidence level.

\section{BACKGROUND NOT ZERO}

All of the above expressions apply equally well to background activity. It is possible that either a background count did not occur during the count interval or that one or more occurred but were not detected. In this case, the upper limit to background activity is the same as that calculated in (9), except that there is no efficiency for background and the background activity is expressed as counts $\sec ^{-1}$. Expression (9) would then become: 


$$
A_{0} G=\frac{3}{\left(3 \times 10^{5}\right)}=1 \times 10^{-5} \text { counts sec. }^{-1}
$$

Then according to the convention at EML, any value of net activity equal to zero would be evaluated in comparison with:

$$
S_{\text {net }}=\sqrt{\frac{1 \times 10^{-5}}{3 \times 10^{-5}}+\frac{1 \times 10^{-5}}{3 \times 10^{-5}}}=8 \times 10^{-6} \text { counts } \sec ^{-1}
$$

and the activity would be reported as:

$$
\frac{\leq 8 \times 10^{-6}}{0.3}
$$

or $\leq 2 \times 10^{-5} \mathrm{~Bq}$ at the $95 \%$ confidence interval. ${ }^{*}$

\section{REFERENCES}

Fisenne, I. M., A. O'Toole and R. Cutler

"Least Squares Analysis and Minimum Detection Levels Applied to Multi-component Alpha Emitting Samples"

Radiochem. Radioanal. Letters, 1, 5-16 (1973)

Harley, N. H. and I. M. Fisenne

"Reporting Results of Radioactivity Measurements at Near Zero Levels of Sample Activity and Background"

USERDA Report HASL-306, pp. 43-50 (1976)

Healy, J. W.

in: Proceedings of the Second Annual Meeting on Bioassay

USAEC Report WASH-736, September (1957)

*This convention would be used for reporting single values. For averaging purposes, the actual value of the net activity, positive or negative, would be used. 
Pasternack, B. S. and N. H. Harley

"Detection Limits for Radionuclides in the Analysis of Multi-Component Gamma Ray Spectrometer Data"

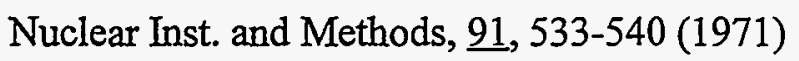




\section{APPENDIX \\ LOWER LIMIT OF DETECTION*}

In the analyses of samples for naturally occurring radionuclides, it is rare that the sample activity and background are the same. Reagent blanks processed through the chemical procedure will show activity in excess of the background of the detection system. Multiple measurements of the background and multiple reagent blank analyses are used to obtain the respective standard errors of these variables. In this case, the equation is

$$
s_{s}=\left[s_{\text {gross }}+s_{\overline{B K}}^{2}+s_{\overline{B I}}^{2}\right]^{1 / 2}
$$

where

$$
\begin{aligned}
s_{\text {gross }}= & \text { the mean background plus mean reagent blank counts for a specified } \\
& \text { counting time, } \\
s_{\mathrm{Bk}}^{-}= & \text {the standard error of the background from multiple measurements }(\mathrm{n}), \\
& \text { corresponding to the same counting time as above, }\left(\left[\mathrm{s}_{\mathrm{BK}}{ }^{2}\right] /[\mathrm{n}]\right)^{1 / 2} \text {, and } \\
\mathrm{s}_{\mathrm{BI}}^{-}= & \text {the standard error of the blank from multiple analyses }(\mathrm{n}) \text {, also with the } \\
& \text { same counting time, }\left(\left[\mathrm{s}_{\mathrm{Bl}}{ }^{2}\right] /[\mathrm{n}]^{1 / 2}\right) .
\end{aligned}
$$

$$
\operatorname{LLD}_{95 \%}=\left(k_{\alpha}+k_{B}\right) S_{s}=3.29 S_{s}
$$

Then, the LLD at the $95 \%$ confidence level is

where

$\mathrm{k}_{\alpha}=$ the preselected risk for concluding falsely that activity present. At the $95 \%$ confidence level $(\alpha)=1.645$, and

\footnotetext{
- Taken from Pasternack and Harley (1971).
} 
$\mathrm{k}_{\beta} \quad=$ the predetermined degree of confidence for detecting the presence of activity. At the $95 \%$ confidence level $(1-\beta)=1.645$.

Thus, at $\mathrm{LLD}_{95 \%}$

and

$$
\mathrm{k}_{\alpha}=\mathrm{k}_{\beta}=1.645
$$

$$
\left(\mathrm{k}_{\alpha}+\mathrm{k}_{\beta}\right)=3.29
$$

The $\mathrm{LLD}_{95 \%}$ must be converted to activity $(\mathrm{Bq})$ using the appropriate conversion factors.

$$
\operatorname{LLD}_{95 \%}(\mathrm{~Bq})=\frac{3.29 \mathrm{~s}_{\mathrm{S}}}{(\mathrm{CT})(\epsilon)(\mathrm{Y})}
$$

where

$\mathrm{CT}=$ the counting time or counting interval in seconds,

$\epsilon=$ the detection efficiency of the measurement system (counts $\sec ^{-1} \mathrm{~Bq}^{-1}$ ), and

$\mathrm{Y}=$ the average radiometric or chemical yield obtained for the separation method.

The applications of Equations 1 and 3 to solid state $\alpha$-spectrometry measurements are illustrated below.

In ${ }^{239} \mathrm{Pu}$ measurements, a manmade radionuclide, there is no measurable blank and the mean background count from multiple measurements is used. Then, the term $\mathrm{s}_{\mathrm{Bl}}$ does not appear and Equation 1 reduces to

$$
\mathrm{s}_{\mathrm{S}}=\left[\mathrm{s}_{\text {gross }}+\mathrm{s}_{\mathrm{Bk}}^{2}\right]^{1 / 2}
$$

For this situation $s_{\text {gross }}$ is the mean background counts and $s_{B k}$ is the standard error of multiple measurements. 
Given the following variables:

$\begin{array}{ll}\text { counter efficiency: } & 40 \% \\ \text { counter background } & \\ \text { (in the }{ }^{239} \mathrm{Pu} \text { energy region): } & 3.3 \times 10^{-5} \pm 1.7 \times 10^{-5}{\text { counts } \mathrm{sec}^{-1}}\left(0.002 \pm 0.001 \text { counts } \mathrm{min}^{-1}\right) \\ \text { tracer yield: } & 75 \% \\ \text { counting time: } & 60,000 \mathrm{~s}(1,000 \mathrm{~min}) \\ & \\ & \\ \mathrm{s}_{\mathrm{S}}=(2+1 \text { counts })^{1 / 2}=(3 \text { counts })^{1 / 2}\end{array}$

and

$$
\operatorname{LLD}_{95 \%}=\frac{3.29(3 \text { counts })^{1 / 2}}{(60000 \mathrm{sec})\left(0.40 \text { counts } \mathrm{sec}^{-1} \mathrm{~Bq}^{-1}\right)(0.75 \text { yield })}=0.0003 \mathrm{~Bq}
$$

In ${ }^{210} \mathrm{Po}$ measurements there usually is a measurable blank and all variables in Equation 1 must be taken into account.

Given the following variables:

counter efficiency: $\quad 40 \%$

counter background

(in the ${ }^{210}$ Po energy region): $\quad 8.3 \times 10^{-5} \pm 5.0 \times 10^{-5}{\text { counts } \mathrm{sec}^{-1}}^{-1}$

blank:

$$
\left(0.005 \pm 0.003 \text { counts } \mathrm{min}^{-1}\right)
$$

tracer yield:

$$
\begin{aligned}
& \left(5.0 \times 10^{-5} \pm 3.3 \times 10^{-5} \text { counts } \mathrm{sec}^{-1}\right) \\
& \left(0.003 \pm 0.002 \text { counts } \mathrm{min}^{-1}\right)
\end{aligned}
$$

counting time:

\section{$80 \%$}

$60,000 \mathrm{~s}(1,000 \mathrm{~min})$

Again, mean background from multiple measurements and the mean reagent blank from multiple analyses are to be used.

$$
s_{S}=\left[(5+3 \text { counts })+(2 \text { counts })^{2}+(3 \text { counts })^{2}\right]^{1 / 2}=4.6 \text { counts }
$$




$$
\operatorname{LLD}_{95 \%}=\frac{3.29(4.6 \text { counts })}{(60000 \mathrm{sec})\left(0.40 \mathrm{cps} \mathrm{Bq}^{-1}\right)(0.80 \text { yield })}=0.0008 \mathrm{~Bq}
$$

The LLD can be reduced by: (1) increasing the number of background and reagent blank measurements, assuming these do not increase over time; (2) increasing the counting time; (3) increasing the counting efficiency; and (4) increasing the sample yield. 


\subsubsection{Radiochemical}




\section{Americium}


AMERICIUM IN SOIL

Contact Person(s) : Anne Berne

\begin{abstract}
APPLICATION
This procedure is applicable to soils which contain americium deposited from worldwide fallout and some nuclear activities.

Americium is leached from the soil with $\mathrm{HNO}_{3}$ and $\mathrm{HCl}$ and simultaneously equilibrated with ${ }^{243} \mathrm{Am}$ tracer. The soil is processed through the plutonium separation steps using ion exchange resin according to Procedure $\mathrm{Pu}-11-\mathrm{RC}$. If determination of plutonium is desired, an appropriate plutonium tracer should be added along with the ${ }^{243} \mathrm{Am}$ tracer. Americium is collected with a calcium oxalate precipitation and finally isolated and purified by ion exchange. After source preparation by microprecipitation, the ${ }^{241} \mathrm{Am}$ is determined by alpha spectrometry using ${ }^{243} \mathrm{Am}$ tracer to provide recovery data.
\end{abstract}

\title{
SPECIAL APPARATUS
}

1. For microprecipitation, see Procedure G-03.

2. Ion-exchange columns - see Specification 7.5.

\section{SPECIAL REAGENTS}

1. Americium-243 tracer solution, about $0.15 \mathrm{~Bq} \mathrm{~g}^{-1}$ in dispensing bottle - standardize for total disintegration rate. Measure purity on an $\alpha$ spectrometer. 
2. Bio-Rad AG 1-X8 resin (100-200 mesh) - see Specification 7.4.

3. Bio-Rad AG 1-X4 resin (100-200 mesh) - see Specification 7.4.

4. $4 \mathrm{M}$ ammonium thiocyanate solution - dissolve $304 \mathrm{~g}$ of $\mathrm{NH}_{4} \mathrm{SCN}$ in deionized distilled water and dilute to $1 \mathrm{~L}$. To purify the $4 \mathrm{M} \mathrm{NH}_{4} \mathrm{SCN}$, place $4 \mathrm{~L}$ of solution in a $5 \mathrm{~L}$ polyethylene beaker. Add $25 \mathrm{~mL}$ of Bio-Rad AG 1-X4 resin (100-200 mesh) ion exchange resin, and $\mathrm{mix}$ for $1 \mathrm{~h}$ with a magnetic stirrer. Allow the resin to settle and filter by gravity through Whatman No. 40 filter paper. Repeat the addition of the resin and filtration steps twice more to remove all $\mathrm{Fe}^{+3}$ from the $4 \mathrm{M} \mathrm{NH}_{4} \mathrm{SCN}$. Store the purified $4 \mathrm{M} \mathrm{NH}_{4} \mathrm{SCN}$ in a polyethylene bottle.

5. $0.4 \mathrm{M} \mathrm{NH}_{4} \mathrm{SCN}-0.3 \mathrm{M} \mathrm{HCl}$ - dilute $100 \mathrm{~mL}$ of purified $4 \mathrm{M} \mathrm{NH}_{4} \mathrm{SCN}$ to $500 \mathrm{~mL}$ with water, then add $25 \mathrm{~mL} \mathrm{HCl}$ and dilute to $1 \mathrm{~L}$. Make $2 \mathrm{~L}$ of solution for 10 samples.

6. Calcium carrier solution, $100 \mathrm{mg} \mathrm{mL}^{-1}$ - dissolve $25 \mathrm{~g} \mathrm{CaCO}_{3}$ in a minimum of $\mathrm{HNO}_{3}$ and dilute to $100 \mathrm{~mL}$.

7. Iron carrier, $100 \mathrm{mg} \mathrm{mL}^{-1}$ - slowly heat $100 \mathrm{~g}$ of iron powder in $500 \mathrm{~mL}$ of $\mathrm{HCl}$ until reaction ceases. Carefully and slowly add $100 \mathrm{~mL}$ of $\mathrm{HNO}_{3}$ while stirring. Cool and dilute to $1 \mathrm{~L}$.

8. Oxalate wash solution - dissolve $10 \mathrm{~g}$ of oxalic acid to make $1 \mathrm{~L}$ of solution $(\sim 1 \%$ solution).

\section{SAMPLE PREPARATION}

1. Weigh $1000 \mathrm{~g}$ of soil into a $4-\mathrm{L}$ beaker. Add a weighed amount (about $0.03 \mathrm{~Bq}$ ) of ${ }^{243} \mathrm{Am}$ tracer.

2. Slowly add $900 \mathrm{~mL}$ of $\mathrm{HNO}_{3}$. Control the foam with the addition of a few drops of $\mathrm{n}$-octyl alcohol. When the reaction subsides, add $300 \mathrm{~mL}$ of $\mathrm{HCl}$. Allow the mixture to react at room temperature, then heat on a low temperature hot plate overnight with occasional stirring. 
3. Dilute to 1:1 $\mathrm{HNO}_{3}$ and filter through Whatman No. 42 filter paper into a 3-L flask. Wash with 1:1 $\mathrm{HNO}_{3}$. Retain the filtrate. Return the residue and filter to the original beaker.

4. Add $900 \mathrm{~mL}$ of $\mathrm{HNO}_{3}$ and wet ash the filter paper. Maintain the $\mathrm{HNO}_{3}$ volume. $\mathrm{Cool}$ and add $300 \mathrm{~mL}$ of $\mathrm{HCl}$ to the residue and heat on a low temperature hot plate for about $3 \mathrm{~h}$ with occasional stirring. Cool and allow to settle overnight.

5. Filter and wash as in Step 3. Combine the filtrate with the filtrate from Step 3. Return the residue and filter to the original beaker.

\section{Repeat Step 4.}

7. Filter and wash as in Step 3. Combine the three filtrates and discard the residue.

8. Decompose any organic matter in the extract by heating with repeated additions of $\mathrm{HNO}_{3}$, covering the sample with a watch cover and letting the sample reflux. Concentrate until salting out begins to occur. Add an equal volume of water. If solution is not clear, proceed to Step 9, otherwise go to Step 14.

9. If any siliceous matter is present, filter by gravity over an $18.5 \mathrm{~cm}$ Whatman No. 42 filter paper. Wash the residue with 1:1 $\mathrm{HNO}_{3}$. Reserve the filtrate.

10. Transfer the filter paper with the residue to the original beaker and ash the paper with $100 \mathrm{~mL}$ of $\mathrm{HNO}_{3}$. Repeat two or three times, then transfer the residue into a $100-\mathrm{mL}$ platinum dish using 1:1 $\mathrm{HNO}_{3}$.

11. Add 5-25 $\mathrm{mL}$ of $\mathrm{HF}$ and 5-25 $\mathrm{mL}$ of $\mathrm{HNO}_{3}$ to the platinum dish and evaporate on a medium temperature hot plate. Repeat the addition of the $\mathrm{HF} / \mathrm{HNO}_{3}$ and the evaporation process two or three times. Rinse the walls of the platinum dish with $1: 1$ $\mathrm{HNO}_{3}$ and evaporate. Repeat three times. Evaporate to dryness. Dissolve with 1:1 $\mathrm{HNO}_{3}$ and evaporate to dryness.

12. Dissolve the residue in $1: 1 \mathrm{HNO}_{3}$ and filter by gravity through a Whatman No. 42 filter paper. Add the filtrate to the solution from Step 9. Discard the filter and any residue. 
13. Heat the combined solution (with the addition of $\mathrm{HNO}_{3}$ if necessary) to complete the oxidation of any organic materials. Evaporate to near dryness. Redissolve in 1:1 $\mathrm{HNO}_{3}$ and stir to get a clear solution, adding $1: 1 \mathrm{HNO}_{3}$ as necessary.

14. Proceed to Procedure $\mathrm{Pu}-11-\mathrm{RC}$, ion-exchange purification saving the column effluents for Americium Determination.

\section{AMERICIUM DETERMINATION}

1. Evaporate the americium effluents to incipient dryness. Redissolve in a minimum amount of 1:1 $\mathrm{HNO}_{3}$, dilute with four volumes of water.

2. Add $5 \mathrm{~mL}$ of calcium carrier solution ( $500 \mathrm{mg}$ of calcium) and $50 \mathrm{~g} \mathrm{~L}^{-1}$ of oxalic acid to the sample while stirring with a magnetic stirrer. (The total volume of the sample solution can be estimated using the markings on the beaker, and the amount of oxalic acid to be added is calculated using that volume.)

3. Adjust the $\mathrm{pH}$ of the solution to $2.0-2.5$ with $\mathrm{NH}_{4} \mathrm{OH}$ using $\mathrm{pH}$ paper as an indicator and continue to stir for $30 \mathrm{~min}$. Remove the magnetic stir bar.

4. Cool and let stand until precipitate settles and solution clears (for more than $6 \mathrm{~h}$ or overnight). Check for completeness of precipitation using a drop of saturated $\mathrm{H}_{2} \mathrm{C}_{2} \mathrm{O}_{4}$ solution. Aspirate (or decant), using a disposable transfer pipette and suction, as much liquid as possible without disturbing the precipitate. Transfer the precipitate to a $250-\mathrm{mL}$ centrifuge bottle using oxalate wash solution (see Note 1). Balance the bottles on a double pan balance and centrifuge for $10 \mathrm{~min}$ at $2000 \mathrm{rpm}$. Decant and discard the supernate.

5. Break up the precipitate with a stirring rod and wash the precipitate with the oxalate wash solution. Centrifuge, decant and discard the wash. Repeat wash. Redissolve the precipitate in a minimal amount $(50-70 \mathrm{~mL})$ of concentrated $\mathrm{HCl}$ (redissolve the precipitate in $\sim 200 \mathrm{~mL}$ of $\mathrm{HNO}_{3}$ a final time and proceed to Step 8). (Note: Dissolution is easier if the centrifuge bottle is placed in a hot water bath and stirred with a glass rod). 
6. Transfer the dissolved precipitate to the original $600-\mathrm{mL}$ beaker. Add enough water to make $\sim 1 \underline{\mathrm{M}}$ solution. Add $50 \mathrm{~g} \mathrm{~L}^{-1}$ of oxalic acid.

7. Repeat Steps 3 through 6 until supernate is colorless.

8. Transfer the dissolved precipitate to the original beaker and heat to destroy the oxalate ion. Evaporate to near dryness. Dissolve in minimum 1:1 $\mathrm{HNO}_{3}$. Transfer to centrifuge bottle using water to complete the transfer.

9. Add enough water to make $\sim 1 \mathrm{M} \mathrm{HNO}_{3}$. Warm the solution in a $90^{\circ}$ hot water bath and add $0.2 \mathrm{~mL}$ iron carrier solution ( $20 \mathrm{mg}$ iron).

10. With the centrifuge bottle in the hot water bath adjacent to a hood, adjust the $\mathrm{pH}$ of the solution to 8-9 with $\mathrm{NH}_{4} \mathrm{OH}$ while stirring with a glass rod. Allow solution to digest in a hot water bath for $20 \mathrm{~min}$.

11. Cool in a cold water bath, rinse, and remove the glass rod. Balance the bottles on a double pan balance and centrifuge for $40 \mathrm{~min}$ at $2000 \mathrm{rpm}$.

12. Decant (or aspirate) and discard the supernate. Add $10 \mathrm{~mL}$ concentrated $\mathrm{HCl}$ to dissolve the $\mathrm{Fe}(\mathrm{OH})_{3}$ pellet. Add four drops $30 \% \mathrm{H}_{2} \mathrm{O}_{2}$ to oxidize any $\mathrm{Mn}$ present, followed by $100 \mathrm{~mL}$ of water. Heat in the water bath for $30 \mathrm{~min}$ to get rid of the excess $\mathrm{H}_{2} \mathrm{O}_{2}$.

13. Repeat Steps 10 to12 three times. Reprecipitate, centrifuge and redissolve. The final precipitate should be redissolved in $\mathrm{HNO}_{3}$.

14. Transfer to a 250 -mL beaker, evaporate to dryness, add $20 \mathrm{~mL} \mathrm{HNO}_{3}$, and evaporate to dryness again.

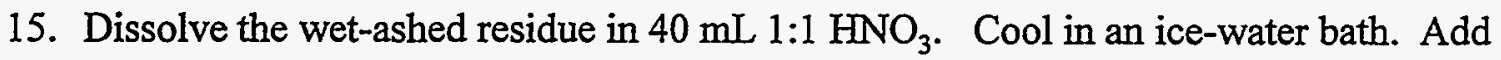
0.6-1.0 $\mathrm{g} \mathrm{NH}_{2} \mathrm{OH} \cdot \mathrm{HCl}$, dissolve, and let the solution react for $15 \mathrm{~min}$. Cover with a watch glass. Heat on a low temperature hot plate to decompose unreacted $\mathrm{NH}_{2} \mathrm{OH}$ $\mathrm{HCl}$, then bring to a gentle boil for 1-2 min. Cool and pass the solution through a 1:1 $\mathrm{HNO}_{3}$ ion-exchange column (see Note 2). Collect the effluent in a 400-mL beaker. Wash the column with $150 \mathrm{~mL}$ of $1: 1 \mathrm{HNO}_{3}$, and collect in the beaker. 
16. Evaporate the sample in the $400-\mathrm{mL}$ beaker to dryness. Convert to $\mathrm{HCl}$ by adding 20 $30 \mathrm{~mL}$ of $\mathrm{HCl}$ at a time, heat to almost dryness, and repeat the $\mathrm{HCl}$ addition and evaporation at least three times. Evaporate again and dissolve the final residue in $30 \mathrm{~mL}$ of $\mathrm{HCl}$. Pass this solution through a $12 \mathrm{~N} \mathrm{HCl}$ ion exchange column (see Note 3). Collect the effluent in a $250-\mathrm{mL}$ beaker. Wash with $100 \mathrm{~mL}$ of $\mathrm{HCl}$, and collect in the $250-\mathrm{mL}$ beaker.

17. Evaporate to dryness. Dissolve in $1-2 \mathrm{~mL}$ of $\mathrm{HCl}$. Cool thoroughly. Add $40 \mathrm{~mL}$ of $4 \mathrm{M} \mathrm{NH}_{4} \mathrm{SCN}$ and stir immediately. Stir the sample and pass the solution through a $4 \mathrm{M} \mathrm{NH}_{4} \mathrm{SCN}$ column (see Note 4). Discard the effluent.

18. Wash the column with $200 \mathrm{~mL}$ of $4 \mathrm{~N} \mathrm{NH}_{4} \mathrm{SCN}$ solution. Discard the wash solution.

19. Elute the americium into a $250-\mathrm{mL}$ beaker with $180 \mathrm{~mL}$ of $0.4 \underline{N} \mathrm{NH}_{4} \mathrm{SCN}-0.3 \underline{\mathrm{N}}$ $\mathrm{HCl}$. Evaporate to dryness on a low temperature hot plate overnight. Discard the resin.

19. To remove $\mathrm{NH}_{4}{ }^{+}$salts, place the beaker on an iron tripod and heat slowly with a cool Bunsen flame. After $\sim 0.5 \mathrm{~h}$, increase the flame temperature and continue heating to remove all $\mathrm{NH}_{4}^{+}$salts and $\mathrm{S}$, then heat briefly to dull red heat. This step requires $\sim 1$ $1.5 \mathrm{~h}$.

20. Cool to room temperature. Add $25 \mathrm{~mL}$ of $\mathrm{HNO}_{3}$ and boil slowly for a few minutes. Cautiously add $1 \mathrm{~mL}$ of $30 \% \mathrm{H}_{2} \mathrm{O}_{2}$ and evaporate the solution to dryness.

21. Convert the residue to $\mathrm{Cl}^{-}$by adding $1 \mathrm{~mL}$ of $\mathrm{HCl}$ and evaporating to dryness twice and proceed to microprecipitation.

\section{Notes:}

1. If a centrifuge is not available, centrifugation can be replaced by filtering and wet ashing the filter paper and precipitate in $\mathrm{HNO}_{3}$

2. Preparation of 1:1 $\mathrm{HNO}_{3}$ Column. Position a plug of glass wool at the base of an 11$\mathrm{mm}$ o.d. column. Transfer with deionized distilled water, $15 \mathrm{~mL}$ of wet settled Bio- 
Rad AG 1-X8 resin (100-200 mesh) to the column and allow it to settle. Place a second plug of glass wool on top of the resin and with the stopcock open allow the $\mathrm{H}_{2} \mathrm{O}$ level to reach the top of the upper plug. Pass $150 \mathrm{~mL}$ of $1: 1 \mathrm{HNO}_{3}$ through the resin bed in three $50-\mathrm{mL}$ portions or enough so that the effluent tests free of $\mathrm{Cl}^{-}$ion using dilute silver nitrate solution, allowing the level of each portion to reach the top of the upper glass wool plug.

3. Preparation of $\mathrm{HCl}$ Column. Position a plug of glass wool at the base of an $11-\mathrm{mm}$ o.d. column. Transfer $10 \mathrm{~mL}$ of wet settled Bio-Rad AG 1-X4 resin (100-200 mesh) with deionized water to the column and allow it to settle. Place a second plug of glass wool on top of the resin and with the stopcock open allow the $\mathrm{H}_{2} \mathrm{O}$ level to reach the top of the upper plug. Pass two 50 -mL volumes of $\mathrm{HCl}$ through the resin bed and allow each to reach the top of the upper glass wool plug.

4. Preparation of $\mathrm{NH}_{4} \mathrm{SCN}$ Column. Position a plug of glass wool at the base of an 11$\mathrm{mm}$ o.d. column. Transfer with deionized distilled water, $15 \mathrm{~mL}$ of wet settled Bio$\operatorname{Rad} A G 1-X 4$ resin (100-200 mesh) to the column and allow it to settle. Place a second plug of glass wool on top of the resin and with the stopcock open allow the $\mathrm{H}_{2} \mathrm{O}$ level to reach the top of the upper plug. Pass $100 \mathrm{~mL}$ of purified $4 \mathrm{M} \mathrm{NH}_{4} \mathrm{SCN}$ through the resin bed in two $50-\mathrm{mL}$ portions, allowing the level of each portion to reach the top of the upper glass wool plug.

\section{MICROPRECIPITATION}

See Microprecipation Source Preparation for Alpha Spectrometry, Procedure G-03.

\section{DATA PROCESSING AND ANALYSES}

For alpha spectrometry measurements, please see Procedure A-01-R. 


\section{LOWER LIMTT OF DETECTION (LLD)}

\begin{tabular}{lll}
\hline & & \\
Counter Efficiency & $(\%)$ & 25 \\
Counter Background & $(\mathrm{cps})$ & $15 \times 10^{-6}$ \\
Yield & $(\%)$ & 50 \\
Blank & $(\mathrm{cps})$ & - \\
& & \\
LLD (400 min) & $(\mathrm{mBq})$ & 1 \\
LLD (1000 min) & $(\mathrm{mBq})$ & 0.5 \\
LLD (5000 min) & $(\mathrm{mBq})$ & 0.3 \\
& & \\
\hline
\end{tabular}




\title{
AMERICIUM-241 IN SOIL - GAMMA SPECTROMETRY
}

\author{
Contact Person(s) : Colin G. Sanderson
}

\section{APPLICATION}

This procedure is capable of determining small amounts of ${ }^{241} \mathrm{Am}$ in large volume soil samples. The lower limit of detection for $600-800 \mathrm{~g}$ of soil in a Marinelli beaker is 0.74 $\mathrm{mBq}$ for a $1000-\mathrm{min}$ count.

Americium-241 decays with the emission of $\gamma$ rays at 11.9, 13.9, 17.8, 20.8, 26.4, and $59.5 \mathrm{keV}$. The $59.5 \mathrm{keV} \gamma$ ray, which has an abundance of $35.9 \%$, can be measured in soil with commercially available germanium $(\mathrm{Ge})$ semiconductor $\gamma$-ray detectors and $600 \mathrm{~mL}$ Marinelli beakers. Gamma-ray attenuation corrections are required if the calibration source and the sample are in a different matrix or are of different densities.

\section{SPECIAL APPARATUS}

1. Hyperpure Ge $\gamma$-ray detector, shield, and associated electronics.

2. Multichannel analyzer.

3. Marinelli beaker - see Specification 7.22.

\section{SPECIAL REAGENT}

Americium-241 calibration solution, about $2.50 \mathrm{~Bq} \mathrm{~mL}^{-1}$. 


\section{SPECTROMETER CALIBRATION}

1. Transfer $600 \mathrm{~mL}$ of standardized ${ }^{241} \mathrm{Am}$ solution to a Marinelli beaker.

2. Set the spectrometer energy calibrations at $0.5 \mathrm{keV} /$ channel and count the standard until 10,000 or more counts are accumulated in the channels corresponding to 58.0 to $61.5 \mathrm{keV}$.

3. Record the count time and the channel-by-channel data corresponding to 56.0 to $63.5 \mathrm{keV}$.

\section{SAMPLE PREPARATION}

1. Transfer $600 \mathrm{~mL}$ of prepared soil to a Marinelli beaker.

2. Accumulate sufficient counts in the $59.5 \mathrm{keV}^{241} \mathrm{Am}$ peak to achieve the desired counting statistics.

3. Record the count time and the channel-by-channel data corresponding to 56.0 to $63.5 \mathrm{keV}$.

\section{DETERMINATION}

If computer calculation techniques are used to determine peak areas from spectral data, the same techniques must be used for both calibration standards and samples. However, in most instances, simple peak integration by channel summing is sufficient.

The net count in the ${ }^{241} \mathrm{Am}$ peak $=\mathrm{A}-\mathrm{B}-\mathrm{C}$.

where
$A=$ sum of counts in the 8 channels from 58.0 to $61.5 \mathrm{keV}$
$\mathrm{B}=$ sum of counts in the 4 channels from 56.0 to $57.5 \mathrm{keV}$ 


$$
\mathrm{C}=\text { sum of counts in the } 4 \text { channels from } 62.0 \text { to } 63.5 \mathrm{keV}
$$

$\mathrm{B}$ and $\mathrm{C}$ are the leading and trailing edges of the photopeak and represent the background upon which the photopeak is superimposed.

The net ${ }^{241} \mathrm{Am}$ counts, AmC, corrected for attenuation equals

$$
\mathrm{A} / \mathrm{e}^{-(\mu \mathrm{d} \cdot \mathrm{w})}
$$

where

$\mathrm{A}=$ observed net counts in the ${ }^{241} \mathrm{Am}$ peak,

$\mu \mathrm{d}=$ attenuation constant for Marinelli beaker (see Note below), for $\mathrm{H}_{2} \mathrm{O}, \mu \mathrm{d}=0.000427 \mathrm{~cm}^{2} \mathrm{~g}^{-1}$

for soil, $\mu \mathrm{d}=0.000533 \mathrm{~cm}^{2} \mathrm{~g}^{-1}$

$\mathrm{w}=$ weight in $\mathrm{g}$ of the standard or sample.

The ${ }^{241} \mathrm{Am}$ detector efficiency at $59.5 \mathrm{keV}$,

$$
\text { Efficiency }=(\mathrm{AmC} / \mathrm{t}) /(\mathrm{Ac} \cdot 600)
$$

where

$$
\begin{aligned}
\text { AmC } & =\text { net }{ }^{241} \mathrm{Am} \text { counts of the standard, corrected for attenuation, and } \\
\mathrm{t} & =\text { standard count time }(\mathrm{sec}) \\
\mathrm{Ac} & ={ }^{241} \mathrm{Am} \text { activity of standard }(\mathrm{Bq} \mathrm{mL}-1)
\end{aligned}
$$

The $\mathrm{Bq}^{241} \mathrm{Am}$ in the sample $=(\mathrm{AmC} / \mathrm{t}) /$ Efficiency 
where

$$
\begin{aligned}
\mathrm{AmC} & =\text { net }^{241} \mathrm{Am} \text { counts of the sample, corrected for attenuation, and } \\
t & =\text { sample count time }(\mathrm{sec}) .
\end{aligned}
$$

Note:

The Marinelli beaker attenuation constant $\left(\mu \mathrm{d}=0.000533 \mathrm{~cm}^{2} \mathrm{~g}^{-1}\right)$ is for soil of normal composition. Soils or ores composed of high atomic number elements will require a different constant. For example, a $1 \% \mathrm{U}$ ore will attenuate the $59.5 \mathrm{keV} \gamma$ ray to $7 \%$ more than normal soil and the correct $\mu \mathrm{d}$ would be $0.000677 \mathrm{~cm}^{2} \mathrm{~g}^{-1}$. 
$A m-03-R C$

AMERICIUM IN WATER, AIR FILTERS, AND TISSUE

(see Volume II) 


\title{
AMERICIUM IN QAP WATER AND AIR FILTERS - EICHROM'S TRU RESIN
}

Contact Person(s) : Anna Berne

\begin{abstract}
APPLICATION
The following procedure has been applied to the preparation, separation, and analysis of spiked water and air filter samples that contain americium but not lanthanides (Berne, 1996). Lanthanides, if present, will not be removed by this method and will significantly reduce the resolution of the $\alpha$-spectrograph. Combined with Procedure Pu-11-RC, this procedure allows for the sequential determination of plutonium and americium. Other researchers have applied TRU Resin methods to other matrices (Horowitz et al., 1990). The procedure is used in the EML Quality Assessment Program (QAP; Sanderson and Greenlaw, 1996).

The water and air filters are equilibrated with ${ }^{243} \mathrm{Am}$ and processed through the plutonium separation steps using ion exchange resin according to Procedure $\mathrm{Pu}-11-\mathrm{RC}$. If determination of plutonium is desired, an appropriate plutonium tracer should be added along with the ${ }^{243} \mathrm{Am}$ tracer. The eluate from the ion exchange column containing americium (and all other ions, except plutonium) is evaporated, redissolved, and loaded onto a TRU Resin extraction column. The americium (and curium, if present) is separated and purified on the column and finally stripped with dilute nitric acid stripping solution. Microprecipitation is used to prepare for $\alpha$ spectrometry.
\end{abstract}

\section{SPECIAL REAGENTS}

1. Americium-243 tracer solution, about $0.15 \mathrm{~Bq} \mathrm{~g}^{-1}$ in dispensing bottle - standardize for total disintegration rate. Measure purity on an $\alpha$ spectrometer. 
2. TRU Resin $2 \mathrm{~mL}$ ion extraction columns or equivalent or can be prepared from TRU Resin, Eichrom Industries, Inc., 8205 Cass AV, Suite 107, Darien, II 60561. Place a plug of glass wool in the bottom of a polyethylene transfer pipette (see Specification 7.7). Add slurried TRU Resin ( $0.5 \mathrm{~g})$. Assemble immediately before use.

3. Column feed solution, $0.5 \underline{\mathrm{M} \mathrm{Al}}\left(\mathrm{NO}_{3}\right)_{3}$ in $2 \mathrm{M} \mathrm{HNO}_{3}$ - place $18.76 \mathrm{~g}$ of $\mathrm{Al}\left(\mathrm{NO}_{3}\right)_{3} \cdot 9 \mathrm{H}_{2} \mathrm{O}$ in a $100-\mathrm{mL}$ volumetric flask and add $2 \mathrm{M} \mathrm{HNO}_{3}$ to the mark. Shake to mix thoroughly.

4. $2 \underline{\mathrm{M} \mathrm{HNO}} \mathrm{HN}_{3}-125 \mathrm{~mL} \mathrm{HNO}$ diluted to $1 \mathrm{~L}$ with water.

5. $1 \mathrm{M} \mathrm{HNO}_{3}-62.5 \mathrm{~mL} \mathrm{HNO}_{3}$ diluted to $1 \mathrm{~L}$ with water.

6. $0.025 \underline{\mathrm{M}} \mathrm{HNO}_{3}-25 \mathrm{~mL} 1 \underline{\mathrm{M}} \mathrm{HNO}_{3}$ diluted to $1 \mathrm{~L}$ with water.

\section{SAMPLE PREPARATION}

See Procedure Pu-01-RC, air filters or Procedure Pu-10-RC, water.

\section{AMERICIUM DETERMINATION}

1. Collect the sample and the wash effluent from Step 4, Ion Exchange Separation, Procedure Pu-11-RC, and evaporate almost to dryness. If necessary, sometime during the evaporation process transfer the solution to a smaller beaker. The final residue should be contained in a beaker not larger than $50 \mathrm{~mL}$. Add $3 \mathrm{~mL}$ of $0.5 \underline{\mathrm{M}}$ $\mathrm{Al}\left(\mathrm{NO}_{3}\right)_{3}$ in $2 \mathrm{M}_{\mathrm{HNO}}$ to each residue and heat very gently to dissolve.

2. Prepare an ion extraction column.

3. Wash the resin with $15 \mathrm{~mL}$ of $2 \mathrm{M} \mathrm{HNO}_{3}$, and discard the effluent.

4. Load the column with the sample solution from Step 1. Wash the beaker with $3 \mathrm{~mL}$ of column-feed solution and add to the column. Discard the effluent. 
5. Rinse the column with $8 \mathrm{~mL}$ of $2 \mathrm{M} \mathrm{HNO}_{3}$, followed by $8 \mathrm{~mL}$ of $1 \underline{\mathrm{M}} \mathrm{HNO}_{3}$, and discard the effluents.

6. Elute the americium fraction with three $3-\mathrm{mL}$ aliquots of $0.025 \mathrm{M} \mathrm{HNO}_{3}$, and collect the eluate in a $50-\mathrm{mL}$ beaker.

7. Evaporate the eluate to dryness. Convert the residue to the chloride form by adding $5 \mathrm{~mL}$ of $\mathrm{HCl}$ three times and evaporating to dryness at a low temperature.

8. Prepare the sample for $\alpha$ spectrometry by microprecipitation (see Procedure G-03).

\section{DATA PROCESSING AND ANALYSIS}

For $\alpha$ spectrometry, see Alpha Radioassay, Procedure A-01-R.

\section{LOWER LIMIT OF DETECTION (LLD)*}

\begin{tabular}{lcc}
\hline Counter Efficiency & $(\%)$ & 30 \\
Counter Background & $(\mathrm{cps})$ & $1.6 \times 10^{-5}$ \\
Recovery & $(\%)$ & 80 \\
Blank & $(\mathrm{cps})$ & - \\
LLD (400 min) & $(\mathrm{mBq})$ & 0.5 \\
LLD (1000 min) & $(\mathrm{mBq})$ & 0.3 \\
LLD (5000 min) & $(\mathrm{mBq})$ & 0.1 \\
& & \\
\hline
\end{tabular}

*Reagent blanks must be analyzed with each set of samples.

\section{REFERENCES}




\section{REFERENCES}

Berne, A.

'Use of EIChrom's TRU Resin in the Determination of Americium, Plutonium and Uranium in Air Filter and water samples."

USDOE Report EML-575, December (1995)

Sanderson, C. G. and P. Greenlaw

"Semi-Annual Report of the Department of Energy, Office of Environmental Restoration and Waste Management, Quality Assessment Program"

USDOE Report EML-581, July (1996) 
$A m-05-R C$

AMERICIUM IN WATER AND AIR FILTERS

(see Volume II) 


\title{
AMERICIUM AND/OR PLUTONIUM IN VEGETATION
}

\author{
Contact Person(s) : Anna Berne
}

\begin{abstract}
APPLICATION
This procedure is applicable to vegetation which contain americium deposited from worldwide fallout and some nuclear activities. It is most effective when used on dried finely powdered samples of vegetation.

The vegetation is either dry ashed in a ceramic crucible using a muffle furnace or wet ashed with nitric acid. Wet ashing requires considerably more time and must be carefully attended to due to the highly reactive nature of vegetation. The sample is further digested with hydrofluoric acid to dissolve silicate compounds. Plutonium is separated by ion exchange and determined by alpha spectrometry. Americium is collected with a calcium oxalate precipitation and finally isolated and purified by ion exchange. After source preparation by microprecipitation, the ${ }^{241} \mathrm{Am}$ is determined by alpha spectrometry using ${ }^{243} \mathrm{Am}$ tracer to provide recovery data.
\end{abstract}

SPECIAL APPARATUS

1. For microprecipitation, see Procedure G-03.

2. Ion-exchange columns - see Specification 7.5. 


\section{SPECIAL REAGENTS}

1. Americium-243 tracer solution, about $0.15 \mathrm{~Bq} \mathrm{~g}^{-1}$ in a dispensing bottle-standardize for total for total disintegration rate (and/or ${ }^{236} \mathrm{Pu}$ tracer solution - a standard solution containing $\sim 0.15 \mathrm{~Bq} \mathrm{~g}^{-1}$ in a dispensing bottle). Measure purity on an $\alpha$ spectrometer.

2. Anion exchange resin, Bio-Rad AG 1-X8 resin (100-200 mesh) - see Specification 7.4 .

3. Anion exchange resin, Bio-Rad AG 1-X4 resin (100-200 mesh) - see Specification 7.4 .

4. TRU Resin $2 \mathrm{~mL}$ ion extraction columns or equivalent or can be prepared from TRU Resin, Eichrom Industries, Inc., 8205 Cass AV, Suite 107, Darien, $\mathbb{L}$ 60561. Place a plug of glass wool in the bottom of a polyethylene transfer pipette (see Specification 7.7). Add slurried TRU Resin (0.5 g). Assemble immediately before use.

5. $0.5 \mathrm{M} \mathrm{Al}\left(\mathrm{NO}_{3}\right)_{3}$ in $2 \mathrm{M} \mathrm{HNO}_{3}$ - place $18.76 \mathrm{~g}$ of $\mathrm{Al}\left(\mathrm{NO}_{3}\right)_{3} \cdot 9 \mathrm{H}_{2} \mathrm{O}$ in a $100-\mathrm{mL}$ volumetric flask and add $2 \mathrm{M} \mathrm{HNO}_{3}$ to the mark. Shake to mix thoroughly.

6. $2 \mathrm{M} \mathrm{HNO}_{3}-125 \mathrm{~mL}$ nitric acid diluted to $1 \mathrm{~L}$ with water.

7. $1 \mathrm{M} \mathrm{HNO}_{3}-62.5 \mathrm{~mL}$ nitric acid diluted to $1 \mathrm{~L}$ with water.

8. $0.025 \mathrm{M} \mathrm{HNO}_{3}-25 \mathrm{~mL} 1 \underline{\mathrm{M} \mathrm{HNO}} \mathrm{HN}_{3}$ diluted to $1 \mathrm{~L}$ with water.

9. Calcium carrier solution, $100 \mathrm{mg} \mathrm{mL}^{-1}$ - dissolve $25 \mathrm{~g} \mathrm{CaCO}_{3}$ in a minimal amount of concentrated $\mathrm{HNO}_{3}$, dilute to $100 \mathrm{~mL}$.

10. Iron carrier, $100 \mathrm{mg} \mathrm{mL}^{-1}$ - slowly heat $100 \mathrm{~g}$ of iron powder in $500 \mathrm{~mL}$ of $\mathrm{HCl}$ until the reaction ceases. Carefully and slowly add $100 \mathrm{~mL}$ of $\mathrm{HNO}_{3}$ while stirring. Cool and dilute to $1 \mathrm{~L}$.

11. Oxalate wash solution - dissolve $10 \mathrm{~g}$ of oxalic acid to make $1 \mathrm{~L}$ of solution $(\sim 1 \%$ solution). 
12. Hydroxylamine hydrochloride, $\mathrm{NH}_{2} \mathrm{OH} \cdot \mathrm{HCl}$.

\section{SAMPLE PREPARATION}

\section{A. Dry ashing}

1. Weigh an aliquot of $<10 \mathrm{~g}$ vegetation into a tared $250-\mathrm{mL}$ porcelain crucible. (Note: After ashing, several aliquots can be combined to provide adequate sample size.) Place each crucible in a muffle furnace with the crucible cover slightly ajar. Increase the temperature of the furnace at a rate of $0.80^{\circ} \mathrm{C} \mathrm{min}$ m $^{-1}$ to $250^{\circ} \mathrm{C}$. Maintain this temperature for 30 minutes. Increase the temperature at a rate of $10^{\circ} \mathrm{C} \mathrm{min}^{-1}$ to $600^{\circ} \mathrm{C}$. Maintain the temperature for $960 \mathrm{~min}$ to completely ash sample. Cool the crucible and weigh to determine percent ash. Ash content for replicate crucibles should vary by not more than $4 \%$. If the ash content of an individual sample is lower by more than $4 \%$, sample loss should be assumed and that sample discarded.

2. Place a known amount (approximately same amount as ${ }^{241} \mathrm{Am}$ in the sample) of ${ }^{243} \mathrm{Am}$ tracer (and/or ${ }^{236} \mathrm{Pu}$ tracer solution) in a 400-mL beaker containing a small amount of 1:1 $\mathrm{HNO}_{3}$. Transfer ashed vegetation to the beaker using $1: 1 \mathrm{HNO}_{3}$ to dissolve the ash and rinse the crucible. Transfer as many aliquots to the beaker as needed to meet the detection requirements.

3. Cover with a watch glass and reflux on a hot plate until there is no evidence of remaining organic matter, adding $\mathrm{HNO}_{3}$ or $\mathrm{H}_{2} \mathrm{O}_{2}$ as necessary.

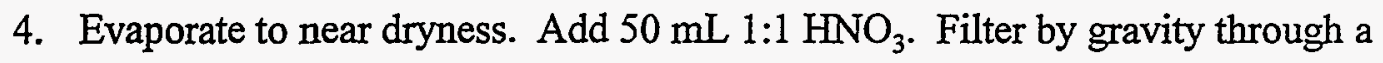
Whatman No. 42 filter paper, washing with $1: 1 \mathrm{HNO}_{3}$ into a beaker. Continue with Step 5 below.

\section{B. Wet ashing}

1. Weigh an aliquot of vegetation into an appropriate sized beaker. (For a 100-300 g sample, use a 3000-mL beaker.) Add a known amount (approximately the same amount as expected of ${ }^{241} \mathrm{Am}$ in the sample) of ${ }^{243} \mathrm{Am}$ tracer solution (and/or ${ }^{236} \mathrm{Pu}$ tracer solution). 
2. Slowly add $500 \mathrm{~mL}$ of $1: 1 \mathrm{HNO}_{3}$. Control the foaming, if necessary, with the addition of a few drops of n-octyl alcohol. Cover with a watch glass and place on a low temperature hot plate overnight, maintaining a slow reaction and stirring as necessary to break up the foam. Gradually increase the temperature of the hot plate, adding $\mathrm{HNO}_{3}$ and maintaining refluxing until the reaction is complete as indicated by the lack of brown nitrogen oxide gas.

3. Slowly add enough $\mathrm{HCl}$ to equal one third the volume of $\mathrm{HNO}_{3}$ still in the beaker. Allow the mixture to react at room temperature for $15 \mathrm{~min}$, cover with a watch glass, then heat on a low temperature hot plate overnight with occasional stirring.

4. Remove the sample from the hot plate, add an equal volume of water. Allow the sample to cool to room temperature. Filter by gravity through a large Whatman No. 42 filter paper into a beaker. Wash with $1: 1 \mathrm{HNO}_{3}$.

5. Retain the filtrate and evaporate to near dryness. Return the residue and filter to the original beaker. Add $100 \mathrm{~mL} \mathrm{HNO}_{3}$, cover with a watch glass and place on a hotplate to reflux until colorless. Change the watch glass to a ribbed watch glass and evaporate to near dryness.

6. Transfer the digested filter with the residue to a 250-mL Teflon beaker using $1: 1$ $\mathrm{HNO}_{3}$. Evaporate to dryness. Add $15 \mathrm{~mL}$ of $\mathrm{HNO}_{3}$ and $15 \mathrm{~mL}$ of $\mathrm{HF}$ to the beaker and evaporate to near dryness on a medium temperature hot plate. Repeat the addition of the $\mathrm{HF} / \mathrm{HNO}_{3}$ and the evaporation process two or three times.

7. Add $30 \mathrm{~mL} \mathrm{HNO}_{3}$ and evaporate to dryness, repeat twice, rinsing the walls of the beaker with acid. Add $20 \mathrm{~mL} \mathrm{HNO}_{3}$. Add $20 \mathrm{~mL}$ of water. Cool. Filter by gravity through a Whatman No. 42 filter paper into the beaker with the filtrate from Step 5 . Rinse with $1: 1 \mathrm{HNO}_{3}$.

8. Evaporate filtrate to dryness. Redissolve in $30 \mathrm{~mL} \mathrm{1:1} \mathrm{HNO}_{3}$. Proceed to Ion Exchange Purification for Plutonium Determination, Procedure Pu-11-RC, saving the column effluents for Americium Determination. 


\section{AMERICIUM DETERMINATION}

1. Evaporate the americium containing effluents in a beaker to incipient dryness. Redissolve in a minimum amount $(20-100 \mathrm{~mL})$ of $1: 1 \mathrm{HNO}_{3}$, dilute with four volumes of water.

2. Add $5 \mathrm{~mL}$ of calcium carrier solution ( $500 \mathrm{mg}$ of calcium) and $50 \mathrm{~g} \mathrm{~L}^{-1}$ of oxalic acid to the sample, while stirring with a magnetic stirrer. The total volume of the sample solution can be estimated using the markings on the beaker, and the amount of oxalic acid to be added is calculated using that volume.

3. Adjust the $\mathrm{pH}$ of the solution to $2.0-2.5$ with $\mathrm{NH}_{4} \mathrm{OH}$ using $\mathrm{pH}$ paper as an indicator and continue to stir for $30 \mathrm{~min}$. Remove the magnetic stir bar.

4. Cool and let stand until precipitate settles and solution clears. Check for completeness of precipitation using a drop of saturated $\mathrm{H}_{2} \mathrm{C}_{2} \mathrm{O}_{4}$ solution. Aspirate as much liquid as possible without disturbing the precipitate. Transfer precipitate to a 250-mL centrifuge bottle using oxalate wash solution (see Note 3). Balance the bottles on a double pan balance and centrifuge for $10 \mathrm{~min}$ at $2000 \mathrm{rpm}$. Discard the supernate.

5. Wash the precipitate with the oxalate wash solution. Centrifuge and discard the wash. Repeat wash. Redissolve the precipitate in a minimal amount (50-70 mL) of concentrated $\mathrm{HCl}$. (Note: Dissolution is easier if the centrifuge bottle is placed in a hot water bath and stirred with a glass stirring rod.)

6. Transfer the precipitate to the original beaker. Add $\sim 3$ volumes of water, $50 \mathrm{~g} \mathrm{~L}^{-1}$ of oxalic acid, and reprecipitate the oxalate with $\mathrm{NH}_{4} \mathrm{OH}$ at a $\mathrm{pH}$ of 2.5-3.5 (see Step 3).

7. Cool the solution, aspirate, transfer to a centrifuge bottle, centrifuge, wash and redissolve the precipitate (repeat Steps 4 and 5).

8. Transfer the solution to original beaker. Add $\sim 3$ volumes of water, $50 \mathrm{~g} \mathrm{~L}^{-1}$ of oxalic acid, and reprecipitate the oxalate at a $\mathrm{pH}$ of 2.5-3.5 with $\mathrm{NH}_{4} \mathrm{OH}$ (see Step 3). 
9. Cool the solution, aspirate, transfer to a centrifuge bottle, centrifuge, wash and redissolve the precipitate in $\sim 200 \mathrm{~mL}$ of concentrated $\mathrm{HNO}_{3}$.

10. Transfer the solution to the original beaker and heat to destroy oxalate ion. Evaporate to near dryness. Dissolve in 1:1 $\mathrm{HNO}_{3}$ and transfer to the centrifuge bottle.

11. Add enough water to make $\sim 1 \underline{\mathrm{M}} \mathrm{HNO}_{3}$. Warm the solution in a $90^{\circ}$ hot water bath and add $200 \mu \mathrm{L}$ iron carrier solution ( $20 \mathrm{mg}$ iron).

12. Adjust the $\mathrm{pH}$ of the solution to 8-9 with $\mathrm{NH}_{4} \mathrm{OH}$, while stirring with a glass rod. Leave the solution in a hot water bath to digest for $20 \mathrm{~min}$.

13. Cool in a cold water bath, rinse and remove the glass rod. Balance the bottles on a double pan balance and centrifuge for $40 \mathrm{~min}$ at $2000 \mathrm{rpm}$.

14. Aspirate the supernate and discard. Add $10 \mathrm{~mL}$ of concentrated $\mathrm{HCl}$ to dissolve the $\mathrm{Fe}(\mathrm{OH})_{3}$ pellet, four drops of $30 \% \mathrm{H}_{2} \mathrm{O}_{2}$ to get rid of any $\mathrm{Mn}$, followed by $100 \mathrm{~mL}$ of water, and heat in the water bath for $30 \mathrm{~min}$ to get rid of excess $\mathrm{H}_{2} \mathrm{O}_{2}$.

15. Reprecipitate, centrifuge and redissolve. Repeat Steps 12 to 14 three times. Reprecipitate, centrifuge and redissolve. The final precipitate should be dissolved in $1: 1 \mathrm{HNO}_{3}$.

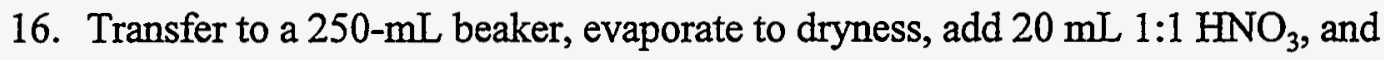
evaporate to dryness again.

17. Dissolve the residue in $40 \mathrm{~mL} \mathrm{1:1} \mathrm{HNO}_{3}$. Cool in an ice-water bath. Add 0.6-1.0 g $\mathrm{NH}_{2} \mathrm{OH} \cdot \mathrm{HCl}$, dissolve, and let react for $15 \mathrm{~min}$. Cover with a watch glass. Heat on a low temperature hot plate to decompose unreacted $\mathrm{NH}_{2} \mathrm{OH} \cdot \mathrm{HCl}$, then bring to gentle boil for 1-2 min. Cool and pass the solution through a 1:1 $\mathrm{HNO}_{3}$ ion-exchange column (see Note 1). Collect the effluent in a $400-\mathrm{mL}$ beaker. Wash with $150 \mathrm{~mL}$ of $1: 1 \mathrm{HNO}_{3}$, and collect in the beaker.

18. Evaporate the sample in the $400-\mathrm{mL}$ beaker to dryness and treat several times with concentrated HCl. Dissolve the residue in $30 \mathrm{~mL} \mathrm{HCl}$. Pass this solution through a 
concentrated $\mathrm{HCl}$ ion exchange column (see Note 2). Collect the effluent in a $250-\mathrm{mL}$ beaker, and wash with $100 \mathrm{~mL}$ of $\mathrm{HCl}$. Evaporate and proceed to microprecipitation if no residue is visible. If residue remains, continue with Step 19.

19. Evaporate to dryness transferring the sample to a $50-\mathrm{mL}$ beaker when volume is sufficiently diminished. Add 10-mL $\mathrm{HNO}_{3}$ and evaporate to dryness. Add $3 \mathrm{~mL}$ $0.5 \mathrm{M} \mathrm{Al}\left(\mathrm{NO}_{3}\right)_{3}$ in $2 \mathrm{M} \mathrm{HNO}_{3}$ to each residue and heat very gently to dissolve.

20. Prepare a TRU Resin column. Wash the resin with $15 \mathrm{~mL} 2 \mathrm{M} \mathrm{HNO}_{3}$, and discard the effluent.

21. Load the sample (see Step 19) on the column. Drain to the top of the resin. Wash the beaker with $3 \mathrm{~mL} 0.5 \mathrm{M} \mathrm{Al}\left(\mathrm{NO}_{3}\right)_{3}$ in $2 \mathrm{M} \mathrm{HNO}_{3}$ and add to the column. Discard the effluent.

22. Rinse the column with $8 \mathrm{~mL} 2 \mathrm{M} \mathrm{HNO}_{3}$, followed by $8 \mathrm{~mL} 1 \mathrm{M} \mathrm{HNO}_{3}$, and discard the effluents.

23. Elute the americium with three $3 \mathrm{~mL}$ aliquots of $0.025 \mathrm{M} \mathrm{HNO}_{3}$ into a $50-\mathrm{mL}$ beaker.

24. Evaporate eluate to dryness. Convert the residue to the chloride form by adding 3-4 $\mathrm{mL} \mathrm{HCl}$. Evaporate to dryness. Redissolve in $\mathrm{HCl}$ and evaporate two more times. Proceed to microprecipitation.

\section{Notes:}

1. Preparation of 1:1 $\mathrm{HNO}_{3}$ Column. Position a plug of glass wool at the base of an 11-mm o.d. column. Transfer $15 \mathrm{~mL}$ of wet settled Bio-Rad AG 1-X8 resin (100200 mesh) to the column with deionized distilled water, and allow to settle. Place a second plug of glass wool on top of the resin, and with the stopcock open allow the $\mathrm{H}_{2} \mathrm{O}$ level to reach the top of the upper plug. Pass $150 \mathrm{~mL}$ (or enough so that the effluent tests free of $\mathrm{Cl}^{-}$ion) of $1: 1 \mathrm{HNO}_{3}$ through the resin bed in three $50-\mathrm{mL}$ portions, allowing the level of each to reach the top of the upper glass wool plug.

2. Preparation of $\mathrm{HCl}$ Column. Position a plug of glass wool at the base of an $11-\mathrm{mm}$ o.d. column. Transfer $10 \mathrm{~mL}$ of wet settled Bio-Rad AG 1-X4 resin (100-200 mesh) 
with deionized water to the column, and allow to settle. Place a second plug of glass wool on top of the resin, and with the stopcock open allow the $\mathrm{H}_{2} \mathrm{O}$ level to reach the top of the upper plug. Pass two $50-\mathrm{mL}$ volumes of $\mathrm{HCl}$ through the resin bed and allow each to reach the top of the upper glass wool plug.

3. If a centrifuge is not available, centrifugation can be replaced by filtering and wet ashing the filter paper and precipitate in $\mathrm{HNO}_{3}$.

\section{MICROPRECIPITATION}

See Microprecipitation Source Preparation for Alpha Spectrometry, Procedure G-03.

LOWER LIMIT OF DETECTION (LLD)

\begin{tabular}{lll}
\hline & & \\
Counter Efficiency & $(\%)$ & 25 \\
Counter Background & $(\mathrm{cps})$ & $15 \times 10^{-6}$ \\
Yield & $(\%)$ & 50 \\
Blank & $(\mathrm{cps})$ & - \\
& & \\
LLD $(400 \mathrm{~min})$ & $(\mathrm{mBq})$ & 1 \\
LLD $(1000 \mathrm{~min})$ & $(\mathrm{mBq})$ & 0.5 \\
LLD $(5000 \mathrm{~min})$ & $(\mathrm{mBq})$ & 0.3 \\
& & \\
\hline
\end{tabular}




\section{Iron}

Environmental Measurements Laboratory U.S. Department of Energy 


\title{
IRON IN AQUEOUS SAMPLES - DUALDPM MODE LIQUDD SCINTILLATION ANALYSIS
}

\section{Contact Person(s) : Salvatore C. Scarpitta}

\begin{abstract}
APPLICATION
The procedure is intended for the determination of ${ }^{55} \mathrm{Fe}$, which decays by electron capture, in spiked aqueous samples that contain various mixed alpha, beta and gamma emitting radionuclides. Beta emitting ${ }^{59} \mathrm{Fe}\left(\mathrm{E}_{\max }=0.475 \mathrm{MeV}\right)$ is added to the samples as the yield determinant prior to $\mathrm{FeOH}_{3}$ scavenging. Following two anion exhange separations that remove most interferences, the samples (as $\mathrm{FePO}_{4}$ ) are counted in a commercially available liquid scintillation counter that is operated in the dual-dpm mode. A single count, in a calibrated instrument, provides the quench corrected activity concentrations of ${ }^{55} \mathrm{Fe}$ in a sample based on recovered ${ }^{59} \mathrm{Fe}$ (Scarpitta and Fisenne, 1996). The procedure is adapted from ASTM (1990) and is used in the EML Quality Assessment Program (QAP, Sanderson and Greenlaw, 1996).
\end{abstract}

\section{SPECIAL APPARATUS}

1. Disposable 3-mL polypropylene mini-columns (Catalog No.257.0119; Whatman Co., 5285 NE Elam Young Pkwy, Suite A-400, Hillsboro, OR 97124).

2. Liquid scintillation counter - Packard Tri-Carb 2250CA, Packard Instrument Co., Meridan, CT 06540 , or equivalent.

3. $20-\mathrm{mL}$ low $\mathrm{K}$ borosilicate glass vials. 
4. Centrifuge and 40-mL plastic tubes.

5. Vortexer.

\section{SPECIAL REAGENTS}

1. Anion exchange resin, Bio-Rad AG1-X8 (50-100 mesh), see Specification 7.4 $25 \mathrm{~mL}$ equilibrated with $125 \mathrm{~mL} 12 \mathrm{M} \mathrm{HCl}$ and $25 \mathrm{~mL}$ equilibrated with $125 \mathrm{~mL}$ of $1: 1 \mathrm{HNO}_{3}$.

2. Cerium carrier: $1 \mathrm{mg} \mathrm{mL}^{-1}-\mathrm{CeCl}$ in dilute $\mathrm{HCl}$.

3. Cesium carrier: $1 \mathrm{mg} \mathrm{mL}^{-1}-\mathrm{CsCl}$ in dilute $\mathrm{HCl}$.

4. Cobalt carrier: $1 \mathrm{mg} \mathrm{mL}-1-\mathrm{CoCl}_{2}$ in dilute $\mathrm{HCl}$.

5. Iron carrier: $5 \mathrm{mg} \mathrm{mL}^{-1}-\mathrm{FeCl}_{3}$ in dilute $\mathrm{HCl}$.

6. Manganese carrier: $1 \mathrm{mg} \mathrm{mL} \mathrm{m}^{-1}-\mathrm{MnCl}_{2}$ in dilute $\mathrm{HCl}$.

7. Strontium carrier: $1 \mathrm{mg} \mathrm{mL}-1-\mathrm{SrNO}_{3}$ in dilute $\mathrm{HCl}$.

8. Zinc carrier: $1 \mathrm{mg} \mathrm{mL}^{-1}-\mathrm{ZnCl}_{2}$ in dilute $\mathrm{HCl}$.

9. Insta-Gel-XF liquid scintillation cocktail - Packard Instrument Co., Downers Grove, II.

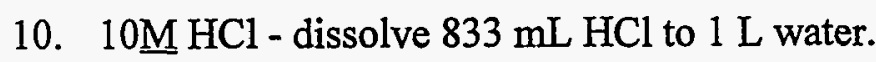

11. $6 \mathrm{M} \mathrm{HCl}$ - dilute $\mathrm{HCl} 1: 1$ in water.

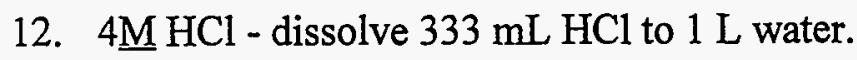

13. $0.5 \mathrm{M} \mathrm{HCl}$ - dissolve $42 \mathrm{~mL} \mathrm{HCl}$ to $1 \mathrm{~L}$ water. 
14. $0.01 \mathrm{M} \mathrm{HCl}$ - dissolve $20 \mathrm{~mL} 0.5 \mathrm{M} \mathrm{HCl}$ to $1 \mathrm{~L}$ water.

15. $8 \underline{\mathrm{N}} \mathrm{HNO}_{3}$ - dilute concentrated $\mathrm{HNO}_{3} 1: 1$ with water.

16. Ammonium phosphate $0.5 \mathrm{M}$ - dissolve $66 \mathrm{~g}$ of $\left(\mathrm{NH}_{4}\right)_{2} \mathrm{HPO}_{4}$ into $1 \mathrm{~L}$ of water.

17. Ammonium hydroxide - concentrated.

18. Quenching agent (e.g., chloroform, nitromethane, carbon tetrachloride, etc.).

\section{SEPARATION}

1. Gravimetrically add $100 \mathrm{dpm}$ of ${ }^{59} \mathrm{Fe}$ yield tracer and $1 \mathrm{~mL}$ of $5 \mathrm{mg} \mathrm{mL}^{-1} \mathrm{Fe}^{+3}$ carrier solution to the sample in a $40-\mathrm{mL}$ plastic centrifuge tube. Add $100 \mathrm{dpm}{ }^{59} \mathrm{Fe}$ tracer and $5 \mathrm{mg}$ of $\mathrm{Fe}^{+3}$ carrier to a 20 -mL glass scintillation vial labeled " $\mathrm{Fe}-59$ Reference Standard." Set the "Fe-59 Reference Standard" aside until Step 12. Prepare two reagent blanks using 40-mL plastic centrifuge tubes, each containing $5 \mathrm{mg}$ of Fe ${ }^{+3}$ carrier but without ${ }^{59} \mathrm{Fe}$ tracer. Add $1 \mathrm{~mL}$ of $\mathrm{NH}_{4} \mathrm{OH}$ to samples and blanks, only, until $\mathrm{pH}>10$ and $\mathrm{Fe}(\mathrm{OH})_{3}$ precipitate forms.

2. Centrifuge samples and blanks at $2000 \mathrm{rpm}$ for $10 \mathrm{~min}$. Decant and wash the precipitate with water.

3. Reconstitute the precipitate with $1 \mathrm{~mL}$ of $\mathrm{HCl}$.

4. Add $1 \mathrm{~mL}$ each of carriers $\left(1 \mathrm{mg} \mathrm{mL}^{-1}\right)$.

5. Prepare 3-mL columns using resin equilibrated in concentrated $\mathrm{HCl}$. Tap columns to speed flow and to assure packing of the resin.

6. Pass solution through the column, flow rate should be $5-8 \mathrm{~mL} \mathrm{~min}^{-1}$.

7. Elute with $10 \mathrm{~mL}$ volumes of each of the following acids $10 \mathrm{M} \mathrm{HCl}, 6 \mathrm{M} \mathrm{HCl}, 4 \mathrm{M} \mathrm{HCl}$ to remove $\mathrm{Mn}, \mathrm{Cs}, \mathrm{Ce}, \mathrm{Sr}$ and $\mathrm{Co}$. Collect the three eluates in the same $50-\mathrm{mL}$ beaker and discard. 
8. Replace the $50-\mathrm{mL}$ beaker with a $20-\mathrm{mL}$ glass scintillation vial. Elute the iron with exactly $10 \mathrm{~mL}$ of $0.01 \mathrm{M} \mathrm{HCl}$. The solution should be yellow. This fraction is used in Step 11.

9. Add $10 \mathrm{~mL}$ of concentrated $\mathrm{HNO}_{3}$ to the eluted sample in Step 8 to make it $8 \underline{\mathrm{N}}$.

10. Prepare a second column with the resin equilabrated with $8 \underline{N} \mathrm{HNO}_{3}$. Place a clean $50-\mathrm{mL}$ labeled beaker under the column.

11. Pass the eluate from the first column through the second column to retain uranium, plutonium and zinc. Wash with 5-10 $\mathrm{mL}$ of $8 \mathrm{~N} \mathrm{HNO}_{3}$ until the yellow colored drops, indicative of $\mathrm{Fe}^{+3}$, are absent from the eluate.

12. Add $10 \mathrm{~mL}$ of $0.5 \mathrm{M}\left(\mathrm{NH}_{4}\right)_{2} \mathrm{HPO}_{4}$ to the $20-\mathrm{mL}$ scintillation vial containing the iron eluate, and add concentrated $\mathrm{NH}_{4} \mathrm{OH}$ dropwise until the $\mathrm{pH}$ is about 3.0. Gently heat on a hot plate to completely precipitate iron as $\mathrm{FePO}_{4}$. Repeat this step using the "Fe59 Reference Standard."

13. Quantitatively transfer the sample to a $40 \mathrm{~mL}$ plastic centrifuge tube, decant and wash the precipitate with hot water.

14. Dissolve the precipitate in $1 \mathrm{~mL}$ of $0.5 \mathrm{M} \mathrm{HCl}$ to obtain a clear solution. (Note: This step is necessary to reduce color quenching of the sample.) Transfer the solution to a clean 20-mL glass scintillation vial, washing the centrifuge tube with two $0.5-\mathrm{mL}$ portions of $0.5 \mathrm{M} \mathrm{HCl}$. The total transferred volume should not exceed $3 \mathrm{~mL}$ of $0.5 \mathrm{M}$ $\mathrm{HCl}$.

15. Dispense $15 \mathrm{~mL}$ of Insta-Gel-XF liquid scintillation cocktail to each vial, cap, and vortex for $20 \mathrm{sec}$. Wipe the external surface of each vial with an alcohol soaked tissue. Let the samples adapt in the dark in the refrigerated liquid scintillation counter for $15 \mathrm{~min}$ prior to counting.

\section{Proceed to Determination}




\section{DETERMINATION}

\section{A. Instrument calibration.}

1. Define the ${ }^{55} \mathrm{Fe}$ and ${ }^{59} \mathrm{Fe}$ spectral ranges or window settings first (see Note 1).

2. Obtain the net activity (cpm) in each window setting by subtracting the background from the activity in the sample.

3. Determine the counting efficiencies (cpm dpm ${ }^{-1}$ ) for ${ }^{55} \mathrm{Fe}$ and ${ }^{59} \mathrm{Fe}$ from two efficiency quench calibration curves (see Note 2).

4. Prepare unquenched ${ }^{55} \mathrm{Fe}$ and ${ }^{59} \mathrm{Fe}$ standards in the same geometry as the analytical samples.

5. Set the energy regions for ${ }^{55} \mathrm{Fe}\left(\right.$ Region $\mathrm{A}$ ) and ${ }^{59} \mathrm{Fe}$ (Region $\left.\mathrm{B}\right)$ using the guidance found in the applicable liquid scintillation counter manual.

6. Optimize the two energy regions (the figure of merit is a common way to optimize the regions of interest) using the guidance found in the operating manual. Alternatively, follow Steps 7 to 9 .

7. Perform a spectrum analysis using the ${ }^{55} \mathrm{Fe}$ standard, and visually adjust the energy range of Region $\mathrm{A}\left(0-14 \mathrm{keV},{ }^{55} \mathrm{Fe}\right)$ to maximize the count rate and to minimize the energy range. (Note: $A$ balance between the count rate and the energy range should be obtained.)

8. Perform a spectrum analysis using the ${ }^{59} \mathrm{Fe}$ standard, and visually adjust the energy range of Region $\mathrm{B}\left(15-500 \mathrm{keV},{ }^{59} \mathrm{Fe}\right)$ to maximize the count rate and to minimize the energy range. (Note: A balance between the count rate and the energy range should be obtained.)

9. Utilizing the full window, set a third region as Region $\mathrm{C}(0-2000 \mathrm{keV})$. 


\section{B. Counting efficiency as a function of quenching.}

1. Establish the efficiency curves (efficiency vs. QIP) for ${ }^{55} \mathrm{Fe}$ and ${ }^{59} \mathrm{Fe}$ using the preset windows for each.

2. Check the efficiency curves annually and regenerate them if any major component of the instrumentation is replaced.

3. Check the liquid scintillation counter's stability first before running the standards and samples by counting the background and the ${ }^{3} \mathrm{H}$ and ${ }^{14} \mathrm{C}$ standards provided by the instrument manufacturer using the "SN" (system normalization) counting plug. (Note: The instrument software contains an appropriate spreadsheet program that compares the count rate of these standards with previously determined standard data according to criteria currently in use at EML.)

\section{Notes:}

1. Before counting samples and blanks, the appropriate window settings are determined for the two regions of interest (i.e., ${ }^{55} \mathrm{Fe},{ }^{59} \mathrm{Fe}$ ). Steps 1 to 8 are performed once, after which the appropriate parameters are manually set in the liquid scintillation counting instrument.

2. The efficiency curve is a plot of the counting efficiency as a function of the quench index parameter (QIP). The QIP is determined internally by the instrument, using a ${ }^{133} \mathrm{~m} \mathrm{Ba}$ source, and is also known as the automatic external standardization (AES) number or the transformed spectral index of the external standard ( $t / \mathrm{SIE})$. A sample aliquot is prepared with a measured volume of a scintillation cocktail that is then placed in a programmed liquid scintillation analyzer, which is operated in the dualdpm counting mode.
CALIBRATION

A. ${ }^{55} \mathrm{Fe}$

1. Use a small volume $(0.1 \mathrm{~mL})$ of ${ }^{55} \mathrm{Fe}$ tracer solution that has sufficient activity (i.e., $1000 \mathrm{dpm}$ ) to give a counting error of less than $1 \%$ for a 15 -min count. 
2. Record tracer information (including volume, activity concentration, reference date, etc.). Pipette various volumes of a quenching agent (i.e., nitromethane) into separately labeled vials to obtain a range of quenching from high to low efficiency. (Note: The following are examples of quenching agent volumes: $0.01,0.02,0.03$, $0.04,0.06,0.08 \mathrm{~mL}$ ).

3. Prepare a vial labeled "zero" that does not contain a quenching agent. (Note: Do not use deionized water to dilute the six aqueous quench standards to a volume consistent with the preparation of the sample set.)

4. Dispense enough scintillation cocktail into each glass vial to make up the final volumes that are consistent with the sample set to be analyzed (e.g., $15 \mathrm{~mL}$ ).

5. Cap and vortex the labeled scintillation vials for 10 to $20 \mathrm{sec}$. Wipe clean the vials with ethanol and a paper towel, and refrigerate in the dark for at least $10 \mathrm{~min}$.

6. Load the six aqueous quench standards from Step 2 into the counting rack with the first sample labeled as "zero." Select the dual-dpm mode from the main program menu. Key in (a) the number of standards per set for ${ }^{55} \mathrm{Fe}$, (b) the activity per sample (dpm), (c ) the reference date of the standards as MM/DD/YY, (d) the half-life of ${ }^{55} \mathrm{Fe}=23976 \mathrm{~d}$, and (e) low-level count mode (optional).

7. Count the quench standards, typically for $15 \mathrm{~min}$ (or until $1 \%$ counting statistics are obtained), in the three regions selected on the liquid scintillation counter. Hit the F2 key to count the data set (the corresponding QIPs given by the instrument are automatically recorded).

8. The net count rate for each quench standard vial is calculated automatically by subtracting the prepared background (zero) count rate in Region A from the measured gross count rates in Region $\mathrm{A}$.

9. The efficiency (Eff) is also determined automatically for each quenched sample in units of counts $\mathrm{min}^{-1} /$ disintegration $\mathrm{min}^{-1}$ by dividing the net activity measured in count $\mathrm{min}^{-1}$ by the calculated activity added in dpm. Also, the uncertainty in the efficiency, sigma (Eff), for each vial should be estimated for each vial. 
10. The efficiency curve is generated by the instrument which plots $\operatorname{Ln}(\mathrm{Eff})$ versus the QIP. Many liquid scintillations systems are equipped with programs to generate efficiency curves. (Note: To use these applications, the operating manual should be referenced.)

11. A least squares fit on the plot may be performed. The coefficients (a) and (b) or the equation

$$
\mathrm{Eff}=\mathrm{a} \exp [\mathrm{b} * \mathrm{QIP}]
$$

(obtained from the intercept $[\operatorname{Ln} a]$ and the slope [b]), and the fitting coefficient, $R^{2}$, should be recorded.

\section{B. ${ }^{59} \mathrm{Fe}$.}

1. Perform the calibration for ${ }^{59} \mathrm{Fe}$ in an identical fashion to ${ }^{55} \mathrm{Fe}$ (Steps A.1 to A.11), except that a tracer solution of ${ }^{59} \mathrm{Fe}$ is used instead of ${ }^{55} \mathrm{Fe}$, and count rates are measured in Region B rather than Region A.

2. Steps 6 and 7 of ${ }^{55} \mathrm{Fe}$ calibration above should be followed using $45 \mathrm{~d}$ as the half-life of ${ }^{59} \mathrm{Fe}$.

\section{PREPARATION OF LIQUID SCINTILATION COUNTING VIALS}

1. Mark the appropriate glass liquid scintillation vials with the sample identification on the cap.

2. Individually pipette a known amount (e.g., $2.0-5.0 \mathrm{~mL}$ ) of each sample into the appropriate scintillation vials.

3. Dispense an appropriate amount (e.g., $15 \mathrm{~mL}$ ) of the scintillation cocktail into each scintillation vial. A separate vial containing 2-5 $\mathrm{mL}$ of water serves as "background" (see Note 1). 
4. Load the samples into the counting rack with the first sample being "zero"from Step A3, Determination.

5. Use the Packard Instrument counting protocol plug No. 1 which is delegated for ${ }^{55} \mathrm{Fe} /{ }^{59} \mathrm{Fe}$ counting.

6. Select the "use curve" option from the count mode menu under the dual-dpm mode when counting samples for ${ }^{55} \mathrm{Fe}$.

Note:

1. Check the samples for phase separation. If phase separation is evident, solubilizing or complexing agents will be required to produce a stable solution.

\section{CALCULATIONS}

When using the "dual-isotope" counting mode, calculations are performed internally by the liquid scintillation software and the results are given in $\mathrm{dpm}$.

\section{REFERENCES}

ASTM Designation: D-4922-89

Standard Test Method for Determination of Radioactive Fe in Water. ASTM Committe D19, 1916 Race Place, Philadelphia PA, 19103, March (1990).

\section{DeFilippis, $S$.}

${ }^{455} \mathrm{Fe}$ and ${ }^{59} \mathrm{Fe}$ : A Qualitative Comparison of Four Methods to Liquid Scintillation Activity Analysis"

Radioactivity Radiochemistry, 2 , 14-21 (1991).

Sanderson, C. G. and P. Greenlaw

"Semi-Annual Report of the Department of Energy, Office of Environmental Restoration and Waste Management, Quality Assessment Program"

USDOE Report EML-581, July (1996) 
Scarpitta, S. C. and I. M. Fisenne

"Calibration of a Liquid Scintillation Counter for Alpha, Beta and Cerenkov Counting" USDOE Report EML-583, July (1996) 


\section{Tritium}

Environmental Measurements Laboratory U. S. Department of Energy 
${ }^{3} \mathrm{H}-01-\mathrm{RC}, \mathrm{Vol}$.II

HASL-300, 28th Edition
Rev. 0 February 1997

${ }^{3} H-01-R C$

TRITIUM IN WATER - ACD ELECTROLYSIS

(see Volume II) 


$$
{ }^{3} H-02-R C
$$

TRITIUM IN WATER - ALKALINE ELECTROLYSIS

(see Volume II) 
${ }^{3} \mathrm{H}-03-\mathrm{RC}$

ORGANICALLY LABELED TRITIUM - COMBUSTION PROCEDURE (see Volume II) 


\title{
TRITIUM IN WATER - LIQUID SCINTILLATION COUNTING
}

\author{
Contact Person(s) : Salvatore C. Scarpitta
}

\begin{abstract}
APPLICATION
The procedure is used for the rapid determination of tritium by liquid scintillation (LS) spectrometry. It applies to all clear liquid samples and it can be completed in a short period ( 1 to $2 \mathrm{~h}$ ) once efficiency curves have been established. The procedure is designed for a Tri-Carb 2250CA LS counter using Insta-Gel XF cocktail and it requires distillation of the samples so that they are free of salts and other interfering radionuclides.

The ${ }^{3} \mathrm{H}$ spectral range or window setting is first defined. The counting efficiencies are then determined by using efficiency curves (quench curves). The efficiency curve is a plot of the counting efficiency as a function of the quench index parameter (QIP). The QIP is also known as the automatic external standardization (AES) number or the transformed spectral index of the external standard (tSIE). A sample aliquot is prepared with a measured volume of a scintillation cocktail that is then placed in a programmed LS counter for spectrum analysis.
\end{abstract}

\section{SPECIAL APPARATUS}

1. Packard Tri-Carb 2250-CA LS spectrometer

2. Low ${ }^{40} \mathrm{~K}$ borosilicate glass scintillation vials

3. Glass distillation apparatus

4. Refrigerator 
5. Tritium source - sealed $20-\mathrm{mL}$ scintillation vial with cocktail.

6. Carbon-14 source - sealed $20-\mathrm{mL}$ scintillation vial with cocktail.

7. Background source - sealed $20-\mathrm{mL}$ scintillation vial with cocktail.

8. Glass containers - where ${ }^{3} \mathrm{H}$ is of concern to minimize evaporative loss of water.

\section{SPECIAL REAGENTS}

1. Standardized solutions of ${ }^{3} \mathrm{H}$ water

2. Insta-gel-XF or equivalent scintillation cocktail

3. $30 \% \mathrm{H}_{2} \mathrm{O}_{2}$

4. Ethanol (95\%)

5. Quenching agent - e.g., chloroform, nitromethane, carbon tetrachloride, nitric acid, etc. (Note: Some of the quenching agents listed are highly toxic and/or are carcinogens, e.g., carbon tetrachloride, and the safety of handling and disposal is the responsibility of the user.)

\section{INTERFERENCES}

1. Perform a ${ }^{3} \mathrm{H}$ distillation if information more accurate than gross tritium is needed.

2. Reanalyze samples with excessively high count rates (e.g., $>1,000,000$ counts min $^{-1}$ ) using less sample material. (Note: When using this procedure, the calculated ${ }^{3} \mathrm{H}$ concentration may be higher than the actual concentration because of possible interferences from other low-energy beta emitters and the beta continuum of highenergy beta particles.) 
3. Add several drops of $30 \% \mathrm{H}_{2} \mathrm{O}_{2}$ to the vial to bleach the sample if quenching causes the efficiency to drop to one fourth of the highest efficiency. (Note: Deep color in the samples may cause severe quenching that will lower the counting efficiency.)

\section{DETERMINATION}

\section{A. Window settings. (see Note 1 )}

1. Prepare unquenched ${ }^{3} \mathrm{H}$ standards in the same geometry as the analytical samples. Set the energy regions for ${ }^{3} \mathrm{H}$ (Region A) using the guidance found in the applicable LS counter manual.

2. Optimize the energy regions using the guidance found in the operating manual (the figure of merit is a common way to optimize the regions of interest). Follow Step 3 as an alternate.

3. Perform a spectrum analysis using the ${ }^{3} \mathrm{H}$ standard, and visually adjust the energy range of Region $\mathrm{A}\left(0\right.$ to $19 \mathrm{keV},{ }^{3} \mathrm{H}$ ) to maximize the count rate and minimize the energy range. A balance between the count rate and the energy range should be obtained (see Note 2).

\section{Notes:}

1. Determine the ${ }^{3} \mathrm{H}$ window settings before counting the samples and backgrounds.

2. If the sample is not distilled there may be interference from low energy beta particles with the same energy as ${ }^{3} \mathrm{H}$, as well as from the beta continuum from high energy beta particles.

\section{B. Efficiency as a function of quenching. (see Note)}

1. Use a small volume (10-50 $\mu \mathrm{L})$ of ${ }^{3} \mathrm{H}$ tracer solution that has sufficient activity to give a counting error of $<1 \%$. Prepare approximately 6-8 standards. 
2. Record the tracer information (including volume, activity concentration, reference date, etc.). In order to obtain a range of quenching from high to low efficiency, pipette various volumes of the quenching agent (i.e., nitromethane) into vials, separately labeled. The following quenching agent volumes are presented as an example: $0,0.01,0.02,0.03,0.05$, and $0.08 \mathrm{~mL}$. (Note: Do not use deionized water to dilute the aqueous standard to a volume consistent with the sample set preparation.)

3. Dispense enough scintillation cocktail into each glass vial to make up the final volumes consistent with the sample set to be analyzed (e.g., $15 \mathrm{~mL}$ ).

4. Cap and shake vigorously the scintillation vials for 10 to $20 \mathrm{sec}$. Wipe the vials clean with $95 \%$ ethanol and a paper towel. Refrigerate the vials for at least $10 \mathrm{~min}$.

5. Check the stability and operational order of the IS counter before running the standards and the samples. This can be done by: counting the background, the ${ }^{3} \mathrm{H}$ standard, and the ${ }^{14} \mathrm{C}$ standard provided by the instrument manufacturer; using an appropriate spreadsheet program; and then comparing the count rate of these standards with previously determined standard data according to criteria currently in use.

6. Count a background sample (prepared similarly to the standards) and the ${ }^{3} \mathrm{H}$ standards for $30 \mathrm{~min}$ (or until 1\% counting statistics are obtained) in the regions selected on the LS counter. Record the corresponding energy regions given by the instrument.

7. Calculate the net count rate for each vial by subtracting the prepared background count rates in Region A from the measured gross count rates in Region A.

8. Determine the beta efficiency $\left(\mathrm{Eff}_{\beta}\right)$ for each vial in units of count per min/ disintegrations per min by dividing the net activity measured in counts $\mathrm{min}^{-1}$ by the calculated activity added in dpm. Also, estimate the uncertainty in the $\beta$-efficiency, $\epsilon$-(Eff) for each vial.

9. Generate the $\beta$-efficiency curve by plotting $\operatorname{Ln}\left(\mathrm{Eff}_{\beta}\right)$ versus the QIP. (Note: Many LS counting systems are equipped with programs to generate efficiency curves. To use these applications, the operating manual should be referenced.) 
10. Perform a least squares fit on the plot. Record the coefficients (a) and (b) for the equation $E f f=a \exp \left(b^{*} \mathrm{QIP}\right)$ and the fitting coefficient, $\mathrm{R}$. Obtain the QIP from the intercept [Ln a] and the slope [b].

Note: Establish efficiency curves (efficiency vs. QIP) by using artificially quenched standards. Check the efficiency curves annually and regenerate them if any major component of the instrumentation is replaced.

\section{SAMPLE PREPARATION}

1. Mark the sample identification on the appropriate scintillation vials with ink that is not removable using ethanol.

2. Pipette a known amount (e.g., $2.0-5.0 \mathrm{~mL}$ ) of each sample into the appropriate scintillation vials.

3. Dispense an appropriate amount (e.g., $15 \mathrm{~mL}$ ) of scintillation cocktail into each scintillation vial.

4. Secure the cap onto each of the scintillation vials, then shake the vials vigorously for 10 to $20 \mathrm{sec}$. Wipe each vial on the outside with a paper towel wet with ethanol to remove any fingerprints.

5. Refrigerate the samples for at least $10 \mathrm{~min}$ before counting. (Note: The samples may also need to be "dark adapted" to minimize the potential for delayed scintillations. This can be accomplished by postponing the start of counting for 10-15 $\mathrm{min}$.)

6. Prepare a background sample in the same manner as the samples. A prepared background sample is one that is developed using stable compounds (e.g., dead water), and has a matrix as similar as possible to that of the samples being analyzed. 
7. Count the sample for an appropriate time (e.g., $10 \mathrm{~min}$ ) in the energy regions specified in Determination, Step 7. Record the QIPs given by the instrument. Return the samples to a well-ventilated area and store until disposal when the sample counts are completed.

\section{CALCULATIONS}

Perform the calculations internally with the LS software when using the DPM mode, or refer to EPA-600 4-80-032, Method 906.0 (Krieger and Whittaker, 1980).

\section{REFERENCE}

Krieger, H. L. and E. L. Whittaker

"Prescribed Procedures for Measurements of Radioactivity in Drinking Water" U. S. Environmental Protection Agency EPA-600 4-80-032, Method 906.0, August (1980) 


\section{Lead}

Environmental Measurements Laboratory U.S. Department of Energy 
LEAD-210 IN BONE, FOOD, URINE, FECES, BLOOD, AIR, AND WATER

Contact Person(s) : Isabel M. Fisenne

\section{APPLICATION}

This procedure is applicable to samples of bone, food, urine, feces, blood, air, and water and is based on the solvent extraction of a lead bromide complex into Aliquat-336 (Petrow and Cover, 1965; Morse and Welford, 1971).

Lead- 210 is isolated from most interferences. Its progeny ${ }^{210} \mathrm{Bi}$ is separated from ${ }^{210} \mathrm{~Pb}$, and the $\beta$ activity is measured radiometrically after ingrowth.

\section{SPECIAL APPARATUS}

1. Atomic absorption (AA) spectrometer.

2. Aluminum foil $-7.2 \mathrm{mg} \mathrm{cm}^{-2}$.

3. Rings and discs - see Specification 7.2.

4. Mylar film - see Specification 7.3.

5. Teflon filter holder.

6. Combination magnetic stirrer and hot plate.

7. Plastic scintillation phosphors - see Specification 7.9. 


\section{SPECIAL REAGENTS}

1. Aliquat-336, methyltricapryl-ammonium chloride (Henkel Corporation, $2430 \mathrm{~N}$. Huachuca Dr., Tucson, AZ 85745-1273), 3:7 in toluene and washed twice with an equal volume of $1.5 \mathrm{M}$ hydrobromic acid.

2. Hydrobromic acid $48 \%$.

3. Hydrobromic acid 3.0M $-340 \mathrm{~mL}$ of $48 \% \mathrm{HBr} \mathrm{L}^{-1}$ of water.

4. Hydrobromic acid $1.5 \mathrm{M}-170 \mathrm{~mL}$ of $48 \% \mathrm{HBr} \mathrm{L}^{-1}$ of water.

5. Hydrobromic acid $0.1 \mathrm{M}-10 \mathrm{~mL}$ of $48 \% \mathrm{HBr} \mathrm{L}^{-1}$ of water.

6. Toluene.

7. Standard $\mathrm{Pb}$ solution $-1000 \mu \mathrm{g} \mathrm{mL} \mathrm{m}^{-1}$.

8. Lead carrier: $20 \mathrm{mg} \mathrm{Pb} \mathrm{mL}-32 \mathrm{~g} \mathrm{~Pb}\left(\mathrm{NO}_{3}\right)_{2} \mathrm{~L}^{-1}$ in $1: 19 \mathrm{HNO}_{3}$.

9. Bismuth carrier: $10 \mathrm{mg} \mathrm{Bi} \mathrm{mL} L^{-1}-23.2 \mathrm{~g} \mathrm{Bi}\left(\mathrm{NO}_{3}\right)_{3} \cdot 5 \mathrm{H}_{2} \mathrm{OL}^{-1}$ in $1: 19 \mathrm{HNO}_{3}$.

\section{SAMPLE PREPARATION}

\section{A. Water.}

1. To daily collections of $20 \mathrm{~L}$ of tap water add $100 \mathrm{~mL}$ of $\mathrm{HNO}_{3}$ and evaporate to about $100 \mathrm{~mL}$ (see Note 1).

2. Add $100 \mathrm{~mL}$ of $\mathrm{HNO}_{3}$ and transfer to a $400-\mathrm{mL}$ beaker. Complete the destruction of organic material and evaporate to near dryness.

3. Add $50 \mathrm{~mL}$ of $10 \% \mathrm{HNO}_{3}$ to the beaker and warm to affect dissolution of any residue. 
4. Cool the solution to room temperature and transfer the sample solution to a $100-\mathrm{mL}$ volumetric flask. Bring the solution to volume with $10 \% \mathrm{HNO}_{3}$.

5. Pipette $1 \mathrm{~mL}$ of sample solution into a $10-\mathrm{mL}$ volumetric flask. Bring the solution to volume with $10 \% \mathrm{HNO}_{3}$. Reserve this solution to determine the stable $\mathrm{Pb}$ content of the sample by AA spectrometry (see Note 2).

6. Return the sample solution in the $100-\mathrm{mL}$ volumetric flask (Step 4) to the $400-\mathrm{mL}$ beaker. Add $1 \mathrm{~mL}$ of $\mathrm{Pb}$ carrier and evaporate the solution to dryness.

7. Add $100 \mathrm{~mL}$ of $3 \mathrm{M} \mathrm{HBr}$ to the sample beaker and warm the solution. Cool the solution to room temperature and proceed with the Determination.

\section{B. Urine, blood, feces and air filters.}

1. Measure 2-L of urine and transfer to a 3-L beaker. Place a measured volume of blood or $24 \mathrm{~h}$ fecal sample in a 1-L beaker. Place the air filter in a $600-\mathrm{mL}$ beaker (see Note 1).

2. Destroy most of the organic material by carefully heating with $\mathrm{HNO}_{3}$. Hydrogen peroxide can be used to complete the oxidation of organic material (see Note 3 ).

3. Add $50-\mathrm{mL}$ of $10 \% \mathrm{HNO}_{3}$ to the beaker and warm to affect dissolution of any residue.

4. Cool the solution to room temperature and transfer the sample solution to a $100-\mathrm{mL}$ volumetric flask. Bring the solution to volume with $10 \% \mathrm{HNO}_{3}$.

5. Pipette $1 \mathrm{~mL}$ of sample solution into a $10-\mathrm{mL}$ volumetric flask. Bring the solution to volume with $10 \% \mathrm{HNO}_{3}$. Reserve this solution to determine the stable $\mathrm{Pb}$ content of the sample by AA spectrometry (see Note 2).

6. Transfer the sample solution in the $100-\mathrm{mL}$ volumetric flask (Step 4) to the $400-\mathrm{mL}$ beaker. Add $1 \mathrm{~mL}$ of $\mathrm{Pb}$ carrier and evaporate the solution to dryness. 
7. Add $100-\mathrm{mL}$ of $3 \mathrm{M} \mathrm{HBr}$ to the sample beaker and warm the solution. Cool the solution to room temperature and proceed with the Determination.

\section{Bone (see Note 4)}

1. Weigh $20 \mathrm{~g}$ of bone ash and transfer to a $400-\mathrm{mL}$ beaker.

2. Dissolve the ash in about $80 \mathrm{~mL}$ of $3 \mathrm{M} \mathrm{HBr}$ and warm to complete the dissolution.

3. Cool the solution to room temperature and transfer the sample solution to a $100-\mathrm{mL}$ volumetric flask. Bring the solution to volume with $3 \mathrm{M} \mathrm{HBr}$.

4. Pipette $1 \mathrm{~mL}$ of sample solution into a $10-\mathrm{mL}$ volumetric flask. Bring the solution to volume with $10 \% \mathrm{HNO}_{3}$. Reserve this solution to determine the stable $\mathrm{Pb}$ content of the sample by AA spectrometry (see Note 2).

5. Transfer the sample solution in the $100-\mathrm{mL}$ volumetric flask (Step 3) to the $400-\mathrm{mL}$ beaker and add $1 \mathrm{~mL}$ of $\mathrm{Pb}$ carrier.

6. Proceed with the Determination.

\section{Food.}

1. Depending upon food type, freeze drying should be used to remove excess water prior to wet ashing the sample (see Note 1).

2. Destroy most of the organic material by carefully heating with $\mathrm{HNO}_{3}$. Hydrogen peroxide can be used to complete the oxidation of organic material (see Note 3 ).

3. Add $50 \mathrm{~mL}$ of $10 \% \mathrm{HNO}_{3}$ to the beaker and warm to affect dissolution of any residue.

4. Cool the solution to room temperature and transfer the sample solution to a $100-\mathrm{mL}$ volumetric flask. Bring the solution to volume with $10 \% \mathrm{HNO}_{3}$. 
5. Pipette $1 \mathrm{~mL}$ of sample solution into a $10-\mathrm{mL}$ volumetric flask. Bring the solution to volume with $10 \% \mathrm{HNO}_{3}$. Reserve this solution to determine the stable $\mathrm{Pb}$ content of the sample by AA spectrometry (see Note 2).

6. Transfer the sample solution in the $100-\mathrm{mL}$ volumetric flask (Step 3) to the $400-\mathrm{mL}$ beaker. Add $1 \mathrm{~mL}$ of $\mathrm{Pb}$ carrier and evaporate the solution to dryness.

7. Add $100-\mathrm{mL}$ of $3 \mathrm{M} \mathrm{HBr}$ to the same beaker and warm the solution. Cool the solution to room temperature and proceed with the Determination.

\section{Notes:}

1. It is necessary to analyze reagent blanks with each batch of samples to correct the ${ }^{210} \mathrm{~Pb}$ results.

2. The stable $\mathrm{Pb}$ content of some samples may be high enough to contribute a significant fraction to the total stable $\mathrm{Pb}$ measured by $\mathrm{AA}$. This would result in an inflated estimate of the $\mathrm{Pb}$ carrier yield.

3. Hydrogen peroxide contains measurable and variable amounts of stable $\mathrm{Pb}$ and should be used sparingly.

4. It has been shown at this Laboratory that no ${ }^{210} \mathrm{~Pb}$ loss occurs from bone dry ashed below $700^{\circ} \mathrm{C}$ (Fisenne, 1994). The absence of ${ }^{210} \mathrm{~Pb}$ loss was determined for three bone types - ribs, vertebrae, and femur. It is the practice at EML to dry ash bones for ${ }^{210} \mathrm{~Pb}$ analyses at $550^{\circ} \mathrm{C}$.

\section{DETERMINATION}

1. Transfer the $3 \mathrm{M} \mathrm{HBr}$ solution to a $250-\mathrm{mL}$ separatory funnel containing $75 \mathrm{~mL}$ of Aliquat-336.

2. Shake for $30 \mathrm{sec}$. Let the phases separate and discard the aqueous (lower) phase. 
3. Wash the organic phase three times with $50-\mathrm{mL}$ portions of $0.1 \mathrm{M} \mathrm{HBr}$ and discard all washes (lower phases).

4. Wash the organic phase twice with an equal volume of water. Transfer the washed organic phase to a suitable disposal container.

5. Combine the strip solutions in a $400-\mathrm{mL}$ beaker and add $100 \mathrm{~mL}$ of $\mathrm{HNO}_{3}$.

6. Wait for any reaction to subside and heat gently until the organic residue is destroyed. Evaporate the solution to $\sim 10 \mathrm{~mL}$.

\section{A. First milking.}

1. Transfer the sample to a $40-\mathrm{mL}$ centrifuge tube with water. Add $1 \mathrm{~mL}$ of Bi carrier.

2. Adjust the $\mathrm{pH}$ of the sample to 8 with $\mathrm{NH}_{4} \mathrm{OH}$.

3. Stir the sample and heat in a hot water bath.

4. Cool and centrifuge the tube for $10 \mathrm{~min}$. Decant and discard the supernate.

5. Dissolve the precipitate with five drops of $\mathrm{HCl}$.

6. Add $40 \mathrm{~mL}$ of water and heat with constant stirring.

7. Cool and centrifuge for $10 \mathrm{~min}$. Decant and reserve the supernate in a $250-\mathrm{mL}$ beaker.

8. Repeat Steps 5-7 twice more, combining the supernates. Discard the precipitate. Record the time and date for ingrowth of ${ }^{210} \mathrm{Bi}$.

9. Add $1 \mathrm{~mL}$ of $\mathrm{Bi}$ carrier and 3-5 $\mathrm{mL}$ of $\mathrm{HCl}$ to the combined supernates. Reduce the volume to $<100 \mathrm{~mL}$.

10. Cool, transfer to a $100-\mathrm{mL}$ volumetric flask and bring to volume with $0.5 \underline{\mathrm{N}} \mathrm{HCl}$. 
11. Pipette $1 \mathrm{~mL}$ of sample into a $10-\mathrm{mL}$ volumetric flask. Bring to volume with $0.5 \underline{\mathrm{N}}$ $\mathrm{HCl}$.

12. Measure the quantity of $\mathrm{Pb}$ in both the sample and the separated $\mathrm{Pb}$ fraction in the 10 $\mathrm{mL}$ volumetric flasks on an AA spectrometer at $283 \mu \mathrm{m}$. (The calibration curve should have a working range of $0-50 \mu \mathrm{g} \mathrm{mL}^{-1}$.)

13. Subtract the $\mathrm{Pb}$ content of the dissolved sample and the reagent blank from the total $\mathrm{Pb}$ content determined in Step 12 to obtain the $\mathrm{Pb}$ carrier yield.

14. Allow 2-3 weeks for ingrowth of ${ }^{210} \mathrm{Bi}$ into the main portion of the sample (Step 10).

\section{B. Second milking.}

1. Transfer the solution from the $100-\mathrm{mL}$ volumetric flask to a $250-\mathrm{mL}$ beaker and evaporate to about $15 \mathrm{~mL}$.

2. Transfer the sample to a $40-\mathrm{mL}$ centrifuge tube and adjust the $\mathrm{pH}$ to 8 with $\mathrm{NH}_{4} \mathrm{OH}$. Centrifuge the tube for $10 \mathrm{~min}$. Decant and discard the supernate.

3. Dissolve the precipitate with five drops of $\mathrm{HCl}$ and bring volume of sample to $30 \mathrm{~mL}$ with $\mathrm{H}_{2} \mathrm{O}$. (Record the time and date for decay of ${ }^{210} \mathrm{Bi}$.)

4. Heat with constant stirring in a hot water bath. Cool and centrifuge the tube for $10 \mathrm{~min}$. Reserve the supernate for additional ${ }^{210} \mathrm{~Pb}$ analysis in a $150-\mathrm{mL}$ beaker.

5. Dissolve the precipitate with five drops of $\mathrm{HCl}$ and dilute to $30 \mathrm{~mL}$ with water.

6. Heat in a hot water bath with constant stirring. Cool and centrifuge the tube for $10 \mathrm{~min}$. Combine the supernate with that from Step 4.

7. Dissolve the precipitate with five drops of $\mathrm{HCl}$. Stir and dilute to $30 \mathrm{~mL}$ with water.

8. Heat the tube in a hot water bath with constant stirring. Cool, filter with suction on a preweighed $2.4 \mathrm{~cm}$ Whatman No. 42 filter paper using a Teflon filter holder. 
9. Wash the tube and the precipitate with water and alcohol. Dry the paper and precipitate for $30 \mathrm{~min}$ at $110^{\circ} \mathrm{C}$ in a drying oven.

10. Cool and reweigh the filter to determine weight of $\mathrm{BiOCl}$ precipitate.

11. Mount the filtered sample on a nylon ring and disc, covering the sample with aluminum foil $\left(7.2 \mathrm{mg} \mathrm{cm}^{-2}\right)$, a plastic scintillation phosphor and Mylar film.

12. Measure the ${ }^{210} \mathrm{Bi}$ on a low-level $\beta$-scintillation counter. (Record the time and date for decay of ${ }^{210} \mathrm{Bi}$.)

13. Standardize the counter with a known amount of ${ }^{210} \mathrm{~Pb}$ from which ${ }^{210} \mathrm{Bi}$ has been separated and prepared in the same way as the sample.

\section{DATA PROCESSING AND ANALYSES}

The ${ }^{210} \mathrm{~Pb}$ activity of the sample is calculated using the following formula:

$$
\mathrm{Bq} \text { of }{ }^{210} \mathrm{~Pb}=\frac{\mathrm{R}_{\mathrm{S}} \mathrm{Y}_{1} \mathrm{Y}_{2} \mathrm{E}}{\mathrm{GD}}
$$

where

$\mathrm{R}_{\mathrm{s}}=$ net counting rate of the sample,

$\mathrm{Y}_{1}=$ yield factor for $\mathrm{Pb}$ carrier,

$\mathrm{Y}_{2}=$ yield factor for $\mathrm{Bi}$ carrier,

$\mathrm{E}=$ counter efficiency factor,

$\mathrm{G}=$ growth factor (growth of ${ }^{210} \mathrm{Bi}$ from first milking to final milking), and

$\mathrm{D}=$ decay factor (decay of ${ }^{210} \mathrm{Bi}$ from final milking to time of counting). 
LOWER LIMIT OF DETECTION (LLD)

\begin{tabular}{lllc}
\hline & & & \\
& & $\mathrm{A}$ & $\mathrm{B}$ \\
\hline & $(\%)$ & 35 & - \\
Counter efficiency & $(\mathrm{cps})$ & 0.005 & - \\
Counter background & $(\%)$ & 80 & 80 \\
Yield & & & \\
& $(\mathrm{Bq})$ & 0.01 & 0.007 \\
LLD (400 min) & $(\mathrm{Bq})$ & 0.005 & 0.005 \\
LLD (1000 min) & & & \\
\hline
\end{tabular}

$\mathrm{A}={ }^{210} \mathrm{~Pb}$ separation, ${ }^{210} \mathrm{Bi}$ ingrowth, ${ }^{210} \mathrm{Bi}$ separation.

$\mathrm{B}={ }^{210} \mathrm{Bi}$ separation only.

\section{REFERENCES}

Fisenne, I. M.

"Lead-210 in Animal and Human Bone: A New Analytical Method"

Env. Int., 20, 627-632 (1994)

Hursh, J. B. (Editor)

USAEC Report AECU-4024, November (1958)

Morse, R. S. and G. A. Welford

"Dietary Intake of ${ }^{210} \mathrm{~Pb}$ "

Health Phys., 21, 53-55 (1971)

Petrow, H. G. and A. Cover

"Direct Radiochemical Determination of Lead-210 in Bone"

Anal. Chem., 37, 1559-1660 (1965) 


\section{DETERMUNATION OF ${ }^{210} \mathrm{~Pb}$ in BONE ASH BY MEASUREMENT OF ${ }^{210} \mathrm{Po}$}

Contact Person(s) : Isabel M. Fisenne

\section{APPLICATION}

The procedure has been tested for sample weights of up to $15 \mathrm{~g}$ of bone ash.

Lead-210 may be determined in bone samples which have been dry ashed at temperatures up to $700^{\circ} \mathrm{C}$. The dry ashed bone samples should be stored for at least $2 \mathrm{y}$ to allow build-up of ${ }^{210} \mathrm{Po}$ (Fisenne, 1994).

Lead, bismuth, polonium and calcium are separated from phosphate and radium as the oxalates. Lead, bismuth and polonium are separated from calcium as the sulfides. Polonium and bismuth are spontaneously deposited on a nickel disc from a weakly acid solution. The ${ }^{210} \mathrm{Po}$ is measured by alpha spectrometry and the chemical yield is determined with the alpha emitting tracer ${ }^{209} \mathrm{Po}$.

Radium-226 is separated from the reserved oxalate supernate as the sulfate. The $\mathrm{Ra} . \mathrm{BaSO}_{4}$ is dissolved in alkaline EDTA and the chemical yield is determined with the gamma-emitting tracer ${ }^{133} \mathrm{Ba}$. The ${ }^{226} \mathrm{Ra}$ is determined by the radon emanation method (see Procedure Ra-03-RC).

The total and unsupported ${ }^{210} \mathrm{~Pb}$ activities are calculated from the ${ }^{210} \mathrm{Po}$ and ${ }^{226} \mathrm{Ra}$ measurements by application of the Bateman equations for the decay of this subseries of the Uranium Series (see Procedure 5.6). 


\section{SPECIAL APPARATUS}

1. Nickel discs $-1.74 \mathrm{~cm}$ diameter by $0.06 \mathrm{~cm}$ thick, "commercial" pure nickel. Prior to use, degrease the disc. [Note: Silver or platinum discs may be used but must be cleaned and recycled. The nickel discs are discarded after measurement of the sample.]

2. Deposition cells - see Specification 7.16.

3. Mechanical stirrers.

4. Teflon stirring rods.

5. Alpha spectrometry system.

6. Radon bubblers - see Specification 7.7.

7. Polyethylene transfer pipettes - see Specification 7.11.

\section{SPECIAL REAGENTS}

1. Polonium-209 tracer solution - $1 \mathrm{~Bq} \mathrm{~g}^{-1}$ of $1 \underline{\mathrm{N}} \mathrm{HNO}_{3}$ solution.

2. Lead carrier: $20 \mathrm{mg} \mathrm{Pb} \mathrm{mL}-1-32 \mathrm{~g} \mathrm{~Pb}\left(\mathrm{NO}_{3}\right)_{2} \mathrm{~L}^{-1}$ of $1 \underline{\mathrm{N}} \mathrm{HNO}_{3}$.

3. Calcium carrier - solid calcium propionate. [Note: Calcium carrier is added only to the reagent blank. The ${ }^{210} \mathrm{Po}$ and ${ }^{226} \mathrm{Ra}$ blank value must be established for each lot of calcium propionate salt prior to its use.]

4. Thioacetamide solution - $100 \mathrm{~g} \mathrm{CH}_{3} \mathrm{CSNH}_{2} \mathrm{~L}^{-1}$ of water.

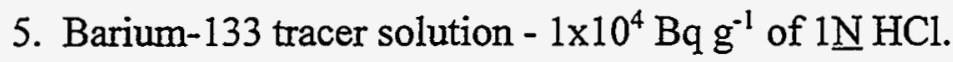

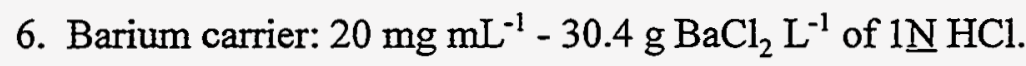


7. Ammonium acetate solution - $15 \mathrm{~g} \mathrm{NH}_{4} \mathrm{OAc} \mathrm{L}^{-1}$ of water.

8. Acetic acid solution - $20 \mathrm{~mL}$ glacial HOAc $\mathrm{L}^{-1}$ of water.

9. Ammonium sulfate solution $-100 \mathrm{~g}\left(\mathrm{NH}_{4}\right)_{2} \mathrm{SO}_{4} \mathrm{~L}^{-1}$ of water.

10. Aerosol OT solution $-0.1 \%$.

11. EDTA solution - $300 \mathrm{~g}$ tetrasodium salt $\mathrm{L}^{-1}$ of water. Filter the solution through a glass fiber filter prior to use.

12. Monoethanolamine - $1: 1$ solution with water.

\section{SAMPLE PREPARATION}

1. Weigh $5 \mathrm{~g}$ of bone ash and transfer to a graduated $1500-\mathrm{mL}$ beaker.

2. Add a weighed aliquot of ${ }^{209} \mathrm{Po}$ (about $0.05 \mathrm{~g}$ ) and ${ }^{133} \mathrm{Ba}$ (about $0.1 \mathrm{~g}$ ) tracer solutions and $1 \mathrm{~mL}$ of $\mathrm{Pb}$ carrier solution to the beaker. [Note: Add $2.5 \mathrm{~g}$ of calcium propionate to the reagent blank beaker. DO NOT ADD CALCIUM PROPIONATE TO THE BONE ASH SAMPLES.]

\section{LEAD SEPARATION}

1. Add $25 \mathrm{~mL}$ of $\mathrm{HNO}_{3}$ and $1 \mathrm{~mL}$ of $\mathrm{HClO}_{4}$ to the samples and reagent blank beakers. [Note: $\mathrm{HClO}_{4}$ is not required for samples dry ashed at $\geq 500^{\circ} \mathrm{C}$.]

2. Place the covered beakers on a medium hotplate. Swirl the beakers to dissolve the bone ash.

3. Evaporate the solution to dense $\mathrm{HClO}_{4}$ fumes. Do not allow the sample to reach dryness. 
4. Convert the sample to chloride form with five successive additions of $25 \mathrm{~mL}$ of $\mathrm{HCl}$. Evaporate the solution to near dryness between additions.

5. Add $25 \mathrm{~mL}$ of $\mathrm{HCl}$ and swirl the beaker to dissolve most of the solids. Remove the beaker from the hotplate.

6. Dilute the solution to $1 \mathrm{~L}$ with water. Stir the solution with a stirring rod to dissolve any remaining solids.

7. Return the beaker to the hotplate and heat the solution for $10 \mathrm{~min}$.

8. Using $\mathrm{NH}_{4} \mathrm{OH}$ in a wash bottle, and stirring constantly, adjust the $\mathrm{pH}$ to about 1.5 to 2 . At this point no permanent hydroxide flock should be present. Any hydroxide flock is dissolved with the addition of $\mathrm{N}$ HCL. [Note: The $\mathrm{pH}$ of the reagent blank is determined with $\mathrm{pH}$ paper.]

9. Weigh $5 \mathrm{~g}$ of solid oxalic acid and add to the sample with stirring. Continue heating the solution for $10 \mathrm{~min}$.

10. Adjust the $\mathrm{pH}$ to 2.0 using $\mathrm{NH}_{4} \mathrm{OH}$ and $\mathrm{pH}$ paper for both the sample and the reagent blank. Stir continuously.

11. Remove the sample from the hotplate. Remove and rinse the stirring rod with water. Allow the oxalate precipitate to settle overnight.

12. Decant the supernate into a 3-L beaker and reserve for Radium Separation.

13. Destroy the oxalates by evaporating with two successive $25-\mathrm{mL}$ portions of $\mathrm{HNO}_{3}$. Do not allow the sample to reach dryness.

14. Convert the sample to the chloride form with five successive $25-\mathrm{mL}$ portions of $\mathrm{HCl}$.

15. Add $25 \mathrm{~mL}$ of $\mathrm{HCl}$ and swirl the beaker to dissolve most of the solids. Add a minimum amount of water to reach complete solution.

16. Remove the beaker from the hotplate and cool. 
17. Transfer the solution with water to a $250-\mathrm{mL}$ centrifuge bottle. Wash the beaker with water and transfer the washings to the centrifuge bottle. Stir the solution. The solution volume should be about $200 \mathrm{~mL}$.

18. Place the centrifuge bottle in a $90^{\circ} \mathrm{C}$ hot water bath. Heat for $10 \mathrm{~min}$.

19. Add $5 \mathrm{~mL}$ of thioacetamide solution to the sample. Stir and heat in the water bath for 1 or $2 \mathrm{~min}$.

20. Remove the sample from the water bath. While stirring continuously, add $\mathrm{NH}_{4} \mathrm{OH}$ to the sample until the black precipitate just persists.

21. Return the sample to the water bath for $1 \mathrm{~h}$.

22. Remove the sample from the water bath. Remove the stirring rod and rinse with water.

23. Dry the outside of the centrifuge bottle. Place the bottle in a centrifuge cup. Tare a pair of samples on a double pan balance.

24. Place the tared samples in the centrifuge. Centrifuge the samples at $1800 \mathrm{rpm}$ for $1 \mathrm{~h}$.

25. Remove the samples from the centrifuge. Add 1 drop of Aerosol OT and decant the sample. Discard the supernate.

26. Add $2 \mathrm{~mL}$ of $\mathrm{HCl}$ to the sample and stir to break up the $\mathrm{PbS}$ precipitate. Place the sample in the water bath. Heat for $5 \mathrm{~min}$.

27. Wash the walls of the bottle with $10 \mathrm{~mL}$ of water and heat for an additional $10 \mathrm{~min}$.

28. Gravity filter the sample through a $12.5-\mathrm{cm}$ diameter Whatman No. 42 paper into a prepared deposition cell.

29. Wash the centrifuge bottle with water and add the washing to the filter. Wash the filter with water. Discard the filter. 
30. Place a cylindrical metal weight over the deposition cell. [Note: A 4-cm diameter by 4-cm height length of galvanized pipe will do.]

31. Place the weighted cell in an $80^{\circ} \mathrm{C}$ water bath. Lower the stirring motor with the Teflon rod in its chuck so that the rod is within $1 \mathrm{~cm}$ of the nickel disc.

32. Turn on the motor and stir for $4 \mathrm{~h}$ at maximum agitation without splashing. Add small quantities of $0.5 \mathrm{~N} \mathrm{HCl}$ to the cell, and water to the water bath to replace evaporative losses.

33. Turn off the motor and raise it to clear the stirring rod from the top of the cell. Remove the cell from the water bath.

34. Pour off the solution and discard. Rinse the cell successively with $0.5 \underline{\mathrm{N}} \mathrm{HCl}$ and water. Discard the rinsing.

35. Dismantle the cell and discard the bottle. Remove the nickel sample disc from the cap and rinse with ethanol.

36. Place the disc on a warm hotplate to dry.

37. Measure the disc in an alpha spectrometry system to determine the ${ }^{209}$ Po yield and the ${ }^{210}$ Po content of the sample. [Note: Record the deposition date so that decay corrections for the unsupported ${ }^{210} \mathrm{Po}$ can be made from separation date to measurement date.]

\section{RADIUM SEPARATION}

1. Evaporate the radium-bearing oxalate solution to dryness on a medium hotplate.

2. Remove the beaker from the hotplate and cool slightly. Add two successive $25-\mathrm{mL}$ portions of $\mathrm{HNO}_{3}$. Heat to destroy the oxalate.

3. Convert the sample to the chloride form with five successive $25-\mathrm{mL}$ portions of $\mathrm{HCl}$. 
4. Add $25 \mathrm{~mL}$ of $\mathrm{HCl}$ to the beaker and warm to dissolve most of the solids. Add about $50 \mathrm{~mL}$ of water and stir to dissolve the remaining solids.

5. Transfer the warm solution to a $250-\mathrm{mL}$ centrifuge bottle. Wash the beaker with water, police and transfer the washing to the centrifuge bottle.

6. Dilute $1 \mathrm{~mL}$ of barium carrier to about $5 \mathrm{~mL}$ with water. Stir the sample thoroughly and continuously while adding the diluted carrier solution dropwise.

7. Place the centrifuge bottle in a hot water bath and warm the solution for about $5 \mathrm{~min}$.

8. With constant stirring, slowly add $\mathrm{NH}_{4} \mathrm{OH}$ until a dense white permanent hydroxide flock forms.

9. Dissolve the flock in a minimum of $\mathrm{HCl}$. [Note: For the blank, adjust the $\mathrm{pH}$ from 1.5 to 2.0 using $\mathrm{pH}$ paper.

10. Add $2 \mathrm{~mL}$ of $\mathrm{NH}_{4} \mathrm{OAc}$ solution and $1 \mathrm{~mL}$ of dilute $\mathrm{HOAc}$ solution to the centrifuge bottle. Stir thoroughly and cool the bottle to room temperature in a water bath.

11. Add $1 \mathrm{~mL}$ of $\left(\mathrm{NH}_{4}\right)_{2} \mathrm{SO}_{4}$ solution to the bottle. Stir, remove the rod and rinse with water. Let the bottle stand at room temperature for $1 \mathrm{~h}$.

12. Dry the outside of the centrifuge bottle. Place the bottle in a centrifuge cup. Tare a pair of samples on a double pan balance.

13. Place the tared samples in the centrifuge. Centrifuge the samples at $1800 \mathrm{rpm}$ for $1 \mathrm{~h}$.

14. Remove the samples from the centrifuge. Add one drop of Aerosol OT to the bottle. Carefully decant and discard the supernate.

15. Heat $5 \mathrm{~mL}$ of EDTA solution $\left(300 \mathrm{~g} \mathrm{~L}^{-1}\right)$ for each sample in a hot water bath.

16. Break up the $\mathrm{BaSO}_{4}$ precipitate with the stirring rod. Add $5 \mathrm{~mL}$ of warm EDTA solution and $1 \mathrm{~mL}$ of 1:1 monoethanolamine. Stir and heat for about $5 \mathrm{~min}$. 
17. Wash down the sides of the centrifuge bottle with about $10 \mathrm{~mL}$ of water. Continue heating the bottle for $15 \mathrm{~min}$, stirring occasionally.

18. Gravity filter the solution through a $12.5-\mathrm{cm}$ diameter Whatman No. 42 filter paper into a $30-\mathrm{mL}$ polyethylene bottle.

19. Wash the centrifuge bottle and the filter paper with water. Discard the filter paper.

20. Dilute the sample to the same liquid level as the ${ }^{133} \mathrm{Ba}$ standard. The standard is prepared by diluting a known aliquot (about $0.1 \mathrm{~g}$ ) of ${ }^{133} \mathrm{Ba}$ solution to $25 \mathrm{~mL}$ in a $30 \mathrm{~mL}$ polyethylene bottle.

21. Gamma count the standard and samples to determine the chemical yield of barium.

22. Transfer the solution to a radon bubbler.

23. Proceed with emanation procedure for ${ }^{226} \mathrm{Ra}(\mathrm{Ra}-03-\mathrm{RC})$ to determine the radium content of the sample.

\section{LOWER LIMIT OF DETECTION (LLD)*}

\begin{tabular}{llcc}
\hline & \multicolumn{2}{c}{${ }^{210} \mathrm{Po}$} & ${ }^{226} \mathrm{Ra}$ \\
\hline & & & \\
Counter efficiency & $(\%)$ & 30. & 56. \\
Counter background & $(\mathrm{cps})$ & $4 \times 10^{-5} \pm 0.7 \times 10^{-5}$ & $2.8 \times 10^{-3} \pm 0.2 \times 10^{-3}$ \\
Yield & $(\%)$ & 85. & 85. \\
Blank & $(\mathrm{cps})$ & $3.5 \times 10^{-4} \pm 0.6 \times 10^{-4}$ & $2.0 \times 10^{-2} \pm 0.8 \times 10^{-2}$ \\
LLD (1000 min) & $(\mathrm{mBq})$ & 1.3 & 1.5 \\
LLD $(2500 \mathrm{~min})$ & $(\mathrm{mBq})$ & 1.0 & $\mathrm{~N} / \mathrm{A}$ \\
& & & \\
\hline
\end{tabular}

*Reagent blanks must be processed with every batch of samples. 


\section{REFERENCE}

Fisenne, I. M.

"Lead-210 in Animal and Human Bone: A New Analytical Method" Env. Int. , 20, 627-632 (1994) 


\section{Polonium}




\begin{abstract}
APPLICATION
This procedure is applicable to water and urine (Hursh, 1958). Organic materials which can be converted to $\mathrm{Cl}^{-}$solutions should also lend themselves to analysis by the procedure given. Reagent blanks must be analyzed along with the samples. [Note: It has been shown (Fellman et al., 1989) that urine samples must be wet ashed to release polonium from metabolically labeled organic compounds. The procedure has been modified to incorporate the destruction of organic matter.]
\end{abstract}

Polonium is quantitatively deposited on a nickel disc from a strong $\mathrm{HCl}$ solution. This is a very specific separation and therefore can be carried out while many other radionuclides are present in the sample.

The plated disc is $\alpha$ counted on a scintillation counter. It is also possible to use a ${ }^{208} \mathrm{Po}$ or ${ }^{209} \mathrm{Po}$ tracer and count on an $\alpha$ spectrometer to measure chemical yield and the activity of the sample.

\title{
SPECIAL APPARATUS
}

1. Nickel discs - made of $0.064-\mathrm{cm}$ thick "commercial pure" nickel sheets. Discs are $2.2 \mathrm{~cm}$ in diameter with a $0.16-\mathrm{cm}$ hole set $0.16 \mathrm{~cm}$ in from the edge. [Note: Coating the disc on one side with an acid resistant paint allows counting time to be cut in half.] 


\section{SAMPLE PREPARATION}

\section{A. Water.}

1. To $1000 \mathrm{~mL}$ of tap water in a $1500-\mathrm{mL}$ beaker, add $50 \mathrm{~mL}$ of $\mathrm{HCl}$.

2. Evaporate to a volume of $20 \mathrm{~mL}$ and transfer to a $250-\mathrm{mL}$ beaker. Add $100 \mathrm{~mL}$ of water and $100 \mathrm{mg}$ of ascorbic acid.

\section{Proceed to Determination.}

\section{B. Urine.}

1. If the time between sample collection and analysis is much greater than $1 \mathrm{~h}$, the urine samples should be preserved by adding $1 \mathrm{mg}$ of sulfamic acid per $\mathrm{mL}$ of urine and storing in a refrigerator at $3^{\circ} \mathrm{C}$.

2. Measure $100 \mathrm{~mL}$ of urine in a graduated cylinder and transfer to a $250-\mathrm{mL}$ beaker. Rinse the graduated cylinder with $20 \mathrm{~mL}$ of $1: 1 \mathrm{HNO}_{3}$ and add to the urine.

3. Evaporate the solution to near dryness and add $5 \mathrm{~mL}$ portions of $\mathrm{HNO}_{3}$ to destroy organic matter.

4. Convert the sample to the $\mathrm{Cl}^{-}$form by evaporating to near dryness with three successive $5-\mathrm{mL}$ portions of $\mathrm{HCl}$.

5. Add $20 \mathrm{~mL}$ of $1: 1 \mathrm{HCl}$ and $100 \mathrm{mg}$ of ascorbic acid to the beaker.

\section{DETERMINATION}

1. Place the beaker in a constant temperature bath at $55^{\circ} \mathrm{C}$.

2. Degrease a nickel disc by dipping in $\mathrm{HNO}_{3}$, followed by dipping in $\mathrm{HCl}$ and rinsing in water. Repeat until the surfaces of the disc are bright and shiny. 
3. Suspend the disc on a glass stirring hook in the solution and stir for $2.5 \mathrm{~h}$ at a speed giving maximum agitation without splashing.

4. Remove the disc, rinse the stirring rod and disc with water and let dry in air.

5. Alpha count each side of the disc. Subtract background from each count and sum the two net cps.

6. Standardize the counter with a known quantity of any $\alpha$ emitter on a metal disc. Natural $U$ plated on a similar disc is a convenient standard.

LOWER LIMIT OF DETECTION (LLD)

\begin{tabular}{llccc}
\hline & & A & B & C \\
\hline Counter Efficiency & $(\%)$ & 50 & 50 & 25 \\
Counter Background & $(\mathrm{cps})$ & $1.675 \times 10^{-5}$ & $1.67 \times 10^{-5}$ & $8.33 \times 10^{-5}$ \\
Yield & $(\%)$ & 70 & 70 & 70 \\
Blank & $(\mathrm{cps})$ & - & - & - \\
& & & & 0.33 \\
LLD (400 min) & $(\mathrm{mBq})$ & 0.5 & 0.17 & 1.5 \\
LLD (1000 min) & $(\mathrm{mBq})$ & 0.33 & 0.10 & 0.5 \\
LLD (5000 min) & $(\mathrm{mBq})$ & 0.17 & & \\
\hline
\end{tabular}

$A=$ alpha scintillation counter (both sides)

$\mathrm{B}=$ alpha scintillation counter (one side)

$\mathrm{C}=$ solid-state alpha spectrometer (one side) 


\section{REFERENCES}

Fellman, A., L. Ralston, D. Hickman, L. Ayres, N. Cohen, H. Spitz and B. Robinson "The Importance Acid Digestion of Urine Prior to Spontaneous Deposition of ${ }^{210} \mathrm{Po}$ " Health Physics, 57, 615-621 (1989)

Hursh, J. B. (Editor)

USAEC Report AECU-4024, November (1958) 
POLONIUM IN WATER, VEGETATION, SOIL, AND AIR FUTERS

Contact Person(s) : Isabel M. Fisenne

\begin{abstract}
APPLICATION
This procedure has been tested for water, vegetation, soil, and Dynaweb filters. Reagent blanks must be analyzed along with the samples.

Polonium is equilibrated with ${ }^{208} \mathrm{Po}$ or ${ }^{209} \mathrm{Po}$ tracer and isolated from most other elements by coprecipitation with lead sulfide. The sulfide precipitate is dissolved in weak $\mathrm{HCl}$ solution. Polonium is quantitatively deposited on a nickel disc. The deposition is very specific and can be carried out in the presence of other radionuclides.

The plated disc is counted on an $\alpha$ spectrometer to measure chemical yield and activity of the sample. The solution from the deposition may be retained and analyzed for ${ }^{210} \mathrm{~Pb}$.
\end{abstract}

\title{
SPECIAL APPARATUS
}

1. Nickel discs $-1.75 \mathrm{~cm}$ diameter x $0.06 \mathrm{~cm}$ thick "commercial pure" nickel. Degrease in acetone, dip in $\mathrm{HCl}$ and rinse with water.

2. Electrolytic cell - see Specification 7.16.

3. Teflon stirring rods. 


\section{SPECIAL REAGENTS}

1. Standardized ${ }^{208} \mathrm{Po}$ or ${ }^{209} \mathrm{Po}$ tracer solution - about $2 \mathrm{~Bq} \mathrm{~g}^{-1}$ in a dispensing bottle.

2. Lead carrier solution: $10 \mathrm{mg} \mathrm{Pb} \mathrm{mL} L^{-1}-15.98 \mathrm{~g} \mathrm{~Pb}\left(\mathrm{NO}_{3}\right)_{2} \mathrm{~L}^{-1}$ of $1: 99 \mathrm{HNO}_{3}$.

3. Thioacetamide solution - $100 \mathrm{~g} \mathrm{CH}_{3} \mathrm{CSNH}_{2} \mathrm{~L}^{-1}$ of water.

4. Saturated ascorbic acid solution.

\section{SAMPLE PREPARATION}

\section{A. Tap water.}

1. Transfer $2.5 \mathrm{~L}$ of tap water to a $3-\mathrm{L}$ beaker.

2. Add $50 \mathrm{~mL}$ of $\mathrm{HNO}_{3}$ and $1 \mathrm{~mL}$ of $\mathrm{Pb}$ carrier solution. Add a weighed aliquot (30-80 $\mathrm{mBq})$ of the ${ }^{208} \mathrm{Po}$ or ${ }^{209} \mathrm{Po}$ tracer solution.

3. Evaporate and add additional aliquots of tap water until a 10-L collection has been obtained. Evaporate gently to about $25 \mathrm{~mL}$.

4. Transfer the solution to a $90-\mathrm{mL}$ centrifuge tube with $\mathrm{H}_{2} \mathrm{O}$. Continue with Determination.

\section{B. Vegetation.}

1. Weigh $100 \mathrm{~g}$ of dried $\left(105-110^{\circ} \mathrm{C}\right)$ material into a $400-\mathrm{mL}$ beaker.

2. Add $1 \mathrm{~mL}$ of $\mathrm{Pb}$ carrier solution and a weighed aliquot $(30-80 \mathrm{mBq})$ of ${ }^{208} \mathrm{Po}$ or ${ }^{209} \mathrm{Po}$ tracer solution.

3. Add $100 \mathrm{~mL}$ of $\mathrm{HNO}_{3}$ with magnetic stirring using a Teflon-coated bar. Digest with gentle heat and stirring for $1 \mathrm{~h}$. 
4. Reduce the volume of the solution to about $25 \mathrm{~mL}$ and transfer the solution to a 90-mL centrifuge tube with water. Continue with Determination.

\section{Soil.}

1. Weigh 1 to $5 \mathrm{~g}$ of soil into a $40-\mathrm{mL}$ platinum dish. Add $1 \mathrm{~mL}$ of $\mathrm{Pb}$ carrier and a weighed aliquot (30-80 $\mathrm{mBq})$ of ${ }^{208} \mathrm{Po}$ or ${ }^{209} \mathrm{Po}$ tracer solution.

2. Add $10 \mathrm{~mL}$ of $\mathrm{HNO}_{3}$ and $10 \mathrm{~mL}$ of $48 \% \mathrm{HF}$. Heat on a medium hot plate. Repeat the additions of $\mathrm{HNO}_{3}$ and $\mathrm{HF}$ until no further dissolution takes place.

3. Add $10 \mathrm{~mL}$ of $\mathrm{HNO}_{3}$ and reduce the volume to about $5 \mathrm{~mL}$.

4. If insoluble material remains, filter the slurry by gravity through a Whatman No. 42 filter paper into a $90-\mathrm{mL}$ centrifuge tube. Wash the filter with hot water. Discard the residue. Continue with Determination.

\section{Dynaweb filter.}

1. To a $8.9 \mathrm{~cm}$ diameter or $1 / 4$ of an $20.3 \mathrm{~cm}$ diameter Dynaweb filter in a $600-\mathrm{mL}$ beaker, add $1 \mathrm{~mL}$ of lead carrier and a weighed aliquot $(30-80 \mathrm{mBq})$ of ${ }^{208} \mathrm{Po}$ or ${ }^{209} \mathrm{Po}$ tracer solution.

2. Add $300 \mathrm{~mL}$ of $\mathrm{HNO}_{3}$ and digest on a medium hot plate.

3. Evaporate to about $25 \mathrm{~mL}$. If the solution is not clear, repeat the evaporation with additional $\mathrm{HNO}_{3}$.

4. Add about $200 \mathrm{~mL}$ of water to polymerize the Dynaweb material.

5. Filter with suction through a Millipore filter and wash with water. Discard the filter and polymerized Dynaweb material.

6. Transfer the filtrate back into the original beaker. 
7. Reduce the volume to $25 \mathrm{~mL}$. Repeat Steps 4-6 until the Dynaweb material is completely removed.

8. Transfer the solution to a $90-\mathrm{mL}$ centrifuge tube. Continue with Determination.

\section{DETERMINATION}

1. Reduce the volume to about $5 \mathrm{~mL}$ in a steam bath. Add $50 \mathrm{~mL}$ of water.

2. Adjust the $\mathrm{pH}$ to $3.5-4$ with $\mathrm{NH}_{4} \mathrm{OH}$. Add $5 \mathrm{~mL}$ of thioacetamide solution. Digest in a steam bath for $1 \mathrm{~h}$.

3. Cool, centrifuge, and decant the supernate. Discard the supernate.

4. Dissolve the precipitate in $2 \mathrm{~mL}$ of $\mathrm{HCl}$. Add $50 \mathrm{~mL}$ of water.

5. Adjust the $\mathrm{pH}$ to $3.5-4$ with $\mathrm{NH}_{4} \mathrm{OH}$. Add $2 \mathrm{~mL}$ of thioacetamide solution. Digest in a steam bath for $1 \mathrm{~h}$.

6. Cool, centrifuge, and decant the supernate. Discard the supernate.

7. Dissolve the precipitate in $1 \mathrm{~mL}$ of $\mathrm{HCl}$. Dilute the solution to $25 \mathrm{~mL}$ with water.

8. Filter the solution by gravity through a Whatman No. 41 filter paper into a prepared

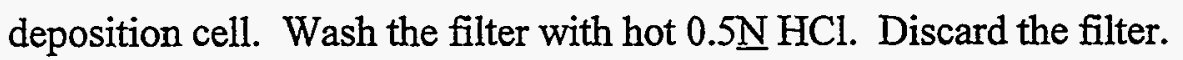

9. Add $1 \mathrm{~mL}$ of saturated ascorbic acid solution to the cell.

10. Place the cell in an $80^{\circ} \mathrm{C}$ water bath.

11. Stir with a Teflon stirrer for $4 \mathrm{~h}$ at a speed giving maximum agitation without splashing. Occasional small additions of $0.5 \mathrm{~N} \mathrm{HCl}$ are necessary to make up for evaporation of the solution. 
12. Remove the cell from the water bath and pour off the solution into a beaker. Reserve for ${ }^{210} \mathrm{~Pb}$ determination if required.

13. Dismantle the cell, rinse the disc with water, then ethanol. Air dry the disc.

14. Place the disc on a warm hotplate to dry.

15. Count the disc on an $\alpha$ spectrometer to resolve the ${ }^{208} \mathrm{Po}$ or ${ }^{209} \mathrm{Po}$ tracer and ${ }^{210} \mathrm{Po}$.

LOWER LIMIT OF DETECTION (LLD)

\begin{tabular}{llllll}
\hline & & A & B & C & D \\
\hline Counter Efficiency & $(\%)$ & 40 & 40 & 40 & 40 \\
Counter Background & $(\mathrm{cps})$ & $8.33 \times 10^{-5}$ & $8.33 \times 10^{-5}$ & $8.33 \times 10^{-5}$ & $8.33 \times 10^{-5}$ \\
Yield & $(\%)$ & 80 & 75 & 60 & 60 \\
Blank & $(\mathrm{cps})$ & 0.01 & 0.01 & 0.01 & 0.01 \\
& & & & & \\
LLD ( 400 min) & $(\mathrm{mBq})$ & 1.5 & 2.0 & 2.0 & 2.0 \\
LLD (1000 min) & $(\mathrm{mBq})$ & 1.0 & 1.3 & 1.3 & 1.3 \\
LLD (5000 min) & $(\mathrm{mBq})$ & 0.4 & 0.6 & 0.6 & 0.6 \\
\hline
\end{tabular}

Solid-state alpha spectrometer:
$\mathrm{A}=\mathrm{H}_{2} \mathrm{O}$
$\mathrm{B}=$ Vegetation
$\mathrm{C}=$ Soil
$\mathrm{D}=$ Dynaweb filter 


\section{Plutonium}




\title{
PLUTONIUM IN AIR FUTERS
}

Contact Person(s) : Anna Berne

\begin{abstract}
APPLICATION
This procedure is applicable to all types of air filters. However, if the filter is made of an organic polymer, it is advisable to first decompose the filter in a muffle furnace at $450^{\circ} \mathrm{C}$ overnight. Samples are then digested in concentrated $\mathrm{HNO}_{3}$, after which the remaining residue and filter material are treated with $\mathrm{HF}$.
\end{abstract}

\section{SPECIAL APPARATUS}

1. Muffle furnace.

2. Pyrex long stem fluted funnel with an inside diameter of $9.5 \mathrm{~cm}$.

\section{SPECIAL REAGENT}

1. Plutonium-236 tracer - a standard solution containing $\sim 0.15 \mathrm{~Bq} \mathrm{~g}^{-1}$ in a dispensing bottle.

\section{SAMPLE PREPARATION}

1. Place the air filter in a 400-mL (or appropriate size) beaker.

2. Add a known amount $(\sim 0.05 \mathrm{~Bq})$ of ${ }^{236} \mathrm{Pu}$ tracer. 
3. Add $150 \mathrm{~mL}$ of concentrated $\mathrm{HNO}_{3}$ and $50 \mathrm{~mL}$ of concentrated $\mathrm{HCl}$ (or appropriate amounts based on air filter size), allow sample to react for about $1 \mathrm{~h}$. Place the sample on a hot plate and cover with a watch glass. Heat the sample overnight on a low setting. Continue heating until the volume is $\sim 100 \mathrm{~mL}$.

4. Remove the sample from the hot plate, add $100 \mathrm{~mL}$ of water and let the sample cool to room temperature. Filter by gravity using a conical funnel with an $18.5 \mathrm{~cm}$ Whatman No. 42 filter paper. Wash with $50-75 \mathrm{~mL}$ of $1: 1 \mathrm{HNO}_{3}$. Collect the filtrate in a 250-mL beaker, evaporate the filtrate to near dryness, add $\sim 35 \mathrm{~mL} \mathrm{1:1} \mathrm{HNO}_{3}$, and save. Cover the beaker with parafilm to prevent changes in the concentration of $1: 1$ $\mathrm{HNO}_{3}$.

5. Transfer the filter and residue to the original beaker and wet ash with $150-200 \mathrm{~mL}$ concentrated $\mathrm{HNO}_{3}$. Allow refluxing to occur on low heat. Do not boil. Evaporate to $\sim 25-30 \mathrm{~mL}$.

6. Transfer the filtered residue to a platinum dish or Teflon beaker. Add $10 \mathrm{~mL}$ of $\mathrm{HF}$, $5 \mathrm{~mL}$ of $\mathrm{HNO}_{3}$, and evaporate to near dryness. Add $10 \mathrm{~mL}$ of $\mathrm{HNO}_{3}$ and $10 \mathrm{~mL}$ of $\mathrm{HF}$, then evaporate to near dryness again. Repeat the addition of $\mathrm{HNO}_{3}$ and $\mathrm{HF}$ two more times. Wash the sides of the vessel three times with $1: 1 \mathrm{HNO}_{3}$, and evaporate to dryness after each addition.

7. Add $20 \mathrm{~mL}$ of $1: 1 \mathrm{HNO}_{3}$ to sample.

8. Filter the sample into the beaker containing the filtrate from Step 4 by gravity filtration using a conical funnel with a $18.5 \mathrm{~cm}$ Whatman No. 40 or No. 42 filter paper. Wash with 1:1 $\mathrm{HNO}_{\hat{3}}(\sim 20 \mathrm{~mL})$.

9. Adjust sample volume to $100 \mathrm{~mL}$ with $1: 1 \mathrm{HNO}_{3}$.

10. Proceed to Plutonium Purification - Ion Exchange Technique, Procedure Pu-11$\mathrm{RC}$. 
$P u-02-R C$

PLUTONIUM IN SOL SAMPLES

(see Volume II) 
PLUTONIUM IN SOIL RESIDUE TOTAL DISSOLUTION METHOD

Contact Person(s) : Anna Berne

\section{APPLICATION}

This procedure is applicable to acid leached soils as well as unleached soils (Krey and Bogen, 1987).

The silica in the sample is removed by the formation of $\mathrm{SiF}_{4}$ using $\mathrm{HF}$. The remaining residue is fused with $\mathrm{KF}$ to decompose the complex silicates, followed by the addition of $\mathrm{H}_{2} \mathrm{SO}_{4}$ to distill the remaining $\mathrm{SiF}_{4}$. This procedure is followed by a pyrosulfate fusion, and finally, coprecipitation with $\mathrm{BaSO}_{4}$.

\section{SPECIAL REAGENTS}

1. Potassium fluoride.

2. Sodium sulfate.

3. Hydroxylamine hydrochloride.

4. $10 \% \mathrm{BaCl}_{2}$ solution $-10 \mathrm{~g}$ of $\mathrm{BaCl}_{2} 100 \mathrm{~mL}^{-1}$ of water.

\section{SAMPLE PREPARATION}

1. Using $100 \mathrm{~mL}$ of concentrated $\mathrm{HNO}_{3}$, wet ash the sample and filter the remaining sample from the leaching of the soil (Steps 2-6 of Sample Preparation, Procedure $\mathrm{Pu}-02-\mathrm{RC})$. 
2. Evaporate the sample on a hot plate making sure the sample is completely dry.

3. Transfer the mixture to a tared plastic zip lock bag. Quantitatively remove the soil residue from the beaker. Reweigh the bag to determine the mass of the residue.

4. Break apart large aggregates by kneading the residue in the bag so that the mixture is homogeneous.

5. Weigh out $50 \mathrm{~g}$ of the soil residue and transfer to a $250-\mathrm{mL}$ platinum crucible.

6. Add a known amount ( $0.05 \mathrm{~Bq}$ or appropriate amount) of ${ }^{236} \mathrm{Pu}$ tracer.

7. Add $5 \mathrm{~mL}$ of $1: 1 \mathrm{HNO}_{3}$ to wet the soil residue. Very slowly add $10 \mathrm{~mL}$ of concentrated HF, it may be necessary to add the HF dropwise. If excessive frothing occurs, wet the sample with $1: 1 \mathrm{HNO}_{3}$ from a wash bottle.

8. Add an additional $10 \mathrm{~mL}$ of HF carefully. Heat the sample at low heat on a hot plate until no liquid remains.

9. When the sample is dry, add another $10 \mathrm{~mL}$ of HF. Heat the sample on a hot plate until the sample is dry.

10. Repeat Step 9 until at least a total of $100 \mathrm{~mL}$ of HF has been added and the sample no longer fumes. During the heating of the sample, the soil residue may move up the walls of the platinum crucible. If this occurs wash the walls with $\mathrm{HF}$.

11. To insure the absence of HF, heat the crucible with a Meker burner until the crucible glows red.

12. Cool the sample and add $20 \mathrm{~g}$ of $\mathrm{KF} \cdot 2 \mathrm{H}_{2} \mathrm{O}$. Heat the sample on a hot plate, mixing with a Teflon stirrer.

13. Continue heating the sample until all the water has evaporated (so that no splattering occurs in the next step). It may be necessary to raise the temperature of the hot plate to assure drying. 
14. Place the sample in a muffle furnace and heat at $950^{\circ} \mathrm{C}$ for $30 \mathrm{~min}$.

15. Remove the crucible from the furnace and cool to room temperature.

16. Slowly add $35 \mathrm{~mL}$ of concentrated $\mathrm{H}_{2} \mathrm{SO}_{4}$ to the crucible on a hot plate at low heat. Heat the sample until the evolution of a small amount of $\mathrm{SO}_{3}$ vapors occurs. After this step, continue heating for $5 \mathrm{~min}$.

17. Cool and add $20 \mathrm{~g}$ of anhydrous $\mathrm{Na}_{2} \mathrm{SO}_{4}$.

18. Place the crucible on a clay triangle mounted on a ring stand and heat the crucible gently with a Meker burner, minimizing bumping and frothing of the sample. Gradually raise the amount of heat while watching for frothing, until the molten mass is dissolved (a clear red color), at this point the temperature is $\sim 700^{\circ} \mathrm{C}$. Remove the Meker burner and cool the crucible to room temperature. (Caution: This fusion is to be performed in a hood because $\mathrm{SO}_{3}$ fumes are emitted.)

19. Flex the walls of the crucible to break apart the fused cake. Transfer the fused material to a $1500-\mathrm{mL}$ beaker and add $200 \mathrm{~mL}$ of water to the crucible. Heat the crucible on a hot plate to dissolve any material in the crucible. Transfer the water wash to the $1500-\mathrm{mL}$ beaker containing the fused material.

20. Perform three additional $200-\mathrm{mL}$ washes of the crucible, each time transferring the solution to the $1500-\mathrm{mL}$ beaker.

21. Add $100 \mathrm{~mL}$ of concentrated $\mathrm{HCl}, 1 \mathrm{~g}$ of $\mathrm{NH}_{2} \mathrm{OH} \cdot \mathrm{HCl}$ to the $1500-\mathrm{mL}$ beaker, which contains the fused material and water, and heat the solution to dissolve the fused material. Cover with a watch glass.

22. Continue to heat the solution until the boiling point is reached. Using a pipette add $5 \mathrm{~mL}$ of $10 \% \mathrm{BaCl}_{2}$ solution and continue boiling the sample for $5 \mathrm{~min}$.

23. Cool to room temperature and filter the solution mixture by gravity through a $15-\mathrm{cm}$ Whatman No. 42 filter paper using a conical funnel. Wash the beaker well with four 50-mL portions of $\mathrm{H}_{2} \mathrm{O}$. Transfer each $\mathrm{H}_{2} \mathrm{O}$ wash to the funnel containing the filtered precipitate. Discard the filtrate. 
24. Transfer the filter paper containing the $\mathrm{BaSO}_{4}$ precipitate to a small platinum crucible $(40 \mathrm{~mL})$. Heat the crucible with a Meker burner to decompose the filter paper.

25. Add $5 \mathrm{~g}$ of anhydrous $\mathrm{Na}_{2} \mathrm{CO}_{3}$ to the crucible and mix the $\mathrm{Na}_{2} \mathrm{CO}_{3}$ with the $\mathrm{BaSO}_{4}$ precipitate. Place the crucible in a muffle furnace at $850^{\circ} \mathrm{C}$ for $30 \mathrm{~min}$.

26. Remove the crucible from the furnace, cool to room temperature, add $5 \mathrm{~mL}$ of water, and heat gently on a hot plate.

27. Transfer the salts and wash the solution into a $40-\mathrm{mL}$ centrifuge tube using a minimum amount of $\mathrm{H}_{2} \mathrm{O}$ to effect the transfer.

28. Centrifuge the sample and discard the supernatant.

29. Dissolve the precipitate in the centrifuge tube with $5 \mathrm{~mL}$ of $6 \mathrm{M} \mathrm{HCl}$. Transfer the solution mixture to a $100-\mathrm{mL}$ Teflon beaker using $6 \mathrm{M} \mathrm{HCl}$. Also, wash the sides of the platinum crucible with $6 \mathrm{M} \mathrm{HCl}$ to remove any remaining residue and transfer to the Teflon beaker.

30. Add $5 \mathrm{~mL}$ of concentrated $\mathrm{HF}$, place the beaker on a hot plate and evaporate the sample to near dryness.

31. Cool, add $5 \mathrm{~mL}$ of $1: 1 \mathrm{HNO}_{3}$, and $5 \mathrm{~mL}$ of concentrated $\mathrm{HF}$, heat on a hot plate and evaporate to near dryness.

32. Add $15 \mathrm{~mL}$ of $1: 1 \mathrm{HNO}_{3}$ and evaporate to near dryness.

33. Repeat Step 32, three to five times, to remove any traces of HF.

34. Add $20 \mathrm{~mL}$ of $1: 1 \mathrm{HNO}_{3}$ and heat gently for a few minutes. Remove the beaker from heat, filter mixture under reduced pressure using a $25-\mathrm{mm}$ Millipore filter with a 0.45 $\mu \mathrm{m}$ pore size or by gravity filtration through a $15-\mathrm{cm}$ Whatman No. 42 filter paper using a conical funnel. 
35. Wash the Teflon beaker with $15 \mathrm{~mL}$ of $1: 1 \mathrm{HNO}_{3}$ and transfer the wash to the filtered precipitate. Discard the precipitate. Proceed to Plutonium Purification - Ion Exchange Technique (see Procedure $P u-11-R C$ ).

\section{REFERENCE}

Krey, P. W. and D. C. Bogen

"Determination of Acid Leachable and Total Plutonium in Large Samples"

J. of Radioanalytical and Nuclear Chemistry, 115, 335-355, December (1987) 
Pu-04-RC, Vol. II

Rev. 0

HASL-300, 28th Edition

February 1997

$P u-04-R C$

PLUTONIUM IN TISSUE

(see Volume II) 
$P u-05-R C$

PLUTONIUM IN TISSUE - SOLVENT EXTRACTION

(see Volume II) 
Pu-06-RC, Vol. II

Rev, 0

HASL-300, 28th Edition

February 1997

$P u-06-R C$

PLUTONIUM IN URINE

(see Volume II) 
Pu-07-RC, Vol. II

HASL-300, 28th Edition

$P u-07-R C$

PLUTONIUM IN LARGE URINE SAMPLES

(see Volume II)
Rev. 0

February 1997 
PLUTONIUM IN VEGETATION AND TISSUE NITRIC/HYDROCHLORIC ACID METHOD (see Volume II) 
PLUTONIUM IN VEGETATION AND TISSUE NITRIC/SULFURIC ACID METHOD

\author{
(see Volume II)
}




\section{PLUTONIUM IN WATER}

\section{Contact Person(s) : Anna Berne}

\section{APPLICATION}

This procedure is used for all types of water samples (i.e., sea water, lake water, tap water, etc.). If the sample contains suspended particulates, they must be removed by filtration. Large volume samples are analyzed after evaporation in an acidic medium.

The sample is heated in $\mathrm{HNO}_{3}$ and then in $3: 1 \mathrm{HNO}_{3}: \mathrm{HCl}$. The volume is then reduced to near dryness and finally the volume of the sample is adjusted with 1:1 $\mathrm{HNO}_{3}$. The sample is then ready to be purified by ion exchange separation (see Procedure $\mathrm{Pu}-11-\mathrm{RC}$ ).

\section{SPECIAI REAGENT}

1. ${ }^{236} \mathrm{Pu}$ tracer - a standard solution containing $0.2 \mathrm{~Bq} \mathrm{~g}^{-1}$ in a dispensing bottle. The purity of the tracer is measured by $\alpha$ spectrometry.

\section{SAMPLE PREPARATION}

1. Transfer $100-1000 \mathrm{~mL}$ of a $\mathrm{H}_{2} \mathrm{O}$ sample to a beaker.

2. To the sample add $\sim 0.05 \mathrm{~Bq}$ (or appropriate amount) of ${ }^{236} \mathrm{Pu}$ tracer.

3. Add an equal amount of concentrated $\mathrm{HNO}_{3}$, cover the beaker with a watch glass and place on a hot plate. Reflux the solution for $4-8 \mathrm{~h}$. 
4. Replace the watch glass with a ribbed watch glass and evaporate the solution to near dryness. When the volume is reduced to $\sim 100 \mathrm{~mL}$, allow the solution to cool to room temperature and transfer to an appropriate size beaker.

5. Continue evaporating the sample to near dryness. Cool, add $75 \mathrm{~mL}$ of concentrated $\mathrm{HNO}_{3}$ and $25 \mathrm{~mL}$ of concentrated $\mathrm{HCl}$. Cover with a watch glass. Allow to react for $30 \mathrm{~min}$. Then place the sample on a hot plate and bring to a boil. After the solution has boiled for $30 \mathrm{~min}$, reduce heat and continue heating overnight. Do not allow the sample to evaporate to dryness.

6. Remove the sample from the hot plate and add $100 \mathrm{~mL}$ of $\mathrm{H}_{2} \mathrm{O}$ to the sample. Allow the sample to cool to room temperature and filter under reduced pressure using a Buchner funnel with a Whatman No. 42 filter paper.

7. Wash with $50 \mathrm{~mL}$ of $1: 1 \mathrm{HNO}_{3}$ and then $50 \mathrm{~mL}$ of $\mathrm{H}_{2} \mathrm{O}$.

8. Transfer the filtrate to a $250-\mathrm{mL}$ beaker, cover the beaker with a ribbed watch glass.

9. (Note: If the filter paper contains a moderate amount of precipitate, it must be treated with HF.) Transfer the filter paper containing the residue from the $\mathrm{HNO}_{3} / \mathrm{HCl}$ digestion to a platinum dish. Place the platinum dish in a muffle furnace and heat at $100^{\circ} \mathrm{C}$, raise the temperature by increments of $100^{\circ} \mathrm{C}$ every hour until a final temperature of $450^{\circ} \mathrm{C}$ is reached. Continue heating at this temperature overnight. Turn off the muffle furnace and let the sample in the platinum dish cool sufficiently to remove it from the furnace. Add $15 \mathrm{~mL}$ of $1: 1 \mathrm{HNO}_{3}$ and $15 \mathrm{~mL}$ of concentrated $\mathrm{HF}$. Heat the sample to neardryness.

10. Repeat Step 9 two times.

11. Add $20 \mathrm{~mL}$ of $1: 1 \mathrm{HNO}_{3}$ to the sample and heat on a hot plate under a low setting until near dryness (to remove traces of $\mathrm{HF}$ ).

12. Repeat Step 11 two times.

13. Add $20 \mathrm{~mL}$ of $1: 1 \mathrm{HNO}_{3}$ to sample. 
14. Using a conical funnel, filter the sample by gravity through an $18.5 \mathrm{~cm}$ Whatman No. 42 filter paper into a beaker containing the filtrate from Step 9. Wash well with 1:1 $\mathrm{HNO}_{3}$.

15. Reduce the volume of the solution to near dryness on a hot plate.

16. Adjust the volume to $100 \mathrm{~mL}$ by the addition of $1: 1 \mathrm{HNO}_{3}$.

17. Proceed to Plutonium Purification - Ion Exchange Technique, Procedure $P u-11-R C$. 


\begin{abstract}
APPLICATION
This procedure has been applied to the leachates derived from the plutonium sample preparation methods described in this Manual. Ion exchange chromatography is used to remove the large amounts of impurities contained in these leachates.
\end{abstract}

SPECIAL APPARATUS

Ion exchange columns - see Specifications 7.5 and 7.6.

\title{
SPECIAL REAGENTS
}

1. $1: 1 \mathrm{HNO}_{3}-500 \mathrm{~mL} \mathrm{HNO}$ diluted to $1 \mathrm{~L}$.

2. Hydroxylamine hydrochloride $-\mathrm{NH}_{2} \mathrm{OH} \cdot \mathrm{HCl}$

3. $0.3 \mathrm{M}$ hydroxylamine hydrochloride $-0.5 \mathrm{M} \mathrm{HNO}_{3}-20.85 \mathrm{~g}$ of $\mathrm{NH}_{2} \mathrm{OH} \cdot \mathrm{HCl}$ diluted to $1 \mathrm{~L}$ with $0.5 \mathrm{M} \mathrm{HNO}_{3}$.

4. Anion exchange resin - Bio-Rad AG 1-X8 (100-200 mesh, $\mathrm{Cl}^{-}$form), see Specification 7.4 


\section{ION EXCHANGE SEPARATION}

1. Cool the sample in an ice bath, add $1 \mathrm{~g}$ of $\mathrm{NH}_{2} \mathrm{OH} \cdot \mathrm{HCl}$, stir, and let stand in an ice bath for $15 \mathrm{~min}$. Remove the sample from the ice bath and heat to boiling on a hot plate with medium heat for 1-3 min. Cool the sample to room temperature.

2. Prepare the ion exchange resin column (see Note).

3. Pass the sample through the resin bed at a flow of $\sim 1 \mathrm{~mL} \mathrm{~min}^{-1}$. Wash the beaker and the column with $30 \mathrm{~mL} \mathrm{1:1} \mathrm{HNO}_{3}$ three times. Allow the liquid to flow until the level reaches the top of the resin bed prior to each wash. Reserve the sample and wash the effluent for ${ }^{241} \mathrm{Am}$ determination (or until yield has been determined as satisfactory).

4. Elute the plutonium with $10 \mathrm{~mL}$ of $0.5 \mathrm{M} \mathrm{HNO}_{3}$ twice - then with $100 \mathrm{~mL}$ of $0.3 \mathrm{M}$ hydroxylamine hydrochloride $-0.5 \mathrm{M} \mathrm{HNO}_{3}$ into a $250-\mathrm{mL}$ beaker. Discard the resin.

5. Slowly add $25-30 \mathrm{~mL} \mathrm{HNO}_{3}$ until effervescence begins, then place on a hot plate and evaporate the eluate to dryness.

6. Dissolve the residue in $30 \mathrm{~mL}$ of $1: 1 \mathrm{HNO}_{3}$ and cool in an ice bath. Add $500-600 \mathrm{mg}$ of $\mathrm{NH}_{2} \mathrm{OH} \cdot \mathrm{HCl}$ and repeat Steps 1-3 using a small column (see Specification 7.6) for all samples.

7. Wash the resin with $100 \mathrm{~mL}$ of $\mathrm{HCl}$ (two $10-\mathrm{mL}$ portions followed by two $40 \mathrm{~mL}$ portions). Wash the resin with two $10-\mathrm{mL}$ portions, followed by one $40-\mathrm{mL}$ portion 1:1 $\mathrm{HNO}_{3}$. Save the effluent until yield determination.

8. Repeat Steps 4 and 5. Discard the resin.

9. Convert the residue to the $\mathrm{Cl}^{-}$form by adding $5 \mathrm{~mL}$ of $\mathrm{HCl}$ and evaporating to dryness three times at a low temperature.

10. See Procedure G-03 for microprecipitation for $\alpha$ spectrometry. 


\section{Note: Preparation of Columns}

1. When preparing a large soil sample use a large column (Specification 7.5), otherwise use the column described in Specification 7.6.

2. Position a plug of glass wool in the base of the column so that no resin will drain out.

3. Add sufficient resin to form a resin bed of $10 \mathrm{~cm}$ in length. Wash the column with sufficient $1: 1 \mathrm{HNO}_{3}$ to remove the $\mathrm{Cl}^{-}$ion from the resin. Test the effluent with a dilute silver nitrate solution. 
PLUTONIUM AND/OR AMERICIUM IN SOIL OR SEDIMENTS

Contact Person(s) : Anna Berne

\begin{abstract}
APPLICATION
This procedure is applicable to soils which contain plutonium and americium deposited from worldwide fallout and some nuclear activities. A total dissolution technique is required for some soil samples for plutonium determination.

Plutonium and americium isotopes are leached and equilibrated with ${ }^{236} \mathrm{Pu}$ and ${ }^{243} \mathrm{Am}$ tracers with nitric and hydrochloric acids from soil samples of up to $100 \mathrm{~g}$ in size. Plutonium is isolated and purified by ion exchange. Americium is collected with a calcium oxalate precipitation, isolated and purified by ion exchange. After source preparation by microprecipitation, the plutonium isotopes and americium are determined by alpha spectrometry.
\end{abstract}

\title{
SPECIAL APPARATUS
}

1. For microprecipitation, see Procedure G-03.

2. Ion-exchange columns - see Specification 7.5.

\section{SPECIAL REAGENTS}

1. Americium-243 tracer solution, $\sim 0.15 \mathrm{~Bq} \mathrm{~g}^{-1}$ in a dispensing bottle. 
2. Plutonium-236 ( ${ }^{242} \mathrm{Pu}$ can also be used) tracer solution, $\sim 0.20 \mathrm{~Bq} \mathrm{~g}^{-1}$ in a dispensing bottle.

3. Bio-Rad AG 1-X8 resin (100-200 mesh) - see Specification 7.4.

4. Bio-Rad AG 1-X4 resin (100-200 mesh) - see Specification 7.4.

5. TEVA resin $2 \mathrm{~mL}$ ion extraction columns (Aliquat 336, methyltricapryl-ammonium chloride, Henkel Corporation, Tucson, AZ 85745-1273, on Amberchrom resin) or equivalent or can be prepared from TEVA resin, Eichrom Industries, 8205 Cass Ave. Suite 107, Darien, IL 60561) - place a plug of glass wool in the bottom of a $2 \mathrm{~mL}$ plastic transfer pipette (see Specification 7.7). Add slurried TEVA resin ( $0.5 \mathrm{~g})$. Place additional glass wool on the top of the resin.

6. $2 \mathrm{M}$ ammonium thiocyanate in $0.1 \mathrm{M}$ formic acid solution - dissolve $152 \mathrm{~g}$ of $\mathrm{NH}_{4} \mathrm{SCN}$ in ASTM Type 2 water, add $4.25 \mathrm{~mL}$ formic acid, and dilute to $1 \mathrm{~L}$.

7. $1 \underline{\mathrm{M}}$ ammonium thiocyanate in $0.1 \mathrm{M}$ formic acid - dissolve $76 \mathrm{~g}$ of $\mathrm{NH}_{4} \mathrm{SCN}$ in ASTM Type 2 water, add $4.25 \mathrm{~mL}$ formic acid, and dilute to $1 \mathrm{~L}$.

8. Calcium carrier solution, $100 \mathrm{mg} \mathrm{mL}^{-1}$ - dissolve $25 \mathrm{~g} \mathrm{CaCO}_{3}$ in a minimal amount of concentrated $\mathrm{HNO}_{3}$, and dilute to $100 \mathrm{~mL}$.

9. Iron carrier, $100 \mathrm{mg} \mathrm{mL}^{-1}$ - slowly heat $100 \mathrm{~g}$ of iron powder in $500 \mathrm{~mL}$ of HCl until reaction ceases. Carefully and slowly add $100 \mathrm{~mL}$ of $\mathrm{HNO}_{3}$ while stirring. $\mathrm{Cool}$ and dilute to $1 \mathrm{~L}$.

10. Oxalate wash solution - dissolve $10 \mathrm{~g}$ of oxalic acid $\left(\mathrm{H}_{2} \mathrm{C}_{2} \mathrm{O}_{4} \cdot 2 \mathrm{H}_{2} \mathrm{O}\right)$ to make $1 \mathrm{~L}$ of solution ( $1 \%$ solution).

11. Hydroxylamine hydrochloride, $\mathrm{NH}_{2} \mathrm{OH} \cdot \mathrm{HCl}$ - solid. 


\section{SAMPLE PREPARATION}

1. Weigh 1-100 g of soil into an appropriate sized beaker. Add weighed amounts of ${ }^{243} \mathrm{Am}$ and ${ }^{236} \mathrm{Pu}$ tracers.

2. Slowly add $100 \mathrm{~mL}$ (Note: volumes are based on $100 \mathrm{~g}$ sample and should be adjusted if sample size is smaller) of $\mathrm{HNO}_{3}$ to the beaker. Control the foaming with the addition of a few drops of n-octyl alcohol. Stir sample with a glass stir rod to mix sample and acid. When the reaction subsides, add $30 \mathrm{~mL}$ of $\mathrm{HCl}$, and stir. Allow the mixture to react at room temperature, rinse and remove stir rod, cover with a watch glass, then reflux on a low temperature hot plate overnight. Remove from hot plate and cool.

3. Dilute the solution in the beaker with water to $1: 1 \mathrm{HNO}_{3}$ and filter the solution with vacuum through 9 or $11 \mathrm{~cm}$ Whatman No. 42 filter paper on a Büchner funnel into a $1 \mathrm{~L}$ flask. Wash with 1:1 $\mathrm{HNO}_{3}$. Retain the filtrate in a 2-L beaker, evaporate the filtrate until salting out begins to occur. Return the residue and filter to the original beaker using $\mathrm{HNO}_{3}$ to complete the transfer.

4. Add $\mathrm{HNO}_{3}$ to the beaker to bring the volume added to $100 \mathrm{~mL}$. Stir with a glass rod to mix sample and acid. Cover with a watch glass and heat until filter is wet ashed. Remove from the hotplate and cool. Add $30 \mathrm{~mL}$ of $\mathrm{HCl}$ to the beaker, cover with the watch glass, and heat on a low temperature hot plate for about $3 \mathrm{~h}$ with occasional stirring. Remove the beaker from the hot plate, and cool.

5. Repeat Step 3; dilute, filter and wash. Combine the filtrates. Return the residue and filter to the original beaker.

6. Repeat Step 4; wet ash filter and leach sample.

7. Repeat Step 3; dilute, filter and wash. Combine the three filtrates in a beaker. Discard the residue and filter paper.

8. Heat the filtrate with repeated $50-\mathrm{mL}$ additions of $\mathrm{HNO}_{3}$, covering the sample with a watch glass and letting the sample reflux until all organic matter is decomposed. Evaporate the solution to incipient dryness. Redissolve in $50-200 \mathrm{~mL}$ of $1: 1 \mathrm{HNO}_{3}$. 
If the solution is not clear, proceed to Step 9, otherwise go to Plutonium Determination.

9. If any siliceous matter is present, filter into a flask by gravity through a Whatman No. 42 filter paper. Wash the residue with $1: 1 \mathrm{HNO}_{3}$, and reserve the filtrate.

10. Transfer the filter paper with the residue to the original beaker and wet ash the paper with $100 \mathrm{~mL}$ of $\mathrm{HNO}_{3}$. Repeat wet ashing two or three times, then transfer the residue in the beaker into a 250-mL Teflon beaker, using 1:1 $\mathrm{HNO}_{3}$. Evaporate to dryness.

11. Add 5-25 mL of $\mathrm{HF}$ and 5-25 $\mathrm{mL}$ of $\mathrm{HNO}_{3}$ to the beaker and evaporate on a medium temperature hot plate. Repeat the addition of the $\mathrm{HF} / \mathrm{HNO}_{3}$ and the evaporation process two or three times. Rinse the walls of the Teflon beaker with $1: 1 \mathrm{HNO}_{3}$ and evaporate, and repeat. Evaporate to dryness. Dissolve with $1: 1 \mathrm{HNO}_{3}$ and evaporate to dryness.

12. Dissolve the residue in $1: 1 \mathrm{HNO}_{3}$ and filter by gravity through a Whatman No. 42 filter paper. Add the filtrate to the solution from Step 9. Discard the filter and any residue. Heat the combined solution to incipient dryness. Redissolve in 50-200 mL $1: 1 \mathrm{HNO}_{3}$.

\section{PLUTONIUM DETERMINATION}

Proceed to Plutonium Purification Ion Exchange Technique Procedure $P u-11-R C$. Save the column effluents for Americium Determination.

\section{AMERICIUM DETERMINATION}

1. Evaporate the americium effluents to incipient dryness. Redissolve in a minimum amount of $1: 1 \mathrm{HNO}_{3}$, dilute with four volumes of water.

2. Add $5 \mathrm{~mL}$ of calcium carrier solution ( $500 \mathrm{mg}$ of calcium) and $50 \mathrm{~g} \mathrm{~L}^{-1}$ of oxalic acid to the sample while stirring with a magnetic stirrer. (Note: The total volume of the 
sample solution can be estimated using the markings on the beaker, and the amount of oxalic acid to be added is calculated using that volume.)

3. Adjust the $\mathrm{pH}$ of the solution to 2.0-2.5 with $\mathrm{NH}_{4} \mathrm{OH}$ using $\mathrm{pH}$ paper as an indicator and continue to stir for $30 \mathrm{~min}$. Remove the magnetic stir bar.

4. Cool the sample and let it stand until precipitate settles and solution clears (for more than $6 \mathrm{~h}$ or overnight ). Check for completeness of precipitation using a drop of saturated $\mathrm{H}_{2} \mathrm{C}_{2} \mathrm{O}_{4}$ solution. Aspirate (or decant), using a disposable transfer pipette and suction, as much liquid as possible without disturbing the precipitate. Transfer the precipitate to a $250-\mathrm{mL}$ centrifuge bottle using oxalate wash solution (see Note 1 ). Balance the bottles on a double pan balance and centrifuge for $10 \mathrm{~min}$ at $2000 \mathrm{rpm}$. Decant and discard the supernate.

5. Break up the precipitate with a stirring rod and wash the precipitate with the oxalate wash solution. Centrifuge, decant and discard the wash. Repeat wash. Redissolve the precipitate in a minimal amount $(50-70 \mathrm{~mL})$ of concentrated $\mathrm{HCl}$ (the final precipitate should be redissolved in $\sim 200 \mathrm{~mL}$ of $\mathrm{HNO}_{3}$, then proceed to Step 8 below). (Note: Dissolution is easier if the centrifuge bottle is placed in a hot water bath and stirred with a glass rod).

6. Transfer the dissolved precipitate to the original $600-\mathrm{mL}$ beaker. Add enough water to make $\sim 1 \underline{\mathrm{M}}$ solution. Add $50 \mathrm{~g} \mathrm{~L}^{-1}$ of oxalic acid.

7. Repeat Steps 3 through 6 until supernate is colorless.

8. Transfer the dissolved precipitate to the original beaker and heat to destroy the oxalate ion. Evaporate to near dryness. Dissolve in a minimum of $1: 1 \mathrm{HNO}_{3}$. Transfer to centrifuge bottle using water to complete the transfer.

9. Add enough water to make $\sim 1 \underline{\mathrm{M}} \mathrm{HNO}_{3}$. Warm the solution in a $90^{\circ}$ hot water bath and add $0.2 \mathrm{~mL}$ iron carrier solution ( $20 \mathrm{mg}$ iron).

10. With the centrifuge bottle in the hot water bath adjacent to a hood, adjust the $\mathrm{pH}$ of the solution to 8-9 with $\mathrm{NH}_{4} \mathrm{OH}$ while stirring with a glass rod. Allow the solution to digest in a hot water bath for $20 \mathrm{~min}$. 
11. Cool in a cold water bath, rinse, and remove the glass rod. Balance the bottles on a double pan balance and centrifuge for $40 \mathrm{~min}$ at $2000 \mathrm{rpm}$.

\section{MICROPRECIPITATION}

See Microprecipitation Source Preparation for Alpha Spectrometry, Procedure G-03.

AMERICIUM

LOWER LIMIT OF DETECTION (LLD)

\begin{tabular}{lll}
\hline $\begin{array}{l}\text { Counter Efficiency } \\
\text { Counter Background }\end{array}$ & $(\%)$ & 25 \\
Yield & $(\mathrm{cps})$ & $15 \times 10^{-6}$ \\
Blank & $(\%)$ & 50 \\
& $(\mathrm{cps})$ & - \\
LLD (400 min) & & \\
LLD (1000 min) & $(\mathrm{mBq})$ & 1 \\
LLD (5000 min) & $(\mathrm{mBq})$ & 0.5 \\
& $(\mathrm{mBq})$ & 0.3 \\
\hline
\end{tabular}

PLUTONIUM

LOWER LIMIT OF DETECTION (LLD)

\begin{tabular}{lll}
\hline Counter Efficiency & $(\%)$ & 25 \\
Counter Background & $(\mathrm{cps})$ & $2 \times 10^{-5}$ \\
Yield & $(\%)$ & 75 \\
Blank & $(\mathrm{cps})$ & - \\
& & \\
LLD $(400 \mathrm{~min})$ & $(\mathrm{mBq})$ & 1 \\
LLD $(1000 \mathrm{~min})$ & $(\mathrm{mBq})$ & 0.5 \\
LLD $(5000 \mathrm{~min})$ & $(\mathrm{mBq})$ & 0.2
\end{tabular}




\section{Radium}




\section{RADIUM-226 IN BONE ASH}

Contact Person(s) : Isabel M. Fisenne

APPLICATION

This procedure is applicable to bone ash only and has been adapted from Hallden et al. (1963).

The separated sample is measured by radon emanation. Only ${ }^{226} \mathrm{Ra}$ yields a radon progeny with suitable characteristics, so the method is specific. Most of the calcium, the alkali metals and phosphates are separated by coprecipitation of the radium as sulfate with barium carrier in a buffered solution. Further purification is obtained by repeating this step in the presence of undissolved $\mathrm{BaSO}_{4}$. The sulfate precipitate is dissolved in alkaline EDTA to prepare the emanating solution. The chemical yield of barium is determined with the $\gamma$-emitting tracer ${ }^{133} \mathrm{Ba}$.

\section{SPECIAL APPARATUS}

Radon bubblers - see Specification 7.7.

\section{SPECIAL REAGENTS}

1. Barium-133 tracer solution - about 50 counts sec $^{-1}$ per 0.1-g aliquot, prepared in 1:99 $\mathrm{HCl}$.

2. Barium carrier solution $\left(20 \mathrm{mg} \mathrm{mL}^{-1}\right)-30.4 \mathrm{~g} \mathrm{BaCl}_{2} \mathrm{~L}^{-1}$ of $1: 99 \mathrm{HCl}$. 
3. Ammonium acetate solution - $15 \mathrm{~g} \mathrm{NH}_{4} \mathrm{C}_{2} \mathrm{H}_{3} \mathrm{O}_{2}\left(\mathrm{NH}_{4} \mathrm{OAc}\right) \mathrm{L}^{-1}$ of water.

4. Acetic acid solution - $20 \mathrm{~mL}$ glacial $\mathrm{CH}_{3} \mathrm{CO}_{2} \mathrm{H}(\mathrm{HOAC}) \mathrm{L}^{-1}$ of water.

5. Ammonium sulfate solution - $100 \mathrm{~g}\left(\mathrm{NH}_{4}\right)_{2} \mathrm{SO}_{4} \mathrm{~L}^{-1}$ of water.

6. Aerosol OT solution $-0.1 \%$.

7. EDTA solution - $300 \mathrm{~g}$ tetrasodium salt of EDTA $\mathrm{L}^{-1}$ of water.

8. EDTA wash solution - 1:9 dilution of EDTA solution.

9. Monoethanolamine $-1: 1(\mathrm{v} / \mathrm{v})$ with water.

\section{DETERMINATION}

1. Weigh $10 \mathrm{~g}$ of bone ash into a $90-\mathrm{mL}$ glass or $100-\mathrm{mL}$ polycarbonate centrifuge tube. Add a weighed aliquot (about $0.1 \mathrm{~g}$ ) of ${ }^{133} \mathrm{Ba}$ tracer solution, and $1 \mathrm{~mL}$ of barium carrier solution.

2. Add $20 \mathrm{~mL}$ of $\mathrm{HCl}$ (slowly at first to prevent foaming). Stir and warm in an $85^{\circ} \mathrm{C}$ water bath for about $5 \mathrm{~min}$.

3. Add $10 \mathrm{~mL}$ of water. Add $\mathrm{NH}_{4} \mathrm{OH}$ until a dense white permanent hydroxide flock forms (about 8-10 $\mathrm{mL}$ ).

4. Dissolve the flock in $\mathrm{HCl}$ (about $4 \mathrm{~mL}$ is required).

5. Add $2 \mathrm{~mL}$ of $\mathrm{NH}_{4} \mathrm{OAc}$ solution and $1 \mathrm{~mL}$ of $\mathrm{HOAc}$ solution. Cool in a water bath to room temperature.

6. Add $1 \mathrm{~mL}$ of $\left(\mathrm{NH}_{4}\right)_{2} \mathrm{SO}_{4}$ solution. Stir and let stand for $0.5 \mathrm{~h}$.

7. Centrifuge at $2000 \mathrm{rpm}$ for $1 \mathrm{~h}$. Add one drop of $0.1 \%$ Aerosol OT. Decant carefully and discard the supernate. 
8. Add $5 \mathrm{~mL}$ of $\mathrm{HCl}$ and warm in an $85^{\circ} \mathrm{C}$ water bath for $5 \mathrm{~min}$. (Most of the $\mathrm{BaSO}_{4}$ remains as a precipitate.)

9. Add $10 \mathrm{~mL}$ of water and then add $\mathrm{NH}_{4} \mathrm{OH}$ until a permanent flock forms.

10. Dissolve the flock in $\mathrm{HCl}$.

11. Add $2 \mathrm{~mL}$ of $\mathrm{NH}_{4} \mathrm{OAc}$ solution and $1 \mathrm{~mL}$ of $\mathrm{HOAc}$ solution. Cool in a water bath to room temperature.

12. Add $1 \mathrm{~mL}$ of $\left(\mathrm{NH}_{4}\right)_{2} \mathrm{SO}_{4}$ solution. Stir and let stand for $0.5 \mathrm{~h}$.

13. Centrifuge at $2000 \mathrm{rpm}$ for $1 \mathrm{~h}$. Add one drop of $0.1 \%$ Aerosol OT. Decant carefully and discard the supernate.

14. Heat a solution of EDTA $\left(300 \mathrm{~g} \mathrm{~L}^{-1}\right)$ and an EDTA wash solution $\left(30 \mathrm{~g} \mathrm{~L}^{-1}\right)$ in an $85^{\circ} \mathrm{C}$ water bath.

15. Break up the $\mathrm{BaSO}_{4}$ precipitate with a stirring rod. Add $1 \mathrm{~mL}$ of $1: 1$ monoethanolamine and $5 \mathrm{~mL}$ of the hot EDTA solution and stir. Let stand for $5 \mathrm{~min}$.

16. Wash down the sides of the tube with about $10 \mathrm{~mL}$ of the hot EDTA wash solution. Let the tube remain in the water bath for $15 \mathrm{~min}$, stirring occasionally.

17. Gravity filter the hot solution through a $12.5 \mathrm{~cm}$ Whatman No. 41 filter paper into a $30 \mathrm{~mL}$ polyethylene bottle.

18. Wash the centrifuge tube and the filter paper with hot EDTA wash solution. Discard the paper and residue.

19. Dilute the sample to the same liquid level as the ${ }^{133} \mathrm{Ba}$ standard. The standard prepared by diluting a known aliquot (about $0.1 \mathrm{~g}$ ) of ${ }^{133} \mathrm{Ba}$ solution to $25 \mathrm{~mL}$ in a $30 \mathrm{~mL}$ polyethylene bottle. Gamma count on a flat crystal to determine the chemical yield of barium.

20. Transfer the sample solution to $\mathrm{a}^{222} \mathrm{Rn}$ bubbler. 
21. De-emanate ${ }^{222} \mathrm{Rn}$ by bubbling with forming gas for about $10 \mathrm{~min}$ at $100 \mathrm{~cm}^{3} \mathrm{~min}^{-1}$ as described in Radium - Emanation Procedure (see Ra-02-RC). Record the time as the starting time for ${ }^{222} \mathrm{Rn}$ buildup. Continue the analysis using the emanation technique.

\section{LOWER LIMIT OF DETECTION (LLD)}

\begin{tabular}{lll}
\hline Counter Efficiency & $(\%)$ & 57.5 \\
Counter Background & $(\mathrm{cps})$ & 0.0028 \\
Yield & $(\%)$ & 90 \\
Blank & $(\mathrm{cps})$ & 0.0012 \\
LLD (400 min) & $(\mathrm{mBq})$ & 3.3 \\
LLD (1000 min) & $(\mathrm{mBq})$ & 1.7 \\
\hline
\end{tabular}

*Reagent blanks must be analyzed with each set of samples.

\section{REFERENCE}

Hallden, N. A., I. M. Fisenne and J. H. Harley

"The Determination of Radium-226 in Human Bone"

Talanta, 10, 1223-1227 (1963) 
RADIUM-226 - EMANATION PROCEDURE

\title{
Contact Person(s) : Isabel M. Fisenne
}

\begin{abstract}
APPLICATION
This procedure is specific and may be applied to almost any matrix which can be converted to a homogeneous solution.

Radium-226 in solution can be determined by de-emanating its ${ }^{222} \mathrm{Rn}$ into an ionization chamber or scintillation cell for measurement. The ${ }^{222} \mathrm{Rn}$ can be de-emanated by bubbling an inert gas through the solution, either after equilibrium has been established or after any known time period. Two half-lives, 7.65 days for example, give $75 \%$ of the maximum buildup.
\end{abstract}

\section{SPECIAL APPARATUS}

1. Radon measuring equipment are described in Radon-222 in Air and Breath Samples, Procedure Rn-01-RC, and in Fisenne and Keller (1985).

2. Radon bubblers - see Specification 7.7.

\section{SAMPLE PREPARATION}

1. The sample with barium carrier should be in homogeneous solution in a volume of 15-25 mL. The solution should be acidic with perchloric acid, neutral or in basic EDTA solution. Hydrochloric acid, $\mathrm{NH}_{3}$ or other volatile materials must be absent. 
2. Detailed preparation procedures for separation of $\mathrm{Ra}$ from sample matrices are presented in other radium procedures in this Manual.

3. Almost all types of samples require simultaneous analysis of reagent blanks, since radium appears in many chemical compounds. Using distilled or organic reagents reduces possible contamination.

\section{DETERMINATION}

1. Transfer the sample solution to ${ }^{222} \mathrm{Rn}$ bubbler.

2. Clamp the bubbler in place to the ${ }^{222} \mathrm{Rn}$ system.

3. Open the forming gas $\left(85 \% \mathrm{~N}_{2}, 15 \% \mathrm{H}_{2}\right)$ tank valve and adjust the line pressure to $70 \mathrm{kPa}$ (10 psi) gauge.

4. Bleed the gas line, the rotometer, and $9.5 \mathrm{~mm}$ diameter rubber tubing.

5. Adjust the flow through the rotometer to a rate of $20 \mathrm{~cm}^{3} \mathrm{~min}^{-1}$.

6. Open both stopcocks on the ${ }^{222} \mathrm{Rn}$ bubbler.

7. Attach the rubber tubing on the exit of the rotometer to the inlet of the ${ }^{222} \mathrm{Rn}$ bubbler. Adjust the forming gas flow rate through the bubbler to $100 \mathrm{~cm}^{3} \mathrm{~min}^{-1}$.

8. Flush the ${ }^{222} \mathrm{Rn}$ from the solution (de-emanate) for $10 \mathrm{~min}$.

9. Close both stopcocks simultaneously. Record the date and time as the beginning of the ${ }^{222} \mathrm{Rn}$ build-up period.

10. Shut off the forming gas and remove the rubber tubing from the bubbler.

11. Place the ${ }^{222} \mathrm{Rn}$ bubbler in a refrigerator for a suitable build-up period, usually 1 week.

12. To emanate ${ }^{222} \mathrm{Rn}$ into a pulse ionization chamber, repeat Steps $2-4$. 
13. Attach the rubber tubing from the rotometer to the inlet of the measurement system.

14. Open the valve to the ionization chamber and close the vacuum valves. Partially fill the chamber with forming gas to a pressure of $-2.7(-50 \mathrm{~cm}$ of $\mathrm{Hg})$ gauge. Close the chamber valve and system entry valve. Open the vacuum valves and detach the rubber tubing from the measurement system.

15. Attach a small drying tube containing about $5 \mathrm{~g}$ of a color indicating drying agent and $4 \mathrm{~cm}$ lengths of $9.5 \mathrm{~mm}$ diameter rubber tubing at both ends to the outlet of the bubbler and the inlet of the measurement system.

16. Open the system entry valve to evacuate the drying tube for $3 \mathrm{~min}$.

17. Adjust the forming gas flow rate to $20 \mathrm{~cm}^{3} \mathrm{~min}^{-1}$ and attach the tubing outlet of the rotometer to the inlet of the bubbler.

18. Close the vacuum valves and open the ionization chamber valve.

19. Open the outlet stopcock of the bubbler and allow the bubbling to subside. Record the date and time as the end of the ${ }^{222} \mathrm{Rn}$ build-up period.

20. Carefully open the bubbler inlet stopcock and adjust the forming gas flow through the bubbler to about $100 \mathrm{~cm}^{3} \mathrm{~min}^{-1}$.

21. When the system gauge indicates that atmospheric pressure has been reached, close both bubbler stopcocks simultaneously. Close the chamber, system entry and forming gas valves. Open the vacuum valves.

22. Remove the bubbler and drying tube from the system. Record the date and time of the end of the emanation as the beginning of the next ${ }^{222} \mathrm{Rn}$ build-up period.

23. Turn on the ionization chamber high voltage. A red LED will light when the chamber is at or above atmospheric pressure.

24. Measure the sample for at least $17 \mathrm{~h}$. 


\section{Notes for Proper Operation:}

1. If the Teflon stopcocks of the bubbler are snug fitting, it is not necessary to grease them. If they do leak, however, a silicone stopcock grease may be used.

2. The glass-to-glass ground joint at the top of the bubbler should also be lightly greased with silicone.

3. During the de-emanation, the gas bubbling should be started slowly to prevent popping of the bubbler top or stopcocks.

4. A better transfer of ${ }^{222} \mathrm{Rn}$ from acid or neutral solutions is obtained if the bubbler is first chilled in a refrigerator. This apparently reduces the bubble size and improves the transfer efficiency. For samples dissolved in alkaline EDTA solution, a drop of octyl alcohol is added to reduce foaming.

\section{STANDARDIZATION}

The chambers are standardized by de-emanating aliquots of a National Institute of Standards and Technology SRM ${ }^{226}$ Ra solution contained in ${ }^{222} \mathrm{Rn}$ bubblers.

\section{DATA PROCESSING AND ANALYSES}

1. For routine work, discard the first $5 \mathrm{~h}$ of counting data (equilibrium for ${ }^{222} \mathrm{Rn}$ and progeny).

2. Determine the total count over the remaining counting period.

3. Calculate the net counts per hour per sample by determining the total sample counts per hour and subtracting the background counts per hour.

4. Divide the net counts per hour by the chamber standardization value of net counts per hour per Bq of ${ }^{222} \mathrm{Rn}$. 
5. Using the midpoint of the counting interval as the time of counting, extrapolate the value obtained to the time noted for the end of ${ }^{222} \mathrm{Rn}$ buildup. Figure 1 may be used for this calculation. This correction is only a few percent. The decay during the counting period is small and is not corrected for.

6. Correct the ${ }^{222} \mathrm{Rn}$ measurement at the end of buildup for the fractional buildup. The figure in Procedure Rn-01-RC may be used to obtain the build-up factor.

7. Subtract the value obtained by carrying a blank determination through the full procedure.

8. Samples are measured twice and the mean and standard deviation of the duplicate emanation results are reported.

9. A computational data sheet is attached for handling routine calculations on a step-bystep basis.

LOWER LIMIT OF DETECTION (LLD)*

\begin{tabular}{lll}
\hline & & \\
Counter Efficiency & $(\%)$ & 57.5 \\
Counter Background & $(\mathrm{cps})$ & 0.0028 \\
Yield & $(\%)$ & 90 \\
Blank & $(\mathrm{cps})$ & 0.0012 \\
LLD (400 min) & $(\mathrm{mBq})$ & 3.3 \\
LLD (1000 min) & $(\mathrm{mBq})$ & 1.7 \\
& & \\
\hline
\end{tabular}

${ }^{*}$ Reagent blank must be analyzed with each set of samples. 


\section{REFERENCE}

Fisenne, I. M. and H. W. Keller

"The EML Pulse Ionization Chamber Systems for the Measurement of Radon-222" USDOE Report EML-437, March (1985) 


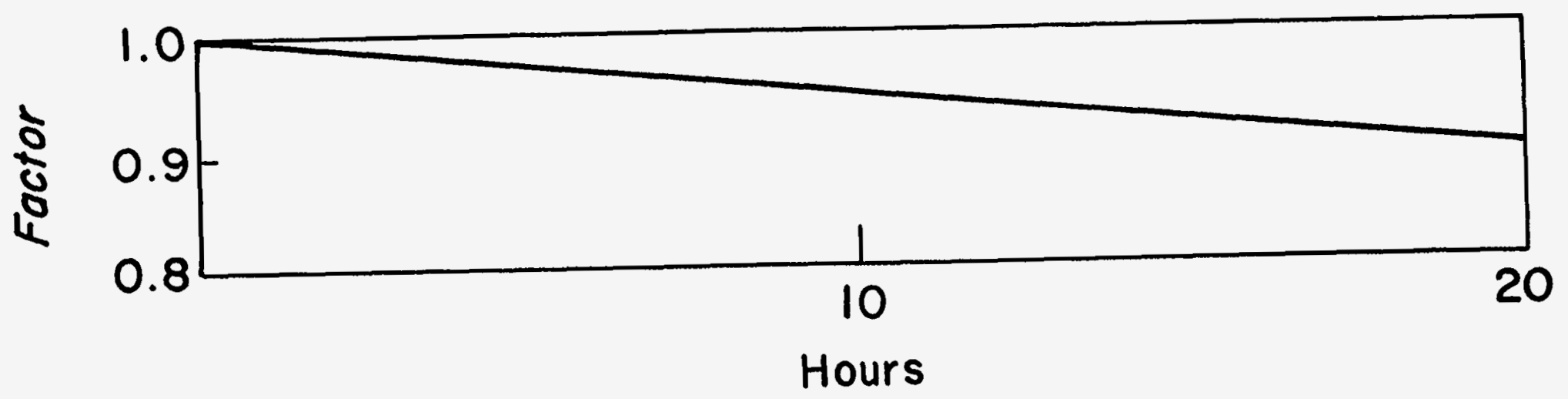

Figure 1. Correction factor from midpoint to the counting interval to the start of radon measurements interval. 
Date

Sample Number

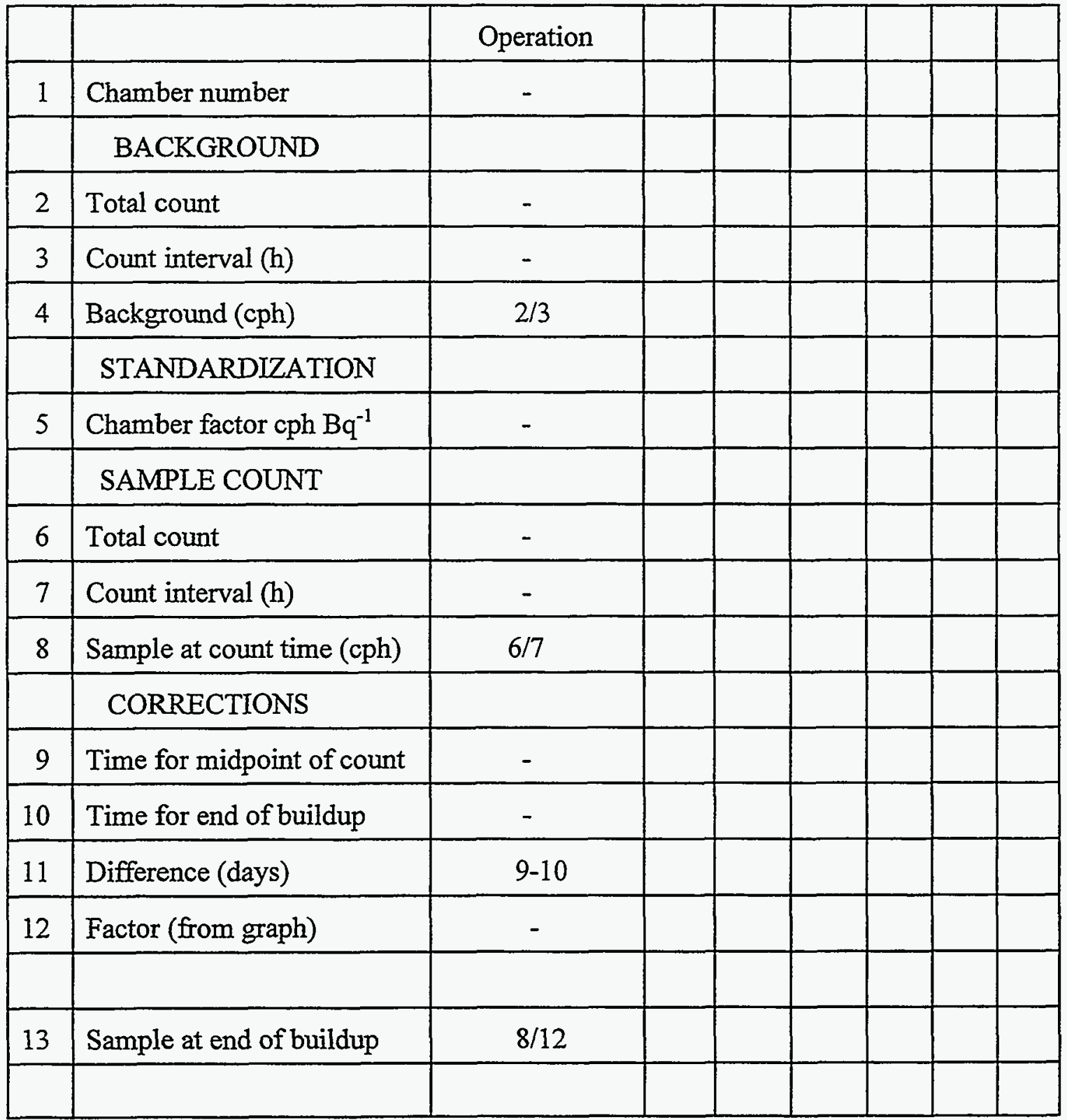


Date



*This sheet is suitable for calculating the blank value. 
RADTUM-226 IN SOL, VEGETATION ASH, AND ION EXCHANGE RESIN

Contact Person(s) : Isabel M. Fisenne

\begin{abstract}
APPLICATION
This procedure is applicable to $5 \mathrm{~g}$ samples of soil and $10 \mathrm{~g}$ of vegetation ash or to ion exchange resin from sampling columns.

Soil, vegetation ash or ion exchange resin are prepared for ${ }^{222} \mathrm{Rn}$ emanation measurement. The sample is pretreated with $\mathrm{HNO}_{3}-\mathrm{HF}$, fused with $\mathrm{KF}$ and transposed to pyrosulfate (Sill, 1961). The cake is dissolved in dilute $\mathrm{HCl} . \mathrm{RaBaSO}_{4}$ is precipitated, filtered, and dissolved in alkaline EDTA. The chemical yield is determined with the $\gamma$-emitting tracer ${ }^{133} \mathrm{Ba}$.
\end{abstract}

\title{
SPECIAI APPARATUS
}

1. Radon bubblers - see Specification 7.7.

2. $100 \mathrm{~mL}$ platinum dishes or $250 \mathrm{~mL}$ platinum crucibles.

3. Millipore filter setup - $47 \mathrm{~mm}$ diameter.

4. Millipore filters $-47 \mathrm{~mm}$ diameter, $0.45 \mu \mathrm{m}$ pore size. 


\section{SPECIAL REAGENTS}

1. Barium-133 tracer solution - about $50 \mathrm{cps}$ per 0.1-g aliquot, prepared in 1:99 $\mathrm{HCl}$.

2. Barium carrier solution $\left(20 \mathrm{mg} \mathrm{mL}^{-1}\right)-30.4 \mathrm{~g} \mathrm{BaCl}_{2} \mathrm{~L}^{-1}$ in $1: 99 \mathrm{HCl}$.

3. EDTA solution - $300 \mathrm{~g}$ tetrasodium salt of EDTA $\mathrm{L}^{-1}$ of water.

4. Triethanolamine - $1: 1$ in water.

\section{SAMPLE PREPARATION}

\section{A. Soil and vegetation.}

1. Weigh $5 \mathrm{~g}$ of soil or $10 \mathrm{~g}$ of vegetation ash into a $100-\mathrm{mL}$ platinum dish. Add a weighed aliquot (about $0.1 \mathrm{~g}$ ) of ${ }^{133} \mathrm{Ba}$ tracer solution and $1 \mathrm{~mL}$ of $\mathrm{Ba}$ carrier solution.

2. Slowly add $10 \mathrm{~mL}$ of $\mathrm{HNO}_{3}$ and $10 \mathrm{~mL}$ of $\mathrm{HF}$ to the sample and evaporate on a hot plate to near dryness.

3. Continue the analysis as described below.

\section{B. Ion exchange resin.}

1. Transfer the resin and paper pulp from the collection column to a $250-\mathrm{mL}$ platinum crucible. Dry under a heat lamp and ash at $500^{\circ} \mathrm{C}$ in a muffle furnace for $48 \mathrm{~h}$.

2. To the cooled crucible, add a weighed aliquot (about $0.1 \mathrm{~g}$ ) of ${ }^{133} \mathrm{Ba}$ tracer solution and $1 \mathrm{~mL}$ of barium carrier solution.

3. Continue the analysis as described below. 


\section{DETERMINATION}

1. Weigh out $15 \mathrm{~g}$ of KF and add to the sample. Press the KF into the sample with a plastic spatula.

Caution - wear rubber gloves and safety glasses during Steps 2-5.

2. Fuse the sample over an air fed Meker burner, gradually increasing the temperature until a clear melt is obtained. Cool the melt.

3. Using a burette, slowly add $17.5 \mathrm{~mL}$ of $\mathrm{H}_{2} \mathrm{SO}_{4}$ to the melt. Heat the dish on a hot plate until a clear melt accompanied by dense fumes is obtained. Cool the melt.

4. Weigh out $10 \mathrm{~g}$ of $\mathrm{Na}_{2} \mathrm{SO}_{4}$, add to the dish and fuse over the Meker burner until a clear melt accompanied by dense fumes is obtained. Cool the melt.

5. Transfer the cake to a $600 \mathrm{~mL}$ beaker containing $350 \mathrm{~mL}$ of hot water and $25 \mathrm{~mL}$ of $\mathrm{HCl}$. Stir the solution to dissolve the cake. Cool for $1 \mathrm{~h}$.

6. Filter the precipitate onto a $47 \mathrm{~mm}$ diameter, $0.45 \mu \mathrm{m}$ pore size Millipore filter into a $1 \mathrm{~L}$ sidearm flask, police the beaker, and wash with water. Add the washing to the funnel. Discard the filtrate.

7. Using a strong stream of water from a wash bottle, transfer the precipitate from the filter into a $150 \mathrm{~mL}$ beaker. Discard the filter.

8. Add $5 \mathrm{~mL}$ of EDTA solution and $1 \mathrm{~mL}$ of $1: 1$ triethanolamine to the beaker. Heat on a warm hot plate for about $15 \mathrm{~min}$, stirring occasionally. Reduce the sample volume to $\sim 15 \mathrm{~mL}$.

9. Gravity filter the warm solution through a Whatman No. 41 filter paper into a $30-\mathrm{mL}$ polyethylene bottle.

10. Wash the beaker dish and filter with hot water. Discard the filter. 
11. Dilute the sample to the same liquid level as a known aliquot (about $0.1 \mathrm{~g}$ ) of the ${ }^{133} \mathrm{Ba}$ tracer solution and dilute to $25 \mathrm{~mL}$ in a $30 \mathrm{~mL}$ polyethylene bottle.

12. Gamma count the samples and standard to determine the chemical yield of barium.

13. Transfer the sample to a ${ }^{222} \mathrm{Rn}$ bubbler with water.

14. De-emanate ${ }^{222} \mathrm{Rn}$ by bubbling with forming gas for about $10 \mathrm{~min}$ at $100 \mathrm{~mL} \mathrm{~min} \mathrm{~m}^{-1}$ as described in ${ }^{226}$ Radium - Emanation Procedure (see Ra-03-RC). Record the time as the starting time for ${ }^{222} \mathrm{Rn}$ buildup. Continue the analysis by the emanation technique.

\section{LOWER LIMIT OF DETECTION (LLD)*}

\begin{tabular}{llc}
\hline & & \\
Counter Efficiency & $(\%)$ & 57.5 \\
Counter Background & $(\mathrm{cps})$ & 0.0028 \\
Yield & $(\%)$ & 90 \\
Blank & $(\mathrm{cps})$ & 0.0020 \\
& & \\
LLD (400 $\mathrm{min})$ & $(\mathrm{mBq})$ & 3.3 \\
LLD $(1000 \mathrm{~min})$ & $(\mathrm{mBq})$ & 1.7 \\
& & \\
\hline
\end{tabular}

${ }^{*}$ Reagent blanks must be analyzed with each set of samples.

\section{REFERENCE}

Sill, C. W.

"Decomposition of Refractory Silicates in Ultramicro Analysis"

Anal. Chem., 33, 1684 (1961) 


\title{
RADIUM-226 IN TAP WATER, URINE, AND FECES
}

\author{
Contact Person(s) : Isabel M. Fisenne
}

\begin{abstract}
APPLICATION
The procedure presented has been applied to tap water, ashed urine, and feces samples. Only ${ }^{226} \mathrm{Ra}$ yields ${ }^{222} \mathrm{Rn}$ progeny that has suitable characteristics for detection by an emanation technique; therefore, the procedure is specific.

After sample preparation, radium is isolated from most other elements by coprecipitation with barium sulfate. Further purification is obtained by the removal of silica with $\mathrm{HF}$ and reprecipitation of the sulfate. The sulfate precipitate is dissolved in alkaline EDTA to prepare the emanating solution. The chemical yield of barium is determined with the $\gamma$-emitting tracer ${ }^{133} \mathrm{Ba}$.
\end{abstract}

\section{SPECIAL APPARATUS}

Radon bubblers - see Specification 7.7.

\section{SPECIAL REAGENT}

1. Barium carrier solution $-20 \mathrm{mg} \mathrm{mL}^{-1}-30.4 \mathrm{~g} \mathrm{BaCl}_{2} \mathrm{~L}^{-1}$ in $1: 99 \mathrm{HCl}$.

2. Ammonium sulfate solution - $100 \mathrm{~g}\left(\mathrm{NH}_{4}\right)_{2} \mathrm{SO}_{4} \mathrm{~L}^{-1}$ in water.

3. Aerosol OT solution $-0.1 \%$. 
4. EDTA solution - $300 \mathrm{~g}$ tetrasodium salt of EDTA $\mathrm{L}^{-1}$ in water.

5. EDTA wash solution - 1:9 dilution of EDTA solution.

6. Ammonium acetate solution $-15 \mathrm{~g} \mathrm{~L}^{-1}$ in water.

7. Acetic acid solution - $20 \mathrm{~mL}$ glacial acetic $\mathrm{L}^{-1}$ in water.

8. Triethanolamine $-1: 1$ in water.

\section{SAMPLE PREPARATION}

\section{A. Tap water.}

1. Transfer two 0.5-L of tap water to a $3 \mathrm{~L}$ beaker.

2. Add $25 \mathrm{~mL}$ of $\mathrm{HCl}$ and $1 \mathrm{~mL}$ of barium carrier solution. Add a weighed aliquot (about $0.1 \mathrm{~g}$ ) of the ${ }^{133} \mathrm{Ba}$ tracer solution.

3. Evaporate and add an additional two 0.5-L aliquots of tap water until a 10-L collection has been obtained. Evaporate gently to about $100 \mathrm{~mL}$.

4. Transfer to a 400-mL beaker with water, policing the sides of the 3-L beaker thoroughly. Evaporate gently to about $100-\mathrm{mL}$.

5. Adjust the $\mathrm{pH}$ to 4 with $1: 1 \mathrm{NH}_{4} \mathrm{OH}$ and proceed with Determination.

\section{B. Urine.}

1. Weigh an aliquot of ${ }^{133} \mathrm{Ba}$ tracer solution (about $0.1 \mathrm{~g}$ ) into a 2-L beaker containing a small amount of water and $1 \mathrm{~mL}$ of barium carrier solution.

2. Add $1500 \mathrm{~mL}$ of urine, then $100 \mathrm{~mL}$ of $\mathrm{HNO}_{3}$ and evaporate to about $1-\mathrm{L}$.

3. Slowly add $100-\mathrm{mL}$ of $\mathrm{HNO}_{3}$. 
4. Repeat the evaporation and addition of acid until a total of 500-mL of $\mathrm{HNO}_{3}$ has been added.

5. Evaporate to about $20 \mathrm{~mL}$. Transfer to a $400-\mathrm{mL}$ beaker with water.

6. Adjust the $\mathrm{pH}$ to 4 with $1: 1 \mathrm{NH}_{4} \mathrm{OH}$ and proceed with Determination.

\section{Feces ash.}

1. Weigh $1 \mathrm{~g}$ of fecal ash into a $150-\mathrm{mL}$ beaker. Add a weighed aliquot (about $0.1 \mathrm{~g}$ ) of ${ }^{133} \mathrm{Ba}$ tracer solution and $1 \mathrm{~mL}$ of barium carrier solution.

2. Cover the ash with a small quantity of water and slowly add $10 \mathrm{~mL}$ of $\mathrm{HNO}_{3}$. Evaporate to a small volume on a medium hot plate.

3. Add about $25 \mathrm{~mL}$ of water. Add $\mathrm{NH}_{4} \mathrm{OH}$ until a permanent hydroxide flock forms (3-5 mL).

4. Dissolve the flock in a few drops of $\mathrm{HNO}_{3}$.

5. Add $2 \mathrm{~mL}$ of $\mathrm{NH}_{4} \mathrm{Ac}$ solution and $1 \mathrm{~mL}$ of acetic acid solution. Dilute to about $100-\mathrm{mL}$ and proceed with Determination.

\section{DETERMINATION}

1. Add $1 \mathrm{~mL}$ of $\left(\mathrm{NH}_{4}\right)_{2} \mathrm{SO}_{4}$ solution and allow the sample to digest for $1 \mathrm{~h}$ at room temperature.

2. Filter by gravity on a $9 \mathrm{~cm}$ Whatman No. 42 filter paper. Wash the paper thoroughly with water. Discard the filtrate and washings.

3. Transfer the paper to a platinum dish. Dry the paper and then ash at $500^{\circ} \mathrm{C}$ for about $1 \mathrm{~h}$.

4. Add $1 \mathrm{~mL}$ of $\mathrm{H}_{2} \mathrm{SO}_{4}$ and $2 \mathrm{~mL}$ of $\mathrm{HF}$. Evaporate to $\mathrm{SO}_{3}$ fumes. 
5. Cool and transfer to a $90 \mathrm{~mL}$ centrifuge tube with water.

6. Police the dish and add the washings to the centrifuge tube.

7. Stir and let stand for $0.5 \mathrm{~h}$.

8. Centrifuge at $2000 \mathrm{rpm}$ for $1 \mathrm{~h}$. Add one drop of $0.1 \%$ Aerosol OT. Decant carefully and discard the supernate.

9. Heat a solution of EDTA ( $300 \mathrm{~g} \mathrm{~L}^{-1}$ EDTA) in an $85^{\circ} \mathrm{C}$ water bath.

10. Break up the $\mathrm{BaSO}_{4}$ precipitate with a stirring rod.

11. Add $1 \mathrm{~mL}$ of $1: 1$ triethanolamine and $5 \mathrm{~mL}$ of the hot EDTA solution, and stir. Wash down the sides of the tube with water.

12. Digest in the steam bath for $15 \mathrm{~min}$, stirring occasionally.

13. Transfer the solution to a $30-\mathrm{mL}$ polyethylene bottle.

14. Dilute the sample to the same liquid level as a known aliquot (about $0.1 \mathrm{~g}$ ) of ${ }^{133} \mathrm{Ba}$ solution diluted to $25 \mathrm{~mL}$ in a $30 \mathrm{~mL}$ polyethylene bottle.

15. Gamma count samples and standard on a flat crystal to determine the chemical yield of barium.

16. Transfer the sample solution to a radon bubbler.

17. De-emanate radon by bubbling with forming gas for about $10 \mathrm{~min}$ at $100 \mathrm{~mL}^{-1} \mathrm{~min}$ as described in Procedure Ra-03-RC. Record the time as the starting time for the radon build-up period. Continue the analysis by the emanation technique. 


\section{LOWER LIMIT OF DETECTION (LLD)*}

\begin{tabular}{lcccc}
\hline & & & & \\
& & & B & C \\
& & & & \\
\hline & & & 57.5 & 57.5 \\
Counter Efficiency & $(\%)$ & 57.5 & 0.0028 & 0.0028 \\
Counter Background & $(\mathrm{cps})$ & 0.0028 & 0.0020 & 0.0012 \\
Yield & $(\%)$ & 90 & 80 & \\
Blank & $(\mathrm{cps})$ & 0.0012 & 0.0020 & 0.39 \\
& & & & 0.15 \\
LLD (400 min) & $(\mathrm{mBq})$ & 0.33 & 0.45 & \\
LLD (1000 min) & $(\mathrm{mBq})$ & 0.17 & 0.29 & \\
& & & & \\
\hline
\end{tabular}

*Reagent blanks must be analyzed with each set of samples.

Pulse ionization chamber:

$$
\begin{aligned}
& A=\text { Tap water } \\
& B=\text { Urine } \\
& C=\text { Feces }
\end{aligned}
$$


Ra-05-RC, Vol. II

HASL-300, 28th Edition
Rev. 0

February 1997

$R a-05-R C$

RADIUM-224 IN URINE

(see Volume II) 



\begin{abstract}
APPLICATION
This procedure is applicable to samples of urine and water. Small amounts of thorium are carried by the $\mathrm{BaSO}_{4}$ and the method fails in the presence of ${ }^{230} \mathrm{Th}$. Natural thorium does not interfere. Other $\alpha$-emitting isotopes of radium also interfere.
\end{abstract}

Radium is initially separated from untreated water or urine by coprecipitation with calcium phosphate. Calcium and most other elements are removed by coprecipitation of radium on barium sulfate. Organic material is removed by ignition, silica is removed by volatilization with $\mathrm{HF}$, and the radium-barium sulfate is reprecipitated.

Radium-226 in the barium sulfate precipitate is stored to allow the buildup of ${ }^{222} \mathrm{Rn}$, ${ }^{218} \mathrm{Po}$, and ${ }^{214} \mathrm{Po}$. The equilibrated $\alpha$ activities are then measured with a scintillation counter. The instrument response is converted to Bq by application of corrections for counter background and efficiency and for self-absorption. Chemical recovery is measured gravimetrically and the ${ }^{226} \mathrm{Ra}$ activity is equivalent to one-fourth of the total $\mathrm{Bq}$ measured.

\title{
SPECIAL APPARATUS
}

1. Rings and discs - see Specification 7.2.

2. Teflon filter holders and filter funnels and sample mounts - see Specification 7.12.

3. Mylar film - see Specification 7.10. 


\section{SPECIAL REAGENTS}

1. Barium carrier solution: $5 \mathrm{mg} \mathrm{Ba} \mathrm{mL}^{-1}-9.51 \mathrm{~g} \mathrm{Ba}\left(\mathrm{NO}_{3}\right)_{2} \mathrm{~L}^{-1}$ of $1: 99 \mathrm{HCl}$.

\section{SAMPLE PREPARATION}

\section{A. Water.}

1. Transfer $4 \mathrm{~L}$ of the sample to a $5 \mathrm{~L}$ stainless steel pot.

2. Add $1 \mathrm{~mL}$ of barium carrier solution and $4 \mathrm{~mL}$ of $\mathrm{HNO}_{3}$.

3. Evaporate to near dryness, add additional aliquots of water and $\mathrm{HNO}_{3}$, and continue until $50 \mathrm{~L}$ of sample have been treated.

4. Evaporate to about $100 \mathrm{~mL}$, add 3-4 $\mathrm{g}$ of $\mathrm{NH}_{4} \mathrm{Ac}$, and adjust the $\mathrm{pH}$ to 5 with $1: 1 \mathrm{NH}_{4} \mathrm{OH}$.

\section{B. Urine.}

1. Transfer a measured volume of urine to a $250-\mathrm{mL}$ centrifuge bottle and add $1 \mathrm{~mL}$ of barium carrier solution.

2. Add $2 \mathrm{~mL}$ of $\mathrm{H}_{3} \mathrm{PO}_{4}$. Adjust the $\mathrm{pH}$ to 9 with $1: 1 \mathrm{NH}_{4} \mathrm{OH}$. Stir, centrifuge, and discard the supernate.

3. Dissolve the precipitate with $10 \mathrm{~mL}$ of $\mathrm{HNO}_{3}$. Dilute to about $100 \mathrm{~mL}$ with water.

4. Add $1 \mathrm{~mL}$ of $\mathrm{H}_{3} \mathrm{PO}_{4}$. Adjust the $\mathrm{pH}$ to 9 with $1: 1 \mathrm{NH}_{4} \mathrm{OH}$. Stir, centrifuge, and discard the supernate.

5. Dissolve the precipitate in a few $\mathrm{mL}$ of acetic acid and dilute to $100 \mathrm{~mL}$ with water. Adjust the pH to 5 with $1: 1 \mathrm{NH}_{4} \mathrm{OH}$. 


\section{DETERMINATION}

1. Add 3-4 $\mathrm{g}$ of $\left(\mathrm{NH}_{4}\right)_{2} \mathrm{SO}_{4}$ with stirring. Digest for $12 \mathrm{~h}$. Cool and filter by gravity on a $9 \mathrm{~cm}$ Whatman No. 42 filter paper. Wash with $0.5 \% \mathrm{H}_{2} \mathrm{SO}_{4}$. Discard the filtrate and washings.

2. Transfer the precipitate and filter to a platinum dish. Dry and ignite at $900^{\circ} \mathrm{C}$.

3. Cool and add $2 \mathrm{~mL}$ of $1: 1 \mathrm{H}_{2} \mathrm{SO}_{4}$ and $5 \mathrm{~mL}$ of $\mathrm{HF}$. Evaporate on a sand bath until $\mathrm{SO}_{3}$ fumes appear.

4. Transfer the solution to a $40 \mathrm{~mL}$, heavy walled centrifuge tube with $30 \mathrm{~mL}$ of $\mathrm{H}_{2} \mathrm{O}$ and digest for $12 \mathrm{~h}$. Centrifuge and discard the supernate.

5. Wash the precipitate with $10 \mathrm{~mL}$ of water. Centrifuge and discard the washings.

6. Slurry the precipitate with water and filter on a tared Whatman No. 42 filter paper.

7. Dry in an oven at about $150^{\circ} \mathrm{C}$ for at least $2 \mathrm{~h}$.

8. Cool, weigh, and mount on a ring and disc with an $\alpha$ phosphor and Mylar.

9. Store for 30 days and count on an $\alpha$-scintillation counter.

\section{DATA PROCESSING AND ANAIYSES}

The ${ }^{226} \mathrm{Ra}$ disintegration rate is obtained from the counting rate of the equilibrated radium fraction through the following calculation:

$$
\mathrm{Bq}=\mathrm{R}_{\mathrm{s}} \mathrm{YTCE}
$$

where $R_{s}$ is the net counting rate of the sample, $Y$ is the recovery factor, $T$ is the selfabsorption correction, $\mathrm{E}$ is the counter efficiency factor, and $\mathrm{C}$ is a theoretical conversion from total $\alpha$ activity to ${ }^{226} \mathrm{Ra} \alpha$ activity. 
Chemical recovery, $\mathrm{Y}$, is obtained by weighing the final $\mathrm{Ba}$ sulfate precipitate. The calculation of the recovery factor is:

$$
Y=\frac{x}{(w-t) f}
$$

where $\mathrm{x}$ is the weight of barium added as carrier, $\mathrm{w}$ is the total weight of the final precipitate and filter paper, $t$ is the weight of the filter paper, and $f$ is the gravimetric factor which equals $0.588 \mathrm{mg}$ barium per $\mathrm{mg}$ of barium sulfate.

Self-absorption correction, $T$, is used to normalize the self-absorption of the ${ }^{226} \mathrm{Ra}$, ${ }^{222} \mathrm{Rn},{ }^{218} \mathrm{Po}$, and ${ }^{214} \mathrm{Po} \alpha$ activities in samples and standards to a common thickness $\left(\mathrm{mg} \mathrm{cm}{ }^{-2}\right)$. T is obtained by counting representative $\alpha$ emitters over the energy range of 4.8-8.8 MeV in precipitates of varying sample thicknesses. The correction is taken as the ratio of counting rates at an arbitrary minimum thickness to other thicknesses over the range of probable sample recoveries. Figure 1 is a typical composite plot of the correction obtained for ${ }^{230} \mathrm{Th},{ }^{212} \mathrm{~Pb}$, and ${ }^{226} \mathrm{Ra}$ activities for varying thicknesses of their oxalate, chromate, and sulfate derivatives, respectively.

Theoretical activity conversion factor, $\mathrm{C}$, equals 0.25 after 30 days of buildup, when the three $\alpha$-emitting progeny are in secular equilibrium with ${ }^{226} \mathrm{Ra}$. However, the ratio of ${ }^{226} \mathrm{Ra}$ to total $\alpha$ activity at any time after the separation of radium may be calculated from the Bateman equation for buildup of ${ }^{222} \mathrm{Rn}$.

Efficiency factor, $\mathrm{E}$, is determined by $\alpha$ counting a known quantity of ${ }^{226} \mathrm{Ra}$ under sample conditions. The correction is taken as the ratio of the theoretical activity to the observed counting rate. The calculation is expressed as:

$$
E=\frac{A}{R_{s}^{\prime} T^{\prime} C^{\prime}}
$$

where $A$ is the theoretical activity of the standard, and $R_{s}^{\prime}$ is the net counting rate of the standard, $T^{\prime}$ is the self-absorption correction for the standard, and $C^{\prime}$ is the theoretical ratio of ${ }^{226} \mathrm{Ra}$ to total $\alpha$ activity in the standard. 
An accompanying computational data sheet illustrates a procedure for routine calculation of ${ }^{226} \mathrm{Ra}$ activity rates. Experimental data are tabulated as they are derived and the calculations are performed on a step-by-step basis.

\section{LOWER LIMIT OF DETECTION (LLD)*}

\begin{tabular}{lll} 
Counter efficiency & $(\%)$ & 50 \\
Counter background & $(\mathrm{cps})$ & $1.67 \times 10^{-5}$ \\
Yield & $(\%)$ & 85 \\
Blank & $(\mathrm{cps})$ & $1.67 \times 10^{3}$ \\
LLD $(400 \mathrm{~min})$ & $(\mathrm{mBq})$ & 3 \\
LLD $(1000 \mathrm{~min})$ & $(\mathrm{mBq})$ & 2 \\
& & \\
\hline
\end{tabular}

*Reagent blank must be analyzed with each set of samples. 


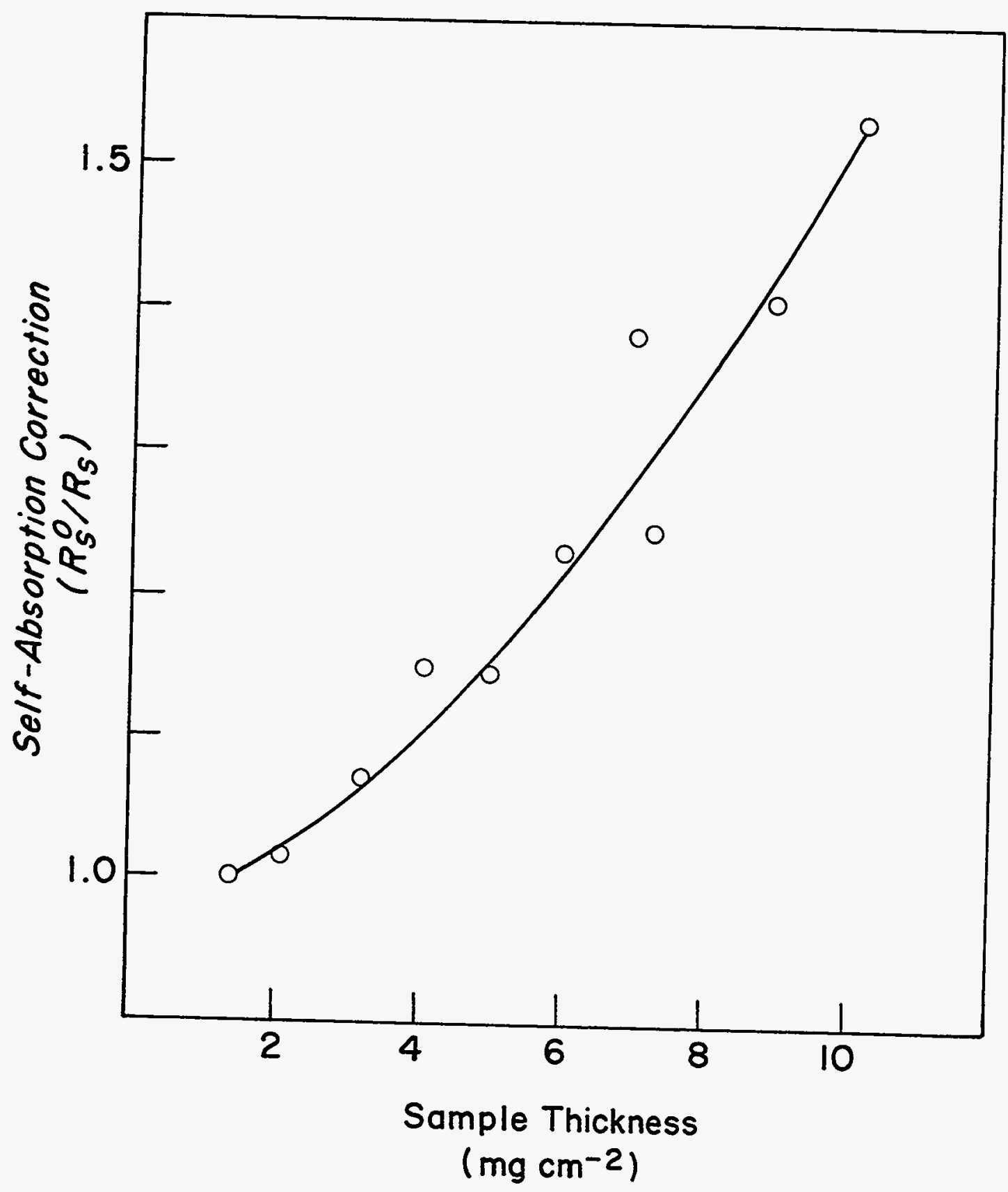

Figure 1. Self-absorption for $\alpha$ activities (4.8-8.9 MeV). 
Date

Sample Number

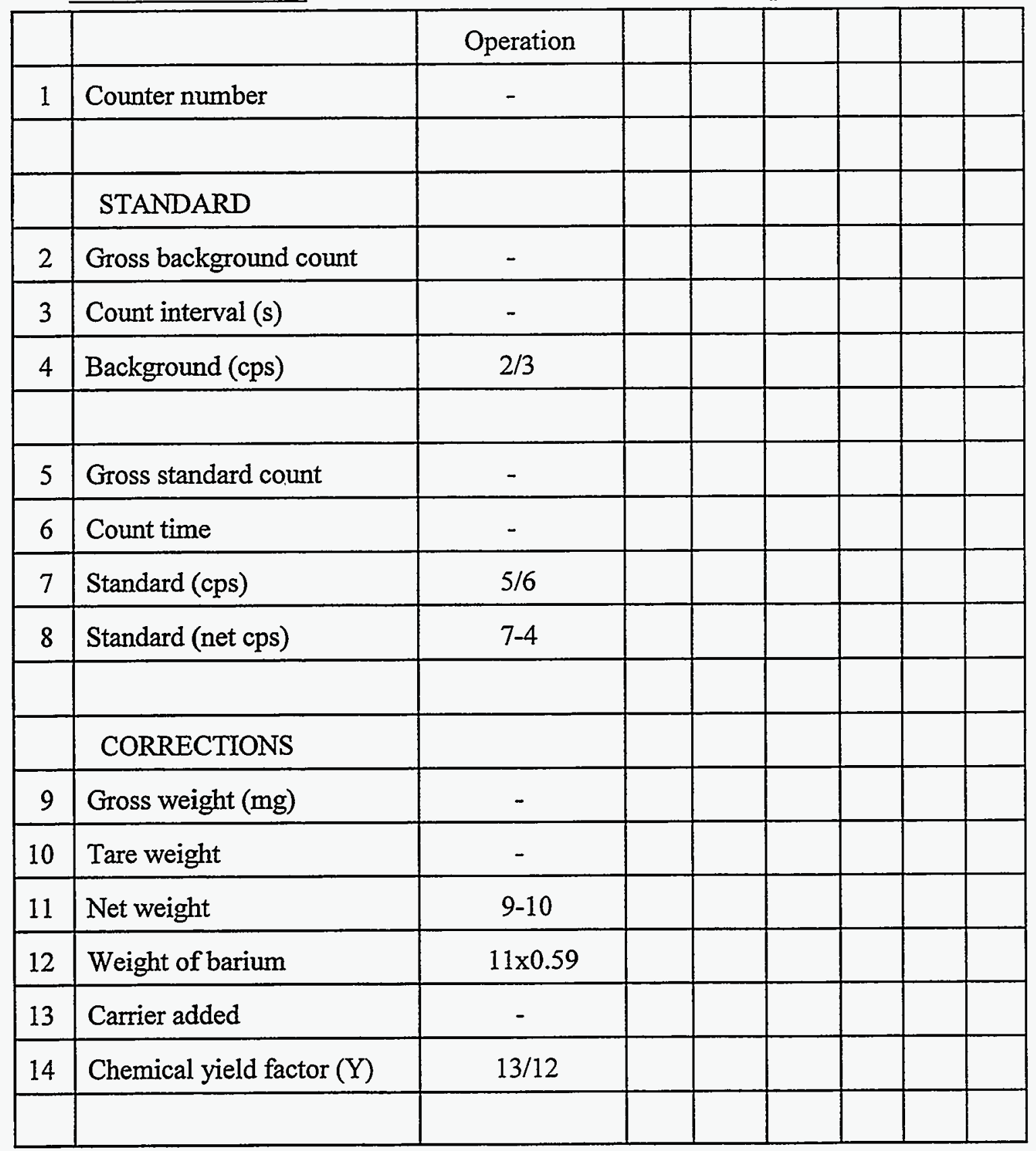




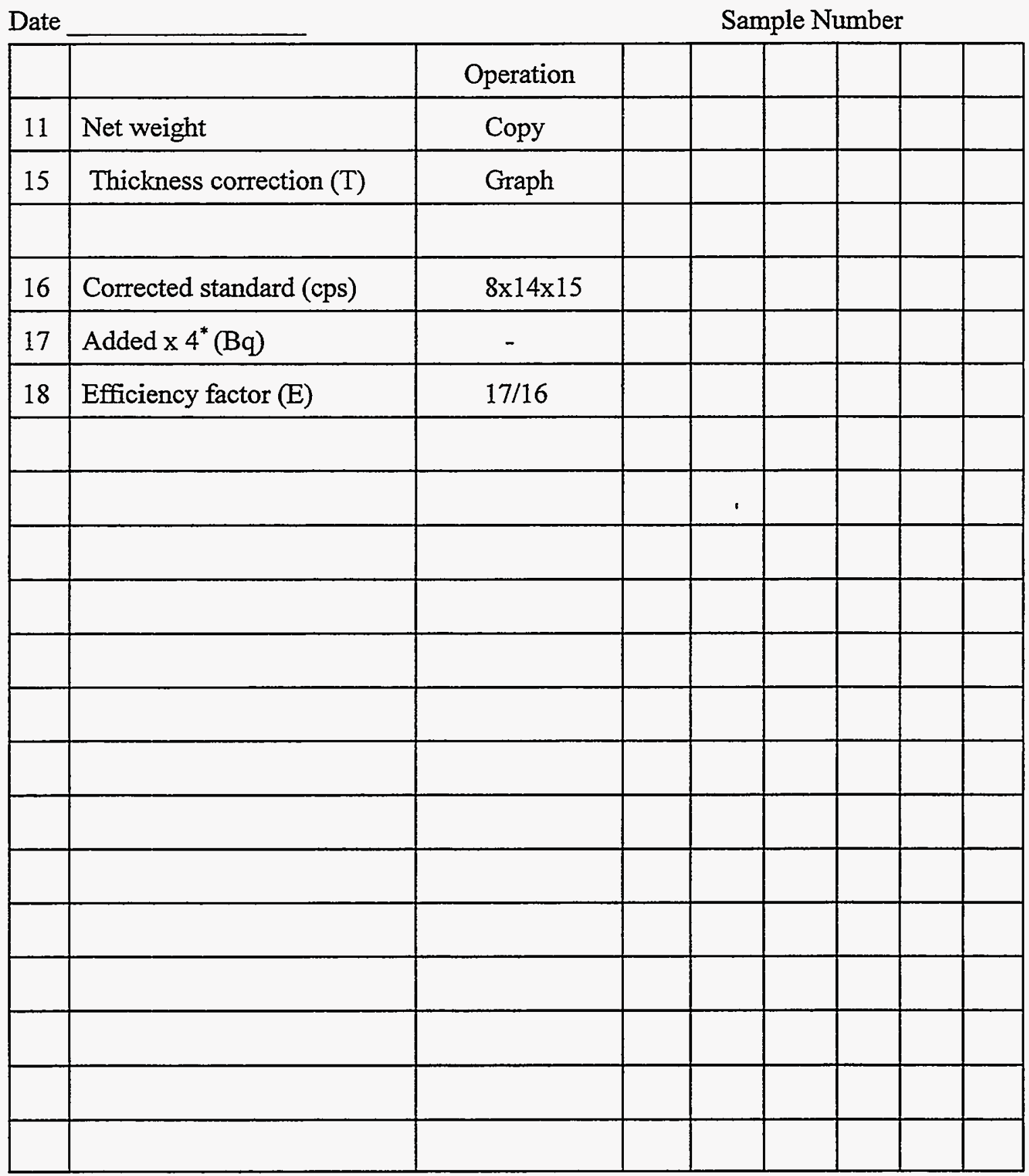

*To include progenys 


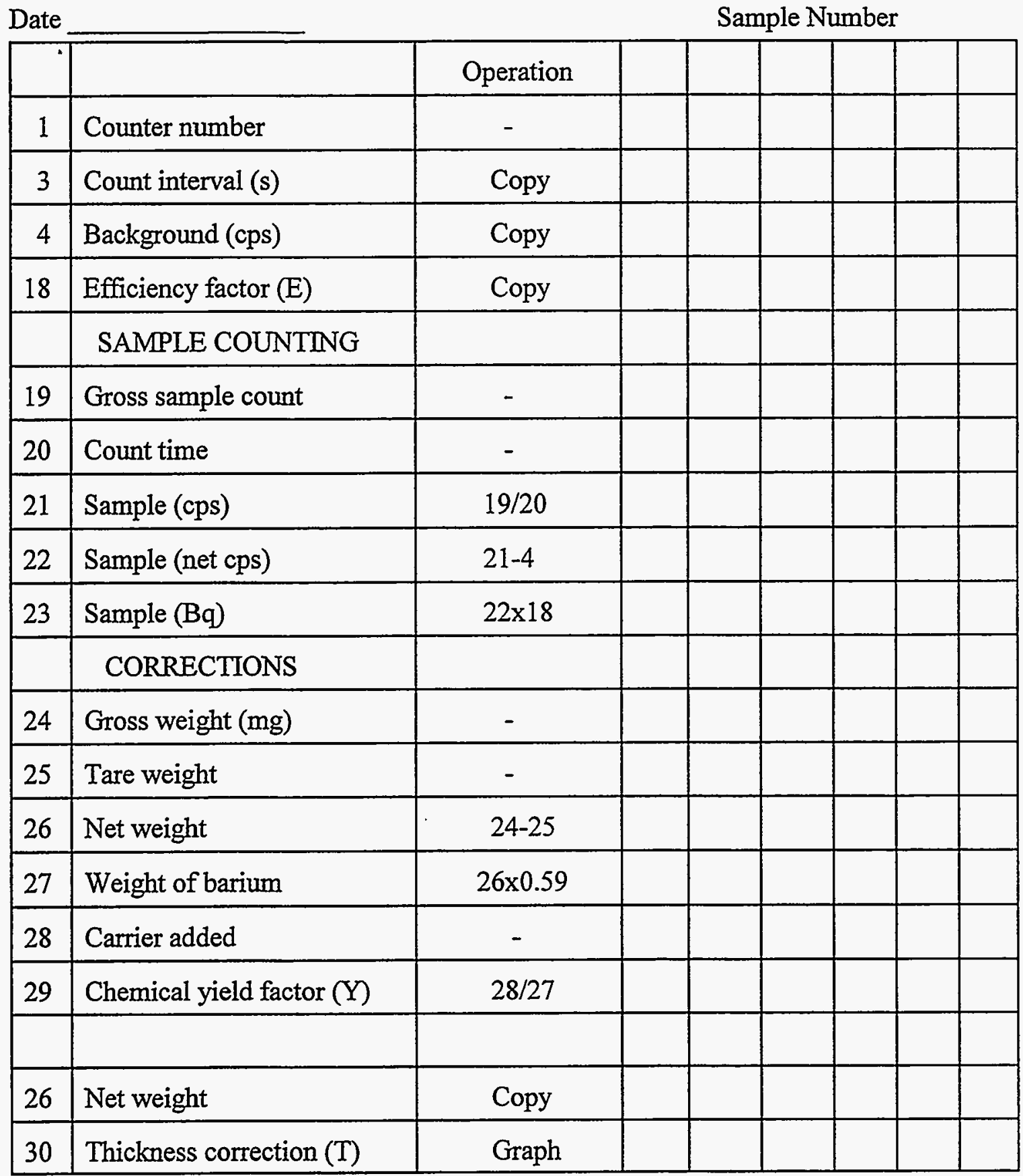


Date

Sample Number

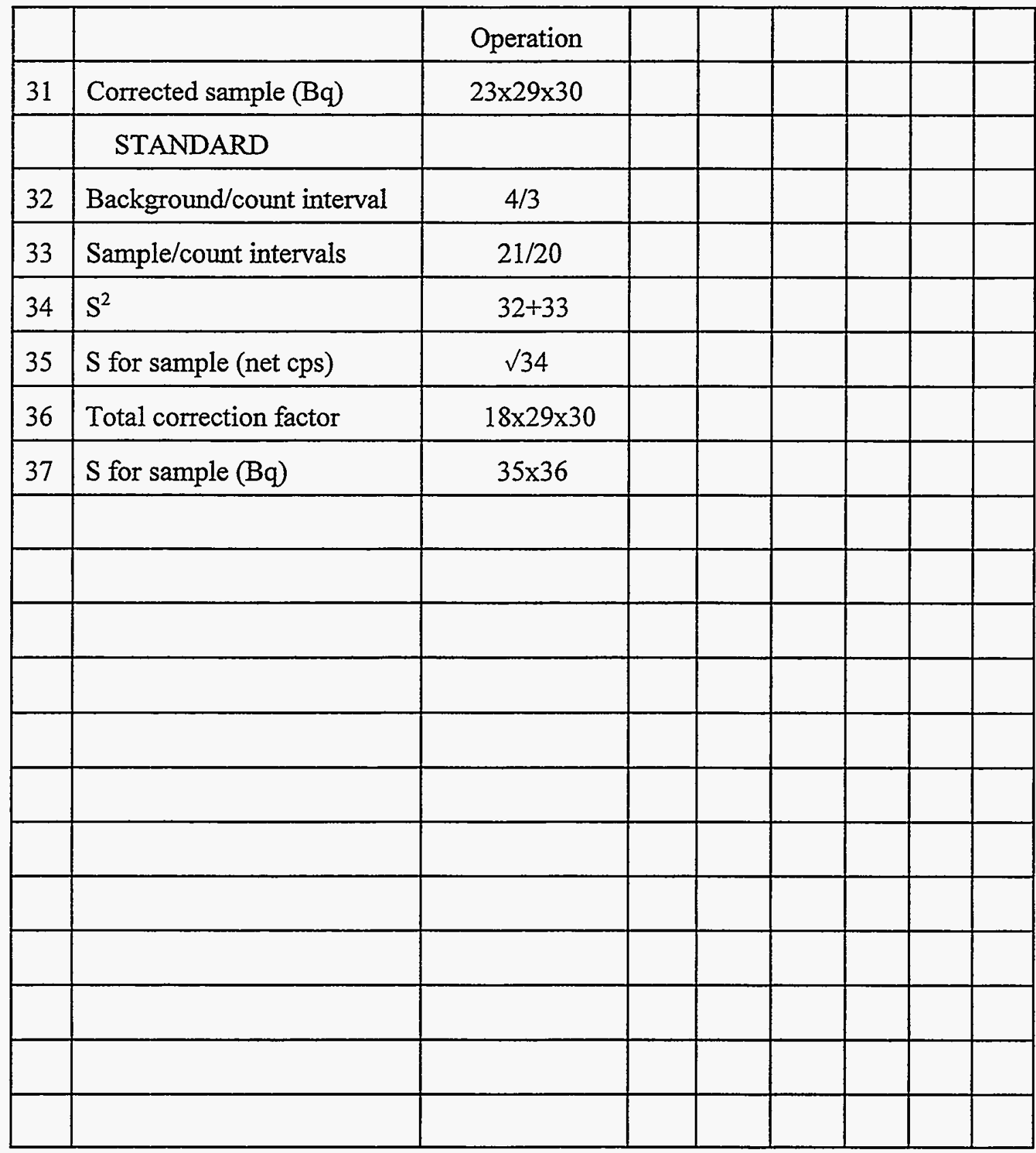


Section 4.5.4, Vol. I

Rev. 0

HASL-300, 28th Edition

February 1997

Radon

Environmental Measurements Laboratory U.S. Department of Energy 


\begin{abstract}
APPLICATION
Procedures are presented which describe EML's method of sampling, counting, and calculating ${ }^{222} \mathrm{Rn}$ concentration in air and breath samples. When radium is present in the body, the gaseous progeny, ${ }^{222} \mathrm{Rn}\left(\mathrm{t}_{1 / 2}=3.825 \mathrm{~d}\right)$, will collect in the lungs and will be eliminated with exhaled breath.
\end{abstract}

One liter glass sampling flasks are provided to field personnel by EML for collecting breath or atmospheric ${ }^{222} \mathrm{Rn}$ samples. The ${ }^{222} \mathrm{Rn}$ sample is transferred to a pulse type ionization chamber, and after it is allowed to come into equilibrium with its progeny products, the sample is $\alpha$ counted. Two of the progeny, ${ }^{218} \mathrm{Po}$ and ${ }^{214} \mathrm{Po}$, are $\alpha$ emitters and contribute to the total count.

\title{
SPECIAL APPARATUS
}

\section{A. Sampling.}

1. 1-L glass flasks with two large bore stopcocks per flask.

2. Tank of compressed, aged air with two-stage regulator.

3. Face mask - No. CS-6772 inhalator, modified to block the emergency intake and with the outlet modified to accept $9.5 \mathrm{~mm} \mathrm{ID}$ rubber tubing (Mine Safety Appliance Co., Pittsburgh, PA). 
4. Demand regulator - No. CS-46516 single stage or equivalent (Mine Safety Appliance Co., Pittsburgh, PA).

\section{B. Analysis.}

1. Platinum black catalyst (Baker and Co., Deoxo Units).

2. Drying tube with Drierite.

3. Flame arresters.

4. Capillary orifice.

5. Vacuum pump.

6. Tank of $\mathrm{H}_{2}$ with two-stage regulator.

7. Tank of forming gas $\left(85 \% \mathrm{~N}_{2}, 15 \% \mathrm{H}_{2}\right)$ with two-stage regulator.

8. Sample introduction system including valves and piping.

9. Pulse type ionization chamber and associated electronic equipment.

\section{SAMPLE COLLECTION}

\section{A. Radon in breath*}

1. Set up the equipment as described in the above reference using $9.5 \mathrm{~mm}$ ( $3 / 8 \mathrm{in})$ rubber tubing on the inhalator outlet. Do not attach to sampling flask.

2. Clean facepiece with cotton and alcohol.

3. Set air pressure on two-stage regulator to $4.5 \mathrm{~kg}(10 \mathrm{lb})$.

- Taken from Harley et al, 1951. 
4. Have the subject hold the facepiece in place while you check for leaks, particularly around the bridge of the nose.

5. Have the subject breathe with the respirator for $5 \mathrm{~min}$ to flush the environmental air from his or her lungs. (This should be regular breathing. Do not ask for deep breaths.)

6. While the subject continues regular breathing, attach the sampling flask (with both stopcocks open) for a 1-min period, remove, and close stopcocks.

7. Repeat Step 6 for a duplicate sample.

B. Radon in the atmosphere.

1. Open both flask stopcocks.

2. Connect about $0.6 \mathrm{~m}$ of $9.5 \mathrm{~mm}$ ( $3 / 8 \mathrm{in})$ rubber tubing to one stopcock.

3. Inhale through the tubing and flask 20 times. Do not exhale through the flask. If convenient, a suction pump may be used.

4. Close both stopcocks.

\section{MEASUREMENT EQUIPMENT PREPARATION}

\section{A. Sample oxidation.}

Before transfer to the counting system, enough $\mathrm{H}_{2}$ is added to the sample flask to completely remove $\mathrm{O}_{2}$ (as $\mathrm{H}_{2} \mathrm{O}$ ) in the platinum catalyst. Because $\mathrm{O}_{2}$ acts to quench the ionization produced by each $\alpha$ disintegration, even small amounts of it in the chamber will seriously affect the counting rate of a sample. Environmental air contains about $20 \%$ $\mathrm{O}_{2}$ and the addition of $40 \mathrm{kPa}(6 \mathrm{psi})$ of $\mathrm{H}_{2}$ is theoretically sufficient for all samples. It has been our practice to add an excess of $\mathrm{H}_{2}$ and therefore $70 \mathrm{kPa}(10 \mathrm{psi})$ is usually added to each sample. 


\section{B. Flame arresters.}

The removal of $\mathrm{O}_{2}$ from the sample takes place in the platinum black catalyst where $\mathrm{O}_{2}$ and $\mathrm{H}_{2}$ combine to form $\mathrm{H}_{2} \mathrm{O}$. This combustion reaction generates a considerable amount of heat, and if allowed to strike back, the sample flask may explode. To prevent such explosions, flame arresters are placed between the catalyst and the sample. The flame arresters consist of a fine mesh copper wire screen and act to dissipate the heat of the reaction.

\section{Capillary orifice.}

A capillary orifice is placed in the line after the catalyst. This slows the passage of gas through the catalyst and insures complete combustion.

\section{Drierite tube.}

Water formed in the $\mathrm{O}_{2}$ removal is collected in a Drierite tube. The Drierite is kept free of $\mathrm{H}_{2} \mathrm{O}$ by evacuating the external piping of the system continuously when not transferring the samples.

\section{E. Counting apparatus.}

The ${ }^{222} \mathrm{Rn}$ counting apparatus consists of a sample introduction system, ionization chamber, preamplifier, amplifier, and count registering device. The ionization chamber counts almost $100 \%$ of the $\alpha$ disintegrations of ${ }^{222} \mathrm{Rn}$ and about $50 \%$ of the disintegrations of its progeny. Each chamber plus its sample introduction system has a capacity of $2 \mathrm{~L}$ and the chamber is operated at a potential of $1000 \mathrm{~V}$. A mixture of $\mathrm{H}_{2}$ $(15 \%)$ and $\mathrm{N}_{2}(85 \%)$ is used as a counting gas. The chambers are constructed of a specially selected, electropolished stainless steel and have a background count of about 10 counts $h^{-1}$ and an efficiency of about 6215 counts $h^{-1}$ for $1 \mathrm{~Bq}$ of ${ }^{222} \mathrm{Rn}$.

A block diagram of the counting system and external apparatus is shown below. 


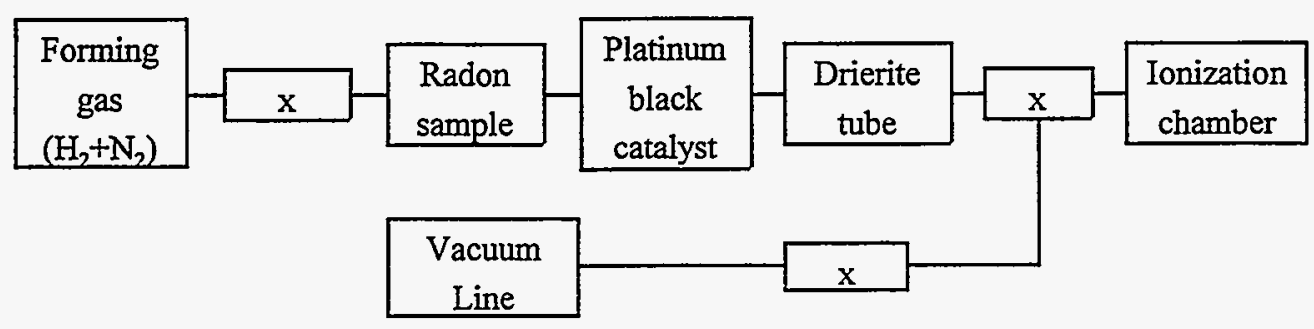

$\mathrm{x}$ indicates valve or stopcock.

The pulses from the ionization chamber are fed to an EML-built preamplifier, amplifier, and control unit. Two Tattletale Clever counters are interfaced to a PC.

\section{DETERMINATION}

1. Connect sample flask to $\mathrm{H}_{2}$ tank with $9.5 \mathrm{~mm}$ (3/8 in) rubber tubing.

2. Raise gauge pressure to $70 \mathrm{kPa}(10 \mathrm{psi})$ gauge.

3. Open flask stopcock to tank to admit $\mathrm{H}_{2}$ to the flask.

4. Close stopcock and tank valve.

5. Raise the forming gas line pressure to $70 \mathrm{kPa}(10 \mathrm{psi})$ gauge and bleed the line and rubber tubing leading to sample inlet.

6. Connect one flask stopcock to the forming gas line with the above rubber tubing, the other to the counting system with another section of $9.5 \mathrm{~mm}$ ( $3 / 8 \mathrm{in})$ rubber tubing.

7. Open the ionization chamber and the vacuum line valve to evacuate the counting chamber, external piping, and the rubber tubing between the counting system and the flask.

8. Close the vacuum line valve when system reaches minus $10.1 \mathrm{kPa}$ of $\mathrm{Hg}$ gauge pressure. 
9. Open the flask stopcock to allow the sample to enter the counting system. Allow pressure equilibration (indicated by pressure gauge).

10. Check platinum catalyst by touch to assure that combination of $\mathrm{H}_{2}$ and $\mathrm{O}_{2}$ has occurred.

11. Close the flask stopcock to the counting system.

12. Open the stopcock to the forming gas line. Allow forming gas to come to pressure in the flask.

13. Repeat Steps 5 and 7 until the gauge indicates atmospheric pressure.

14. Open the stopcock to the forming gas line, then attach the stopcock to the counting system. Allow forming gas to flow until the gauge pressure reaches $35 \mathrm{kPa}$ (5 psi).

15. Close all valves and remove flask and tubing from system.

16. Turn on ionization chamber high voltage.

17. Count the sample for at least $14 \mathrm{~h}$.

\section{DATA PROCESSING AND ANALYSES}

1. Discard the first $5 \mathrm{~h}$ of counting data (equilibration period for ${ }^{222} \mathrm{Rn}$ and progeny).

2. Determine the gross count over the remaining counting period.

3. Calculate net counts per hour per sample by determining gross sample counts per hour and subtracting background counts per hour.

4. Divide net counts per hour by the chamber standardization value of net counts per hour per $\mathrm{Bq}$ of ${ }^{222} \mathrm{Rn}$. 
5. Using the midpoint of the counting interval as the time of counting, extrapolate the value obtained to the time of sampling. Figure 1 may be used for this calculation.

\section{STANDARDIZATION}

Each unit is standardized several times a year with ${ }^{222} \mathrm{Rn}$ from a radium solution obtained from the National Institute of Standard and Technology (NIST). This solution is diluted and split into aliquots which are placed in ${ }^{222} \mathrm{Rn}$ bubblers (see Specification 7.8). The ${ }^{222} \mathrm{Rn}$ is allowed to build up for a known period before the standard is used.

The ${ }^{222} \mathrm{Rn}$ standard is transferred to the chamber by emanation. The bubbler is first attached to the external feed system. When the ionization chamber and external system are evacuated, the vacuum line is shut off from the system and the bubbler outlet stopcock opened. The inlet stopcock is then opened and forming gas flushes the ${ }^{222} \mathrm{Rn}$ into the chamber until the system is brought to atmospheric pressure.

At equilibrium, there are three $\alpha$ disintegrations per $^{222} \mathrm{Rn}$ disintegration, however, two of these are from the particulate $\alpha$-emitting progeny. Since these $\alpha$ disintegrations deposit on the walls of the ionization chamber, they are counted with a maximum efficiency of $50 \%$. One becquerel of ${ }^{222} \mathrm{Rn}$ in the ionization chamber thus has a theoretical counting rate of 7190 counts $\mathrm{h}^{-1}$. Actually, the ionization chambers in this Laboratory yield a counting rate of 6215 counts h $\mathrm{h}^{-1} \mathrm{~Bq}^{-1}$ of ${ }^{222} \mathrm{Rn}$ in equilibrium with its progeny or an overall efficiency of $86 \%$.

\section{LOWER LIMT OF DETECTION (LLD)}

\begin{tabular}{lcc}
\hline & & \\
Counter Efficiency & $(\%)$ & 57.5 \\
Counter Background & $(\mathrm{cps})$ & 0.0028 \\
Yield & $(\%)$ & - \\
Blank & $(\mathrm{cps})$ & - \\
LLD (400 min) & (Bq) & 0.01 \\
LLD (1000 min) & $(\mathrm{Bq})$ & 0.07 \\
\hline
\end{tabular}




\section{REFERENCE}

Harley, J. H., E. Jetter and M. Eisenbud

"A Method of Obtaining Reproducible Breath Radon Samples"

Arch. Ind. Hyg. Occ. Med., 4, 1-9 (1951) 


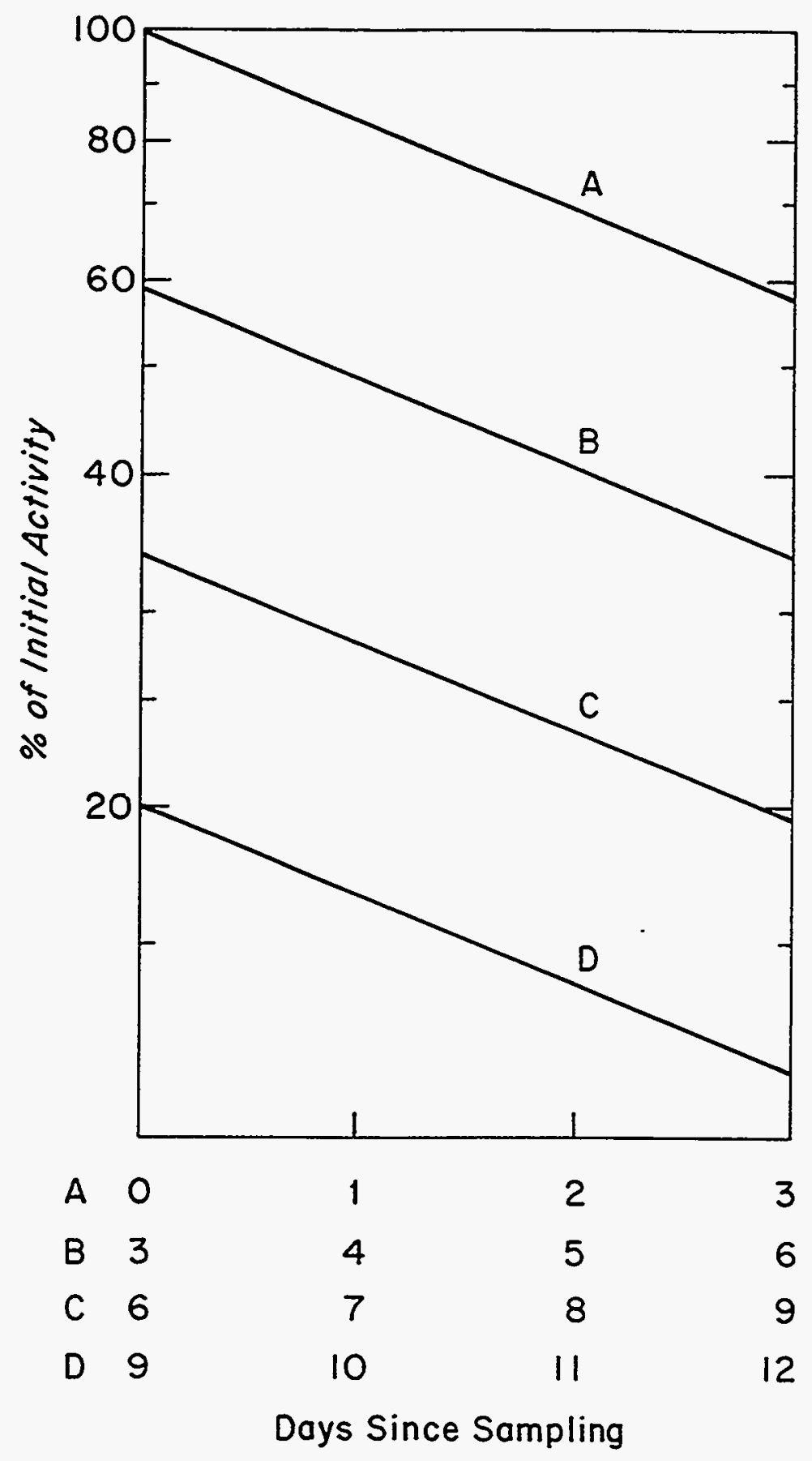

Figure 1. Decay correction for radon from the midpoint of the counting interval to collection time. 


\section{Strontium}

Environmental Measurements Laboratory U.S. Department of Energy 
$S r=01-R C$

STRONTIUM-89

(see Volume II) 
$S r-02-R C$

STRONTIUM-90

(see Volume II) 


\title{
STRONTIUM-90 IN ENVIRONMENTAL MATRICES
}

Contact Person : Marie Lawrence

\begin{abstract}
APPLICATION
This procedure is applicable to the preparation, separation, and analysis of vegetation, water, air filters and soil.

Strontium is separated from calcium, other fission products and natural radioactive elements. Fuming $\mathrm{HNO}_{3}$ separations remove the calcium and most of the other interfering ions. Radium, lead and barium are removed with barium chromate. Traces of other fission products are scavenged with iron hydroxide. After the ${ }^{90} \mathrm{Sr}+{ }^{90} \mathrm{Y}$ equilibrium has been attained, the ${ }^{90} \mathrm{Y}$ is precipitated as the hydroxide and converted to the oxalate for counting on a low-background gas proportional beta counter. Chemical yield is determined with ${ }^{85} \mathrm{Sr}$ tracer by counting in a gamma well detector.
\end{abstract}

\section{SPECIAL APPARATUS}

1. Teflon filter holder or filter funnel and sample mount - see Specification 7.12.

2. Rings and discs - see Specification 7.2.

3. Magnetic stirrers with Teflon-coated magnet bars.

4. Mylar film - see Specification 7.3.

5. Glass fiber filters - see Specification 7.8.

6. Fisher filtrator, Fisher Chemical Company, Pittsburgh, PA 15219-4785. 
7. Polyethylene reference bottles, $30-\mathrm{mL}$ narrow mouth to fit in a gamma well detector.

\section{SPECIAL REAGENTS}

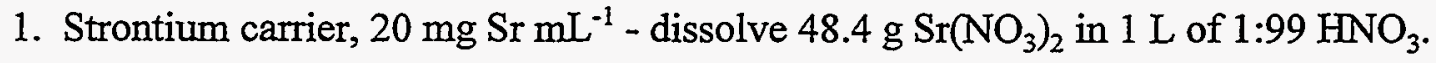

2. Yttrium carrier, $10 \mathrm{mg} \mathrm{Y} \mathrm{mL}^{-1}$ - dissolve $12.7 \mathrm{~g}$ of highest purity $\mathrm{Y}_{2} \mathrm{O}_{3}$ in a minimal amount of $\mathrm{HNO}_{3}$; use heat if necessary. Filter, if necessary, and add water to make $1 \mathrm{~L}$ of solution. See the APPENDIX for the yttrium carrier counting check.

3. Iron carrier, $5 \mathrm{mg} \mathrm{Fe} \mathrm{mL}^{-1}$ - dissolve $5 \mathrm{~g} \mathrm{Fe}$ wire in $1: 1 \mathrm{HCl}$ and dilute to $1 \mathrm{~L}$ with $1: 99 \mathrm{HCl}$, or dissolve $34.7 \mathrm{~g} \mathrm{Fe}\left(\mathrm{NO}_{3}\right)_{3} \cdot 9 \mathrm{H}_{2} \mathrm{O}$ in $1 \mathrm{~L}$ of $1: 99 \mathrm{HNO}_{3}$.

4. Barium carrier, $10 \mathrm{mg} \mathrm{Ba} \mathrm{mL}^{-1}$ - dissolve $9.5 \mathrm{~g} \mathrm{Ba}\left(\mathrm{NO}_{3}\right)_{2}$ in water and dilute to $0.5 \mathrm{~L}$.

5. Barium buffer solution - $500 \mathrm{~mL} 6 \mathrm{M}$ acetic acid (glacial HOAC) plus $1 \mathrm{~L}$ of $6 \underline{\mathrm{M}} \mathrm{NH}_{4} \mathrm{OAc}$ plus $0.5 \mathrm{~L} \mathrm{Ba}$ carrier $\left(10 \mathrm{mg} \mathrm{mL}^{-1}\right)$.

6. Calcium carrier, $200 \mathrm{mg} \mathrm{Ca} \mathrm{mL}^{-1}$ - dissolve $500 \mathrm{~g}$ calcium carbonate $\left(\mathrm{CaCO}_{3}\right)$ in a minimum of $\mathrm{HCl}$ and dilute to $1 \mathrm{~L}$ with water.

7. ${ }^{85} \mathrm{Sr}$ tracer, about $7 \times 10^{5} \mathrm{~Bq} \mathrm{~L}^{-1}$, in a well counter, this tracer should provide about 150 counts $\mathrm{sec}^{-1} \mathrm{~mL}^{-1}$.

8. Sodium carbonate solution, $1 \mathrm{M}$ - dissolve $106 \mathrm{~g} \mathrm{Na}_{2} \mathrm{CO}_{3}$ in $\mathrm{H}_{2} \mathrm{O}$ and dilute to $1 \mathrm{~L}$.

9. Sodium carbonate solution, $2 \mathrm{M}$ - dissolve $212 \mathrm{~g} \mathrm{Na}_{2} \mathrm{CO}_{3}$ in $\mathrm{H}_{2} \mathrm{O}$ and dilute to $1 \mathrm{~L}$.

10. Sodium chromate solution, $0.3 \mathrm{M}$ - dissolve $50 \mathrm{~g} \mathrm{Na}_{2} \mathrm{CrO}_{4}$ in $\mathrm{H}_{2} \mathrm{O}$ and dilute to $1 \mathrm{~L}$.

11. $50 \%$ sodium hydroxide solution - slowly dissolve $500 \mathrm{~g} \mathrm{NaOH}$ in $500 \mathrm{~mL}$ of $\mathrm{H}_{2} \mathrm{O}$ in a plastic liter bottle immersed in a beaker of ice water. 


\section{SAMPLE PREPARATION}

\section{A. Water}

1. Transfer an aliquot of sample water to an appropriate sized beaker (use deionized water for the blank).

2. Add $1 \mathrm{~mL}(20 \mathrm{mg})$ strontium carrier to the blank and the sample.

3. Add exactly $1.00 \mathrm{~mL}$ of ${ }^{85} \mathrm{Sr}$ tracer to three $30-\mathrm{mL}$ plastic bottles, the blank and the sample.

4. Fill the plastic bottles with equal volumes of $1 \underline{\mathrm{M}} \mathrm{HCl}$. Set bottles aside. They will serve as references when determining the strontium yield.

5. Evaporate the samples to dryness. Add $10-\mathrm{mL}$ volumes of concentrated nitric acid to the dried residue, and evaporate repeatedly to remove any trace of $\mathrm{HCl}$ due to the reagents added.

6. Dissolve the final residue in $8 \mathrm{M} \mathrm{HNO}_{3}$. If the sample is not in a $400-\mathrm{mL}$ beaker, quantitatively transfer the sample with water to a $400-\mathrm{mL}$ beaker containing a Teflon-coated magnetic stirring bar. Dilute the sample to $200 \mathrm{~mL}$ with additional water.

7. Place the beaker on a magnetic stirrer/hot plate and stir. Adjust the $\mathrm{pH}$ to 5-6 with $50 \% \mathrm{NaOH}$. While continuing to stir, add $15 \mathrm{~mL}$ of $2 \mathrm{M} \mathrm{Na}_{2} \mathrm{CO}_{3}$. Heat the sample to just below boiling and stir for $30 \mathrm{~min}$.

8. Remove the sample from the hot plate and allow the precipitate to settle overnight.

9. Place a small glass fiber filter backed with a Whatman No. 42 filter paper of the same size into a Büchner funnel. Mount the funnel on a $500 \mathrm{~mL}$ filter flask.

10. Filter the sample by vacuum through the funnel. Wash the carbonates retained on the filter with $1 \mathrm{M} \mathrm{Na}_{2} \mathrm{CO}_{3}$ solution. Discard the filtrate. 
11. Resease the vacuum and transfer the funnel to a clean filter flask. Apply the vacuum. Dissolve the carbonates on the filter with hot $8 \mathrm{M} \mathrm{HNO}_{3}$. Wash the filter with water.

12. Transfer the filtrate to a $400 \mathrm{~mL}$ beaker, rinsing with $8 \mathrm{M} \mathrm{HNO}_{3}$. Evaporate the solution to dryness.

\section{Proceed to Determination, Fuming $\mathrm{HNO}_{3}$ Separation}

\section{B. Air Filters}

1. Place the air filter in a 400-mL beaker (use a dry beaker for the blank).

2. Add exactly $1.00 \mathrm{~mL}$ of ${ }^{85} \mathrm{Sr}$ to three $30-\mathrm{mL}$ plastic reference bottles, the blank and the sample.

3. Fill the plastic bottles with equal volumes of $1 \mathrm{M} \mathrm{HCl}$. Set bottles aside. (The same three reference bottles may be used for water and air filters if the analyses are done simultaneously.)

4. Add $20 \mathrm{mg}(1 \mathrm{~mL})$ strontium carrier to the blank and the sample.

5. Add $150 \mathrm{~mL} \mathrm{HNO}_{3}$ and $50 \mathrm{~mL} \mathrm{HCl}$. Reflux on a hot plate until clear and colorless. Evaporate to a volume of $\sim 100 \mathrm{~mL}$.

6. Add $100 \mathrm{~mL}$ of deionized water. Cool the sample to room temperature. Filter by gravity through a Whatman No. 42 filter. Wash the filter with $8 \mathrm{M} \mathrm{HNO}_{3}$.

7. Evaporate the filtrate to dryness. Add $20 \mathrm{~mL}$ volumes of concentrated nitric acid to the dried residue, and evaporate repeatedly to remove $\mathrm{HCl}$. Continue with Step 6, Section A, Water. 


\section{Soil (NaOH-HCI method) - see Note 1}

1. Weigh out enough soil to generate an activity at least 10 times background (ideally 100 times) into an appropriate sized beaker containing a Teflon-coated magnetic stirring bar (see chart below). Add water to about a quarter of the beaker's volume and add 5-10 $\mathrm{mL}(100-200 \mathrm{mg})$ strontium carrier solution. Place the beaker on a magnetic stirrer.

2. To each of three $30-\mathrm{mL}$ plastic reference bottles and to the sample add $1.00 \mathrm{~mL}$ of ${ }^{85} \mathrm{Sr}$ tracer solution. Fill the reference bottles to the same level with $1 \mathrm{M} \mathrm{HCl}$.

3. Stir the sample. While continuing to stir, add a sufficient amount of $50 \% \mathrm{NaOH}$ to make the solution $1 \mathrm{~N} \mathrm{NaOH}$. (see chart below)

4. Cover with a watch glass and stir for $10 \mathrm{~min}$. Reflux overnight on a warm hot plate.

5. Remove the beaker from the hot plate and allow to cool. While stirring, cautiously add $\mathrm{HCl}, 1 \mathrm{~mL}$ at a time until the reaction slows, to make the solution $6 \mathrm{M}$ acidic (see chart below). If analyzing highly calcareous soils, an additional quantity of $\mathrm{HCl}$ should be added to replace the acid required to decompose the carbonates. If necessary, add a few drops of n-octyl alcohol to reduce foaming.

$\begin{array}{ccccccc}\begin{array}{c}\text { Activity } \\ \left(\mathrm{Bq} \mathrm{kg}^{-1}\right)\end{array} & \begin{array}{c}\text { Sample size } \\ (\mathrm{g})\end{array} & \begin{array}{c}\text { Beaker size } \\ (\mathrm{mL})\end{array} & \begin{array}{c}\text { Water } \\ (\mathrm{mL})\end{array} & \begin{array}{c}\text { Carrier } \\ (\mathrm{mL})\end{array} & \begin{array}{c}50 \% \mathrm{NaOH} \\ (\mathrm{mL})\end{array} & \begin{array}{c}\mathrm{HCl} \\ (\mathrm{mL})\end{array} \\ \sim 500 & 5-10 & 250 & 70 & 5 & 4 & 90 \\ \sim 100 & 15-20 & 400 & 100 & 5 & 6 & 130 \\ \sim 50 & 30-40 & 800 & 200 & 5 & 11 & 260 \\ \sim 10 & 100 & 1000 & 250 & 5 & 14 & 320\end{array}$

6. Digest the sample overnight on a warm hot plate. Remove the beaker from the hot plate and cool. 
7. Filter the sample under vacuum using a Whatman No. 42 filter paper backed by a glass fiber filter. Wash with approximately $100 \mathrm{~mL}$ hot $6 \mathrm{M} \mathrm{HCl}$, followed by $60 \mathrm{~mL}$ hot $\mathrm{H}_{2} \mathrm{O}$.

8. Turn off the vacuum. Return the soil residue and the filter paper to the original beaker.

9. Quantitatively transfer the filtrate and washes to an appropriate sized beaker, rinsing with water, and place on a warm hot plate to reduce the volume.

10. Remove the filtrate from the hot plate. Add 20-50 $\mathrm{mL}$ of $\mathrm{HNO}_{3}$. Cover with a watch glass and place back on the hot plate. Turn the heat up to high. Continue adding $\mathrm{HNO}_{3}$ until the conversion is complete, as indicated by the absence of brown fumes after the addition of $\mathrm{HNO}_{3}$.

11. Add water to about a quarter of the beaker's volume to the beaker containing the filter paper and soil residue. Repeat Steps 3-7.

12. Transfer the second filtrate to the beaker containing the original filtrate. Convert to $\mathrm{HNO}_{3}$ as in Step 10 .

13. Reduce the volume of the combined filtrates to $\sim 50 \mathrm{~mL}$ or until salting out begins to occur. Add $\sim 100 \mathrm{~mL}$ (or twice the volume) of water, stir and cool to room temperature. If cloudy, filter under vacuum through two glass fiber filters, washing with hot $1-2 \mathrm{M} \mathrm{HNO}_{3}$. Discard the filters.

14. Quantitatively transfer the filtrate to a large $(800-1500 \mathrm{~mL})$ beaker containing a magnetic stirring bar, rinsing with water. Place the beaker on a magnetic stirrer/hot plate and stir while warming the solution.

15. Add approximately $5 \mathrm{~g}$ of $\mathrm{H}_{2} \mathrm{C}_{2} \mathrm{O}_{4}$ (oxalic acid) $\mathrm{L}^{-1}$ and continue to stir until the salt completely dissolves.

16. While stirring, adjust the $\mathrm{pH}$ to 5.5-6.0 with $\mathrm{NH}_{4} \mathrm{OH}$. If the mixture turns brown due to the presence of $\mathrm{FeO}(\mathrm{OH})$, add just enough $\mathrm{H}_{2} \mathrm{C}_{2} \mathrm{O}_{4}$ to bring back the green color and readjust the $\mathrm{pH}$. Repeat this process, using decreasing quantities of $\mathrm{H}_{2} \mathrm{C}_{2} \mathrm{O}_{4}$, until 
the last $\mathrm{pH}$ adjustment does not result in the brown color. (Note: At this point, there should be enough $\mathrm{H}_{2} \mathrm{C}_{2} \mathrm{O}_{4}$ to precipitate the insoluble white oxalates and to complex the $\mathrm{Fe}^{+3}$ ion, but not enough to cause crystallization of the $\left(\mathrm{NH}_{4}\right)_{2} \mathrm{C}_{2} \mathrm{O}_{4}$ upon cooling.) Finish by adding several grams of oxalic acid as excess and adjust the $\mathrm{pH}$ again. Stir for $30 \mathrm{~min}$ on a warm hot plate.

17. Turn off the stirrer, remove the beaker from the hot plate, and allow the precipitate to settle overnight.

18. Add $5 \mathrm{~mL}$ of $\mathrm{Ca}$ carrier solution ( $1 \mathrm{~g} \mathrm{Ca}$ ), stir the supernatant very gently and allow the fresh precipitate to settle for 15-20 $\mathrm{min}$.

19. Filter the sample by gravity through a large Whatman No. 42 filter paper. Wash the beaker with $\mathrm{H}_{2} \mathrm{O}$, adding the washes to the funnel. Wash the precipitate with water until the filtrate is colorless.

20. Transfer the filter paper and precipitate to a $600-\mathrm{mL}$ beaker. Add $100 \mathrm{~mL}$ of $\mathrm{HNO}_{3}$. Cover with a watch glass and wet ash the oxalates until clear and colorless or oxidation seems complete, adding more $\mathrm{HNO}_{3}$ as necessary.

21. Add an equal volume of water and stir on a magnetic stirrer. If cloudy, filter through two glass fiber filters washing with $8 \mathrm{M} \mathrm{HNO}_{\hat{3}}$, followed by water. Evaporate to dryness.

\section{Proceed to Determination, Fuming $\mathrm{HNO}_{3}$ Separations.}

Note: This method was developed at the U.S. Department of Agriculture Soil Survey Laboratory, Soil Conservation Service, Beltsville, MD. Comparative soil analyses at EML showed that the ${ }^{85} \mathrm{Sr}$ tracer could be completely equilibrated with ${ }^{90} \mathrm{Sr}$ present in the soils when consecutively treated with $\mathrm{NaOH}$ and $\mathrm{HCl}$. The $\mathrm{NaOH}-\mathrm{HCl}$ method yielded results equal to those obtained with the complete dissolution method.

\section{Vegetation (dry ashing).}

1. Weigh an aliquot of up to $10 \mathrm{~g}$ of vegetation into a tared $250-\mathrm{mL}$ porcelain crucible. (Note: After ashing, several aliquots can be combined to provide the desired sample 
size.) Place each crucible in a muffle furnace with the crucible cover slightly ajar. Increase the temperature of the furnace at a rate of $0.80^{\circ} \mathrm{C} \mathrm{min}{ }^{-1}$ to $250^{\circ} \mathrm{C}$. Maintain this temperature for 30 minutes. Increase the temperature at a rate of $10^{\circ} \mathrm{C} \mathrm{min}^{-1}$ to $600^{\circ} \mathrm{C}$. Maintain this temperature for $960 \mathrm{~min}$ to completely ash the sample. Cool the crucible and weigh it to determine the percent ash. Ash content for replicate crucibles should vary by not more than $4 \%$.

2. Transfer the ashed vegetation to a beaker using $8 \mathrm{M} \mathrm{HNO}_{3}$ to dissolve the ash and rinse the crucible. Add $1 \mathrm{~mL}$ of $\mathrm{Sr}$ carrier $(20 \mathrm{mg})$.

3. Add $1.00 \mathrm{~mL}$ of ${ }^{85} \mathrm{Sr}$ tracer to the blank, the sample and each of three $30-\mathrm{mL}$ plastic reference bottles. Fill the bottles to the same level with $1 \mathrm{M} \mathrm{HCl}$.

4. Cover with a watch glass and reflux on a hot plate until there is no evidence of remaining organic matter, adding $\mathrm{HNO}_{3}$ or $\mathrm{H}_{2} \mathrm{O}_{2}$ as necessary.

5. Evaporate to near dryness. Add $50 \mathrm{~mL}$ of $8 \mathrm{M} \mathrm{HNO}_{3}$. Filter by gravity through a Whatman No. 42 filter paper into a beaker, washing with $8 \mathrm{M} \mathrm{HNO}_{3}$. Continue with Step 6 below.

\section{E. Vegetation (wet ashing).}

1. Weigh an aliquot of vegetation into an appropriate sized beaker. (For 100-300 g, use a 3000-mL beaker.) Add $1 \mathrm{~mL}$ of $\mathrm{Sr}$ carrier $(20 \mathrm{mg}$ ).

2. Add $1.00 \mathrm{~mL}$ of ${ }^{85} \mathrm{Sr}$ tracer to the blank, the sample and each of three $30-\mathrm{mL}$ plastic reference bottles. Fill the bottles to the same level with $1 \underline{\mathrm{M}} \mathrm{HCl}$.

3. Slowly add $500 \mathrm{~mL}$ of $8 \mathrm{M} \mathrm{HNO}_{3}$. Control the foaming, if necessary, by adding a few drops of n-octyl alcohol. Cover with a watch glass and place on a low temperature hot plate overnight to maintain a slow reaction, stirring as necessary to break up the foam. Gradually increase the temperature of the hot plate, adding $\mathrm{HNO}_{3}$ and continuing to reflux until the reaction is complete, as indicated by the absence of brown nitrogen oxide gas. 
4. Remove from the hot plate. Slowly add enough $\mathrm{HCl}$ to equal one third the volume of $\mathrm{HNO}_{3}$ still in the beaker. Allow the mixture to react at room temperature for $15 \mathrm{~min}$, then cover with a watch glass and heat on a low temperature hot plate overnight with occasional stirring.

5. Remove the sample from the hot plate and add an equal volume of water. Allow the sample to cool to room temperature. Filter by gravity through a large Whatman No. 42 filter paper into a beaker. Wash with $8 \underline{\mathrm{M}} \mathrm{HNO}_{3}$.

6. Evaporate the filtrate to dryness. Dissolve the residue in a minimum of $8 \mathrm{M} \mathrm{HNO}_{3}$. Quantitatively transfer the solution to a $400-\mathrm{mL}$ beaker containing a Teflon-coated magnetic stirring bar, rinsing with $\mathrm{H}_{2} \mathrm{O}$. Dilute the solution to $200 \mathrm{~mL}$ with additional $\mathrm{H}_{2} \mathrm{O}$.

7. Place the beaker on a magnetic stirrer/hot plate and stir. Adjust the $\mathrm{pH}$ to 5-6 with $50 \% \mathrm{NaOH}$. While continuing to stir, add $15 \mathrm{~mL}$ of $2 \mathrm{M} \mathrm{Na}_{2} \mathrm{CO}_{3}$. Heat the sample to just below boiling and stir for $30 \mathrm{~min}$.

8. Remove the sample from the hot plate and allow the precipitate to settle overnight.

9. Place a small glass fiber filter backed by a Whatman No. 42 filter paper of the same size into a Büchner funnel. Mount the funnel on a $500 \mathrm{~mL}$ filter flask.

10. Filter the sample by vacuum through the funnel. Wash the carbonates retained on the filter with $1 \underline{\mathrm{M} \mathrm{Na}} \mathrm{CO}_{3}$ solution. Discard the filtrate.

11. Release the vacuum and transfer the funnel to a clean filter flask. Apply the vacuum. Dissolve the carbonates on the filter with hot $8 \underline{\mathrm{M}} \mathrm{HNO}_{3}$. Wash the filter with water.

12. Transfer the filtrate to a $400 \mathrm{~mL}$ beaker, rinsing with $8 \mathrm{M} \mathrm{HNO}_{3}$. Evaporate to dryness.

\section{Proceed to Determination, Fuming $\mathrm{HNO}_{3}$ Separations.}




\section{DETERMONATION}

\section{A. Fuming $\mathrm{HNO}_{3}$ separations.}

1. Dissolve the residual salt in $\mathrm{H}_{2} \mathrm{O}$ and some fuming $\mathrm{HNO}_{3}$, while stirring on a magnetic stirrer. When dissolved, add additional fuming $\mathrm{HNO}_{3}$ to precipitate $\mathrm{Sr}\left(\mathrm{NO}_{3}\right)_{2}$. The first two separations require concentrations of $>75 \% \mathrm{HNO}_{3}$, subsequent separations require less $\mathrm{HNO}_{3}$ (see chart below). Water and air filters usually require two separations. Large quantities of soils with a high $\mathrm{Ca}$ content may require up to five or more fuming $\mathrm{HNO}_{3}$ separations.

$\begin{array}{rrccc}\text { Separation } & \text { Water }(\mathrm{mL}) & \text { Fuming } \mathrm{HNO}_{3}(\mathrm{~mL}) & \% \mathrm{HNO}_{3} & \text { final volume }(\mathrm{mL}) \\ \text { 1st } & 40 & 25+195 & 80.1 & 260 \\ \text { 2nd } & 60 & 25+195 & 76.0 & 280 \\ \text { 3rd } & 40 & 25+115 & 75.4 & 180 \\ \text { 4th } & 30 & 105 & 75.4 & 135 \\ \text { 5th } & 23 & 77 & 74.9 & 100\end{array}$

2. Place a dry (very important to prevent sample loss) $5.5-\mathrm{cm}$ glass fiber filter (for smaller volumes a $4.25-\mathrm{cm}$ filter) in a dry Büchner funnel and mount the funnel in a 1-L filter flask.

3. Suction filter the sample into the flask. Turn off the vacuum. Transfer the funnel to a Fisher filtrator, placing an appropriate sized beaker underneath (for the last filtration, use a 40-mL heavy-wall conical centrifuge tube (C-tube)). Apply a vacuum while dissolving the precipitate on the filter with water into the beaker. Use additional water to complete the transfer of any residue in the original beaker to the funnel and subsequently into the beaker or C-tube. Proceed with Step 4 or 5.

4. Evaporate the sample solution to dryness if another fuming $\mathrm{HNO}_{3}$ separation is desired, and repeat Steps 1 to 3 using smaller volumes as indicated in the chart. 
5. If the sample solution is now in a C-tube, place the tube in a hot water bath and adjust the volume to $\sim 20 \mathrm{~mL}$. Proceed with First Milking.

\section{B. First milking.}

1. Add $1 \mathrm{~mL}$ of iron carrier solution to the separated strontium fraction in the centrifuge tube. Stir the solution and place the tube in a $90^{\circ} \mathrm{C}$ water bath to warm.

2. While stirring, adjust the $\mathrm{pH}$ of the sample to 8 with $\mathrm{NH}_{4} \mathrm{OH}$. Remove the stirring rod, rinsing with $\mathrm{H}_{2} \mathrm{O}$. Remove the centrifuge tube from the water bath and cool to room temperature in a cold water bath.

3. Centrifuge the sample at $2000 \mathrm{rpm}$ for $5 \mathrm{~min}$. Decant the supernate into a second 40 $\mathrm{mL}$ centrifuge tube. Reserve the supernate for Step 6 and note the hour and date of this initial $\mathrm{OH}^{-1}$ precipitation as first milk separation time.

4. Dissolve the precipitate in the first centrifuge tube in a few drops of $\mathrm{HCl}$ and dilute to $10 \mathrm{~mL}$ with $\mathrm{H}_{2} \mathrm{O}$. Stir the solution and warm the tube in the hot water bath.

5. While stirring, adjust the $\mathrm{pH}$ of the sample to 8 with $\mathrm{NH}_{4} \mathrm{OH}$. Remove the stirring rod, rinsing with $\mathrm{H}_{2} \mathrm{O}$. Remove the centrifuge tube from the water bath and cool to room temperature in a cold water bath.

6. Centrifuge the sample at $2000 \mathrm{rpm}$ for $5 \mathrm{~min}$. Decant and combine the supernate with the supernate reserved from Step 3. Evaporate to reduce the volume to $20 \mathrm{~mL}$.

Discard the precipitate.

7. While stirring, add $4 \mathrm{~mL}$ of barium buffer solution to the sample. If necessary, adjust the $\mathrm{pH}$ of the sample to 5.5 with either $6 \mathrm{M} \mathrm{HCl}$ or $\mathrm{NH}_{4} \mathrm{OH}$ (see Note 1 ).

8. Return the centrifuge tube to the hot water bath. While stirring vigorously, add $1 \mathrm{~mL}$ of $0.3 \mathrm{M} \mathrm{Na}_{2} \mathrm{CrO}_{4}$ dropwise to the sample (see Note 2). Allow the sample to digest in the hot water bath for $10 \mathrm{~min}$ or longer to allow a good precipitate to form.

9. Remove the stirring rod, rinsing with $\mathrm{H}_{2} \mathrm{O}$. Remove the sample tube from the hot water bath and cool in a cold water bath. 
10. Centrifuge the tube at $2000 \mathrm{rpm}$ for $5 \mathrm{~min}$. Decant the supernate into a $30-\mathrm{mL}$ polyethylene bottle. Discard the precipitate.

11. Add 10-15 drops of $\mathrm{HCl}$ and exactly $1.00 \mathrm{~mL}$ of yttrium carrier solution to the sample in the polyethylene bottle and enough water to bring the volume of the solution to the same level as in the reference bottles.

12. Proceed to Strontium-85 yield determination.

Notes:

1. The $\mathrm{pH}$ of the solution is critical at this point. Complete precipitation of $\mathrm{BaCrO}_{4}$ will not occur in a more acidic solution and strontium will partially precipitate in more basic solutions.

2. If large quantities of barium are present in the sample, only a partial precipitation of the $\mathrm{Ba}$ as $\mathrm{BaCrO}_{4}$ may occur. The sample is centrifuged and the supernate decanted into another $40-\mathrm{mL}$ centrifuge tube. The precipitation is completed by the dropwise addition of $0.3 \mathrm{M} \mathrm{Na}_{2} \mathrm{CrO}_{4}$ to the supernate and the analysis is continued with Step 10 .

\section{Strontium-85 yield measurement.}

1. Measure the activity of the three reference aliquots, the blank and the sample with a $\mathrm{NaI}(\mathrm{Tl})$ crystal gamma detector, collecting at least $10^{4}$ counts.

2. After subtracting the background counts, calculate the ${ }^{85} \mathrm{Sr}$ yield of the sample by dividing the sample counts by the average of the three reference counts.

3. Store the sample for 2 weeks to allow ${ }^{90} \mathrm{Y}$ to reach secular equilibrium with ${ }^{90} \mathrm{Sr}$ (see Note).

Note: The first milk separation time noted in Step 3, First milking, is the start of the yttrium ingrowth period. In order to correct for less than complete buildup of ${ }^{90} \mathrm{Y}$, a correction factor is included in the calculations. 


\section{Second milking.}

1. Quantitatively transfer the sample from the polyethylene bottle to a 40-mL, heavywalled, conical centrifuge tube with a minimum of $\mathrm{H}_{2} \mathrm{O}$. Stir the solution and place the tube in a $90^{\circ} \mathrm{C}$ water bath to warm.

2. While stirring, adjust the $\mathrm{pH}$ of the sample to 8 with $\mathrm{NH}_{4} \mathrm{OH}$. Add six drops of $\mathrm{H}_{2} \mathrm{O}_{2}$ and heat for $1 \mathrm{~h}$. Remove and rinse the stirring rod. Remove the centrifuge tube from the water bath and cool to room temperature in a cold water bath.

3. Centrifuge the sample for $5 \mathrm{~min}$ at $2000 \mathrm{rpm}$. Decant the supernate into another 40-mL centrifuge tube. Record the hour and date of the precipitation as second milk separation time.

4. Dissolve the precipitate in the centrifuge tube with a few drops of $\mathrm{HCl}$ and stir. Dilute the sample to $15 \mathrm{~mL}$ with $\mathrm{H}_{2} \mathrm{O}$. Stir the solution and warm the tube in the hot water bath.

5. While stirring, adjust the $\mathrm{pH}$ of the sample to 8 with $\mathrm{NH}_{4} \mathrm{OH}$. Remove and rinse the stirring rod. Remove the centrifuge tube from the water bath and cool to room temperature in a cold water bath.

6. Centrifuge the sample for $5 \mathrm{~min}$. Decant and combine the supernate with the supernate reserved from Step 3. Return the combined supernates to the hot water bath and reduce the volume to $20 \mathrm{~mL}$. Transfer to a $30-\mathrm{mL}$ polyethylene bottle and set aside for possible future milking.

7. Add four drops of $\mathrm{HCl}$ to the precipitate and stir until it dissolves. Add $25 \mathrm{~mL}$ of $\mathrm{H}_{2} \mathrm{O}$, stir and heat in the hot water bath.

8. Add $1 \mathrm{~mL}$ of strontium carrier ( $20 \mathrm{mg} \mathrm{Sr}$ ) to serve as a holdback carrier. While stirring, adjust the $\mathrm{pH}$ to 8 with $\mathrm{NH}_{4} \mathrm{OH}$.

9. Remove and rinse the stirring rod. Remove the sample tube from the hot water bath and cool in a cold water bath. 
10. Centrifuge the tube at $2000 \mathrm{rpm}$ for $5 \mathrm{~min}$. Decant and discard the supernate.

11. Add three drops of $\mathrm{HCl}$ to dissolve the precipitate, and $25 \mathrm{~mL}$ of $\mathrm{H}_{2} \mathrm{O}$.

12. Stir the sample and place the tube in a hot water bath. Add $1 \mathrm{~mL}$ of saturated $\mathrm{H}_{2} \mathrm{C}_{2} \mathrm{O}_{4}$ (oxalic acid) solution.

13. Adjust the $\mathrm{pH}$ to $2-3$ by the dropwise addition of $\mathrm{NH}_{4} \mathrm{OH}$ with vigorous stirring. Digest the sample in the hot water bath for $1 \mathrm{~h}$.

14. Rinse and remove the stirring rod from the tube, and cool it to room temperature in cold water.

15. Centrifuge the tube at $2000 \mathrm{rpm}$ for $10 \mathrm{~min}$. Decant and discard most of the supernate.

16. Dry a $2.8-\mathrm{cm}$ Whatman No. 42 filter paper on a $110^{\circ} \mathrm{C}$ hot plate or in an $110^{\circ} \mathrm{C}$ oven, cool and weigh to the nearest $0.1 \mathrm{mg}$.

17. Using a Teflon filter funnel assembly, filter the precipitate by suction through the weighed $2.8-\mathrm{cm}$ Whatman No. 42 filter paper, backed by a $2.8-\mathrm{cm}$ glass fiber filter, completing the transfer with a minimum amount of water. Wash the precipitate with $95 \%$ ethanol.

18. With the suction on, remove the filter funnel. Carefully separate the Whatman filter with the precipitate from the glass fiber filter backing. Place the filter paper with the precipitate on $110^{\circ} \mathrm{C}$ hot plate. Turn off the vacuum and discard the glass fiber filter. Discard the filtrate.

19. Dry the filter with precipitate to a constant weight to determine the gravimetric yield.

20. Carefully mount the filtered precipitate on a nylon disc, cover with Mylar, and fasten the assembly with a nylon ring.

21. Measure the sample in a low-level gas flow proportional counter, recording the hour and date of the beginning of the measurement period. 
22. Collect at least 10,000 counts, disregarding the first $200 \mathrm{~min}$ ( 2 cycles) of counting to eliminate possible interference from any ${ }^{222} \mathrm{Rn}$ progeny present due to the filtration process. Proceed to calculations.

\section{E. Gravimetric yttrium yield measurement.}

1. Standardize triplicate $10-\mathrm{mL}$ aliquots of the original yttrium carrier solution each time a fresh batch is made by precipitating the oxalate as described above and filtering through a fine (grade F), tared, sintered, glass filter crucible that has been dried to a constant weight.

2. Determine the weight of the yttrium oxalate precipitated from the sample as described in Steps 15-19 of Second Milking. The yttrium yield is the ratio of the weight of the sample oxalate to the standardized weight of the oxalate precipitated from the carrier solution.

\section{CALCULATIONS}

The $\beta$ counting data obtained from the ${ }^{90} \mathrm{Y}$ precipitate must be corrected to give the activity of the ${ }^{90} \mathrm{Sr}$ in the sample. The corrections include those for ingrowth of ${ }^{90} \mathrm{Y}$, counter background, ${ }^{90} \mathrm{Y}$ efficiency, strontium yield, yttrium yield, and ${ }^{90} \mathrm{Y}$ decay. ${ }^{90} \mathrm{Y}$ beta emissions are very energetic and are always counted with approximately the same mass of precipitate, so no correction for self-absorption is necessary.

The strontium yield is ordinarily determined by measuring the recovery of ${ }^{85} \mathrm{Sr}$ tracer added to the sample. Since the ratio of sample counts to counts from an aliquot of the original ${ }^{85} \mathrm{Sr}$ tracer solution is used to determine yield, there is no need to know the radioactivity rate of the tracer or to apply decay corrections for ${ }^{85} \mathrm{Sr}$.

The activity of a sample of ${ }^{90} \mathrm{Sr}$ over a time interval, $t$, is

$$
\int_{0}^{t} A d t^{\prime}=\int_{0}^{t} A_{0} e^{-\lambda s^{\prime}} d t^{\prime}
$$


where $A_{0}$ is the initial activity of the sample. Integrating and rearranging to solve for $A_{0}$ yields:

$$
A_{0}=\left(\int_{0}^{t} A d t^{\prime}\right) \cdot \frac{\lambda}{1-e^{-\lambda t}}
$$

The half-life of ${ }^{90} \mathrm{Sr}$ is quite large $(29 \mathrm{y})$, so $\mathrm{A}_{0}$ is essentially constant throughout the period of chemical separation and analysis. After 2 weeks, a sample of ${ }^{90} \mathrm{Sr}$ will be in secular equlibrium with its daughter, ${ }^{90} \mathrm{Y}$, and the activities of the two nuclides will be equal. The quantity under the integral sign in the last equation above is the (corrected) measured activity of the separated ${ }^{90} \mathrm{Y}$ over the time period from separation to end of counting:

$$
\int_{0}^{t} A d t^{\prime}=\frac{N_{y}-B \cdot d t_{c}}{R_{y} \cdot R_{S r} \cdot I_{y} \cdot D_{y} \cdot E_{c}}
$$

To obtain $A_{0}$, this quantity is multiplied by the factor

$$
\frac{\lambda_{Y}}{1-e^{-\lambda_{\gamma} \cdot d t_{c}}}
$$

where:

$$
\begin{aligned}
& \lambda_{\mathrm{Y}}=\text { decay constant of }{ }^{90} \mathrm{Y}\left(0.0108 \mathrm{~h}^{-1}\right) \\
& \mathrm{dt}_{\mathrm{c}}=\text { total count time minus two } 100 \text {-minute cycles (see Note) } \\
& \mathrm{N}_{\mathrm{y}}=\text { total counts from all cycles except the first two (see Note) } \\
& \mathrm{B}=\text { counter background for the matrix used ( }{ }^{90} \mathrm{Y} \text {-oxalate) } \\
& \mathrm{R}_{\mathrm{y}}=\text { yttrium yield fraction }
\end{aligned}
$$


$\mathrm{R}_{\mathrm{sr}}=$ strontium yield fraction

$\mathrm{I}_{\mathrm{y}}={ }^{90} \mathrm{Y}$ ingrowth fraction $=1-\mathrm{e}^{-0.0108 \cdot \mathrm{dtl}-2}=$ fraction of ${ }^{90} \mathrm{Y}$ produced during the time from extraction of ${ }^{90} \mathrm{Sr}$ ("1st milk") to separation of ${ }^{90} \mathrm{Y}$ from ${ }^{90} \mathrm{Sr}$ ("2nd milk").

$D_{y}={ }^{90} Y$ decay fraction $=\mathrm{e}^{-0.0108 \cdot \mathrm{dt} 2-\mathrm{c}} 0$

$E_{c}=$ counter efficiency for ${ }^{90} \mathrm{Y}$-oxalate (counts $\min ^{-1} \mathrm{dpm}^{-1}$ )

$\mathrm{dt} 1 \rightarrow 2=1$ st milk to 2 nd milk time

$\mathrm{dt} 2 \rightarrow \mathrm{c}_{0}=2$ nd milk time to start of counting plus two 100 -min cycles (see Note)

Note: The first two cycles are ignored to allow for the decay of short-lived beta-emitting daughters from any radon-222 that may have attached to the Y-oxalate mount during preparation.

The calculated activity of the blank is subtracted from the calculated activity of the sample. The result is converted to appropriate units and divided by the sample size to obtain the activity concentration of the sample.

To check the radiochemical purity of the ${ }^{90} Y$-oxalate precipitate, a weighted linear regression analysis is done on the counting data, with Ln (counts-background counts) plotted against time. The weighting factor is the variance of the dependent variable:

$$
\text { Weighting factor }=\operatorname{Var}\left(\ln \left(c-c_{b k g}\right)\right)=\frac{c+\left(\sigma_{c_{b k g}}\right)^{2}}{\left(c-c_{b k g}\right)^{2}}
$$


where:

$$
\begin{aligned}
\mathrm{C} & =\text { sample counts } \\
\mathrm{C}_{\mathrm{bkg}} & =\text { background counts } \\
\sigma_{\mathrm{Cbkg}} & =\text { standard deviation of background counts }
\end{aligned}
$$

The slope of the weighted regression line is equal to $\lambda_{\mathrm{Y}}$, the decay constant of ${ }^{90} \mathrm{Y}$. The value for $\lambda_{Y}$ obtained from the regression analysis is compared to the known value of $0.0108 \mathrm{~h}^{-1}$.

All calculations are done by computer.

LOWER LIMIT OF DETECTION (LLD)

\begin{tabular}{llc}
\hline & & \\
Counter Efficiency & $(\%)$ & 40 \\
Counter Background & $(\mathrm{cps})$ & 0.005 \\
Yield (Sr) & $(\%)$ & 80 \\
Yield (Y) & $(\%)$ & 95 \\
Blank & $(\mathrm{cps})$ & \\
& & \\
LLD (400 min) & $(\mathrm{Bq})$ & 0.007 \\
LLD (1000 min) & $(\mathrm{Bq})$ & 0.004 \\
\hline
\end{tabular}




\section{APPENDIX \\ YTTRIUM CARRIER COUNTING CHECK}

To varify that the carrier solution contains only stable yttrium, complete the following procedure:

1. Pipette $1 \mathrm{~mL}$ of yttrium carrier into each of three $40-\mathrm{mL}$ centrifuge tubes. Dilute to $20 \mathrm{~mL}$ with $\mathrm{H}_{2} \mathrm{O}$.

2. Heat in a water bath to about $90^{\circ} \mathrm{C}$. While stirring, adjust the $\mathrm{pH}$ to 8 with $\mathrm{NH}_{4} \mathrm{OH}$. Digest for $10 \mathrm{~min}$ and cool in a cold water bath.

3. Centrifuge for $5 \mathrm{~min}$. Decant and discard the supernate. Proceed with Steps 11-22 of Second Milking. 


\title{
STRONTIUM-90 IN WATER CONTAINING OTHER RADIOISOTOPES BY CERENKOV COUNTING
}

\author{
Contact Person(s): Salvatore C. Scarpitta
}

\section{APPLICATION}

The following procedure is used in the EML Quality Assessment Program (QAP; Sanderson and Greenlaw, 1996) for water or gross alpha/beta samples containing ${ }^{90} \mathrm{Sr}$. This procedure was developed by Jimmy Chang, Institute of Nuclear Energy Research, Taiwan, and was tested at EML using NIST ${ }^{90} \mathrm{Sr} / \mathrm{Y}$ reference materials and ${ }^{90} \mathrm{Sr}$ contained in QAP water samples. It allows for the rapid determination of ${ }^{90} \mathrm{Sr}\left(\beta_{\max }=0.546 \mathrm{MeV}\right)$ via its progeny, ${ }^{90} \mathrm{Y}\left(\beta_{\max }=2.27 \mathrm{MeV}\right)$ in aqueous solutions by Cerenkov counting (see Procedure Ba-01-R; Scarpitta and Fisenne, 1996).

Cerenkov counting is applicable for $\beta$ particles with maximum energies $>0.263$ $\mathrm{MeV}$. Alpha and gamma emitting nuclides are not detected. Immediately following separation of ${ }^{90} \mathrm{Y}$ from ${ }^{90} \mathrm{Sr}$, a baseline count rate, $\mathrm{C}_{\mathrm{b}}$, is obtained to quantify any Cerenkov contribution by other nuclides that may be present in the sample.

Yield recovery is determined by adding $30 \mathrm{mg}$ of $\mathrm{Sr}^{+2}$ carrier to the sample or by gamma counting the added ${ }^{85} \mathrm{Sr}$ tracer. The sample is Cerenkov counted at any two time intervals, $t_{1}$ and $t_{2}$, to measure the ingrowth of ${ }^{90} \mathrm{Y}$ above $\mathrm{C}_{\mathrm{b}}$. Strontium-90 is calculated from the ingrowth of ${ }^{90} \mathrm{Y}$. The Cerenkov counting efficiency for ${ }^{90} \mathrm{Y}$ in water is about $65-$ $70 \%$, whereas that of ${ }^{90} \mathrm{Sr}$ is $<0.3 \%$ for a $0-15 \mathrm{keV}$ Cerenkov counting window. For a 20 -min count time, the detection limit is about $6 \mathrm{mBq} \mathrm{mL}^{-1}\left(0.16 \mathrm{pCi} \mathrm{mL}^{-1}\right)$ or $0.35 \mathrm{dpm}$ $\mathrm{mL}^{-1}$ with a relative standard deviation $<3 \%$. 


\section{SPECIAL APPARATUS}

Packard Tri-Carb 2250-CA liquid scintillation spectrometer

\section{SPECIAL REAGENTS}

1. NIST traceable ${ }^{90} \mathrm{Sr} / Y$ reference standard of known activity (about $1000 \mathrm{dpm} \mathrm{g}^{-1}$ ) to determine the ${ }^{90} \mathrm{Y}$ Cerenkov counting efficiency.

2. TRU Resin - Eichrom Industries, 8205 Cass Ave., Suite 107, Darien, II 606651.

\section{DETERMINATION}

1. Dispense about $8-16 \mathrm{~Bq}$ of ${ }^{90} \mathrm{Sr}$ gravimetrically into either a $20 \mathrm{~mL} \mathrm{low}{ }^{40} \mathrm{~K}$ borosilicate glass or plastic scintillation vial (see Note 1 ).

2. Add $10 \mathrm{~mL}$ of deionized water.

3. Prepare a blank using $10 \mathrm{~mL}$ of water.

4. Count both samples three times for $10-\mathrm{min}$ each using the net average count rate to determine the ${ }^{90} \mathrm{Y}$ counting efficiency, $\mathrm{E}^{90} \mathrm{Y}$ (counts $\mathrm{min}^{-1} \mathrm{dpm}^{-1}$ ).

5. Preset the Packard Tri-Carb to Protocol 4 for Cerenkov determinations (Scarpitta and Fisenne, 1996). (Note: The Cerenkov counting window is typically $0-15 \mathrm{keV}$, although the full window, $0-2000 \mathrm{keV}$, may be used with a $50 \%$ increase in background.)

6. Obtain a sample containing an unknown amount of ${ }^{90} \mathrm{Sr}$.

7. Add $30 \mathrm{mg}$ of $\mathrm{Sr}^{++}$carrier (as nitrate) to the sample for yield recovery.

8. Prepare an identical vial containing water as a $\mathrm{Sr}^{++}$reference standard. 
9. Reduce a premeasured amount, $\mathrm{M}_{\mathrm{g}}(\mathrm{g})$, of sample to be tested to $10 \mathrm{~mL}$ to improve counting statistics.

\section{Note :}

1. A wavelength shifter, ANSA (7-Amino 1,3 Naphthalene di-Sulfonic Acid) can be used to enhance the Cerenkov counting efficiency but is not recommended if strontium yield recovery is to be determined gravimetrically. Nuclides that produce a Cerenkov signal in $25 \mathrm{mM}$ ANSA are shown in Figure 1 (see Scarpitta and Fisenne, 1996).

\section{SEPARATION}

1. Separate the ${ }^{90} \mathrm{Y}$ from the ${ }^{90} \mathrm{Sr}$ by either oxalate precipitation (see Procedure Sr-03RC) or EiChrom's TRU Spec extraction chromatographic resin. Record the separation date and time, $t_{\mathrm{o}}$.

2. Obtain a net baseline count rate for $C_{b}$ immediately following ${ }^{90} Y$ separation, using Protocol No. 4 on the Packard Tri-Carb $2250 \mathrm{CA}$ counter and the Cerenkov counting window (0-15 keV).

\section{CALCULATIONS}

1. Recount the ${ }^{90} \mathrm{Sr}$ fraction three times a day over a 2-day period using the count rates (counts $\mathrm{min}^{-1}$ ) $\mathrm{CT}_{1}, \mathrm{CT}_{2}$ and $\mathrm{CT}_{3}$ to calculate the ${ }^{90} \mathrm{Sr}$ activity in Step 2. The times $\mathrm{t}_{1}$, $t_{2}$ and $t_{3}$ are the number of hours after ${ }^{90} Y$ separation in Step 1 of Separation.

2. Use the ${ }^{90} \mathrm{Sr}$ calculation as follows when ${ }^{89} \mathrm{Sr}$ is not present in the sample. (Note: $\mathrm{A}$ Basic computer program is provided in the Appendix to perform the ${ }^{90} \mathrm{Sr}$ calculations.)

$$
A_{1}{ }^{90} \mathrm{Sr}\left(B q \mathrm{~kg}^{-1}\right)=\frac{\left(C T_{2}-C T_{1}\right)-C_{b}}{60 \times M_{S} \times E^{90} Y \times\left[\exp \left\{-\lambda\left(t_{1}-t_{0}\right)\right\}-\exp \left\{-\lambda\left(t_{2}-t_{0}\right)\right\}\right]}
$$




$$
A_{2}{ }^{90} S r\left(B q \mathrm{~kg}^{-1}\right)=\frac{\left(C T_{3}-C T_{1}\right)-C_{b}}{60 \times M_{S} \times E^{90} Y \times\left[\exp \left\{-\lambda\left(t_{1}-t_{0}\right)\right\}-\exp \left\{-\lambda\left(t_{3}-t_{0}\right)\right\}\right]}
$$

where

$$
\begin{aligned}
\lambda & ={ }^{90} \mathrm{Y} \text { decay constant }-0.01083 \mathrm{~h}^{-1} \\
\mathrm{E}^{90} \mathrm{Y} & \left.={ }^{90} \mathrm{Y} \text { Cerenkov counting efficiency (counts } \min ^{-1} \mathrm{dpm}^{-1}\right) \\
\mathrm{M}_{\mathrm{s}} & =\text { mass of sample }(\mathrm{kg})
\end{aligned}
$$

3. Obtain the average of the two ${ }^{90} \mathrm{Sr}$ activity concentrations, $\mathrm{A}_{1}$ and $\mathrm{A}_{2}$ from Step 2 . (Note: A third count could be obtained with Equation 2 if modified accordingly.)

4. Using the sample vial and the $\mathrm{Sr}^{+2}$ reference standard, precipitate the strontium as the carbonate, filter, dry and weigh each to obtain the yield recovery. Correct the value . obtained in Step 3, dividing by the yield recovery factor. (Note: Gamma emitting ${ }^{85} \mathrm{Sr}$ can be added to the sample in Step 7 of Determination instead of $\mathrm{Sr}^{+2}$.)

\section{REFERENCES}

Sanderson, C. G. and P. Greenlaw

"Semi-Annual Report of the Department of Energy, Office of Environmental

Management, Quality Assessment Program"

USDOE Report EML-581, July (1996)

Scarpitta, S. C. and I. M. Fisenne

"Cerenkov Counting as a Complement to Liquid Scintillation Counting"

Appl. Radiat. Isot., 47, 795-800 (1996)

Scarpitta, S. C. and I. M. Fisenne

"Calibration of a Liquid Scintillation Counter for Alpha, Beta and Cerenkov Counting" USDOE Report EML-583, July (1996) 


\section{Cerenkov Efficiency in $10 \mathrm{ml}$ of $25 \mathrm{mM}$ ANSA} (Plastic Vials)

\section{\% Efficiency (0-50 keV)}
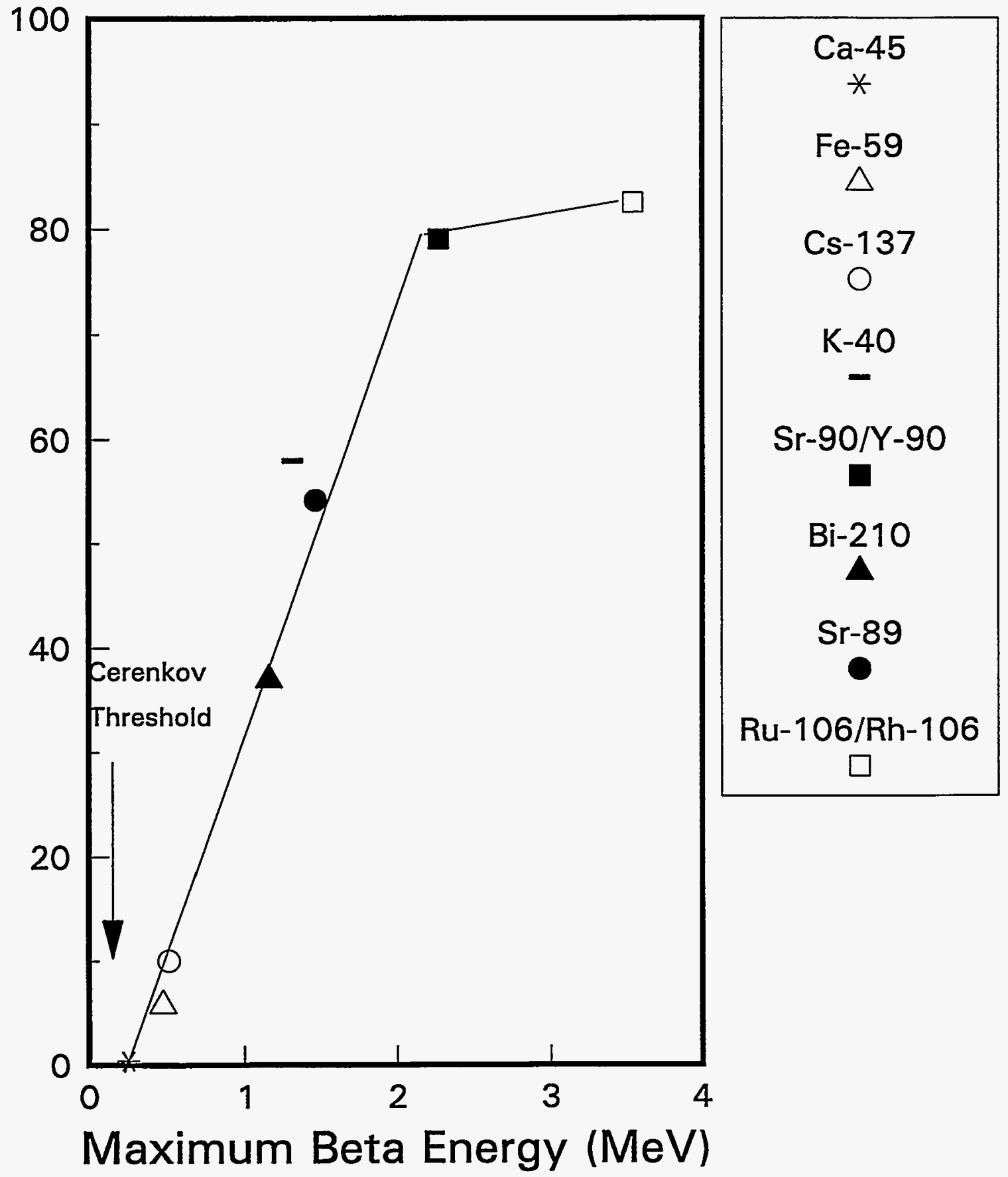

ANSA $=$ 7-Amino 1,3 Naphthalene di-Sulfonic Acid

Figure 1. Cerenkov efficiency in $10 \mathrm{~mL}$ of $25 \mathrm{mM}$ ANSA (plastic vials). 


\section{APPENDIX \\ Basic Computer Program}

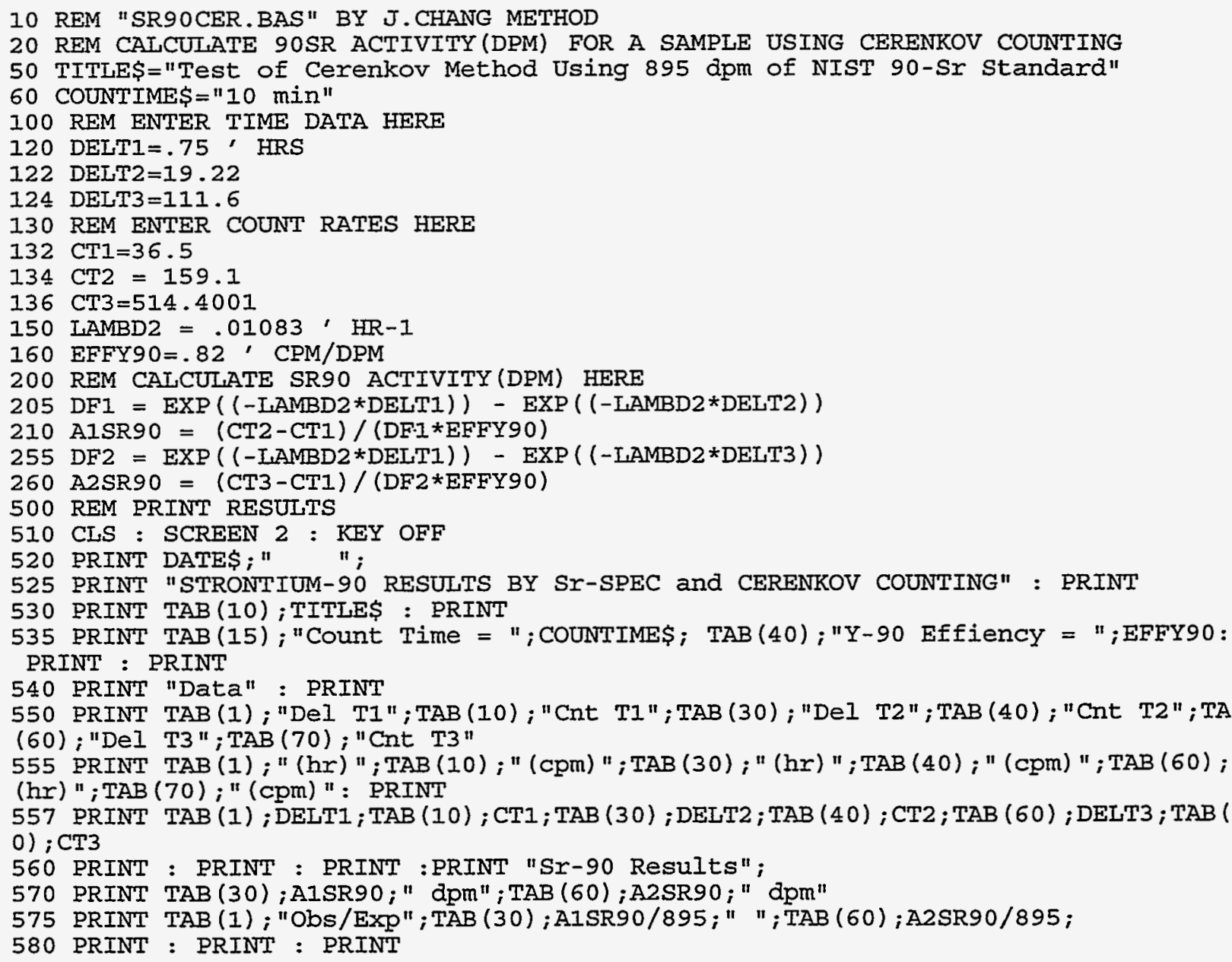

02-21-1995 STRONTIUM-90 RESULTS BY SY-SPEC and CERENKOV COUNTING

Test of. Cerenkov Method Using 895 dpm of NIST 90-Sr standard

Count Time $=10 \mathrm{~min} \quad \mathrm{Y}-90$ Effiency $=.82$

Data

\begin{tabular}{|c|c|c|c|c|c|}
\hline$\underset{(\mathrm{hr})}{\operatorname{Del}} \mathrm{T1}$ & $\begin{array}{l}\text { Cnt } \mathrm{T} 1 \\
\text { (cpm) }\end{array}$ & $\underset{(\mathrm{hr})}{\operatorname{Del}} \mathrm{T} 2$ & $\begin{array}{l}\text { Cnt T2 } \\
\text { (cpm) }\end{array}$ & $\underset{(\mathrm{hr})}{\operatorname{Del} \mathrm{T} 3}$ & $\begin{array}{l}\text { Cnt T3 } \\
\text { (cpm) }\end{array}$ \\
\hline .75 & 36.5 & 19.22 & 159.1 & 111.6 & 514.4001 \\
\hline $\begin{array}{l}\mathrm{Sr}-90 \\
\mathrm{Obs} / \mathrm{Ex}\end{array}$ & Its & $\begin{array}{l}831.42 \\
.9289\end{array}$ & dpm & $\begin{array}{l}840.6 \\
.9392\end{array}$ & dpm \\
\hline
\end{tabular}

Ok 
Section 4.5.4, Vol.

HASL-300, 28th Edition

\section{Technetium}

Environmental Measurements Laboratory U.S. Department of Energy 
$T C-01-R C$

TECHNETIUM-99 IN WATER AND VEGETATION (see Volume II) 
Section 4.5.4, Vol. I

Rev. 0

HASL-300, 28th Edition

February 1997

\section{Thorium}

Environmental Measurements Laboratory U.S. Department of Energy 
Th-01-RC, Vol. II

HASL-300, 28th Edition
Rev. 0

February 1997

Th-01-RC

\section{THORIUM IN URINE}

(see Volume II) 


\section{Uranium}

Environmental Measurements Laboratory U.S. Department of Energy 


\section{ENRICHED URANIUM IN URINE}

(see Volume II) 
Contact Person(s) : Isabel M. Fisenne

\begin{abstract}
APPLICATION
This procedure has been used to analyze soft tissue, vegetation, water, and air filter samples (Hindman, 1983; Sill and Williams, 1981; Welford et al., 1960).

Uranium from acid leached, dry-ashed and wet-ashed materials is equilibrated with ${ }^{232} \mathrm{U}$ tracer, and is isolated by anion exchange chromatography. The separated $\mathrm{U}$ isotopes are microprecipitated for $\alpha$ spectrometry.
\end{abstract}

\title{
SPECIAL APPARATUS
}

1. Ion exchange columns (see Specification 7.5).

2. Polyethylene dispensing bottles (see Specification 7.11).

3. Special apparatus for the microprecipitation of $U$ are listed under the generic procedure, G-03.

\section{SPECIAL REAGENTS}

1. Uranium-232 tracer solution - about $0.3 \mathrm{~Bq} \mathrm{~g}^{-1}$ of solution in a dispensing bottle.

2. Bio Rad AG 1-X4 (100-200 mesh), anion exchange resin (see Specification 7.4). 


\section{SAMPLE PREPARATION}

\section{A. Vegetation and soft tissue.}

1. Dry ash the sample according to the method described in Procedure Sr-02-RC (see Note 1).

2. Weigh out $10 \mathrm{~g}$ of ash and transfer to a $400-\mathrm{mL}$ beaker.

3. Add a weighed amount of ${ }^{232} \mathrm{U}$ tracer solution $(\sim 0.03 \mathrm{~Bq})$ from the dispensing bottle (see Note 2).

4. Add $200 \mathrm{~mL}$ of $\mathrm{HNO}_{3}$ to the beaker and evaporate slowly to dryness.

5. Add $25 \mathrm{~mL}$ of $\mathrm{HNO}_{3}$ to the beaker. Repeat the acid addition and evaporation until a white residue is obtained. (Note: If silicious material is present, transfer the sample to a $100 \mathrm{~mL}$ platinum dish or a $100 \mathrm{~mL}$ Teflon beaker with $\mathrm{HNO}_{3}$. Add $10 \mathrm{~mL}$ of $\mathrm{HF}$ to the vessel and evaporate to dryness. Repeat additions of $25 \mathrm{~mL} \mathrm{HNO}_{3}-10 \mathrm{~mL} \mathrm{HF}$ as necessary to volatilize the silica. Remove the HF by adding three successive 10$\mathrm{mL}$ volumes of $\mathrm{HNO}_{3}$ to the vessel and evaporate to dryness.)

6. Add $25 \mathrm{~mL}$ of $\mathrm{HCl}$ and evaporate to dryness. Repeat the acid addition and evaporation twice more.

7. Heat to dissolve the residue in $50-100 \mathrm{~mL}$ of $7 \mathrm{~N} \mathrm{HCl}$.

8. Continue with Determination.

\section{B. Water.}

1. Evaporate the $\mathrm{H}_{2} \mathrm{O}$ sample to a small volume.

2. Add a weighed amount of ${ }^{232} \mathrm{U}$ tracer solution $(\sim 0.017 \mathrm{~Bq})$ from a dispensing bottle and evaporate slowly to dryness (see Note 2 ). 
3. Add $50 \mathrm{~mL}$ of $\mathrm{HNO}_{3}$ and evaporate to dryness. Add $25 \mathrm{~mL}$ of $\mathrm{HNO}_{3}$ and evaporate twice more.

4. Dissolve the residue in $25 \mathrm{~mL}$ of $\mathrm{HCl}$ and evaporate to dryness. Repeat the $\mathrm{HCl}$ addition and evaporation.

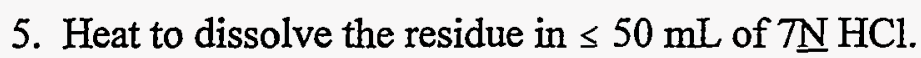

6. Continue with Determination.

\section{Air filters.}

\section{Cellulose filters:}

1. Add a weighed amount of ${ }^{232} \mathrm{U}$ tracer solution $(\sim 0.017 \mathrm{~Bq})$ from a dispensing bottle to the filter in a platinum dish and dry ash in an electric muffle at $550^{\circ} \mathrm{C}$ (see Note 2).

2. Dissolve the residue in $\mathrm{HNO}_{3}$ and transfer to a 250 -mL beaker.

3. Add $25 \mathrm{~mL}$ of $\mathrm{HNO}_{3}$ and evaporate to dryness. Repeat the acid addition and evaporation twice more.

4. Dissolve the residue in $25 \mathrm{~mL}$ of $\mathrm{HCl}$ and evaporate to dryness. Repeat the $\mathrm{HCl}$ addition and evaporation twice more.

5. Heat and dissolve the residue in $\leq 50 \mathrm{~mL}$ of $7 \underline{\mathrm{N}} \mathrm{HCl}$.

\section{Continue with Determination.}

\section{Glass fiber filters:}

1. Place the filter and a magnetic stirring bar in a 400-mL Teflon beaker. Add a weighed amount of ${ }^{232} \mathrm{U}$ tracer solution $(\sim 0.033 \mathrm{~Bq})$ from a dispensing bottle.

2. Add $100 \mathrm{~mL}$ of $\mathrm{HNO}_{3}$, mechancially stir while heating for $1 \mathrm{~h}$. Reduce the solution volume to $\sim 25 \mathrm{~mL}$. Remove the stirring bar and rinse with $\mathrm{H}_{2} \mathrm{O}$. 
3. Add $10 \mathrm{~mL}$ of $\mathrm{HF}$ and evaporate to dryness.

4. Repeat the $25 \mathrm{~mL} \mathrm{HNO}_{3}-10 \mathrm{~mL} \mathrm{HF}$ additions and evaporations as necessary to volatilize the silica.

5. Add $25 \mathrm{~mL}$ of $\mathrm{HNO}_{3}$ to the beaker and evaporate to dryness. Repeat twice more.

6. Heat and dissolve the residue in $25 \mathrm{~mL}$ of $\mathrm{HCl}$ and evaporate to dryness. Repeat the $\mathrm{HCl}$ addition and evaporation twice more.

7. Dissolve the residue in $\leq 50 \mathrm{~mL}$ of $7 \underline{\mathrm{N}} \mathrm{HCl}$.

8. Continue with Determination.

\section{DETERMINATION}

1. Pass the $7 \underline{\mathrm{N}} \mathrm{HCl}$ sample solution obtained during sample preparation through a prepared anion exchange column (see Note 3). Discard the column effluent.

2. Wash the column with $400 \mathrm{~mL}$ of $\underline{7 \mathrm{~N} \mathrm{HCl}}$. Discard the washings.

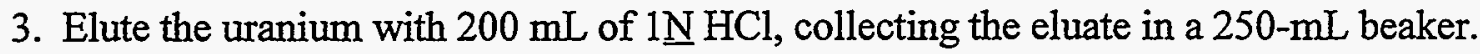
Discard the resin.

4. Evaporate the eluate to near dryness.

5. Destroy any residual organic material with dropwise additions of $\mathrm{HNO}_{3}$.

6. Evaporate the solution to dryness. Dissolve the residue in a few drops of $\mathrm{HCl}$.

7. Convert the solution to the chloride with three 5-mL additions of $\mathrm{HCl}$.

8. Add 1-2 $\mathrm{mL}$ of $1 \underline{\mathrm{N}} \mathrm{HCl}$, prepared with filtered water (see Procedure G-03, Microprecipitation Source Preparation for Alpha Spectrometry). Cool to room temperature. 
9. Continue the analysis under Procedure G-03, Microprecipitation Source Preparation for Alpha Spectrometry.

\section{Notes:}

1. Freeze-dried or wet tissue may be wet ashed directly in $\mathrm{HNO}_{3}$. Proceed with Step 3 of Vegetation and Soft Tissue.

2. It is necessary to analyze reagent blanks with each batch of samples to correct the $U$ results.

3. $20 \mathrm{~mL}$ of Bio-Rad AG1-X4, prepared according to Specification 7.4 are conditioned with $500 \mathrm{~mL}$ of $7 \mathrm{~N} \mathrm{HCl}$.

\section{LOWER LIMT OF DETECTION (LLD)}

\begin{tabular}{lll} 
Uranium Isotopes & & \\
Counter Efficiency & $(\%)$ & 40 \\
Counter Background & $(\mathrm{cps})$ & $3.33 \times 10^{-6}$ for ${ }^{238} \mathrm{U}$ \\
& & $3.33 \times 10^{-6}$ for ${ }^{234} \mathrm{U}$ \\
Yield & $(\%)$ & 85 \\
Blank & $(\mathrm{cps})$ & $3.33 \times 10^{-6}$ for ${ }^{238} \mathrm{U}$ \\
& & $3.33 \times 10^{-5}$ for ${ }^{234} \mathrm{U}$ \\
LLD (400 min) & $(\mathrm{mBq})$ & 0.0002 for ${ }^{238} \mathrm{U}$ \\
& & 0.0005 for ${ }^{234} \mathrm{U}$ \\
LLD (1000 min) & $(\mathrm{mBq})$ & 0.0001 for ${ }^{238} \mathrm{U}$ \\
& & 0.0003 for ${ }^{234} \mathrm{U}$ \\
LLD (5000 min) & $(\mathrm{mBq})$ & 0.0005 for ${ }^{238} \mathrm{U}$ \\
& & 0.0001 for ${ }^{234} \mathrm{U}$ \\
\hline
\end{tabular}




\section{REFERENCES}

Hindman, F. D.

"Neodymium Fluoride Mounting for Alpha Spectrometric Determination of Uranium, Plutonium and Americium"

Anal. Chem., 55, 2460-2461 (1983)

Sill, C. W. and R. L. Williams

"Preparation of Actinides for Alpha Spectrometry without Electrodeposition"

Anal. Chem., 53, 412-415 (1981)

Welford, B. A. , R. S. Morse and J. S. Alercio

"Urinary Uranium Levels in Non-Exposed Individuals"

Am. Ind. Hyg. Asso. J., 21 (1960) 


\begin{abstract}
APPLICATION
This procedure has been used to analyze $50 \mathrm{~g}$ human bone ash samples (Fisenne et al., 1980; Hindman, 1983; Sill and Williams, 1981).

Bone ash is dissolved in acid, and the $U$ is equilibrated with ${ }^{232} U$ tracer and isolated by solvent extraction. The purified $U$ isotopes are microprecipitated for $\alpha$ spectrometry.
\end{abstract}

\title{
SPECIAL APPARATUS
}

1. Mechanical shaker.

2. Polyethylene dispensing bottle - see Specification 7.10.

3. Special apparatus for the microprecipitation of $U$ are listed under the generic procedure, G-03.

\section{SPECIAL REAGENTS}

1. Uranium-232 tracer solution - about $0.1 \mathrm{~Bq} \mathrm{~g}^{-1}$ of solution in a dispensing bottle.

2. Alamine-336, tertiary tricaprylyl amine (Henkel Corporation, 2430 N. Huachuca Dr., Tucson, AZ 85745-1273) 3:7 in toluene. Wash twice with an equal volume of 1:1 $\mathrm{HCl}$. Prepare $100 \mathrm{~mL}$ of acid-washed 3:7 Alamine-336 for each sample. 
3. Standardized sodium hydroxide $0.1 \underline{\mathrm{N}}$ - dissolve $4 \mathrm{~g}$ of $\mathrm{NaOH}$ in $\mathrm{H}_{2} \mathrm{O}$ and dilute to $1 \mathrm{~L}$. Standardize the solution against potassium acid phthalate.

4. Phenolphthalein indicator - dissolve $0.5 \mathrm{~g}$ of reagent in $100 \mathrm{~mL}$ of $95 \%$ ethanol.

\section{SAMPLE PREPARATION}

1. Weigh $50 \mathrm{~g}$ of ground, dry-ashed bone and transfer to a $400-\mathrm{mL}$ beaker.

2. Add a weighed amount of ${ }^{232} \mathrm{U}$ tracer ( $0.01 \mathrm{~Bq}$ per sample) from the dispensing bottle. (Note: It is necessary to analyze reagent blanks with each batch of samples to correct the U results.)

3. Add $100 \mathrm{~mL}$ of $\mathrm{HCl}$ and heat gently on a hot plate for $10 \mathrm{~min}$ with occasional stirring.

4. Add $70 \mathrm{~mL}$ of $\mathrm{H}_{2} \mathrm{O}$ and stir to obtain a clear solution. If insoluble material is present, filter the sample through a glass fiber filter. Wash the filter with $1: 1 \mathrm{HCl}$ and discard the residue.

5. Cool the solution. Transfer a $100-\mu \mathrm{L}$ aliquot of the sample into a $150-\mathrm{mL}$ beaker containing $25 \mathrm{~mL}$ of $\mathrm{H}_{2} \mathrm{O}$. Add two to three drops of $0.5 \%$ phenolphthalein indicator. Stir and titrate the solution with $0.1 \underline{\mathrm{N}} \mathrm{NaOH}$ to the pink endpoint. Calculate the normality of the sample solution.

6. If the normality is $>5.8 \mathrm{~N}$ in $\mathrm{HCl}$, proceed directly to the extraction. If the normality is $<5.8 \underline{\mathrm{N}}$, transfer the sample to a $250-\mathrm{mL}$ graduated cylinder and record the volume. Return the sample to the beaker and add an appropriate volume of $\mathrm{HCl}$ to the cylinder to increase the sample acid concentration to $\underline{N}$. Transfer the acid to the sample beaker and proceed with the extraction. 


\section{DETERMMNATION}

1. Transfer $50 \mathrm{~mL}$ of acid-washed Alamine-336 into each of two $500-\mathrm{mL}$ separatory funnels.

2. Transfer the sample to the first separatory funnel. Wash the beaker with $1: 1 \mathrm{HCl}$ and add the washings to the funnel.

3. Shake the separatory funnel for $5 \mathrm{~min}$. Allow the phases to separate and draw off the aqueous (lower) phase into the second separatory funnel. Retain the organic phase in the first funnel.

4. Shake the second separatory funnel for $5 \mathrm{~min}$. Allow the phases to separate, draw off, and then discard the aqueous (lower) phase.

5. Combine the two organic phases in one of the separatory funnels.

6. Wash the organic phase four times for $5 \mathrm{~min}$ with equal volumes of $1: 1 \mathrm{HCl}$. Discard the washings.

7. Strip the U from the organic phase by shaking twice for $5 \mathrm{~min}$ with $100-\mathrm{mL}$ portions of $0.1 \mathrm{~N} \mathrm{HCl}$. Combine the strip solutions in a $400-\mathrm{mL}$ beaker.

8 Evaporate the solution to near dryness.

9. Destroy any residual organic material with dropwise additions of $\mathrm{HNO}_{3}$.

10. Evaporate the solution to dryness. Dissolve the residue in a few drops of $\mathrm{HCl}$.

11. Convert the solution to the chloride with three $5-\mathrm{mL}$ additions of $\mathrm{HCl}$.

12. Add 1-2 $\mathrm{mL}$ of $1 \underline{\mathrm{N}} \mathrm{HCl}$, prepared with filtered water (see Procedure G-03, Microprecipitation Source Preparation for Alpha Spectrometry). Cool to room temperature. 
13. Continue the analysis under Procedure G-03, Microprecipitation Source Preparation for Alpha Spectrometry.

\section{LOWER LIMT OF DETECTION (LLD)}

\begin{tabular}{lll}
\hline Counter Efficiency & $(\%)$ & 40 \\
Counter Background & $(\mathrm{cps})$ & $3.33 \times 10^{-6}$ for ${ }^{238} \mathrm{U}$ \\
& & $6.67 \times 10^{-6}$ for ${ }^{234} \mathrm{U}$ \\
Yield & $(\%)$ & 90 \\
Blank & $(\mathrm{cps})$ & $3.33 \times 10^{-6}$ for ${ }^{238} \mathrm{U}$ \\
& & $3.30 \times 10^{-5}$ for ${ }^{234} \mathrm{U}$ \\
LLD (400 min) & $(\mathrm{mBq})$ & 0.2 for ${ }^{238} \mathrm{U}$ \\
& & 0.5 for ${ }^{234} \mathrm{U}$ \\
LLD (1000 min) & $(\mathrm{mBq})$ & 0.1 for ${ }^{238} \mathrm{U}$ \\
& & for ${ }^{234} \mathrm{U}$ \\
LLD (5000 min $)$ & $(\mathrm{mBq})$ & 0.05 for ${ }^{238} \mathrm{U}$ \\
& & 0.1 for ${ }^{234} \mathrm{U}$ \\
\end{tabular}




\section{REFERENCES}

Fisenne, I. M., P. M. Perry, and G. A. Welford

"Determination of Uranium Isotopes in Human Bone Ash"

Anal. Chem., $\underline{52}, 777-779$ (1980)

Hindman, F. D.

"Neodymium Fluoride Mounting for Alpha Spectrometric Determination of Uranium, Plutonium and Americium"

Anal. Chem., 55, 2460-2461 (1983)

Sill, C. W. and R. L. Williams

"Preparation of Actinides for Alpha Spectrometry without Electrodeposition"

Anal. Chem., 53, 412-415 (1981) 
URANIUM IN BIOLOGICAL AND ENVIRONMENTAL MATERIALS

(see Volume II) 


\subsubsection{Generic Procedures}


ELECTRODEPOSITION OF THE ACTINIDES: MITCHELL METHOD

Contact Person(s) : Isabel M. Fisenne

\begin{abstract}
APPLICATION
This method describes the preparation of separated actinide fractions for $\alpha$-spectrometry measurement (Mitchell, 1960). It is applicable to any of the actinides that can be dissolved in $\mathrm{HCl}$ solution. The actinide fractions would be either the final elution from an ion exchange separation or the final strip from a solvent extraction separation.
\end{abstract}

\title{
SPECIAL APPARATUS
}

1. Electrodeposition apparatus - see Specification 7.15.

2. Electrolytic cells - see Specification 7.16.

3. Platinum - iridium stirrer - see Specification 7.15.

4. Virgin platinum discs $-17.6 \mathrm{~mm} \times 0.006 \mathrm{~mm}$; mirrored finish on one side.

5. Ring and discs - Specification 7.2.

6. Mylar film - see Specification 7.3.

\section{SPECIAL REAGENTS}

Methyl red indicator solution $0.1 \%$ in ethanol - Fisher Chemical Co. 


\section{ELECTRODEPOSITION}

1. Add $5 \mathrm{~mL}$ of concentrated $\mathrm{HNO}_{3}$ to the separated actinide fraction. Evaporate the solution to dryness but do not bake.

2. Dissolve the residue in $2 \mathrm{~mL}$ of $\mathrm{HCl}$ and evaporate to near dryness. Repeat the $\mathrm{HCl}$ and evaporate to near dryness. Repeat the $\mathrm{HCl}$ addition and evaporation twice more.

3. Add $1 \mathrm{~mL}$ of $\mathrm{HCl}$ and heat the solution gently. Transfer the solution to an electrodeposition cell.

4. Rinse and police the beaker with two successive $1-\mathrm{mL}$ portions of water. Add the rinsing to the cell.

5. Add one drop of methyl red indicator solution to the cell and swirl the solution to $\operatorname{mix.}$

6. While swirling the solution in the cell, add 7.5 $\mathrm{N} \mathrm{NH}_{4} \mathrm{OH}$ until the pink color just disappears.

7. Adjust the solution to a persistent pink color by dropwise additions of $2 \mathrm{~N} \mathrm{HCl}$ with swirling to mix. Add two drops of $2 \mathrm{~N} \mathrm{HCl}$ in excess.

8. Dilute the solution in the cell to $5 \mathrm{~mL}$ with water.

9. With the platinum anode in the chuck of the stirring motor, lower the anode to about $1 \mathrm{~cm}$ above the platinum disc in the electrodeposition cell. Turn on the stirring motor and adjust the speed to prevent splattering.

10. Turn on the current and adjust the flow to $1.2 \mathrm{~A}$. Electrodeposit with the rotating anode for $1 \mathrm{~h}$. (Note: At EML the electrodeposition cell is supported on a lucite pedestal which is immersed in an ice water bath to minimize evaporation of the sample solution.)

11. Add $1 \mathrm{~mL}$ of $\mathrm{NH}_{4} \mathrm{OH}$ to the cell to quench the electrolyte. 
12. Turn off the stirring moter. Raise the stirring motor and anode assembly, and turn off the power supply. Pour off and discard the electrolyte solution. Rinse the cell with water and discard the rinse.

13. Disassemble the cell and rinse the disc with ethyl alcohol solution. Touch the edge of the disc with a tissue to absorb the excess alcohol.

14. Place the disc on a 200 to $250^{\circ} \mathrm{C}$ hot plate to dry.

15. Submit the sample for alpha spectrometry measurement.

\section{INTERFERENCES}

Any element present in the separated fraction that is able to be electrodeposited will be present on the metal disc. In particular, ${ }^{210} \mathrm{~Pb}(5.30 \mathrm{MeV})$ deposited on the disc would interfere with the yield determination of ${ }^{232} \mathrm{U}(5.32 \mathrm{MeV})$ or ${ }^{243} \mathrm{Am}(5.28 \mathrm{MeV})$ tracers used in the determination of isotopic $U$ and ${ }^{241} \mathrm{Am}$, respectively.

Incomplete separation of rare earth elements or incomplete wet ashing for the removal of organic materials will decrease the efficiency of the electrodeposition and may result in a thick deposit unsuitable for $\alpha$-spectrometry measurement.

Samples containing more than $20 \mu \mathrm{g}$ of $\mathrm{U}$ are unsuitable for measurement by $\alpha$ spectrometry due to the thickness of the deposit.

\section{REFERENCE}

Mitchell, R. F.

"Electrodeposition of Actinide Elements at Tracer Concentrations"

Anal. Chem., 32, 326-328 (1960) 


\title{
ELECTRODEPOSITION OF THE ACTINDES: TALVITIE METHOD
}

Contact Person(s) : Isabel M. Fisenne and Pamela M. Perry

\begin{abstract}
APPLICATION
This method describes the preparation of separated actinide fractions $\alpha$-spectrometry measurement (Talvitie, 1972). It is applicable to any of the actinides that can be dissolved in dilute ammonium sulfate solution. Examples of applicable actinide fractions would be the final elution from an ion exchange separation or the final strip from a solvent extraction separation.
\end{abstract}

\section{SPECIAL APPARATUS}

1. Electrodeposition apparatus - see Specification 7.15.

2. Electrolytic cells - see Specification 7.16.

3. Platinum - iridium stirrer - see Specification 7.15.

4. Virgin platinum discs $-17.6 \mathrm{~mm} \times 0.006 \mathrm{~mm}$; mirrored finish on one side.

\section{SPECIAL REAGENTS}

Thymol blue indicator, sodium salt solution ( $0.04 \%)$ - dissolve $0.04 \mathrm{~g}$ of the salt in $100 \mathrm{~mL}$ of $\mathrm{H}_{2} \mathrm{O}$. 


\section{ELECTRODEPOSITION}

1. Add $2 \mathrm{~mL}$ of $0.36 \mathrm{M} \mathrm{NaHSO}_{4}$ to the separated actinide fraction. Add $5 \mathrm{~mL}$ of concentrated $\mathrm{HNO}_{3}$, swirl to mix, and evaporate the solution to dryness but do not bake.

2. Dissolve the residue in $5 \mathrm{~mL}$ of electrolyte solution and warm the solution.

3. Transfer the sample solution to the electrodeposition cell. Rinse the beaker with 5 to $10 \mathrm{~mL}$ of electrolyte solution and add the rinsing to the electrodeposition cell.

4. Add tbree to four drops of thymol blue indicator to the sample and adjust the $\mathrm{pH}$ with $1.8 \mathrm{M} \mathrm{H}_{2} \mathrm{SO}_{4}$ or concentrated $\mathrm{NH}_{4} \mathrm{OH}$, or both, until a salmon pink color persists.

5. With the platinum anode in the chuck of the stirring motor, lower the anode to about 1 $\mathrm{cm}$ above the stainless steel disc in the electrodeposition cell. Turn on the stirring motor and adjust the speed to prevent spattering.

6. Turn on the current and adjust the flow to $1.2 \mathrm{~A}$. Electrodeposit with the rotating anode for 1 hour. (Note: At EML the electrodeposition cell is supported on a lucite pedestal which is immersed in an ice water bath to minimize evaporation of the sample solution.)

7. After $1 \mathrm{~h}$, add $1 \mathrm{~mL}$ of $\mathrm{NH}_{4} \mathrm{OH}$ to the cell to quench the electrolyte.

8. Turn off the stirring motor. Raise the stirring motor and anode assembly and turn off the power supply. Pour off and discard the electrolyte solution. Rinse the cell with three successive portions of $0.15 \underline{\mathrm{M} \mathrm{HN}} \mathrm{H}_{4} \mathrm{OH}$. Discard the rinsings.

9. Disassemble the cell and rinse the disc with ethyl alcohol. Touch the edge of the disc with a tissue to absorb the excess alcohol.

10. Place the disc on a 200 to $250^{\circ} \mathrm{C}$ hot plate to dry.

11. Submit the sample for $\alpha$-spectrometry measurement. 


\section{INTERFERENCES}

1. Any element present in the separated fraction that is able to be electrodeposited will be present on the metal disc. In particular, ${ }^{210} \mathrm{~Pb}(5.30 \mathrm{MeV})$ deposited on the disc would interfere with the yield determination of ${ }^{232} \mathrm{U}(5.32 \mathrm{MeV})$ or ${ }^{243} \mathrm{Am}(5.28 \mathrm{MeV})$ tracers used in the determination of isotopic $U$ and ${ }^{241} \mathrm{Am}$, respectively.

2. Incomplete separation of rare earth elements or incomplete wet ashing for the removal of organic material will decrease the efficiency of the electrodeposition and may result in a thick deposit unsuitable for $\alpha$-spectrometry measurement.

3. Samples containing more than $20 \mu \mathrm{g}$ of $U$ are unsuitable for measurement by $\alpha$ spectrometry due to the thickness of the deposit.

\section{REFERENCE}

Talvitie, N. A.

"Electrodeposition of Actinides for Alpha Spectrometric Determination"

Anal. Chem., 44, 280-283 (1972) 
G-03

MICROPRECIPITATION SOURCE PREPARATION

FOR ALPHA SPECTROMETRY

Contact Person(s) : Isabel M. Fisenne

\begin{abstract}
APPLICATION
Microprecipitates of Th, $\mathrm{U}, \mathrm{Pu}, \mathrm{Am}$, and $\mathrm{Cm}$, suitable for $\alpha$-spectrometry measurements, are prepared by coprecipitation with $\mathrm{Nd}$ as the $\mathrm{F}$. [Adapted from Sill and Williams (1981) and Hindman (1983).]
\end{abstract}

\title{
SPECIAL APPARATUS
}

1. Ultrasonic bath.

2. Millipore $47 \mathrm{~mm}$ diameter Pyrex glass filtration chimney, fitted glass support and metal clamp.

3. Millipore $47 \mathrm{~mm}$ diameter filters, $0.45 \mu \mathrm{m}$ pore size.

4. Gelman $25 \mathrm{~mm}$ diameter polysulfone filtration chimney, stem support and stainless steel screen.

5. Gelman $25 \mathrm{~mm}$ Metricel filter, $0.1 \mu \mathrm{m}$ pore size.

6. Eppendorf $100 \mu \mathrm{L}$ pipette or equivalent.

7. $100 \mu \mathrm{L}$ disposable pipette tips. 
8. $10 \mathrm{~mL}$ plastic pipette.

9. $10 \mathrm{~mL}$ plastic culture tubes.

10. Pipetting bulb.

11. $50 \mathrm{~mL}$ plastic graduated cylinder.

12. $10 \mathrm{~mL}$ plastic graduated cylinder.

13. $20 \mathrm{~L}$ plastic carboy with spigot.

14. $2 \mathrm{~L}$ vacuum filtration flask.

15. $250 \mathrm{~mL}$ vacuum filtration flask.

Special Precautions - Due to the use of HF in the preparation of the reagents and in the precipitation procedure, rubber gloves must be worn and plasticware must be used as noted above.

\section{SPECIAL REAGENTS}

1. Filtered deionized water - filter $20 \mathrm{~L}$ of deionized water through $0.45 \mu \mathrm{m}$ pore size Millipore filters. Store the filtered water in a 20-L capacity plastic carboy with a spigot (see Note 1).

2. $1 \mathrm{~N} \mathrm{HCl}$ - add $83 \mathrm{~mL}$ of concentrated $\mathrm{HCl}$ to $917 \mathrm{~mL}$ of filtered deionized water and store in a plastic bottle.

3. Neodymium carrier solution, $1000 \mu \mathrm{g} \mathrm{mL}^{-1}$ (Spex Industries, Wayne, NJ), or equivalent (see Note 2).

4. Neodymium carrier solution, $0.5 \mathrm{mg} \mathrm{mL}^{-1}$. Dilute $10 \mathrm{~mL}$ of the $1000 \mu \mathrm{g} \mathrm{mL}^{-1} \mathrm{Nd}$ carrier solution to $20 \mathrm{~mL}$ with filtered deionized water. 
5. $48 \% \mathrm{HF}$.

6. Neodymium fluoride substrate solution - $10 \mu \mathrm{g} \mathrm{mL}^{-1}$ - pipette $5 \mathrm{~mL}$ of Nd carrier $\left(1000 \mu \mathrm{g} \mathrm{mL}^{-1}\right)$ into a $500-\mathrm{mL}$ plastic bottle. Add $460 \mathrm{~mL}$ of $1 \underline{\mathrm{N}} \mathrm{HCl}$ to the plastic bottle. Cap the bottle and shake to mix. Measure $40 \mathrm{~mL}$ of $48 \% \mathrm{HF}$ in a plastic graduated cylinder. Uncap the bottle and add the HF. Recap the bottle and shake to mix thoroughly.

7. $0.58 \mathrm{~N} \mathrm{HF}$ - pour $980 \mathrm{~mL}$ of filtered deionized water into a $1 \mathrm{~L}$ pastic bottle. Measure $20 \mathrm{~mL}$ of $48 \% \mathrm{HF}$ in a plastic graduated cylinder. Uncap the bottle and add the HF. Recap the bottle tightly and shake to mix.

8. Ethyl alcohol, $100 \%$.

9. Ethyl alcohol, $80 \%-\mathrm{mix} 800 \mathrm{~mL}$ of $100 \%$ ethyl alcohol and $200 \mathrm{~mL}$ of filtered deionized water. Store in a $1 \mathrm{~L}$ plastic bottle.

10. Titanium trichloride, $20 \%$ solution (see Note 3 ).

Notes:

1. Deionized water may contain a sufficient quantity of solid material to adversely effect the resolution of the final filtered sample.

2. Neodymium is preferred as a carrier for the determination of thorium. Cerium compounds tend to contain variable and measurable quantities of thorium.

3. Titanium trichloride is an extremely powerful reducing agent, which should be used in a well-ventilated hood.

\section{PRECIPITATION OF Th, Pu, Am, AND Cm}

1. The separated $\mathrm{Th}, \mathrm{Pu}, \mathrm{Am}$ or $\mathrm{Cm}$ solution for precipitation should be in a 1-2 $\mathrm{mL}$ volume of $1 \underline{\mathrm{N}} \mathrm{HCI}$ or $1 \underline{\mathrm{N} \mathrm{HNO}} \mathrm{HN}_{3}$ solution. (The conditions for the precipitation of $\mathrm{U}$ are noted separately.) 
2. Transfer the solution to a $10-\mathrm{mL}$ plastic culture tube. Wash the original sample vessel twice with 1-mL washes of the same concentration acid as the sample. Transfer the washings to the culture tube. Mix by gently shaking the tube.

3. Add $100 \mu \mathrm{L}$ of the $0.5 \mathrm{mg} \mathrm{mL}^{-1} \mathrm{Nd}$ carrier solution to the tube with an Eppendorf pipette. Gently shake the tube to mix the solution.

4. Add 10 drops $(0.5 \mathrm{~mL})$ of $48 \% \mathrm{HF}$ to the tube and $\mathrm{mix}$ well by gentle shaking.

5. Place the tube in a cold-water ice bath for at least $30 \mathrm{~min}$.

6. Insert the polysulfone filter stem in the $250-\mathrm{mL}$ vacuum flask. Place the stainless steel screen on top of the fitted plastic filter stem.

7. Place a $25-\mathrm{mm}$ Metricel filter on the stainless steel screen. Caution - place the less glossy side of the Metricel filter face up. The filters are usually shipped in the box in this manner, but the analyst should check each filter visually.

8. Wet the filter with $100 \%$ ethyl alcohol. Center the filter on the stainless steel screen support and apply a vacuum.

9. Lock the filter chimney firmly in place on the filter stem. Open the system to full vacuum.

10. Wash the filter with $100 \%$ ethyl alcohol, followed by a filtered deionized water wash.

11. Draw $5000 \mu \mathrm{L}(5 \mathrm{~mL})$ of $\mathrm{Nd}$ substrate solution into a plastic pipette.

12. Add $5 \mathrm{~mL}$ of the Nd substrate solution down the side of the filter chimney. Apply a vacuum to the filter for at least $15 \mathrm{sec}$.

13. Repeat Steps 11 and 12 with an additional $5000 \mu \mathrm{L}$ of the substrate solution.

14. Place the sample to be filtered in a $150 \mathrm{~mL}$ beaker containing $25 \mathrm{~mL}$ of $\mathrm{H}_{2} \mathrm{O}$. Set the beaker in an ultrasonic unit containing about a $2.54 \mathrm{~cm}$ depth of $\mathrm{H}_{2} \mathrm{O}$. 
15. Ultrasonicate the sample tube for about $1 \mathrm{~min}$ to suspend the $\mathrm{NdF}_{3}$ precipitate.

16. Pour the sample down the side of the filter chimney and apply a vacuum.

17. Add about $2 \mathrm{~mL}$ of $0.58 \mathrm{~N}$ HF to the tube and ultrasonicate briefly. Pour the wash down the side of the filter chimney.

18. Repeat Step 17.

19. Add about $2 \mathrm{~mL}$ of filtered deionized water to the tube and ultrasonicate briefly. Pour the wash down the side of the filter chimney.

20. Repeat Step 19.

21. Add about $2 \mathrm{~mL}$ of $80 \%$ ethyl alcohol to the tube and ultrasonicate briefly. Pour the wash down the side of the filter chimney.

22. Repeat Step 21.

23. Wash any drops remaining on the sides of the chimney down toward the filter with $80 \%$ ethyl alcohol. Caution - Directing of a stream of liquid onto the filter will disturb the distribution of the precipitate on the filter and render the sample unsuitable for $\alpha$-spectrometry resolution.

24. Without turning off the vacuum, remove the filter chimney.

25. Reduce or turn off the vacuum to remove the filter. Discard the filtrate. (Caution - If the filtrate is to be retained, it should be placed in a plastic container to avoid dissolution of the glass vessel by dilute HF.)

26. Place the filter directly on a suitable mounting disc. Secure with a mounting ring.

27. Place the mounted sample under a heat lamp (sample to lamp distance should be about $10 \mathrm{~cm}$ ) for $10 \mathrm{~min}$ prior to $\alpha$-spectrometry measurement. 


\section{PRECIPITATION OF U}

1. The $\mathrm{U}$ bearing solution must be in $\mathrm{HCl}$ solution.

2. Transfer $1-2 \mathrm{~mL}$ of the $U$ bearing solution ( $1 \underline{\mathrm{N}} \mathrm{HCl}$ ) to a $10-\mathrm{mL}$ plastic culture tube. Wash the original sample vessel twice with $1-\mathrm{mL}$ portions of $1 \underline{\mathrm{N}} \mathrm{HCl}$. Transfer the washings to the culture tube. Mix by gently shaking the tube.

3. Add $100 \mu \mathrm{L}$ of the $0.5 \mathrm{mg} \mathrm{mL}^{-1} \mathrm{Nd}$ carrier with an Eppendorf pipette. Gently shake the tube to mix the solution.

4. Add four drops of $20 \% \mathrm{Ti}$ trichloride to the tube and gently shake the tube. A strong permanent violet color should appear. If the color fails to appear, add a few more drops of Ti trichloride.

5. Continue the precipitation from Step 4, Precipitation of $\mathrm{Th}, \mathrm{Pu}, \mathrm{Am}$, and $\mathrm{Cm}$.

\section{REFERENCES}

Hindman, F. D.

"Neodymium Fluoride Mounting for Alpha Spectrometric Determination of Uranium, Plutonium, and Americium"

Anal. Chem., 55, 2460-2461 (1983)

Sill, C. W. and R. L. Williams

"Preparation of Actinides for Alpha Spectrometry Without Electrodeposition"

Anal. Chem., $\underline{53}, 421-415$ (1981) 


\subsubsection{Sequential Analyses}




\begin{abstract}
APPLICATION
This procedure is applicable to $5 \mathrm{~g}$ soil samples, $10 \mathrm{~g}$ vegetation and fecal ash samples and ion exchange resin from sampling columns. [Adapted from Fisenne et al. (1980), Hindman (1983), Sill (1961), and Sill and Williams (1981).]

Tracers and carriers are added to the sample which is pretreated with $\mathrm{HNO}_{3}-\mathrm{HF}$, fused with $\mathrm{KF}$, and transposed to pyrosulfate. The cake is dissolved in $\mathrm{HCl}$. Radium/ barium sulfate is precipitated, filtered, and dissolved in alkaline EDTA. The chemical yield for $\mathrm{Ra}$ is determined with the $\gamma$-emitting tracer ${ }^{133} \mathrm{Ba}$. Radium-226 is determined by ${ }^{222} \mathrm{Rn}$ emanation.
\end{abstract}

Uranium is isolated by solvent extraction. The $U$ isotopes are coprecipitated with $\mathrm{Nd}$ as the $\mathrm{F}^{-}$for measurement by $\alpha$ spectrometry.

\title{
SPECIAL APPARATUS
}

1. $100-\mathrm{mL}$ platinum dishes or $250-\mathrm{mL}$ platinum crucibles.

2. Millipore glass filtration chimney, fritted glass support, and metal clamp - $47 \mathrm{~mm}$ diameter.

3. Millipore filters $-47 \mathrm{~mm}$ diameter, $0.45 \mu \mathrm{m}$ pore size.

4. Polyethylene dispensing bottle or similar - see Specification 7-11.

5. Mechanical shaker. 
6. Special apparatus for the microprecipitation of $U$ are listed under the generic procedure, G-03.

\section{SPECIAL REAGENTS}

1. ${ }^{133} \mathrm{Ba}$ tracer solution - about $50 \mathrm{cps}$ per $0.1 \mathrm{~g}$ aliquot, prepared in $1: 99 \mathrm{HCl}$.

2. Barium carrier solution $\left(20 \mathrm{mg} \mathrm{mL}^{-1}\right)-30.4 \mathrm{~g} \mathrm{BaCl}_{2} \mathrm{~L}^{-1}$ in $1: 99 \mathrm{HCl}$.

3. EDTA solution - $300 \mathrm{~g}$ tetrasodium salt of EDTA $\mathrm{L}^{-1}$ in $\mathrm{H}_{2} \mathrm{O}$.

4. Triethanolamine $-1: 1$ in $\mathrm{H}_{2} \mathrm{O}$.

5. ${ }^{232} \mathrm{U}$ tracer solution - about $0.17 \mathrm{~Bq} \mathrm{~g}^{-1}$ of solution in a dispensing bottle.

6. Alamine-336, tertiary tricaprylyl amine (Henkel Company, 2430 N. Huachuca Dr., Tucson, AZ 85745-1273) 3:7 in toluene. Wash twice with an equal volume of 1:1 $\mathrm{HCl}$. Prepare $100 \mathrm{~mL}$ of acid-washed 3:7 Alamine-336 for each sample.

\section{SAMPLE PREPARATION}

\section{A. Soil and vegetation.}

1. Weigh $5 \mathrm{~g}$ of soil or $10 \mathrm{~g}$ of fecal or vegetation ash into a $100-\mathrm{mL}$ platinum dish. Add a weighed aliquot (about $0.1 \mathrm{~g}$ ) of ${ }^{133} \mathrm{Ba}$ tracer solution and $1 \mathrm{~mL}$ of $\mathrm{Ba}$ carrier solution. Add a weighed aliquot of ${ }^{232} \mathrm{U}$ tracer solution (about $1 \mathrm{~g}$ for soil and about $0.5 \mathrm{~g}$ for fecal or vegetation ash).

2. Slowly add $10 \mathrm{~mL}$ of $\mathrm{HNO}_{3}$ and $10 \mathrm{~mL}$ of $\mathrm{HF}$ to the sample and evaporate on a hot plate to near dryness.

3. Continue the analysis as described under Determination. 


\section{B. Ion exchange resin.}

1. Transfer the resin and paper pulp from the collection column (see Specification 7.4) to a $250-\mathrm{mL}$ platinum crucible. Dry under a heat lamp and ash at $500^{\circ} \mathrm{C}$ in a muffle furnace for $48 \mathrm{~h}$.

2. To the cooled crucible, add a weighed aliquot (about $0.1 \mathrm{~g}$ ) of ${ }^{133} \mathrm{~B}$ a tracer solution and $1 \mathrm{~mL}$ of Ba carrier solution. Add a weighed aliquot (about $0.1 \mathrm{~g}$ ) of ${ }^{232} \mathrm{U}$ tracer solution.

3. Continue the analysis as described under Determination.

\section{DETERMINATION}

\section{A. Radium separation.}

1. Weigh out $15 \mathrm{~g}$ of $\mathrm{KF}$ and add to the sample. Press the $\mathrm{KF}$ into the sample with a plastic spatula.

Caution: Wear rubber gloves and safety glasses during Steps 2-5.

2. Fuse the sample over an air-fed Meker burner, gradually increasing the temperature until a clear melt is obtained. Cool the melt.

3. Using a burette, slowly add $17.5 \mathrm{~mL}$ of $\mathrm{H}_{2} \mathrm{SO}_{4}$ to the melt. Heat the dish on a hot plate until the cake dissolves and evaporate until $\mathrm{SO}_{3}$ fumes appear.

4. Weigh out $10 \mathrm{~g}$ of $\mathrm{Na}_{2} \mathrm{SO}_{4}$, add to the dish and fuse over a blast burner until a clear melt accompanied by dense fumes is obtained. Cool the melt.

5. Transfer the cake to a $600-\mathrm{mL}$ beaker containing $350 \mathrm{~mL}$ of hot water and $25 \mathrm{~mL}$ of $\mathrm{HCl}$. Stir the solution to dissolve the cake. Cool for $1 \mathrm{~h}$. 
6. Filter the precipitate onto a $47 \mathrm{~mm}$ diameter $0.45 \mu \mathrm{m}$ pore size Millipore filter, police the beaker and wash with $\mathrm{H}_{2} \mathrm{O}$. Reserve the filtrate for $\mathrm{U}$ separation.

7. Using a strong stream of $\mathrm{H}_{2} \mathrm{O}$ from a wash bottle, transfer the precipitate to a $150-\mathrm{mL}$ beaker. Discard the filter.

8. Add $5 \mathrm{~mL}$ of EDTA solution and $1 \mathrm{~mL}$ of $1: 1$ triethanolamine to the beaker. Heat on a warm hot plate for about $15 \mathrm{~min}$, adding $10 \mathrm{~mL}$ of $\mathrm{H}_{2} \mathrm{O}$ and stirring occasionally. Reduce the sample volume to about $15 \mathrm{~mL}$.

9. Filter the warm solution by gravity through a Whatman No. 42 filter paper into a $30-\mathrm{mL}$ polyethylene bottle.

10. Wash the beaker and filter with hot water. Discard the filter.

11. Dilute the sample to the same liquid level as a known aliquot (about $0.1 \mathrm{~g}$ ) of the ${ }^{133} \mathrm{Ba}$ tracer solution diluted to $25 \mathrm{~mL}$ in a $30-\mathrm{mL}$ polyethylene bottle.

12. Gamma count the samples and standard to determine the chemical yield of Ba.

13. Transfer the sample to a ${ }^{222} \mathrm{Rn}$ bubbler with $\mathrm{H}_{2} \mathrm{O}$.

14. De-emanate ${ }^{222} \mathrm{Rn}$ by bubbling with forming gas for about $10 \mathrm{~min}$ at $100 \mathrm{~mL} \mathrm{~min} \mathrm{~m}^{-1}$ as described in the Emanation Procedure for ${ }^{226} \mathrm{Ra}$, Ra-03. Record the time as the starting time for ${ }^{222} \mathrm{Rn}$ buildup. Continue the analysis by the emanation technique.

\section{B. Uranium separation.}

1. Evaporate the reserved filtrate (Radium Separation, Step 6) to a 150-mL volume.

2. Add $150 \mathrm{~mL}$ of $\mathrm{HCl}$ to the sample solution and stir.

3. Remove the sample from the hot plate and cool to room temperature.

4. Transfer $100 \mathrm{~mL}$ of acid washed Alamine-336 into a 500-mL separatory funnel. 
5. Transfer the sample solution into the separatory funnel. Wash the beaker with $1: 1$ $\mathrm{HCl}$ and add the washings to the funnel.

6. Shake the separatory funnel for $5 \mathrm{~min}$. Allow the phases to separate. Draw off the aqueous (lower) phase and discard.

7. Wash the organic phase four times for $5 \mathrm{~min}$ with equal volumes of $1: 1 \mathrm{HCl}$. Discard the washings.

8. Strip the $U$ from the organic phase by shaking twice for $5 \mathrm{~min}$ with $100-\mathrm{mL}$ portions of $0.1 \underline{\mathrm{N}} \mathrm{HCl}$. Combine the strip solutions in a $400-\mathrm{mL}$ beaker. Place the organic phase in a suitable container for disposal.

9. Evaporate the strip solution to near dryness.

10. Destroy any residual organic material with dropwise additions of $\mathrm{HNO}_{3}$.

11. Evaporate the solution to dryness. Dissolve the residue in a few drops of $\mathrm{HNO}_{3}$.

12. Convert the solution to the chloride with three $5-\mathrm{mL}$ additions of $\mathrm{HCl}$.

13. Add 1-2 $\mathrm{mL}$ of $1 \underline{\mathrm{N}} \mathrm{HCl}$, prepared with filtered water (see Procedure G-03, Microprecipitation Source Preparation for Alpha Spectrometry).

14. Continue the analysis under Procedure G-03, Microprecipitation Source Preparation for Alpha Spectrometry. 


\section{LOWER LIMIT OF DETECTION (LLD)*}

\begin{tabular}{|c|c|c|}
\hline \multicolumn{3}{|l|}{$\underline{226} \underline{\mathrm{Ra}}$} \\
\hline Counter Efficiency & $(2 \%)$ & 57.5 \\
\hline Counter Background & (cps) & 0.0028 \\
\hline Yield & $(\%)$ & 90 \\
\hline Blank & (cps) & 0.0020 \\
\hline LLD (400 min) & $(\mathrm{mBq})$ & 3.3 \\
\hline $\operatorname{LLD}(1000 \mathrm{~min})$ & $(\mathrm{mBq})$ & 1.7 \\
\hline \multicolumn{3}{|l|}{ Uranium Isotopes } \\
\hline Counter Efficiency & $(\%)$ & 40 \\
\hline Counter Background & (cps) & $\begin{array}{l}3.33 \times 10^{-6} \text { for }{ }^{238} \mathrm{U} \\
3.33 \times 10^{-6} \text { for }{ }^{234} \mathrm{U}\end{array}$ \\
\hline Yield & $(\%)$ & 85 \\
\hline Blank & (cps) & $\begin{array}{l}3.33 \times 10^{-6} \text { for }{ }^{238} \mathrm{U} \\
3.00 \times 10^{-5} \text { for }{ }^{234} \mathrm{U}\end{array}$ \\
\hline LLD (400 min) & $(\mathrm{mBq})$ & $\begin{array}{l}0.3 \text { for }{ }^{238} U \\
0.8 \text { for }{ }^{234} U\end{array}$ \\
\hline LLD (1000 min) & $(\mathrm{mBq})$ & $\begin{array}{l}0.2 \text { for }{ }^{238} \mathrm{U} \\
0.5 \text { for }{ }^{234} \mathrm{U}\end{array}$ \\
\hline (5000 min) & $(\mathrm{mBq})$ & $\begin{array}{l}0.1 \text { for }{ }^{238} U \\
0.2 \text { for }{ }^{234} U\end{array}$ \\
\hline
\end{tabular}

"It is necessary to analyze the reagents used with each batch of samples so that blank corrections can be made for $U$ and ${ }^{226} \mathrm{Ra}$.

\section{REFERENCES}

Fisenne, I. M., P. M. Perry, and G. A. Welford . "Determination of Uranium Isotopes in Human Bone Ash" Anal. Chem., 52, 777-779 (1980)

Hindman, F. D.

"Neodymium Fluoride Mounting for Alpha Spectrometric Determination of Uranium, Plutonium and Americium"

Anal. Chem., 55, 2460-2461 (1983) 


\section{Sill, C. W.}

"Decomposition of Refractory Silicates in Ultramicro Analysis"

Anal. Chem., 33, 1684 (1961)

Sill, C. W. and R. L. Williams

"Preparation of Actinides for Alpha Spectrometry without Electrodeposition" Anal. Chem., 53, 412-415 (1981) 


\begin{abstract}
APPLICATION
The method is applicable to $5 \mathrm{~g}$ soil samples, $20 \mathrm{~g}$ vegetation ash samples and $50 \mathrm{~g}$ bone ash samples.

Soil, vegetation ash and bone ash samples are spiked with ${ }^{229} \mathrm{Th},{ }^{232} \mathrm{U}$ and ${ }^{133} \mathrm{Ba}$ tracer solutions. The soil and vegetation ash samples are pretreated with $\mathrm{HNO}_{3}-\mathrm{HF}$ fused with potassium fluoride and transposed to pyrosulfate. The cake is dissolved in $\mathrm{HCl}$. Bone ash samples are dissolved directly in $\mathrm{HCl}$. Uranium isotopes are isolated by solvent extraction and are coprecipitated with neodymium as the fluoride for alpha spectrometry measurement. Thorium is coprecipitated with calcium as the acidic oxalate. The thorium is separated by solvent extraction and coprecipitated with neodymium as the fluoride for alpha spectrometry measurement. Barium carrier is added and $\mathrm{Ra} \cdot \mathrm{BaSO}_{4}$ is precipitated, filtered and dissolved in alkaline EDTA. The chemical yield is determined with the $\gamma$-emitting tracer ${ }^{133} \mathrm{Ba}$ and ${ }^{226} \mathrm{Ra}$ is determined by radon emanation.
\end{abstract}

\title{
SPECIAL APPARATUS ${ }^{*}$
}

1. 100-mL platinum dishes.

2. Polyethylene transfer pipettes.

3. Separatory funnels $-125,250,500$ and $1000 \mathrm{~mL}$.

* Special apparatus and special reagents for the microprecipitation of uranium and thorium with neodymium as the fluoride are listed in Microprecipitation of the Actinides, G-03. 
4. Wrist action separatory funnel shaker unit.

5. Millipore filtration unit - $47 \mathrm{~mm}$ diameter Pyrex glass filtration chimney, fritted glass support and metal clamp.

6. Millipore filters $-47 \mathrm{~mm}$ diameter, $0.45 \mu \mathrm{m}$ pore size.

7. Radon bubblers - see Specification 7.7.

\section{SPECIAL REAGENTS}

1. Uranium-232 tracer solution - about $0.2 \mathrm{~Bq} \mathrm{~g}^{-1}$ of solution in a polyethylene transfer pipette (see Note 1).

2. Thorium-229 tracer solution - about $0.2 \mathrm{~Bq} \mathrm{~g}^{-1}$ of solution in a polyethylene transfer pipette.

3. Barium-133 tracer solution - about $10 \mathrm{kBq} \mathrm{g}^{-1}$ of solution in a polyethylene transfer pipette. Add $0.1 \mathrm{~g}$ of tracer to each sample.

4. Alamine-336, tertiary tricapryl amine (Henkel Corp., 2430 N. Huachuca Dr., Tucson, AZ 85745-1273) 3:7 in toluene. Wash twice with an equal volume of $1: 1 \mathrm{HCl}$. Prepare $100 \mathrm{~mL}$ of acid-washed 3:7 Alamine-336 for each sample.

5. Aliquat-336, methyltricapryl-ammonium chloride (Henkel Corp., 2430 N. Huachuca Dr., Tucson, AZ 85745-1273) 4:6 in toluene. Wash four times with an equal volume of $2 \mathrm{~N} \mathrm{HNO}_{3}$ and once with an equal volume of $1: 1 \mathrm{HNO}_{3}$. Prepare $50 \mathrm{~mL}$ of acid-washed 4:6 Aliquat-336 for each soil and vegetation ash sample and $100 \mathrm{~mL}$ for each bone ash sample.

6. Calcium propionate $\left(\left(\mathrm{CH}_{3} \mathrm{CH}_{2} \mathrm{COO}\right)_{2} \mathrm{Ca}\right)$ - solid (see Note 2$)$.

7. Oxalic acid $\left(\mathrm{CHO}_{2} \mathrm{COOH}\right)$ - solid (see Note 2).

8. Barium carrier solution ( $20 \mathrm{mg} \mathrm{Ba} \mathrm{mL}^{-1}$ ) $-30.4 \mathrm{~g}$ of $\mathrm{BaCl}_{2} \mathrm{~L}^{-1}$ of $1: 99 \mathrm{HCl}$. 
9. Ammonium sulfate solution $\left(100 \mathrm{mg} \mathrm{mL}^{-1}\right)-100 \mathrm{~g}$ of $\left(\mathrm{NH}_{4}\right)_{2} \mathrm{SO}_{4} \mathrm{~L}^{-1}$ of $\mathrm{H}_{2} 0$.

10. EDTA solution - $500 \mathrm{~g}$ tetrasodium salt of EDTA $\mathrm{L}^{-1}$ of $\mathrm{H}_{2} \mathrm{O}$.

11. Triethanolamine - $1: 1$ with $\mathrm{H}_{2} 0$.

\section{SAMPLE PREPARATION}

\section{A. Soil, milk, and vegetation ash.}

1. Weigh $5 \mathrm{~g}$ of soil or up to $20 \mathrm{~g}$ of milk or vegetation ash into a $100-\mathrm{mL}$ platinum dish. Add weighed aliquots of ${ }^{133} \mathrm{Ba},{ }^{229} \mathrm{Th}$ and ${ }^{232} \mathrm{U}$ tracer solutions (see Note 3 ).

2. Slowly add $10 \mathrm{~mL}$ of $\mathrm{HNO}_{3}$ and $10 \mathrm{~mL}$ of $\mathrm{HF}$ to the sample and evaporate on a hot plate to near dryness.

3. Continue the analysis as described under Sample Dissolution.

\section{B. Bone ash.}

1. Weigh $50 \mathrm{~g}$ of ground, dry ashed bone into a $400-\mathrm{mL}$ beaker. Add weighed aliquots of ${ }^{133} \mathrm{Ba},{ }^{229} \mathrm{Th}$ and ${ }^{232} \mathrm{U}$ tracer solutions (see Note 3 ).

2. Continue the analysis as described under Sample Dissolution.

\section{SAMPLE DISSOLUTION ${ }^{*}$}

\section{A. Soil, milk, and vegetation ash.}

1. Weigh $15 \mathrm{~g}$ of $\mathrm{KF}$ and sprinkle over the surface of the sample.

\footnotetext{
- Based upon Sill (1981).
} 
2. Fuse the sample over a Meker burner, gradually increasing the temperature until a clear melt is obtained. Cool the melt.

3. Slowly add $17.5 \mathrm{~mL}$ of $\mathrm{H}_{2} \mathrm{SO}_{4}$ to the melt. Heat the dish on a high temperature hot plate until the cake dissolves and heat until sulfur trioxide fumes appear.

4. Weigh $10 \mathrm{~g}$ of $\mathrm{Na}_{2} \mathrm{SO}_{4}$, add to the dish and fuse over the Meker burner until a clear melt, accompanied by dense fumes, is obtained. Cool the melt.

5. Transfer the cake to a $600-\mathrm{mL}$ beaker containing $250 \mathrm{~mL}$ of hot 1:1 $\mathrm{HCl}$. Stir the solution to dissolve the cake. If necessary, add additional $\mathrm{HCl}$ to completely dissolve the cake. Cool for $1 \mathrm{~h}$.

6. Continue the analysis as described under Uranium Separation.

\section{B. Bone ash.}

1. Add $100 \mathrm{~mL}$ of $\mathrm{HCl}$ and heat gently on a hot plate for $10 \mathrm{~min}$ with occasional stirring.

2. Add $70 \mathrm{~mL}$ of water and stir to obtain a clear solution. Cool the solution for $1 \mathrm{~h}$.

3. Continue the analysis as described under Uranium Separation.

\section{URANIUM SEPARATION*}

1. Transfer $50 \mathrm{~mL}$ of acid-washed Alamine- 336 into each of two $500-\mathrm{mL}$ separatory funnels.

2. Transfer the sample to the first separatory funnel. Wash the beaker with $1: 1 \mathrm{HCl}$ and add the washings to the funnel.

"Based on Fisenne et al. (1980) 
3. Shake the separatory funnel for $5 \mathrm{~min}$. Allow the phases to separate and draw off the aqueous (lower) phase into the second separatory funnel. Retain the organic phase in the first funnel.

4. Shake the second separatory funnel for $5 \mathrm{~min}$. Allow the phases to separate, draw off the aqueous phase into a $2000-\mathrm{mL}$ beaker for subsequent analyses of thorium and radium.

5. Combine the two organic phases in one of the separatory funnels.

6. Wash the organic phase four times for $5 \mathrm{~min}$ with an equal volumes of $1: 1 \mathrm{HCl}$. Combine the washings in the $2000-\mathrm{mL}$ beaker (Step 4).

7. Strip the uranium from the organic phase by shaking twice for $5 \mathrm{~min}$ with $100-\mathrm{mL}$ portions of $0.1 \mathrm{~N} \mathrm{HCl}$. Combine the strip solutions in a $400-\mathrm{mL}$ beaker. The organic phase is placed and retrained in a suitable disposal container (see Note 4).

8. Add $1 \mathrm{~mL}$ of $\mathrm{H}_{2} \mathrm{SO}_{4}$ to the strip solution and evaporate the solution to sulfur trioxide fumes.

9. Destroy any residual organic material with dropwise additions of $\mathrm{HNO}_{3}$.

10. Evaporate the solution to dryness. Dissolve the residue in a few drops of $\mathrm{HCl}$.

11. Convert the solution to the chloride with three 5-mL additions of $\mathrm{HCl}$.

12. Add 1-2 $\mathrm{mL}$ of $1 \underline{\mathrm{N}} \mathrm{HCl}$, prepared with filtered water (see Microprecipitation). Cool to room temperature.

13. Continue the analysis under Microprecipitation of the Actinides, G-03. 


\section{THORIUM SEPARATION*}

\section{A. Calcium oxalate collections.}

1. Add $0.5 \mathrm{~g}$ of calcium carrier (2.5 $\mathrm{g}$ of calcium propionate) to the sample waste and wash solutions reserved from Steps 4 and 6 in Uranium Separation.

Note: DO NOT ADD CALCIUM CARRIER TO BONE ASH OR MUK SAMPLES.

2. Evaporate the solution volume to $100-200 \mathrm{~mL}$.

3. Dilute the solution to $1400 \mathrm{~mL}$ with deionized water. Heat the solution to near boiling.

4. Adjust the $\mathrm{pH}$ to 2.5 with $\mathrm{NH}_{4} \mathrm{OH}$.

5. Add $10 \mathrm{~g}$ of oxalic acid to soil, milk, and vegetation ash samples. Add $25 \mathrm{~g}$ of oxalic acid to bone ash samples.

6. Stir and heat the solution to a gentle boil. Continue heating for $5 \mathrm{~min}$.

7. Adjust the $\mathrm{pH}$ to 2.5 with $\mathrm{NH}_{4} 0 \mathrm{H}$. Gently boil the solution for $10 \mathrm{~min}$.

8. Remove the sample from the hot plate, check the $\mathrm{pH}$ and allow the sample to stand overnight at room temperature.

9. Decant the supernate into a $3000-\mathrm{mL}$ beaker and reserve for radium analysis. Reduce the volume of the decanted solution to $500 \mathrm{~mL}$ or less.

10. Add $100 \mathrm{~mL}$ of $\mathrm{HNO}_{3}$ to the calcium oxalate precipitate and heat to destroy the oxalate. Repeat the $\mathrm{HNO}_{3}$ addition, if necessary.

11. Convert the sample solution to the chloride with three successive additions of $50 \mathrm{~mL}$ of $\mathrm{HCl}$.

*Based upon Fisenne and Perry (1978). 
12. Repeat Steps 5-10 for soil, milk, and vegetation ash samples. Bone ash samples require four calcium oxalate collections to remove most of the phosphate ions.

13. Wet ash the oxalate precipitate with repeated additions of $50-100 \mathrm{~mL}$ of $\mathrm{HNO}_{3}$ for soil and vegetation samples. Bone samples initially require $200 \mathrm{~mL}$ of $\mathrm{HNO}_{3}$.

14. Reduce the sample solution to near dryness and add $25 \mathrm{~mL}$ of $1: 1 \mathrm{HNO}_{3}$ to the soil and vegetation ash samples. Add $100 \mathrm{~mL}$ of $1: 1 \mathrm{HNO}_{3}$ to the bone ash sample.

15. Warm to dissolve the calcium salts. Cool the solution to room temperature.

\section{B. Solvent extraction separation.}

1. For soil and vegetation ash samples, transfer $25 \mathrm{~mL}$ of acid-washed Aliquat-336 into each of two $125-\mathrm{mL}$ separatory funnels. For bone ash samples, add $50 \mathrm{~mL}$ of acid-washed Aliquat-336 into each of two 250-mL separatory funnels.

2. Transfer the sample to the first separatory funnel. Wash the beaker with $1: 1 \mathrm{HNO}_{3}$ and add the washings to the funnel.

3. Shake the separatory funnel for $5 \mathrm{~min}$. Allow the phases to separate and draw off the aqueous (lower) phrase into the second separatory funnel. Retain the organic phase in the first funnel.

4. Shake the second separatory funnel for $5 \mathrm{~min}$. Allow the phases to separate, draw off the aqueous phase into the beaker with the reserved radium fraction.

5. Combine the two organic phases in one of the separatory funnels.

6. Wash the combined organic phases twice for $5 \mathrm{~min}$ with half volumes of $1: 1 \mathrm{HNO}_{3}$. Add the washings to the reserved radium fraction.

7. Strip the thorium from the organic phase by shaking for $5 \mathrm{~min}$ with $25 \mathrm{~mL}$ of concentrated $\mathrm{HCl}$. Allow the phases to separate and draw off the aqueous phase into the empty separatory funnel. 
8. Repeat Step 7, combining the thorium bearing aqueous phases. The organic phase should be placed and retained in a suitable disposal container (see Note 4).

9. Add $25 \mathrm{~mL}$ of toluene to the separatory funnel and shake for $5 \mathrm{~min}$.

10. Allow the phases to separate and draw off the aqueous phase into a $150-\mathrm{mL}$ beaker. Discard the organic phase.

11. Heat the solution gently to drive off organic vapors.

12. Reduce the sample solution volume to $10 \mathrm{~mL}$. Wet ash the solution with repeated $10-$ $\mathrm{mL}$ additions of $\mathrm{HNO}_{3}$ to remove organics. Caution - Do not allow the solution to reach dryness.

13. Convert the solution to the chloride with three 5-mL additions of $\mathrm{HCl}$. Reduce the acid volume to near dryness.

14. Add 1-2 $\mathrm{mL}$ of $1 \underline{\mathrm{N}} \mathrm{HCl}$, prepared with filtered water (see Microprecipitation of the Actinides, G-03). Cool to room temperature.

15. Continue the analysis under Microprecipitation of the Actinides, G-03.

\section{RADIUM SEPARATION ${ }^{*}$}

1. Add $1 \mathrm{~mL}$ of barium carrier $\left(20 \mathrm{mg}\right.$ barium $\left.\mathrm{mL}^{-1}\right)$ to the reserved radium fraction.

2. Reduce the sample solution to dryness and wet ash with repeated $50-100 \mathrm{~mL}$ additions of $\mathrm{HNO}_{3}$ to remove organics.

3. Convert the sample to the chloride with repeated $50-100 \mathrm{~mL}$ additions of $\mathrm{HCl}$.

4. Dilute the sample solution to $1000 \mathrm{~mL}$ with water. Heat and stir to dissolve the salts. Add additional water, if necessary.

"Based upon Hallden et al. (1963). 
5. Adjust the solution $\mathrm{pH}$ to 2.5 with $\mathrm{NH}_{4} \mathrm{OH}$. Cool the beaker to room temperature in a water bath.

6. Add $5 \mathrm{~mL}$ of $\left(\mathrm{NH}_{4}\right)_{2} \mathrm{SO}_{4}$ solution $\left(100 \mathrm{mg}\left(\mathrm{NH}_{4}\right)_{2} \mathrm{SO}_{4} \mathrm{~mL}^{-1}\right)$. Stir and let stand at room temperature overnight.

7. Filter the solution through $47 \mathrm{~mm}$ diameter, $0.45 \mu \mathrm{m}$ pore size Millipore filter.

8. Police and wash the beaker with water. Add the washings to the filter chimney. Wash the filter with water.

9. Place the filter with the Ra.BaSO 4 in a $150-\mathrm{mL}$ beaker. Discard the filtrate.

10. Add $5 \mathrm{~mL}$ of warm EDTA solution and $1 \mathrm{~mL}$ of $1: 1$ triethanolamine to the beaker. Heat on a warm hot plate for about $5 \mathrm{~min}$. Add $10 \mathrm{~mL}$ of water and stir occasionally. Continue to heat for $15 \mathrm{~min}$.

11. Gravity filter the warm solution through Whatman No. 42 filter paper into a $30-\mathrm{mL}$ (1 oz) polyethylene bottle.

12. Wash the beaker and filter with hot water. Discard the filter paper.

13. Dilute the sample solution to the same liquid level as a known aliquot of the ${ }^{13 \hat{3}} \mathrm{Ba}$ tracer solution diluted to $25 \mathrm{~mL}$ in a $30-\mathrm{mL}(1 \mathrm{oz})$ polyethylene bottle.

14. Gamma count the samples and standard to determine the chemical yield of barium.

15. Transfer the sample to a radon bubbler with water.

16. Determine the ${ }^{226} \mathrm{Ra}$ content of the sample as described in Emanation Procedure for Radium-226, Ra-03.

\section{Notes:}

1. The ${ }^{232} \mathrm{U}$ decays to ${ }^{228} \mathrm{Th}$ and its daughters. Therefore the activity of the thorium subseries increases with time in the ${ }^{232} \mathrm{U}$ spike. Thus, a blank correction for ${ }^{228} \mathrm{Th}$ 
from the added ${ }^{232} \mathrm{U}$ tracer is required, resulting in an increasing lower limit of detection (LLD) for ${ }^{228} \mathrm{Th}$.

2. Calcium compounds and oxalic acid contain variable and measurable quantities of ${ }^{226} \mathrm{Ra}$. Each lot of these reagents should be checked prior to use to obtain the lowest possible reagent blank value.

3. It is necessary to analyze reagent blank samples along with each batch of samples to determine the proper blank corrections.

4. The organic phase is washed twice for $5 \mathrm{~min}$ with an equal volume of water and placed in a suitable disposal container.

\section{LOWER LIMIT OF DETECTION}

Based upon Fisenne et al. (1987) and Harley and Fisenne (1990).

\section{REFERENCES}

Fisenne, I. M. and P. M. Perry

"Determination of Plutonium in Tissue by Aliquat-336 Extraction"

Radiochemical and Radioanalytical Letters, 33, 259 (1978)

Fisenne, I. M., P. M. Perry and G. A. Welford

"Determination of Uranium Isotopes in Human Bone Ash"

Analytical Chemistry, 52, 777 (1980)

Fisenne, I. M., P. M. Perry, K. M. Decker and H. W. Keller

"The Daily Intake of ${ }^{234,235,238} \mathrm{U},{ }^{228,230,232} \mathrm{Th}$ and ${ }^{226,228} \mathrm{Ra}$ by New York City

Residents"

Health Physics, 푸, 357 (1987) 
Hallden, N. A., I. M. Fisenne and J. H. Harley

"Determination of Radium-226 in Human Bone"

Talanta, 10, 1223 (1963)

Harley, N. H. and I. M. Fisenne

"Distribution and $\alpha$ Radiation Dose from Naturally Occurring, $U, T h$ and $R a$ in the Human Skeleton"

Health Physics, $\underline{58}, 515$ (1990)

Sill, C. W.

"Decomposition of Refractory Silicates in Ultramicro Analysis"

Analytical Chemistry, 33, 1684 (1981) 


\title{
AMERICIUM, PLUTONIUM AND URANIUM IN WATER
}

\section{Contact Person(s) : Anna Berne}

\begin{abstract}
APPLICATION
This procedure describes a method for the separation and measurement of americium, plutonium and uranium in water (adapted from Eichrom Industries, Inc., Procedure ACW03, Rev. 1.5). Americium, plutonium and uranium are separated by Eichrom resins prior to measurements by alpha spectrometry. Tracers are used to monitor chemical recoveries and to correct the results to improve precision and accuracy. This is a rapid, reliable method for the measurement of actinides in water samples that is more costeffective and efficient than traditional ion exchange, solvent extraction and precipitation techniques.
\end{abstract}

\section{INTERFERENCES}

Actinides with unresolvable alpha energies such as ${ }^{241} \mathrm{Am}$ and ${ }^{238} \mathrm{Pu}$ or ${ }^{237} \mathrm{~Np}$ and ${ }^{234} \mathrm{U}$ must be chemically separated to enable measurement. This method effectively separates these isotopes.

\section{SPECIAL APPARATUS}

1. Column rack

2. Filter -0.45 micron 


\section{SPECIAL REAGENTS}

1. Ammonium hydrogen oxalate $(0.1 \underline{\mathrm{M}})$ - dissolve $6.31 \mathrm{~g}$ of $\mathrm{H}_{2} \mathrm{C}_{2} \mathrm{O}_{4} \cdot 2 \mathrm{H}_{2} \mathrm{O}$ and $7.11 \mathrm{~g}$ of $\left(\mathrm{NH}_{4}\right)_{2} \mathrm{C}_{2} \mathrm{O}_{4} \cdot \mathrm{H}_{2} \mathrm{O}$ in $900 \mathrm{~mL}$ of water, filter (Whatman No. 4 suggested) and dilute to $1 \mathrm{~L}$ with water.

2. Ammonium hydroxide ( $5 \mathrm{wt} \%$ ) - dissolve $50 \mathrm{~g}$ ammonium hydroxide in $950 \mathrm{~g}$ of water.

3. Appropriate tracers or standards.

4. Ascorbic acid.

5. Ferrous sulfamate solution (0.6M) - add $57 \mathrm{~g}$ of $\mathrm{NH}_{2} \mathrm{SO}_{3} \mathrm{H}$ to $150 \mathrm{~mL}$ of water, heat to $70^{\circ} \mathrm{C}$, add $7 \mathrm{~g}$ of iron, in small increments until dissolved, filter (Whatman No. 4 suggested), transfer to flask and dilute to $200 \mathrm{~mL}$ with water. Prepare fresh weekly.

6. Hydrochloric acid $(0.01 \mathrm{M} \mathrm{HCl})$ - add $0.8 \mathrm{~mL}$ of $\mathrm{HCl}$ to $900 \mathrm{~mL}$ of water and dilute to $1 \mathrm{~L}$ with water.

7. Hydrochloric acid (4M HCl) - add $333 \mathrm{~mL}$ of $\mathrm{HCl}$ to $500 \mathrm{~mL}$ of water and dilute to 1 $\mathrm{L}$ with water.

8. Hydrochloric acid ( $5 \underline{\mathrm{M}})$, oxalic acid $(0.05 \underline{\mathrm{M}})$ solution - Dissolve $6.3 \mathrm{~g}$ oxalic acid dihydrate in $400 \mathrm{~mL}$ of water. Add $417 \mathrm{~mL} \mathrm{HCl}$. Cool to room temperature and dilute to $1 \mathrm{~L}$ with water.

9. Hydrochloric acid ( $9 \mathrm{M} \mathrm{HCl})$ - add $750 \mathrm{~mL}$ of $\mathrm{HCl}$ to $100 \mathrm{~mL}$ of water and dilute to $1 \mathrm{~L}$ with water.

10. Iron powder - a fine mesh powder dissolves faster in sulfamic acid.

11. Nitric acid (2M) - sodium nitrite (0.1 $\underline{M}$ solution) - add $32 \mathrm{~mL}$ of $\mathrm{HNO}_{3}$ to $200 \mathrm{~mL}$ of water, dissolve $1.72 \mathrm{~g}$ of sodium nitrite in the solution and dilute to $250 \mathrm{~mL}$ with water. Prepare fresh daily. 
12. Nitric acid solution (0.5 $\mathrm{M})$ - add $32 \mathrm{~mL}$ of $\mathrm{HNO}_{3}$ to $900 \mathrm{~mL}$ of water and dilute to $1 \mathrm{~L}$ with water.

13. Nitric acid solution (2M) - add $127 \mathrm{~mL}$ of $\mathrm{HNO}_{3}$ to $800 \mathrm{~mL}$ of water and dilute to $1 \mathrm{~L}$ with water.

14. Nitric acid solution (3M $)$ - add $191 \mathrm{~mL}$ of $\mathrm{HNO}_{3}$ to $700 \mathrm{~mL}$ of water and dilute to $1 \mathrm{~L}$ with water.

15. Nitric acid ( $3 \mathrm{M})$ - Aluminum nitrate (1M) solution -dissolve $212 \mathrm{~g}$ of anhydrous aluminum nitrate in $700 \mathrm{~mL}$ of water, add $191 \mathrm{~mL}$ of $\mathrm{HNO}_{3}$ and dilute to $1 \mathrm{~L}$ with water.

16. Hydrochloric acid (4⿳一巛工M HCl) - hydrofluoric acid $(0.1 \underline{\mathrm{M}})$ - add $333 \mathrm{~mL}$ of $\mathrm{HCl}$ and $3.6 \mathrm{~mL} \mathrm{HF}$ to $500 \mathrm{~mL}$ of water and dilute to $1 \mathrm{~L}$ with water. Prepare fresh daily.

23. TRU Resin - prepacked column, $0.7 \mathrm{~g} 100-150$ micron particle size resin.

24. U/TEVA Resin - prepacked column, 0.7 g 100-150 micron particle size resin.

\section{SAMPLE PREPARATION}

1. If not already prefiltered, filter the sample through a 0.45 micron filter.

2. If samples larger than $1 \mathrm{~L}$ are analyzed, evaporate the sample to $\sim 1 \mathrm{~L}$.

3. Aliquot 500 to $1000 \mathrm{~mL}$ of the filtered sample (or enough to meet the required detection limit) into an appropriate size beaker.

4. Add $5 \mathrm{~mL}$ of $\mathrm{HCl}$ per liter of sample $(0.5 \mathrm{~mL}$ per $100 \mathrm{~mL})$ to acidify each sample.

5. Add the appropriate tracers. 
6. Evaporate sample to $<50 \mathrm{~mL}$ and transfer to a $100-\mathrm{mL}$ beaker. (Note: For some water samples, calcium sulfate formation may occur during evaporation.) Gently evaporate the sample to dryness and redissolve in approximately $5 \mathrm{~mL}$ of $\mathrm{HNO}_{3}$. Evaporate to dryness and redissolve in $\mathrm{HNO}_{3}$ two more times, evaporate to dryness and go to Actinide Separation Using Eichrom's Resins.

\section{SEPARATION}

1. Dissolve each precipitate from Step 6, Sample Preparation, in $10 \mathrm{~mL}$ of $3 \mathrm{M} \mathrm{HNO}_{3}$ $1.0 \mathrm{M} \mathrm{Al}\left(\mathrm{NO}_{3}\right)_{3}$. (Note: An additional $5 \mathrm{~mL}$ may be necessary if the volume of precipitate is large.)

2. Add $2 \mathrm{~mL}$ of $0.6 \mathrm{M}$ ferrous sulfamate to each solution. Swirl to mix. (Note: If the additional $5 \mathrm{~mL}$ was used to dissolve the sample in Step 1, add a total of $3 \mathrm{~mL}$ of ferrous sulfamate solution.)

3. Add $200 \mathrm{mg}$ of ascorbic acid to each solution, swirling to mix. Wait for 2-3 min. (Note: If particles are observed to be suspended in the solution, centrifuge the sample. The supernatant will be transferred to the column in Step 5, Uranium separation from plutonium, americium using U/TEVA resin. The precipitates will be discarded.)

\section{A. Uranium separation from plutonium, americium using U/TEVA resin}

1. For each sample solution, place a U/TEVA Resin column in the column rack.

2. Place a beaker below each column, remove the bottom plug from each column and allow to drain.

3. Pipette $5 \mathrm{~mL}$ of $3 \mathrm{M} \mathrm{HNO}_{3}$ into each column to condition the resin and allow to drain.

4. Place a clean, labeled $50-\mathrm{mL}$ beaker below each column. 
5. Transfer each solution from Step 3 into the appropriate U/TEVA Resin column by pouring or by using a plastic transfer pipette and collect the eluate.

6. Add $5 \mathrm{~mL}$ of $3 \mathrm{M} \mathrm{HNO}_{3}$ to rinse to each beaker and transfer each solution into the appropriate U/TEVA Resin column and collect eluate.

7. Add $5 \mathrm{~mL}$ of $3 \mathrm{M} \mathrm{HNO}_{3}$ into each column and collect eluate.

8. Set aside the solutions collected in Steps 5,6 and 7 for americium and plutonium separations.

9. Pipette $4 \mathrm{~mL}$ of $9 \mathrm{M} \mathrm{HCl}$ into each column and allow to drain. Discard this rinse.

(Note: The rinse converts the resin to the chloride system. Some neptunium may be removed here.)

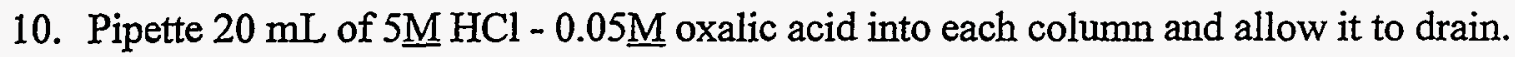
Discard eluate. (Note: This rinse removes neptunium and thorium form the column. The $9 \mathrm{M} \mathrm{HCl}$ and $5 \underline{\mathrm{M}} \mathrm{HCl}-0.05 \mathrm{M}$ oxalic acid rinses also removes any residual ferrous ion that might interfere.)

11. Place a clean, labeled beaker below each column.

12. Pipette $15 \mathrm{~mL}$ of $0.01 \mathrm{M} \mathrm{HCl}$ into each column to strip the uranium. Allow to drain.

13. Evaporate to dryness. Treat with $5 \mathrm{~mL}$ of $\mathrm{HNO}_{3}$ several times to remove traces of oxalic acid. Convert to $\mathrm{HCl}$.

14. Set beakers aside for Procedure G-03, Microprecipitation Source Preparation for Alpha Spectrometry.

\section{B. Plutonium and americium separation using TRU resin}

1. Place a TRU Resin column in the column rack for each sample dissolved.

2. Remove the bottom plug from each column and allow each column to drain. 
3. Pipette $5 \mathrm{~mL}$ of $2 \mathrm{M} \mathrm{HNO}_{3}$ into each column to condition resin and allow to drain (just prior to sample loading).

4. Transfer each solution from Step 8 of Uranium Separation into the appropriate TRU Resin column by pouring and/or using a plastic transfer pipette.

5. Allow the load solution to drain through the column.

6. Pipette $5 \mathrm{~mL}$ of $2 \mathrm{M} \mathrm{HNO}_{3}$ into the sample beaker and transfer this rinse to the appropriate column using the same plastic pipette.

7. Allow the initial rinse solution to drain through each column.

8. Pipette $5 \mathrm{~mL}$ of $2 \mathrm{M} \mathrm{HNO}_{3}-0.1 \mathrm{M} \mathrm{NaNO}$ directly into each column, rinsing each column reservoir while adding the $2 \mathrm{M} \mathrm{HNO}_{3}-0.1 \mathrm{MNaNO}_{2}$. (Note: Sodium nitrite is used to oxidize $\mathrm{Pu}^{+3}$ to $\mathrm{Pu}^{+4}$ and to enhance the plutonium/americium separation).

9. Allow the rinse solution to drain through each column.

10. Add $5 \mathrm{~mL}$ of $0.5 \mathrm{M} \mathrm{HNO}_{3}$ to each column and allow to drain. (Note: $0.5 \mathrm{M} \mathrm{HNO}_{3}$ is used to lower the nitrate concentration prior to conversion to the chloride system.)

11. Discard the load and rinse solutions.

12. Ensure that clean, labeled beakers or vials are below each column.

13. Add $3 \mathrm{~mL}$ of $9 \mathrm{M} \mathrm{HCl}$ to each column to convert to $\mathrm{HCl}$. Collect the eluate.

14. Add $20 \mathrm{~mL}$ of $4 \underline{\mathrm{M} \mathrm{HCl}}$ to elute americium. Collect the eluate in the same beaker. Evaporate to dryness. Treat with $5 \mathrm{~mL} \mathrm{HNO}_{3}$ several times until wet-ashing of the residue is complete. Convert to $\mathrm{HCl}$. Set beakers aside for Procedure $\mathrm{G}-03$, Microprecipitation Source Preparation for Alpha Spectrometry.

15. Rinse the columns with $25 \mathrm{~mL}$ of $4 \mathrm{M} \mathrm{HCl}-0.1 \mathrm{M} \mathrm{HF}$. Discard eluate. 
16. Ensure the clean, labeled beakers or vials are below each column. Add $10 \mathrm{~mL}$ of $0.1 \mathrm{M} \mathrm{NH}_{4} \mathrm{HC}_{2} \mathrm{O}_{4}$ to elute plutonium from each column.

17. Evaporate to dryness. Treat with $5 \mathrm{~mL} \mathrm{HNO}_{3}$ several times until wet-ashing of the residue is complete. Convert to $\mathrm{HCl}$. Set beakers aside for Procedure G-03, Microprecipitation Source Preparation for Alpha Spectrometry.

\section{PRECISION AND BIAS}

1. Precision - A relative standard deviation of $4.2 \%$ at the $0.42 \mathrm{~Bq}$ level has been reported for uranium. A relative standard deviation of $3.2 \%$ at the $1 \mathrm{~Bq}$ level has been reported for plutonium.

2. Bias - Mean chemical recoveries of $95 \%$ for americium, $93 \%$ for plutonium and $86 \%$ for uranium have been reported. Since results are corrected based on spike recovery, no significant bias exists for the method.

\section{LOWER LIMIT OF DETECTION (LLD)} NATURAL URANIUM

\begin{tabular}{lcl}
\hline Counter Efficiency & $(\%)$ & 30 \\
Counter Background & $(\mathrm{cps})$ & $3.33 \times 10^{-6}$ for $^{238} \mathrm{U}$ \\
& & $6.67 \times 10^{-6}$ for $^{234} \mathrm{U}$ \\
Recovery : & $(\%)$ & 85 \\
LLD (400 min) & $(\mathrm{m} \mathrm{Bq})$ & 0.2 for ${ }^{238} \mathrm{U}$ \\
& & 0.3 for ${ }^{234} \mathrm{U}$ \\
LLD (1000 min) & $(\mathrm{m} \mathrm{Bq})$ & 0.1 for ${ }^{238} \mathrm{U}$ \\
& & 0.2 for ${ }^{234} \mathrm{U}$ \\
LLD (5000 min) & $(\mathrm{m} \mathrm{Bq})$ & 0.06 for ${ }^{238} \mathrm{U}$ \\
& & 0.09 for ${ }^{234} \mathrm{U}$ \\
\hline
\end{tabular}




\section{PLUTONIUM}

\begin{tabular}{lll}
\hline Counter Efficiency & $(\%)$ & 30 \\
Counter Background & $(\mathrm{cps})$ & $2 \times 10^{-5}$ \\
Recovery & $(\%)$ & 75 \\
Blank & $(\mathrm{cps})$ & - \\
LLD $(400 \mathrm{~min})$ & $(\mathrm{mBq})$ & 0.6 \\
$\operatorname{LLD}(1000 \mathrm{~min})$ & $(\mathrm{mBq})$ & 0.4 \\
$\operatorname{LLD}(5000 \mathrm{~min})$ & $(\mathrm{mBq})$ & 0.2 \\
\hline
\end{tabular}

\section{AMERICIUM}

\begin{tabular}{lll}
\hline Counter Efficiency & $(\%)$ & 30 \\
Counter Background & $(\mathrm{cps})$ & $1.6 \times 10^{-5}$ \\
Recovery & $(\%)$ & 80 \\
LLD $(400 \mathrm{~min})$ & $(\mathrm{mBq})$ & 0.5 \\
$\operatorname{LLD}(1000 \mathrm{~min})$ & $(\mathrm{mBq})$ & 0.3 \\
$\operatorname{LLD}(5000 \mathrm{~min})$ & $(\mathrm{mBq})$ & 0.1 \\
\hline
\end{tabular}




\section{RADIONUCLIDE DATA}

U.S. Department of Energy 201 Varick Street, 5th Floor

New York, NY 10014-4811 


\subsection{Alpha}

5.2.1 Scope

5.3 Beta

5.3.1 Scope

5.4 Gamma

5.4.1 Scope

5.5 X-Ray

5.5.1 Scope

5.6 Natural Decay Series

5.6.1 Scope $5.6-1$ 


\section{RADIONUCLIDE DATA}

\subsection{OVERVIEW}

This section describes current radionuclide measurement techniques in use at EML and their associated data. A radionuclide is identified by its three principal characteristics: half-life, type of emission, and energy of the emission. Sometimes overlooked in the process are progeny radionuclides with their own particular characteristics.

In the 1960s, routine measurements were being performed at EML for $\alpha, \beta$, and $\gamma$ radionuclides. Measurements in both the Frisch grid ion chamber and the solid-state surface barrier detection systems were used to identify $\alpha$ energies in sources in properly calibrated systems. Contained in this section is a table of $\alpha$ emitters by energy, especially linked to the charts of the naturally occurring radionuclides, which assists in radionuclide identification in separated samples and in radionuclide purity checks.

Routine measurement of $\beta$ emitters is performed on counters designed at EML. These counters have a very low background count rate due to anticoincidence counting techniques. The overall counting efficiencies are comparable to proportional counters. Radiochemical purity is typically assessed by decay measurements.

Use of $\mathrm{NaI}(\mathrm{T} 1) \gamma$ spectrometry detection systems provides poorly resolved spectra compared with the $\mathrm{Ge}(\mathrm{Li})$ and intrinsic $\mathrm{Ge}$ systems presently in use. The resolution of these present systems require sophisticated computer algorithms to identify energies associated with particular radionuclides. It is most important that the energies and the branching ratios used at any facility be documented and a table of $\gamma$ emitters in the environment is included.

Contained in the natural decay series are charts of three natural radionuclide series and the man-made americium series. Pertinent data are included in these charts. 


\subsection{ALPHA}

\section{Contact Person(s) : Isabel M. Fisenne}

\subsubsection{SCOPE}

The tables on $\alpha$ emitters by atomic number and by energy have been developed for internal use at EML. The present tables are revisions compiled from the data given in Table of Radioactive Isotopes by Browne et al. (1986).

\section{REFERENCE}

Browne, E., R. B. Firestone and V. S. Shirley (Editors)

Table of Radioactive Isotopes

John Wiley and Sons, Inc., New York (1986) 


\begin{tabular}{|c|c|c|c|c|}
\hline Radionuclide & $t_{1 / 2}$ & $\begin{array}{l}\text { Isotopic } \\
\text { Abundance } \\
\quad(\%)\end{array}$ & $\begin{array}{c}\alpha \text { Emission } \\
\text { (when not } \\
100 \% \text { ) }\end{array}$ & $\mathrm{E}_{\mathrm{MeV}}$ \\
\hline $85^{211}$ At & $7.215 \mathrm{~h}$ & & $41.7 \%$ & 5.868 \\
\hline $85^{217}$ At & $32.3 \mathrm{~ms}$ & & & 7.067 \\
\hline $86^{219} \mathrm{Rn}\left({ }^{219} \mathrm{Em}\right)$ & $3.96 \mathrm{~s}$ & & & $\begin{array}{l}6.819(80.9 \%), 6.553(12.2 \%) \\
6.425(7.5 \%)\end{array}$ \\
\hline $86^{220} \mathrm{Rn}\left({ }^{220} \mathrm{Em}, \mathrm{Tn}\right)$ & $55.6 \mathrm{~s}$ & & & 6.288 \\
\hline $86^{222} \mathrm{Rn}(222 \mathrm{Em}, \mathrm{Rn})$ & $3.825 \mathrm{~d}$ & & & 5.490 \\
\hline $87^{221} \mathrm{Fr}$ & $4.9 \mathrm{~m}$ & & & $\begin{array}{l}6.341(83.4 \%), 6.243(1.3 \%) \\
6.127(15.1 \%)\end{array}$ \\
\hline $88^{223} \mathrm{Ra}(\mathrm{AcX})$ & $11.43 \mathrm{~d}$ & & & $\begin{array}{l}5.747(9.5 \%), 5.716(52.5 \%) \\
5.607(24.2 \%), 5.540(9.2 \%) \\
\text { others }\end{array}$ \\
\hline $88{ }^{224} \mathrm{Ra}(\operatorname{ThX})$ & $3.66 \mathrm{~d}$ & & & $5.686(95.1 \%), 5.449(4.9 \%)$ \\
\hline $88^{226} \mathrm{Ra}$ & $1600.0 y$ & & & $4.784(94.5 \%), 4.601(5.6 \%)$ \\
\hline $89^{225} \mathrm{Ac}$ & $10.0 \mathrm{~d}$ & & & $\begin{array}{l}5.829(51.6 \%), 5.793(18.1 \%) \\
5.791(8.6 \%), \text {, } \\
5.631(10 \%), \\
\text {. }\end{array}$ \\
\hline $90^{227} \mathrm{Th}(\mathrm{Rd} \mathrm{Ac})$ & $18.718 \mathrm{~d}$ & & & $\begin{array}{l}6.038 \%(24.5 \%) \\
5.978(23.4 \%), \\
5.757(20.3 \%), 5.714(4.9 \%) \text {, } \\
5.710(8.2 \%) \text {, others }\end{array}$ \\
\hline $90{ }^{228} \mathrm{Th}(\mathrm{Rd} \mathrm{Th})$ & $1.913 \mathrm{y}$ & & & $5.423(72.7 \%), 5.341(26.7 \%)$ \\
\hline $90^{229} \mathrm{Th}$ & $7340.0 y$ & & & $\begin{array}{l}5.050(5.2 \%), 4.968(6.0 \%) \\
4.901(10.2 \%), 4.845(56.2 \%) \\
4.814(9.3 \%), \text { others }\end{array}$ \\
\hline $90^{230} \mathrm{Th}(\mathrm{Io})$ & $7.54 \times 10^{4} y$ & & & $4.688(76.3 \%), 4.621(23.4 \%)$ \\
\hline $90^{232} \mathrm{Th}(\mathrm{Th})$ & $1.405 \times 10^{10} \mathrm{y}$ & 100. & & $4.010(77 \%), 3.952(23 \%)$ \\
\hline $91^{231} \mathrm{~Pa}(\mathrm{~Pa})$ & $3.276 \times 10^{4} y$ & & & $\begin{array}{l}5.059(11 \%), 5.029(20 \%) \\
5.013(25.4 \%), 4.951(22.8 \%), \\
4.734(8.4 \%) \text {, others }\end{array}$ \\
\hline $92^{250} \mathrm{U}$ & $20.8 \mathrm{~d}$ & & & $\begin{array}{l}5.889(67.4 \%), 5.818(32 \%) \\
\text { others }\end{array}$ \\
\hline $92{ }^{232} \mathrm{U}$ & $68.9 \mathrm{y}$ & & & $5.320(68.6 \%), 5.264(31.2 \%)$ \\
\hline $92^{233} \mathrm{U}$ & $1.592 \times 10^{5} \mathrm{y}$ & & & $\begin{array}{c}4.825(84.4 \%), 4.783(13.2 \%) \text {, } \\
\text { others }\end{array}$ \\
\hline $92{ }^{234} \mathrm{U}$ (UII) & $2.454 \times 10^{5} \mathrm{y}$ & 0.0055 & & $4.776(72.5 \%), 4.724(27.5 \%)$ \\
\hline
\end{tabular}




\begin{tabular}{|c|c|c|c|c|}
\hline Radionuclide & $t_{1 / 2}$ & $\begin{array}{l}\text { Isotopic } \\
\text { Abundance } \\
(\%)\end{array}$ & $\begin{array}{c}\alpha \text { Emission } \\
\text { (when not } \\
100 \% \text { ) }\end{array}$ & $E_{M e V}$ \\
\hline $92{ }^{235} \mathrm{U}(\mathrm{AcU})$ & $7.037 \times 10^{8} y$ & 0.7200 & & $\begin{array}{l}4.597(5 \%), 4.556(4.2 \%) \\
4.395(55 \%), 4.364(\sim 11 \%), \\
4.218(5.7 \%), \text { others }\end{array}$ \\
\hline $92{ }^{236} \mathrm{U}$ & $2.342 \times 10^{7} y$ & & & $4.494(74 \%), 4.445(26 \%)$ \\
\hline $92{ }^{238}$ U(U) & $4.468 \times 10^{9} \mathrm{y}$ & 99.2745 & & $4.196(77 \%), 4.147(23 \%)$ \\
\hline $93{ }^{237} \mathrm{~Np}$ & $2.14 \times 10^{6} \mathrm{y}$ & & & $\begin{array}{l}4.873(2.6 \%), 4.988(47 \%), \\
4.772(25 \%), 4.766(8 \%) \\
4.640(6.2 \%), \text { others }\end{array}$ \\
\hline $94{ }^{236} \mathrm{Pu}$ & $2.851 \mathrm{y}$ & & & $5.768(68.1 \%), 5.721(31.7 \%)$ \\
\hline $94{ }^{238} \mathrm{Pu}$ & $87.74 y$ & & & $5.499(71.6 \%), 5.456(28.3 \%)$ \\
\hline $94{ }^{239} \mathrm{Pu}$ & $2.411 \times 10^{4} \mathrm{y}$ & & & $\begin{array}{l}5.156(73.2 \%), 5.143(15.1 \%) \\
5.105(10.6 \%)\end{array}$ \\
\hline $94^{240} \mathrm{Pu}$ & $6563.0 y$ & & & $5.168(73.5 \%), 5.124(26.4 \%)$ \\
\hline $94^{242} \mathrm{Pu}$ & $3.763 \times 10^{5} y$ & & & $4.901(78 \%), 4.856(22.4 \%)$ \\
\hline $94{ }^{244} \mathrm{Pu}$ & $8.26 \times 10^{7} y$ & & & $4.589(80.5 \%), 4.546(19.4 \%)$ \\
\hline $95^{241} \mathrm{Am}$ & $432.7 y$ & & & $\begin{array}{l}5.486(85.2 \%), 5.443(12.8 \%) \\
5.388(1.4 \%)\end{array}$ \\
\hline $95^{243} \mathrm{Am}$ & $7380.0 y$ & & & $\begin{array}{l}5.277(88 \%), 5.234(11 \%) \\
5.180(1.1 \%)\end{array}$ \\
\hline $96^{240} \mathrm{Cm}$ & $27.0 \mathrm{~d}$ & & & $6.291(70.6 \%), 6.248(28.8 \%)$ \\
\hline $96^{242} \mathrm{Cm}$ & $162.94 \mathrm{~d}$ & & & $6.113(74 \%), 6.070(25 \%)$ \\
\hline $96{ }^{243} \mathrm{Cm}$ & $28.5 y$ & & & $\begin{array}{l}6.067(1.5 \%), 6.059(5 \%) \\
5.992(6.5 \%), 5.786(73.3 \%) \\
5.742(10.6 \%), \text { others }\end{array}$ \\
\hline $96{ }^{244} \mathrm{Cm}$ & $18.11 \mathrm{y}$ & & & $\begin{array}{c}5.805(76.4 \%), 5.763(23.6 \%) \\
\text { others }\end{array}$ \\
\hline $96{ }^{245} \mathrm{Cm}$ & $8500.0 \mathrm{y}$ & & & $\begin{array}{c}5.362(93.2 \%), 5.304(5.0 \%) \\
\text { others }\end{array}$ \\
\hline $96^{246} \mathrm{Cm}$ & $4730.0 y$ & & & $5.386(79 \%), 5.343(21 \%)$ \\
\hline $96{ }^{247} \mathrm{Cm}$ & $1.56 \times 10^{7} \mathrm{y}$ & & & $\begin{array}{l}5.266(13.8 \%), 5.210(5.7 \%) \\
4.869(71 \%), 4.818(4.7 \%) \\
\text { others }\end{array}$ \\
\hline
\end{tabular}




\begin{tabular}{|c|c|c|c|c|}
\hline Radionuclide & $t_{1 / 2}$ & $\begin{array}{c}\text { Isotopic } \\
\text { Abundance } \\
(\%)\end{array}$ & $\begin{array}{c}\alpha \text { Emission } \\
\text { (when not } \\
100 \% \text { ) }\end{array}$ & $\mathrm{E}_{\mathrm{MeV}}$ \\
\hline $96{ }^{248} \mathrm{Cm}^{*}$ & $3.40 \times 10^{5} \mathrm{y}$ & & $91.7 \%$ & $5.078(81.9 \%), 5.035(18 \%)$ \\
\hline $97^{247} \mathrm{Bk}$ & $1380.0 \mathrm{y}$ & & & $\begin{array}{l}5.795(5.5 \%), 5.712(17 \%), \\
5.687(13 \%), 5.532(45 \%), \\
5.501(7 \%) \text {, others }\end{array}$ \\
\hline $98{ }^{246} \mathrm{Cf}$ & $1.487 \mathrm{~d}$ & & & $6.750(78.0 \%), 6.709(21.8 \%)$ \\
\hline $98^{248} \mathrm{Cf}$ & $334.0 \mathrm{~d}$ & & & $6.262(83.0 \%), 6.220(17.0 \%)$ \\
\hline $98{ }^{249} \mathrm{Cf}$ & $350.6 y$ & & & $\begin{array}{l}6.194(2.2 \%), 6.140(1.1 \%) \text {, } \\
5.945(4 \%), 5.903(2.8 \%) \text {, } \\
5.812(84.4 \%), \text { others }\end{array}$ \\
\hline $98{ }^{250} \mathrm{Cf}$ & $13.09 \mathrm{y}$ & & & $6.031(84.5 \%), 5.989(15.1 \%)$ \\
\hline $98^{251} \mathrm{Cf}$ & $898.0 y$ & & & $\begin{array}{l}\text { 6.074(2.7\%), 6.014(11.6\%), } \\
5.851(27 \%), 5.812(4.2 \%), \\
5.677(35 \%), \text { others }\end{array}$ \\
\hline $98^{252} \mathrm{Cf}^{*}$ & $2.646 \mathrm{y}$ & & $96.9 \%$ & $6.118(84 \%), 6.076(15.8 \%)$ \\
\hline $99^{252} \mathrm{Es}^{*}$ & $1.291 \mathrm{y}$ & & $76.4 \%$ & $\begin{array}{l}\text { 6.632(79.8\%), } 6.562(13.5 \%) \\
6.483(2.2 \%) \text {, others }\end{array}$ \\
\hline $99^{253} \mathrm{Es}$ & $20.4 \mathrm{~d}$ & & & $\begin{array}{c}6.633(89.8 \%), 6.592(6.6 \%) \\
\text { others }\end{array}$ \\
\hline $99^{254}$ Es & $275.7 d$ & & & $\begin{array}{l}\text { 6.427(93.1\%), 6.416(1.8\%), } \\
6.357(2.6 \%)\end{array}$ \\
\hline $99^{255}$ Es* & $39.8 \mathrm{~d}$ & & $8.0 \%$ & $\begin{array}{l}6.300(87.5 \%), 6.260(10.0 \%) \\
6.213(2.5 \%)\end{array}$ \\
\hline $100^{52} \mathrm{Fm}$ & $1.058 \mathrm{~d}$ & & & $7.04(\sim 85 \%), 6.999(\sim 15 \%)$ \\
\hline $100^{253} \mathrm{Fm}^{*}$ & $3.00 \mathrm{~d}$ & & $12.0 \%$ & $\begin{array}{l}7.024(6.7 \%), 6.943(42.7 \%) \\
6.901(9.8 \%), 6.847(8.5 \%) \\
6.674(23.3 \%), \text { others }\end{array}$ \\
\hline $100{ }^{255} \mathrm{Fm}$ & $20.07 \mathrm{~h}$ & & & $\begin{array}{c}\text { 7.022(93.4\%), } 6.963(5.0 \%) \\
\text { others }\end{array}$ \\
\hline $100^{257} \mathrm{Fm}$ & $100.5 \mathrm{~d}$ & & & $\begin{array}{l}6.696(3.4 \%), 6.519(93.5 \%) \\
6.441(2.0 \%)\end{array}$ \\
\hline $101{ }^{258} \mathrm{Md}$ & $55.4 d$ & & & $6.790(28 \%), 6.716(72 \%)$ \\
\hline
\end{tabular}

*Relative $\alpha$-intensity normalized to $100 \alpha$-decays. 


\section{ALPHA EMITTERS BY ENERGY (MeV)*}

\begin{tabular}{|c|c|c|c|}
\hline $3.8-4.0$ & $4.0-4.2$ & $4.2-4.4$ & $4.4-4.6$ \\
\hline \multicolumn{4}{|c|}{ Uranium Series: } \\
\hline & ${ }^{238} \mathrm{U}$ & & \\
\hline \multicolumn{4}{|c|}{ Thorium Series: } \\
\hline${ }^{232} \mathrm{Th}$ & ${ }^{232} \mathrm{Th}$ & & \\
\hline
\end{tabular}

$4.64 .8 \quad 4.8-5.0 \quad 5.0-5.2 \quad 5.2-5.4 \quad 5.4-5.6$

${ }^{234} \mathrm{U}$

${ }^{230} \mathrm{Th}_{2}$

${ }^{226} \mathrm{Ra}$

Thorium Serie:

Actinium Series:

${ }^{235} \mathrm{U}$

${ }^{235} \mathrm{U}$

${ }^{231} \mathrm{~Pa}$

${ }^{231} \mathrm{~Pa}$

${ }^{231} \mathrm{~Pa}$

Americium Series:

$\begin{array}{ll}{ }^{237} \mathrm{~Np} & \\ { }^{233} \mathrm{U} & { }^{233} \mathrm{U} \\ & { }^{229} \mathrm{Th}\end{array}{ }^{229} \mathrm{Th}$

All Emitters:

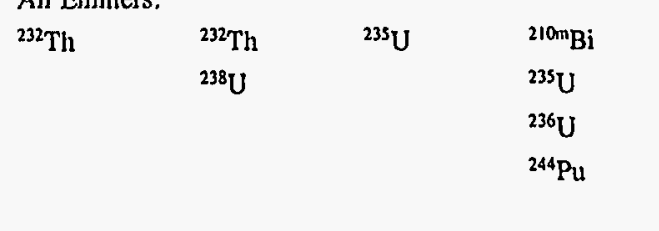

$\begin{array}{lll}{ }^{226} \mathrm{Ra} & { }^{209} \mathrm{Po} & { }^{208} \mathrm{Po} \\ { }^{230} \mathrm{Th} & { }^{210 \mathrm{~m}} \mathrm{Bi} & { }^{229} \mathrm{Th} \\ { }^{231} \mathrm{~Pa} & { }^{229} \mathrm{Th} & { }^{231} \mathrm{~Pa} \\ { }^{233} \mathrm{U} & { }^{231} \mathrm{~Pa} & { }^{239} \mathrm{Pu} \\ { }^{234} \mathrm{U} & { }^{233} \mathrm{U} & { }^{240} \mathrm{Pu} \\ { }^{237} \mathrm{~Np} & { }^{237} \mathrm{~Np} & { }^{243} \mathrm{Am} \\ & { }^{242} \mathrm{Pu} & { }^{248} \mathrm{Cm} \\ & { }^{247} \mathrm{Cm} & \end{array}$

${ }^{229} \mathrm{Th}$

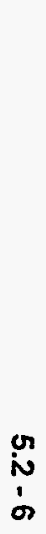

*A listing may denote more than one energy.

$5.4-5.6$

${ }^{222} \mathrm{Rn}$

${ }^{228} \mathrm{Th}$

${ }^{212} \mathrm{Bi}$

${ }^{223} \mathrm{Ra}$

${ }^{227} \mathrm{Th} \quad{ }^{227} \mathrm{Th} \quad{ }^{227} \mathrm{Th}$

${ }^{241} \mathrm{Am}$

${ }^{241} \mathrm{Am}$

${ }^{225} \mathrm{Ac}$

${ }^{225} \mathrm{Ac}$

${ }^{221} \mathrm{Fr}$

${ }^{221} \mathrm{Fr}$

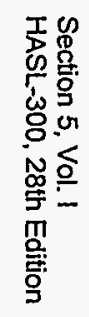

$\begin{array}{llllll}{ }^{206} \mathrm{Po} & { }^{222} \mathrm{Rn} & { }^{212} \mathrm{Bi} & { }^{211} \mathrm{At} & { }^{212} \mathrm{Bi} & { }^{211} \mathrm{Bi} \\ { }^{210} \mathrm{Po} & { }^{223} \mathrm{Ra} & { }^{223} \mathrm{Ra} & { }^{225} \mathrm{Ac} & { }^{218} \mathrm{Po} & { }^{220} \mathrm{Rn} \\ { }^{228} \mathrm{Th} & { }^{224} \mathrm{Ra} & { }^{224} \mathrm{Ra} & { }^{227} \mathrm{Th} & { }^{221} \mathrm{Fr} & { }^{221} \mathrm{Fr} \\ { }^{232} \mathrm{U} & { }^{228} \mathrm{Th} & { }^{225} \mathrm{Ac} & { }^{230} \mathrm{U} & { }^{227} \mathrm{Th} & { }^{240} \mathrm{Cm} \\ { }^{241} \mathrm{Am} & { }^{238} \mathrm{Pu} & { }^{227} \mathrm{Th} & { }^{243} \mathrm{Cm} & { }^{242} \mathrm{Cm} & { }^{248} \mathrm{Cf} \\ { }^{243} \mathrm{Am} & { }^{241} \mathrm{Am} & { }^{236} \mathrm{Pu} & { }^{244} \mathrm{Cm} & { }^{243} \mathrm{Cm} & { }^{254} \mathrm{Es} \\ { }^{245} \mathrm{Cm} & { }^{247} \mathrm{Bk} & { }^{243} \mathrm{Cm} & { }^{249} \mathrm{Cf} & { }^{249} \mathrm{Cr} & { }^{255} \mathrm{Es} \\ { }^{246} \mathrm{Cm} & & { }^{244} \mathrm{Cm} & { }^{250} \mathrm{Cf} & { }^{250} \mathrm{Cf} & \\ { }^{247} \mathrm{Cm} & & { }^{247} \mathrm{Bk} & { }^{251} \mathrm{Cf} & { }^{251} \mathrm{Cf} & \\ & & & & & \\ & & & & & \end{array}$


ALPHA EMITTERS BY ENERGY (MeV) -- (Cont'd)

\begin{tabular}{|c|c|c|c|c|c|c|c|c|c|c|c|}
\hline $6.4-6.6$ & $6.6-.68$ & $6.8-7.0$ & $7.0-7.2$ & 7.2-7.4 & 7.4-7.6 & 7.6-7.8 & $7.8-8.0$ & $8.0-8.2$ & $8.2-8.4$ & $8.4-.86$ & $8.6-8.8$ \\
\hline \multicolumn{12}{|c|}{ Uranium Series:: } \\
\hline Thorium & $:{ }^{216} \mathrm{Po}$ & & & & & & & & & & ${ }^{212} \mathrm{Po}$ \\
\hline \multicolumn{12}{|c|}{ Actinium Series: } \\
\hline Americi & & & ${ }^{217} \mathrm{At}$ & & & & & & ${ }^{213} \mathrm{p}_{\mathrm{O}}$ & & \\
\hline $\begin{array}{l}\text { All Emr } \\
{ }^{219} \mathrm{Rn} \\
{ }^{252} \mathrm{Es} \\
{ }^{253} \mathrm{Es} \\
{ }^{254} \mathrm{Es} \\
{ }^{255} \mathrm{Fmn}\end{array}$ & $\begin{array}{l}{ }^{211} \mathrm{Bi} \\
{ }^{216} \mathrm{Po} \\
{ }^{246} \mathrm{Cr} \\
{ }^{252} \mathrm{Es} \\
{ }^{253} \mathrm{Es} \\
{ }^{253} \mathrm{Fm} \\
{ }^{257} \mathrm{Fm} \\
{ }^{258} \mathrm{Md}\end{array}$ & $\begin{array}{l}{ }^{219} \mathrm{Rn} \\
{ }^{252} \mathrm{Fm} \\
{ }^{253} \mathrm{Fm} \\
{ }^{255} \mathrm{Fm}\end{array}$ & $\begin{array}{l}{ }^{217} \mathrm{At} \\
{ }^{252} \mathrm{Fm} \\
{ }^{253} \mathrm{Fm} \\
{ }^{255} \mathrm{Fm}\end{array}$ & ${ }^{215} \mathrm{Po}$ & & ${ }^{214} \mathrm{Po}$ & & & ${ }^{213} \mathrm{Po}_{0}$ & & ${ }^{212} \mathrm{Po}$ \\
\hline
\end{tabular}

iv 
SPECIFIC ACTIVITY OF SELECTED ALPHA EMITTERS (in order of ascending atomic numbers)

\begin{tabular}{|c|c|c|c|}
\hline Nuclide & $t_{1 / 2}(y)$ & $\mathrm{mCi} \mathrm{mg}$ & $\mathrm{MBq} \mathrm{mg}^{-1}$ \\
\hline${ }^{210 m} \mathrm{Bi}$ & $3.00 \times 10^{6}$ & $5.7 \times 10^{-4}$ & $2.11 \times 10^{-2}$ \\
\hline${ }^{208} \mathrm{Po}$ & 2.898 & 593.1 & $2.19 \times 10^{4}$ \\
\hline${ }^{209} \mathrm{Po}$ & 102 & 16.8 & $6.22 \times 10^{2}$ \\
\hline${ }^{226} \mathrm{Ra}$ & 1600 & 0.989 & $3.66 \times 10^{1}$ \\
\hline${ }^{228} \mathrm{Th}$ & 1.913 & 819.6 & $3.03 \times 10^{4}$ \\
\hline${ }^{229} \mathrm{Th}$ & 7340 & 0.213 & 7.88 \\
\hline${ }^{230} \mathrm{Th}$ & $7.54 \times 10^{4}$ & $2.062 \times 10^{-2}$ & $7.63 \times 10^{-1}$ \\
\hline${ }^{232} \mathrm{Th}$ & $1.405 \times 10^{10}$ & $1.0969 \times 10^{-7}$ & $4.06 \times 10^{-6}$ \\
\hline${ }^{231} \mathrm{~Pa}$ & $3.276 \times 10^{4}$ & $4.724 \times 10^{-2}$ & 1.75 \\
\hline${ }^{232} \mathrm{U}$ & 68.9 & 22.4 & $8.29 \times 10^{2}$ \\
\hline${ }^{233} \mathrm{U}$ & $1.592 \times 10^{5}$ & $9.64 \times 10^{-3}$ & $3.57 \times 10^{-1}$ \\
\hline${ }^{234} \mathrm{U}$ & $2.454 \times 10^{5}$ & $6.225 \times 10^{-3}$ & $2.30 \times 10^{-1}$ \\
\hline${ }^{235} \mathrm{U}$ & $7.037 \times 10^{8}$ & $1.922 \times 10^{-6}$ & $7.11 \times 10^{-5}$ \\
\hline${ }^{236} \mathrm{U}$ & $2.342 \times 10^{7}$ & $6.508 \times 10^{-5}$ & $2.41 \times 10^{-3}$ \\
\hline${ }^{238} \mathrm{U}$ & $4.468 \times 10^{9}$ & $3.36237 \times 10^{-7}$ & $1.24 \times 10^{-5}$ \\
\hline${ }^{237} \mathrm{~Np}$ & $2.140 \times 10^{6}$ & $7.05 \times 10^{-4}$ & $2.61 \times 10^{-2}$ \\
\hline${ }^{256} \mathrm{Pu}$ & 2.851 & 531.3 & $1.97 \times 10^{4}$ \\
\hline${ }^{238} \mathrm{Pu}$ & 87.74 & 17.119 & $6.33 \times 10^{2}$ \\
\hline${ }^{239} \mathrm{Pu}$ & $2.411 \times 10^{4}$ & $6.204 \times 10^{-2}$ & 2.30 \\
\hline${ }^{240} \mathrm{Pu}$ & 6563 & 0.22696 & 8.40 \\
\hline
\end{tabular}


SPECIFIC ACTIVITY OF SELECTED ALPHA EMITTERS (Cont'd)

(in order of ascending atomic numbers)

\begin{tabular}{|c|c|c|c|}
\hline Nuclide & $t_{1 / 2}(y)$ & $\mathrm{mCi} \mathrm{mg}^{-1}$ & $\mathrm{MBq} \mathrm{mg}^{-1}$ \\
\hline${ }^{242} \mathrm{Pu}$ & $3.763 \times 10^{5}$ & $3.962 \times 10^{-3}$ & $1.45 \times 10^{-1}$ \\
\hline${ }^{241} \mathrm{Am}$ & 432.7 & 3.428 & $1.27 \times 10^{2}$ \\
\hline${ }^{243} \mathrm{Am}$ & 7380 & 0.1993 & 7.37 \\
\hline${ }^{242} \mathrm{Cm}$ & $4.461 \times 10^{-1}$ & 3311.4 & $1.23 \times 10^{5}$ \\
\hline${ }^{243} \mathrm{Cm}$ & 28.5 & 51.6 & $1.91 \times 10^{3}$ \\
\hline${ }^{244} \mathrm{Cm}$ & 18.11 & 80.90 & $2.99 \times 10^{3}$ \\
\hline${ }^{245} \mathrm{Cm}$ & 8500 & 0.1717 & 6.35 \\
\hline${ }^{248} \mathrm{Cm}$ & $3.40 \times 10^{5}$ & $4.24 \times 10^{-3}$ & $1.57 \times 10^{-1}$ \\
\hline${ }^{247} \mathrm{Bk}$ & 1380 & 1.05 & $3.89 \times 10^{1}$ \\
\hline${ }^{248} \mathrm{Cf}$ & $9.1444 \times 10^{-1}$ & 1579 & $5.84 \times 10^{4}$ \\
\hline${ }^{249} \mathrm{Cf}$ & 350.6 & 4.095 & $1.52 \times 10^{2}$ \\
\hline${ }^{250} \mathrm{Cf}$ & 13.08 & 109.3 & $4.04 \times 10^{3}$ \\
\hline${ }^{251} \mathrm{Cf}$ & 898 & 1.59 & $5.88 \times 10^{1}$ \\
\hline${ }^{252} \mathrm{Cf}$ & 2.645 & 536.3 & $1.98 \times 10^{4}$ \\
\hline${ }^{252} \mathrm{Es}$ & 1.291 & 1098 & $4.06 \times 10^{4}$ \\
\hline${ }^{254} \mathrm{Es}$ & $7.54825 \times 10^{-1}$ & 1865 & $6.90 \times 10^{4}$ \\
\hline
\end{tabular}




\subsection{BETA}

Contact Person(s) : Isabel M. Fisenne

\subsubsection{SCOPE}

The table of $\beta$ emitters presented here is useful in identifying unknown $\beta$ emitters whose energies and possibly half-lives have been determined by standard laboratory techniques, including the Harley-Hallden method (see Procedure A-01-R). It is also a handy guide to $\beta$ emitting isotopes for applications requiring specific half-lives and/or energies.

The original table was developed by Hallden (1955). Since that time there have been three revisions based on "current" published compilations. The present table was compiled from the Table of Isotopes (Lederer and Shirley, 1978).

The emitter energy listings are the maximum $\beta$ energy $\left(\mathrm{E}_{\max }\right)$. Isotopes decaying the emission of $\beta$ particles of different energies are listed in the energy group corresponding to each $E_{\max }$, provided that the branch contributes $>5 \%$ of the total $\beta$ emission. Isotopes with longer-lived parents are also listed under the parent half-life. In the case of multiple long-lived parents, the nuclide is listed under the half-life of the nearest long-lived parent, as well as its own half-life.

\section{REFERENCES}

Hallden, N. A.

"Beta Emitters by Energy and Half-life"

Nucleonics, 13, 78-79 (1955)

Lederer, C. and V. S. Shirley (Editors)

Table of Isotopes - Seventh Edition

John Wiley and Sons, New York, NY (1978) 


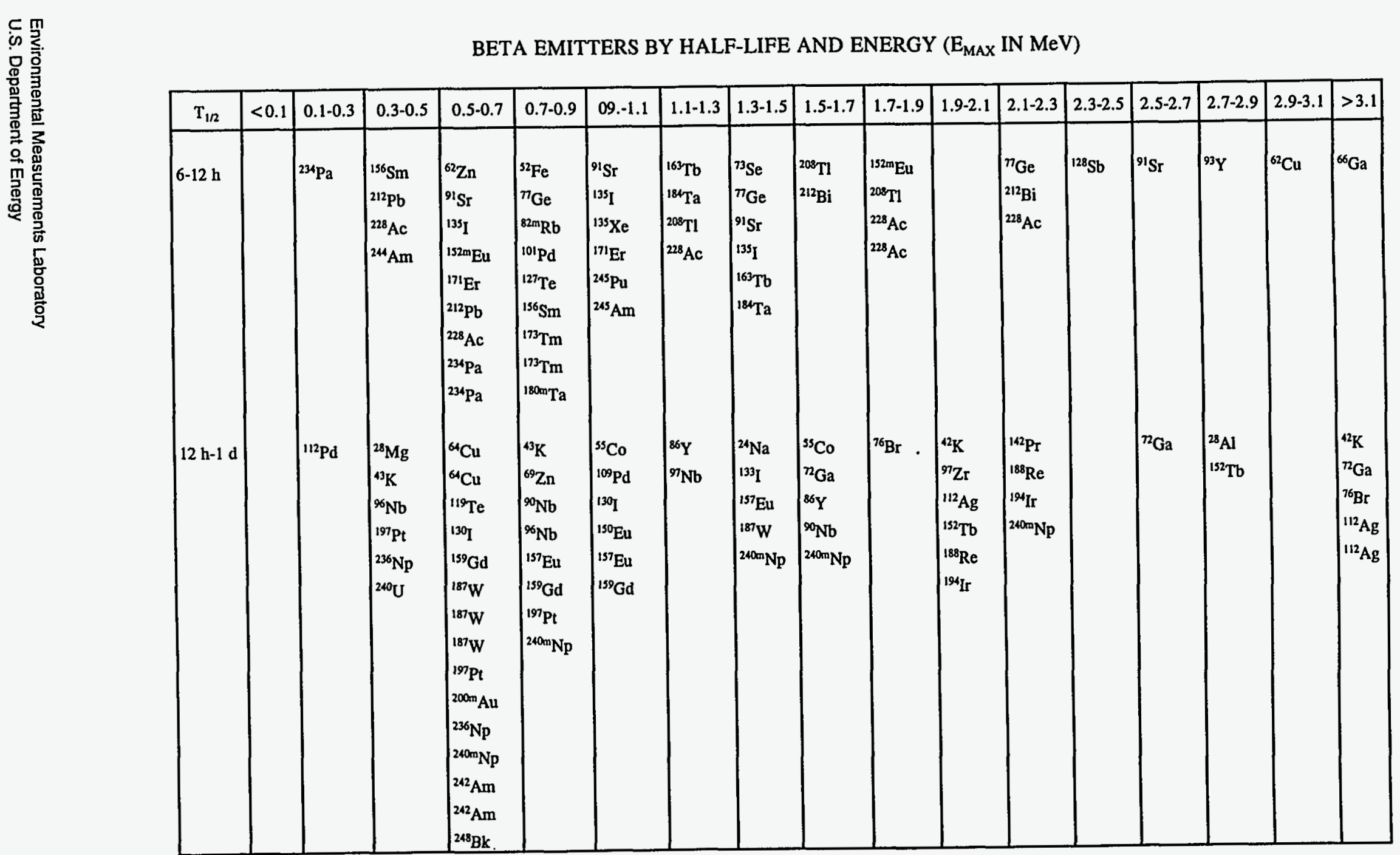


BETA EMITTERS BY HALF-LIFE AND ENERGY (E MAX $\left._{\text {IN }} \mathrm{MeV}\right)$ - (Cont'd)

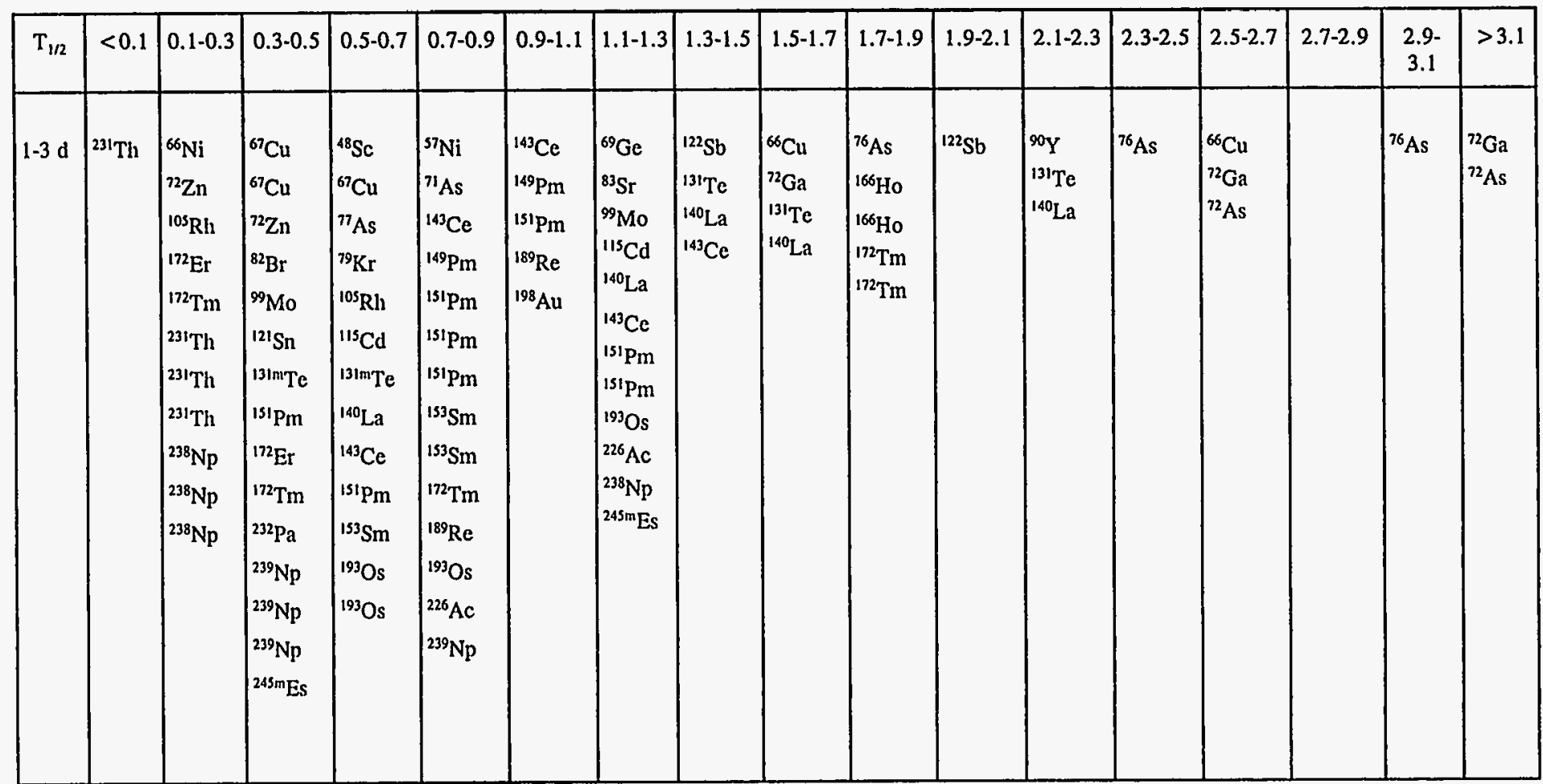

Note: Where the symbol for a nuclide appears more than once in any grouping it indicates emission of more than one energy. 
BETA EMITTERS BY HALF-LIFE AND ENERGY (E $\mathrm{E}_{\mathrm{MAX}}$ IN MeV) - (Cont'd)

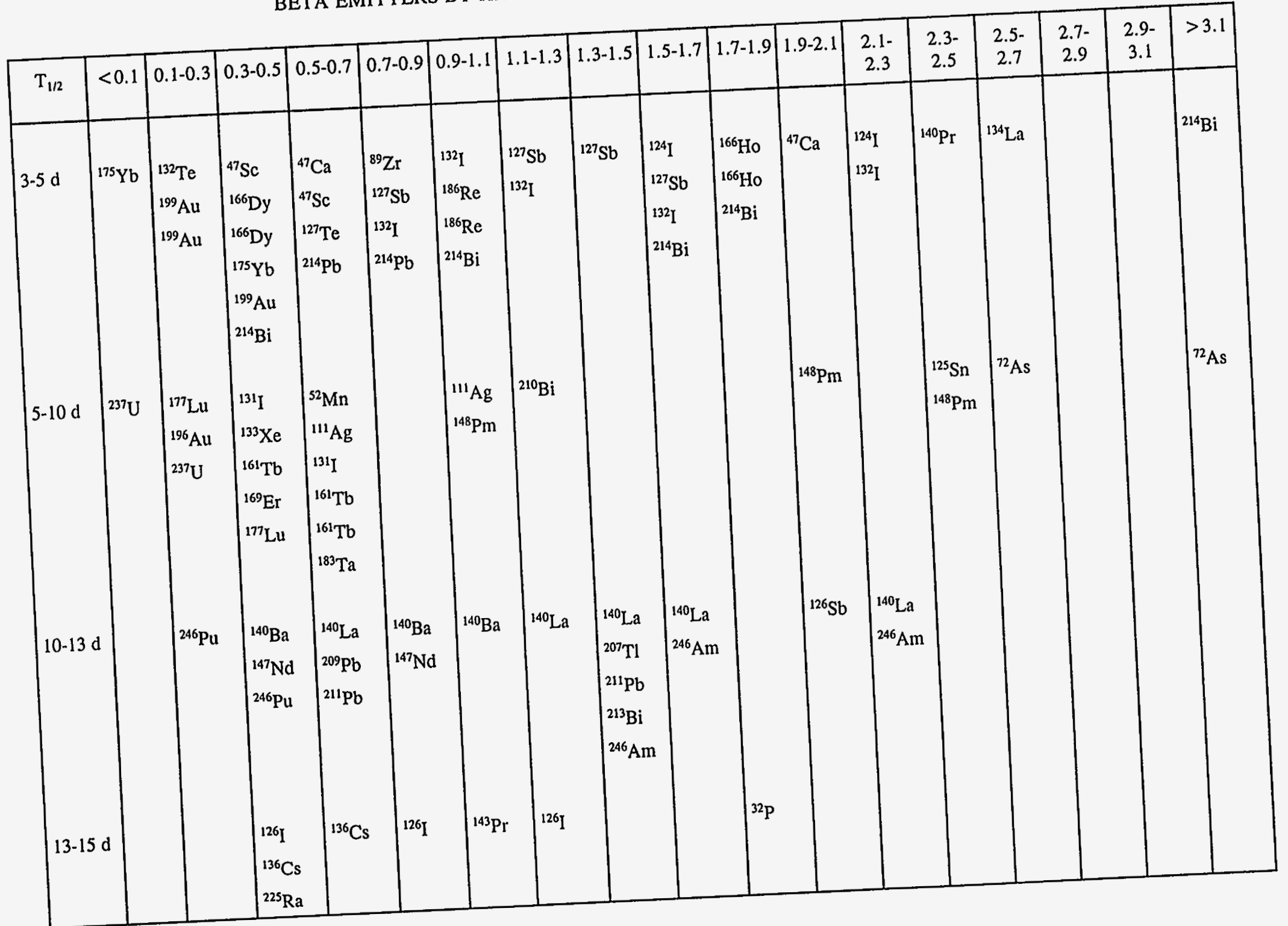




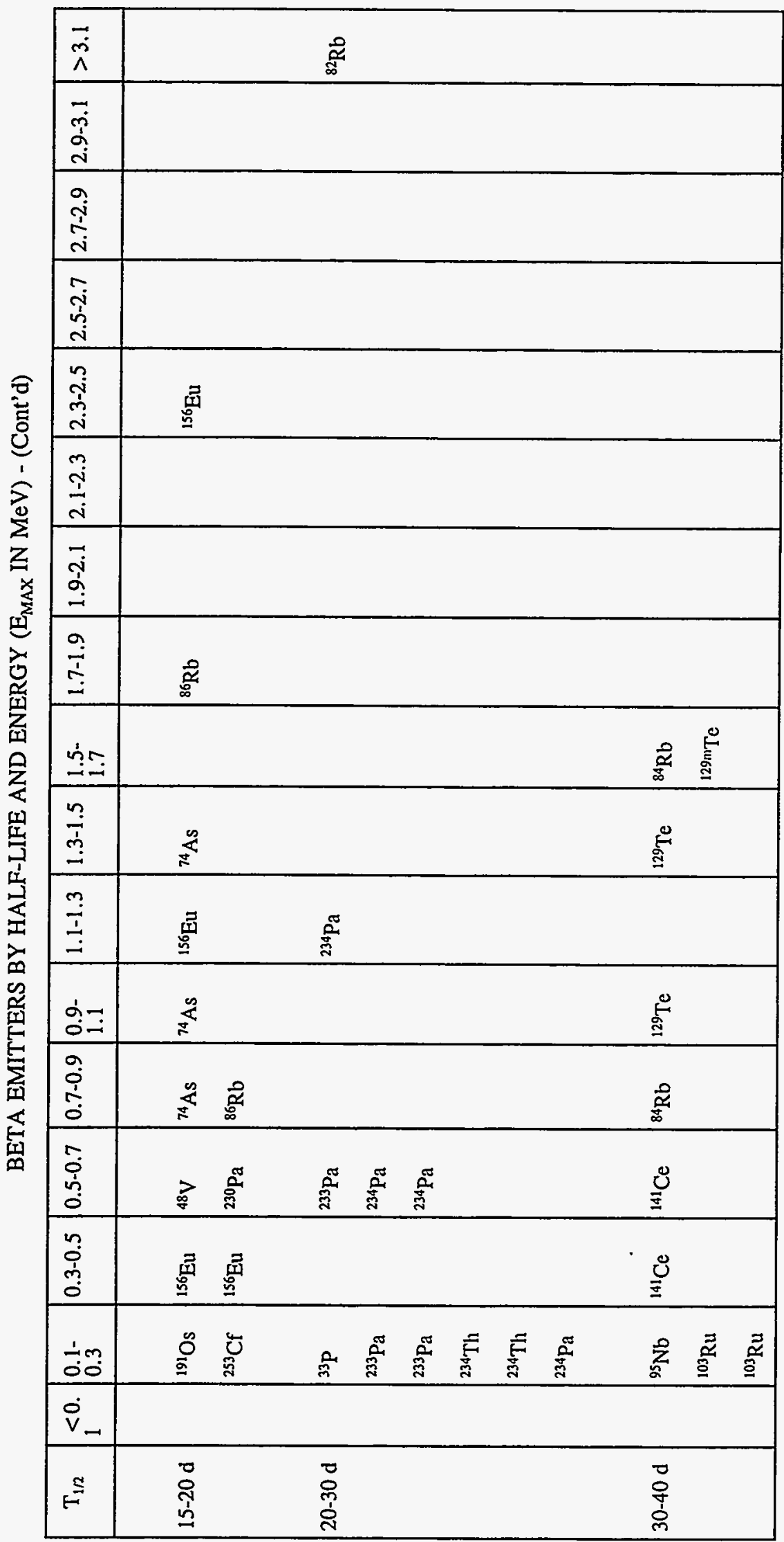


BETA EMITTERS BY HALF-LIFE AND ENERGY (E

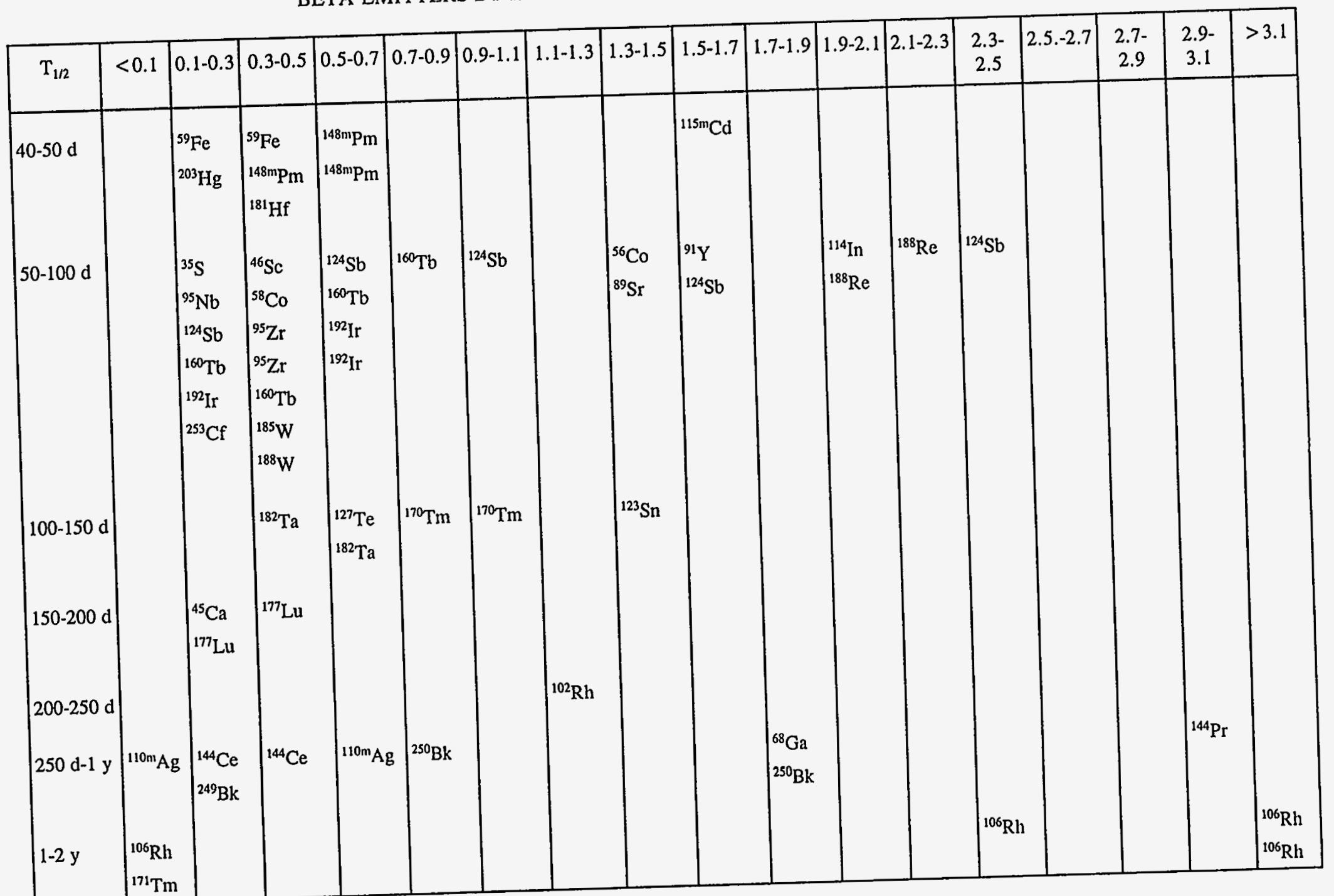




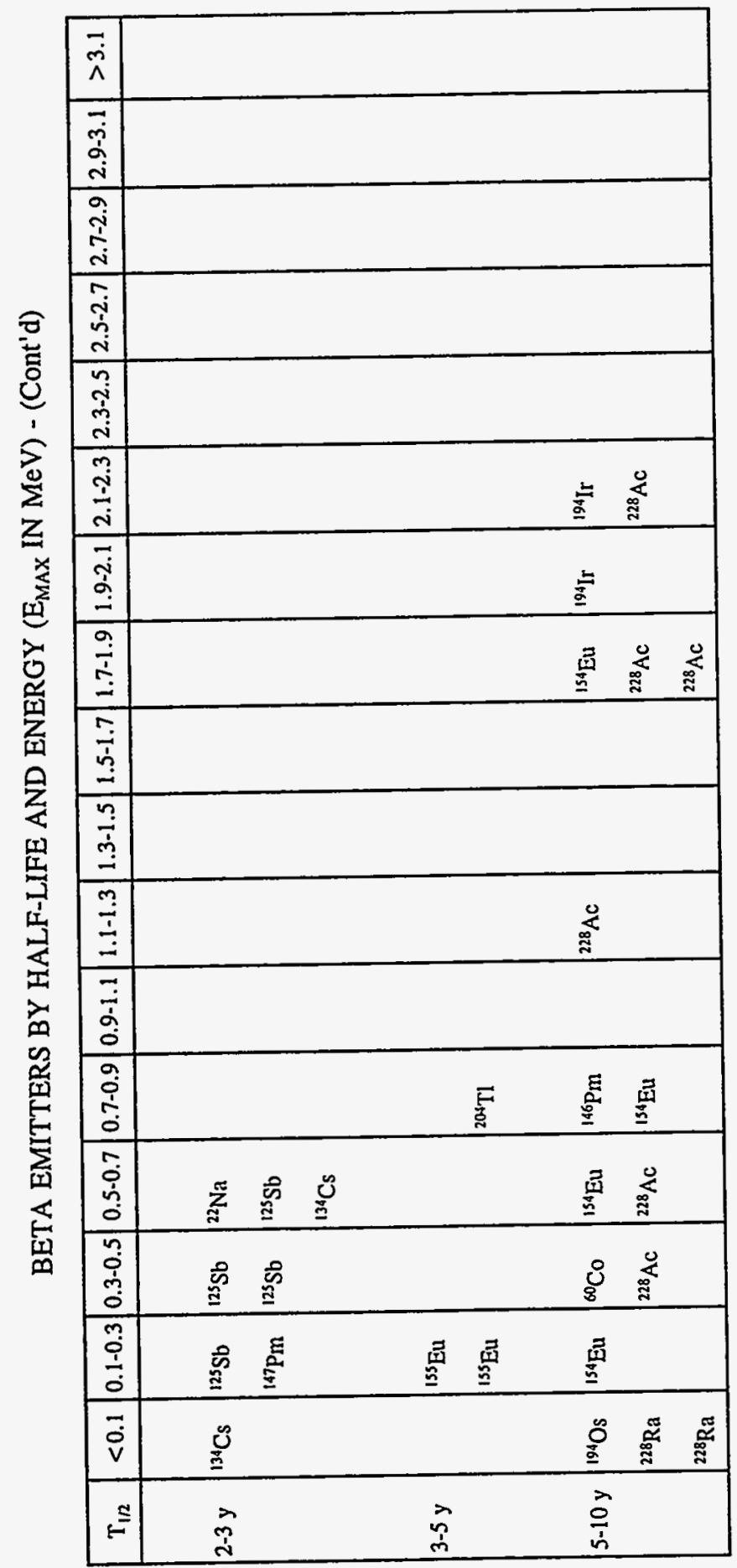




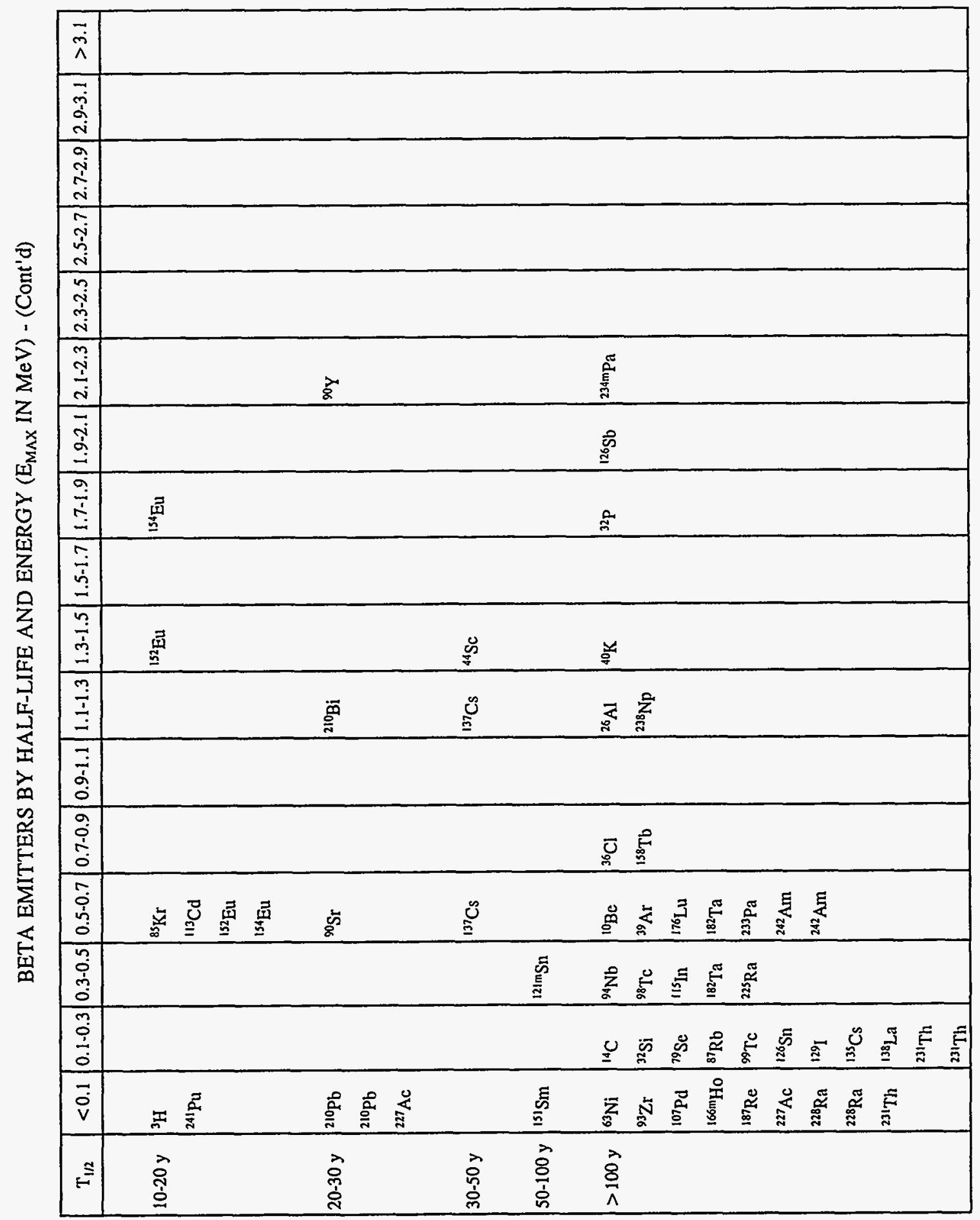


BETA EMITTERS BY HALF-LIFE AND ENERGY (E $E_{\text {MAX }}$ IN MeV) - (Cont'd)

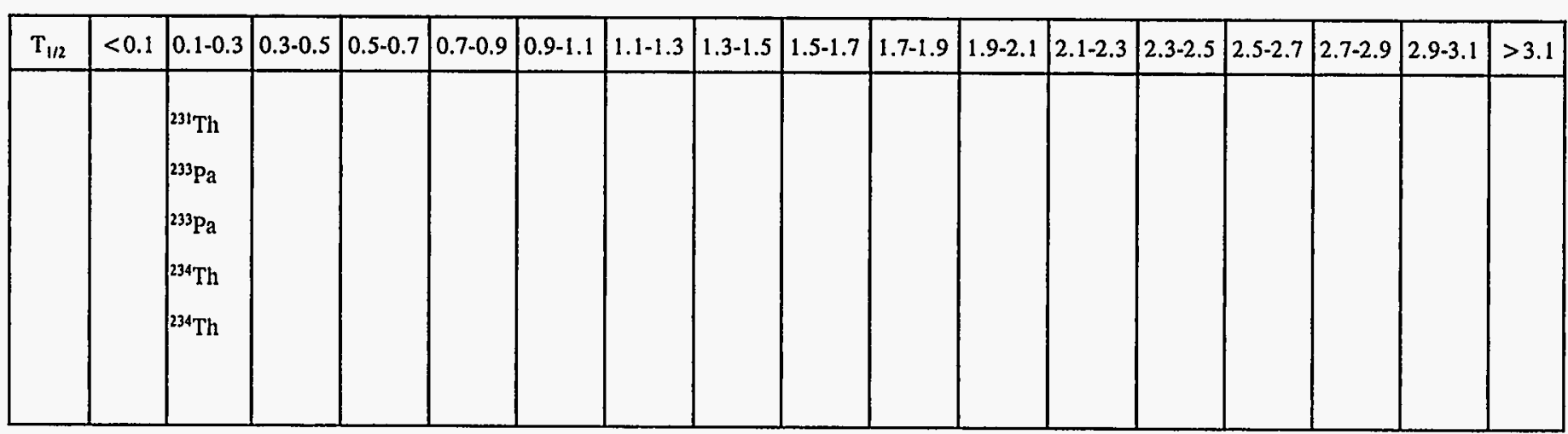

Note: Where the symbol for a nuclide appears more than once in any grouping it indicates emission of more than one energy. 


\subsection{GAMMA}

Contact Person(s) : Colin G. Sanderson

\subsubsection{SCOPE}

Presented here is a tabulation of $\gamma$ rays and nuclides that are common to environmental analyses. These data may be useful for interpreting either field or laboratory measurements.

As an aid in verifying nuclide identifications, two additional $\boldsymbol{\gamma}$-ray lines are included when possible as E2 and E3.

The following special notations are used in the table:

\begin{tabular}{cl}
\hline Symbol & \multicolumn{1}{c}{ Meaning } \\
\hline$\%$ & ratio of $\gamma$-rays to disintegrations. \\
$\mathrm{X}$ & indicates X-ray. \\
$\mathrm{KX}, \mathrm{LX}$ & sum of K or L X-rays. \\
$\mathrm{D}$ & indicates a doublet. \\
$\mathrm{T}$ & indicates a triplet. \\
$*$ & indicates annihilation radiation. \\
Long & $\begin{array}{l}\text { in half-life column is used for all } \\
\text { members of the primordial series, } \\
\%\end{array}$ \\
\hline
\end{tabular}




\section{REFERENCE}

Browne, E., R. B. Firestone and V. S. Shirley (Editors)

Table of Radioactive Isotopes

John Wiley and Sons, Inc., New York (1986) 
TABLE OF $\gamma$ RAYS AND NUCLIDES COMMON TO ENVIRONMENTAL ANALYSES

\begin{tabular}{|c|c|c|c|c|c|c|c|c|}
\hline Energy & Nuclide & $\%$ & $T_{1 / 2}$ & E2 & $\%$ & E3 & $\%$ & Origin \\
\hline 14.4 & ${ }^{57} \mathrm{Co}$ & 9.5 & $272 \mathrm{D}$ & 122.1 & 85.5 & 136.5 & 10.7 & Activation \\
\hline 26.3 & ${ }^{24 t} \mathrm{Am}$ & 2.4 & $433 Y$ & 59.5 & 35.7 & 17.0LX & 38.7 & ${ }^{241} \mathrm{Pu}$ \\
\hline 30.0 & ${ }^{140} \mathrm{Ba}$ & 13.6 & $12.8 \mathrm{D}$ & 162.7 & 6.2 & 304.9 & 4.3 & Fallout \\
\hline 39.6 & ${ }^{129} \mathrm{I}$ & 7.5 & $1.6 \mathrm{E} 7$ & $30.0 \mathrm{KX}$ & 70.8 & & & Fission \\
\hline 39.9 & ${ }^{212} \mathrm{Bi}$ & 1.1 & Long & 727.3 & 6.7 & 1620.7 & 1.5 & ${ }^{232} \mathrm{Th}$ \\
\hline 40.6 & ${ }^{99} \mathrm{Mo}$ & 1.1 & $65.9 \mathrm{H}$ & $18.3 \mathrm{X}$ & 3.2 & 140.5 & 3.5 & Fallout \\
\hline 46.5 & ${ }^{210} \mathrm{~Pb}$ & 4.1 & $22.3 Y$ & & & & & ${ }^{238} \mathrm{U}$ \\
\hline 49.8 & ${ }^{132} \mathrm{Te}$ & 14.4 & $78.2 \mathrm{H}$ & $30.0 \mathrm{KX}$ & 70.9 & 111.9 & 1.9 & Fallout \\
\hline 53.2 & ${ }^{133} \mathrm{Ba}$ & 2.2 & $10.5 Y$ & 81.0 & 34.2 & $31.0 \mathrm{KX}$ & 101.3 & Activation \\
\hline 59.5 & ${ }^{237} \mathrm{U}$ & 32.8 & $6.75 D$ & 101.1 & 26.0 & 208.0 & 22.0 & Fallout \\
\hline 59.5 & ${ }^{241} \mathrm{Am}$ & 35.7 & $433 Y$ & 26.3 & 2.4 & $17.0 \mathrm{LX}$ & 38.7 & ${ }^{241} \mathrm{Pu}$ \\
\hline 60.0 & ${ }^{155} \mathrm{Eu}$ & 1.1 & $4.96 \mathrm{Y}$ & 86.5 & 30.4 & 105.3 & 20.6 & Fallout \\
\hline 61.5 & ${ }^{239} \mathrm{~Np}$ & 1.0 & $2.36 \mathrm{D}$ & $14.3 \mathrm{LX}$ & 56.1 & $101.0 \mathrm{KX}$ & 38.9 & Fallout \\
\hline 63.3 & ${ }^{234} \mathrm{Th}$ & 3.8 & Long & $92.6 \mathrm{D}$ & 5.4 & & & ${ }^{238} \mathrm{U}$ \\
\hline 66.9 & ${ }^{136} \mathrm{Cs}$ & 12.5 & $13.2 \mathrm{D}$ & $34.0 \mathrm{KX}$ & 17.6 & 86.4 & 6.3 & Fission \\
\hline $74.8 \mathrm{X}$ & ${ }^{214} \mathrm{~Pb}$ & 6.5 & Long & $77.1 \mathrm{x}$ & 11.0 & $87.3 \mathrm{X}$ & 3.9 & ${ }^{238} \mathrm{U}$ \\
\hline $74.8 \mathrm{X}$ & ${ }^{212} \mathrm{~Pb}$ & 10.5 & Long & $77.1 \mathrm{X}$ & 17.7 & $87.2 \mathrm{X}$ & 6.3 & ${ }^{232} \mathrm{Th}$ \\
\hline $75.0 \mathrm{X}$ & ${ }^{208} \mathrm{TI}$ & 3.6 & Long & $72.8 \mathrm{X}$ & 2.1 & $84.8 X$ & 1.3 & ${ }^{232} \mathrm{Th}$ \\
\hline $77.1 X$ & ${ }^{214} \mathrm{~Pb}$ & 11.0 & Long & $74.8 \mathrm{X}$ & 6.5 & $87.2 X$ & 3.9 & ${ }^{238} \mathrm{U}$ \\
\hline $77.1 \mathrm{X}$ & ${ }^{212} \mathrm{~Pb}$ & 17.7 & Long & $74.8 \mathrm{X}$ & 10.5 & $87.2 \mathrm{X}$ & 6.3 & ${ }^{232} \mathrm{Th}$ \\
\hline 79.6 & ${ }^{133} \mathrm{Ba}$ & 3.2 & $10.5 \mathrm{Y}$ & 53.2 & 2.2 & & & Activation \\
\hline 80.1 & ${ }^{144} \mathrm{Ce}$ & 1.1 & $285 \mathrm{D}$ & 133.5 & 11.1 & 696.5 & 1.3 & Fallout \\
\hline 80.2 & ${ }^{131} I$ & 2.6 & $8.04 \mathrm{D}$ & 364.5 & 81.2 & 284.3 & 6.1 & Fission \\
\hline 81.0 & ${ }^{133} \mathrm{Ba}$ & 34.2 & $10.5 Y$ & 276.4 & 7.3 & 79.6 & 3.2 & Activation \\
\hline 81.0 & ${ }^{133} \mathrm{Xe}$ & 37.0 & $5.25 \mathrm{D}$ & 79.6 & 0.2 & $31.0 \mathrm{KX}$ & 40.1 & Fission \\
\hline $84.3 \mathrm{X}$ & ${ }^{228} \mathrm{Th}$ & 1.2 & $1.91 Y$ & $12.3 \mathrm{X}$ & 3.1 & & & ${ }^{232} \mathrm{Th}$ \\
\hline 86.4 & ${ }^{136} \mathrm{Cs}$ & 6.3 & $13.2 \mathrm{D}$ & 66.9 & 12.5 & 153.3 & 7.5 & Fission \\
\hline 86.5 & ${ }^{155} \mathrm{Eu}$ & 34.0 & $4.96 Y$ & 105.3 & 20.6 & 60.0 & 1.1 & Fallout \\
\hline $87.2 \mathrm{X}$ & ${ }^{214} \mathrm{~Pb}$ & 3.9 & Long & $77.1 \mathrm{X}$ & 11.0 & 241.9 & 7.5 & ${ }^{238} \mathrm{U}$ \\
\hline $87.2 \mathrm{X}$ & ${ }^{212} \mathrm{~Pb}$ & 6.3 & Long & 238.6 & 43.6 & $77.1 \mathrm{X}$ & 17.7 & ${ }^{232} \mathrm{Th}$ \\
\hline 88.0 & ${ }^{109} \mathrm{Cd}$ & 3.6 & $463 D$ & $23.0 \mathrm{KX}$ & 99.8 & & & Activation \\
\hline $90.0 \mathrm{X}$ & ${ }^{228} \mathrm{Ac}$ & 3.4 & Long & $93.4 \mathrm{X}$ & 5.6 & 99.6 & 1.3 & ${ }^{232} \mathrm{Th}$ \\
\hline 91.1 & ${ }^{147} \mathrm{Nd}$ & 28.0 & $11.0 \mathrm{D}$ & $38.5 \mathrm{KX}$ & 37.4 & 319.4 & 2.0 & Fallout \\
\hline $92.6 \mathrm{D}$ & ${ }^{234} \mathrm{Th}$ & 5.4 & Long & 63.3 & 3.8 & & & ${ }^{238} \mathrm{U}$ \\
\hline $93.4 \mathrm{X}$ & ${ }^{228} \mathrm{AC}$ & 5.6 & Long & $90.0 \mathrm{X}$ & 3.4 & 99.6 & 1.3 & ${ }^{232} \mathrm{Th}$ \\
\hline 97.1 & ${ }^{237} \mathrm{U}$ & 16.0 & $6.75 \mathrm{D}$ & 101.0 & 26.0 & 208.0 & 22.0 & Fallout \\
\hline 99.6 & ${ }^{228} \mathrm{AC}$ & 1.3 & Long & 129.0 & 2.9 & 209.4 & 4.1 & ${ }^{232} \mathrm{Th}$ \\
\hline
\end{tabular}


TABLE OF $\gamma$ RAYS AND NUCLIDES COMMON TO ENVIRONMENTAL ANALYSES (Cont'd)

\begin{tabular}{|c|c|c|c|c|c|c|c|c|}
\hline Energy & Nuclide & $\%$ & $T_{1 / 2}$ & E2 & $\%$ & E3 & $\%$ & Origin \\
\hline 101.1 & ${ }^{237} \mathrm{U}$ & 26.0 & $6.75 \mathrm{D}$ & 59.5 & 32.8 & 208.0 & 22.0 & Fallout \\
\hline 105.3 & ${ }^{155} \mathrm{Eu}$ & 20.6 & $4.96 \mathrm{Y}$ & 86.5 & 34.0 & 60.0 & 1.1 & Fallout \\
\hline $105.4 \mathrm{X}$ & ${ }^{228} \mathrm{AC}$ & 2.0 & Long & 99.6 & 1.3 & 129.0 & 2.9 & ${ }^{232} \mathrm{Th}$ \\
\hline 106.1 & ${ }^{239} \mathrm{~Np}$ & 22.7 & $2.36 \mathrm{D}$ & 61.5 & 1.0 & $117.0 \mathrm{KX}$ & 11.6 & Fallout \\
\hline 109.2 & ${ }^{235} \mathrm{U}$ & 1.5 & 70E7Y & $93.4 \mathrm{KX}$ & 5.5 & 143.8 & 10.5 & Natural \\
\hline 111.9 & ${ }^{132} \mathrm{Te}$ & 1.9 & $78.2 \mathrm{H}$ & 49.8 & 14.4 & 116.4 & 1.9 & Fallout \\
\hline 113.9 & ${ }^{237} \mathrm{U}$ & 25.0 & $6.75 \mathrm{D}$ & 101.1 & 26.0 & 208.0 & 22.0 & Fallout \\
\hline 116.3 & ${ }^{132} \mathrm{Te}$ & 1.9 & $78.2 \mathrm{H}$ & 111.9 & 1.9 & 228.3 & 88.2 & Fallout \\
\hline 121.8 & ${ }^{152} \mathrm{Eu}$ & 28.4 & $13.3 Y$ & 344.3 & 26.6 & 244.7 & 7.5 & Fallout \\
\hline 122.1 & ${ }^{57} \mathrm{Co}$ & 85.5 & 273D & 136.5 & 10.7 & 14.4 & 9.5 & Activation \\
\hline 123.1 & ${ }^{154} \mathrm{Eu}$ & 40.5 & $8.8 Y$ & 248.0 & 6.6 & 591.8 & 4.8 & Fallout \\
\hline 127.2 & ${ }^{10} \mathrm{Rh}$ & 73.0 & $3.3 Y$ & 198.0 & 70.8 & 325.2 & 13.4 & Fallout \\
\hline 129.0 & ${ }^{228} \mathrm{Ac}$ & 2.9 & Long & 99.6 & 1.3 & 209.4 & 4.1 & ${ }^{232} \mathrm{Th}$ \\
\hline 133.5 & ${ }^{144} \mathrm{Ce}$ & 11.1 & $285 \mathrm{D}$ & 696.5 & 1.3 & 80.1 & 1.1 & Fallout \\
\hline 136.5 & ${ }^{57} \mathrm{Co}$ & 10.7 & $272 \mathrm{D}$ & 122.1 & 85.5 & 14.4 & 9.5 & Activation \\
\hline 138.0 & ${ }^{138} \mathrm{Cs}$ & 1.5 & $32.2 \mathrm{M}$ & 227.7 & 1.5 & 462.8 & 30.7 & ${ }^{138} \mathrm{Xe}$ \\
\hline 140.5 & ${ }^{99} \mathrm{Mo}$ & 3.5 & $65.9 \mathrm{H}$ & 40.6 & 1.1 & 181.1 & 6.1 & Fallout \\
\hline 140.5 & ${ }^{99^{m}} \mathrm{Tc}$ & 87.2 & $6.01 \mathrm{H}$ & $18.4 \mathrm{X}$ & 6.1 & $20.6 \mathrm{X}$ & 1.2 & ${ }^{99} \mathrm{Mo}$ \\
\hline 143.8 & ${ }^{235} \mathrm{U}$ & 10.5 & $70 \mathrm{E} 7 \mathrm{Y}$ & 109.2 & 1.5 & 163.4 & 4.7 & Natural \\
\hline 145.4 & ${ }^{141} \mathrm{C}$ & 48.4 & $32.5 \mathrm{D}$ & $37.0 \mathrm{KX}$ & 17.4 & & & Fission \\
\hline 151.2 & ${ }^{85 \mathrm{~m}} \mathrm{Kr}$ & 75.2 & $4.48 \mathrm{H}$ & 304.9 & 13.7 & & & Fission \\
\hline 153.3 & ${ }^{136} \mathrm{Cs}$ & 7.5 & $13.2 \mathrm{D}$ & 86.4 & 6.3 & 164.0 & 4.6 & Fission \\
\hline 153.9 & ${ }^{138} \mathrm{Xe}$ & 6.0 & $14.1 \mathrm{M}$ & 242.7 & 3.5 & 258.4 & 31.5 & Fission \\
\hline 162.7 & ${ }^{140} \mathrm{Ba}$ & 6.2 & $12.8 \mathrm{D}$ & 304.9 & 4.3 & 30.0 & 13.6 & Fallout \\
\hline 163.4 & ${ }^{235} \mathrm{U}$ & 4.7 & 70E7Y & 143.8 & 10.5 & 185.7 & 53.0 & Natural \\
\hline 164.0 & ${ }^{136} \mathrm{Cs}$ & 4.6 & $13.2 \mathrm{D}$ & 153.3 & 7.5 & 176.6 & 13.6 & Fission \\
\hline 165.9 & ${ }^{139} \mathrm{Ce}$ & 79.9 & $138 \mathrm{D}$ & $34.0 \mathrm{KX}$ & 79.5 & & & Activation \\
\hline 166.0 & ${ }^{88} \mathrm{Kr}$ & 3.1 & $2.84 \mathrm{H}$ & 196.3 & 26.0 & 362.3 & 2.3 & Fission \\
\hline 176.3 & ${ }^{125} \mathrm{Sb}$ & 6.8 & $2.73 \mathrm{Y}$ & 427.9 & 29.4 & 380.4 & 1.5 & Fallout \\
\hline 176.6 & ${ }^{136} \mathrm{Cs}$ & 13.6 & $13.2 \mathrm{D}$ & 164.0 & 4.6 & 273.7 & 12.7 & Fission \\
\hline 181.1 & ${ }^{99} \mathrm{Mo}$ & 6.1 & $65.9 \mathrm{H}$ & 140.5 & 3.5 & 366.4 & 1.2 & Fallout \\
\hline 185.7 & ${ }^{235} \mathrm{U}$ & 53.0 & 70 E7Y & 143.8 & 10.5 & 205.3 & 4.7 & Natural \\
\hline 186.1 & ${ }^{226} \mathrm{Ra}$ & 3.3 & $1600 Y$ & & & & & Natural \\
\hline 192.3 & ${ }^{59} \mathrm{Fe}$ & 3.1 & 44.5D & 1099.3 & 56.5 & 1291.6 & 43.2 & Activation \\
\hline 196.3 & ${ }^{88} \mathrm{Kr}$ & 26.0 & $2.84 \mathrm{H}$ & 362.3 & 2.3 & 166.0 & 3.1 & Fission \\
\hline 198.0 & ${ }^{101} \mathrm{Rh}$ & 70.8 & $3.3 \mathrm{Y}$ & 127.2 & 73.0 & 325.2 & 13.4 & Fallout \\
\hline 205.3 & ${ }^{235} \mathrm{U}$ & 4.7 & 70E7Y & 185.7 & 53.0 & 143.8 & 10.5 & Natural \\
\hline 208.0 & ${ }^{237} \mathrm{U}$ & 22.0 & $6.75 \mathrm{D}$ & 59.5 & 32.8 & 101.1 & 26.0 & Fallout \\
\hline
\end{tabular}


TABLE OF $\gamma$ RAYS AND NUCLIDES COMMON TO ENVIRONMENTAL ANALYSES (Cont'd)

\begin{tabular}{|c|c|c|c|c|c|c|c|c|}
\hline Energy & Nuclide & $\%$ & $T_{1 / 2}$ & E2 & $\%$ & E3 & $\%$ & Origin \\
\hline 209.4 & ${ }^{228} \mathrm{Ac}$ & 4.1 & Long & 129.0 & 2.9 & 270.3 & 3.8 & ${ }^{232} \mathrm{Th}$ \\
\hline 227.7 & ${ }^{138} \mathrm{Cs}$ & 1.5 & $32.2 \mathrm{M}$ & 138.0 & 1.5 & 409.0 & 4.7 & ${ }^{138} \mathrm{Xe}$ \\
\hline 228.2 & ${ }^{239} \mathrm{~Np}$ & 10.7 & $2.36 \mathrm{D}$ & 106.1 & 22.7 & 277.6 & 14.2 & Fallout \\
\hline 228.3 & ${ }^{132} \mathrm{Te}$ & 88.2 & $78.2 \mathrm{H}$ & 116.4 & 1.9 & 111.9 & 1.9 & Fallout \\
\hline 233.2 & ${ }^{133 \mathrm{~m}} \mathrm{Xe}$ & 10.3 & $2.19 \mathrm{D}$ & $30.0 \mathrm{KX}$ & 56.3 & & & Fission \\
\hline 238.6 & ${ }^{212} \mathrm{~Pb}$ & 43.6 & Long & 300.0 & 3.3 & & & ${ }^{232} \mathrm{Th}$ \\
\hline 240.8 & ${ }^{224} \mathrm{Ra}$ & 3.9 & Long & & & & & ${ }^{232} \mathrm{Th}$ \\
\hline 241.9 & ${ }^{214} \mathrm{~Pb}$ & 7.5 & Long & 295.1 & 19.2 & 352.0 & 37.1 & ${ }^{238} \mathrm{U}$ \\
\hline 242.7 & ${ }^{138} \mathrm{Xe}$ & 3.5 & $14.1 \mathrm{M}$ & 153.9 & 6.0 & 258.4 & 31.5 & Fission \\
\hline 244.7 & ${ }^{152} \mathrm{Eu}$ & 7.5 & $13.3 Y$ & 121.8 & 28.4 & 344.3 & 26.6 & Fallout \\
\hline 248.0 & ${ }^{154} \mathrm{Eu}$ & 6.6 & $8.8 \mathrm{Y}$ & 123.1 & 40.5 & 591.8 & 4.8 & Fallout \\
\hline 249.8 & ${ }^{135} \mathrm{Xe}$ & 90.0 & $9.10 \mathrm{H}$ & 608.2 & 2.9 & $31.0 \mathrm{KX}$ & 5.2 & Fission \\
\hline 258.4 & ${ }^{138} \mathrm{Xe}$ & 31.5 & $14.1 \mathrm{M}$ & 242.7 & 3.5 & 396.6 & 6.3 & Fission \\
\hline 262.8 & ${ }^{132} \mathrm{I}$ & 1.4 & $2.28 \mathrm{H}$ & 505.9 & 5.0 & 522.7 & 16.1 & ${ }^{132} \mathrm{Te}$ \\
\hline 270.3 & ${ }^{228} \mathrm{AC}$ & 3.8 & Long & 209.4 & 4.1 & 328.0 & 3.5 & ${ }^{232} \mathrm{Th}$ \\
\hline 273.7 & ${ }^{136} \mathrm{Cs}$ & 12.7 & $13.2 \mathrm{D}$ & 176.6 & 13.6 & 340.6 & 48.6 & Fission \\
\hline 276.4 & ${ }^{133} \mathrm{Ba}$ & 7.1 & $10.5 \mathrm{Y}$ & 302.9 & 18.4 & 81.0 & 34.2 & Activation \\
\hline 277.3 & ${ }^{208} \mathrm{TI}$ & 2.4 & Long & 510.6 & 7.8 & 583.0 & 30.9 & ${ }^{232} \mathrm{Th}$ \\
\hline 277.6 & ${ }^{239} \mathrm{~Np}$ & 14.2 & $2.36 \mathrm{D}$ & 228.2 & 10.7 & 315.9 & 1.6 & Fallout \\
\hline 279.2 & ${ }^{203} \mathrm{Hg}$ & 81.5 & 46.ळD & $74.6 \mathrm{X}$ & 12.9 & & & Fallout \\
\hline 284.3 & ${ }^{131} I$ & 6.1 & $8.04 \mathrm{D}$ & 364.5 & 81.2 & 80.2 & 2.6 & Fission \\
\hline 295.1 & ${ }^{214} \mathrm{~Pb}$ & 19.2 & Long & 351.9 & 37.1 & 241.9 & 7.5 & ${ }^{238} \mathrm{U}$ \\
\hline 300.0 & ${ }^{212} \mathrm{~Pb}$ & 3.3 & Long & 238.6 & 43.6 & & & ${ }^{232} \mathrm{Th}$ \\
\hline 302.9 & ${ }^{133} \mathrm{Ba}$ & 18.4 & $10.5 \mathrm{Y}$ & 276.4 & 7.1 & 356.0 & 62.2 & Activation \\
\hline 304.9 & ${ }^{140} \mathrm{Ba}$ & 4.3 & $12.8 \mathrm{D}$ & 162.7 & 6.2 & 423.7 & 3.1 & Fallout \\
\hline 304.9 & ${ }^{85 \mathrm{~m}} \mathrm{Kr}$ & 13.7 & $4.48 \mathrm{H}$ & 151.2 & 75.1 & & & Fission \\
\hline 315.9 & ${ }^{239} \mathrm{~Np}$ & 1.6 & $2.36 \mathrm{D}$ & 277.6 & 14.2 & 334.3 & 2.1 & Fallout \\
\hline 319.4 & ${ }^{147} \mathrm{Nd}$ & 2.0 & $11.0 \mathrm{D}$ & 439.9 & 1.2 & 91.1 & 28.0 & Fallout \\
\hline 320.1 & ${ }^{s !} \mathrm{Cr}$ & 9.8 & 27.7D & & & & & Activation \\
\hline 325.2 & ${ }^{101} \mathrm{Rh}$ & 13.4 & $3.3 Y$ & 127.2 & 73.0 & 198.0 & 70.8 & Fallout \\
\hline 328.0 & ${ }^{228} \mathrm{Ac}$ & 3.5 & Long & 270.3 & 3.8 & 338.4 & 12.4 & ${ }^{232} \mathrm{Th}$ \\
\hline 328.8 & ${ }^{140} \mathrm{La}$ & 20.7 & $40.3 \mathrm{H}$ & 432.5 & 3.0 & 487.0 & 45.9 & Fallout \\
\hline 334.3 & ${ }^{239} \mathrm{~Np}$ & 2.1 & $2.36 \mathrm{D}$ & 315.9 & 1.6 & 61.5 & 1.0 & Fallout \\
\hline 338.4 & ${ }^{228} \mathrm{Ac}$ & 12.4 & Long & 328.0 & 3.5 & 409.6 & 2.2 & $232 \mathrm{Th}$ \\
\hline 340.6 & ${ }^{136} \mathrm{Cs}$ & 48.6 & $13.2 \mathrm{D}$ & 273.7 & 12.7 & 818.6 & 99.8 & Fission \\
\hline 344.3 & ${ }^{152} \mathrm{Eu}$ & 26.6 & $13.3 Y$ & 244.7 & 7.5 & 411.1 & 2.2 & Fallout \\
\hline 352.0 & ${ }^{214} \mathrm{~Pb}$ & 37.1 & Long & 241.9 & 7.5 & 295.1 & 19.2 & ${ }^{238} \mathrm{U}$ \\
\hline 356.0 & ${ }^{133} \mathrm{Ba}$ & 62.2 & $10.5 Y$ & 302.9 & 18.4 & 383.8 & 8.9 & Activation \\
\hline
\end{tabular}


TABLE OF $\gamma$ RAYS AND NUCLIDES COMMON TO ENVIRONMENTAL ANALYSES (Cont'd)

\begin{tabular}{|c|c|c|c|c|c|c|c|c|}
\hline Energy & Nuclide & $\%$ & $\mathrm{~T}_{1 / 2}$ & E2 & $\%$ & E3 & $\%$ & Origin \\
\hline 362.3 & ${ }^{88} \mathrm{Kr}$ & 2.3 & $2.84 \mathrm{H}$ & 196.3 & 26.0 & 834.9 & 13.0 & Fission \\
\hline 364.5 & ${ }^{131} I$ & 81.2 & $8.04 \mathrm{D}$ & 637.0 & 7.3 & 284.3 & 6.1 & Fission \\
\hline 366.4 & ${ }^{99} \mathrm{Mo}$ & 1.2 & $65.9 \mathrm{H}$ & 181.1 & 6.1 & 739.5 & 12.1 & Fallout \\
\hline 380.4 & ${ }^{125} \mathrm{Sb}$ & 1.5 & $2.73 Y$ & 176.3 & 6.8 & 427.9 & 29.4 & Fallout \\
\hline 383.8 & ${ }^{133} \mathrm{Ba}$ & 8.9 & $10.5 Y$ & 356.0 & 62.2 & 302.9 & 18.4 & Activation \\
\hline 396.6 & ${ }^{138} \mathrm{Xe}$ & 6.3 & $14.1 \mathrm{M}$ & 258.4 & 31.5 & 401.5 & 2.2 & Fission \\
\hline 401.5 & ${ }^{138} \mathrm{Xe}$ & 2.2 & $14.1 \mathrm{M}$ & 434.6 & 20.3 & 396.6 & 6.3 & Fission \\
\hline 402.6 & ${ }^{87} \mathrm{Kr}$ & 49.6 & $76.3 \mathrm{M}$ & 845.5 & 7.3 & 673.9 & 1.9 & Fission \\
\hline 409.0 & ${ }^{138} \mathrm{Cs}$ & 4.7 & $32.2 \mathrm{M}$ & 227.7 & 1.5 & 462.8 & 30.7 & ${ }^{138} \mathrm{Xe}$ \\
\hline 409.6 & ${ }^{228} \mathrm{AC}$ & 2.2 & Long & 338.4 & 12.4 & 463.1 & 4.6 & ${ }^{232} \mathrm{Th}$ \\
\hline 411.1 & ${ }^{152} \mathrm{Eu}$ & 2.2 & $13.3 \mathrm{Y}$ & 344.3 & 26.6 & $444.0 \mathrm{D}$ & 3.1 & Fallout \\
\hline 415.3 & ${ }^{102} \mathrm{Rh}$ & 2.1 & $2.89 \mathrm{Y}$ & 418.5 & 10.6 & 420.4 & 3.2 & Fallout \\
\hline 418.5 & ${ }^{102} \mathrm{Rh}$ & 10.6 & $2.89 \mathrm{Y}$ & 415.3 & 2.1 & 420.4 & 3.2 & Fallout \\
\hline 420.4 & ${ }^{102} \mathrm{Rh}$ & 3.2 & $2.89 \mathrm{Y}$ & 418.5 & 10.6 & 475.1 & 95.0 & Fallout \\
\hline 423.7 & ${ }^{140} \mathrm{Ba}$ & 3.1 & $12.8 \mathrm{D}$ & 437.6 & 1.9 & 304.9 & 4.3 & Fallout \\
\hline 427.9 & ${ }^{125} \mathrm{Sb}$ & 29.4 & $2.73 Y$ & 380.4 & 1.5 & 463.4 & 10.5 & Fallout \\
\hline 432.5 & ${ }^{140} \mathrm{La}$ & 3.0 & $40.3 \mathrm{H}$ & 487.0 & 45.9 & 328.8 & 20.7 & Fallout \\
\hline 434.6 & ${ }^{138} \mathrm{Xe}$ & 20.3 & $14.1 \mathrm{M}$ & 401.5 & 2.2 & 1114.3 & 1.5 & Fission \\
\hline 437.6 & ${ }^{140} \mathrm{Ba}$ & 1.9 & $12.8 \mathrm{D}$ & 537.3 & 24.4 & 423.7 & 3.1 & Fallout \\
\hline 439.9 & ${ }^{147} \mathrm{Nd}$ & 1.2 & $11.0 \mathrm{D}$ & 319.4 & 2.0 & 531.0 & 13.1 & Fallout \\
\hline $444.0 \mathrm{D}$ & ${ }^{152} \mathrm{Eu}$ & 3.1 & $13.3 Y$ & 411.1 & 2.2 & 778.9 & 13.0 & Fallout \\
\hline 446.8 & ${ }^{110 \pi} \mathrm{Ag}$ & 3.8 & $250 \mathrm{D}$ & 657.8 & 94.6 & 620.4 & 2.8 & Activation \\
\hline 462.8 & ${ }^{138} \mathrm{Cs}$ & 30.7 & $32.2 \mathrm{M}$ & 547.0 & 10.8 & 409.0 & 4.7 & ${ }^{138} \mathrm{Xe}$ \\
\hline 463.1 & ${ }^{228} \mathrm{Ac}$ & 4.6 & Long & 409.6 & 2.2 & 755.3 & 1.3 & ${ }^{232} \mathrm{Th}$ \\
\hline 463.4 & ${ }^{125} \mathrm{Sb}$ & 10.5 & $2.73 \mathrm{Y}$ & 427.9 & 29.4 & 600.5 & 17.8 & Fallout \\
\hline 468.7 & ${ }^{102 \mathrm{~m}} \mathrm{Rh}$ & 2.9 & $207 \mathrm{D}$ & 475.1 & 46.0 & 556.6 & 1.9 & Fallout \\
\hline 475.1 & ${ }^{102 \mathrm{~m}} \mathrm{Rh}$ & 46.0 & $207 \mathrm{D}$ & 468.7 & 2.9 & 556.6 & 1.9 & Fallout \\
\hline 475.1 & ${ }^{102} \mathrm{Rh}$ & 95.0 & $2.89 \mathrm{Y}$ & 628.1 & 8.5 & 420.5 & 3.2 & Fallout \\
\hline 475.4 & ${ }^{134} \mathrm{Cs}$ & 1.5 & $2.06 Y$ & 563.2 & 8.4 & 569.3 & 15.4 & Fission \\
\hline 477.6 & ${ }^{7} \mathrm{Be}$ & 10.3 & $53.2 \mathrm{D}$ & & & & & Cosmic \\
\hline 487.1 & ${ }^{140} \mathrm{La}$ & 45.5 & $40.2 \mathrm{H}$ & 751.9 & 4.3 & 432.6 & 2.9 & Fallout \\
\hline 497.1 & ${ }^{103} \mathrm{Ru}$ & 89.5 & $39.6 \mathrm{D}$ & 610.3 & 5.6 & & & Fallout \\
\hline 505.9 & ${ }^{132} I$ & 5.0 & $2.28 \mathrm{H}$ & 262.8 & 1.4 & 522.7 & 16.1 & ${ }^{132} \mathrm{Te}$ \\
\hline 510.6 & ${ }^{208} \mathrm{Tl}$ & 7.8 & Long & 277.3 & 2.4 & 583.0 & 30.9 & ${ }^{232} \mathrm{Th}$ \\
\hline 511.0 & ${ }^{65} \mathrm{Zn}$ & 2.9 & $244 \mathrm{D}$ & 1115.5 & 50.8 & & & Activation \\
\hline 511.0 & ${ }^{58} \mathrm{Co}$ & 30.0 & $70.9 \mathrm{D}$ & 810.8 & 99.5 & & & Activatior \\
\hline
\end{tabular}


TABLE OF $\gamma$ RAYS AND NUCLIDES COMMON TO ENVIRONMENTAL ANALYSES (Cont'd)

\begin{tabular}{|c|c|c|c|c|c|c|c|c|}
\hline Energy & Nuclide & $\%$ & $T_{1 / 2}$ & $\mathrm{E} 2$ & $\%$ & E3 & $\%$ & Origin \\
\hline 511.0 & ${ }^{22} \mathrm{Na}$ & 180.8 & $2.60 Y$ & 1274.5 & 99.9 & & & Cosmic \\
\hline 511.9 & ${ }^{106} \mathrm{Ru}$ & 20.7 & $372 \mathrm{D}$ & 1050.4 & 1.5 & 621.9 & 9.8 & Fallout \\
\hline 514.0 & ${ }^{85} \mathrm{Sr}$ & 99.3 & $64.8 \mathrm{D}$ & $13.4 \mathrm{KX}$ & 50.6 & $15.0 \mathrm{KX}$ & 8.7 & Activation \\
\hline 522.7 & ${ }^{132} \mathrm{I}$ & 16.1 & $2.28 \mathrm{H}$ & 505.9 & 5.0 & 547.0 & 1.3 & ${ }^{132} \mathrm{Te}$ \\
\hline 526.6 & ${ }^{135 \mathrm{~m}} \mathrm{Xe}$ & 81.2 & $15.7 \mathrm{M}$ & $30.0 \mathrm{KX}$ & 14.0 & & & Fission \\
\hline 531.0 & ${ }^{147} \mathrm{Nd}$ & 13.1 & $11.0 \mathrm{D}$ & 439.9 & 1.2 & 319.4 & 2.0 & Fallout \\
\hline 537.3 & ${ }^{140} \mathrm{Ba}$ & 24.4 & $12.8 \mathrm{D}$ & 437.6 & 1.9 & 423.7 & 3.1 & Fallout \\
\hline 547.0 & ${ }^{138} \mathrm{Cs}$ & 10.8 & $32.2 \mathrm{M}$ & 462.8 & 30.7 & 871.7 & 5.1 & ${ }^{138} \mathrm{Xe}$ \\
\hline 547.0 & ${ }^{132} \mathrm{I}$ & 1.3 & $2.28 \mathrm{H}$ & 522.7 & 16.1 & 621.2 & -2.0 & $132 \mathrm{Te}$ \\
\hline 556.6 & ${ }^{102 \mathrm{~m}} \mathrm{Rh}$ & 1.9 & $207 D$ & 475.1 & 46.0 & 628.1 & 5.5 & Fallout \\
\hline 563.2 & ${ }^{134} \mathrm{Cs}$ & 8.4 & $2.06 \mathrm{Y}$ & 475.4 & 1.5 & 569.3 & 15.4 & Fission \\
\hline 569.3 & ${ }^{134} \mathrm{Cs}$ & 15.4 & $2.06 \mathrm{Y}$ & 563.2 & 8.4 & 604.7 & 97.6 & Fission \\
\hline 569.2 & ${ }^{207} \mathrm{Bi}$ & 97.8 & $32.2 Y$ & 1063.1 & 74.9 & 1769.7 & 6.9 & Fallout \\
\hline 583.0 & ${ }^{208} \mathrm{Tl}$ & 30.9 & Long & 510.6 & 7.8 & 860.3 & 4.3 & ${ }^{232} \mathrm{Th}$ \\
\hline 591.8 & ${ }^{154} \mathrm{Eu}$ & 4.8 & $8.8 Y$ & 248.0 & 6.6 & 692.5 & 1.7 & Fallout \\
\hline 600.5 & ${ }^{125} \mathrm{Sb}$ & 17.8 & $2.73 Y$ & 463.4 & 10.5 & 606.6 & 5.0 & Fallout \\
\hline 602.7 & ${ }^{124} \mathrm{Sb}$ & 97.8 & $60.2 \mathrm{D}$ & 645.9 & 7.4 & 709.3 & 1.4 & Fallout \\
\hline 604.7 & ${ }^{134} \mathrm{Cs}$ & 97.6 & $2.06 \mathrm{Y}$ & 795.9 & 85.4 & 569.3 & 15.4 & Fission \\
\hline 606.6 & ${ }^{125} \mathrm{Sb}$ & 5.0 & $2.73 \mathrm{Y}$ & 600.5 & 17.8 & 635.9 & 11.3 & Fallout \\
\hline 608.2 & ${ }^{135} \mathrm{Xe}$ & 2.9 & $9.10 \mathrm{H}$ & 249.8 & 90.0 & $31.6 \mathrm{KX}$ & 5.2 & Fission \\
\hline 609.3 & ${ }^{214} \mathrm{Bi}$ & 46.1 & Long & 665.4 & 1.6 & 768.4 & 4.9 & ${ }^{238} U$ \\
\hline 610.3 & ${ }^{103} \mathbf{R u}$ & 5.6 & $39.3 \mathrm{D}$ & 497.1 & 88.7 & & & Fallout \\
\hline 620.4 & ${ }^{110 m} \mathrm{Ag}$ & 2.8 & $250 D$ & 657.8 & 94.6 & 446.8 & 3.8 & Activation \\
\hline 621.2 & ${ }^{132} \mathrm{I}$ & $\sim 2.0$ & $2.28 \mathrm{H}$ & 547.1 & 1.3 & 630.3 & 13.8 & ${ }^{132} \mathrm{Te}$ \\
\hline 621.9 & ${ }^{106} \mathrm{Ru}$ & 9.8 & $372 \mathrm{D}$ & 511.9 & 20.7 & 1050.4 & 1.5 & Fallout \\
\hline 628.1 & ${ }^{102 \pi} \mathrm{Rh}$ & 5.5 & $207 D$ & 556.6 & 1.9 & 1103.2 & 2.9 & Fallout \\
\hline 628.1 & ${ }^{102} \mathrm{Rh}$ & 8.5 & $\sim 2.9 Y$ & 475.1 & 95.0 & 631.3 & 56.0 & Fallout \\
\hline 630.3 & ${ }^{132} Y$ & 13.8 & $2.28 \mathrm{H}$ & 621.2 & $\sim 2.0$ & 650.6 & 2.7 & ${ }^{132} \mathrm{Te}$ \\
\hline 631.3 & ${ }^{102} \mathrm{Rh}$ & 56.0 & $\sim 2.9 \mathrm{Y}$ & 628.1 & 8.5 & 692.4 & 1.8 & Fallout \\
\hline 635.9 & ${ }^{125} \mathrm{Sb}$ & 11.3 & $2.73 \mathrm{Y}$ & 606.6 & 5.0 & 671.4 & 1.8 & Fallout \\
\hline 637.0 & ${ }^{131} I$ & 7.3 & $8.04 \mathrm{D}$ & 364.5 & 81.2 & 722.9 & 1.8 & Fission \\
\hline 645.9 & ${ }^{124} \mathrm{Sb}$ & 7.4 & $60.2 \mathrm{D}$ & 602.7 & 97.8 & 709.3 & 1.4 & Fallout \\
\hline 650.6 & ${ }^{132} \mathrm{I}$ & 2.7 & $2.28 \mathrm{H}$ & 630.3 & 13.8 & 667.7 & 98.7 & ${ }^{132} \mathrm{Te}$ \\
\hline 657.8 & ${ }^{110 \mathrm{~m}} \mathrm{Ag}$ & 94.6 & $250 \mathrm{D}$ & 620.4 & 2.8 & 677.6 & 10.4 & Activation \\
\hline 661.7 & ${ }^{137} \mathrm{Cs}$ & 85.2 & $30.0 \mathrm{Y}$ & $33.0 \mathrm{KX}$ & 7.1 & & & Fallout \\
\hline 665.4 & ${ }^{214} \mathrm{Bi}$ & 1.6 & Long & 609.3 & 46.1 & 768.4 & 4.9 & ${ }^{238} \mathrm{U}$ \\
\hline 667.7 & ${ }^{132} I$ & 98.7 & $2.28 \mathrm{H}$ & 650.6 & 2.7 & 669.9 & 4.9 & ${ }^{132} \mathrm{Te}$ \\
\hline
\end{tabular}


TABLE OF $\gamma$ RAYS AND NUCLIDES COMMON TO ENVIRONMENTAL ANALYSES (Cont'd)

\begin{tabular}{|c|c|c|c|c|c|c|c|c|}
\hline Energy & Nuclide & $\%$ & $T_{1 / 2}$ & $\mathrm{E} 2$ & $\%$ & E3 & $\%$ & Origin \\
\hline 669.9 & ${ }^{132} \mathrm{I}$ & 4.9 & $2.28 \mathrm{H}$ & 667.7 & 98.7 & 671.6 & 5.2 & ${ }^{132} \mathrm{Te}$ \\
\hline 671.4 & ${ }^{125} \mathrm{Sb}$ & 1.8 & $2.73 \mathrm{Y}$ & 635.9 & 11.3 & 606.6 & 5.0 & Fallout \\
\hline 671.6 & ${ }^{132} I$ & 5.2 & $2.28 \mathrm{H}$ & 669.9 & 4.9 & 727.D & 5.4 & ${ }^{132} \mathrm{Te}$ \\
\hline 673.9 & ${ }^{87} \mathrm{Kr}$ & 1.9 & $76.3 \mathrm{M}$ & 845.5 & 7.3 & 402.6 & 49.6 & Fission \\
\hline 677.6 & ${ }^{110 m} \mathrm{Ag}$ & 10.4 & $250 \mathrm{D}$ & 657.8 & 94.6 & 687.0 & 6.4 & Activation \\
\hline 687.0 & ${ }^{110 \mathrm{~m}} \mathrm{Ag}$ & 6.4 & $250 \mathrm{D}$ & 677.6 & 10.4 & 706.7 & 16.4 & Activation \\
\hline 692.4 & ${ }^{102} \mathrm{Rh}$ & 1.8 & $-2.9 Y$ & 631.3 & 56.0 & 695.6 & 2.7 & Fallout \\
\hline 692.5 & ${ }^{154} \mathrm{Eu}$ & 1.7 & $8.8 \mathrm{Y}$ & 591.8 & 4.8 & 723.4 & 19.7 & Fallout \\
\hline 695.6 & ${ }^{102} \mathrm{Rh}$ & 2.7 & $\sim 2.9 \mathrm{Y}$ & 692.4 & 1.8 & 697.6 & 45.7 & Fallout \\
\hline 696.5 & ${ }^{144} \mathrm{Ce}$ & 1.3 & $285 \mathrm{D}$ & 133.5 & 11.1 & 80.1 & 1.1 & Fallout \\
\hline 697.6 & ${ }^{102} \mathrm{Rh}$ & 45.7 & $\sim 2.9 Y$ & 766.9 & 34.0 & 695.6 & 2.7 & Fallout \\
\hline 706.7 & ${ }^{110 \mathrm{~m}} \mathrm{Ag}$ & 16.4 & $250 \mathrm{D}$ & 687.0 & 6.4 & 744.3 & 4.7 & Activation \\
\hline 709.3 & ${ }^{124} \mathrm{Sb}$ & 1.4 & $60.2 \mathrm{D}$ & 645.9 & 7.4 & 713.8 & 2.3 & Fallout \\
\hline 713.8 & ${ }^{124} \mathrm{Sb}$ & 2.3 & $60.2 \mathrm{D}$ & 709.3 & 1.4 & 722.8 & 10.9 & Fallout \\
\hline 722.8 & ${ }^{124} \mathrm{Sb}$ & 10.9 & $60.2 \mathrm{D}$ & 713.8 & 2.3 & 968.2 & 1.9 & Fallout \\
\hline 722.9 & ${ }^{131} \mathrm{I}$ & 1.8 & $8.04 \mathrm{D}$ & 364.5 & 81.2 & 637.0 & 7.3 & Fission \\
\hline 723.4 & ${ }^{154} \mathrm{Eu}$ & 19.7 & $8.8 Y$ & 692.5 & 1.7 & 756.8 & 4.3 & Fallout \\
\hline 724.2 & ${ }^{95} \mathrm{ZI}_{\mathrm{I}}$ & 44.1 & $64.0 \mathrm{D}$ & 756.7 & 54.5 & & & Fallout \\
\hline $727.0 \mathrm{D}$ & ${ }^{132} I$ & 5.4 & $2.28 \mathrm{H}$ & 671.6 & 5.2 & 728.7 & 1.1 & ${ }^{132} \mathrm{Te}$ \\
\hline 727.3 & ${ }^{212} \mathrm{Bi}$ & 6.7 & Long & 39.9 & 1.1 & 1620.7 & 1.5 & ${ }^{232} \mathrm{Th}$ \\
\hline 728.7 & ${ }^{132} I$ & 1.1 & $2.28 \mathrm{H}$ & $727.0 \mathrm{D}$ & 5.4 & 772.7 & 76.2 & ${ }^{132} \mathrm{Te}$ \\
\hline 739.5 & ${ }^{99} \mathrm{Mo}$ & 12.1 & $65.9 \mathrm{H}$ & 366.4 & 1.2 & 777.9 & 4.4 & Fallout \\
\hline 744.3 & ${ }^{110 m} \mathrm{Ag}$ & 4.7 & $250 D$ & 706.7 & 16.4 & 763.9 & 22.3 & Activation \\
\hline 751.7 & ${ }^{140} \mathrm{La}$ & 4.3 & $40.3 \mathrm{H}$ & 487.0 & 45.9 & 815.8 & 23.6 & Fallout \\
\hline 755.3 & ${ }^{228} \mathrm{Ac}$ & 1.3 & Long & 463.1 & 4.6 & 772.3 & 1.1 & ${ }^{232} \mathrm{Th}$ \\
\hline 756.7 & ${ }^{95} \mathrm{Zr}$ & 54.5 & $64.0 \mathrm{D}$ & 724.2 & 44.1 & & & Fallout \\
\hline 756.8 & ${ }^{154} \mathrm{Eu}$ & 4.3 & $8.8 \mathrm{Y}$ & 723.4 & 19.7 & 873.2 & 11.5 & Fallout \\
\hline 763.1 & ${ }^{208} \mathrm{Tl}$ & 0.6 & Long & 583.0 & 30.9 & 860.3 & 4.3 & ${ }^{232} \mathrm{Th}$ \\
\hline 763.9 & ${ }^{110 m} \mathrm{Ag}$ & 22.3 & $250 \mathrm{D}$ & 744.3 & 4.7 & 818.0 & 7.3 & Activation \\
\hline 765.8 & ${ }^{95} \mathrm{Nb}$ & 99.8 & $35.0 \mathrm{D}$ & & & & & Fallout \\
\hline 766.9 & ${ }^{102} \mathrm{Rh}$ & 34.0 & $\sim 2.9 \mathrm{Y}$ & 697.6 & 45.7 & 1046.6 & 34.0 & Fallout \\
\hline 768.4 & ${ }^{214} \mathrm{Bi}$ & 5.0 & Long & 665.6 & 1.6 & $786.4 \mathrm{D}$ & 0.3 & ${ }^{238} \mathrm{U}$ \\
\hline 772.3 & ${ }^{228} \mathrm{AC}$ & 1.1 & Long & 755.3 & 1.3 & 794.8 & 4.6 & ${ }^{232} \mathrm{Th}$ \\
\hline 772.7 & ${ }^{132} I$ & 76.2 & $2.28 \mathrm{H}$ & 728.7 & 1.1 & 780.1 & 1.2 & ${ }^{132} \mathrm{Te}$ \\
\hline 777.9 & ${ }^{99} \mathrm{Mo}$ & 4.4 & $65.9 \mathrm{H}$ & 739.5 & 12.1 & 366.4 & 1.2 & Fallout \\
\hline 778.9 & ${ }^{152} \mathrm{Eu}$ & 13.0 & $13.3 Y$ & $444.0 \mathrm{D}$ & 3.1 & 867.4 & 4.2 & Fallout \\
\hline 780.1 & ${ }^{132} \mathrm{I}$ & 1.2 & $2.28 \mathrm{H}$ & 772.7 & 76.2 & 809.8 & 2.9 & ${ }^{132} \mathrm{Te}$ \\
\hline
\end{tabular}


TABLE OF $\gamma$ RAYS AND NUCLIDES COMMON TO ENVIRONMENTAL ANALYSES (Contd)

\begin{tabular}{|c|c|c|c|c|c|c|c|c|}
\hline Energy & Nuclide & $\%$ & $\mathrm{~T}_{1 / 2}$ & E2 & $\%$ & E3 & $\%$ & Origin \\
\hline 785.5 & ${ }^{212} \mathrm{Bi}$ & 1.1 & Long & 727.3 & 6.7 & 1620.7 & 1.5 & ${ }^{232} \mathrm{Th}$ \\
\hline 786.4 & ${ }^{214} \mathrm{Bi}$ & 0.3 & Long & 768.4 & 4.9 & 806.2 & 1.2 & ${ }^{238} \mathrm{U}$ \\
\hline 794.8 & ${ }^{228} \mathrm{Ac}$ & 4.6 & Long & 772.3 & 1.1 & 830.6 & 0.6 & ${ }^{232} \mathrm{Th}$ \\
\hline 795.8 & ${ }^{134} \mathrm{Cs}$ & 85.4 & $2.06 \mathrm{Y}$ & 604.7 & 97.8 & 801.9 & 8.7 & Fission \\
\hline 802.0 & ${ }^{134} \mathrm{Cs}$ & 8.7 & $2.06 Y$ & 795.9 & 85.4 & 1038.6 & 1.0 & Fission \\
\hline 806.2 & ${ }^{214} \mathrm{Bi}$ & 1.2 & Long & 786.4 & 0.3 & 934.0 & 3.2 & ${ }^{238} \mathrm{U}$ \\
\hline 809.8 & ${ }^{132} I$ & 2.9 & $2.28 \mathrm{H}$ & 780.1 & 1.2 & 812.3 & 5.6 & ${ }^{132} \mathrm{Te}$ \\
\hline 810.8 & ${ }^{58} \mathrm{Co}$ & 99.5 & $70.9 \mathrm{D}$ & 511.0 & 30.0 & & & Activation \\
\hline 812.3 & ${ }^{132} \mathrm{I}$ & 5.6 & $2.28 \mathrm{H}$ & 809.8 & 2.9 & 877.2 & 1.1 & ${ }^{132} \mathrm{Te}$ \\
\hline 815.8 & ${ }^{140} \mathrm{La}$ & 23.6 & $40.3 \mathrm{H}$ & 751.7 & 4.3 & 867.8 & 5.6 & Fallout \\
\hline 818.0 & ${ }^{10 m} \mathrm{Ag}$ & 7.3 & $250 \mathrm{D}$ & 763.9 & 22.3 & 884.7 & 72.7 & Activation \\
\hline 818.6 & ${ }^{136} \mathrm{Cs}$ & 99.8 & $13.2 \mathrm{D}$ & 340.6 & 48.6 & 1048.1 & 79.7 & Fission \\
\hline 830.6 & ${ }^{228} \mathrm{Ac}$ & 0.6 & Long & 794.8 & 4.6 & 835.6 & 1.7 & ${ }^{232} \mathrm{Th}$ \\
\hline 834.8 & ${ }^{54} \mathrm{Mn}$ & 100.0 & $312.2 \mathrm{D}$ & & & & & Fallout \\
\hline 834.9 & ${ }^{88} \mathrm{Kr}$ & 13.0 & $2.84 \mathrm{H}$ & 362.3 & 2.3 & 985.8D & 1.3 & Fission \\
\hline 835.6 & ${ }^{228} \mathrm{Ac}$ & 1.7 & Long & 830.6 & 0.6 & 840.4 & 0.9 & ${ }^{232} \mathrm{Th}$ \\
\hline 840.4 & ${ }^{228} \mathrm{Ac}$ & 0.9 & Long & 835.6 & 1.7 & 904.3 & 0.9 & ${ }^{232} \mathrm{Th}$ \\
\hline 845.5 & ${ }^{87} \mathrm{Kr}$ & 7.3 & $76.3 \mathrm{M}$ & 673.9 & 1.9 & 1175.5 & 1.1 & Fission \\
\hline 860.3 & ${ }^{208} \mathrm{TI}$ & 4.3 & Long & 2614.4 & 35.8 & 583.0 & 30.9 & ${ }^{232} \mathrm{Th}$ \\
\hline 867.4 & ${ }^{152} \mathrm{Eu}$ & 4.2 & $13.3 \mathrm{Y}$ & 778.9 & 13.0 & 964.1 & 14.5 & Fallout \\
\hline 867.8 & ${ }^{140} \mathrm{La}$ & 5.6 & $40.3 \mathrm{H}$ & 815.8 & 23.6 & 919.6 & 2.7 & Fallout \\
\hline 871.7 & ${ }^{138} \mathrm{Cs}$ & 5.1 & $32.2 \mathrm{M}$ & 547.0 & 10.8 & 1009.8 & 29.8 & ${ }^{138} \mathrm{Xe}$ \\
\hline 873.2 & ${ }^{154} \mathrm{Eu}$ & 11.5 & $8.8 \mathrm{Y}$ & 756.8 & 4.3 & 996.3 & 10.3 & Fallout \\
\hline 877.2 & ${ }^{132} I$ & 1.1 & $2.28 \mathrm{H}$ & 812.3 & 5.6 & 954.6 & 18.1 & ${ }^{132} \mathrm{Te}$ \\
\hline 884.7 & ${ }^{110 \mathrm{~m}} \mathrm{Ag}$ & 72.7 & $250 \mathrm{D}$ & 818.0 & 7.3 & 937.5 & 34.4 & Activation \\
\hline 898.1 & ${ }^{88} \mathrm{Y}$ & 92.7 & $107 \mathrm{D}$ & 1836.1 & 99.4 & & & Activation \\
\hline 898.0 & ${ }^{88} \mathrm{Rb}$ & 14.1 & $17.8 \mathrm{M}$ & 1836.1 & 21.4 & 2677.9 & 2.0 & ${ }^{88} \mathrm{Kr}$ \\
\hline 904.3 & ${ }^{228} \mathrm{Ac}$ & 0.9 & Long & 840.4 & 0.9 & 911.2 & 29.0 & ${ }^{232} \mathrm{Th}$ \\
\hline 911.2 & ${ }^{228} \mathrm{Ac}$ & 29.0 & Long & $966.0 \mathrm{D}$ & 23.2 & 840.4 & 0.9 & ${ }^{232} \mathrm{Th}$ \\
\hline 919.6 & ${ }^{140} \mathrm{La}$ & 2.7 & $40.3 \mathrm{H}$ & 867.8 & 5.6 & 925.2 & 7.0 & Fallout \\
\hline 925.2 & ${ }^{140} \mathrm{La}$ & 7.1 & $40.3 \mathrm{H}$ & 487.0 & 45.9 & 919.6 & 2.7 & Fallout \\
\hline 934.0 & ${ }^{214} \mathrm{Bi}$ & 3.2 & Long & 1120.3 & 15.0 & 806.2 & 1.2 & ${ }^{238} \mathrm{U}$ \\
\hline 937.5 & ${ }^{110 \mathrm{~m}} \mathrm{Ag}$ & 34.4 & $250 \mathrm{D}$ & 1384.3 & 24.3 & 884.7 & 72.7 & Activation \\
\hline 954.6 & ${ }^{132} \mathrm{I}$ & 18.1 & $2.28 \mathrm{H}$ & 877.2 & 1.1 & 1136.2 & 3.0 & ${ }^{132} \mathrm{Te}$ \\
\hline 964.1 & ${ }^{152} \mathrm{Eu}$ & 14.5 & $13.3 \mathrm{Y}$ & 1085.9 & 9.9 & 867.4 & 4.2 & Fallout \\
\hline 964.6 & ${ }^{228} \mathrm{Ac}$ & 5.8 & Long & 969.0 & 17.4 & 911.2 & 29.0 & ${ }^{232} \mathrm{Th}$ \\
\hline 968.2 & ${ }^{124} \mathrm{Sb}$ & 1.9 & $60.2 \mathrm{D}$ & 1045.1 & 1.9 & 722.8 & 10.9 & Fallout \\
\hline
\end{tabular}


TABLE OF $\gamma$ RAYS AND NUCLDES COMMON TO ENVIRONMENTAL ANALYSES (Cont'd)

\begin{tabular}{|c|c|c|c|c|c|c|c|c|}
\hline Energy & Nuclide & $\%$ & $T_{1 / 2}$ & $\mathrm{E} 2$ & $\%$ & E3 & $\%$ & Origin \\
\hline 969.0 & ${ }^{228} \mathrm{Ac}$ & 17.4 & Long & 911.2 & 29.0 & 1459.2 & 1.1 & ${ }^{232} \mathrm{Th}$ \\
\hline 985.8 & ${ }^{88} \mathrm{Kr}$ & 1.3 & $2.84 \mathrm{H}$ & 1141.4 & 1.3 & 834.9 & 13.0 & Fission \\
\hline 996.3 & ${ }^{154} \mathrm{Eu}$ & 10.3 & $8.8 Y$ & 1004.8 & 17.9 & 873.2 & 11.5 & Fallout \\
\hline 1001.0 & ${ }^{234 \mathrm{~m}} \mathrm{~Pa}$ & 0.7 & Long & 766.4 & 0.2 & 742.8 & 0.1 & ${ }^{238} \mathrm{U}$ \\
\hline 1004.8 & ${ }^{154} \mathrm{Eu}$ & 17.9 & $8.8 Y$ & 1274.5 & 35.5 & 996.3 & 10.3 & Fallout \\
\hline 1009.8 & ${ }^{138} \mathrm{Cs}$ & 29.8 & $32.2 \mathrm{M}$ & 1147.3 & 1.2 & 871.7 & 5.1 & ${ }^{138} \mathrm{Xe}$ \\
\hline 1038.6 & ${ }^{134} \mathrm{Cs}$ & 1.0 & $2.06 Y$ & 1167.9 & 1.8 & 802.0 & 8.7 & Fission \\
\hline 1045.1 & ${ }^{124} \mathrm{Sb}$ & 1.9 & $60.2 \mathrm{D}$ & 1325.5 & 1.6 & 968.2 & 1.9 & Fallout \\
\hline 1046.6 & ${ }^{102} \mathrm{Rh}$ & 33.0 & $2.9 \mathrm{Y}$ & 1103.2 & 4.4 & 766.9 & 34.0 & Fallout \\
\hline 1048.1 & ${ }^{136} \mathrm{Cs}$ & 79.7 & $13.2 \mathrm{D}$ & 818.6 & 99.8 & 1235.4 & 19.8 & Fission \\
\hline 1050.4 & ${ }^{106} \mathrm{Ru}$ & 1.5 & $372 \mathrm{D}$ & 511.9 & 20.7 & 621.9 & 9.8 & Fallout \\
\hline 1063.1 & ${ }^{207} \mathrm{Bi}$ & 74.9 & $32.2 Y$ & 569.2 & 97.8 & 1769.7 & 6.9 & Fallout \\
\hline 1085.9 & ${ }^{152} \mathrm{Eu}$ & 9.9 & $13.3 Y$ & 1112.1 & 13.6 & 964.1 & 14. & Fallout \\
\hline 1099.3 & ${ }^{59} \mathrm{Fe}$ & 56.5 & $44.5 \mathrm{D}$ & 1291.6 & 43.2 & 192.3 & 3.1 & Fallout \\
\hline 1103.2 & ${ }^{102 m} \mathrm{Rh}$ & 2.9 & $207 \mathrm{D}$ & 556.6 & 1.9 & 628.1 & 5.5 & Fallout \\
\hline 1103.2 & ${ }^{102} \mathrm{Rh}$ & 4.4 & $2.9 Y$ & 1046.6 & 33.0 & 1112.9 & 18.9 & Fallout \\
\hline 1112.1 & ${ }^{152} \mathrm{Eu}$ & 13.6 & $13.3 \mathrm{Y}$ & 1085.9 & 9.9 & 1212.9 & 1.4 & Fallout \\
\hline 1112.9 & ${ }^{102} \mathrm{Rh}$ & 18. & $2.9 Y$ & 1046.6 & 33.0 & 1103.2 & 4.4 & Fallout \\
\hline 1114.3 & ${ }^{138} \mathrm{Xe}$ & 1.5 & $14.1 \mathrm{M}$ & 1768.4 & 16.7 & 434.6 & 20.3 & Fission \\
\hline 1115.5 & ${ }^{65} \mathrm{Zn}$ & 50.8 & $244 D$ & 511.0 & 2.9 & & & Activation \\
\hline 1120.3 & ${ }^{214} \mathrm{Bi}$ & 15.0 & Long & 1155.2 & 1.7 & 934.0 & 3.2 & ${ }^{238} \mathrm{U}$ \\
\hline 1136.2 & ${ }^{132} I$ & 3.0 & $2.28 \mathrm{H}$ & 954.6 & 18.1 & 1143.6 & 1.4 & ${ }^{132} \mathrm{Te}$ \\
\hline 1141.4 & ${ }^{88} \mathrm{Kr}$ & 1.3 & $2.84 \mathrm{H}$ & 1369.4 & 1.5 & 985.8D & 1.3 & Fission \\
\hline 1143.6 & ${ }^{132} I$ & 1.4 & $2.28 \mathrm{H}$ & 1136.2 & 3.0 & 1173.3 & 1.1 & ${ }^{132} \mathrm{Te}$ \\
\hline 1147.3 & ${ }^{138} \mathrm{Cs}$ & 1.2 & $32.2 \mathrm{M}$ & 1009.8 & 29.8 & 1343.6 & 1.1 & ${ }^{138} \mathrm{Xe}$ \\
\hline 1155.2 & ${ }^{214} \mathrm{Bi}$ & 1.7 & Long & 1238.1 & 5.9 & 1120.3 & 15.0 & ${ }^{238} \mathrm{U}$ \\
\hline 1167.9 & ${ }^{134} \mathrm{Cs}$ & 1.8 & $2.06 \mathrm{Y}$ & 1038.6 & 1.0 & 1365.2 & 3.0 & Fission \\
\hline 1173.2 & ${ }^{60} \mathrm{Co}$ & 99.9 & $5.27 Y$ & 1332.5 & 100.0 & & & Activation \\
\hline 1173.3 & ${ }^{132} \mathrm{I}$ & 1.1 & $2.28 \mathrm{H}$ & 1143.6 & 1.4 & 1290.8 & 1.1 & ${ }^{132} \mathrm{Te}$ \\
\hline 1175.5 & ${ }^{87} \mathrm{Kr}$ & 1.1 & $76.3 \mathrm{M}$ & 1740.6 & 2.0 & 845.5 & 7.3 & Fission \\
\hline 1212.9 & ${ }^{152} \mathrm{Eu}$ & 1.4 & $13.3 \mathrm{Y}$ & 1112.1 & 13.6 & 1299.2 & 1.6 & Fallout \\
\hline 1235.4 & ${ }^{136} \mathrm{Cs}$ & 19.8 & $13.2 \mathrm{D}$ & 818.6 & 99.8 & 1048.1 & 79.7 & Fission \\
\hline 1238.1 & ${ }^{214} \mathrm{Bi}$ & 5.9 & Long & 1155.2 & 1.7 & 1281.0 & 1.5 & ${ }^{238} \mathrm{U}$ \\
\hline 1274.5 & ${ }^{22} \mathrm{Na}$ & 99.9 & $2.60 Y$ & 511.0 & 181.0 & & & Cosmic \\
\hline 1274.5 & ${ }^{154} \mathrm{Eu}$ & 35.5 & $8.8 Y$ & 1004.8 & 17.9 & 1596.6 & 1.8 & Fallout \\
\hline 1281.0 & ${ }^{214} \mathrm{Bi}$ & 1.5 & Long & 1238.1 & 5.9 & 1377.7 & 4.0 & ${ }^{238} \mathrm{U}$ \\
\hline 1290.8 & ${ }^{132} I$ & 1.1 & $2.28 \mathrm{H}$ & 1173.3 & 1.1 & 1295.4 & 2.0 & ${ }^{132} \mathrm{Te}$ \\
\hline 1291.6 & ${ }^{59} \mathrm{Fe}$ & 43.2 & $44.5 \mathrm{D}$ & 1099.3 & 56.5 & 192.3 & 3.1 & Activation \\
\hline
\end{tabular}


TABLE OF $\gamma$ RAYS AND NUCLIDES COMMON TO ENVIRONMENTAL ANALYSES (Cont'd)

\begin{tabular}{|c|c|c|c|c|c|c|c|c|}
\hline Energy & Nuclide & $\%$ & $T_{1 / 2}$ & E2 & $\%$ & E3 & $\%$ & Origin \\
\hline 1293.6 & ${ }^{41} \mathrm{Ar}$ & 99.2 & $1.83 \mathrm{H}$ & & & & & Activation \\
\hline 1295.4 & ${ }^{132} I$ & 2.0 & $2.28 \mathrm{H}$ & 1290.8 & 1.1 & 1372.1 & 2.5 & ${ }^{132} \mathrm{Te}$ \\
\hline 1299.2 & ${ }^{152} \mathrm{Eu}$ & 1.6 & $13.3 Y$ & 1212.9 & 1.4 & 1408.0 & 20.8 & Fallout \\
\hline 1325.5 & ${ }^{124} \mathrm{Sb}$ & 1.6 & $60.2 \mathrm{D}$ & 1045.1 & 1.9 & 1368.2 & 2.7 & Fallout \\
\hline 1332.5 & ${ }^{60} \mathrm{Co}$ & 100.0 & $5.27 \mathrm{Y}$ & 1173.2 & 99.9 & & & Activation \\
\hline 1343.6 & ${ }^{138} \mathrm{Cs}$ & 1.1 & $32.2 \mathrm{M}$ & 1147.3 & 1.2 & 1435.8 & 76.3 & ${ }^{138} \mathrm{Xe}$ \\
\hline 1365.2 & ${ }^{134} \mathrm{Cs}$ & 3.0 & $2.06 Y$ & 1167.9 & 1.8 & 1038.6 & 1.0 & Fission \\
\hline 1368.2 & ${ }^{124} \mathrm{Sb}$ & 2.7 & $60.2 \mathrm{D}$ & 1325.5 & 1.6 & 1436.7 & 1.3 & Fallout \\
\hline 1368.6 & ${ }^{24} \mathrm{Na}$ & 100.0 & $14.7 \mathrm{H}$ & 2754.1 & 99.9 & & & Activation \\
\hline 1369.4 & ${ }^{88} \mathrm{Kr}$ & 1.5 & $2.84 \mathrm{H}$ & 1141.4 & 1.3 & 1518.4 & 2.2 & Fission \\
\hline 1372.1 & ${ }^{132} \mathrm{I}$ & 2.5 & $2.28 \mathrm{H}$ & 1295.4 & 2.0 & 1398.6 & 7.1 & ${ }^{132} \mathrm{Te}$ \\
\hline 1377.7 & ${ }^{214} \mathrm{Bi}$ & 4.0 & Long & 1281.0 & 1.5 & 1401.5 & 1.4 & ${ }^{238} \mathrm{U}$ \\
\hline 1384.3 & ${ }^{110 m} \mathrm{Ag}$ & 24.3 & $250 \mathrm{D}$ & 1475.8 & 4.0 & 937.5 & 34.4 & Activation \\
\hline 1398.6 & ${ }^{132} \mathrm{I}$ & 7.1 & $2.28 \mathrm{H}$ & 1372.1 & 2.5 & 1442.5 & 1.4 & ${ }^{132} \mathrm{Te}$ \\
\hline 1401.5 & ${ }^{214} \mathrm{Bi}$ & 1.4 & Long & 1377.7 & 4.0 & 1408.0 & 2.5 & ${ }^{238} \mathrm{U}$ \\
\hline 1408.0 & ${ }^{214} \mathrm{Bi}$ & 2.5 & Long & 1401.5 & 1.4 & 1509.2 & 2.2 & ${ }^{238} \mathrm{U}$ \\
\hline 1408.0 & ${ }^{152} \mathrm{Eu}$ & 20.8 & $13.3 \mathrm{Y}$ & 1299.2 & 1.6 & 1212.9 & 1.4 & Fallout \\
\hline 1435.8 & ${ }^{138} \mathrm{Cs}$ & 76.3 & $32.2 \mathrm{M}$ & 1343.6 & 1.1 & 2218.0 & 15.2 & ${ }^{138} \mathrm{Xe}$ \\
\hline 1436.6 & ${ }^{124} \mathrm{Sb}$ & 1.3 & $60.2 \mathrm{D}$ & 1368.2 & 2.7 & 1691.0 & 47.1 & Fallout \\
\hline 1442.5 & ${ }^{132} \mathrm{I}$ & 1.4 & $2.28 \mathrm{H}$ & 1398.6 & 7.1 & 1921.1 & 1.2 & ${ }^{132} \mathrm{Te}$ \\
\hline 1459.2 & ${ }^{228} \mathrm{Ac}$ & 1.1 & Long & 1499.0D & 1.6 & 969.0 & 17.4 & ${ }^{232} \mathrm{Th}$ \\
\hline 1460.8 & ${ }^{40} \mathrm{~K}$ & 10.7 & $1.3 \mathrm{E} 9$ & & & & & Natural \\
\hline 1475.8 & ${ }^{110 \mathrm{~m}} \mathrm{Ag}$ & 4.0 & 250D & 1384.3 & 24.3 & 1505.0 & 13.0 & Activation \\
\hline 1499.0D & ${ }^{228} \mathrm{AC}$ & 1.6 & Long & 1459.2 & 1.1 & 1588.2 & 3.6 & ${ }^{232} \mathrm{Th}$ \\
\hline 1505.0 & ${ }^{110 \mathrm{~m}} \mathrm{Ag}$ & 13.0 & $250 \mathrm{D}$ & 1475.8 & 4.0 & 1562.3 & 1.0 & Activation \\
\hline 1509.2 & ${ }^{214} \mathrm{Bi}$ & 2.2 & Long & 1408.0 & 2.5 & 1661.3 & 1.2 & ${ }^{238} \mathrm{U}$ \\
\hline 1518.4 & ${ }^{88} \mathrm{Kr}$ & 2.2 & $2.84 \mathrm{H}$ & 1369.4 & 1.5 & 1529.8 & 10.9 & Fission \\
\hline 1529.8 & ${ }^{88} \mathrm{Kr}$ & 10.9 & $2.84 \mathrm{H}$ & 1518.4 & 2.2 & 2029.9 & 4.5 & Fission \\
\hline 1588.2 & ${ }^{228} \mathrm{Ac}$ & 3.6 & Long & 1499.0D & 1.6 & 1630.5 & 2.0 & ${ }^{232} \mathrm{Th}$ \\
\hline 1596.5 & ${ }^{140} \mathrm{La}$ & 95.4 & $40.3 \mathrm{H}$ & 487.0 & 45.9 & 2521.7 & 3.4 & Fallout \\
\hline 1596.6 & ${ }^{154} \mathrm{Eu}$ & 1.7 & $8.8 \mathrm{Y}$ & 1274.5 & 35.5 & 1004.8 & 17.9 & Fallout \\
\hline 1620.7 & ${ }^{212} \mathrm{Bi}$ & 1.5 & Long & 727.3 & 6.7 & 785.5 & 1.1 & ${ }^{232} \mathrm{Th}$ \\
\hline 1630.5 & ${ }^{228} \mathrm{Ac}$ & 2.0 & Long & 1588.2 & 3.6 & $1499.0 \mathrm{D}$ & 1.6 & ${ }^{232} \mathrm{Th}$ \\
\hline 1661.3 & ${ }^{214} \mathrm{Bi}$ & 1.2 & Long & 1509.2 & 2.2 & 1729.6 & 3.1 & ${ }^{238} \mathrm{U}$ \\
\hline 1691.0 & ${ }^{124} \mathrm{Sb}$ & 47.1 & $60.2 \mathrm{D}$ & 2090.9 & 5.5 & 1436.7 & 1.3 & Fallout \\
\hline
\end{tabular}


TABLE OF $\gamma$ RAYS AND NUCLIDES COMMON TO ENVIRONMENTAL ANALYSES (Cont'd)

\begin{tabular}{|c|c|c|c|c|c|c|c|c|}
\hline Energy & Nuclide & $\%$ & $\mathrm{~T}_{1 / 2}$ & E2 & $\%$ & E3 & $\%$ & Origin \\
\hline 1729.6 & ${ }^{214} \mathrm{Bi}$ & 3.1 & Long & 1764.5 & 15.9 & 1661.3 & 1.2 & ${ }^{238} \mathrm{U}$ \\
\hline 1740.6 & ${ }^{87} \mathrm{Kr}$ & 2.0 & $76.3 \mathrm{M}$ & 1175.5 & 1.1 & 2011.9 & 2.9 & Fission \\
\hline 1764.5 & ${ }^{214} \mathrm{Bi}$ & 15.9 & Long & 1729.6 & 3.1 & 1847.4 & 2.1 & ${ }^{238} \mathrm{U}$ \\
\hline 1768.4 & ${ }^{138} \mathrm{Xe}$ & 16.7 & $14.1 \mathrm{M}$ & 1114.3 & 1.5 & 1850.9 & 1.4 & Fission \\
\hline 1769.7 & $207 \mathrm{Bi}$ & 6.9 & $32.2 \mathrm{Y}$ & 1063.1 & 74.9 & 569.2 & 97.8 & Fallout \\
\hline 1836.1 & ${ }^{88} \mathrm{Rb}$ & 21.4 & $17.8 \mathrm{M}$ & 2677.9 & 2.0 & 898.1 & 14.1 & ${ }^{88} \mathrm{Kr}$ \\
\hline 1836.1 & ${ }^{88} \mathrm{Y}$ & 99.4 & $107 \mathrm{D}$ & 898.1 & 92.7 & & & Other \\
\hline 1847.4 & ${ }^{214} \mathrm{Bi}$ & 2.1 & Long & 1764.5 & 15.9 & 2118.5 & 1.2 & ${ }^{238} \mathrm{U}$ \\
\hline 1850.9 & ${ }^{138} \mathrm{Xe}$ & 1.4 & $14.1 \mathrm{M}$ & 1768.4 & 16.7 & 2004.8 & 5.4 & Fission \\
\hline 1921.1 & ${ }^{132} \mathrm{I}$ & 1.2 & $2.28 \mathrm{H}$ & 1442.5 & 1.4 & 2002.4 & 1.1 & ${ }^{132} \mathrm{Te}$ \\
\hline 2002.4 & ${ }^{132} I$ & 1.1 & $2.28 \mathrm{H}$ & 1921.1 & 1.2 & 1442.5 & 1.4 & ${ }^{132} \mathrm{Te}$ \\
\hline 2004.8 & ${ }^{138} \mathrm{Xe}$ & 5.4 & $14.1 \mathrm{M}$ & 1850.9 & 1.4 & 2015.9 & 12.3 & Fission \\
\hline 2011.9 & ${ }^{87} \mathrm{Kr}$ & 2.9 & $76.3 \mathrm{M}$ & 1740.6 & 2.0 & $2556.0 \mathrm{D}$ & 13.1 & Fission \\
\hline 2015.9 & ${ }^{138} \mathrm{Xe}$ & 12.3 & $14.1 \mathrm{M}$ & 2004.8 & 5.4 & 2079.3 & 1.4 & Fission \\
\hline 2029.9 & ${ }^{88} \mathrm{Kr}$ & 4.5 & $2.84 \mathrm{H}$ & 1529.8 & 10.9 & 2035.5 & 3.7 & Fission \\
\hline 2035.5 & ${ }^{88} \mathrm{Kr}$ & 3.7 & $2.84 \mathrm{H}$ & 2029.9 & 4.5 & 2195.8 & 13.2 & Fission \\
\hline 2079.3 & ${ }^{138} \mathrm{Xe}$ & 1.4 & $14.1 \mathrm{M}$ & 2015.9 & 12.3 & 2252.3 & 2.3 & Fission \\
\hline 2090.9 & ${ }^{124} \mathrm{Sb}$ & 5.5 & $60.2 \mathrm{D}$ & 1436.6 & 1.3 & 1691.0 & 47.1 & Fallout \\
\hline 2118.5 & ${ }^{214} \mathrm{Bi}$ & 1.2 & Long & 1847.4 & 2.1 & 2204.1 & 5.0 & ${ }^{238} \mathrm{U}$ \\
\hline 2195.8 & ${ }^{88} \mathrm{Kr}$ & 13.2 & $2.84 \mathrm{H}$ & 2035.5 & 3.7 & 2231.8 & 3.4 & Fission \\
\hline 2204.1 & ${ }^{214} \mathrm{Bi}$ & 5.0 & Long & 2447.7 & 1.6 & 2118.5 & 1.2 & ${ }^{238} \mathrm{U}$ \\
\hline 2217.8 & ${ }^{138} \mathrm{Cs}$ & 15.2 & $32.2 \mathrm{M}$ & 1435.8 & 76.3 & 2639.4 & 7.6 & ${ }^{138} \mathrm{Xe}$ \\
\hline 2231.8 & ${ }^{88} \mathrm{Kr}$ & 3.4 & $2.84 \mathrm{H}$ & 2195.8 & 13.2 & 2392.1 & 34.6 & Fission \\
\hline 2252.3 & ${ }^{138} \mathrm{Xe}$ & 2.3 & $14.1 \mathrm{M}$ & 2079.3 & 1.4 & 2015.9 & 12.3 & Fission \\
\hline 2392.1 & ${ }^{88} \mathrm{Kr}$ & 34.6 & $2.84 \mathrm{H}$ & 2231.8 & 3.4 & 2195.8 & 13.2 & Fission \\
\hline 2447.7 & ${ }^{214} \mathrm{Bi}$ & 1.6 & Long & 2204.1 & 5.0 & 2118.5 & 1.2 & ${ }^{238} \mathrm{U}$ \\
\hline 2521.7 & ${ }^{140} \mathrm{La}$ & 3.4 & $40.3 \mathrm{H}$ & 1596.5 & 96.4 & 487.0 & 45.9 & Fallout \\
\hline $2556 \mathrm{D}$ & ${ }^{87} \mathrm{Kr}$ & 13.1 & $76.3 \mathrm{M}$ & 2011.9 & 2.9 & 1740.6 & 2.0 & Fission \\
\hline 2614.4 & ${ }^{208} \mathrm{TI}$ & 35.8 & Long & 860.3 & 4.3 & 583.0 & 30.9 & ${ }^{232} \mathrm{Th}$ \\
\hline 2639.4 & ${ }^{138} \mathrm{Cs}$ & 7.6 & $32.2 \mathrm{M}$ & 2217.8 & 15.2 & 1435.8 & 76. & ${ }^{138} \mathrm{Xe}$ \\
\hline
\end{tabular}


TABLE OF $\gamma$ RAYS AND NUCLIDES COMMON TO ENVIRONMENTAL ANALYSES (Cont'd)

\begin{tabular}{|c|c|c|c|c|c|c|c|c|}
\hline Energy & Nuclide & $\%$ & $\mathrm{~T}_{1 / 2}$ & E2 & $\%$ & E3 & $\%$ & Origin \\
\hline 2677.9 & ${ }^{88} \mathrm{Rb}$ & 2.0 & $17.8 \mathrm{M}$ & 1836.1 & 21.4 & 898.1 & 14.1 & ${ }^{88} \mathrm{Kr}$ \\
\hline 2754.0 & ${ }^{24} \mathrm{Na}$ & 99.9 & $14.7 \mathrm{H}$ & 1368.6 & 100.0 & & & Activation \\
\hline 6129.2 & ${ }^{16} \mathrm{~N}$ & 68.8 & $7.13 \mathrm{~S}$ & 7115.2 & 4.7 & & & Other \\
\hline 7115.2 & ${ }^{16} \mathrm{~N}$ & 4.7 & $7.13 \mathrm{~S}$ & 6129.2 & 68.8 & & & Other \\
\hline
\end{tabular}




\subsection{X-RAY}

Contact Person(s) : Colin G. Sanderson

\subsubsection{SCOPE}

This section presents a table of X-ray energies which are useful for radiochemical analyses.

A number of nuclides emit X-rays as part of their decay scheme. These $\mathrm{X}$-rays may be counted with Ar proportional counters with $\mathrm{Ge}$ planar or n-type $\mathrm{Ge}$ co-axial detectors or with thin crystal $\mathrm{NaI}(\mathrm{Tl})$ scintillation counters. In both cases, spectral measurements can be made and both qualitative and quantitative information obtained on the sample.

$\mathrm{K}$ Shell vacancies are filled by a higher shell election. In the process an energy $E_{k}-E_{x}$ is liberated either as an X-ray or an Auger electron. The most important X-ray transitions are designated as,

$$
\begin{aligned}
& \mathrm{K}_{\alpha 1}=\mathrm{K}-\mathrm{L}_{\mathrm{II}} \\
& \mathrm{K}_{\alpha 2}=\mathrm{K}-\mathrm{L}_{\mathrm{II}} \\
& \mathrm{K}_{B 1}=\mathrm{K}-\mathrm{M}_{\mathrm{II}} \\
& \mathrm{K}_{B 2}=\mathrm{K}-\mathrm{N}_{\mathrm{III}} \\
& \mathrm{K}_{B 3}=\mathrm{K}-\mathrm{M}_{\mathrm{II}} \\
& \mathrm{K}_{B 4}=\mathrm{K}-\mathrm{N}_{\mathrm{II}} \\
& \mathrm{K}_{B 5}=\mathrm{K}-\mathrm{M}_{\mathrm{IV}}
\end{aligned}
$$


With moderate resolution only $\mathrm{K}_{B 1}{ }^{\prime}$ and $\mathrm{K}_{B 2}{ }^{\prime}$ can be resolved,

$$
\begin{gathered}
\mathrm{K}_{B 1}{ }^{\prime}=\mathrm{K}_{B 1}+\mathrm{K}_{B 3}+\mathrm{K}_{B 5} \\
\mathrm{~K}_{B 2}{ }^{\prime}=\mathrm{K}_{B 2}+\mathrm{K}_{B 4}
\end{gathered}
$$

The same is true for $K_{\alpha}$

$$
\mathrm{K}_{\alpha}=\mathrm{K}_{\alpha 1}+\mathrm{K} \alpha_{2}
$$

The present table lists the values for $\mathrm{K}_{\alpha}, \mathrm{K}_{\mathrm{B} 1}{ }^{\prime}$ and $\mathrm{K}_{\mathrm{B2}}{ }^{\prime}$. Electron binding energies used in this table were based on the tabulations of Wapstra et al. (1959) and Siegbahn (1965).

\section{REFERENCES}

Browne, E. and R. B. Firestone

Table of Radioactive Isotopes

Shirley, V. S. (Editor)

John Wiley and Sons, Inc., New York (1986)

Siegbahn, K.

Alpha, Beta, and Gamma Ray Spectroscopy

North-Holland Publishing, Co., Amsterdam (1965)

Wapstra, A. H., G. J. Nijgh and R. Van Lieshout

Nuclear Spectroscopy Tables

North-Holland Publishing, Co., Amsterdam (1959) 


\begin{tabular}{|c|c|c|c|}
\hline Z & Element & $\mathrm{K}_{\alpha}^{-}$ & $\begin{array}{c}\text { Energy } \\
(\mathrm{keV}) \\
\mathrm{K}_{\mathrm{BI}} \\
\end{array}$ \\
\hline 3 & $\mathrm{Li}$ & 0.05 & \\
\hline 4 & $\mathrm{Be}$ & 0.11 & \\
\hline 5 & $\mathrm{~B}$ & 0.18 & \\
\hline 6 & C & 0.28 & \\
\hline 7 & $\mathrm{~N}$ & 0.40 & \\
\hline 8 & 0 & 0.53 & \\
\hline 9 & F & 0.68 & \\
\hline 10 & $\mathrm{Ne}$ & 0.85 & \\
\hline 11 & $\mathrm{Na}$ & 1.04 & \\
\hline 12 & $\mathrm{Mg}$ & 1.25 & \\
\hline 13 & $\mathrm{Al}$ & 1.49 & \\
\hline 14 & $\mathrm{Si}$ & 1.74 & \\
\hline 15 & $P$ & 2.01 & \\
\hline 16 & $S$ & 2.31 & \\
\hline 17 & $\mathrm{C} 1$ & 2.62 & \\
\hline 18 & $\mathrm{Ar}$ & 2.96 & 3.19 \\
\hline 19 & $\mathrm{~K}$ & 3.31 & 3.59 \\
\hline 20 & $\mathrm{Ca}$ & 3.69 & 4.01 \\
\hline 21 & $\mathrm{Sc}$ & 4.09 & 4.46 \\
\hline 22 & $\mathrm{Ti}$ & 4.51 & 4.93 \\
\hline 23 & $\mathrm{~V}$ & 4.95 & 5.43 \\
\hline 24 & $\mathrm{Cr}$ & 5.42 & 5.95 \\
\hline 25 & $\mathrm{Mn}$ & 5.90 & 6.49 \\
\hline 26 & $\mathrm{Fe}$ & 6.40 & 7.06 \\
\hline 27 & Co & 6.93 & 7.65 \\
\hline 28 & $\mathrm{Ni}$ & 7.47 & 8.26 \\
\hline 29 & $\mathrm{Cu}$ & 8.03 & 8.91 \\
\hline 30 & $\mathrm{Zn}$ & 8.63 & 9.57 \\
\hline 31 & $\mathrm{Ga}$ & 9.24 & 10.3 \\
\hline 32 & $\mathrm{Ge}$ & 9.88 & 11.1 \\
\hline
\end{tabular}




\begin{tabular}{|c|c|c|c|c|}
\hline Z & Element & $\mathrm{K}_{\alpha}^{-}$ & $\begin{array}{c}\text { Energy } \\
(\mathrm{keV}) \\
\mathrm{K}_{\mathrm{B1}}{ }^{-} \\
\end{array}$ & $K_{B 2}{ }^{\circ}$ \\
\hline 33 & As & 10.5 & 11.7 & \\
\hline 34 & $\mathrm{Si}$ & 11.2 & 12.5 & \\
\hline 35 & $\mathrm{Br}$ & 11.9 & 13.3 & \\
\hline 36 & $\mathrm{Kr}$ & 12.6 & 14.1 & \\
\hline 37 & $\mathrm{Rb}$ & 13.4 & 15.0 & \\
\hline 38 & $\mathrm{Sr}$ & 14.1 & 15.8 & 16.1 \\
\hline 39 & $\mathrm{Y}$ & 14.9 & 16.7 & 17.0 \\
\hline 40 & $\mathrm{Zr}$ & 15.7 & 17.7 & 18.0 \\
\hline 41 & $\mathrm{Nb}$ & 16.6 & 18.6 & 19.0 \\
\hline 42 & Mo & 17.4 & 19.6 & 20.0 \\
\hline 43 & $\mathrm{Te}$ & 18.3 & 20.6 & 21.0 \\
\hline 44 & $\mathrm{Ru}$ & 19.2 & 21.6 & 22.1 \\
\hline 45 & $\mathrm{Rh}$ & 20.2 & 22.7 & 23.2 \\
\hline 46 & $\mathrm{Pd}$ & 21.1 & 23.8 & 24.3 \\
\hline 47 & $\mathrm{Ag}$ & 22.1 & 24.9 & 25.5 \\
\hline 48 & $\mathrm{Cd}$ & 23.1 & 26.1 & 26.6 \\
\hline 49 & In & 24.1 & 27.3 & 27.9 \\
\hline$\underline{50}$ & $\mathrm{Sn}$ & 25.1 & 28.4 & 29.1 \\
\hline 51 & $\mathrm{Sb}$ & 26.3 & 29.7 & 30.4 \\
\hline 52 & $\mathrm{Te}$ & 27.3 & 31.0 & 31.7 \\
\hline 53 & I & 28.5 & 32.3 & 33.0 \\
\hline 54 & $\mathrm{Xe}$ & 29.6 & 33.6 & 34.4 \\
\hline 55 & Cs & 30.8 & 34.9 & 35.8 \\
\hline 56 & $\mathrm{Ba}$ & 32.0 & 36.4 & 37.2 \\
\hline 57 & $\mathrm{La}$ & 33.3 & 37.8 & 38.7 \\
\hline 58 & $\mathrm{Ce}$ & 34.5 & 39.3 & 40.2 \\
\hline 59 & $\operatorname{Pr}$ & 35.9 & 40.7 & 41.8 \\
\hline 60 & $\mathrm{Nd}$ & 37.2 & 42.3 & 43.3 \\
\hline 61 & $\mathrm{Pm}$ & 38.5 & 43.8 & 44.9 \\
\hline 62 & $\mathrm{Sm}$ & 39.8 & 45.4 & 46.6 \\
\hline
\end{tabular}




\begin{tabular}{lcccc} 
& & & \multicolumn{3}{c}{$\begin{array}{c}\text { Energy } \\
(\mathrm{keV})\end{array}$} & \\
$\mathrm{Z}$ & Element & $\mathrm{K}_{\alpha}^{-}$ & $\mathrm{K}_{\mathrm{BI}}$ & $\mathrm{K}_{\mathrm{B} 2}$ \\
\hline 63 & $\mathrm{Eu}$ & 41.3 & 47.0 & 48.2 \\
64 & $\mathrm{Cd}$ & 42.7 & 48.7 & 49.9 \\
65 & $\mathrm{~Tb}$ & 44.1 & 50.4 & 51.7 \\
\hline & & & & \\
66 & $\mathrm{Dy}$ & 45.6 & 52.1 & 53.4 \\
67 & $\mathrm{Ho}$ & 47.1 & 53.8 & 55.3 \\
68 & $\mathrm{Er}$ & 48.7 & 55.6 & 57.1 \\
\hline & & & & \\
69 & $\mathrm{Tm}$ & 50.3 & 57.5 & 59.0 \\
70 & $\mathrm{Yb}$ & 51.9 & 59.4 & 60.9 \\
71 & $\mathrm{Lu}$ & 53.5 & 61.3 & 62.9 \\
\hline & & & & \\
72 & $\mathrm{Hf}$ & 55.2 & 63.2 & 64.9 \\
73 & $\mathrm{Ta}$ & 57.1 & 65.2 & 67.0 \\
74 & $\mathrm{~W}$ & 58.8 & 67.2 & 69.1 \\
\hline & & & & \\
75 & $\mathrm{Re}$ & 60.6 & 69.3 & 71.2 \\
76 & $\mathrm{Os}$ & 62.4 & 71.4 & 73.4 \\
77 & $\mathrm{Ir}$ & 64.3 & 73.6 & 75.6 \\
\hline & & & & \\
78 & $\mathrm{Pt}$ & 66.2 & 75.7 & 77.8 \\
79 & $\mathrm{Au}$ & 68.2 & 78.0 & 80.1 \\
80 & $\mathrm{Hg}$ & 70.1 & 80.1 & 82.5 \\
\hline & & & & \\
81 & $\mathrm{Tl}$ & 72.1 & 82.4 & 84.9 \\
82 & $\mathrm{~Pb}$ & 74.2 & 84.7 & 87.3 \\
83 & $\mathrm{Bi}$ & 76.3 & 87.1 & 89.8 \\
\hline & & & & \\
84 & $\mathrm{Po}$ & 78.4 & 89.6 & 92.3 \\
85 & $\mathrm{At}$ & 80.5 & 92.7 & 95.0 \\
86 & $\mathrm{Rn}$ & 82.8 & 94.7 & 97.5 \\
\hline & & & & \\
87 & $\mathrm{Fr}$ & 85.0 & 97.3 & 100.2 \\
88 & $\mathrm{Ra}$ & 87.3 & 99.9 & 103.0 \\
89 & $\mathrm{Ac}$ & 89.7 & 102.6 & 105.7 \\
\hline & & & & \\
\hline
\end{tabular}




\begin{tabular}{rcrcr} 
& & & & $\begin{array}{c}\text { Energy } \\
(\mathrm{keV})\end{array}$ \\
$\mathrm{Z}$ & Element & $\mathrm{K}_{\alpha}{ }^{-}$ & $\mathrm{K}_{\mathrm{B} 1}{ }^{-}$ & $\mathrm{K}_{\mathrm{B} 2}{ }^{\circ}$ \\
\hline 90 & $\mathrm{Th}$ & 92.1 & 105.3 & 108.6 \\
91 & $\mathrm{~Pa}$ & 94.5 & 108.1 & 111.4 \\
92 & $\mathrm{U}$ & 97.0 & 111.0 & 114.5 \\
\hline & & & & \\
93 & $\mathrm{~Np}$ & 99.5 & 113.9 & 117.5 \\
94 & $\mathrm{Pu}$ & 102.1 & 116.9 & 120.5 \\
95 & $\mathrm{Am}$ & 104.7 & 119.9 & 123.6 \\
\hline & & & & \\
96 & $\mathrm{Cm}$ & 107.5 & 123.0 & 126.9 \\
97 & $\mathrm{Bk}$ & 110.2 & 126.2 & 130.2 \\
98 & $\mathrm{Cf}$ & 113.0 & 129.4 & 133.5 \\
\hline & & & & \\
99 & Es & 115.9 & 132.7 & 136.9 \\
100 & $\mathrm{Fm}$ & 118.8 & 136.0 & 140.4 \\
101 & $\mathrm{Md}$ & 122.8 & 139.4 & 144.9 \\
\hline & & & & \\
102 & $\mathrm{No}$ & 124.8 & 142.7 & 147.5 \\
103 & $\mathrm{Lw}$ & 127.9 & 146.2 & 151.2 \\
104 & & 130.5 & 149.7 & 154.5
\end{tabular}




\title{
5.6 NATURAL DECAY SERIES
}

\author{
Contact Person(s) : Isabel M. Fisenne
}

\subsubsection{SCOPE}

Charts of the four heavy element series are given in this section. These are the three natural series and the artificial Am series.

Data for half-lives and energies have been taken from Browne et al. (1986). Energies are given in order of abundance and include only the major emissions. Branching in the chains that amount to one percent or less are also omitted.

\section{REFERENCE}

Browne, E. and R. B. Firestone

Table of Radioactive Isotopes

Shirley, V. S. (Editor)

John Wiley and Sons, Inc. (1986) 


\section{Principal Members of the Uranium Series}

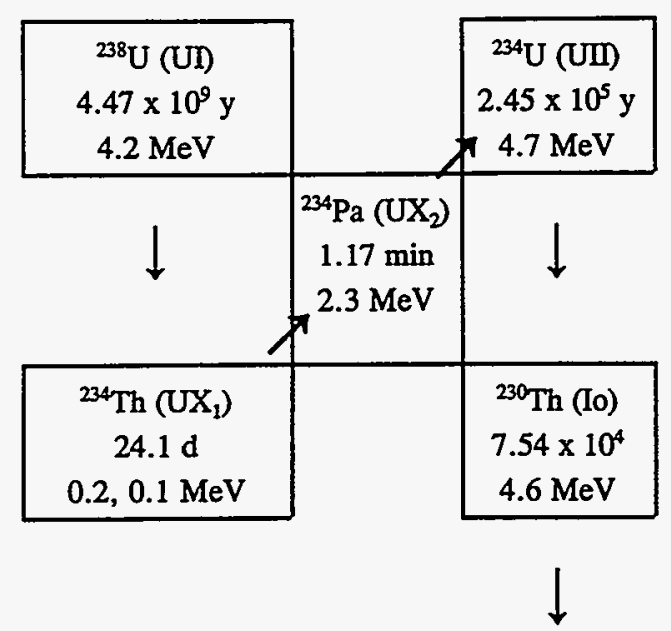

${ }^{226} \mathrm{Ra}(\mathrm{Ra})$ $1600 \mathrm{y}$

$4.8 \mathrm{MeV}$

$\downarrow$

\begin{tabular}{|c|}
\hline${ }^{222} \mathrm{Rn}(\mathrm{Rn})$ \\
$3.825 \mathrm{~d}$ \\
$5.5 \mathrm{MeV}$ \\
\hline
\end{tabular}
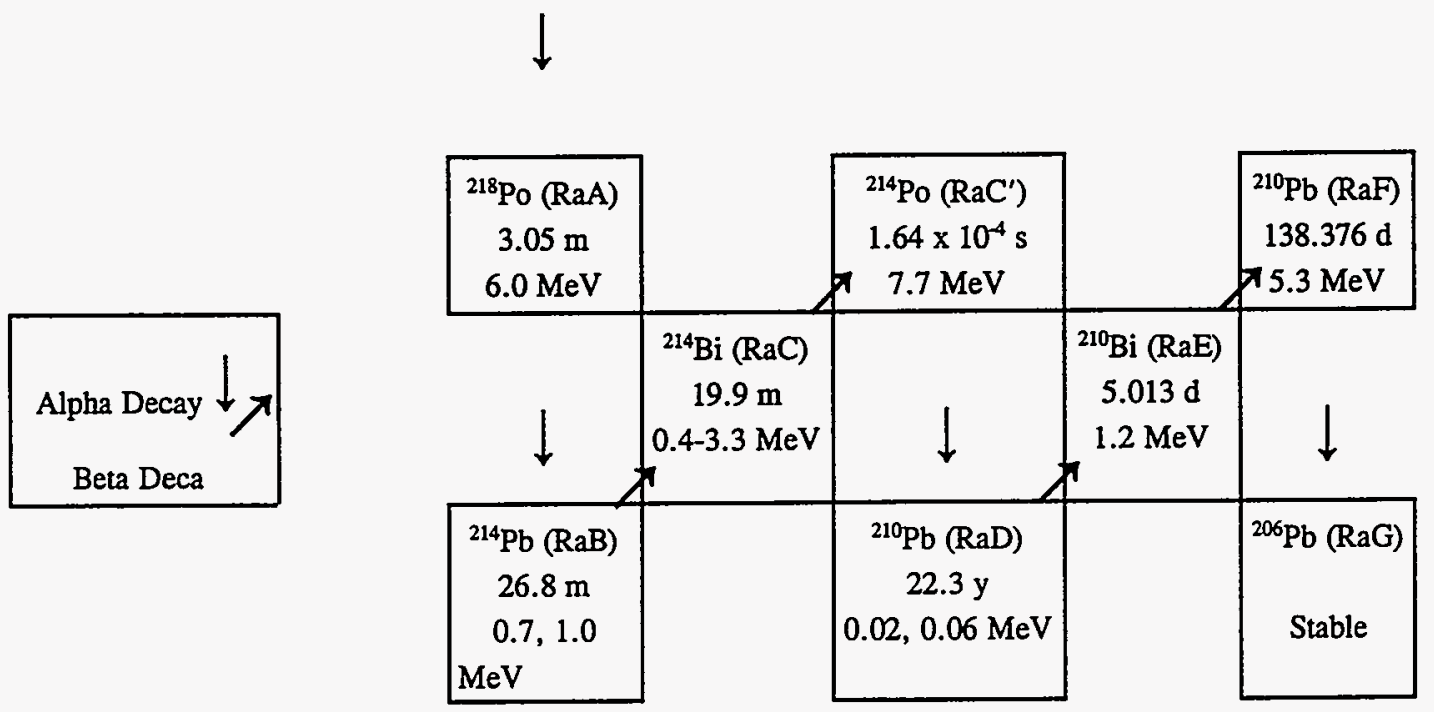
Principal Members of the Thorium Series

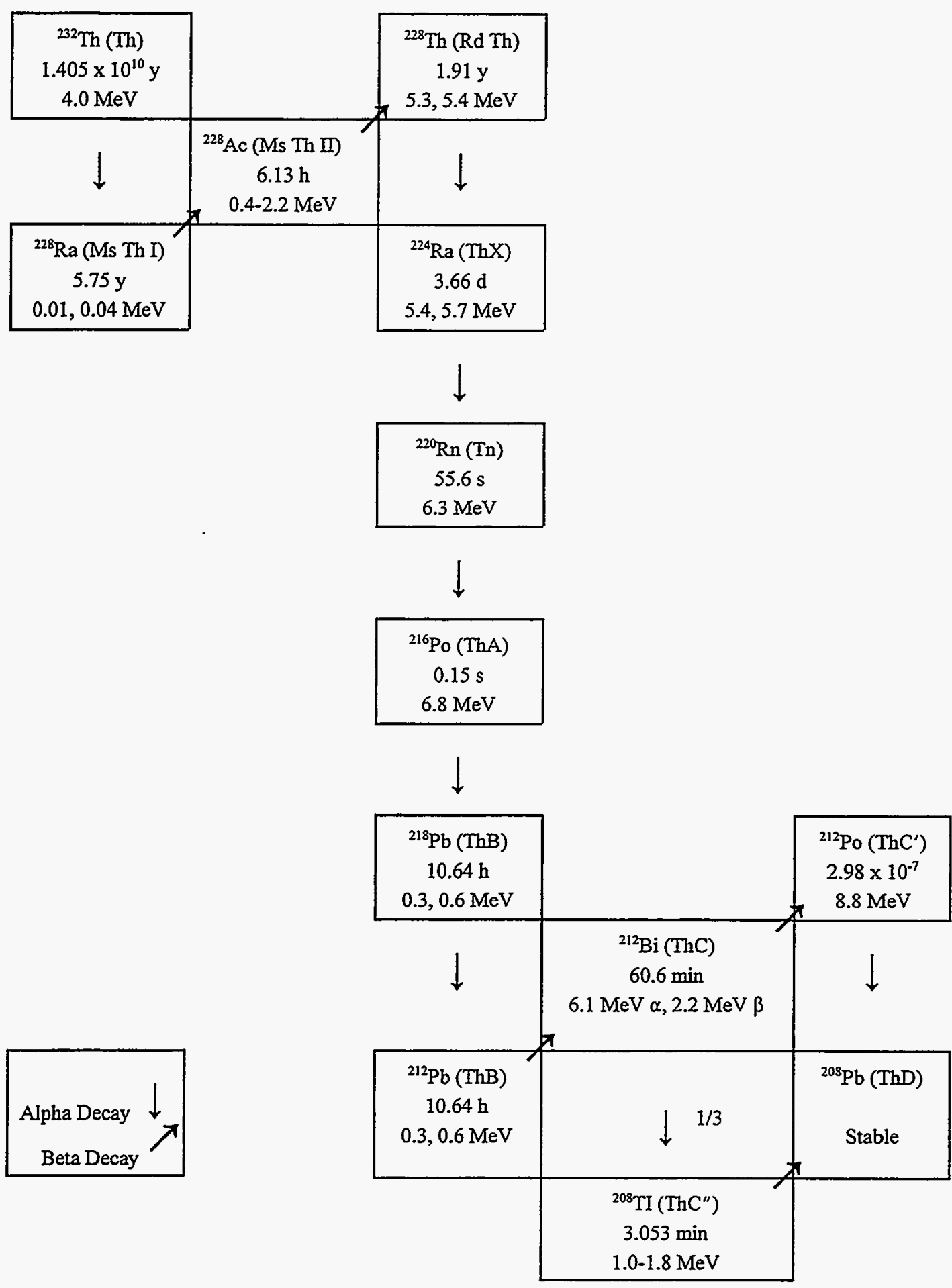


Principal Members of the Actinium Series

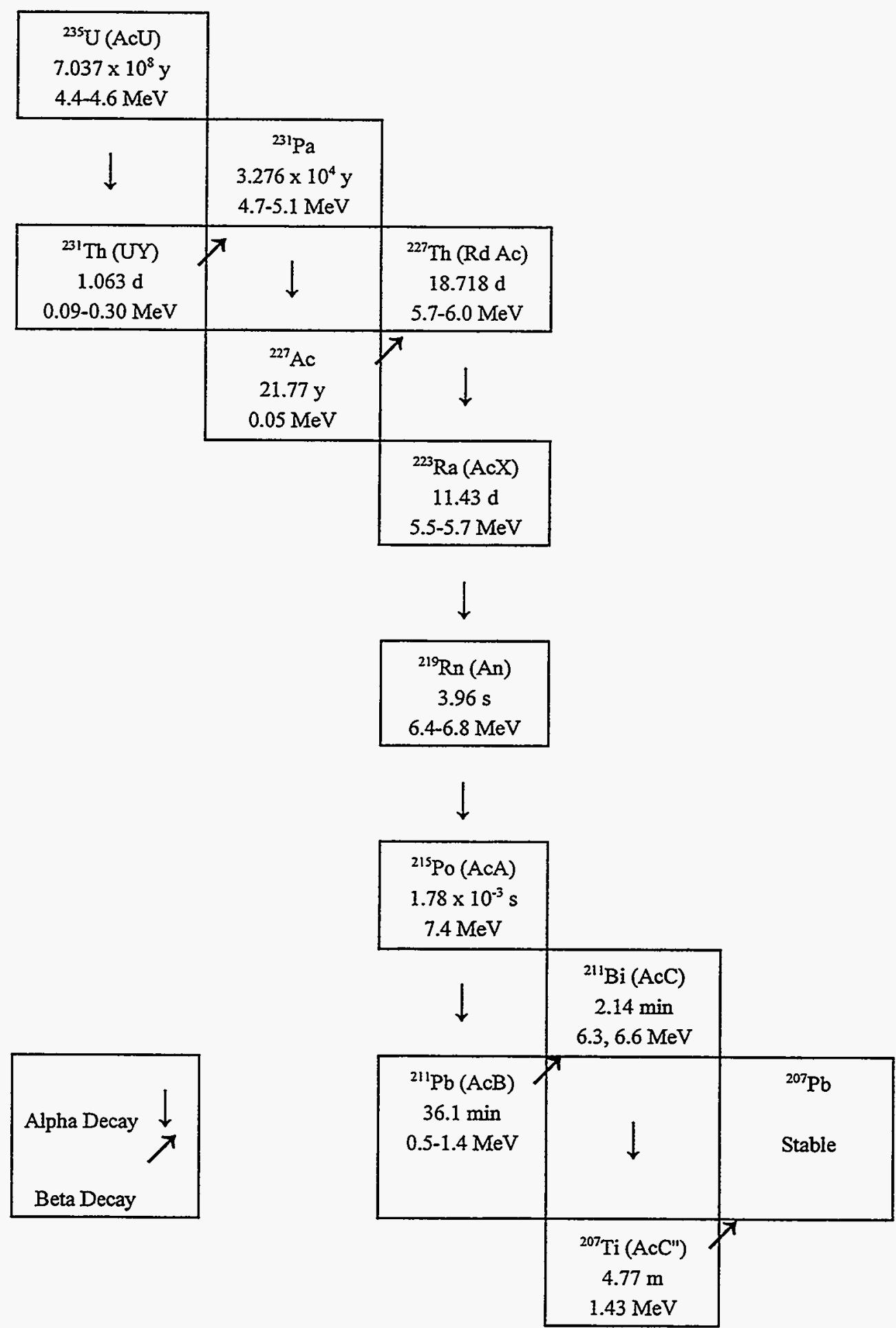




\section{Principal Members of the Americium Series}
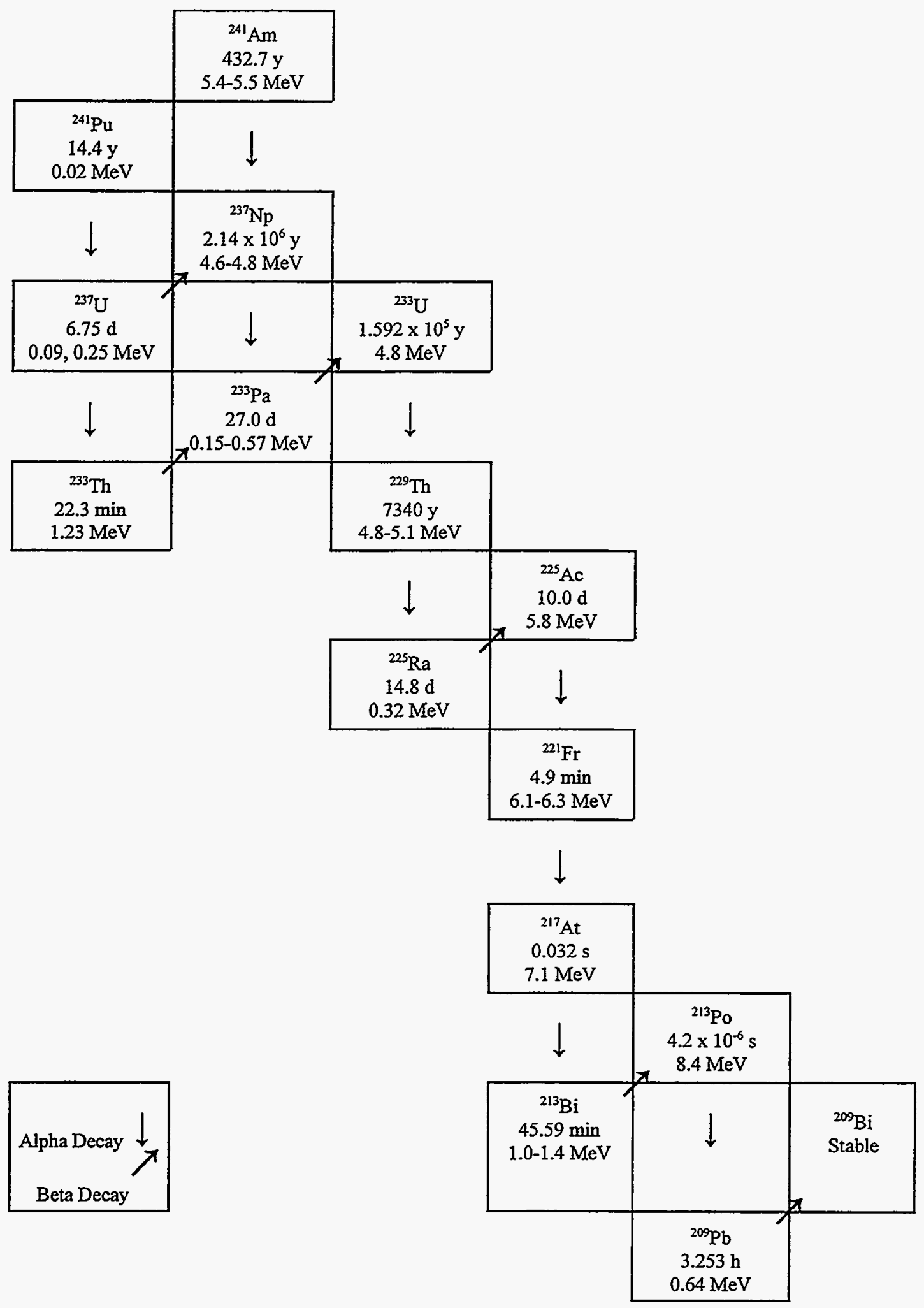


\section{SPECIAL FACILITIES}

U.S. Department of Energy 201 Varick Street, 5th Floor New York, NY 10014-4811 


\section{Special Facilities}

Page

6.1 Overview

\subsection{Radon Gas and Radon/Thoron Progeny Facilities for Testing and Research}

6.2.1 Scope $\ldots \ldots \ldots \ldots \ldots \ldots \ldots \ldots \ldots \ldots \ldots \ldots \ldots .6 .2-1$

6.2.2 The EML Pulse Ionization Chamber Facility for Radon-222

Measurements .............................. 6.2-1

6.2 .2 .1 Introduction $\ldots \ldots \ldots \ldots \ldots \ldots \ldots \ldots \ldots \ldots \ldots \ldots, 6.2-1$

6.2.2.2 Principle of Operation ...................... 6.2-2

6.2 .2 .3 Design $\ldots \ldots \ldots \ldots \ldots \ldots \ldots \ldots \ldots \ldots \ldots \ldots \ldots .6 .2 .2$

6.2.2.4 Procedure $\ldots \ldots \ldots \ldots \ldots \ldots \ldots \ldots \ldots \ldots \ldots \ldots, 6.2-2$

6.2.2.5 Calibration.............................. 6.2-3

6.2.2.6 Routine Chamber Checks and Maintenance .......... 6.2-3

6.2.3 Radon, Thoron, and Progeny Exposure Facility ........... 6.2-4

6.2.3.1 Introduction .............................. $6.2-4$

6.2.3.2 Description of the Exposure Facility $\ldots \ldots \ldots \ldots \ldots \ldots .6 .2-4$

\subsection{Chemistry Clean Room Facility $\ldots \ldots \ldots \ldots \ldots \ldots \ldots .6 .3-1$}

6.3.1 Scope $\ldots \ldots \ldots \ldots \ldots \ldots \ldots \ldots \ldots \ldots \ldots \ldots \ldots \ldots \ldots .6 .3-1$

6.3 .2 Facility Layout $\ldots \ldots \ldots \ldots \ldots \ldots \ldots \ldots \ldots \ldots \ldots \ldots \ldots \ldots \ldots \ldots \ldots \ldots \ldots .3 .1$



6.3.4 Monitoring of Clean Room Operation $\ldots \ldots \ldots \ldots \ldots \ldots \ldots .6 .3-2$

6.3.5 Permanent Apparatus $\ldots \ldots \ldots \ldots \ldots \ldots \ldots \ldots \ldots \ldots . \ldots . . .6 .2$

6.4 Scanning Electron Microscope Facility ............ 6.4-1

6.4.1 Scope $\ldots \ldots \ldots \ldots \ldots \ldots \ldots \ldots \ldots \ldots \ldots \ldots \ldots \ldots .6 .6 .4 .1$

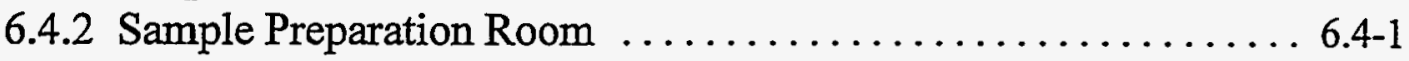

6.4.3 Microscope Room .......................... 6.4-1

6.4.3.1 Scanning Electron Microscope $\ldots \ldots \ldots \ldots \ldots \ldots \ldots .6 .4 .2$

6.4.3.2 Microanalysis System .................... 6.4-2

6.4.3.3 Sample Critical Point Drying and Coating Equipment ..... 6.4-4

6.4.4 Quality Control ............................. 6.4-4 


\subsection{Mobile Radiation Measurements}

$$
\text { Laboratory } \ldots \ldots \ldots \ldots \ldots \ldots \ldots \ldots \ldots \ldots \ldots \ldots, 6.5-1
$$

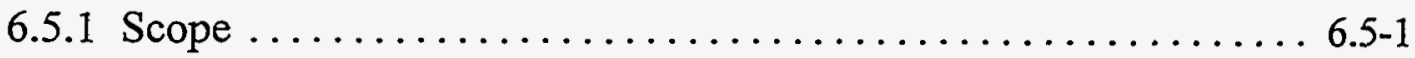

6.5 .2 Base Vehicle .............................. 6.5-1

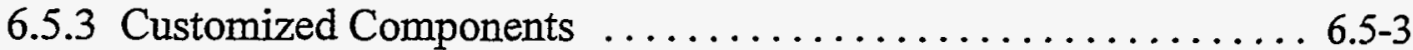

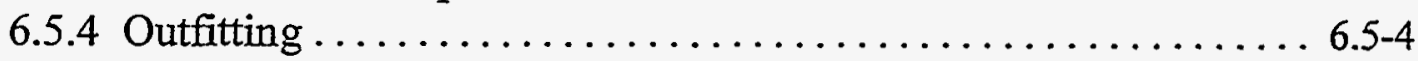

6.6 Work Boat and Tripod Corer ................... 6.6-1

6.6 .1 Scope $\ldots \ldots \ldots \ldots \ldots \ldots \ldots \ldots \ldots \ldots \ldots \ldots \ldots \ldots .6 .6 .1$

6.6.2 Research Vessel Sedimental Journey $\ldots \ldots \ldots \ldots \ldots \ldots \ldots .6 .6 .6 .1$

6.6.2.1 Dimensions and Configurations ..................6.6.1

6.6.2.2 Power Equipment ........................... 6.6-2

6.6.2.3 Depth Recording Gear .................... 6.6-2

6.6.3 Trailer and Towing Vehicle $\ldots \ldots \ldots \ldots \ldots \ldots \ldots \ldots .6 .6-3$

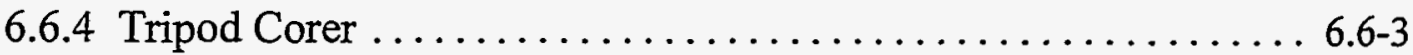

6.6.4.1 Coring Tube and Attachments ................. 6.6-4

6.6 .4 .2 Frame .............................. $6.6-4$

6.6.4.3 Weight Basket $\ldots \ldots \ldots \ldots \ldots \ldots \ldots \ldots \ldots \ldots \ldots .6 .6 .5$

6.6.4.4 Core Extruder ...........................6. 6.5

6.6.5 Assembly, Launching and Retrieval Procedure ........... 6.6-5

6.7 Chester Regional Baseline Station ............... 6.7-1

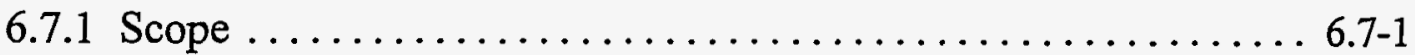

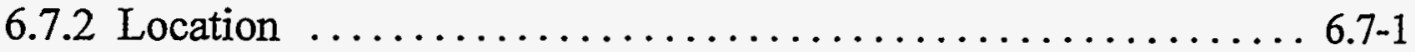

6.7.3 Local Terrain, Soil, and Vegetation................. 6.7-1

6.7.4 Site Characteristics and Permanent Facilities ............. 6.7-2

6.7.5 Meteorological Data Instrumentation ................. 6.7-3

6.7.6 Acknowledgements ............................ 6.7 


\section{SPECIAL FACILITIES}

\subsection{OVERVIEW}

Seven major facilities that are used in research programs at EML are described in this section. Four of these are stationary, and are located in EML's quarters in New York City. Two of the facilities are mobile and are used in field sampling/measurement work all over the U.S. The seventh facility is an outdoor laboratory located in rural New Jersey. 


\title{
6.2 RADON GAS AND RADON/THORON PROGENY FACILITIES FOR TESTING AND RESEARCH
}

\author{
Contact Person(s) : Isabel M. Fisenne and Alfred J. Cavallo
}

\subsubsection{SCOPE}

Two major on-site EML facilities that are used for research on $\mathrm{Rn}$ gas measurements, instruments, and methods are described here (the pulse ionization chamber facility and the $\mathrm{Rn}, \mathrm{Th}$, and progeny exposure facilities).

\subsubsection{THE EML PULSE IONIZATION CHAMBER FACILITY FOR RADON-222 MEASUREMENTS}

\subsubsection{1}

\section{INTRODUCTION}

The pulse ionization chamber facility is used for the direct measurement of environmental levels of ${ }^{222} \mathrm{Rn}$ in air and breath samples. The chambers are calibrated periodically by de-emanating $\mathrm{Rn}$ gas from $\mathrm{Ra}$ solutions certified by the National Institute of Standards and Technology (NIST). Thus, the facility also serves as the EML reference for $\mathrm{Rn}$ gas measurements, and values so obtained are considered to be EML's best estimate of the $\mathrm{Rn}$ activity in a sample.

The following discussion will be confined to the direct measurement of ${ }^{222} \mathrm{Rn}$ in air samples as part of an integrated calibration facility. A fuller description of the EML pulse ionization chamber facility can be found in Fisenne and Keller (1985). 


\subsubsection{2}

PRINCIPLE OF OPERATION

The EML pulse ionization chamber is operated on the principle of fast pulse counting (Curtiss and Davis, 1943). Fast pulse counting is based on direct detection of the $\alpha$ ionization produced in a single ionization chamber. To accomplish fast pulse counting, it is mandatory that $\mathrm{O}_{2}$ and water vapor be removed before a sample is introduced into the chamber.

\section{DESIGN}

The EML pulse ionization chambers, which are shown in Figure 6.1, are constructed of stainless steel. The interior surface is electropolished to remove surface contamination and to lower the background count rate, which is the limiting factor for measurement sensitivity. Clean chambers have a background of about 10 counts $\mathrm{h}^{-1}$.

The EML chambers are constructed with a plug in the baseplate to accommodate an electrodeposited standard source for the determination of the $\alpha$ counting plateau. The $\alpha$ detection efficiency of the chambers with such a source is $52 \%$.

The transfer systems for the removal of $\mathrm{O}_{2}$ and water vapor are designed to meet three criteria: simplicity; a small ratio of dead space to chamber volume; and dichotomy, that is, access to one or two ionization chambers.

\section{PROCEDURE}

For direct transfer and measurement to a single chamber, the air or breath sample volume is restricted to $1 \mathrm{~L}$ or less. Sample transfer is accomplished by evacuating the entire system, including the ionization chambers. Pure $\mathrm{H}_{2}$ gas is added to the sample container ( $35 \mathrm{kPa}$ of $\mathrm{H}_{2}$ ). The sample is transferred through the system with forming gas 
$\left(85 \% \mathrm{~N}_{2}, 15 \% \mathrm{H}_{2}\right)$. The gas flow rate through the system is regulated by a capillary orifice and small particles are removed by a filter paper. The added excess $\mathrm{H}_{2}$ and the $\mathrm{O}_{2}$ in the sample react in the platinum black catalyst cartridges to produce $\mathrm{H}_{2} \mathrm{O}$ and excess heat. The $\mathrm{H}_{2} \mathrm{O}$ formed in the catalytic reaction is removed in a Drierite column. The gas is collected in a single ionization chamber which is pressurized to $35 \mathrm{kPa}$ with forming gas. A block diagram of the ionization chamber and external transfer apparatus is shown in Section 4, Procedure $\mathrm{Rn}-01-\mathrm{RC}$ in which the details of the procedure for the measurement of air samples are described.

The EML pulse ionization chambers are calibrated with NIST standard reference material (SRM) ${ }^{226} \mathrm{Ra}$ solutions using the Rn emanation technique (see Section 4, Procedure Ra-07-RC). In April 1984, NIST issued a new series of SRM ${ }^{226} \mathrm{Ra}$ solutions for Rn emanation measurements. As part of EML's internal quality control program, a major effort was undertaken to determine the calibration factor for the nine ionization chambers currently in use. The overall mean and standard deviation of 81 emanations into the nine chambers was $6245 \pm 135$ counts $\mathrm{h}^{-1}$ per Bq of ${ }^{222} \mathrm{Rn}$ in equilibrium with its short-lived progeny. This value was in excellent agreement with previous calibrations.

\section{ROUTINE CHAMIBER CHECKS AND MAINTENANCE}

The backgrounds of the nine pulse ionization chambers are measured with forming gas each weekend and occasionally during the work week to ensure against temporal biases. The backgrounds for the nine chambers in service range from 8-16 counts $\mathrm{h}^{-1}$. A control chart of the weekend background count rates is maintained for each chamber. At the beginning of the calendar year, the yearly average and running average background rates from previous years are calculated and control limits are established for the year. 
Over a period of years, the background of any chamber increases due to the buildup of long-lived $\mathrm{Rn}$ progeny on the interior surfaces. The background increase is a function of exposure in terms of ${ }^{222} \mathrm{Rn} \mathrm{Bq} \mathrm{h}$ in the chamber, that is, $0.037 \mathrm{~Bq}$ of ${ }^{222} \mathrm{Rn}$ in a chamber for $17 \mathrm{~h}$ will produce $2.2 \times 10^{-6} \mathrm{~Bq}$ of ${ }^{210} \mathrm{~Pb}$. After $1 \mathrm{y}$, the ${ }^{210} \mathrm{Po}$ will have reached $82 \%$ of the ${ }^{210} \mathrm{~Pb}$ activity and will contribute an additional $3 \times 10^{-3}$ counts $\mathrm{h}^{-1}$ to the chamber background. Fractions or multiples of the $.037 \mathrm{~Bq}$ of ${ }^{222} \mathrm{Rn}$ example are additive in the total temporal increase of the chamber background count rate. The background count rate is reduced by dismantling and electropolishing the chamber.

The platinum black catalyst and Drierite are kept free of water vapor by maintaining these cartridges under vacuum, except during sample introduction into a chamber.

The chamber systems are checked occasionally for electrically generated noise by filling the chambers with room air. The $\mathrm{O}_{2}$ in the air effectively reduces the pulse sizes below the $0.75 \mathrm{~V}$ tripping level of the electronic system and only electrically generated pulses are registered during the overnight measurement period. The electrical noise in the chamber system is $<0.25$ counts $\mathrm{h}^{-1}$.

Throughout the year the calibration factor of each chamber is checked by emanating $\mathrm{Rn}$ from a standard $\mathrm{Ra}$ emanation flask.

\subsubsection{RADON, THORON, AND PROGENY EXPOSURE FACILITY}

6.2.3.1

INTRODUCTION

The EML radon, thoron, and progeny exposure facility consists of a walk-in chamber that is used for research, testing, calibration, and for the evaluation of measuring instruments.

The $30 \mathrm{~m}^{3}$ chamber, installed in 1993, provides for a well-controlled, clean, airtight, and uniform test environment. Research with inert aerosols and other pollutants can also be carried out. Figure 6.2 shows the major features of the exposure facility. 


\section{A. Radon and thoron gas sources.}

Radon is generated from a Pylon (Model RN-1025, Ottawa, Ontario, Canada) ${ }^{226} \mathrm{Ra}$ source with $3810 \mathrm{kBq}$ of radium. The source is sealed hermetically inside a container located on top of the chamber. The injection of radon into the mixing chamber is computer controlled to obtain the desired concentrations of radon inside the main chamber.

Thoron is generated from a Pylon (Model TH-1025) ${ }^{228} \mathrm{Th}$ source. The strength of the source was $1960 \mathrm{kBq}$ at the time of acquisition. The source has to be recharged every $5 \mathrm{yr}$ because of its short half-life. The thoron generator can be installed in different locations inside the main chamber in order to achieve the desired airborne thoron concentrations.

\section{B. Design characteristics of the chamber.}

A schematic diagram of the chamber is shown in Figure 6.2. The interior dimensions of the main exposure room are $3.3 \mathrm{~m} \mathrm{x} 3.83 \mathrm{~m} \times 2.59 \mathrm{~m}$ with a total volume of $30.75 \mathrm{~m}^{3}$. The wall panels are made of enameled aluminum on the exterior, and 22 gauge stainless steel on the inside. The floor is constructed of 16 gauge stainless steel. The floors, walls, and ceiling are insulated to ensure minimum heating and cooling requirements.

The mixing room has a volume of $3.5 \mathrm{~m}^{3}$. The anteroom, which has a volume of $5.8 \mathrm{~m}^{3}$, serves as a means to transfer instruments in and out of the main chamber and as a buffer between the main chamber and the adjacent laboratory space. Two viewing windows, with triple panes, are located on the west wall. On the same wall there are 10 sampling ports that are $10 \mathrm{~cm}$ in diameter at a height of $1 \mathrm{~m}$ off the floor. A two-way audio system is provided for communication purposes. 


\section{Environmental control characteristics.}

The environmental conditioning system (Fidelity Engineering, Hunt Valley, MD) consists of the following systems: refrigeration, heating, humidification, dehumidification, air delivery, control, and pressurization air.

The refrigeration system consists of a single air-cooled condensing unit, two refrigeration evaporator coils, and a refrigerant piping network. The cooling coils are made of copper with aluminum fins; the refrigerant is HCFC-22. All of the elements of the air conditioning system are constructed to minimize contamination. The compressor, condenser coils receiver, and filter dryer are located outside the chamber, adjacent to the mixing room (see Figure 6.2). The evaporator coils are inside the mixing room. The temperature in the main test chamber can be controlled from $5^{\circ} \mathrm{C}-40^{\circ} \mathrm{C}$. The difference in temperature anywhere within the chamber is no more than $1^{\circ} \mathrm{C}$.

For humidification of the environment, water vapor is generated by an evaporative type system which is installed inside the mixing room. The system runs hot water across a screen-like surface and allows the water to evaporate into the air. The temperature of the water is kept below boiling. Dehumidification is accomplished by a refrigeration coil that becomes active only on call. It is isolated by a damper for the defrost cycle to avoid reintroduction of humidity during defrosting. The humidity is variable between a minimum that is determined by $\mathrm{a}-10^{\circ} \mathrm{C}$ dew point temperature and a maximum of $95 \%$. The humidity inside the chamber is controlled to within 2 units of the set point during steady conditions.

The environmental conditioning system also has a hydronic hot water system located outside the test chamber. It consists of a small glass electric hot water heater of about $2000 \mathrm{~W}$, a water circulation pump, a diaphragm expansion tank, and a filter. The hot water system is used to heat the test chamber air as needed.

The air delivery system, located in the mixing room, uses an in-line axial fan that can deliver a maximum of $30 \mathrm{~m}^{3} \mathrm{~min}^{-1}$. A typical operating range is $3-10 \mathrm{~m}^{3} \mathrm{~min}^{-1}$. In addition, a filtered pressurization air system is used to create a slight positive pressure in the main chamber to prevent uncontrolled infiltration. 


\section{Aerosol generator systems.}

A TSI Model 3470 condensation monodisperse aerosol generator (TSI, St. Paul, MI) located outside the main chamber, is used to produce particles of the desired concentration and size. Vaporized Carnauba wax condenses on $\mathrm{NaCl}$ nuclei to produce monodisperse aerosols with a geometric standard deviation $\left(\sigma_{g}\right)$ of around 1.1 or less. The concentration of the aerosols inside the test chamber can be controlled from 1,000 to $30,000 \mathrm{~cm}^{3}$. If polydisperse aerosols are desired, the concentration can be increased to more than $100,000 \mathrm{~cm}^{-3}$. The generated aerosols are injected through a sampling port hole into the main chamber. Aerosols from a burning candle, an electric heater, a kerosene lamp, or from cigarette smoke, can be generated individually or in combination by placing the generators inside the test chamber. The particle concentration inside the main chamber without any generated aerosol is $<200 \mathrm{~cm}^{3}$.

\section{E. Aerosol and vapor monitoring systems.}

The aerosol concentration inside the main chamber is measured with an Environment One Rich Model 200 condensation nucleus counter, or a TSI Model 3025 ultra-fine particle counter. The size distribution of the test aerosol is measured with a TSI scanning mobility particle sizer. A Bruel \& Kjaer Multi-gas Monitor Type 1302 is used to measure key organic pollutants. The detection limit depends on the type of pollutant and ranges from $0.001-1.0 \mathrm{ppm}$.

\section{F. Radon control and monitoring systems.}

The concentration of radon, thoron and progeny is regulated by adjusting the output from the radon generator. Radon is measured with two continuous scintillation cell monitors (cell volume $=0.096$ and $3.3 \mathrm{~L}$ ). The monitors are interfaced to a computer located at the control panel outside the chamber. The calibration and the accuracy of the radon monitors are based on intercomparisons made with pulse ionization chambers (see Section 6.2.2). The data are logged to a computer at $15-\mathrm{min}$ intervals, and may be recalled at any time. Radon and thoron concentrations in the main chamber are adjustable in the range from $100-5000 \mathrm{~Bq} \mathrm{~m}^{-3}$, and $50-5000 \mathrm{~Bq} \mathrm{~m}^{-3}$, respectively.

The radon and thoron progeny concentrations are regulated by the dilution of the parent gases and by the presence of aerosols. The progeny concentrations are measured 
inside the main chamber by grab, integrating, and continuous monitoring devices. The standard monitoring instruments for radon and thoron progeny are the AlphaSmart-760 (Alpha Nuclear, Missisauga, Ontario, Canada) and the GRI-1100 monitor. The analyses for the individual radon and thoron progeny and the potential alpha energy concentrations are performed using the methods of Thomas (1972), Nazaroff (1983), and Raabe and Wrenn (1969). All three methods are computerized and the data are available on an hourly basis. The concentrations in the main chamber range from $5.6 \times 10^{-9} \mathrm{~J} \mathrm{~m}^{-3}$ to $1.4 \mathrm{x}$ $10^{-5} \mathrm{~J} \mathrm{~m}^{-3}$ for radon progeny, and $1 \times 10^{-5}$ to $7 \times 10^{-5} \mathrm{~J} \mathrm{~m}^{-3}$ for thoron progeny.

Because of the capability to maintain the test chamber at less than 200 particle $\mathrm{cm}^{-3}$, the equilibrium factor as low 0.01 is achievable. At very high particle concentrations, an equilibrium factor of 0.5 is obtainable.

The sensors for temperature and humidity are located in the main test chamber and in the mixing room. The static pressure in the main chamber and the speed of the conditioned air exiting from the mixing room are monitored continuously. The data are automatically logged on the control computer.

\section{G. Radon progeny particle size distribution measurements.}

The test chamber particle size conditions can be measured with the micro-orifice uniform deposit impactor (MOUDI - see Section 2.2.2.5), EML developed screen diffusion batteries (see Section 2.2.2.6), and graded screen array (see Section 2.2.2.7). When used in combination, these instruments can measure radon and thoron progeny particle sizes ranging from 0.5 to $5000 \mathrm{~nm}$ (Tu and Knutson, 1994).

\section{REFERENCES}

Curtiss, L. F. and F. J. Davis

"A Counting Method for the Determination of Small Amounts of Radium and Radon" J. Res. Nat. Bur. Stand., 31, 181-195 (1943)

Fisenne, I. M. and H. W. Keller

"The EML Pulse Ionization Chamber System for ${ }^{222} \mathrm{Rn}$ Measurements"

USDOE Report EML-437 (1985) 
Nazaroff, W. W.

"Optimizing the Total Alpha Three Count Technique for Measuring Concentrations of

Radon Progeny in Residences"

Health Phys., 46, 395-405 (1983)

Raabe, O. G. and M. E. Wrenn

"Analyses of the Activity of Radon Daughter Sampler by Weighted Least Squares"

Health Phys., 17, 593-605 (1969)

Thomas, J. W.

"Measurements of Radon Daughters in Air"

Health Phys., 23, 783-789 (1972)

$\mathrm{Tu}, \mathrm{K}$. W. and E. O. Knutson

"Measurements of Radon Progeny Size Distribution with a MOUDI and a Graded Screen Array"

Richard C. Flagan (Editor)

in: Proceedings of the 4th International Aerosol Conference, Vol. 2, p. 767 (1994) 


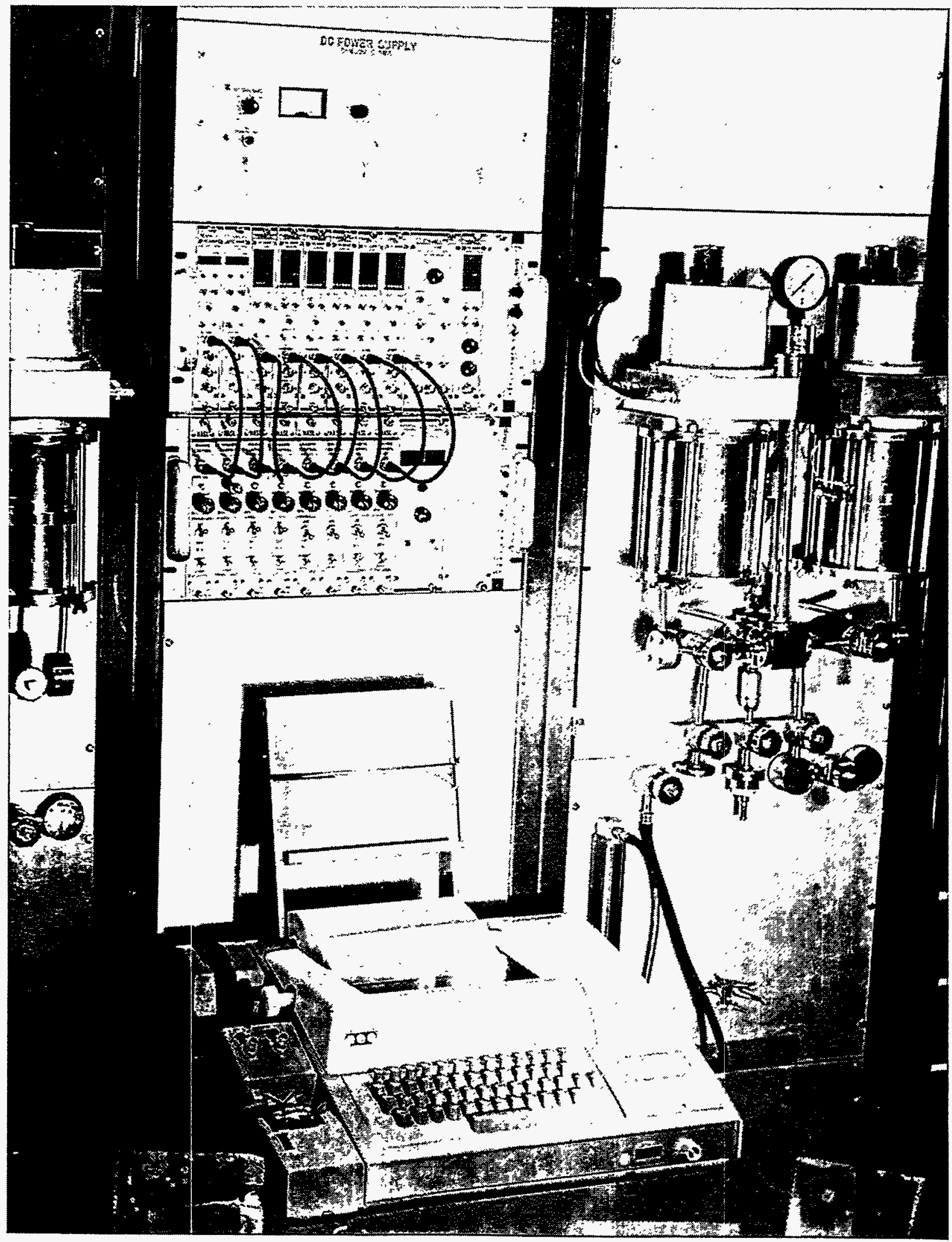

Figure 6.1 Photograph of pulse ionization chamber facility. 


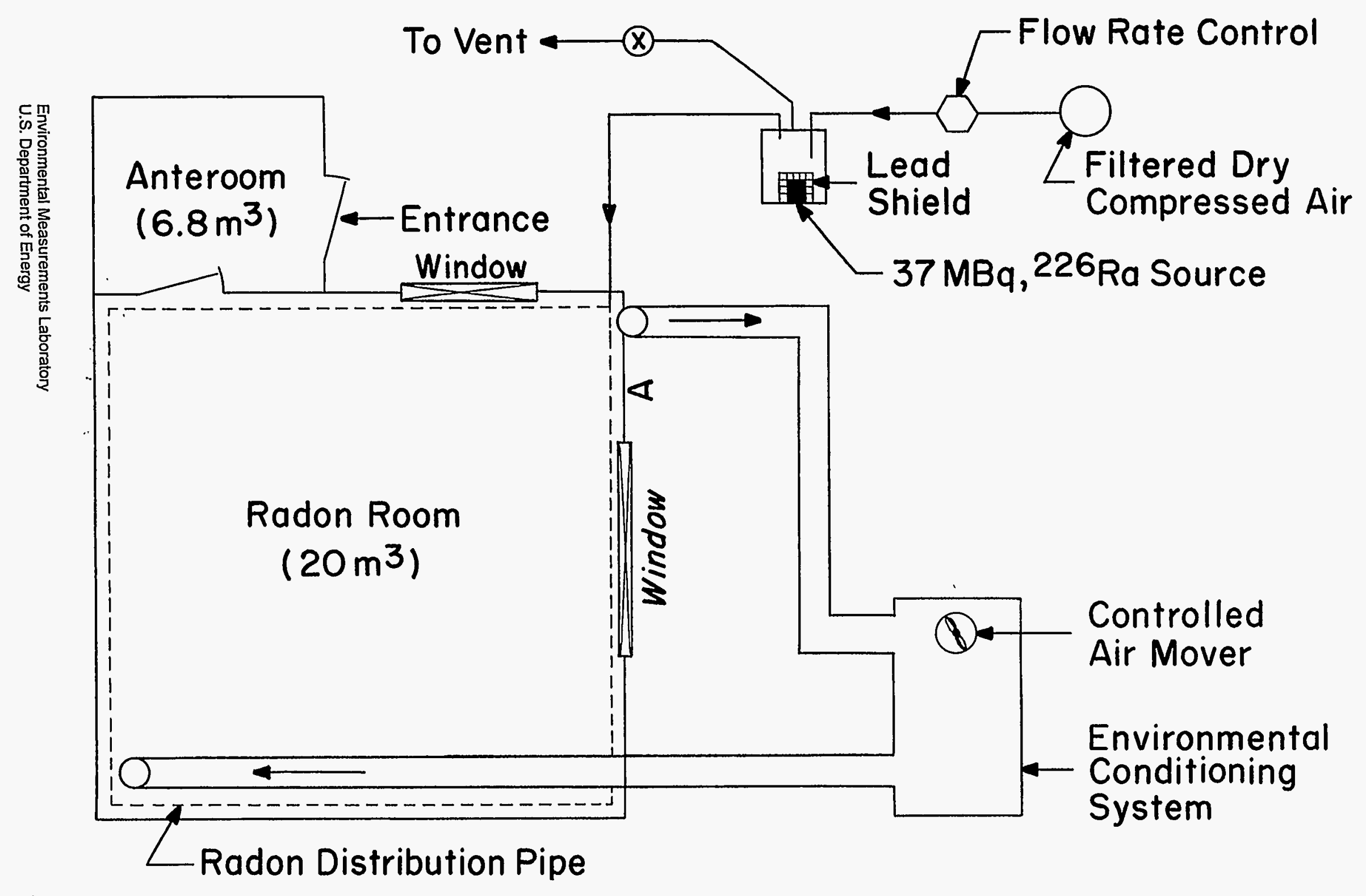




\title{
6.3 CHEMISTRY CLEAN ROOM FACILITY
}

\author{
Contact Person(s) : Thomas M. Beasley
}

\subsubsection{SCOPE}

Presented here is a description of the clean room chemical laboratory that has been in operation at EML since 1976. The facility is used for low-level trace metal analyses of biological and environmental samples for various on-going and past programs, such as, metabolic balance studies, geochronology of pollution, and atmospheric transport and deposition phenomena.

Generally, these programs require the analyses to be performed in an ultraclean environment because of the low concentrations found in many of the specimen. Typically specimens of urine, excreta, diet, fish, vegetation, air, filters, water (tap, lake, rain), sediments, and soil have been analyzed for more than 20 environmentally significant trace metals.

\subsubsection{FACILITY LAYOUT}

A diagram of the facility, its equipment, and instrumentation is shown in Figure 6.3. Note that one entry serves as the change room and also houses the air handling system. The other entry houses the gas cylinders that are required by the instruments.

\subsubsection{AIR CLEANING SYSTEM}

Clean air is supplied to the room through 16 high efficiency particulate air filters suspended directly over the workbench, located in the center of the room. This down 
ward flow of air maintains Class 100 conditions (as defined in Federal Standard 209B, 1973) at the top of the workbench. The same air flow maintains Class 2000 conditions in other areas of the facility.

The air handler supplies sufficient clean air to maintain positive pressure in the clean room, even with the fume hood and the furnace hood in operation.

\title{
6.3.4 MONITORING OF CLEAN ROOM OPERATION
}

To check that the facility is operating properly, particle concentrations are monitored with a Royco particle counter. In accordance with Federal Standard 209B, this counter is set to measure the concentration of particles with diameters larger than $0.5 \mu \mathrm{m}$.

\subsubsection{PERMANENT APPARATUS}

The clean room chemical laboratory is equipped for all stages of analysis of trace metals in various biological matrices from sample preparation to final measurements.

The following are the major instruments used in the clean room.

\author{
Atomic Absorption Spectrometer \\ Model 603 \\ Perkin-Elmer Corp. \\ Norwalk, CT 06859-0001 \\ Direct Current Plasma Emission Spectrometer \\ Model SMI II \\ Spectrametric, Inc. \\ Valencia, CA 91355
}

The basic attributes of both instruments are well-documented in the scientific literature; e.g., Dean and Rains (1971), and details are given in the manufacturer's instrument manuals. At EML, both instruments are used to verify the results by two 
distinct techniques and where the matrix and/or the concentration range of the specimen prohibits accurate and precise measurement on one instrument.

The clean room can produce both ultrapure deionized water and a sub-boiling distillation apparatus. The clean room is also equipped with a freezer, refrigerator, muffle, drying oven, and several analytical balances.

\section{REFERENCES}

Dean, F. and T. Rains

"Flame Emission and Atomic Absorption Spectrometry"

Marcel Dekker Inc., New York (1971)

Federal Standard 209B

"Federal Standard Clean Room and Work Station Requirements, Controlled Environment"

Available from: General Services Administration, Specifications Activity, Printed Materials Supply Division, Washington, DC 20407 


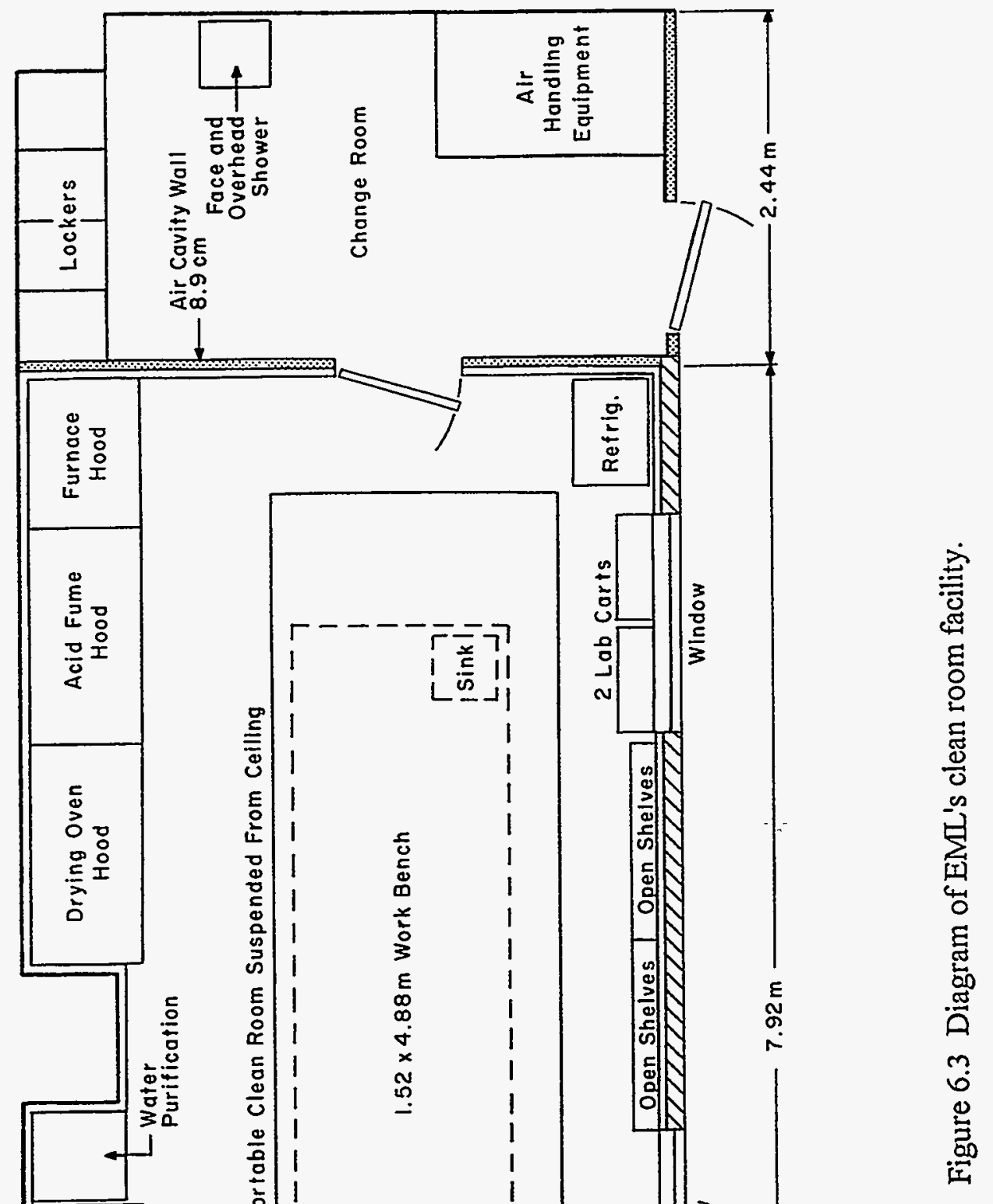




\title{
6.4 SCANNING ELECTRON MICROSCOPE FACILITY
}

\author{
Contact Person(s) : Robert Leifer
}

\subsubsection{SCOPE}

This section describes EML's scanning electron microscope (SEM) facility. The physical layout of this facility is shown in Figure 6.4. The SEM is used to: 1) analyze aerosols collected from the troposphere and the stratosphere (Kromidas et al., 1996); 2) characterize laboratory-generated aerosol; and 3) analyze biological samples.

\subsubsection{SAMPLE PREPARATION ROOM}

The sample preparation room is maintained under clean-room conditions using high efficiency particulate air (HEPA) ceiling filters. The air is recirculated and controlled with a system that is independent of the building air delivery system. There are two Class-100 benches to provide additional cleanliness for sample preparation and for the preparation of sampling equipment. The first, an exhausting laminar bench (Advanced Purification Inc., Hauppauge, NY 11788; Model G68-PD) allows the use of various solvents and provides a Class 100 environment for equipment setup and preparation of biological samples. The second bench (Laminar Flow Inc., Ivyland, PA 18974; special order) is a recirculating vertical laminar flow system with two cartridges that contain molecular sieve 5A and activated charcoal. These cartridges remove substances from the airstream that may interfere with aerosols. Such interfering substances include ammonia, water vapor, and organics.

\subsubsection{MICROSCOPE ROOM}

The microscope room is also maintained under clean-room conditions using HEPA ceiling filters. 
The SEM is an AMRAY-1820 (AMRAY, Bedford, MA 01730) and has a 150-mm specimen chamber and a resolution of $5 \mathrm{~nm}$. The SEM may be operated at low voltages $(\sim 1 \mathrm{kV})$, thus allowing imaging of samples without prior preparation. Microprocessor controlled operations permit automatic image focusing and stigmation and provides push button digital magnification from $25 \mathrm{X}-400,000 \mathrm{X}$ at a $12 \mathrm{~mm}$ working distance. However, just how much of this magnification is usable depends heavily on the contrast characteristics of the sample. The SEM is supplied with a solid-state backscatter detector. This detector produces images which emphasize maximum atomic number differences. The resulting image shows contrast differences that can be related to differences in trace element composition. The SEM is also supplied with an Xray/imaging system (described below). This system controls the sample stage automatically and thus allows for unattended $\mathrm{X}$-ray analyses, imaging, and sample characterization of more than 100 different fields.

The whole microscope assembly is isolated on a Micro-g vibration isolation table (TMC, Peabody, MA 01960).

\subsubsection{2}

MICROANALYSIS SYSTEM

The microanalysis system used in conjunction with the AMRAY 1820 SEM is the PGT IMIX system with a two position light element $\mathrm{Si}(\mathrm{Li})$ energy dispersive spectrometer (Model 4200 EDS, Princeton Gamma-Tech, Princeton, NJ 08540). The PGT IMIX system is equipped with:

1. An ultrahigh resolution 19-in color display.

2. Dec $11 / 73$ high performance processor with floating point. 
3. SPARC station 1+ (SUN Microsystems) with a built-in, 3 1/2" floppy disk drive, external CD ROM drive, and a 1.27 gigabyte Fujitsu drive.

4. Two thousand channel data collection system.

5. Pulse processing electronics.

6. EDS X-ray analysis software.

7. Qualitative and quantitative analysis software.

8. Chemical classification software.

9. PGT beam control package.

10. Advanced feature analysis package.

11. Mega plane option ( $800 \times 1040$ pixel image).

The microanalysis system allows detailed chemical and size classifications of samples. In the windowless mode, the model 4200 EDS allows the analysis of X-rays with energies down to $700 \mathrm{eV}$. In this position, detection of $\mathrm{B}, \mathrm{C}, \mathrm{N}$, and $\mathrm{O}$ is possible, as well as enhanced detection of $\mathrm{F}, \mathrm{Na}, \mathrm{Mg}$, and $\mathrm{Al}$. The PGT beam control package allows automated analysis of specific points on a collection surface by controlling the scan coils of the microscope column. Up to 300 points can be automatically stored for later use.

The PGT imaging system is connected to the Tissue Culture Facility via a video cable that is attached to a CCD video camera and can capture images from the optical microscopes. 
The sample coating equipment consists of a Denton high vacuum evaporator (Denton Vacuum, Inc., Cherry Hill, NJ 08003), an EFFA Carbon Coater (Ernest E. Fullam Co., Latham, NY 12110; Model No. 12560), and a sputter coater (Polaron, Warrington, PA; Model E5000). The sputter coater is supplied with platinum, gold, and silver targets. The carbon coater and sputter coater, plus an optical microscope are housed in a vertical flow class 100 laminar clean bench (Laminaire Corp., Rahway, NJ 07065; Model DWS630).

Critical point drying of biological samples is accomplished via the Balzers CPD 030 using acetone as the transfer medium and liquid $\mathrm{CO}_{2}$ as the transition liquid. The $\mathrm{CPD}$ 030 is located in the Sample Preparation Room with access to the exhaust laminar flow bench.

\subsubsection{QUALITY CONTROL}

When using an SEM, several things should be kept in mind. The sampling process must be conducted very carefully to provide the microscopist with a representative sample of the material of interest. Many methods of sampling introduce bias in that their collection efficiency varies with particle size. The internal environment of the microscope should also be taken into account. In order to function, the column is kept at a high vacuum $\left(10^{-5}\right.$ Torr) and there can be large amounts of heat generated in the sample by electron bombardment. These conditions can alter or destroy susceptible samples. Contamination is always a concern. Considering the size of the particles of interest, unless strict sample handling procedures are followed, contributions from contaminating sources may outweigh the sample material. 


\section{REFERENCE}

Kromidas, L., and R. Leifer

"An Innovative Application of a Commercially Available Double-sided Adhesive for the Collection of Aerosols by Impaction"

Atmospheric Environment, 30, 1177-1180 (1996) 


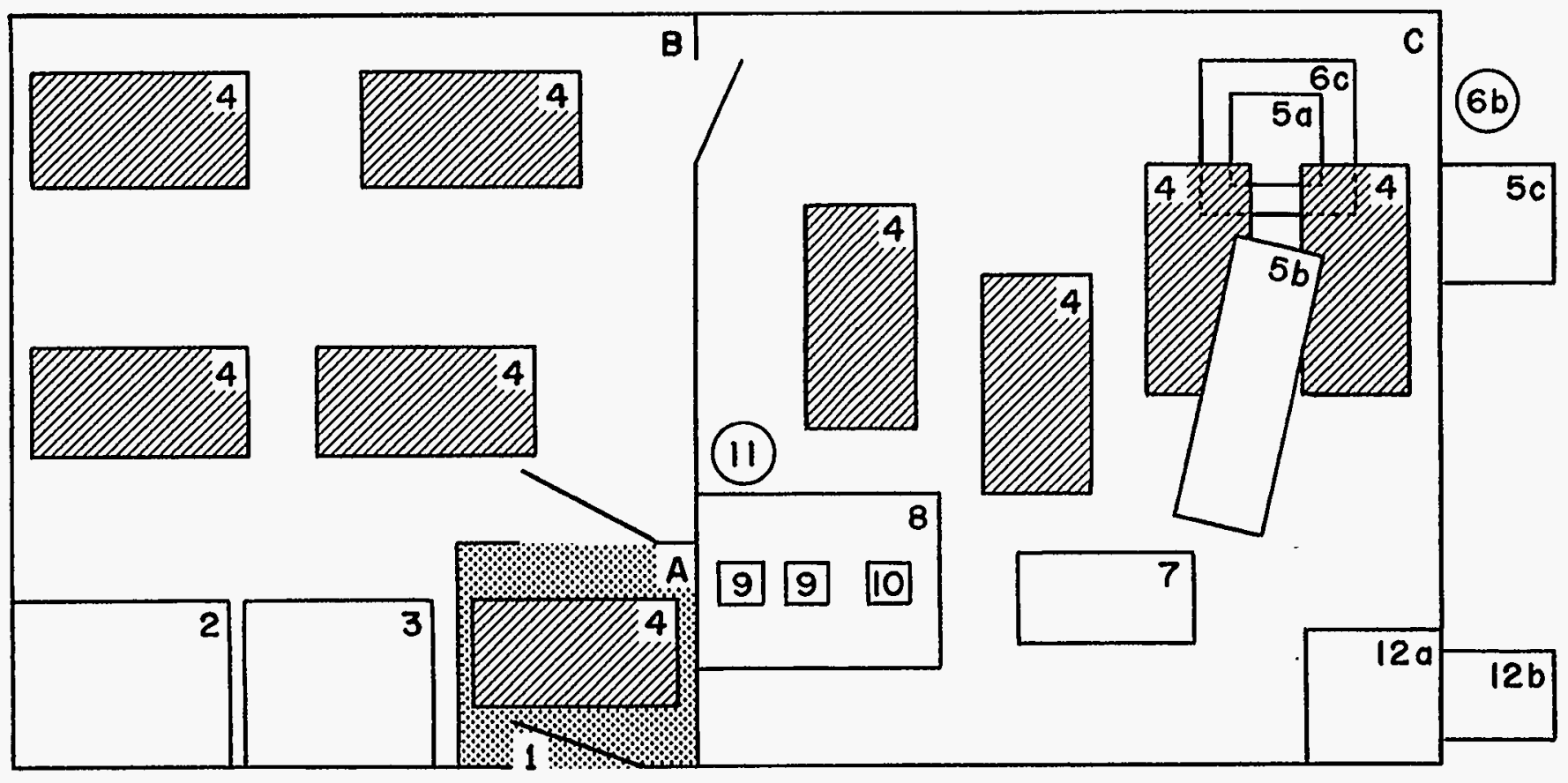

Figure 6.4 SEM facility: A) clothing change room; B) sample preparation room; C) SEM room. Areas in rooms: 1) sticky floor mat; 2) exhaust laminar flow bench; 3) recirculating laminar flow bench; 4) HEPA ceiling filters with blowers; 5a) column and stage; 5b) SEM electronic console; 5c) SEM vacuum, air and water support module (equipment placed outside microscope room); 6a) Micro-g vibration table; 6b) air supply for Micro-g table; 7) PGT microanalysis system; 8) vertical laminar flow bench; 9) particle coating devices; 10) optical microscope; 11) tank of Argon; 12a) Denton high vacuum evaporator; 12b) evaporator and coating device vacuum, air and water module. 


\title{
6.5 MOBILE RADIATION MEASUREMENTS LABORATORY
}

\author{
Contact Person(s) : Kevin M. Miller
}

\subsubsection{SCOPE}

We describe here a customized motor vehicle that is used by EML as a self-contained work station during field trips. Its primary function is for conducting radiation measurements in the environment, the main feature being a shielded Ge detector for $\gamma$-ray spectral analyses of collected samples. A certain flexibility in design is achieved with the use of roll out electronic equipment racks which facilitate adaptation to different projects and/or changes in instrumentation.

\subsubsection{BASE VEHICLE}

The base vehicle was purchased in two parts: the van chassis, which includes the cab and engine, and the truck body, which is essentially the box placed on the rear end of the chassis. A photograph of the complete vehicle is shown is Figure 6.5. A breakdown of the major specifications follows.

\section{A. Chassis.}

1. 1982 Ford E-350 dual rear wheel chassis, $4.01 \mathrm{~m}$ wheel-base (Ford Motor Company, Dearborn, MI 48121)

2. $7.5 \mathrm{~L}$ V-8 gasoline engine

3. Automatic transmission

4. Power steering

5. Power brakes (front disc)

6. Front stabilizer bar

7. Heavy duty springs and shock absorbers

8. $100 \mathrm{~A}$ alternator

9. $80 \mathrm{~A}-\mathrm{hr}$ battery 
10. Heavy duty cooling system

11. Tinted glass

12. Auxiliary fuel tank [with main tank - 152 L (40 gal) total capacity]

13. Transmission cooler

14. External oil cooler

15. Cruise control

16. Reclining swivel captain seats (two)

17. Recreational-type swing out side mirrors

18. Interval wipers

19. Trailer hitch

20. AM/FM radio

21. 'Digital clock

22. $\mathrm{C} / \mathrm{B}$ radio

\section{B. Body.}

1. Grumman-Olson Kurbette (Grumman Olson, Division Grumman Allied Industries, Inc., Melville, NY 11747)

2. Interior length, $3.96 \mathrm{~m}$

3. Interior width, $2.29 \mathrm{~m}$

4. Interior height, $1.85 \mathrm{~m}$

5. Double rear doors with windows

6. Single curb side door with window

\section{Full vehicle specifications.}

1. External length, $6.68 \mathrm{~m}$

2. Overall height, $2.72 \mathrm{~m}$

3. Gross vehicle weight, $4649 \mathrm{~kg}$

4. Tare weight (before customizing), $2492 \mathrm{~kg}$

5. Load capacity, $2157 \mathrm{~kg}$ 


\subsubsection{CUSTOMIZED COMPONENTS}

A commercial van customizer (East Coast Vans and Campers, Inc., West Babylon, NY 11704) was used to finish the interior of the cargo section of the vehicle and to provide the electrical systems, climate control, cabinetry, and mounting arrangements for equipment. Interior layout is shown in Figure 6.6. Specific features are as follows.

\section{A. Interior finish.}

1. Wall and ceiling - lined with plywood and covered with washable white marlite board

2. Insulation (walls, doors, and ceiling) - R-11 foiled fiberglass wool

3. Floor - sheet vinyl with metal bright molding

\section{B. Electrical.}

1. Generator - Kohler model 4.5CKM21-RV, 1800 rpm, 2 cylinder, 4500 W, $120 \mathrm{~V} \mathrm{AC}, 37.5 \mathrm{~A}, 60 \mathrm{~Hz}$ with high temperature-low oil shutdown, battery charger, air-vac cooling (Kohler Company, Kohler, WI 53044). Starting is electrical using vehicle battery via interior panel that features elapsed time of operation. Unit is mounted in a sound absorbent enclosure and is serviceable from both interior and exterior panels. Fuel is gasoline, fed from the vehicle's main tank.

2. Circuit box panel with four breakers

3. Four duplex $120 \mathrm{~V}$ wall outlets

4. Outside $120 \mathrm{~V}$ shoreline weather protected input box with $15 \mathrm{~m}$ extension line (provides power when available from an external source)

5. One 1.2-m dual, and two 0.6-m quadruple fluorescent light fixtures

6. Rotary warning lamp mounted on cab roof

7. Two outside $12 \mathrm{~V}$ spot lamps installed on front pillar posts

\section{Climate control.}

1. Coleman TSR Mach 3 13,500 BTU rotary compressor type, $115 \mathrm{~V}, 60$ cycle, 1 phase air conditioner (Coleman Company, Inc., Wichita, KS 67201) mounted on roof with added support beam and columns. Unit also provides $5600 \mathrm{BTU}$ heating.

2. $0.3 \mathrm{~m}$ square crank operated screened roof vent

3. One $0.35 \mathrm{~m} \times 0.46 \mathrm{~m}$ and one $0.35 \mathrm{~m} \times 0.76 \mathrm{~m}$ sliding screened side windows 


\section{Cabinetry (formica covered).}

1. Overhead steel storage rack $1.83 \mathrm{~m}$ long $\times 0.3 \mathrm{~m}$ deep

2. Counter top $1.83 \mathrm{~m}$ long $\times 0.6 \mathrm{~m}$ deep, desk height with drawer and cabinets $\left(0.281 \mathrm{~m}^{3}\right)$, and motor generator compartment $\left(0.4 \mathrm{~m}^{3}\right)$ underneath

3. Floor to ceiling assorted cabinet units over each rear wheel well $\left(0.7 \mathrm{~m}^{3}\right.$ each)

4. Floor to ceiling cabinet $0.38 \mathrm{~m}$ deep $\times 0.61 \mathrm{~m}$ wide in rear $\left(0.4 \mathrm{~m}^{3}\right)$

\section{E. Miscellaneous.}

1. Swivel passenger-work seat

2. Steel frame for $135 \mathrm{~kg}$ radiation shield

3. Wall and floor mounts for roll out electronic equipment racks

4. Floor mounted rings for detector and storage dewar placement

5. Temperature and humidity gauge (inside)

6. Fore and aft wall mounted fire extinguishers (type A, B, C)

\subsubsection{OUTFITTING}

A photograph of the finished interior with some of the instrumentation carried is shown in Figure 6.7. Typical instrumentation and equipment carried on an extended field trip for environmental radiation measurements include the following.

\section{A. Instrumentation.}

1. Ge detector (17 L dewar) in shield

2. One roll out cabinet containing

a. Tracor-Northern TN-1750 4000 channel pulse height analyzer

b. Hewlett-Packard $9825 \mathrm{~B}$ computer

c. Computer interfaces

d. Disk drive for above

e. NIM power supply with $\mathrm{HV}$ and spectroscopy amplifier modules

3. Hand-held Ge detector

4. Canberra Series 10,8000 channel portable battery powered pulse height analyzer

5. Battery charger for above 
6. Portable cassette data recorder and tapes for Series 10 analyzer

7. Ionization chamber survey instrument (SPICER, Latner et al., 1983)

8. Electronic analytical balance $(0-30 \mathrm{~kg} \pm 1 \mathrm{~g})$

9. Tracor-Northern TN-1705 1000 channel pulse height analyzer

10. Portable cassette data recorder and tapes for above

11. Oscilloscope modules (adapts to TN-1705 analyzer)

12. Porta-Nim power supply

13. Multitester

14. Portable scintillation exposure ratemeter (KLERUS, Latner et al., 1985)

15. Miscellaneous cables and connectors

16. Instruction and service manuals

17. Calibration and radiation data tables

18. Check sources

19. Computer analysis software and data storage media (diskettes and tapes)

20. Magnetic shielded diskette and tape storage box

21. $120 \mathrm{~V} \mathrm{AC}$ power extension lines

\section{B. Miscellaneous equipment.}

1. 50 and/or $30 \mathrm{~L}$ liquid $\mathrm{N}_{2}$ storage tanks with transfer hoses

2. Soil sampling gear (corers, augers, templates, mallets, bags, pails)

3. Soil preparation gear (pans, spatula, mixing claw, Marinelli beakers)

4. Stakes, rope, tape measurer

5. Pressure meter (barometer)

6. Tool kit

7. Water jugs

8. Ice chest

9. Electric fan

10. Large plastic bags

11. $300 \mathrm{~W}$ portable back-up motor generator

12. Assorted stationery and office supplies

13. Maps

14. Data log books

15. Hot air gun

16. Vacuum cleaner

17. Freon solvent 
18. Binoculars

19. Camera, film

20. Wet suits, gloves

\section{Safety and emergency equipment.}

1. First aid kit

2. Snake bite kit

3. Fire rescue blanket

4. Road hazard markers

5. Tow line

6. Hydraulic jack

7. Spare tire

8. Flashlights

9. Battery charger

10. Booster cables

11. Air compressor

\section{REFERENCES}

Latner, N., K. M. Miller, S. Watnick and R. T. Graveson "SPICER: A Sensitive Radiation Survey Instrument" Health Phys., Vol. 44, 4, 379-386, April (1983)

Latner, N., S. Watnick, V. C. Negro and R. T. Graveson "KLERUS: An Energy Independent Gamma Survey Meter" USDOE Report EML-446, October (1985) 


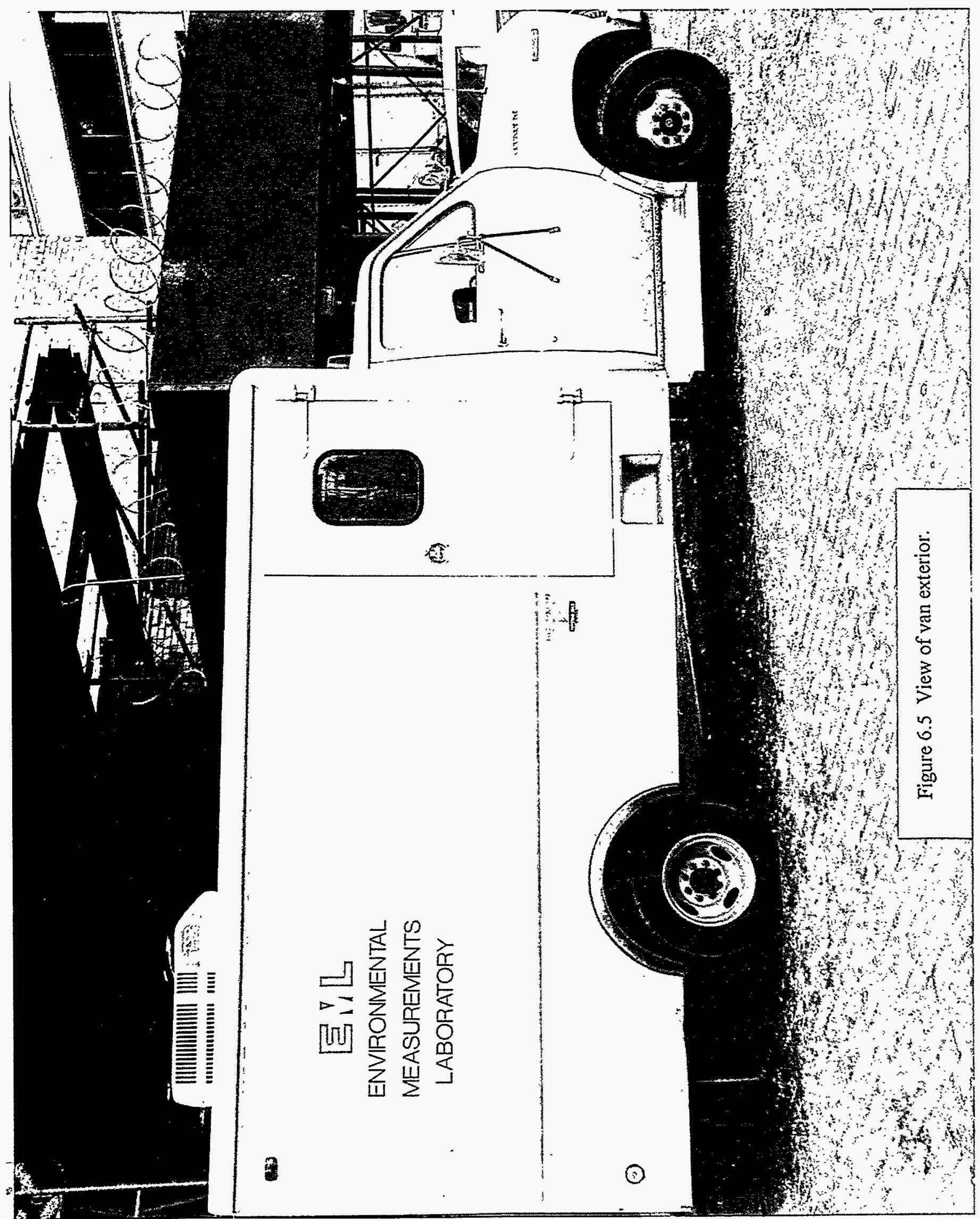




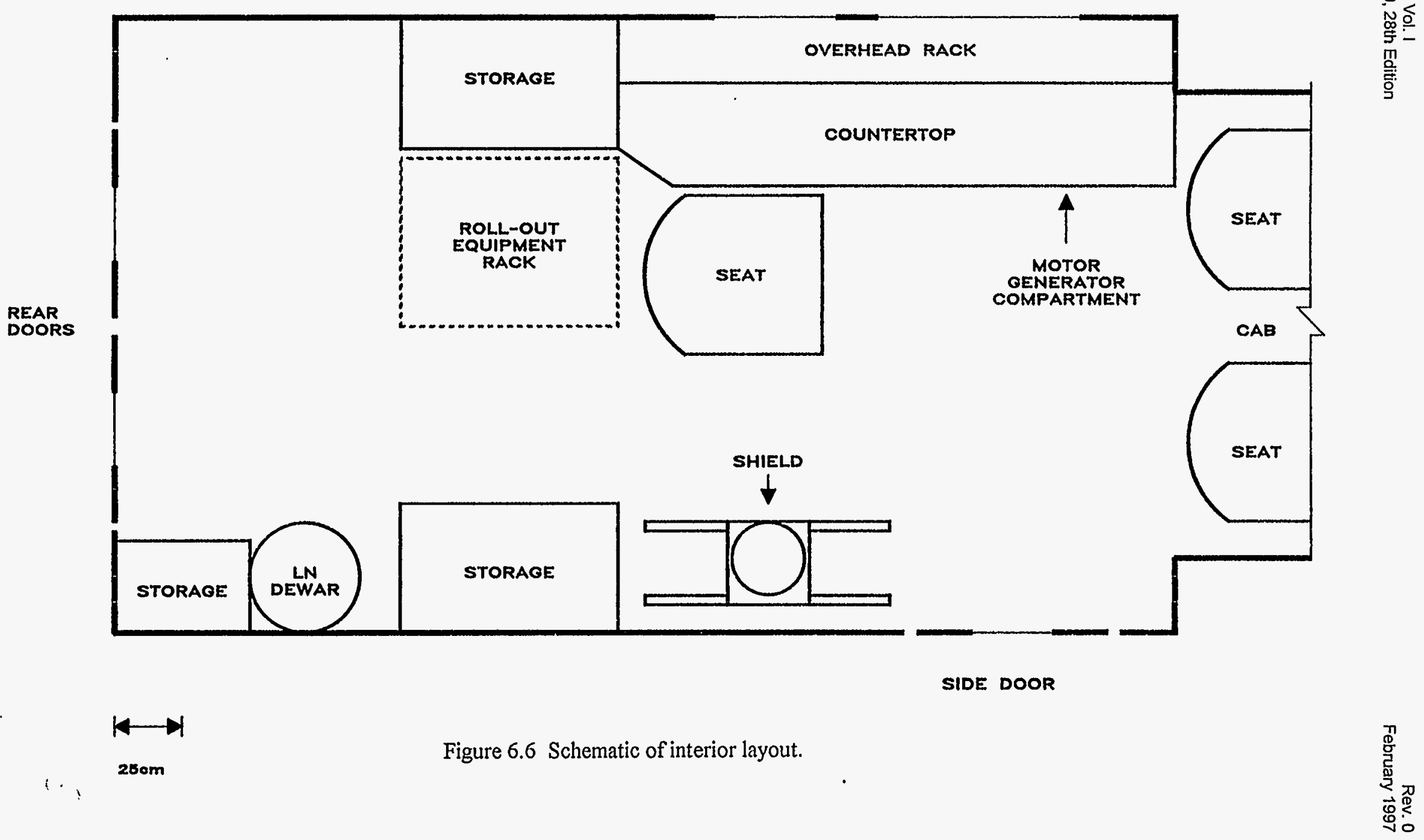




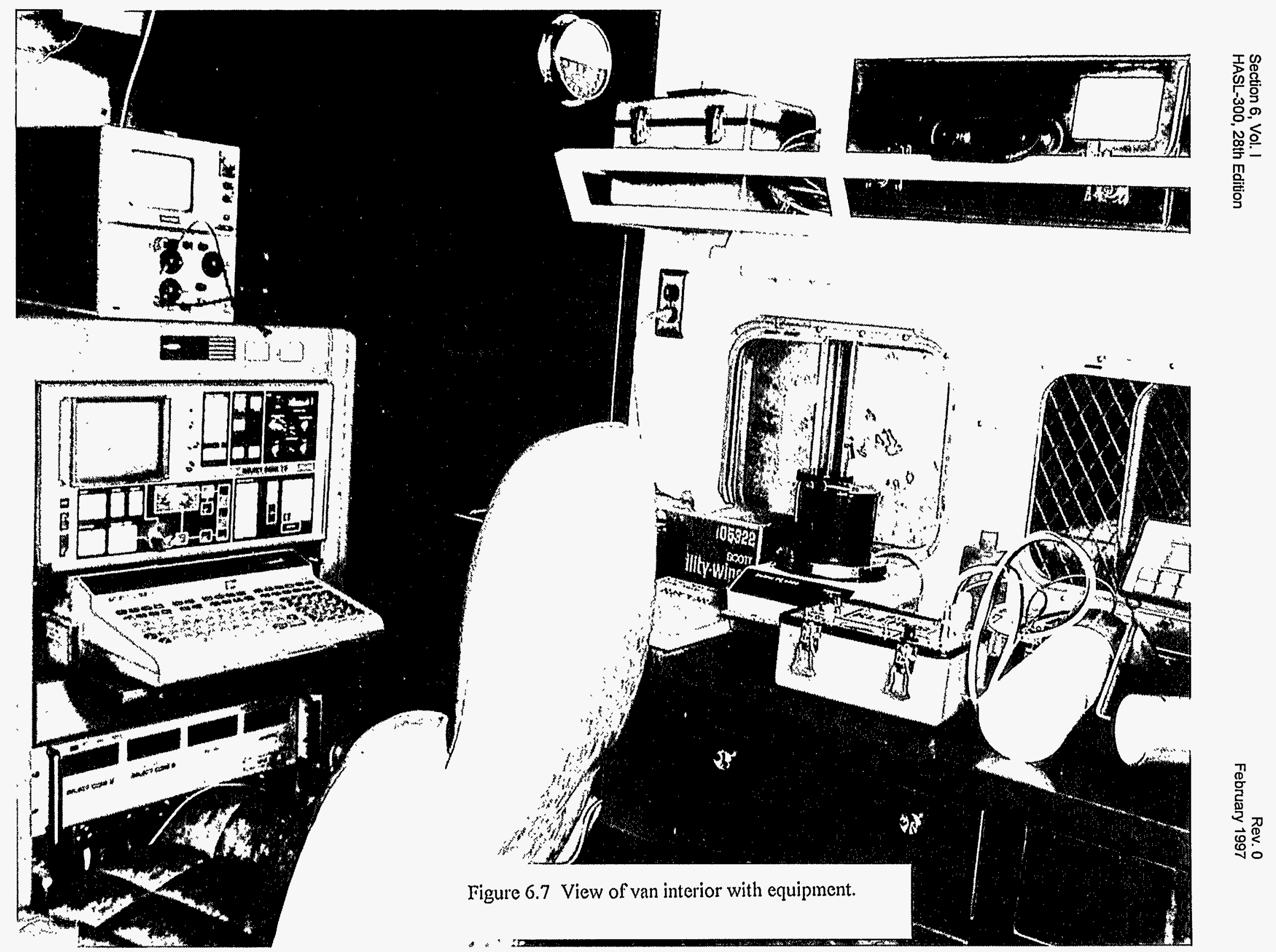




\title{
6.6 WORK BOAT AND TRIPOD CORER
}

\author{
Contact Person(s) : Mathew A. Monetti
}

\subsubsection{SCOPE}

The main focus of this section is to present an overall description of the EML research vessel, Sedimental Journey, including its associated trailer and large diameter sphincter corer. Sedimental Journey is used for taking sediment cores from inland lakes as a means of reconstructing the deposition history of energy-related pollutants in different regions of the U.S. (Heit et al., 1981). The sampling procedure itself is discussed in Section 2.5 of this Manual.

\subsubsection{RESEARCH VESSEL SEDIMENTAL JOURNEY}

\author{
6.6.2.1
}

DIMENSIONS AND CONFIGURATIONS

Sedimental Journey is an outboard-powered aluminum boat. It was specially designed and constructed by Winninghoff Boats, Inc. (Warehouse Lane, Rowley, MA 01969). The principal dimensions of the boat and some accessory items are listed in Table 6.1. A photograph of Sedimental Journey is shown in Figure 6.8.

The main feature that distinguishes this boat as a sediment coring research vessel is the well in the stern section of the hull. This craft has the maximum beam size $(2.6 \mathrm{~m})$ that can legally be trailered by someone with a standard driver's license. There is some cabin space in the bow that is primarily used for dry storage. The captain's chair and steering wheel are located on the forward starboard side. This midship area is protected by a permanently mounted windshield, as well as a collapsible canvas cover. There are storage brackets available for the disassembled coring device along each side of the boat. 
The winch motor and spool are bolted directly onto a plate on the hull. The boat has four mounts along the gunnels in the stern (two on each side) which are used to attach the quadrapod.

\subsubsection{2}

\section{POWER EQUTPMENT}

Sedimental Journey is powered by a pair of $30 \mathrm{~kW}$ ( 40 horsepower) Evinrude outboard engines. The controls for these motors are visible on the starboard deck in Figure 6.8.

A gasoline powered winch is installed on the starboard deck in front of the control console. Information about the winch is given in Table 6.1. The winch, together with the quadrapod mounted over the well, are used to lower the tripod corer to the lake bottom for sampling, and to retrieve it after sampling. The quadrapod is clearly visible as the tallest structure on the deck in Figure 6.8.

\subsubsection{3}

\section{DEPTH RECORDING GEAR}

Two devices are available for measuring depths. One is a mechanical system mounted on the cable sheave at the top of the quadrapod, which records the length of cable payed out. The second device on board is a recording fathometer (Low Range Model X-16). This unit is capable of measuring depths to $300 \mathrm{~m}$. The transducer is mounted on the rear underside of the hull. The display is placed on a bracket so that it is visible from the captain's chair. The boat has a Loran unit for locating geographical positions and determining bearing. This micrologic explorer model unit is mounted next to the fathometer display. The receiving antenna is positioned along the port side. For emergency communications, Sedimental Journey is also equipped with an ICOM VHF radio. The radio is mounted beside the Loran unit and has an antenna off the starboard side. 


\subsubsection{TRAILER AND TOWING VEHICLE}

The trailer used to transport the boat is a commercially available E-Z loader, with a maximum weight capacity of $2,000 \mathrm{~kg}$. The towing vehicle is a four-wheel drive Chevrolet $4 \times 4$ pickup truck with a $5.7 \mathrm{~L} \mathrm{V-8} \mathrm{engine} \mathrm{and} \mathrm{an} \mathrm{automatic} \mathrm{transmission} \mathrm{with}$ four-wheel driver transfer case. A Class III load-equalizing type hitch and antisway bars are used to stabilize the ride.

\subsubsection{TRIPOD CORER}

Figure 6.9 shows the assembly that is lowered through the well in the hull of Sedimental Journey, to the bottom of the lake or reservoir to be sampled. It consists of three main subassemblies: the coring tube and its attachments, the frame, and the weight stand.

The operation of the sampling assembly is described in Section 2.5.3.3, but a brief overview is useful here. When the tripod contacts the bottom, a catch is released and gravity drives the coring tube (marked "core barrel" in Figure 6.9) into the sediment. The depth of penetration depends on the nature of the sediment and on the number of lead weights placed in the weight basket. When the winch has started to raise the assembly (usually after $1 \mathrm{~min}$ of penetration), the initial tension on the cable acts to close the sphincter valves at both ends of the coring tube. This done, the cable pulls the coring tube out of the sediment back into the frame. Finally, continued pulling on the cable raises the entire assembly back to the deck of Sedimental Journey.

Sampling operations are most convenient with a crew of three or four. The skipper maneuvers the boat on the sampling station and operates the recorder, while one crew member operates the winch which has separate throttle, brake, and clutch controls and thus requires their full attention, and the remaining crew members handle the sampling gear. 
This subassembly consists of a $1 \mathrm{~m}$ or $1.5 \mathrm{~m}$ length of tubing, labeled "core barrel" in Figure 6.9, with fittings at both ends. The tube is made of fiberglass, although Lucite tubes can also be used. The outside diameter is $21.9 \mathrm{~cm}$ and the wall thickness is $3 \mathrm{~mm}$.

The fitting at the lower end is called the "core catcher" and consists of a stainless steel sleeve fitted over the coring tube. The sleeve provides the edge that cuts into the sediment, as well as the mechanism to close the bottom of the tube after coring.

The fitting at the upper end of the coring tube is also a stainless steel sleeve. It carries the valve mechanism used to close off the top end of the coring tube after the core is taken.

A further description of this subassembly can be found in Burke (1968) and in Burke et al. (1983).

6.6.4.2

FRAME

The frame consists of a top plate, a guide ring, and three legs, and is assembled from its components before launching Sedimental Journey. Assembly involves installing 15 bolts. Two of the legs fold so the corer can fit through the well in the hull. Once the corer is in the water, but before the corer is lowered to the sediment, the collapsed legs are manually swung out and locked with a spring-loaded pin.

The guide ring, together with the vertical support shown in Figure 6.9, serve to steer the coring tube vertically into the sediment during sampling. 
Up to 14 lead weights, each $13.6 \mathrm{~kg}$, can be loaded onto the tripod corer to provide the force needed to drive the coring tube into the sediment. These weights are specially shaped to fit into the bucket-like structure labeled "weight basket" in Figure 6.9.

6.6.4.4

CORE EXTRUDER

The extrusion sampling method is described in Section 2.5.4.4. The core extruder mechanism is simple and easy to use (see Figure 6.10). The basic components consist of the following. A flat aluminum plate $(50 \mathrm{~cm} \times 50 \mathrm{~cm}$ ) with two pins (one in the center and one off to the side) is used as the base. Two pieces of galvanized pipe $(5 \mathrm{~cm}$ in diameter) are used for support. One piece is $1.5 \mathrm{~m}$ long and is fitted over the centered base pin. The other piece ( $2 \mathrm{~m}$ ) is marked with a scale of holes drilled down the length of the pipe, and it fits over the side pin. The core with the piston in place on the bottom is positioned onto the center pipe. A clamp with a loop is placed over the large pipe and tightened around the core. As the core barrel is pulled down, sediment is forced out of the top. The scale on the large pipe is used to regulate the depth of each section removed from the core. Locking pliers can be used to mark the stop points on the scaled pipe so that sections are one or more centimeters thick.

\subsubsection{ASSEMBLY, LAUNCHING AND RETRIEVAL PROCEDURE}

The quadrapod and corer are assembled before the boat is launched. Sedimental Journey is launched by backing the trailer down a ramp and into the water. Once the boat contacts the water, the brakes are applied and the boat gently slips into the water. The trailer is then pulled out of the water and parked. The launching procedure takes about one-half hour to complete. Retrieval of Sedimental Journey is essentially a reversal of the launching, except that the boat is pulled onto the submerged trailer with a manual winch. The coring gear is then disassembled and stored before transporting the boat. 


\section{REFERENCES}

Burke, J. C.

"A Sediment Coring Device of 21-cm Diameter With Sphincter Core Retainer" Limnology and Oceanography, 13 , 714-718 (1968)

Burke, J. C., R. E. Hamblin and S. A. Casso

"Tripod Modification of Sphincter Corer: Construction, Operation, Core Extrusion and Sampling Efficiency"

Woods Hole Oceanographic Institution Technical Report WHOI-83-36, Woods Hole, MA 02543,1-13 (1983)

Heit, M., Y. L. Tan and C. S. Klusek

"Anthropogenic Trace Elements and Polycyclic Aromatic Hydrocarbon Levels in Sediment Cores From Two Lakes In The Adirondack Acid Lake Region"

Water, Air, and Soil Pollution, 15, 441-464 (1981) 


\section{TABLE 6.1}

\section{NUMERICAL DATA ON THE RESEARCH VESSEL SEDIMENTAL JOURNEY}

Principal Dimensions:

Hull

$\begin{array}{ll}\text { Length } & 9.0 \mathrm{~m} \\ \text { Weight } & 900 \mathrm{~kg} \\ \text { Material } & 3 / 16 " \\ \text { Draft } & 7.0 \mathrm{~cm} \\ \text { Beam size } & 2.6 \mathrm{~m} \\ \text { Gunnel height } & 70.0 \mathrm{~cm} \\ & \\ \text { Length } & 1.8 \mathrm{~m} \\ \text { Width } & 0.8 \mathrm{~m}\end{array}$

\section{Accessory Equipment:}

Propulsion

Outboard Motors (2)

Evinrude Outboards

$18.6 \mathrm{~kW}(40 \mathrm{Hp})$

Winch (modified Hydro Products Model HR-35, gasoline-engine powered,engine)

Cable diameter

Cable length

Rated load capacity

$4.8 \mathrm{~mm}$

$305 \mathrm{~m}$

Lifting speed

$726 \mathrm{~kg}$

$20 \mathrm{~m} \mathrm{~min}^{-1}$

Quadrapod (mounted on deck centered over well; used with a winch for raising/lowering

Diameter of legs samplers; disassembles for over the road travel)

Length of legs

$0.05 \mathrm{~m}$

Safe working load

$3.05 \mathrm{~m}$

Quadrapod (mounted on deck centered over well; used with a winch for raising/lowering samplers; disassembles for over the road travel)

Diameter of legs

Length of legs

$0.05 \mathrm{~m}$

Safe working load

Depth Recording Gear:

Fathometer (Low Range Model X-16)

Depth measuring range

Loran Unit: Micrologic explorer

VHF Radio: ICOM

Trailer: Weight $2000 \mathrm{~kg}$ 


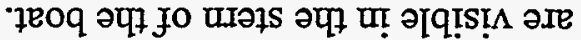

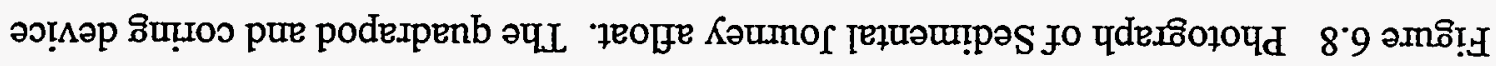

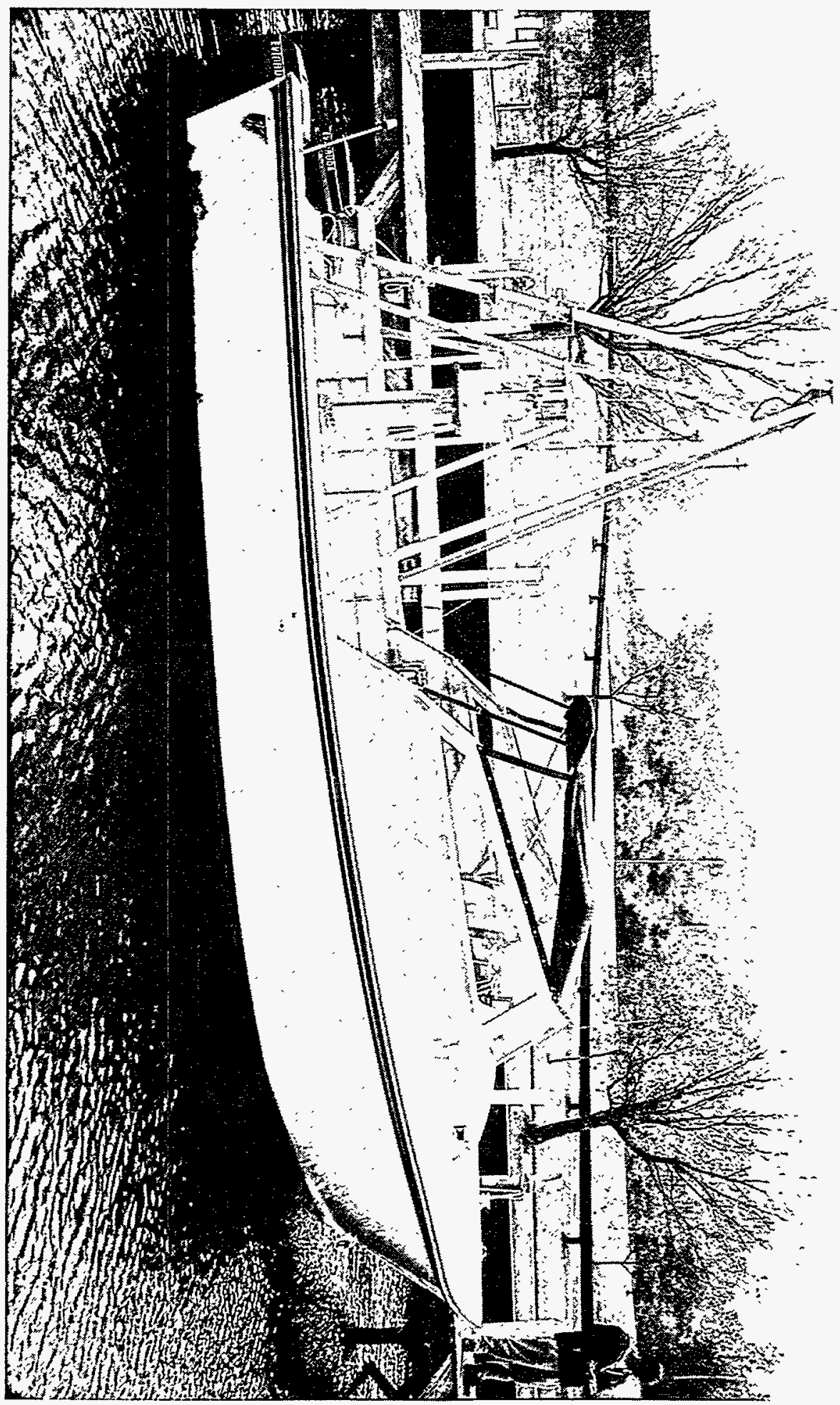




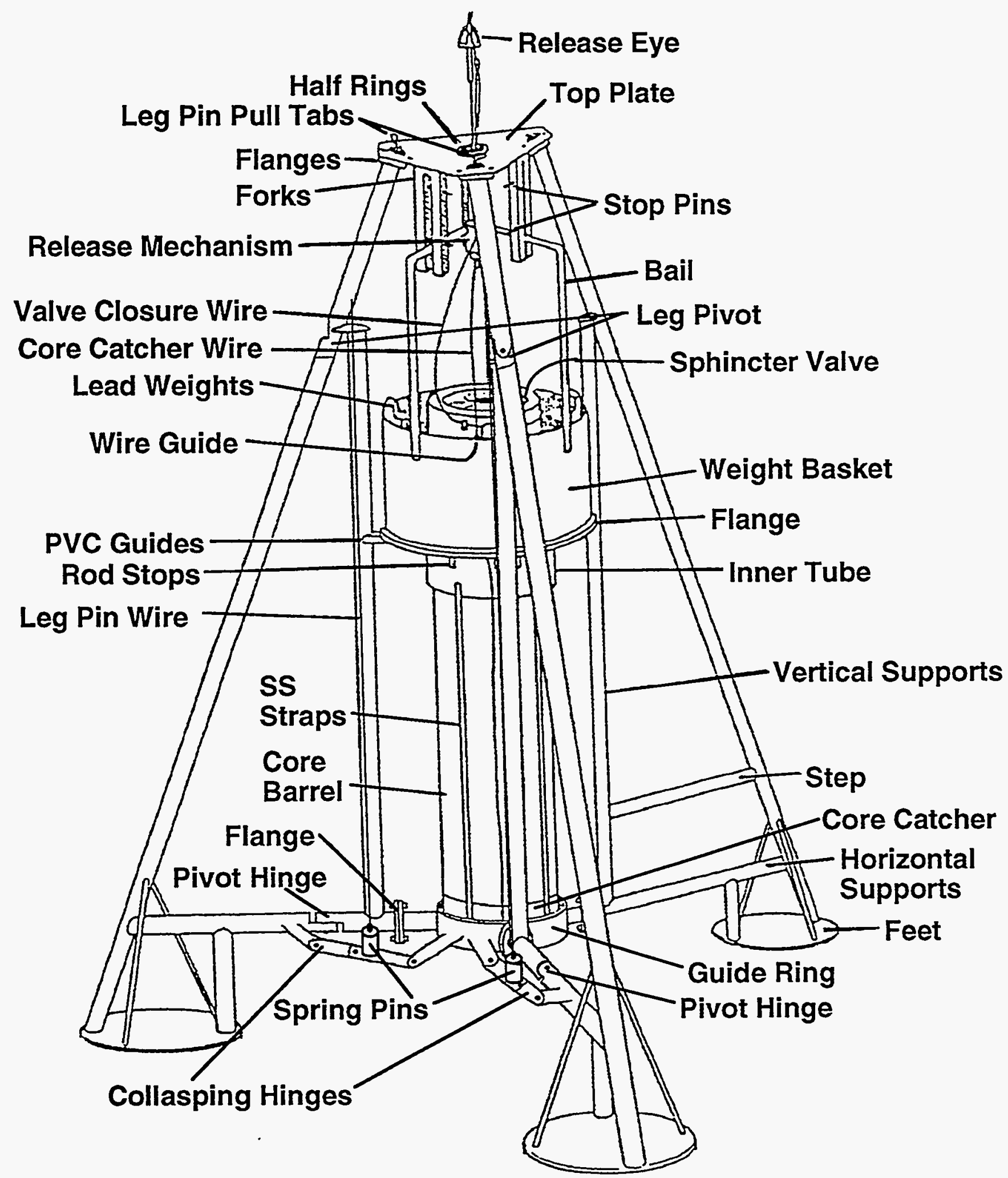

Figure 6.9 Detailed schematic of tripod corer. 


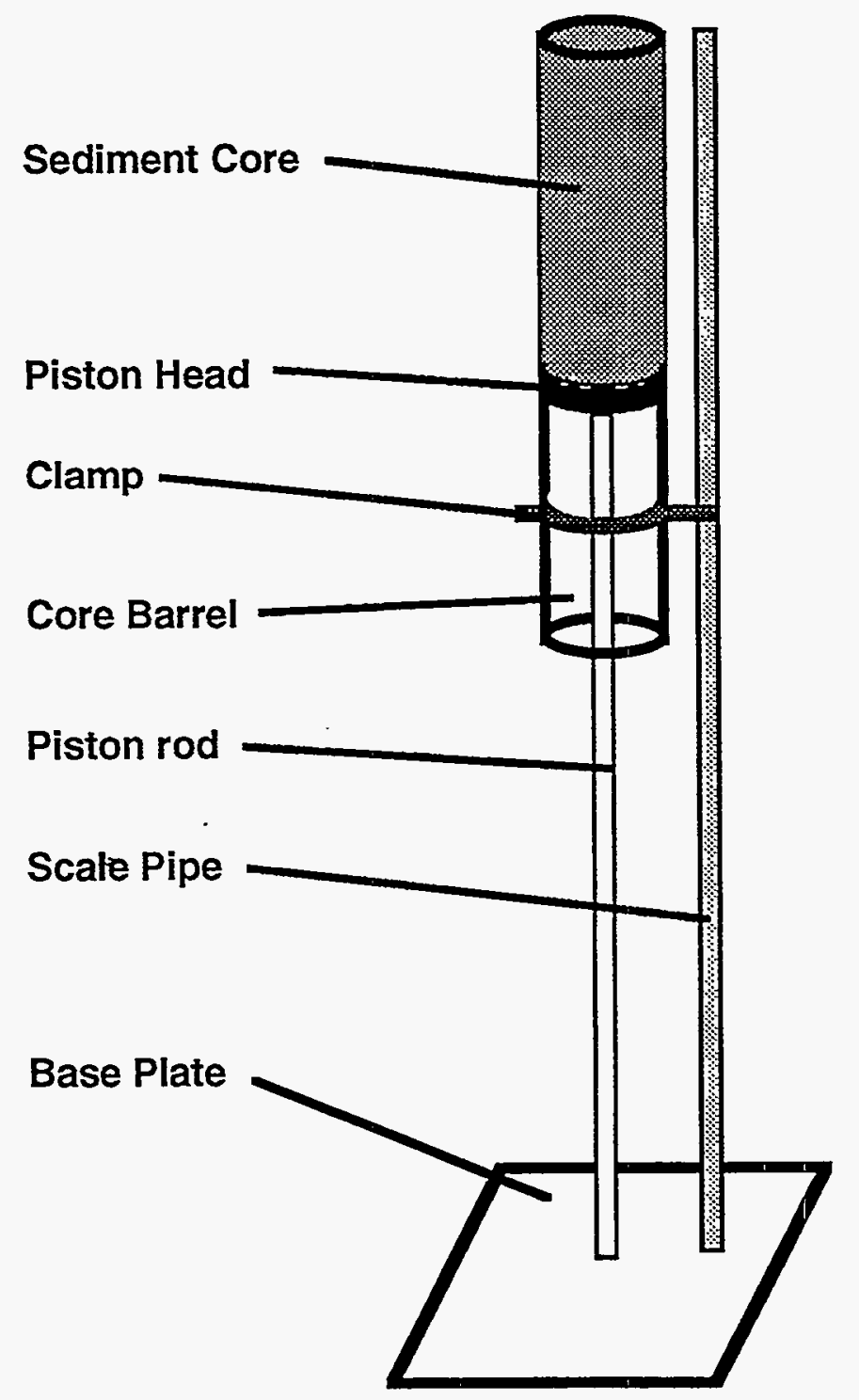

Figure 6.10 Diagram of extruder used to section sediment cores. 


\title{
6.7 CHESTER REGIONAL BASELINE STATION
}

\author{
Contact Person(s) : Brian Albert
}

\subsubsection{SCOPE}

The location, site characteristics, and permanent facilities of the field station operated by EML at Chester, NJ, are described here. The station is used by EML as a site for environmental research and for field testing of environmental instruments. Both shortand long-term research and testing programs are carried out, but our purpose here is to describe the site and the facilities - not the programs. Reports on the programs can be found in a series of USDOE Reports, and the latest publication in the series is USDOE Report EML-538.

\subsubsection{LOCATION}

The EML Regional Baseline Station, henceforth called EML/Chester, is located about $64 \mathrm{~km}$ west of the Laboratory, on the grounds of the Chester, NJ, unit of Bell Communications Research (BCR). Table 6.2 shows the latitude, longitude, and elevation of EML/Chester, and the distance to nearby areas. Table 6.3 gives the distance and direction from EML/Chester to major metropolitan areas. Similar information for coalburning electric generating stations is shown in Table 6.4.

\subsubsection{LOCAL TERRAIN, SOIL, AND VEGETATION}

Figure 6.11 is an aerial photograph showing EML/Chester and the surrounding area. The view is westward, across the station which is the fenced rectangular area in the center foreground. It is seen that the general environs consist of rolling hills with a mixture of fields and woods. 
The ground slopes at about $12 \%$ from the upper right to the lower left (NW to SE). The research station is located about $200 \mathrm{~m}$ south and about $10 \mathrm{~m}$ below the nearest high ground.

Figure 6.11 also shows that the research station is located at the southern edge of a cleared field, with a wooded area about $15 \mathrm{~m}$ to the south. There is also a wooded area about $75 \mathrm{~m}$ to the north-northeast. To the west, the direction of the prevailing winds, there is a fetch of about $150 \mathrm{~m}$ to the line of trees along Dover-Chester Road. The trees in all cases are deciduous and their average height is about $15 \mathrm{~m}$.

The cleared area around the station is grass-covered. BCR personnel keep the grass cut to a height not exceeding $20 \mathrm{~cm}$. The grass within the station fence is kept to about $10 \mathrm{~cm}$ by cutting with conventional power lawn mowers (also by BCR personnel).

Information of the radionuclide content of the soil at EML/Chester can be found in EML-347 (1978, p. 146).

\subsubsection{SITE CHARACTERISTICS AND PERMANENT FACULITIES}

EML/Chester is situated on a $15.2 \times 61 \mathrm{~m}$ area of ground, and is surrounded by a 2.4 $\mathrm{m}$ high chain-link fence. The fence provides additional security for the site. (The first line of security is provided by the fact that the site is on property owned by BCR.)

Figure 6.12 is a plan view of EML/Chester. A $2.4 \times 6 \mathrm{~m}$ enclosed trailer serves as a communications center and staging area for experiments at the research station. Electric service totaling $45 \mathrm{~kW}$ is brought into the trailer via underground cables; both 120 and $240 \mathrm{~V}$ are available. The trailer's interior is temperature-controlled to protect the most delicate equipment.

A computer link using commercial telephone lines is available between the EML/Chester site and the Laboratory in New York. This provides semicontinuous communication, particularly important after installation of new or critical experiments and to promptly alert the researchers to events of interest or problems. 
Six redwood platforms are provided for mounting long- and short-term outdoor experiments. Each platform has electric service and wiring for data communications. High volume aerosol samplers and an environmental $R n$ monitor are examples of the experiments mounted on these platforms (see Section 6.8.5).

The southwest end of the enclave is set aside for measurements of environmental radiation. High pressure pulse ionization chambers for measuring the $\gamma$ field are an example of the equipment installed here. A telephone pole $(11.5 \mathrm{~m})$ is provided for use in measuring vertical gradients.

At the northeast end of the enclave there is a $10 \mathrm{~m}$ meteorological tower, with instruments mounted at the $1 \mathrm{~m}$ and $10 \mathrm{~m}$ levels. The meteorological instruments, which comprise the bulk of the permanent instrumentation at the site, are described in the next section.

\subsubsection{METEOROLOGICAL DATA INSTRUMENTATION}

The instrumentation presently being used at the site, see Table 6.5, include rain gauges, a solar pyranometer, a wind vector transmitter, a temperature and dew point sensor, and a barometric pressure sensor. The wind instrument is located atop a $10 \mathrm{~m}$ tower and provides the $X$ and $Y$ components of the wind vector. In an area free from shadows is a solar pyranometer which measures the total solar radiation reaching the earth's surface. Precipitation amounts are also continuously monitored using two heated tipping bucket rain gauges and one weighing bucket rain gauge. An aspirated temperature and dew point sensor has been installed at a height of $1 \mathrm{~m}$. Signals for all sensors are recorded on magnetic cassette tape using a data acquisition system designed by EMIL. The data acquisition system is operated in a 6 min integration cycle, thus providing 10 average values per hour.

\subsubsection{ACKNOWLEDGEMENTS}

We acknowledge the support and cooperation of the Bell Communications Research management and personnel in all aspects of operation of the Regional Baseline Station. 


\section{REFERENCES}

"EML Regional Baseline Station at Chester, NJ"

USDOE Report EML-347, October (1978)

"EML Regional Baseline Station at Chester, NJ"

USDOE Report EML-538, December (1991)

Groff, J. A. and Gratch, S.

Trans. Amer. Soc. Heat. Vent. Eng., 52, 95 (1946) 


\section{LATITUDE, LONGITUDE, ELEVATION, AND IMMEDIATE} SURROUNDINGS OF EML/CHESTER

Latitude $40^{\circ} 47^{\prime} \mathrm{N}$

Longitude $74^{\circ} 40^{\prime} \mathrm{W}$

Elevation $.268 \mathrm{~m} \mathrm{MSL}$

Distance and direction to--

BCR Admin building $\ldots \ldots \ldots \ldots \ldots \ldots \ldots \ldots 244 \mathrm{~m} \mathrm{SSW}$

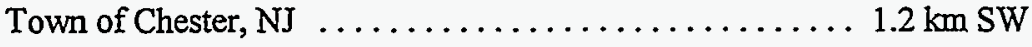

Dover - Chester Road ................... $150 \mathrm{~m}$ NW

TABLE 6.3

DISTANCE AND DIRECTION TO MAJOR METROPOLITAN AREAS FROM EMI/CHESTER

Manhattan, NY . $64 \mathrm{~km} \mathrm{E}$

Newark, NJ $44 \mathrm{~km}$ ESE

Trenton, NJ $66 \mathrm{~km} \mathrm{~S}$

Philadelphia, PA $.94 \mathrm{~km} \mathrm{SSW}$

Allentown and

Bethlehem, PA .....................6 $66 \mathrm{~km} \mathrm{WSW}$

Scranton, PA $112 \mathrm{~km} \mathrm{NW}$ 
TABLE 6.4

DISTANCE AND DIRECTION TO MAJOR COAL-FIRED POWER PLANTSFROM EML/CHESTER

Plant Name Distance and Direction

\begin{tabular}{|c|c|}
\hline New Jersey: & \\
\hline Hudson . & $51 \mathrm{~km}$ ESE \\
\hline Mercer .... & $\ldots \ldots 63 \mathrm{~km} \mathrm{~s}$ \\
\hline Vineland ... & $\ldots \ldots 151 \mathrm{~km} \mathrm{~S}$ \\
\hline Pennsylvania: & \\
\hline Eddystone .... & . . $117 \mathrm{~km} \mathrm{SSW}$ \\
\hline Hatfield & $80 \mathrm{~km} \mathrm{SW}$ \\
\hline Keystone..... & . $211 \mathrm{~km} \mathrm{WSW}$ \\
\hline Martin's Creek & $\ldots .43 \mathrm{~km} \mathrm{~W}$ \\
\hline Sunbury ..... & $\ldots 181 \mathrm{~km} \mathrm{~W}$ \\
\hline Hunlock Creek & . $129 \mathrm{~km}$ WNW \\
\hline Montaur & $201 \mathrm{~km}$ WNW \\
\hline Portland & $\ldots 38 \mathrm{~km} \mathrm{NW}$ \\
\hline
\end{tabular}


TABLE 6.5

\section{METEOROLOGICAL INSTRUMENTS}

\section{Precipitation}

a. Belfort Instrument Company: Model 5-405-1 - heated tipping bucket rain gauge (two).

b. Belfort Instrument Company: Model 5-780 - universal recording rain gauge.

c. Environmental Measurements Laboratory - trace precipitation sensor.

\section{Solar Radiation}

Eppley Laboratory Inc.: Eppley black and white pyranometer, Model 8-48 (horizontal surface receiver $\left.-180^{\circ}\right)$.

\section{Temperature and Relative Humidity}

Model 8151-A - aspirated temperature and humidity shield, with Model 4480-A temperature sensor and Model 5120-D Humidity - Qualimetrics, Inc.

Temperature, Dew Point Temperature, and Relative Humidity

Climatronics Corp.: A Model TS-10WA aspirated temperature and dew point shield, with Model 100093 temperature sensor and Model 101197 lithium chloride dew point sensor.

The relative humidity is derived from the measurements of the temperature and dew point temperature and the use of the Groff-Gratch formulation for the saturation vapor pressure in the pure phase over plane surfaces of pure water (Groff and Gratch, 1946).

\section{Wind Speed and Wind Direction}

Vertical wind sensor Model F460 - Climatronics Corp.

Low Threshold Wind Vane Model 2005 and Low Threshold Anemometer Model 2010 - Qualimetrics, Inc.

\section{Barometric Pressure}

Analog output barometer, Model 7105-A - Qualimetrics, Inc. 




Environmental Measurements Laboratory 


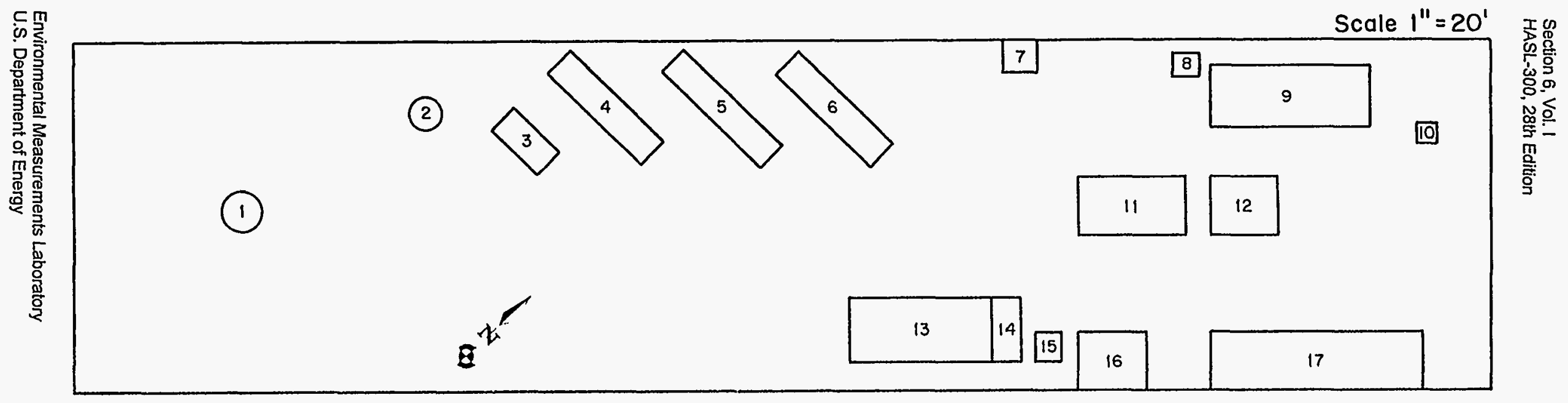

1. HPIC's, TLD's

2. Nominal $11.5 \mathrm{~m}$ utility pole

3. Bureau of Mines experiments

4. Bureau of Mines experiments

5. National Parks Service experiments

6. Environmental Protection Agency experiments

7. 45 KVA XMFR (200 amp. service)

8. Radiometer
9. Short Term Experiments

Particle size samplers

Particle efficiency samplers

10. $10 \mathrm{~m}$ Tower

$10 \mathrm{~m}$ Anemometer

Im Temp.-D/P Temp.

11. Deposition Collectors Wet/Dry Collectors

Total Fallout Collectors

Trace Precipitation Sensor
12. Precipitation Collectors Rain Gauges

13. Instrument Trailer Power Distribution Center Data Acquisition Systems

14. Utility Platform

15. Storage Shelter

16. EMI 2 Filter Radon Monitor

17. Surface Air Samplers

$\stackrel{9}{i}$

¿́

Figure 6.12 Plan view of Regional Baseline Station. 


\section{SPECIFICATIONS}

U.S. Department of Energy 201 Varick Street, 5th Floor New York, NY 10014-4811 


\section{Specifications}

7.1 Overview

7.2 Ring and Disc Mounts $7.2-1$

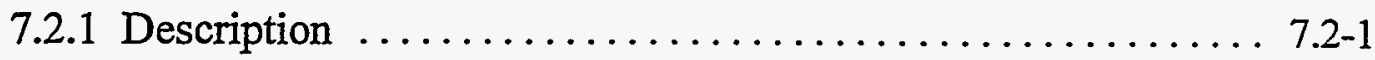

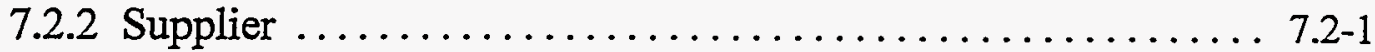

7.2 .3 Costs. ............................. 2 . 2

7.3 Mylar Film $\ldots \ldots \ldots \ldots \ldots \ldots \ldots \ldots \ldots \ldots \ldots \ldots \ldots \ldots \ldots \ldots$

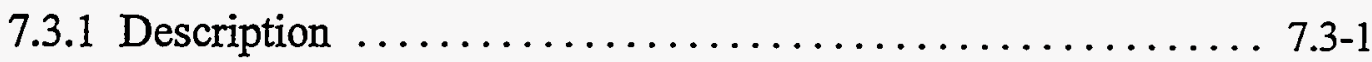



7.4 Ion Exchange Resins ........................... 7.4

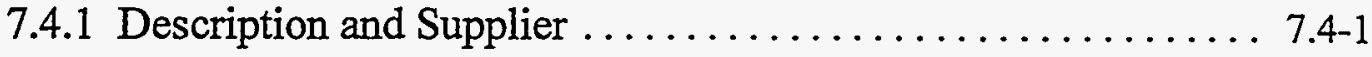

7.5 Large Ion-exchange Columns .................. 7.5-1



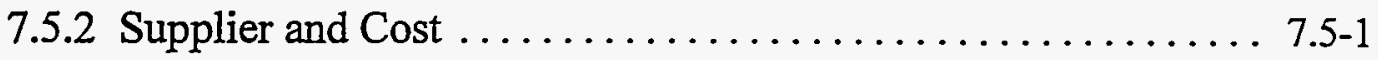

7.6 Small Ion-exchange Columns ................. $7.6-1$



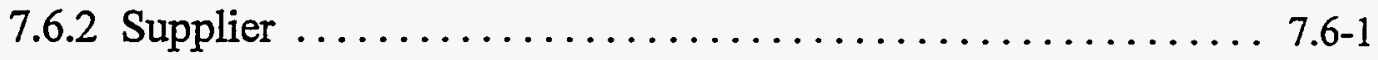

7.7 Radon Bubblers - Pyrex ................. $7.7-1$

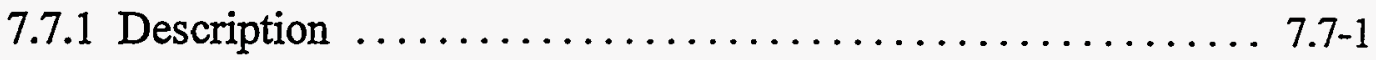

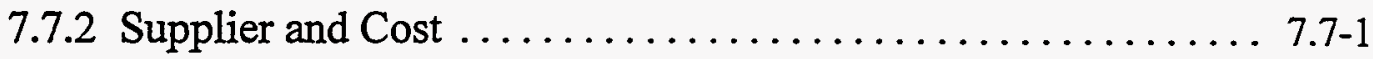

7.8 Glass Fiber Filter Paper $\ldots \ldots \ldots \ldots \ldots \ldots \ldots \ldots \ldots \ldots \ldots$

7.8.1 Description ....................... 
7.9 Beta Phosphors

7.9.1 Description

7.9.2 Supplier and Cost $7.9-1$

7.10 Alpha Phosphor on Mylar $7.10-1$



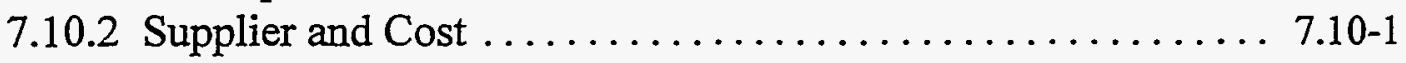

7.11 Polyethylene Dispensing Bottles

7.11.1 Description ............................... 7.11-1

7.11.2 Supplier and Cost $\ldots \ldots \ldots \ldots \ldots \ldots \ldots \ldots \ldots \ldots \ldots \ldots \ldots \ldots \ldots \ldots \ldots .11-3$

7.12 Filter Funnels and Sample Mounts

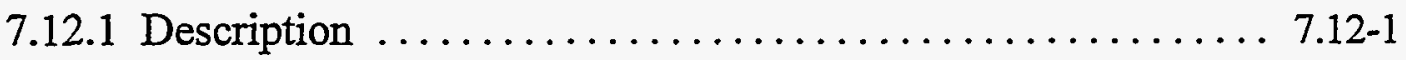

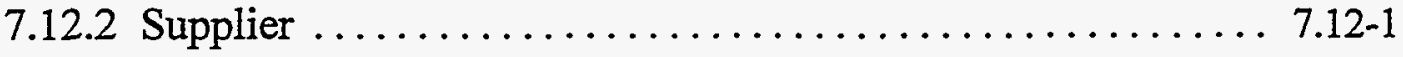

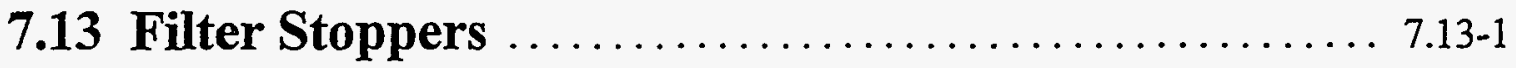



7.13 .2 Supplier $\ldots \ldots \ldots \ldots \ldots \ldots \ldots \ldots \ldots \ldots \ldots \ldots \ldots \ldots \ldots \ldots \ldots \ldots \ldots \ldots .13-1$

7.14 Ion-exchange Fallout Collectors ................ 7.14-1





7.15 Electrolysis Electrode $\ldots \ldots \ldots \ldots \ldots \ldots \ldots \ldots \ldots \ldots \ldots . .15-1$

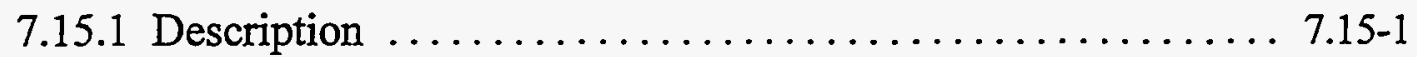




7.16 Plating Cells .................................. 7.16-1

7.16.1 Description $\ldots \ldots \ldots \ldots \ldots \ldots \ldots \ldots \ldots \ldots \ldots \ldots \ldots \ldots .1 .16-1$

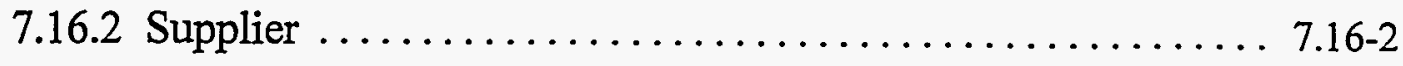

7.17 Air Sampling Filters ........................ 7.17 .1

7.17.1 Dynaweb DW7301L Filters ................... 7.17-1

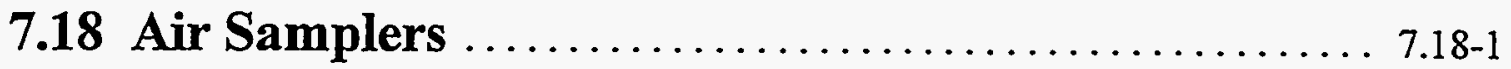

7.18.1 Roots System $\ldots \ldots \ldots \ldots \ldots \ldots \ldots \ldots \ldots \ldots \ldots \ldots \ldots \ldots \ldots \ldots \ldots \ldots \ldots .18 .1$

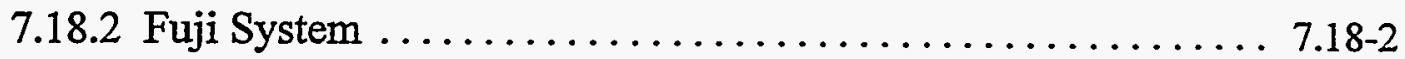

7.19 Gamma Planchets $\ldots \ldots \ldots \ldots \ldots \ldots \ldots \ldots \ldots \ldots \ldots \ldots .19-1$

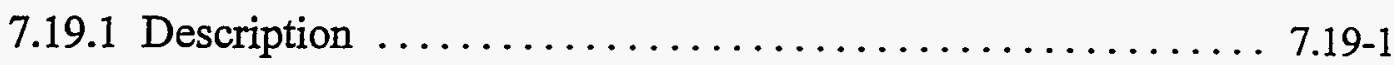

7.19.2 Supplier $\ldots \ldots \ldots \ldots \ldots \ldots \ldots \ldots \ldots \ldots \ldots \ldots \ldots \ldots \ldots \ldots \ldots .19 .1$

7.20 Electrolysis Cells $\ldots \ldots \ldots \ldots \ldots \ldots \ldots \ldots \ldots \ldots \ldots \ldots .20,1$



7.21 Aluminium Sample Cans for Gamma Counting .... 7.21-1



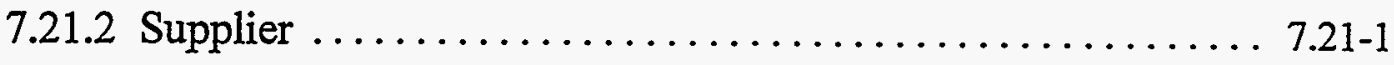

7.22 Molded Marinelli Beakers ................... 7.22-1

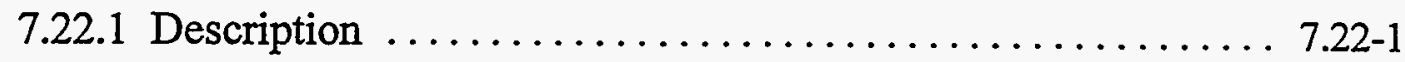



7.23 Radon Sampling and Counting Flasks ........... 7.23-1

7.23.1 Description $\ldots \ldots \ldots \ldots \ldots \ldots \ldots \ldots \ldots \ldots \ldots \ldots \ldots \ldots \ldots \ldots \ldots \ldots .23-1$

7.23 .2 Coating Flasks $\ldots \ldots \ldots \ldots \ldots \ldots \ldots \ldots \ldots \ldots \ldots \ldots \ldots \ldots \ldots .23-1$ 


\subsection{Containers for the Collection of Fallout and} Atmospheric Deposition ........................ 7.24-1

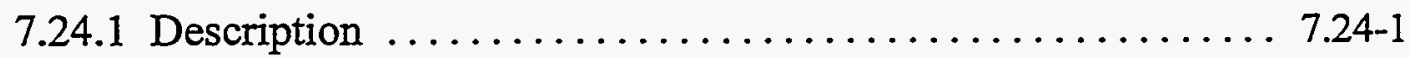

7.25 Fallout Collection and Shipping Container ......... 7.25-1

7.25.1 Description $\ldots \ldots \ldots \ldots \ldots \ldots \ldots \ldots \ldots \ldots \ldots \ldots \ldots \ldots \ldots \ldots \ldots \ldots \ldots \ldots \ldots \ldots \ldots .25-1$

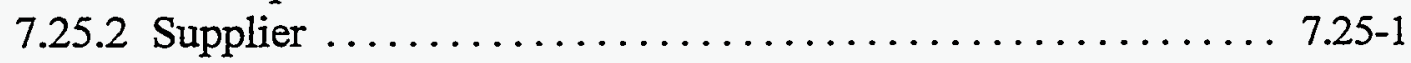

7.25 Disposable Chromatographic Column .......... 7.26-1

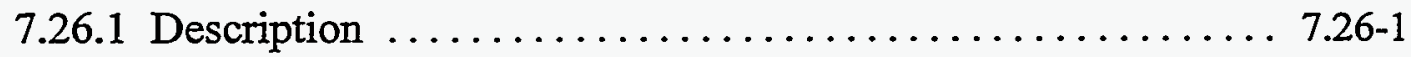

7.26 .1 Supplier $\ldots \ldots \ldots \ldots \ldots \ldots \ldots \ldots \ldots \ldots \ldots \ldots \ldots \ldots \ldots \ldots \ldots \ldots \ldots .26-1$ 


\section{SPECIFICATIONS}

\section{I OVERVIEW}

This section describes materials used in some of the procedures. These materials are unique in that they are not necessarily available from the usual scientific vendors. Consequently, an attempt was made to provide all pertinent information in order to enable the user of this Manual to obtain the items referred to.

Special attention is drawn to Section 7.2 since it provides information on several items from a single vendor. 



\subsection{RING AND DISC MOUNTS}

\subsubsection{DESCRIPTION}

Plastic rings and discs in nominal 1 in $(2.54 \mathrm{~cm})$ and 2 in $(5.08 \mathrm{~cm})$ diameters are molded of nylon. The 1-in units are made according to dimensions given in Figure 7.1, and the 2-in units follow the same form with an overall diameter of 2.03 in $(5.16 \mathrm{~cm})$.

The discs are molded as cups to allow use of the discs either for mounting solid samples using the ring or to evaporate liquid samples in the cup.

Ring

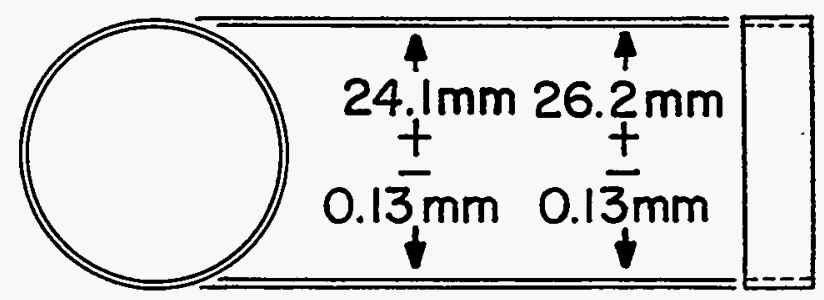

Figure 7.1. Plastic ring and disc mounts.
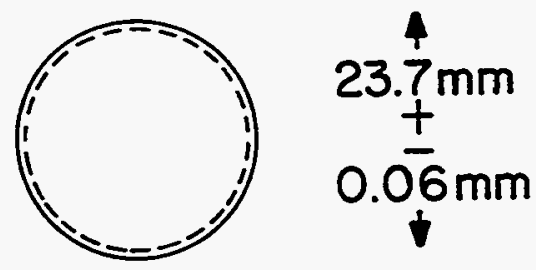

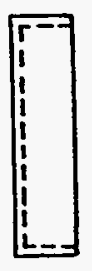

\subsubsection{SUPPLIER}

The present source of supply is:

Control Molding Corporation

Smith and Canal Streets

P.O. Box 70

Franklin, $\mathrm{NH} 03235$

(603) 934-6103

the price list, as of June 1995, is reproduced and included as Specification 7.2.3. 


\subsubsection{COSTS}

RINGS AND DISCS: Prices are per set (one set = one ring and disc) in lots of:

$\underline{500} \quad \underline{1000} \quad \underline{2500} \quad \underline{5000} \quad \underline{10000} \quad \underline{25000}$

1 in dia. plain*

.42

.34

.32

.22

2 in dia. plain**

.50

.40

.36

.26

1 in dia. funnel

1.20

1.13

.67

.55

.42

.37

disc and ring***

Minimum Order $\$ 350.00$

*EML Manual, Specification 7.2, Control Molding \#J-356 Color Natural

**EML Manual, Specification 7.2, Control Molding \#J-424 Color Natural

***EML Manual, Specification 7.12, Control Molding \#J-444 Color Black

FILTERS: Prices are cents per each in lots of:

$\begin{array}{llll} & \underline{100} & \underline{250} & \underline{1000} \\ \begin{array}{l}\text { Polyethylene } \\ \text { filter chimney* }\end{array} & 4.50 & 2.25 & 1.03 \\ \text { Geon filter stopper** } & 4.50 & 2.25 & 1.03\end{array}$

*EMIL Manual, Specification 7.12, Control Molding \#J-443

**EML Manual, Specification 7.13, Control Molding \#J-483 
PLATNNG CEL CAP: (20-mm thread to fit Polyethylene bottles, with brass bushing for mounting and electrical connection)
Dollars each in lot of:
$\underline{25}$
$\underline{50}$
$\underline{100}$
$\underline{500}$
18.50
11.10
6.65
3.46

EML Manual, Specification 7.16, Control Molding \#J-604

GAMMA PLANCHETS: Prices are per set in lots of:

$\begin{array}{lllllll} & \underline{100} & \underline{250} & \underline{500} & \underline{1000} & \underline{2500} & \underline{5000} \\ \begin{array}{l}2 \text { in dia. } x 1 \text { in deep } \\ \text { with .030 in disc }\end{array} & 5.00 & 3.00 & 1.50 & 1.15 & .91 & .68\end{array}$

Minimum Order $\$ 350.00$

EML Manual, Specification 7.19, Control Molding \#J-608

MARINELLI BEAKER AND COVER: Unit price in lots of:

$\begin{array}{lllllll} & \underline{100} & \underline{250} & \underline{500} & \underline{1000} & \underline{2500} & \underline{5000} \\ \text { Black Butyrate } & 4.07 & 3.19 & 2.88 & 2.73 & 2.65 & 2.61\end{array}$

Minimum Order $\$ 350.00$

EML Manual, Specification 7.22, Control Molding \#J-762. Other materials and colors quoted on request.

Prices are in U.S. dollars, F.O.B. Franklin, NH. For shipments outside the U.S. add 5\% to prices listed and $\$ 45.00$ for bank transferral charges. Unless otherwise specified, all items are bulk packed in poly bags. All orders are subject to $\$ 350.00$ minimum. Blanket orders welcome. Discounts available for volume buyers. 


\subsection{MYLAR* FILM}

\subsubsection{DESCRIPTION}

Mylar film is used to cover precipitates to protect them during counting and storage. The film is obtained in $38.1 \mathrm{~mm}$ width rolls. The most useful films are $.013 \mathrm{~mm}$ thick, weighing about $1.8 \mathrm{mg} \mathrm{cm}^{-2}$ and $.006 \mathrm{~mm}$ thick, weighing about $0.9 \mathrm{mg} \mathrm{cm}^{-2}$.

\subsubsection{SUPPLIER}

Retail quantities may be obtained from:

Spex Sample Preparation

203 Norcross Ave.

Metuchen, NJ 08840

1-800-LAB-SPEX

"Mylar is the registered trademark of E.I. du Pont de Nemours \& Co. for its polyester film. 


\title{
7.4 ION EXCHANGE RESINS
}

\subsubsection{DESCRIPTION AND SUPPLIER}

Analytical grade anion and cation exchange resins are required to concentrate trace amounts of radionuclides from environmental samples. They are available from:

\author{
Bio-Rad Laboratories \\ 2000 Alfred Nobel Drive \\ Hercules, CA 94547 \\ (510) $741-1000$ \\ FAX: (510) 741-1060
}

or

Eichrom Industries, Inc.

8205 S. Cass Ave., Suite 107

Darien, II 60561

(800) 422-6693

FAX: (708) 963-1928

and may be used as received. 


\subsection{LARGE ION-EXCHANGE COLUMNS}

\subsubsection{DESCRIPTION}

Fabricated from borosilicate glass tubing.

\subsubsection{SUPPLIER AND COST}

Ace Glass, Inc.

1430 Northwest Boulevard

P.O. Box 688

Vineland, NJ 08360

(609) 692-3333

Ace Glass, Inc., supplies large ion-exchange columns as specialty items. Costs will be quoted by the supplier. 


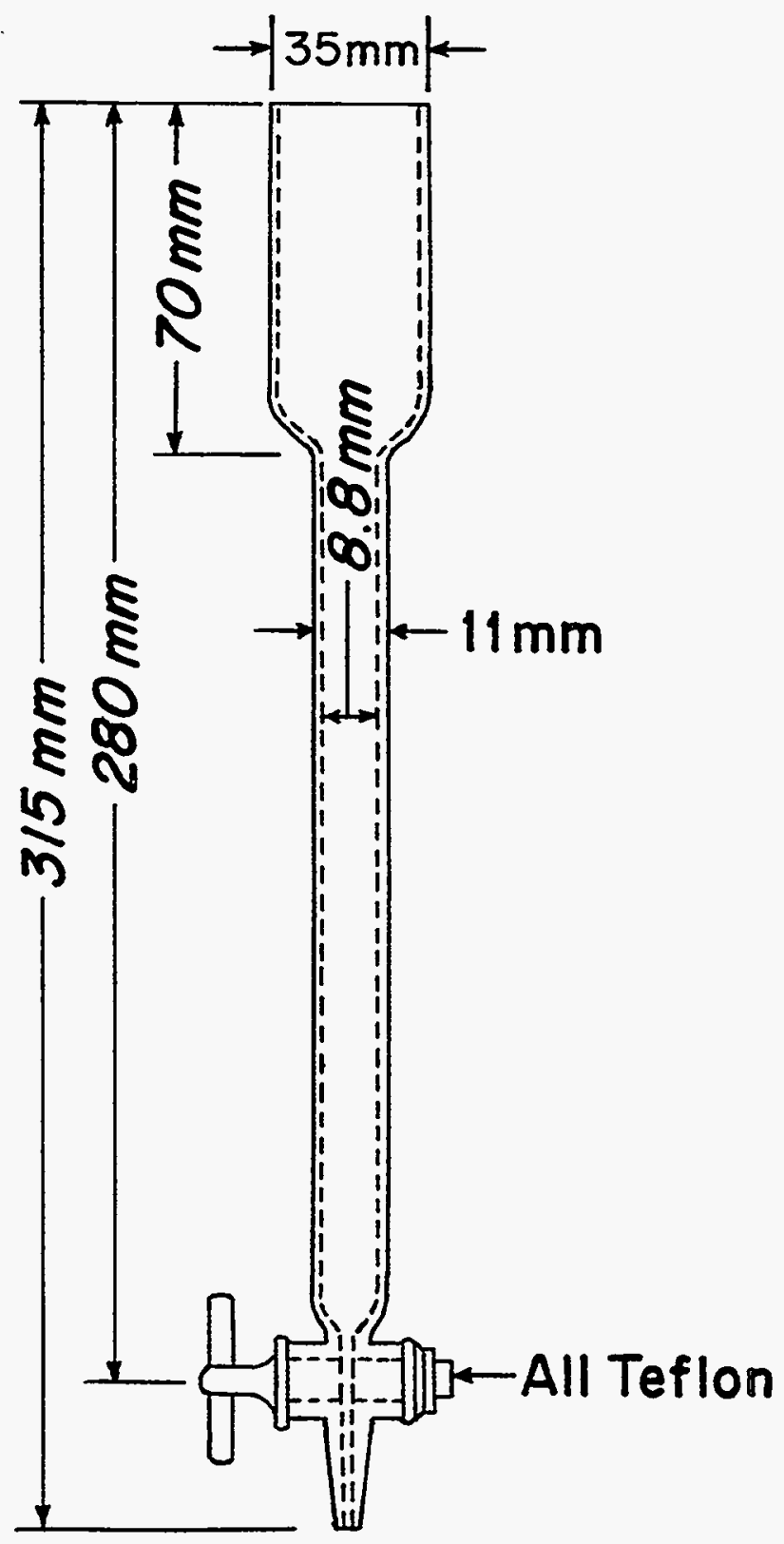




\subsection{SMALL ION-EXCHANGE COLUMNS}

\subsubsection{DESCRIPTION}

Fabricated from borosilicate glass tubing.

\subsubsection{SUPPLTER}

Ace Glass, Inc.

1430 Northwest Boulevard

P.O. Box 688

Vineland, NJ 08360

(609) 692-3333

Ace Glass, Inc., supplies small ion-exchange columns as a specialty item. Costs will be quoted by the supplier. 


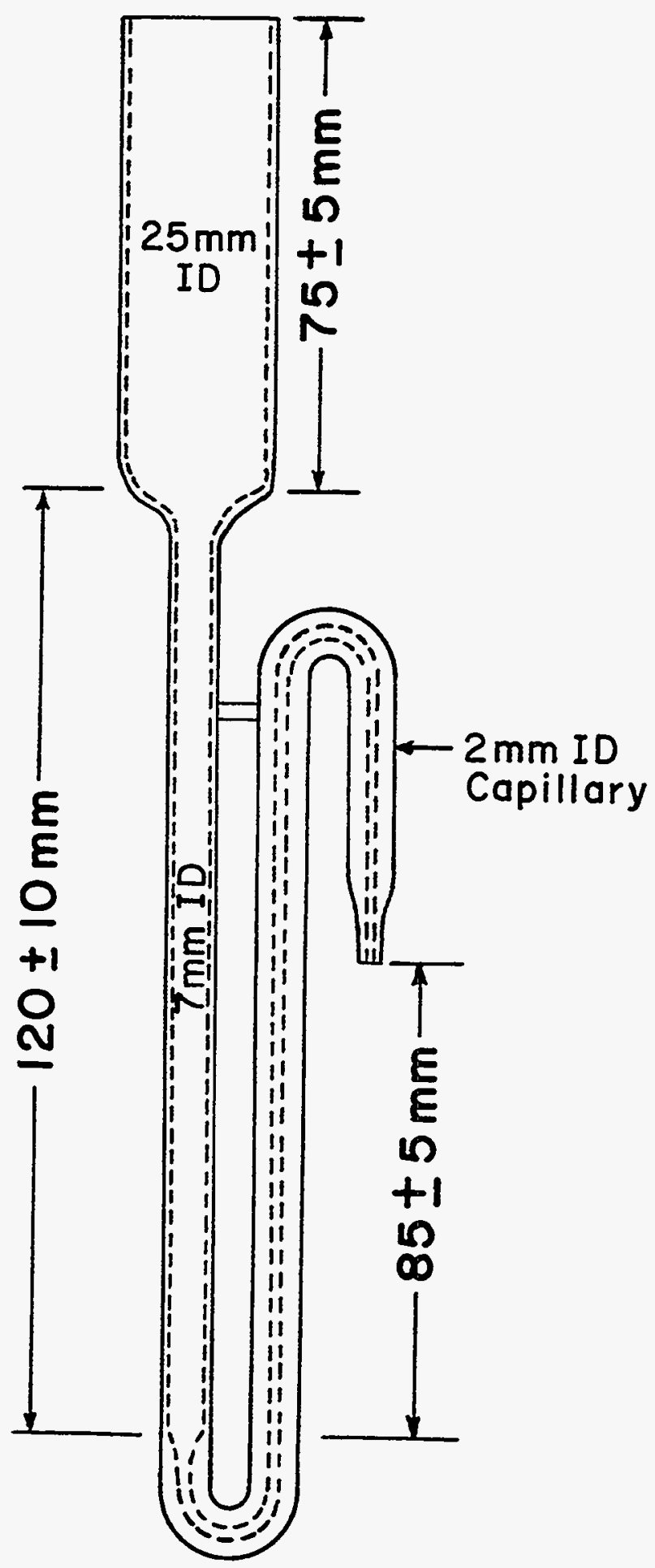

Figure 7.3 Small ion-exchange columns. 


\subsection{RADON BUBBLERS - PYREX}

\subsubsection{DESCRIPTION}

1. Main chamber about $150-\mathrm{mm}$ long, base to neck, with capacity about $50 \mathrm{~mL}$.

2. Top of chamber to have $19 / 38$ standard taper joint.

3. Stopcocks 2-mm bore with tapered Teflon plug - side arms $7 \mathrm{~mm}$ O.D.

4. All side tubing to be $7 \mathrm{~mm}$ (not as shown).

5. Glass rod structural support between main chamber and side tube, $15 \mathrm{~mm}$ in length, 4-mm diameter.

6. Medium porosity glass frit in bottom of chamber.

7. Glass tubing from stopcocks $25-\mathrm{mm}$ long.

\subsubsection{SUPPLIER AND COST}

Ace Glass, Inc.

1430 Northwest Boulevard

P.O. Box 688

Vineland, NJ 08360

(609) $692-3333$

Cost: $\sim \$ 69.00$ 


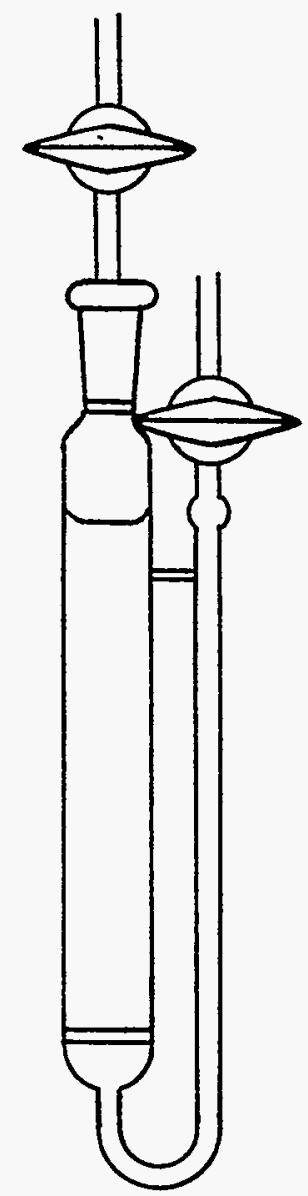

Figure 7.4 Radon bubbler. 


\subsection{GLASS FIBER FILTER PAPER ${ }^{*}$}

\subsubsection{DESCRIPTION}

Two equivalent materials are available. One is No. X-934-AH made by Hurlburt Paper Company, the other is Whatman glass fiber paper GF/C. These are available from laboratory supply houses. Gelman Instrument Company sells a "Spectro Grade Type A" filter with the following typical analyses.

$\mu \mathrm{g}$ per $203 \mathrm{~mm} \times 254 \mathrm{~mm}$

Sheet Less Than:

$\begin{array}{llr}\text { Antimony } & \mathrm{Sb} & 50 \\ \text { Arsenic } & \mathrm{As} & 50 \\ \text { Beryllium } & \mathrm{Be} & 10 \\ \text { Bismuth } & \mathrm{Bi} & 50 \\ \text { Cadmium } & \mathrm{Cd} & 15 \\ \text { Chromium } & \mathrm{Cr} & 50 \\ \text { Cobalt } & \mathrm{Co} & 15 \\ \text { Copper } & \mathrm{Cu} & 10 \\ \text { Iron } & \mathrm{Fe} & 400 \\ \text { Lead } & \mathrm{Pb} & 25 \\ \text { Manganese } & \mathrm{Mn} & 50 \\ \text { Mercury } & \mathrm{Hg} & 100 \\ \text { Molybdenum } & \mathrm{Mo} & 15 \\ \text { Nickel } & \mathrm{Ni} & 100 \\ \text { Selenium } & \mathrm{Se} & 2000 \\ \text { Tin } & \mathrm{Sn}^{-} & 50 \\ \text { Titanium } & \mathrm{Ti} & 15 \\ \text { Vanadium } & \mathrm{V} & 15 \\ \text { Zinc } & \mathrm{Zn}^{-} & 60 \\ & & \\ \text { Water Extractable } & & 150 \\ \text { Ions } & \mathrm{SO}_{4}^{-} & 10 \\ & \mathrm{NO}_{3}^{-} & 10 \\ & \mathrm{NH}_{4}^{+} & 120 \\ & \mathrm{~F}^{-} & 1650\end{array}$

Most glass fiber filters contain traces of alpha and beta activity. Any procedure where the final sample is mounted on a glass filter for counting should be carefully checked for possible interference. 


\subsection{BETA PHOSPHORS}

\subsubsection{DESCRIPTION}

This technique has been described by Harley et al. (1962). Phosphor discs $.2540-\mathrm{mm}$ thick and $23.8 \mathrm{~mm}$ in diameter and other sizes are available in lots of 1000 , as NE-102 clear to as NE-102A, at about $\$ 1.50$ each.

\subsubsection{SUPPLIER AND COST}

Bicron Corp.

12345 Kinsman Rd.

Newbury, $\mathrm{OH} 44065$

(216) 564-2251

Approximate cost: $\$ 1,600 / 1000$ sq. in.

\section{REFERENCE}

Harley, J. H., N. A. Hallden and I. M. Fisenne

"Beta Scintillation Counting with Thin Plastic Phosphors"

Nucleonics, Vol. 20, 1, 59-61, January (1962) 


\subsection{ALPHA PHOSPHOR ON MYLAR}

\subsubsection{DESCRIPTION}

The phosphor is the standard silver-activated zinc sulfide (Sylvania Type 130 [7] or DuPont 1101 phosphor) coated on one side of a Mylar film. The material is sprayed onto the backing using the zinc sulfide as a pigment in a paint vehicle.

A fresh die-cut phosphor disc, $23.8 \mathrm{~mm}$ in diameter, is used for each sample. The sample filter paper is laid on a nylon disc, the phosphor is placed against the sample, a strip of .0076-mm Mylar is overlaid, and the assembly is locked together with a nylon ring.

Counting is performed with a bare phototube on either manual or automatic counters.

\subsubsection{SUPPLIER AND COSTS}

The phosphor is available in strips or discs from:

William B. Johnson \& Associates, Inc.

P.O. Box 472

Ronceverte, WV 24970

(304) 645-6568

FAX: (304) 645-2182

The zinc sulfide is sprayed on $.076-\mathrm{mm}$ Mylar. The catalog numbers are:

\section{Price}

ASP-3, $305 \mathrm{~mm}$ wide strip

ASP-4, $23.8 \mathrm{~mm}$ discs

ASP-5, $49.2 \mathrm{~mm}$ discs

ASP-6, $50.8 \mathrm{~mm}$ dișcs
$\$ 27 /$ sheet

$\$ 32 / \mathrm{C}$

$\$ 61 / \mathrm{C}$

$\$ 61 / \mathrm{C}$ 


\subsection{POLYETHYLENE DISPENSING BOTTLES}

\subsubsection{DESCRIPTION}

Commercially available disposable plastic transfer pipettes (DPTP), made from low density polyethylene (LDPE) are used for weighing and dispensing small aliquots of radionuclide solutions.

The DPTPs may be purchased in capacities of 1 to $10 \mathrm{~mL}$. All are pre-drawn with capillary tips of various diameters. The DPTP may be completely or partially filled by squeezing the pipette to expel the air, inserting the tip under the surface of the solution and releasing the vacuum in the pipette. The pipette tip is wiped with a disposable tissue. Single drops or a larger volume may be dispensed by squeezing the pipette with the tip down. Depending on the size of the capillary tip, individual drops may range from 0.10 to $0.05 \mathrm{~g}$.

A radioactive solution may be sealed in the DPTP by heating the tip of the capillary and squeezing it with forceps. The tip may then be cut below the seal when it is desired to deliver another portion of the solution.

Similar polyethylene dispensing bottles ( $5 \mathrm{~cm}^{3}$ ampules) were tested for loss of liquid over a storage period up to 6 months. The loss from $5.5 \mathrm{~g}$ of distilled water was $0.042 \mathrm{~g}$. The loss from $4.0 \mathrm{~g}$ of ethyl alcohol was $0.136 \mathrm{~g}$ and the loss from $6.7 \mathrm{~g}$ of concentrated $\mathrm{HCl}$ was $0.188 \mathrm{~g}$. Intermediate measurements showed a roughly linear rate of weight loss.

In general, it appears that the losses from the sealed dispensing bottles are small for short-time periods, but that they must be taken into consideration if long-time storage is required.

The stability of nuclide solutions in polyethylene bottles and possible losses through absorption on the walls have been questioned many times. A series of tests were performed to determine if appreciable losses occurred in the normal use of these bottles for solution storage and handling. The data obtained should be considered as a guide and appropriate tests carried out as needed. 
Six isotopes $\left({ }^{137} \mathrm{Cs},{ }^{144} \mathrm{Ce},{ }^{95} \mathrm{Zr},{ }^{131} \mathrm{I},{ }^{106} \mathrm{Ru},{ }^{140} \mathrm{Ba}\right)$ were obtained from Oak Ridge National Laboratory, split, diluted, and stored in sealed polyethylene dispensing bottles for a period of from 10-63 days.

In each case, duplicate samples were prepared in water, carrier $\left(1 \mathrm{mg} \mathrm{mL}^{-1}\right)$ or $\mathrm{HCl}$ (1N) solution, except in the case of ${ }^{131} \mathrm{I}$ where $\mathrm{Na}_{2} \mathrm{SO}_{3}\left(\mathrm{pH}\right.$ at 8 ) was used, and ${ }^{95} \mathrm{Zr}$ where oxalic acid was used.

The samples were gamma counted in a $\mathrm{NaI}$ well-crystal so that a counting error of at least $1 \%$ was maintained. After the initial count, the drawn stems were cut off, the bottles rinsed four times with warm water, and then recounted. In the case of residual activity,


shown in Table 7.1. Ruthenium adsorbed appreciably on polyethylene, but the other nuclides were kept in solution with carrier or acid (Harley et al., 1963).

Testing of additional nuclides and various solution/carrier combinations were carried out in subsequent years. These results are shown in Table 7.2, where even ruthenium is kept is solution with both carrier and acid.

\section{REFERENCE}

Harley, J. H., N. A. Hallden and I. M. Fisenne

"Storage of Standardized Radioactive Solutions"

Nature, 197, 1230 (1963) 


\title{
7.11.2 SUPPLIER AND COST
}

The DPTPs are available from several supply houses, including:

\author{
Bio-Rad Chemical Division \\ 2000 Alfred Nobel Drive \\ Hercules, CA 94547 \\ (510) 741-1000 \\ FAX: (510) 741-1060
}

The most useful sizes are sold in boxes of 400 to $500 /$ box at prices ranging from $\$ 18$ to $\$ 30 / \mathrm{box}$. 
TABLE 7.1.

STORAGE CONDITIONS FOR NUCLIDES IN POLYETHYLENE AMPOULES ${ }^{\star}$

\begin{tabular}{|c|c|c|c|c|}
\hline \multirow[b]{2}{*}{ Nuclide } & \multicolumn{4}{|c|}{$\%$ Retained after Rinse } \\
\hline & Carrier & Acid & Water & Acid \\
\hline \multirow[t]{3}{*}{${ }^{137} \mathrm{Cs}$} & - & - & 0 & \\
\hline & $\mathrm{x}$ & - & 0 & \\
\hline & - & $x$ & 0 & \\
\hline \multirow[t]{3}{*}{${ }^{144} \mathrm{Ce}$} & - & - & 5 & 0.6 \\
\hline & $\mathrm{x}$ & - & 0 & \\
\hline & - & $\mathrm{x}$ & 0 & \\
\hline \multirow[t]{3}{*}{${ }^{95} \mathrm{Zr}$} & - & - & 48 & 33 \\
\hline & $\mathrm{x}$ & - & $0^{* *}$ & \\
\hline & - & $\mathrm{x}$ & 1.2 & 0.8 \\
\hline \multirow[t]{3}{*}{${ }^{131} \mathbf{I}$} & - & - & 25 & 25 \\
\hline & $\mathrm{x}$ & - & 17 & 17 \\
\hline & - & $\mathrm{x}$ & 26 & 26 \\
\hline $\begin{array}{l}{ }^{131} \mathrm{I}_{\text {with }} \mathrm{Na}_{2} \mathrm{SO}_{3} \\
\text { at } \mathrm{pH} 8\end{array}$ & & & 0 & \\
\hline \multirow[t]{3}{*}{${ }^{106} \mathrm{Ru}$} & - & - & 35 & 15 \\
\hline & $x$ & - & 25 & 26 \\
\hline & - & $x$ & 29 & 28 \\
\hline \multirow[t]{3}{*}{${ }^{140} \mathrm{Ba}$} & - & - & 0 & \\
\hline & $\mathrm{x}$ & - & 0 & \\
\hline & - & $\mathrm{x}$ & - & \\
\hline
\end{tabular}

"Taken from Harley et al., 1963.

"With either $\mathrm{Zr}$ or Nb or with both as carriers. 
TABLE 7.2

ADDITIONAL NUCLIDES SUCCESSFULLY STORED IN POLYETHYLENE

\begin{tabular}{|c|c|}
\hline Nuclide & Conditions \\
\hline $\begin{array}{l}{ }^{125} \mathrm{Sb} \\
{ }^{241} \mathrm{Am}\end{array}$ & $\begin{array}{l}5 \mu \mathrm{g} \mathrm{Sb} \mathrm{g}^{-1} 4 \underline{\mathrm{N} \mathrm{HCl}} \\
0.1 \underline{\mathrm{N}} \mathrm{HNO}_{3}\end{array}$ \\
\hline $\begin{array}{l}{ }^{7} \mathrm{Be} \\
{ }^{109} \mathrm{Cd}\end{array}$ & $\begin{array}{l}0.1 \underline{\mathrm{N}} \mathrm{HCl} \\
1 \underline{\mathrm{N}} \mathrm{HCl}\end{array}$ \\
\hline $\begin{array}{l}{ }^{45} \mathrm{Ca} \\
{ }^{51} \mathrm{Cr}\end{array}$ & $\begin{array}{l}0.1 \underline{\mathrm{N}} \mathrm{HCl} \\
1 \underline{\mathrm{N}} \mathrm{HCl}\end{array}$ \\
\hline $\begin{array}{l}{ }^{60} \mathrm{Co} \\
{ }^{244} \mathrm{Cm}\end{array}$ & $\begin{array}{l}0.1 \underline{\mathrm{N}} \mathrm{HCl} \\
3 \underline{\mathrm{N}} \mathrm{HCl}\end{array}$ \\
\hline${ }^{198} \mathrm{Au}$ & $4 \underline{\mathrm{N} \mathrm{HCl}}$ \\
\hline${ }^{59} \mathrm{Fe}$ & $0.1 \underline{\mathrm{N}} \mathrm{HCl}$ \\
\hline $\begin{array}{l}{ }^{210} \mathrm{~Pb}+\text { Progeny } \\
{ }^{54} \mathrm{Mn}\end{array}$ & $\begin{array}{l}3 \underline{\mathrm{N}} \mathrm{HNO}_{3} \\
1 \underline{\mathrm{N} \mathrm{HCl}}\end{array}$ \\
\hline${ }^{63} \mathrm{Ni}$ & $\begin{array}{l}0.1 \underline{\mathrm{N}} \mathrm{HCl} \\
0.1 \underline{\mathrm{N}} \mathrm{HCl}\end{array}$ \\
\hline $\begin{array}{l}{ }^{239} \mathrm{Pu} \\
{ }^{210} \mathrm{Po}\end{array}$ & $\begin{array}{l}0.1 \mathrm{NHNO}_{3} \\
1 \underline{N} \mathrm{HNO}_{3}\end{array}$ \\
\hline${ }^{147} \mathrm{Pm}$ & $\begin{array}{l}1 \underline{\mathrm{N} \mathrm{HCl}} \\
0.1 \underline{\mathrm{N}} \mathrm{HCl}\end{array}$ \\
\hline $\begin{array}{l}{ }^{106} \mathrm{Ru} \\
{ }^{22} \mathrm{Na}\end{array}$ & $\begin{array}{l}25 \mu \mathrm{g} \mathrm{Ru} \mathrm{g}^{-1} 1 \underline{\mathrm{N}} \mathrm{HCl} \\
0.1 \underline{\mathrm{N} \mathrm{HCl}}\end{array}$ \\
\hline $\begin{array}{l}{ }^{90} \mathrm{Sr} \\
{ }^{204} \mathrm{Tl}\end{array}$ & $\begin{array}{l}0.1 \underline{\mathrm{NHCl}} \\
1 \underline{\mathrm{NHNO}}\end{array}$ \\
\hline $\begin{array}{l}{ }^{228} \mathrm{Th} \\
{ }^{232} \mathrm{U}\end{array}$ & $\begin{array}{l}3 \underline{\mathrm{N}} \mathrm{HNO}_{3} \\
1 \underline{\mathrm{N}} \mathrm{HCl}\end{array}$ \\
\hline${ }^{88} \mathrm{Y}$ & $\begin{array}{l}0.1 \underline{\mathrm{N}} \mathrm{HCl} \\
0.1 \underline{\mathrm{N}} \mathrm{HCl}\end{array}$ \\
\hline
\end{tabular}




\subsection{FILTER FUNNELS AND SAMPLE MOUNTS}

\subsubsection{DESCRIPTION}

A simple and inexpensive combined filter funnel and sample mount is shown in Figure 7.5. The funnel chimney is molded of polyethylene and may be reused. It is sufficiently inexpensive that it could be discarded after each use, but the smooth surface should not retain any material to cross-contaminate the samples. The nylon funnel base is designed to be used as a sample mounting disc.

In the filtration, a double paper or glass fiber filter is used with a diameter of 23.5-24 mm. A short piece of $3.1750 \mathrm{~mm}$ I.D. plastic tubing is attached to the base tube and the base is put in place on a rubber stopper mounted on a standard filter flask. The rubber stopper can be bored out to about $1 \mathrm{~cm}$. No seal is required between the plastic tubing and the stopper as the suction is maintained between the funnel base and the stopper. After filtration, the funnel chimney is removed and the base mounted as with the standard disc.

\subsubsection{SUPPLIER}

The present source of supply is:

Control Molding Corporation

Smith and Canal Streets

P.O. Box 70

Franklin, NH 03235

(603) $934-6103$ 
This vendor is retaining the molds for EML. They have been instructed to accept orders from any source. They may be ordered as:

1. Polyethylene funnel chimney, and

2. Funnel disc and ring. 


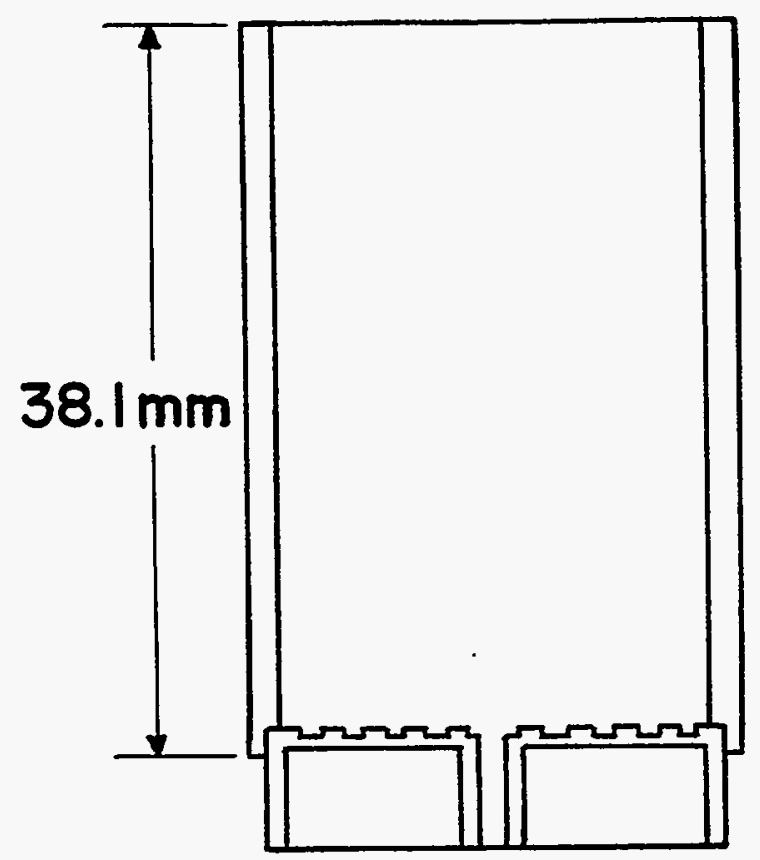

Polyethylene Funnel Chimney

Nylon Sample Mount

Nylon Sample Mount Top View

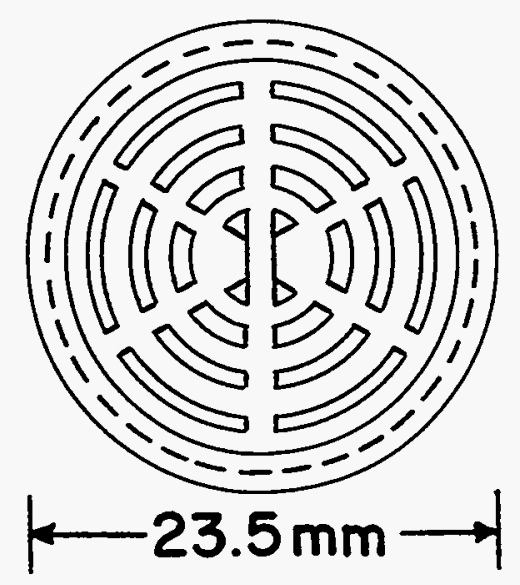

Figure 7.5 Filter funnels and sample mounts. 


\subsection{FHTER STOPPERS}

\subsubsection{DESCRIPTION}

The stopper sketched below is molded from "Geon" which is reasonably inert to common chemicals. It is intended for use in supporting the filter discs described in Section 7.12.

If the filtrate is not to be saved, the stopper may be used as is with a filter flask. If the filtrate is to be saved, a length of thin plastic tubing may be attached to the bottom of the filter disc and passed through the stopper hole for use with a Fisher filtrator.

\subsubsection{SUPPLIER}

These stoppers are available from the supplier who furnishes the filter discs (Control Molding Corporation, see Specification 7.2).

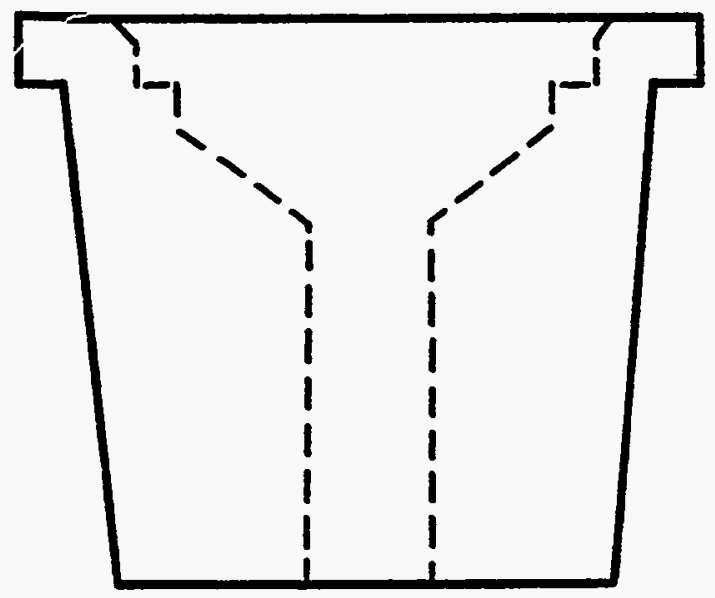

Figure 7.6 Filter stopper, twice full size (corresponds to a No. 6 rubber stopper). 


\subsection{ION-EXCHANGE FALLOUT COLLECTORS}

\subsubsection{DESCRIPTION}

A schematic diagram of the ion exchange fallout collector is shown in Figure 7.7. This unit consists of a funnel, ion-exchange column, leveling device, wooden housing with a clamp, and electric heater (optional). The funnel is welded to a threaded cap which may be attached to the top of the ion exchange column. The bottom of the column is also threaded for a tapered fitting to attach the leveling tube. The device consists of a polyethylene tube extending from the bottom of the column to a $\mathrm{T}$-connector above the height of the paper pulp in the column. This prevents the column from running dry during the collection period. Water flows out of the $\mathrm{T}$-connector and is directed out of the wooden housing through a flexible hose. The tapered fitting and the funnel are replaced with standard bottle caps prior to shipment.

\subsubsection{SUPPLIER}

Aside from the housing unit and electric heater, the various components of the ionexchange fallout collector can be obtained from

$$
\begin{aligned}
& \text { Bel-Art Products } \\
& \text { Pequannock, NJ } 07440 \\
& \text { (201) 694-0500 }
\end{aligned}
$$

Figure 7.8 is a schematic diagram showing the dimensions of the funnel, column, and tapered cap which EML uses.

We at EML assemble all of the components before placing the unit in the field. 


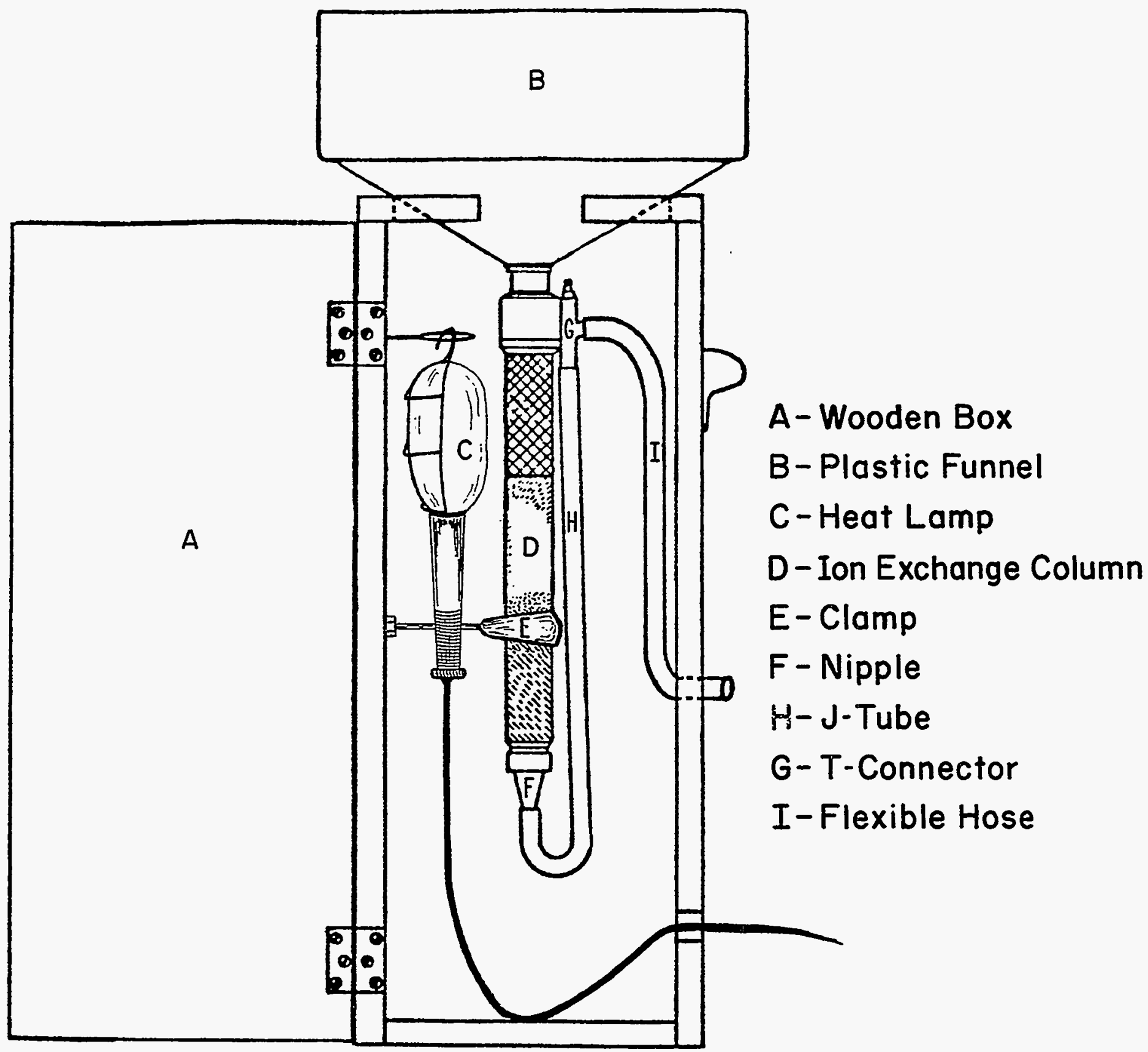

Figure 7.7 Ion-exchange fallout collector unit. 


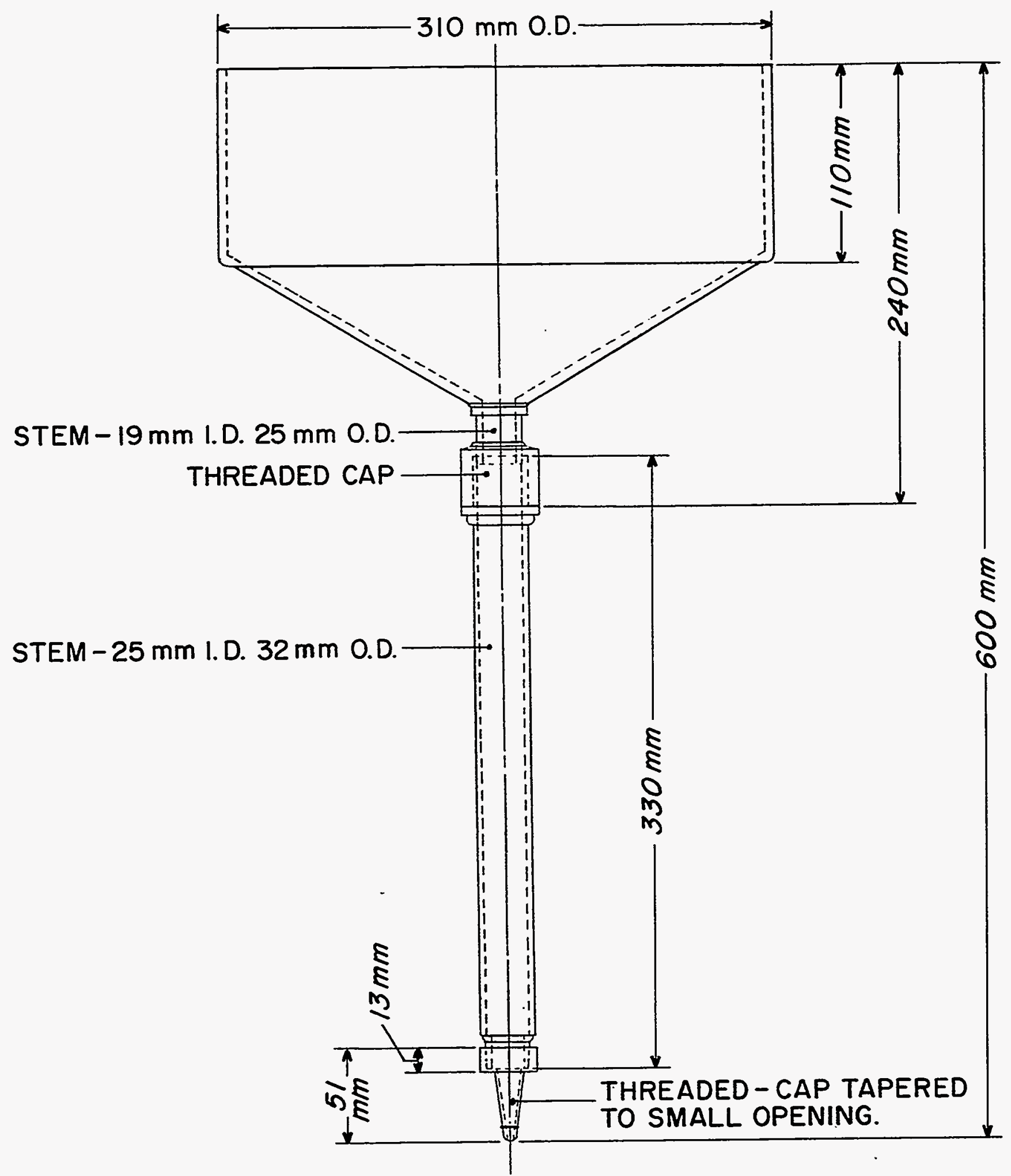

Figure 7.8 Schematic diagram of funnel, column, and tapered cap. 


\subsection{ELECTROLYSIS ELECTRODE}

\subsubsection{DESCRIPTION}

The platinum electrolysis electrode is used in the electrodeposition procedure (see Figure 7.9). It is attached to a rotary motor and intended for use in the plating cell described in Specification 7.16. It is separated from the platinum electrodeposition disk by $1 \mathrm{~cm}$.

\subsubsection{SUPPLIER}

Johnson-Matthey, Inc.

2001 Nolte Drive

West Deptford, NJ 08066

(609) 853-8000 


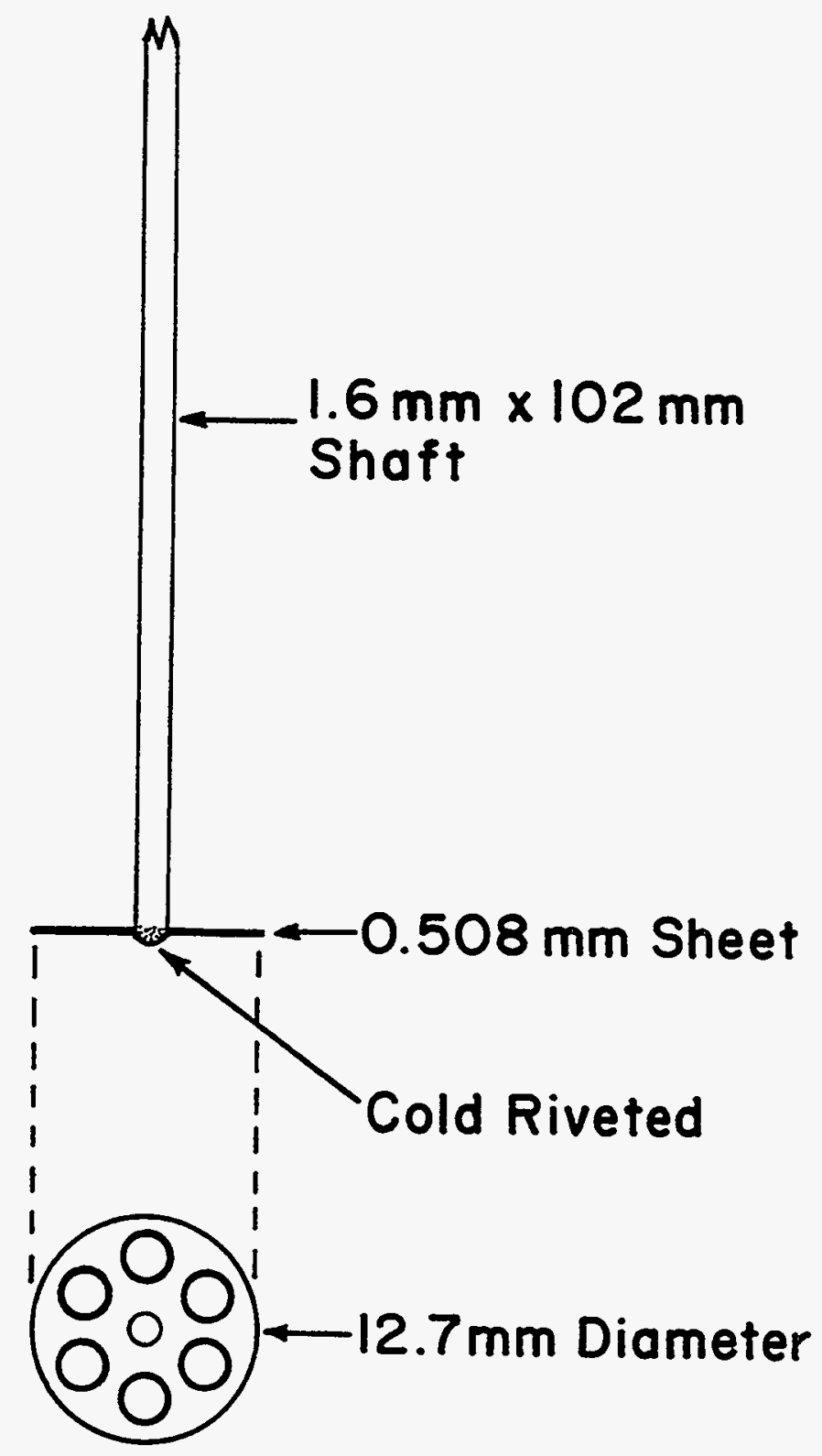

Figure 7.9 Platinum anode for electrolysis (drawn twice actual size). 


\subsection{PLATING CELLS}

\subsubsection{DESCRIPTION}

The plating cell used at EML for electroplating on platinum discs is based on numerous other designs, chiefly the one used at the Laboratory of Radiation Biology, University of Washington (see Figure 7.10).
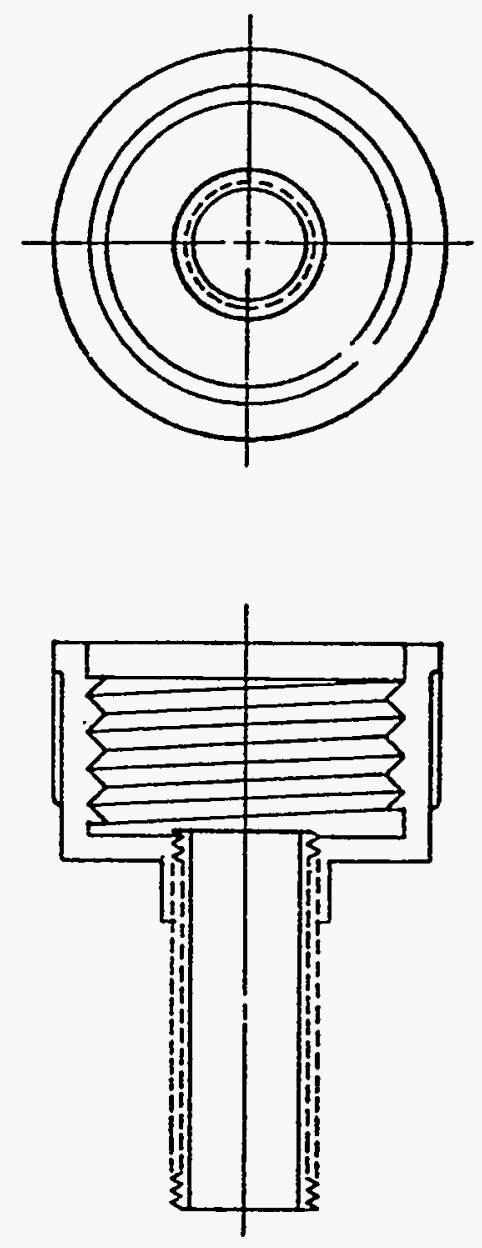

Figure 7.10 Plating cell. 
The major feature of the cell is an elongated 20 -mm cap for either a $30 \mathrm{~mL}(1 \mathrm{oz})$ or a $60 \mathrm{~mL}(2 \mathrm{oz})$ polyethylene bottle. This cap has space for an $18-\mathrm{mm}$ diameter plating disc and has a threaded brass bushing for making electrical connection.

In use, the bottom is cut off either a $30-\mathrm{mL}$ or $60-\mathrm{mL}$ bottle (20-mm cap size), and any flashing or other roughness on the top of the bottle is smoothed by rubbing once or twice over a piece of sandpaper if necessary. The plating disc is placed on the cap and the bottle is screwed in firmly.

Electrical connection may be made by a clip, or by threading the bushing into a metal plate which then also acts as a support for the cell.

\subsubsection{SUPPLIER}

Control Molding Corporation ${ }^{*}$

Smith and Canal Streets

P.O. Box 70

Franklin, NH 03235

(603) 934-6103

"See Specification 7.2.3. 


\subsection{AIR SAMPLING FILTERS}

\subsubsection{DYNAWEB DW7301L FILTERS}

Since the end of 1992, all sites in SASP and RAMP have been supplied with Dynaweb filters. The filter is composed of a $100 \%$ polypropylene web that is $100 \%$ binderless. Three layers of this web are collated and sandwiched between two sheets of a protective DuPont Reeme ( $100 \%$ polyester) scrim. The top scrim is removed prior to sampling at RAMP sites because after their return to EML for analysis these samples are compressed into a pellet and the scrim hinders compression. At all other sites, the filter can be used in sampling with both the top and bottom scrim in place. The filter medium weighs about $20 \mathrm{mg} \mathrm{cm}^{-2}$, has an ash weight ranging from $0.1-1 \%$ of the total weight, and about $65 \mathrm{~cm}^{2}$ of the filter can be compressed to $1.3 \mathrm{~cm}^{3}$ at 5 tons pressure. Radiochemical analyses of blank filters for ${ }^{90} \mathrm{Sr},{ }^{238} \mathrm{Pu}$, and ${ }^{239} \mathrm{Pu}$ indicate values that are near or below the lower limits of detection for these analyses. Gamma-ray spectrometry on blank filters indicates the absence of the gamma-ray emitting isotopes that we routinely report.

The collection efficiency of the Dynaweb filter media was determined using electrically classified monodisperse aerosols of dioctyl sebacate (DOS) liquid and sodium chloride $(\mathrm{NaCl})$ solid particles. The test aerosol particle sizes and face velocities ranged from 0.015 to $0.5 \mu \mathrm{m}$ at 30 and $100 \mathrm{~cm} \mathrm{sec}^{-1} \mathrm{NaCl}$, and from 0.05 to $0.5 \mu \mathrm{m}$ at 50 to $100 \mathrm{~cm} \mathrm{sec}^{-1}$ for DOS. The submicron particle penetration data are summarized in Figure 7.11. The most penetrating particle size is in the 0.07 to $0.1 \mu \mathrm{m}$ range. The minimum collection efficiency, using a test aerosol of $\mathrm{NaCl}$ at a face velocity in the vicinity of 100 $\mathrm{cm} \mathrm{sec}^{-1}$ was about $87 \%$. The average pressure drops at face velocities of 50 and $100 \mathrm{~cm}$ were 2.26 and $3.92 \mathrm{kPa}$, respectively.

Filter intercomparisons are conducted using Microdon LM2020 and Dynaweb DW7301L materials at EML's Regional Baseline Station at Chester, NJ, and in Barbados by the University of Miami. The filter samples collected at Chester, NJ, were measured for ${ }^{7} \mathrm{Be}$ and ${ }^{210} \mathrm{~Pb}$, while those from Barbados were measured for dust loading, sodium, chloride, nitrate, and non-sea salt sulfate. Statistical t-tests and Sign tests were performed on the paired data to determine if the measured differences were significant. At the $95 \%$ probability level, in all but one case $\left({ }^{210} \mathrm{~Pb}\right)$, no t-test or Sign test indicated a significant difference between the two filters. 
Dynaweb DW7301L is manufactured and distributed by:

\section{WEB Dynamics}

1 Forge Road

East Stroudsburg, PA 18301 


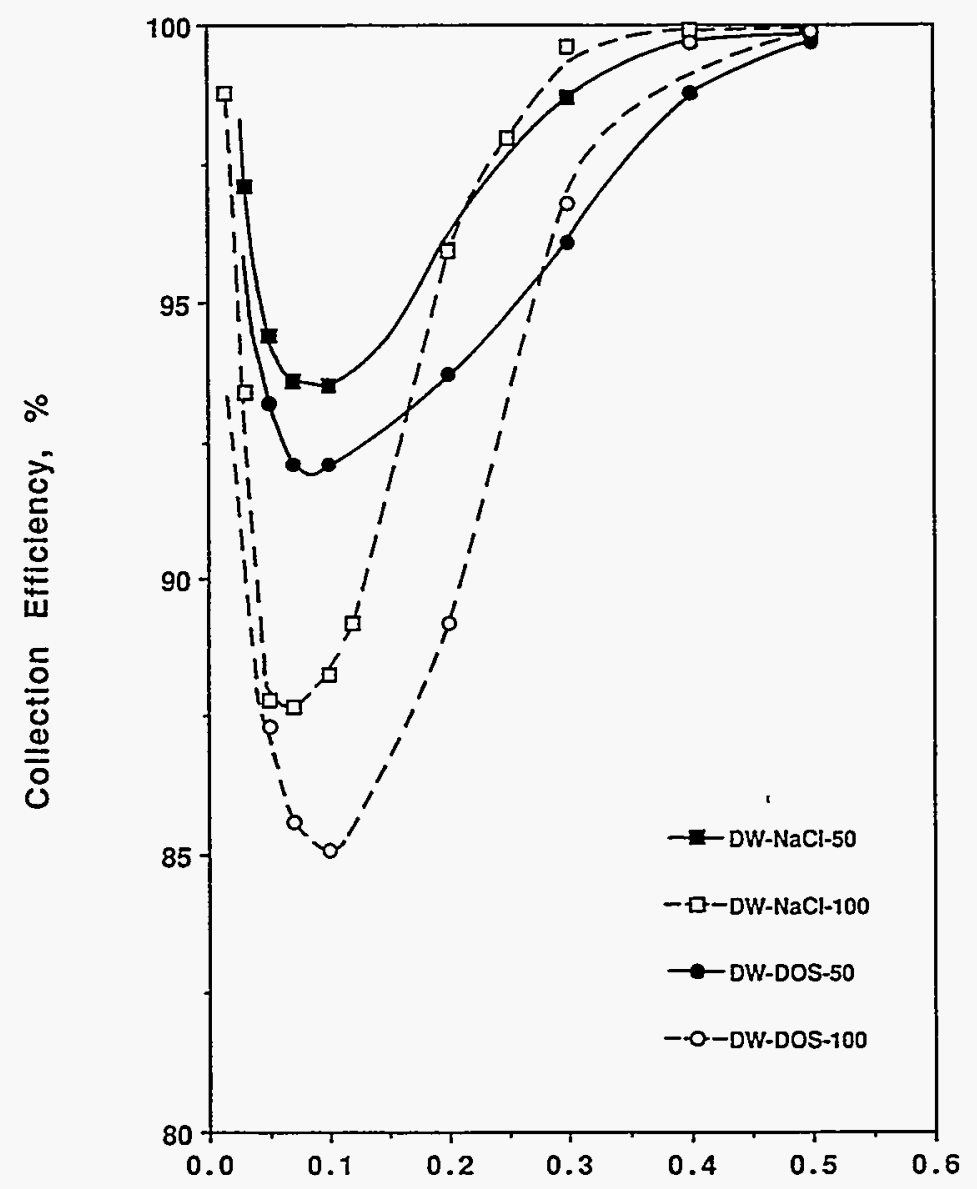

Particle Size, $\mu \mathrm{m}$

Figure 7.11 Submicron particle penetration data. 


\section{REFERENCES}

Cambray, R. S. and K. Playford

Unpublished data

AERE Harwell, Oxfordshire, England, December (1985)

Lockhart, L., R. Patterson and W. Anderson

"Characteristics of Air Filter Media Used for Monitoring Airborne Radioactivity"

Naval Research Laboratory Report NRL-6054, June (1964) 


\subsection{AIR SAMPLERS}

\subsubsection{ROOTS SYSTEM}

The SASP sites are equipped with a Roots Rotary Lobe Blower Type 24 AF connected to a $1 \mathrm{HP}$ electric motor by a fan belt. Roots lobe pumps fall into a category of pumps where volumetric displacement is the means by which flow is induced. Lobe pumps use two counter-rotating impellers to provide flow. As each impeller passes over the inlet, a volume of air is trapped, carried through the blower to the discharge and is expelled against the discharge pressure. Figure 7.12 shows a sketch of the Roots pump and motor. Figures 7.13 and 7.14 show a detailed and field view of the Roots system. The Roots rotary is manufactured by:

Dresser Industries Incorporated

Roots Blower and Vacuum Pump Division

Connersville, $\mathbb{N} 47331$

The local distributor is:

Hayes Machine Co.

11-03 43rd Road

Long Island City, NY 11101

The electric motor which is used to drive the blower is purchased from:

Harry Lumb and Associates, Inc.

39 Golden Star

Irvine, CA 92714 
The specifications on the electric motor are:

$$
\begin{aligned}
& \text { 60/50 Hz, } 115 / 230 \mathrm{~V}, 15 / 7.5 \text { Amps } \\
& \text { Single Phase, } 1 \mathrm{HP}, 1725 \mathrm{RPM} \\
& \text { TEFC, Frame ELX-182T, Design B } \\
& \text { Code J, Ambient } 40^{\circ} \mathrm{C} \text {, Insulation Class B } \\
& \text { SF1.2, Continuous Duty }
\end{aligned}
$$

The Roots system, composed of blower, motor, frame, plumbing, and accessories costs $\sim \$ 2500$ including labor. The wooden shelter and metal stand both cost about $\$ 500$ each.

\subsubsection{FUJ SYSTEM}

The RAMP sites are equipped with a Fuji ring compressor Model 302P which is directly connected to a $0.5 \mathrm{HP}$ electric motor. Fuji ring compressors induce flow through momentum transfer. Air enters through an inlet port and is accelerated by the impeller. Through centrifugal force, energy is imparted to the inlet air which is discharged out of the pump outlet. Figure 7.15 presents a sketch of the Fuji system. Figures 7.16 and 7.17 show a detailed and field view of the Fuji system. The Fuji Ring Compressors are manufactured by:

Fuji Electric Corp. of America

Frassetto Industrial Park

6A Frassetto Way

Lincoln Park, NJ 07035

These compressors are distributed by:

The Knotts Company, Inc.

350 Snyder Avenue

Berkeley Heights, NJ 07922 
The Fuji Compressor motor has the following specifications:

$50 / 60 \mathrm{~Hz}, 115 / 230 \mathrm{~V}$

5.0/2.5 Amp, Single Phase

$0.51 \mathrm{HP}$

The Fuji system containing the ring compressor, aluminum housing, sampling manifold and accessory equipment is purchased from:

Dr. Joseph Prospero

University of Miami

Rosenstiel School of Marine and Atmospheric Science

Miami, FL 33181

The cost is $\sim \$ 2500$ per system. 
THERMAL OVERLOAD

SWITCH TO MAIN

POWER SUPPLY

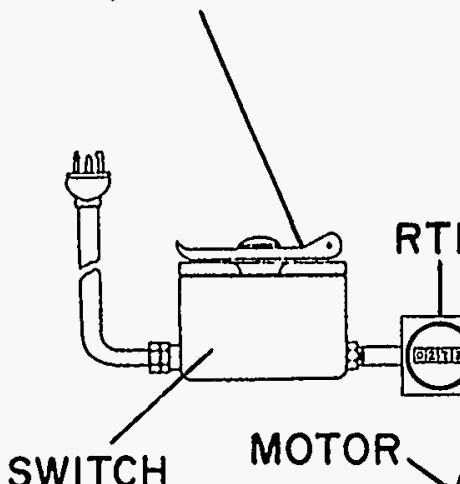

SWITCH

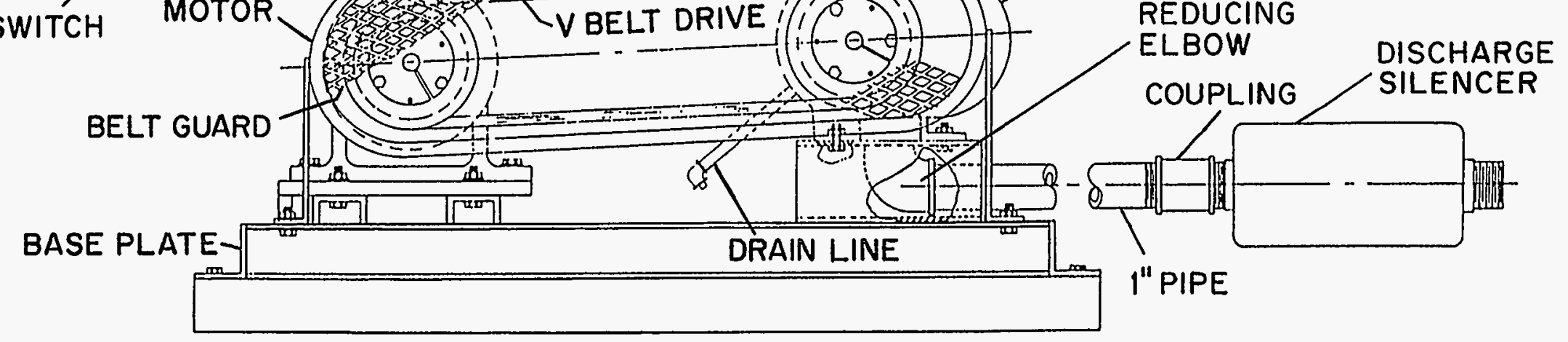

Figure 7.12 Sketch of the Roots system.

MAGNEHELIC

RELIEF VALVE CONDUIT BOX



PUMP<smiles>[Mg][Te]</smiles>

REDUCING

ELBOW DISCHARGE NIPPLE


CLAMPS (6) 


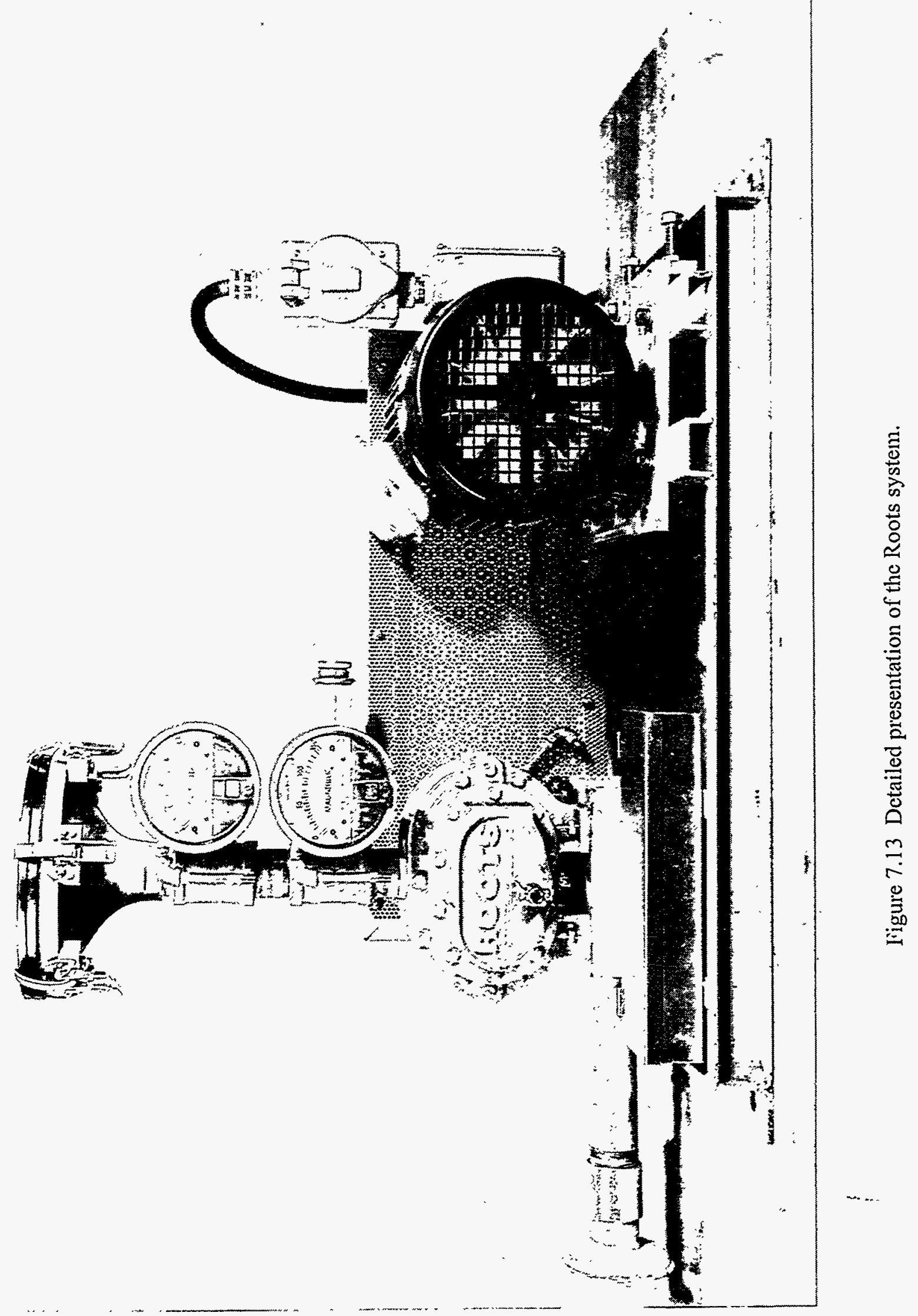




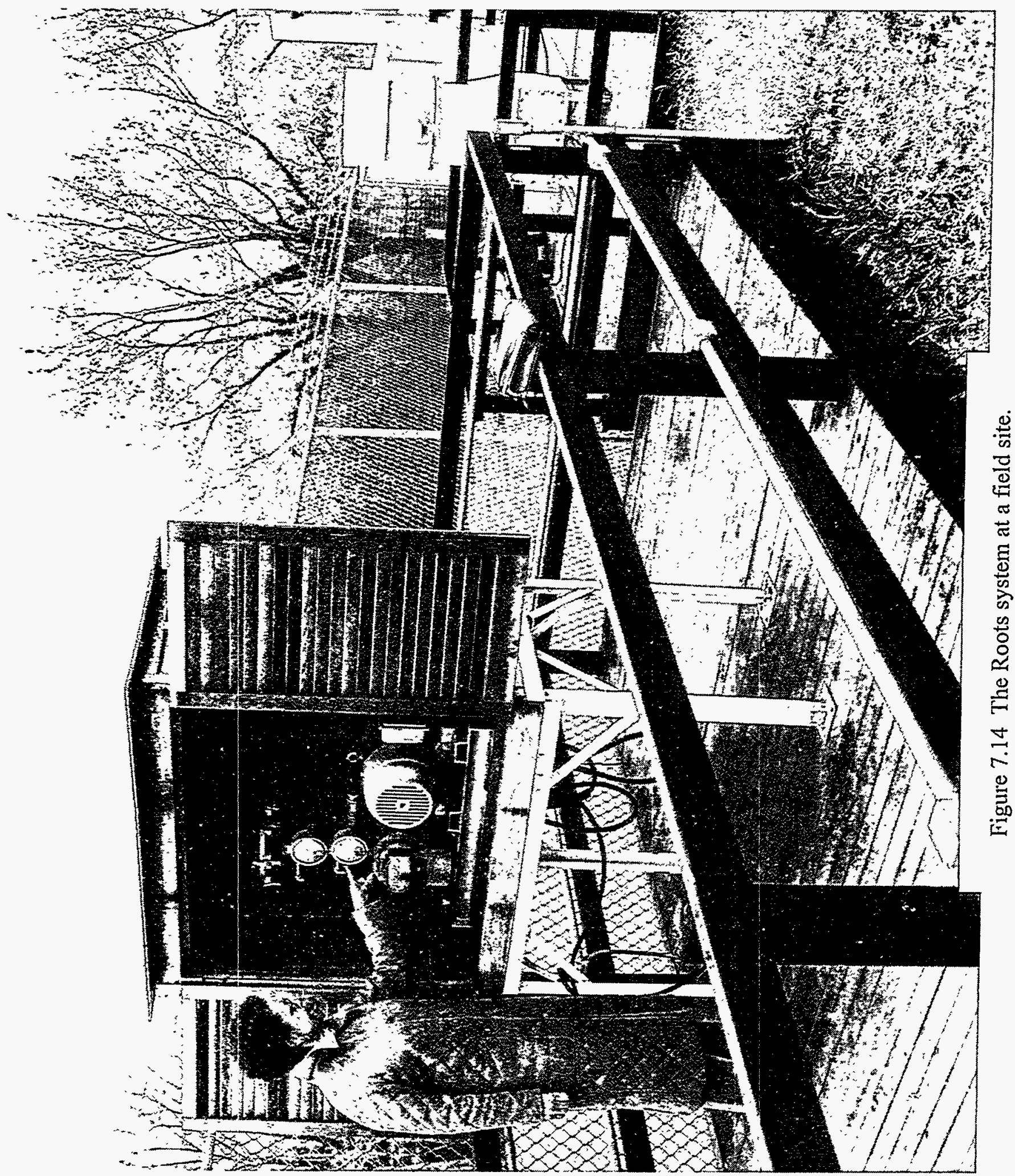




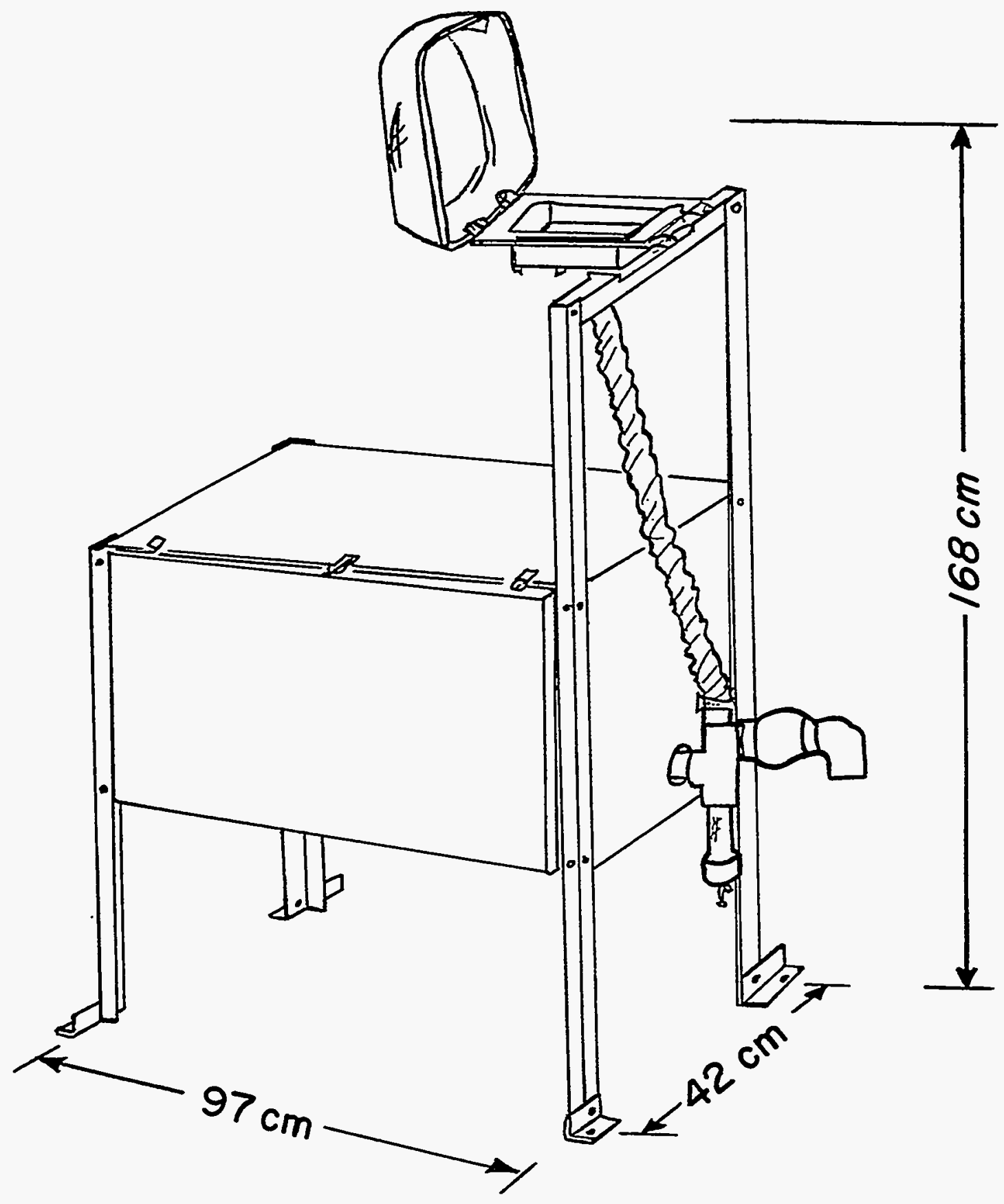

Figure 7.15 Sketch of the Fuji system. 







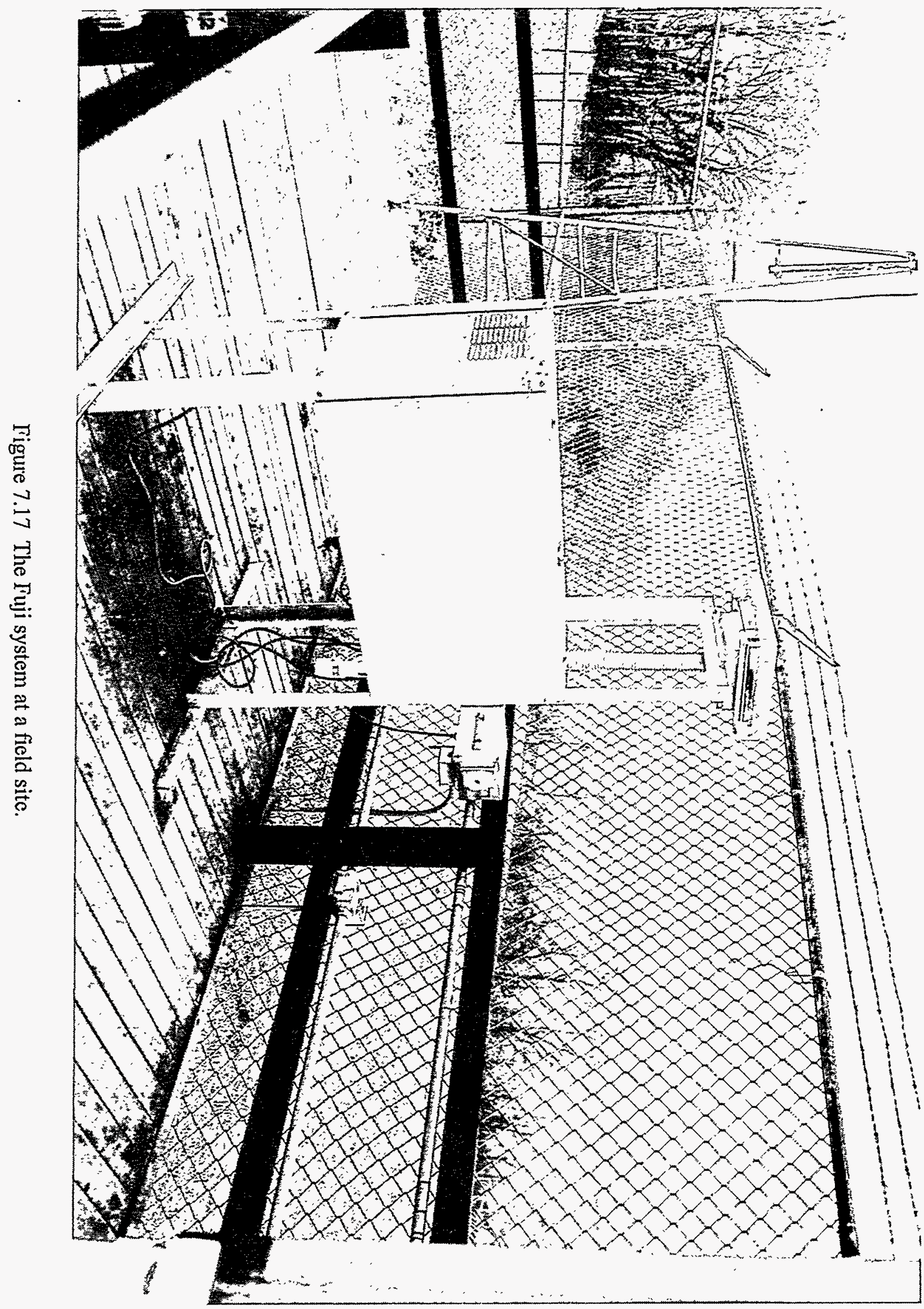




\subsection{GAMMA PLANCHETS}

\subsubsection{DESCRIPTION}

This is a planchet molded of nylon. The inside diameter is $50.8 \mathrm{~mm}$, the depth is 25.4 $\mathrm{mm}$, and the mean wall thickness is $0.64 \mathrm{~mm}$. A flat circular cover is also provided.

These planchets are used to provide a uniform geometry for gamma counting air particulate filter samples and other similar materials. The filters are pressed into the planchet with a hand-operated hydraulic press using a steel die. When used in this way, a $6.35-\mathrm{mm}$ hole is cut in the bottom of the planchet to allow the filter to be punched out for radiochemical analyses after counting.

\subsubsection{SUPPLIER}

The planchets are available from:

Control Molding Corporation ${ }^{*}$

Smith and Canal Streets

P.O. Box 70

Franklin, NH 03235

(603) $934-6103$

"See Specification 7.2.3. 


\subsection{ELECTROLYSIS CELLS ${ }^{*}$}

\subsubsection{DESCRIPTION}

A. This electrolysis cell was developed at New York University for the electrolytic enrichment of tritium in acid solution. The electrodes were subsequently modified at EML by replacing a coiled platinum wire with platinum sheet.

The cells that were used at EML were made by a commercial glass blower from the drawing shown in Figure 7.18, using standard glass tubing and standard taper joints.

B. This electrolysis cell (Figure 7.19) was developed at the University of Miami by Dr. Gote Ostlund for the electrolytic enrichment of tritium in alkaline solution. The cell is also used for the final distillation of the enriched sample.

For distillation, an adapter and receiver were substituted for the funnel top.

No longer used at EML. 




Figure 7.18 Electrolysis cell. 


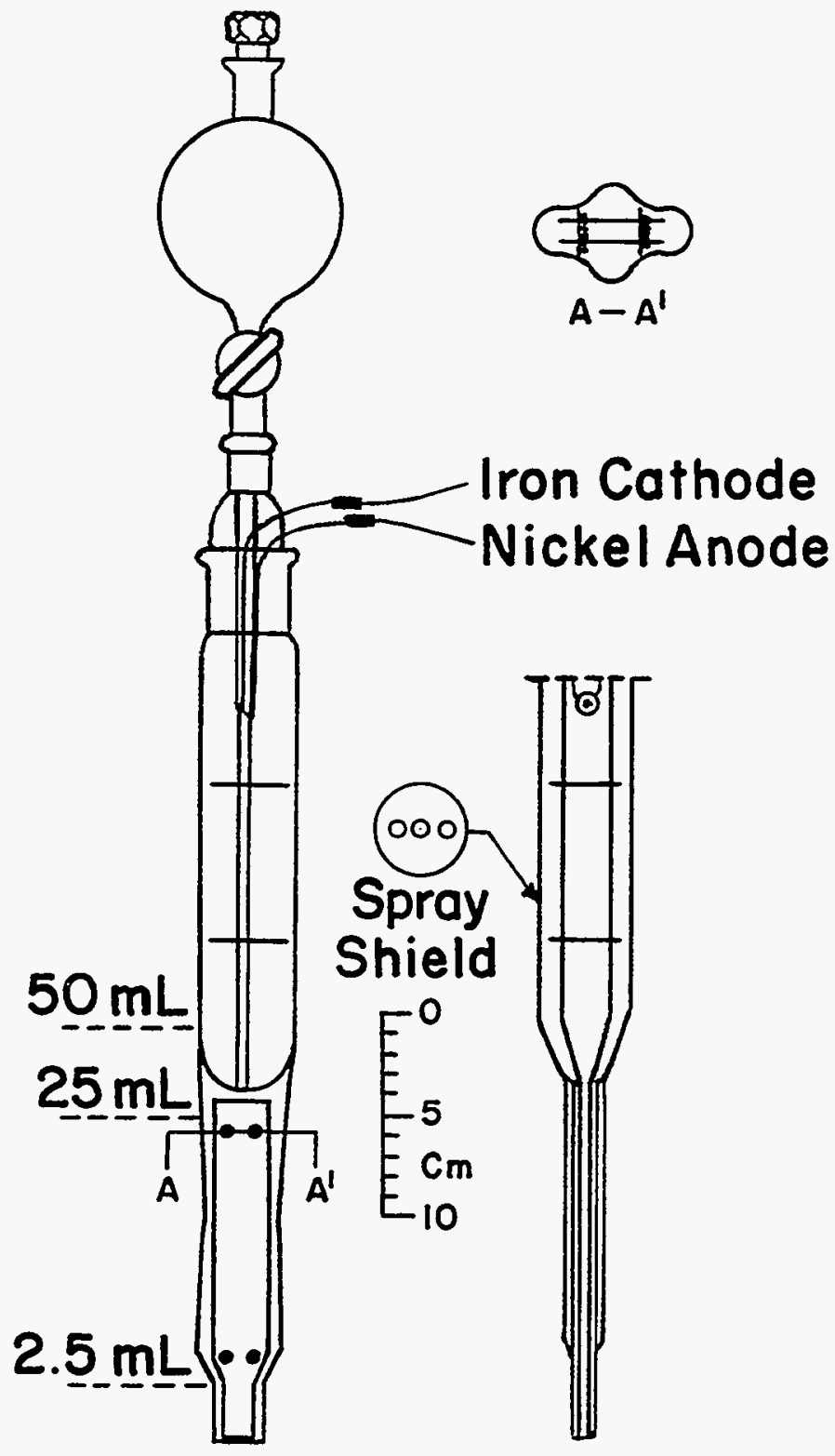

Figure 7.19 Alkaline electrolysis cell. 


\subsection{ALUMINUM SAMPLE CANS FOR GAMMA COUNTING}

\subsubsection{DESCRIPTION}

The sample containers for gamma counting and gamma spectrometry measurements are drawn, straight-walled, "step shoulder" aluminum can bodies and accompanying "full panel pull out" lids. The body and lid are fabricated from $.254 \mathrm{~mm}$ and $.305 \mathrm{~mm}$ thickness, respectively, of various alloy-temper combinations of aluminum, and their inside surfaces are enameled.

One size is in current use. It holds about $100 \mathrm{~mL}$ and is about $60.325 \mathrm{~mm}$ by $31.8 \mathrm{~mm}$. It is described as:

$208 \times 107$ can body

209.5 lid

\subsubsection{SUPPLIER}

They are available from:



The device for sealing the lid to the can is the Automatic Portable Electric Can Sealer, No. EL-12253-120V available from:

Wisconsin Aluminum Foundry Co., Inc.

P.O. Box 246

838 South 16th Street

Manitowac, WI 54220

(414) 682-8627

FAX: (414) 682-4090 


\subsection{MOLDED MARINELLI BEAKERS}

\subsubsection{DESCRIPTION}

The annular sample containers for gamma counting and gamma spectrometry of soil samples are molded from black Butyrate (see Figure 7.20). They are fitted with a snap cap and the wall thickness is $\sim 0.18 \mathrm{~cm}$. The capacity of the beaker is about $600 \mathrm{~mL}$.

\subsubsection{SUPPLIER}

They are available from:

$$
\begin{aligned}
& \text { Control Molding Corporation } \\
& \text { Smith and Canal Streets } \\
& \text { P.O. Box } 70 \\
& \text { Franklin, NH } 03235 \\
& \text { (603) 934-6103 }
\end{aligned}
$$

in lots of 100 or more.

Similar molded sample containers with $1 \mathrm{~L}$ and $4 \mathrm{~L}$ capacities (see Figure 7.21), having a $7.6 \mathrm{~cm}$ deep well to fit standard $7.6 \mathrm{~cm}$ x $7.6 \mathrm{~cm}$ NaI crystals are available from:

Ga-Ma and Associates, Inc.

P.O. Box 522115

Miami, FL 33152-2115

(305) 888-0383

in lots of 25 or more.

These containers were specifically designed to fit the current $\mathrm{Ge}(\mathrm{Li})$ detectors. They will also fit some integral line $\mathrm{NaI}(\mathrm{T} 1)$ detectors, but will not fit most of the standard crystals.

"See Specification 7.2.3. 


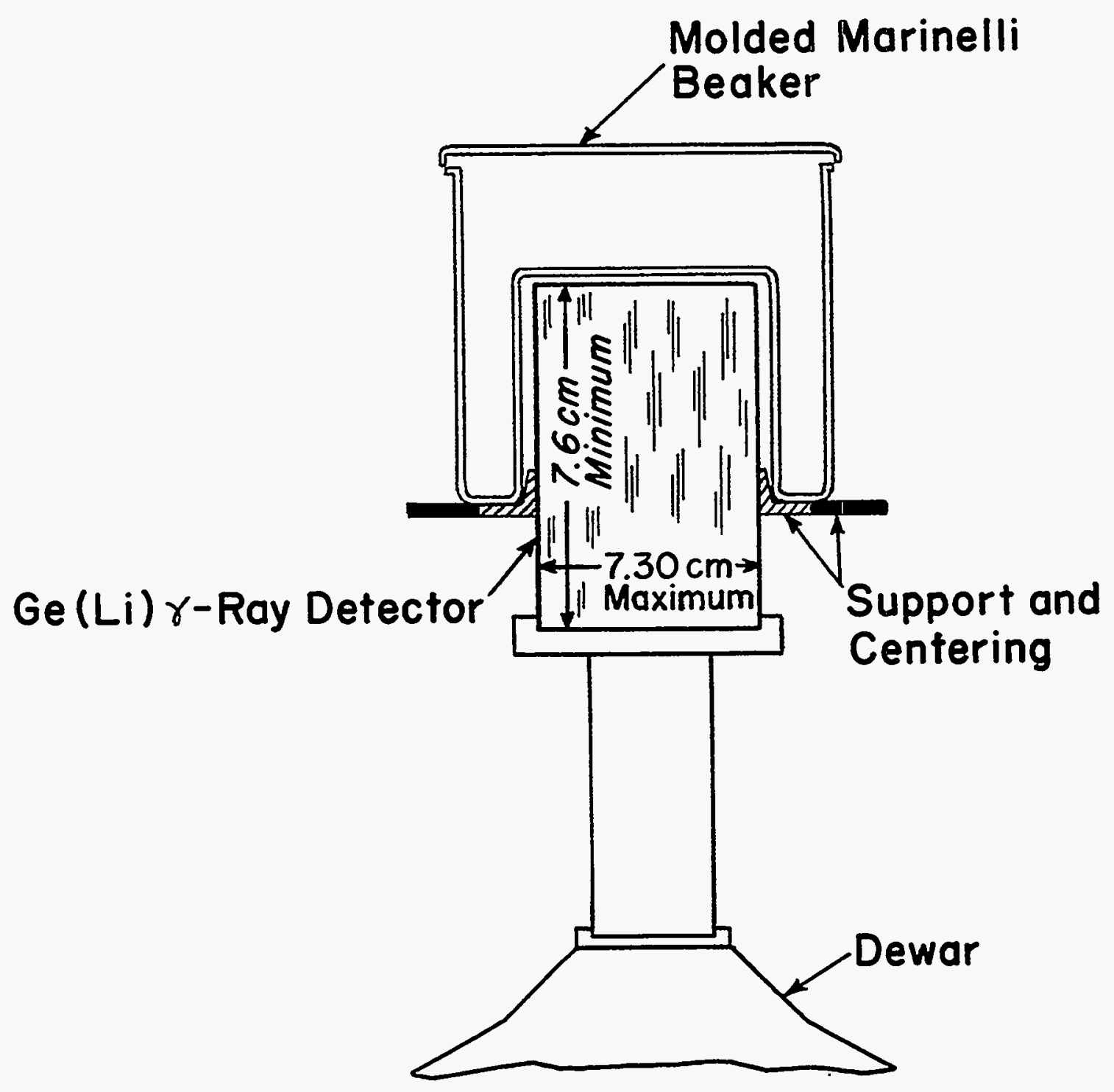

Figure 7.20 Molded Marinelli beaker. 


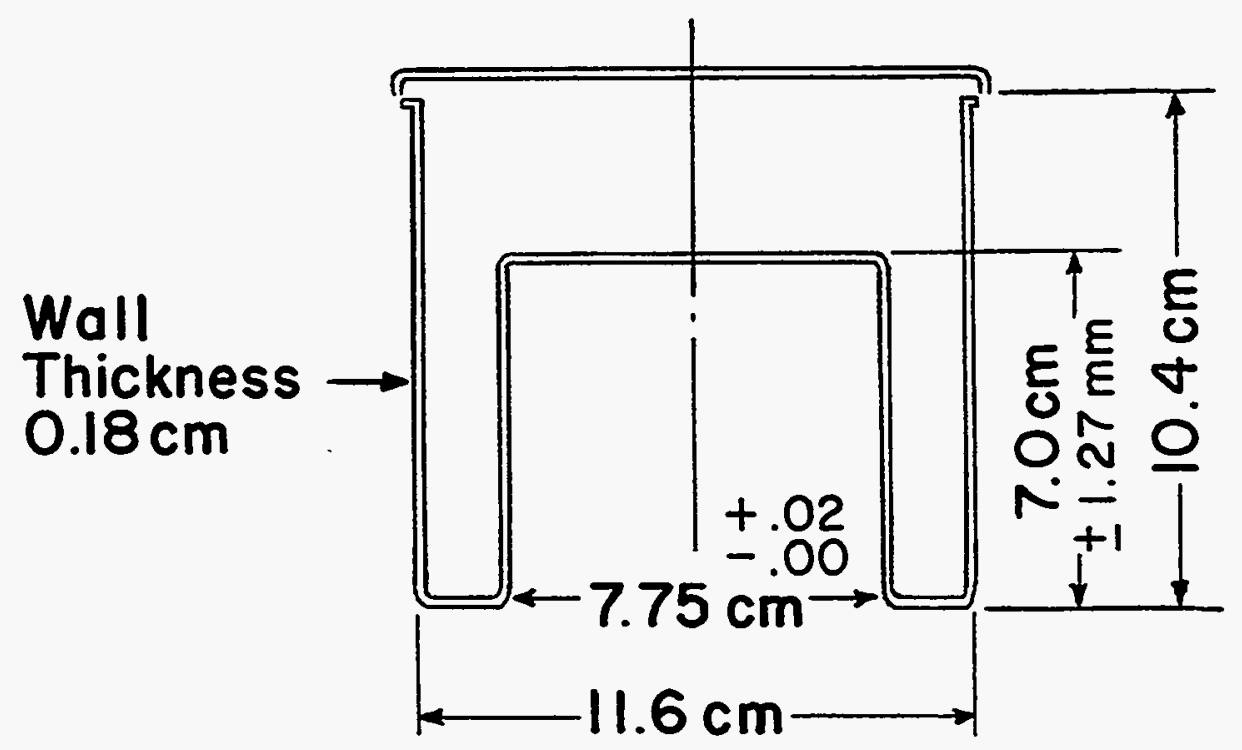

Figure 7.21 Molded Marinelli beaker. 


\subsection{RADON SAMPLING AND COUNTING FLASKS}

\subsubsection{DESCRIPTION}

Scintillation flasks are made up of methyl-methacrylate tubing and sheet. They are fitted with simple valves for flow-through samplings. The bottoms of the flasks are cemented in, while the tops slide in with O-ring seals. They range in size from 165-2.0 L. A schematic drawing and photograph of the 415-mL scintillation flask are shown in Figures 7.22 and 7.23.

\subsubsection{COATING FLASKS}

\section{$\underline{\text { Materials }}$}

1. DuPont 1101 silver-activated zinc sulfide phosphor.

2. Bonding solution (caution - flammable). Dissolve $30 \mathrm{~mL}$ of Dow Corning 200 silicone fluid $(200,000 \mathrm{cs})$ in $285 \mathrm{~mL}$ of reagent benzene plus $285 \mathrm{~mL}$ of reagent cyclohexane. This is enough for about 100 flasks.

\section{Procedure (Use a Hood)}

1. Clean the flask with a nonabrasive cleaner. A plastic cleaning foam (Ren RP-70 cleaner) has been found to be excellent.

2. Remove cover, add $50 \mathrm{~mL}$ of the bonding solution, close both valves, replace cover and rotate the flask slowly until all surfaces are coated. Open valves and remove cover.

3. Pour out bonding mixture into a second clean flask or return to the stock bottle. Air dry the flask for a few minutes.

4. Add 15-20 g of phosphor, close both valves and replace cover. 
5. Shake gently until all surfaces are coated. Open valves and remove cover.

6. Pour the loose powder into a second flask or a clean container. Blow off excess phosphor from flask.

7. Replace cover and purge the flask with aged air or nitrogen for a few minutes. Close the valves and store the flask for use.

8. When background becomes excessive through use, wipe out phosphor with tissues, clean, and rephosphor. 




Figure 7.22 Schematic drawing of scintillation flask. 


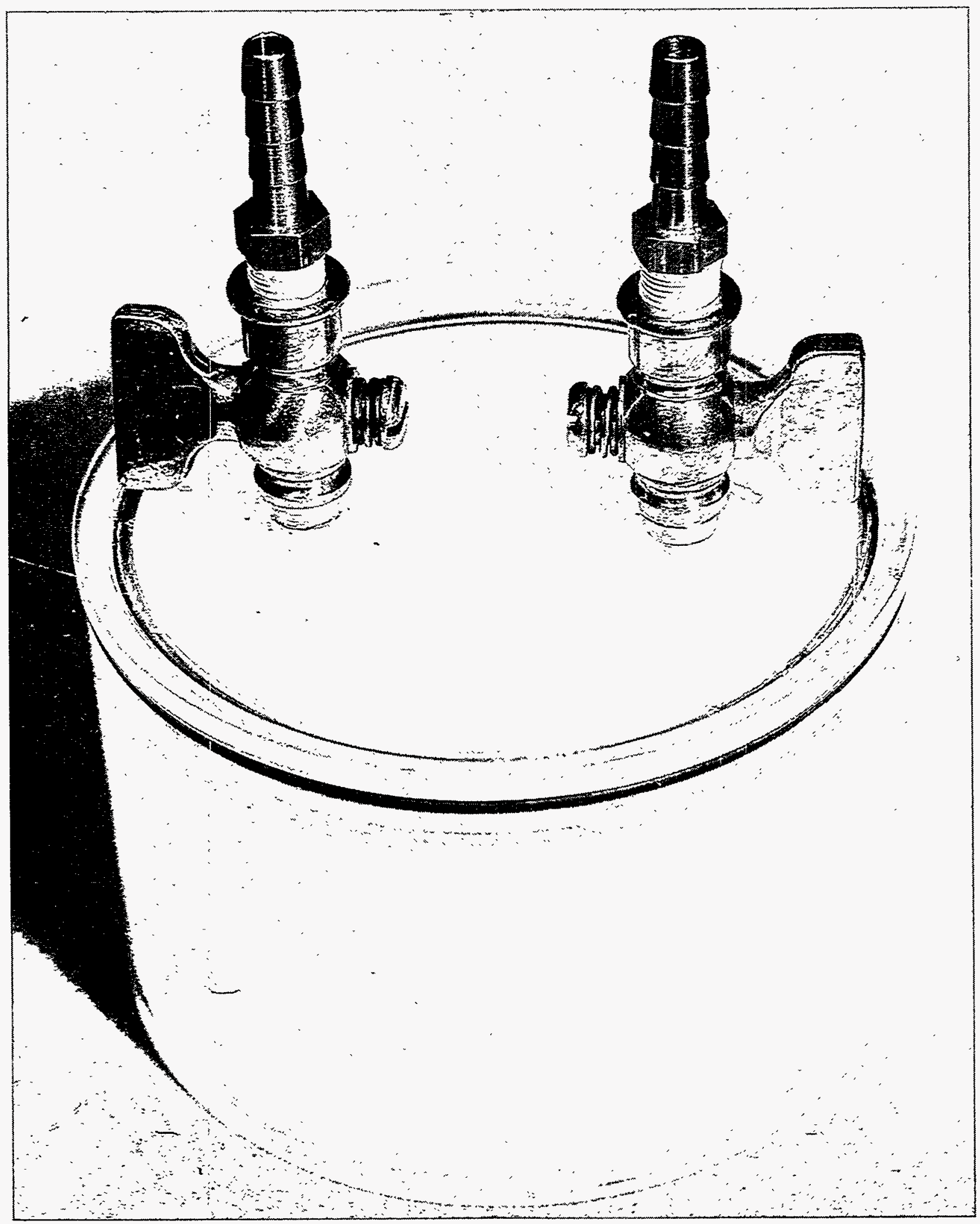

Figure 7.23 Photograph of scintillation flask. 


\subsection{CONTAINERS FOR THE COLLECTION OF FALLOUT AND ATMOSPHERIC DEPOSITION}

\subsubsection{DESCRIPTION}

Samples of atmospheric deposition that are obtained using a wet/dry collector (see Section 2.3.4) and that are to be measured for radioactive fallout or for the chemical composition of the deposited material are usually collected using a polyethylene bucket of the type that has been used by the National Atmospheric Deposition Program/National Trends Network (NADP/NTN).

These ( 3.5 gallon) buckets, which will hold about $13 \mathrm{~kg}$ of water, are constructed of high density polyethylene. The ears for the wire bail are integrally molded near the top of the container. The bucket is molded in one piece and with two reinforcing rings around the outside diameter above the ears to provide additional container strength. The body of the bucket is tapered to permit one pail to nest inside another for economical shipping and storage of empty buckets. The inner rim of the plastic cover interlocks with the upper rim of the bucket. An O-ring seal in a groove just inside of the rim of the cover presses down against the bucket rim and prevents leakage after the cover has been hammered into place, using a rubber mallet. The covers must be discarded after one use because their rims need to be cut in order to remove them from the buckets. The buckets may be cleaned and reused for most purposes. We obtain the buckets and covers from:

Southcorp Packaging Bennett Industries

Peotone, Il 60468

(708) 258-3211

We use custom made cubic fiber boxes for mailing the buckets to and from distant sampling sites. For mailing the boxes, we use a reversible, plastic covered address card, with the site address on one side and our address on the other side. We commonly glue a return address label to the box within the frame that holds the reversible address label so that the box can be returned to us even if the reversible label is lost during shipment. 
There are a few problems that have been encountered in the use of these sample buckets. When the buckets are cleaned routinely for reuse, some contamination may remain in them. The cleaning process has to be designed and monitored to prevent cross contamination of samples. When the rims of the lids are cut to facilitate their removal from the buckets, care must be taken not to cut the surface of the bucket also, if the buckets are to be reused. During the NADP/NTN it has been shown that the O-rings are a potential source of contamination even if they have been cleaned before use. If the buckets are upside down or lying on their sides for some period during shipment, the water sample may dissolve small amounts of sulfate and other materials from the O-rings. For most uses to which the buckets may be put, this should not be a major problem. 


\title{
7.25 FALLOUT COLLECTION AND SHIPPING CONTAINER
}

\subsubsection{DESCRIPTION}

The sampling containers for the EML wet/dry collectors are polyethylene pails (see Section 2.3.4) particularly well suited for shipping because of the design of the lip and cover. On the underside rim of the cover there is a groove with an O-ring seal. When the cover is securely hammered onto the pot with a rubber mallet, no leakage will occur. In most cases when the cover is removed back at the laboratory, it is destroyed.

The samples are shipped in fiber mailing boxes.

\subsubsection{SUPPLIER}

They are available from:

\author{
FIBERBILT \\ 601 West 26th Street, 15th Floor \\ New York, NY 10001 \\ (212) 675-5820
}




\subsection{DISPOSABLE CHROMATOGRAPHIC COLUMN}

\subsubsection{DESCRIPTION}

The column is prepared from a disposable polyethylene transfer pipette. The pipette used here (Bio-Rad Cat \#223-9527, Style EE) has a working capacity of $\sim 4.5 \mathrm{~mL}$ and a barrel $\sim 100 \mathrm{~mm}$ long and $6.5 \mathrm{~mL}$ O.D.

\subsubsection{SUPPLIER}

Bio-Rad Laboratories

2000 Alfred Nobel Drive

Hercules, CA 94547

(510) $741-1000$

FAX: (510) 741-1060 